Gauss, Carl Friedrich

\title{
Disquisitiones arithmeticae
}

Fleischer

Lipsiae

1801 

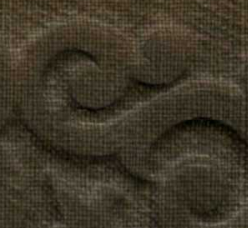

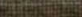

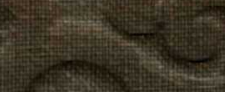

$x^{4} \rightarrow$

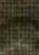

\section{${ }^{4}+y^{2}$}

$46+2+2=$

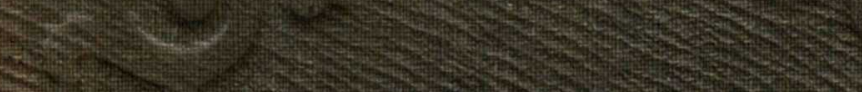

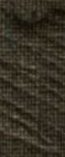

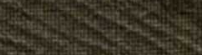

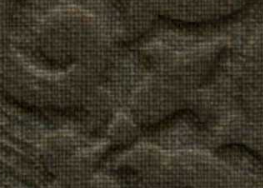

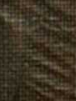

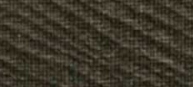

\section{$+\sin ^{2}$}

Thto

(1)

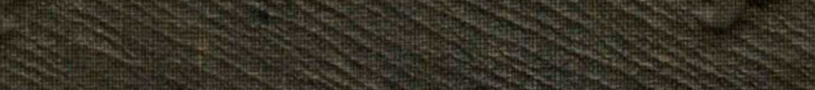

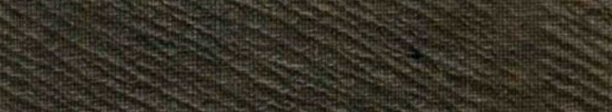

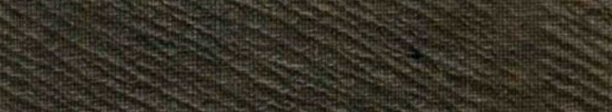

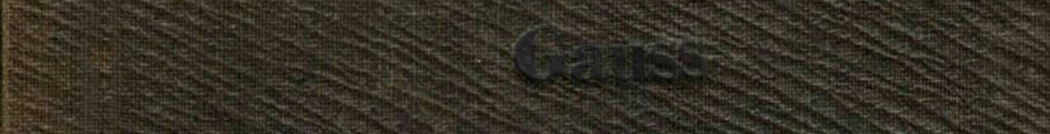

Whas

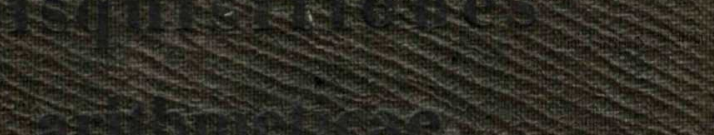

Whe

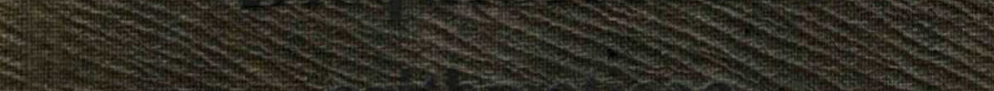

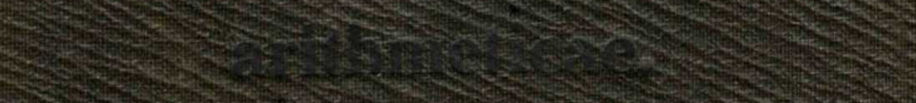

sing
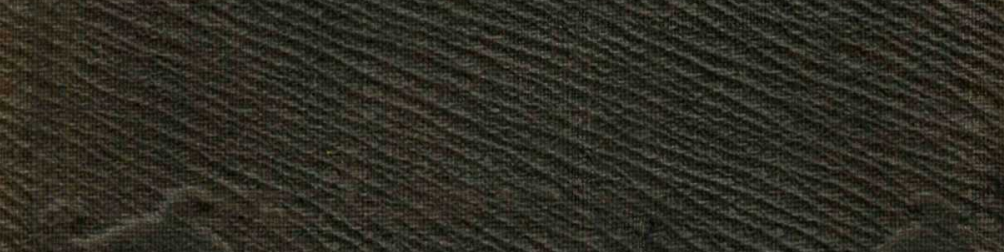

$\frac{d y}{5}$

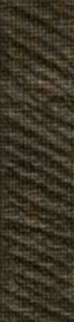




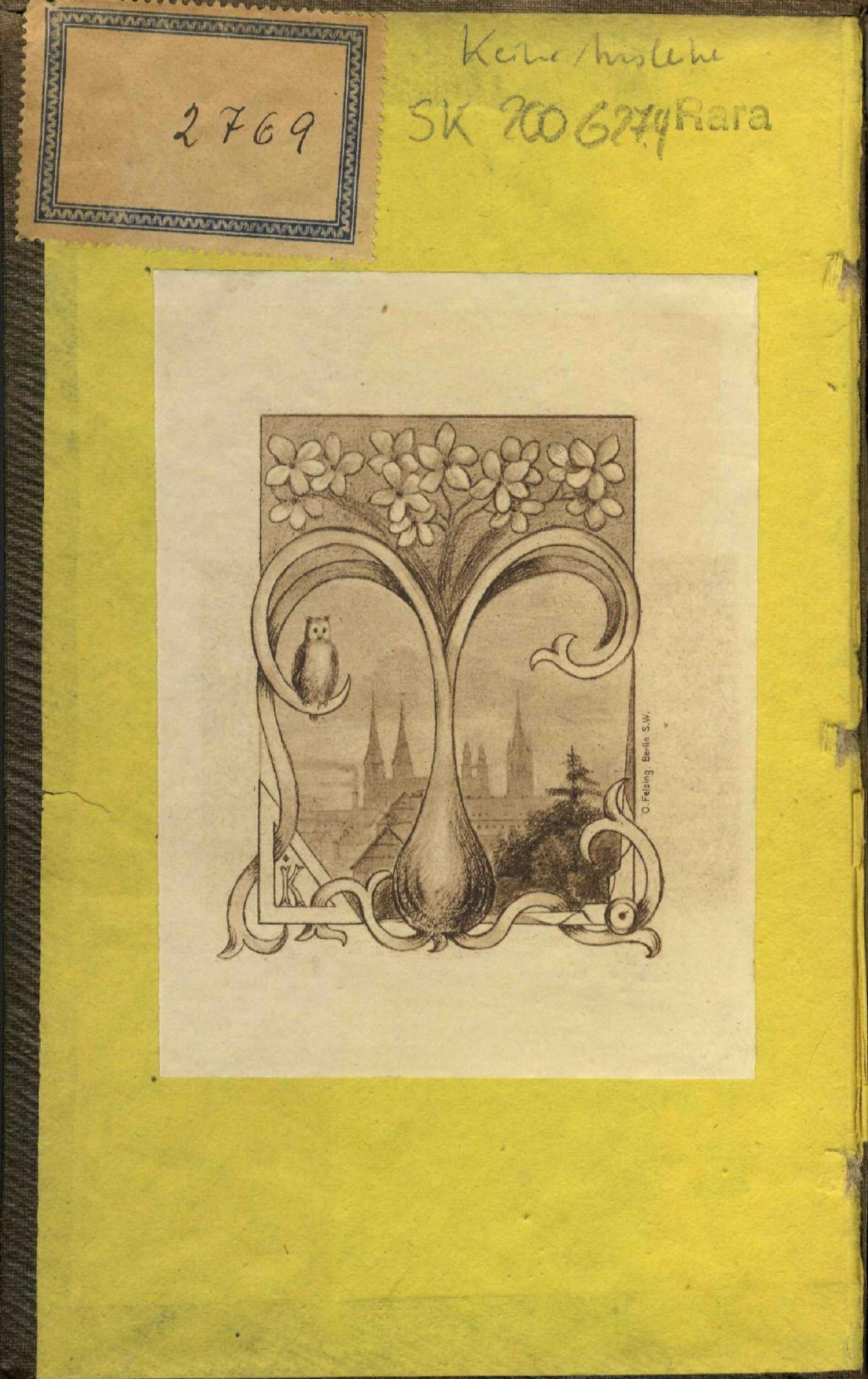


Wg 10

Grome profipour Listing

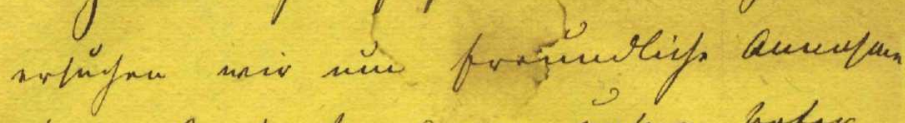

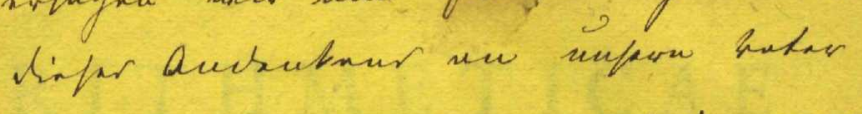
Goithingne 22: funi 1856 Eygaupis, Therese Gaufs

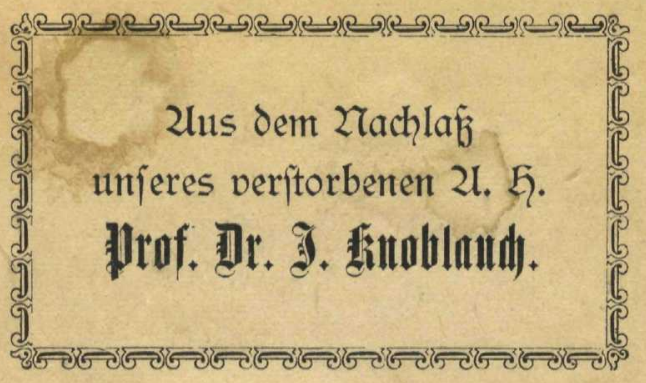

Sk200 G274

$\operatorname{Rer} 76$ Rara 


\section{DISQVISITIONES}

\section{A RITH METICAE}

A V TOAE

D. CAROLO FRIDERICO GAVSS

\section{LIPSI A E}

TCOAMISISA VDGERIT. FIEISCIER, Jun 


\title{
SERENISSIM O
}

\section{PRINCIPI AC DOMINO}

\section{CAROLO GVILIELMO FERDINANDO}

\author{
BRVNOVICENSTVM AC INVEBVRGENSIVM \\ DVCr
}




\title{
PRINCEPS SERENISSIME
}

\begin{abstract}
Summae equidem felicitati mihi duco, quod Celsissimo nomini Tuo hoc opus inscribere mihi permittis, quod vt Tibi offeram sancto pietatis officio obstringor. Nisi enim Tua gratia, Serenissime Princeps, introitum mibi ad scientias primum aperuisset, nisi perpetua Tua beneficia studia mea vsque sustentauissent, scientiae mathematicae, ad quam vehementi serrper amore delatus sum, totum me deuauere non potuissem. Quin adeo eas ipsas meditationes, quarum par tem hoc volumen exhibet, vt suscipere, per plures annos continuare literisque consignare liceret, Tua sola benignitas effecit, quae vi, ceterarum curarum expers, huic imprimis incumbere possem praestitit. Quas quum tandem in lucem emittere cuperem, Tua munificentia cuncta, quae editionem remorabantur, obstacula remouit. Haec Tua tanta de me meisque conatibus merita gratissima potius mente tacitaque admiratione reuoluere, quam iustis dignisque laudibus celebrare


possum. Namque non solum tali me muneri haud parem sentio, sed et neminem ignorare puto, solennem Tibi esse tam insignem liberalitatem in omnes qui ad optimas disciplinas excolerndas conferre videntur, neque eas scientias, quae vulgo abstrusiores et a vitae communis vtilitate remotiores creduntur, a patrocinio Tuo exclusas esse, quum $T u$ ipse intimum scientiarum omnium inter se et necessarium vinculum mente illa sapientissima omniumque quae ad humanae. societatis prosperitatem augendam pertinent peritissima, penitus perspexeris. Quodsi Tu, Princeps Serenissime, hunc librum, et gratissimi in $T e$ animi et laborum nobilissimae scientiae dicatorum testem, insigni illo fauore, quo me tamdiu amplexus es, haud indignum indicaueris, operam meam me non inutiliter collocasse, eiusque honoris, quem prae omnibus in rotis habui, compotem me factum esse, mihi gratulabor

\section{PRINCEPS SERENISSIME}

Brunouici mense Julio 1801.

Celsitudinis Tuae seruus addictissimiss

C. F. Gauss. 


\section{PRAEFATIO}

Disquisitiones in hoc opere contentae ad eam Matheseos partem pertinent, quae circa numseros integros versatur, fractis plerumque, surdis semper exclusis. Analysis indeterminata quam vocant seu Diophantaea, quae ex infinitis solutionibus problemati indeterminato satisfacientibus eas seligere docet, quae pe: numeros integros aut saltem rationales absolumntwr (plerumque ea quoque conditione adiecta it sint positiui 2 non est ilia disciplina ipsa, sed potiks pars eius valde specialis, ad eamque ita fere se habet, vt ars aequationes reducendi et soluendi (Algelíra, ad vaiuersam Analysin. Nimirum quewradinodum ad Analyseos ditionem refertuntur omnes quae circa quantitatum affectiones generales institui possunt disquisitiones: ita numeri integri (fractique quatenus per integros determinantur) obiectum proprium AnITHMeticar consilumit. Sed quim ea, quae Arithmetices nomine vulgo- traduntur, vix vltra artem numerandi et calculandi (i. e. numeros per signa idonea e. g. secunúum systema decadicum exhibendi, operationesque arithmeticas perficiendi) extendantur, adiectis nonnullis quae vel ad Arithmeticam omnino non pertinent (vt doctrina de logarithmis) vel saltem 
numeris interris non sunt propria sed ad omnes qumtiates patent: e re esse vidatur, duas Arithmaticae partes distinguere, illaque at srithmeticam elementarem referre, omnes autem disquisitiones generales de numerorum integromum affectionibus propris Anthmeticat Sublimiori, de qua sola hic sermo erit, vindicare.

Pertinent ad Arithmoticam Sublimiorem ea, quae Euclides in Elementis L. VII sqq. elegantia at rigore apud veleres consuetis tradidit: attimen ad prima initia huius scientiae limitantur. Diophanti opus celebre, quod toum problematis in determinatis dicatum est, multas quatstiones continet, quae propter difficultatem suam artificiorumque subtilitatem de auctoris ingentio th actmine existimationem hand mediocrem ancoitantry praesertin si subsidiorum quibus illi sti lituit temutatem consileres. At quum hase problowata dexteritatem quandan potius seitamquat tratutio-

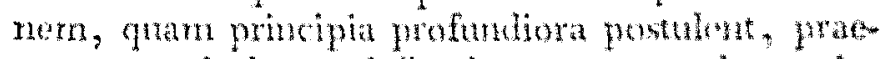

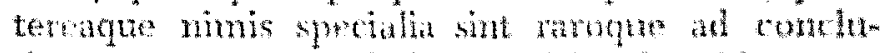

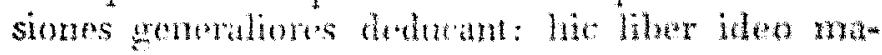

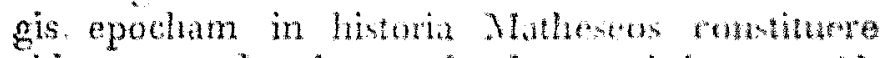
villatur, quod prina amis characteristicas at Atgebrie vestigia sistit, quam quah Arithmetioum

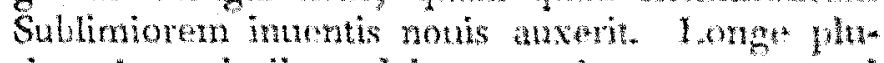
rima "recentionibus debentur, inter puos pauct

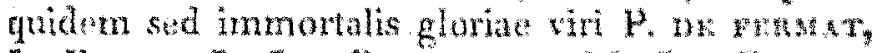

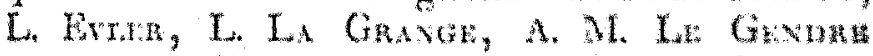
(vt paucos allos praptertam) intruatun at wethetraila huis dizinae scimtias aperthertut, quantisque dintis abundent patefecerunt. Qmacham vero inuenta a singulis his geometris profecta 
sint, hic enarrare supersedeo, quum e praefationibus Additamentorum quibus ill. La Grange Enleri Algebram ditauit operisque mox mernorandi $a b$ ill. Le Gendre nuper editi cognosci possint, insuperque pleraque locis suis in his Disquisitionibus Arithmeticis Jaudentur.

Propositum huius operis, ad quod edendum Iam amos abhinc quinque publice fidem dederam, id fuit, vt disquisitiones ex Arithmetica Sublimiori, quas partim ante id tempus partim postea institui, diuulgarem. Ne quis vero miretur, scientiam hic a primis propernodum initis repetitam, multasque disquisitiones hic denuo resurntas esse, quibus alii operam suam iam nauarunt, monendum esse duxi, me, quum primum initio a. 1795 huic disquisitionum generi animum applicax, omnium quae quidem a recemtioribus in hac arena elaboratn fuerint ignarum, omniumque subsidiorum per quae de his quidpinm comperire potuissem expertem fuisse. Scilicet in alio forte labore tunc occupatus, casu incidi in eximiam quardam veritatem arithmeticam (fuit autem ni fallor theorema art. 108), quam quum et per se pulcherrimam aestimarem et cun mairibus comexam esse suppicarer, summa qua potui contentione in id incubui, vt principia quibus inniteretur perspicerem, demonstrationemque rigorosam nanciscerer. Quod postquam tandem ex voto successisset, illecebris harum quaestionum ita fui implicatus, yt eas deserere non potuerim; quo pacto, dum alia semper ad alia viam sternebant, ea quae in quatuor primis Sectionibus hüus opexis traduntur ad maxi* 5 
mam partem absoluta erant, antequam de alionum geometrarum laboribus similibus quidquam vidissern. Dein copia mihi facta, horum summorum ingeniorum scripta euoluendi, maiorem quidem partem meditationurn mearum rebus dudum transactis impensam esse agnoui: sed eo alacrior, illorum vestigis insistens, Arithmetican vlterius excolere studui, ita variae disquisitiones institutae sunt $_{2}$, quarum partem Sectiones $V, V I$ et VII tradunt. Postquam interiecto tempere consilium de fructibus vigiliarum in publicum edendis cepi: eo lubentius, quod plares optabant, mihi persuaderi passus sum, ne quid vel ex illis inuestigationibus prioribus supprimerem, quod tum temporis liber non habebatur, ex quo aliorum geometrarum labores de his rebus, in Academiarum Commentaris sparsi, edisci patuissent; quod multae ex illis omnino nouae et pleraeque per methodos nouas tractatas erant; denique quod ornnes tum inter se tum cum disquisitionibus posterioribus tam arcto nexu conaerebant, vt ne noua quidem satis commode explicari possent, nisi reliquis ab initio repetitis.

Prodit interea opus egregium viri iam antea de Arithmetica Sublimiori magnopere meriti, Le Gendre Essai d' zune theorie des nombres, Paris a. VI, th quo non modo ommia quae hactenus in hac scientia elaborata sunt diligenter collegit et in ordinem redegit, sed permulta insuper noua de suo adiecit. Quum hic liber serius ad manum mihi peruenerit, postquam maxima operis pars typis ian exscripta esset; nullibi, vbi rerum analogia occasionem dare potuisset, 


\section{$\pm \quad x x \cdot-$}

eius mentionem iniicere licuit; de paucis tantummodo locis quaedam obsemationes in Additamentis adiungere necessarium videbatur, quas vir humanissimus et candidissimus benigne vt spero interpretabitur.

Inter impressionem huius operis, quae pluries interrupta variisque impedimentis vsque in quartum annum protracta est, non. modo eas inuestigationes, quas quidem iam antea susceperam, sed quarum promulgationem in aliud tempus differre constitueram, ne liber nimis magnus euaderet, viterius continuaui, sed plures etiam alias nouas aggressus sum. Plures quoque, quas ex eadem ratione leuiter tantum attigi, quum tractatio vberior minus necessaria videretur (e. g. eae quae in artt. $37.82 \mathrm{sqq}$, alisque locis traduntur), postea resumtae sunt, disquisitionibusque generalioribus quae luce perdignae videntur locum dederunt (Conf. etiam quae in Additamentis de art 306 dicuntur $)$. Denique quum liber praesertim propter arnplitudinem Sect. $V$ in longe maius quam exspectaueram volumen excresceret, plura quae ab initio ei destinata erant, interque ea totam Secionem octauam (quae passim iam in hoc volumine commemoratur, atque tractationem generalem de congruentiis algebraicis cuiusuis gradus continet) resecare oportuit. Haec omnia, quae volumen huic aequale facile explebunt, publici iuris fient, quamprimum occasio aderit.

Quod, in pluribus quaestionibus difficilibus, demonstrationibus syntheticis vsus sum, analysinque per quam erutae sunt suppressi, imprimis 
breuitatis studio tribuendum est, cui quantum fieri poterat consulere oportebat.

Theoria diuisionis circuli, siue polygonorum regularium, quae in Sect. VII tractatur, ipsa quidem per se ad Arithmeticam non pertinet, attamen eils principia vnice ex Arithmetica Sublimiori petenda sunt: quod forsan geometris tam inexspectalum erit, quantum veritates nouas, quas ex hoc fonte haurire licuit, ipsis gratas fore spero.

Haec sunt, de quibus lectorem praemonere volui. De rebus ipsis non meum est iudicare. Nihil equidem magis opto, quam vt is, quibus scientiarum incrementa cordi sunt, placeant, quae vel hactenus desiderata explent, vel aditum ad noua aperiunt. 
Sectio prima, De numerorum congruentia in genere p. 1.

Numeri congrui, moduli, residuá et non residua, art. I sq. Residua minima, 4. Propositiones elementares de congruis, 5. Quaedam applicationes, Ia.

Sectio secunda. De congruentiis primi gradus p. 8. Theoremata praeliminaria de numeris primis, factoribus etc. I3. Solutio congruentiarum primi gradus, 26. De inueniendo numero secundum moduIos datos residuis datis congruo 32 . Congruentiae lineares quae plures incognitas implicant 37 . Theoremata varia 38.

Sectio tertia De residuis potestatum P. 4.

Resida terminorum progressionis geometricae ab vnitate incipientis constitnuni seriem periodicam, 45. Considercantur primo moduli qui sems anmeri 
primi. Ponendo modulum $=p$, multitudo terminorum in periodo metitur numerum $p$ - I art. $49 *$ Fermatii theorema, 50. Quot numeris respondeant periodi, in quibus terminorum multitndo est dinisor datus numeri $p-I$ art. 52. Radices primitinae, bases, indices, 5\%. Algorithmus indicum, 58. De radicibus congruentiae $x^{\mathrm{n}} \equiv A$, art. 60 . Nexus indicum in systematibus diuersis, 6́. Bases vsibus pecnliaribus accommodatae, 72. Methodus radices primitiuas assignandi, 73 . Theoremata varia de periodis et radicibus primitinis, 75. (Theorema Wilsonianum, 76). De modulis qui sunt numerorum primorm potestates, 82. Modubi qui sunt potestates binarii, go. Moduli e pluvibus pri. mis compositi, 92.

Sectio quarta. De congruentiis secundi gradus p. $9^{2}$.

Residua et non-residua quadratica art. 94. Qnoties modulus est numerus primus, multitudo residuorum ipso minorum multitudini nonresiduorum aequalis, 96. Quaestio, vtrum numerus compositus residuum numeri primi dati sit an nonresiduum, $a b$ indole factorum pendet, 98 . De moäulis, qui sunt numeri compositi, roo. Criterium generale, vtrum numerus datus numeri primi dati residuum sit an nonresidun, Io6. Disquisitiones de mimeris prinis

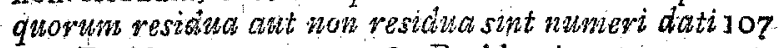
sqq. Residuum - $\mathrm{I}$ art, Io8. Residua +2 et 2 , art. II2. Residua +3 et -3 , art $11 \%$ Residua +5 et -5 art. 12x. De $=7$ art. 124. Praeparatio ad disquisitionem generalem, $125^{\circ}$ Per inductionem. theorema generale (frandamentalo) stabilitar, conclusionesque inde deducuntur 130 . Demonstratio rigorosa huius theorematis, I35. Methodus analoga, theorena art. II4 demonstrandi, I45. Solutio problematis generalis $\mathrm{I} 46$. De formis linearibus omnes numeros primos continentibus, quorum vel residuum vel non residunin est numerps quicunque datus 147. De aliorum laboribus circa has inuesti- 
gationes I5I. De congruentiis secundi gradus non puris 152 .

Sectio quinta. De formis aequationibusque indeterminatis secundi gradus p. 165 .

Disquisitionis propositum; formarum definitio et signum 153. Numerorum repraesentatio; detarminans 154- Valores expr. $r(b b-a c)$ (mod. $M)$ ad quos repraesentatio numeri $M I$ per formam $(a$, $b, c)$ pertinet, $155^{\circ}$. Forma aliam implicans, siue sub alia concenta; transformatio, propria et impropria, 157. Aequitualentia, propria et impropria I58. Formae oppositas I59, contiguae 160. Diuisores communes coëfficientium formarum 16r. Nexus ornnium transformationum similium formae datae in formam datan I62. Formae ancipites 163. Theorema circa casum vbi forma sub alia simul proprie et improprie contenta est 164 . Generalia de repraesentationibns numerorum per formas, earumque néxu curn- transformationibus I66. De formis determinantis negatini i 7 x. Applicationes speciales ad discerptionem numerorum in quadrata duo, in quadratum simplex et duplex, in simplex et triplex 182. De formis determinantis positivi non-quadrati 183. De formis determinantis quadrati 206. Formae sub allis contentae quibus tamen non aequitalent 2I3. Format determinantis o art. 2I5. Solutio generalis omnium aequationum indeterminatarum secundi gradus duas incognitas implicantium per numeros integros 216. Annotationes historicae 222.

DISQVISITIONES VITERIORES DE FORMIS. Distributio formarum determinantis dati in classes $223 ;$ classium in ordines 226 . Ordinum partitio in genera 228 . De compositione forriorum 238. Compusitio ordinum 245 , generum 246 , classium 249 . Pro determinante dato in singulis generibus eiusdem ordinis classes asque muitae continentur 252 . 
Comparantur multitudines classium in singulis gew neribus ordinum diuersorum contentarum 253 . De multitudine classium ancipitam 257. Certe semissi omnium characterum pro determinante dato assignabilium genera proprie primitiua (positiua pro det. neg.) respoudere nequeunt $26 \mathrm{r}$. Theorematis fundamentalis et reliquorum theorematum ad residua $-1,+2,-2$ pertinentium demonstratio secunda 262. Ea characterum semissis, quibus genera respondere nequeunt, propins determinantur 263 . Methodus peculiaris, numeros primos in duo quadrata decomponendi 265. Draressio contrinens TRACTATVM DE FORMIS TERNARIIS 266 sqq. Quaedam applicationes ad theoriam formarum binatiarum. De inuenienda forma e crius duplicatione forma binaria data generis principalis oriatur 286 . Omnibus characteribus, praeter eos, qui in artt. 262,263 impossibiles inuenti sunt, genera reuera respondent 287 , III. Theoria decompositionis tum numerorum tum formarum binariarum in tria quadrata 288. Demonstratio theorematum Fermatianorum, quemuis integrum in tres numeros trigonales vel quatuor quadrata discerpi posse 293. SoIutio aequationis $a x x+b y y+c z z=0$ art. 294. De methodo per quam ill. Le Gendre theorema funw damentale tractanit 296. Repraesentatio cifrae per formas ternarias quascunque 299. Solutio generalis aequationum indeterminatarum secundi gradus duas incognitas implicantium per quantitates rationales 300. De multitudine mediocri generum 3or, classium 302. Algorithmus singularis classium proprie primitinarum; determinantes regulares et irregulares etc. art. 305 .

Sectio sexta. Variae applicationes disquisitionum praecedentium

p. 540 .

Resolutio fractionum in simpliciores 309 . Conuersio fractionum com:aunium in decimales 3 12. Solatio congruentiae $x x \equiv A$ per methodum exclu 
sionis 3 I9. Solutio aequationis indeterminatae mxx + nyy $=A$ per exclusiones 323 . Alia methodus congrnentiam $x x \equiv A$ soluendi pro eo casu vbi $A$ est negatious 327. Duae methodi; numeros compositos a primis dignoscendi, illorumque factores inuestigandi, 320 .

Sectio septima: De asquationibus, circuli sectiones definientibus.

Disquisitio reducitur ad casum simplicissimum, vbi multitudo partium, in quas circulum secare oportet, est numerns primus 336. Aequationes pro functionibus trigonometricis arcnum qui sunt pars aut partes totins peripheriae; redactio functionum trigonometricarum ad radices aequationis $x^{n}-$ I $=0$ art. 337. Theoria radicum husus aequationis (vbi supponitut, $x$ esse numerum primum). Onittendo radicem $I$, reliquae $(\Omega)$ continentur in $a e-$ quatione $X=x^{n-1}+x^{n-2}+$ etc. $+x+r=0$. Functio $X$ resolui nequit in factores inferiores, in quibus omnes coëfficientes sint rationales $34 \mathrm{I}$. Propositum disquisitionam sequentium declaratur 342. Omnes radices $\Omega$ in certas classes (periodos) distribuuntur 343. Varin thecremata de his periodis 344 sqq. His disquisitionibus superstruitar solutio aequationis $X=0$ art. 352 . Exempla pro $n=19$, vbi negotium ad duas aequationes cubicas vnamque quadraticam, et pro $n=87, \quad \mathrm{vbi}$ ad quatuor quadraticas reducitur arti. 353,354 . Disquisitiones vileriores de hor argmmento. Aggregata, in quibus terminorum multitudo par, sunt quantitates reales 355 . De aequatione, per quam distributio radictm $\Omega$ in dus periodos definitur 356 . Demonstratio theorematis in sect. IV commenorati 357. De aequatione pro distributione radicum $\Omega$ in tres periodos 338. "Aequationum, per quas radices $\Omega$ innerinntur redurtio ad puras 359. Applicatio disçusitionum praecedentium ad fumitiones trigonometricas. Methodus, angulos 
quibus singulae radices $\Omega$ respondeant dignoscendi 36r: Tangentes, cotangentes, secantes et cosecantes e sinubus et cosinubus absque diuisione deriuantar 362 . Methodus, aequationes pro functionibus trigonometricis successiue deprimendi 363 . Sectiones circuli, quas per aequationes quadraticas siue per constructiones geometricas perficere licet $3^{6} 5$.

Idditamenta.

p. 666.

\section{aabulae.}




\title{
DISQVISITIONES ARITHMETICAE
}

\author{
SeCta PaIMA
}

DE

NVMERORTM COXGRIENTA IS GENERE.

\begin{abstract}
x. $S_{i}$ numerus a, mumatormm $n, c$ diferen.

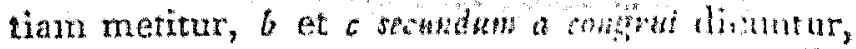
sin minus, incongrai ipsum a modmam appalla, mus. Vterque numerurum b. $c$, priori in cast alterius vesidum, in posterioni vero non residum yocatur.

Hae notiones de omnilus mumeris integris tam positiuis quan negatiuis") valent, netue
\end{abstract}

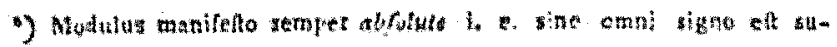
14: hisu. 
vero ad fractos sunt extendendae. E. g* -9 et +16 secundum modulum 5 sunt congrui; -7 ipsius +15 secundum modulum a residuum, secundum modulum 3 vero nonresiduum. Ceterum quoniam cifram numerus quisclue metitur, omnis numerus tamquam sibi ipsi congruus secundum modulum quemcunque est spectandus.

2. Omnia numeri dai a residua secundum modulum $m$ sub formula $a+k m$ comprehenduntur, designante $k$ numerum integrum indeterminatum. Propositionum quas post trademus faciliores nullo negotio hinc demonstrari possunt: sed istarum quidem veritatem aeque facile quiuis intuendo poterit perspicere.

Numerorum congruentiam hoc signo, $\Longrightarrow$ in posterum denotabimus, modulam vbi opus erit in clausulis adiungentes, $-16 \equiv 9$ (mod.5), $-7 \equiv 5$ (mod. I 1$\left.)^{\star}\right)$.

3. THeOR Propositis m numeris integris suc. cessiuis, $a, a+\mathrm{I}, a+2 \ldots a+m-\mathrm{I}$, alioque $A$. illowm aliquis huic secundum moduinm $m$ congrass erit, et quidem unicus tantum.

Si enim $\frac{n_{-}}{m}$ integer, erit $a=A$, sin fractus, sit integer proxime maior, (aut quando est negatiuus, proxime minor, si ad signum non respiciatur) $=\dot{k}$, cadetque $A+k m$ inter $a$ et

- Hoc signurn propter magnath alalogiam quae inter aequalitatem atque congruentiam incebitut adoptauimus. ob eandem caulsam ill. Le Genure in comment. infra satuluc laudanda ipsum aequalitatis signum pro congruentia retinuit, quod hos ne ambiguitas oriatur imiknti dubitunimus. 


\section{$-3-$}

$a \mp m$, quare erit numerus quaesitus. Et manifestum est omnes quotientes $\frac{x-a}{m}, \frac{a+1-A}{n}$, $\frac{\dot{a}+\frac{3}{m}-A}{m}$ etc: inter $k-1$ " et $k+1+1$ sitos esse; quare plures quam vnus integri esse nequeunt.

4. Quisrue igitur numerus residum habebit tum in hac serie, $o, f, 2, \ldots m \ldots$, tum in hac, $0,-i,-2, \ldots-(n-1)$, quae residua minima dicemus, patetque, nisi o fuerit residuun; bina semper dari, positiunim alterum, alterum negatinum. Quae si magnirudine sunt inaequalia, alterum erit $<\frac{\mathrm{m}}{2}, \sin$ sécus vtrumque $={ }_{2}^{12}$, signi repecte nom haibito. Vride patet, quemuis numerum residum habere moduli semissem non superans quod absolute minisinim vocabitur.

E. g. -13 secundim modulum 5 , habet residurim minimum positiuun 2 , quod simul est absolute miniminin, -3 vero residuam minimun nềgatiutum $;+5$ secundum modnlum 7 sui ipsius est residuum minimum positiuum, - z negatiuum, simuilque absolute irinimum.

5." His notionibus stabilitis eas numerorum congruorum proprietates quae prinia fron. te se offerunt colligamus.

Qui numeri secundam noodulum confositum sünt

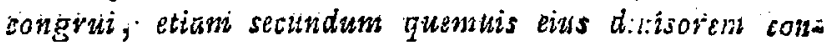
grui.

Si plures numeri eideni nuniero secundzm eurdem modulum sunt congruti, inter se erunt ivingrai (seoundum eundem modulum):

Haec modulorum identitás etiain in so quentibus est subinteligenda; 


\section{- $4-$}

Numeri congrui residut minina iabent oxdin, incangrui dittersin.

6. Si habntur qrotounque ummer $A, B, C$,

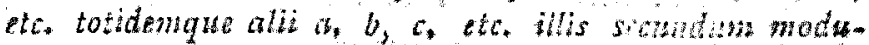
lom quencunque congrat, $A=a, B=b$, etc bit $A+$ $B+C+e t a=a+b+c+i t s$.

Si $A=a, B=b$ ifit $A-B \quad a \cdots b$.

7. Si $A= \pm a$, erit quoque is $A$ ka.

Si $k$ numerus positiuus, hoc est tantummodo casus particularis propos. art. praec., ponendo ibi $A=B=C$ etc., $a-b=0$ etc. Sí a negatiuns, erit - positiuus, adeoque -

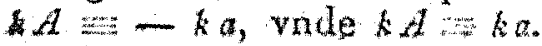

Si $A=a, B=b$, crit $A B=$ ab. Namque $A B=A b=b a$.

8. Si habentu quatewnqu numeri $A, B, G$ atc. totidemque ulis $a, b, c$ etw, his congtat, $A, a, B$

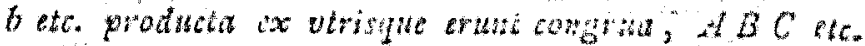
$=a b c a t c$.

Exartic. praec. $A$ a a a et ob eandem rationem $A B C$ abs, edemque molo quotcuncue alii lactores atcedere po-siut.

Si omnes numeri $A, B, C$, etc, aequabes assumuntur, nec non respondentes $a_{3} b_{3}, c$, th.

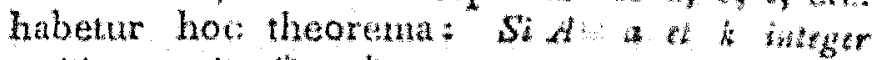
posititus, erit $A^{\text {ta }} a^{\text {th }}$.

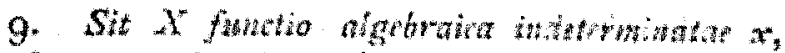

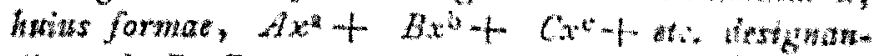

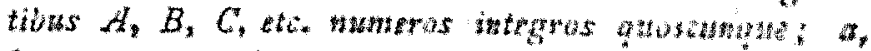

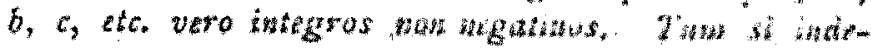

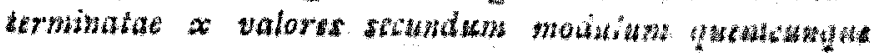




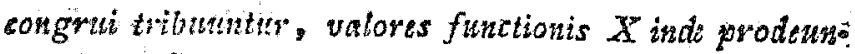
tes congrui erint.

Sint $f, g$, valores congrui ipsius $x$. Tum ex art. praec $f^{a} \equiv g^{a}$ et $A f^{a} \equiv A g^{a}$, eodemque modo $B f^{b}=B g^{b}$ etc. Hinc $A f^{a}+B f^{b}+$ $C f^{\mathrm{c}}+$ etc. $=A g^{\mathrm{a}}+B \mathrm{~g}^{\mathrm{b}}+C g^{\mathrm{c}}+$ etc. Q. E. D.

Ceterum facile intelligitur, quomodo hoc theorema ad functiones plurium indeterminata, rum extendi possit.

ro. Quodsi igitur pro $x$ omnes numeri integri consecutitui substituuntur, valorescue functionis $x$ ad residun minima reducuntur, haec seriem constituent, in qua post interuallum $n$ terminorum (designante $m$ modulum) iidem termini iterum recurrunt; siue haec series ex pcrioto $m$ terminorum infinities repetita, erit formata, Sit e. g. $X=x^{3}-8 x+6$ et $=5$; tum pro $x=0,1,2,3$ etc., valores ipsius $X$ haec residua minima positiua suppeditant, $I, 4,5,4,3, \mathrm{r}, 4$, etc., vbi quina priora I, $4,3,4,3$ in infinitum repetuntur; atque si series retro continuatur, i. e. ipsi $x$ valores negatiui tribuuntur, eadem periodus ordine terminorum inuerso prodit: vnde manifesturl est, terminos alios quam qui hanc periodum constituant in tota serie locum habere non posse.

Ir. In hoc igitur exemplo $X$ neque $\equiv$ 0 , neque $=2(\bmod .5)$ fieri potest, multoque minus $=0$, aut $=2$. Vnde sequitur, aequationes $x^{3}-8 x+6=0$, et $x^{3}-8 x+4=0$ per numeros integros et proin, vti notum est, per numeros rationales solui non posse: $\mathrm{Ge}_{\text {* }}$ 


\section{$-6^{n}-$}

neraliter perspicum est, aeduationem $x=0$, quando $x$ functio incognitae $x$, huisus formae, $x^{\mathrm{n}}+A x^{q}+B x^{2}=+\operatorname{etc}+N ; A, B, C$, etc integri, atque " integer positiuus, (ad quam fornam onnes aequationes algebracas reduci posse constat) radicem rationalem mullam habere, si congruentiae $x=0$ secundum vllum modulum salisfieri nequeat. Sel hoc criterium, guod hic sponte se nobis obtulit, in sect. VIIE fusius pertractabitur. Poterit certe ex hoc specimine notiuncula qualiscunque de harum inuestigationum vtilitate efformari.

12. Theorematibus in hoc capite traditis complura quae in arithmeticis doceri solent innituntur, e. E. regulae ad explorandam diusibilitatem nuneri propositi per $9 \times 1$ i aut alios numeros. Secudum nodulum 9 onnes numeri to potestates vnitati sunt congruate quare si numerus propositus habet lormam $a+10 b+100$ ft atc., idem residanm minimum secundum modulum 9 dabil, ynod $a+b+c+e t c$ Hitc manifistum est, si figurae singule numefi cecadice expressi sine resprocti loti quem occirant addartur, summam hanc numerumque propesituni ęadem residua minima praebere, adeorgue hunc per 9 diuidi pose , si illa per 9 sit diuisibilis, et contra. liem etiam de divisore 5 tenendrom. Quoniam sevwdum moduhut $x, 100=x$ erit generaliter $1025=5$, $10^{\circ k}=10^{\circ}=-1$, et numerus formae +10 $b+100 c+e t c$ secundun modulum $x \mathrm{x}$ idera yesidutum minimun dabit quod $a-b+c$ etc; vade regula nota protinus dexiuatur. Ex eo. 


\section{- $7-$}

dem principio omnia similia praeccpta facile deducuntur.

Nec minus ex praecedentilus petenda est ratio regularum, quae ad verificationem operationum arithneticarum vulgo commendantur. Scilicet si ex numeris datis alii per additionem, subtractionen, multiplicationem aut eleuationem ad potestates sunt deducendi: substiuuntur datorum loco residua ipsorum minima secundum modulum arbitrarimm (valgo 9 aut $\&$, quoniam in nostro systemate decadico secundum hos, vti molo ostendimus, residua tam facile possunt inueniri). Numeri hinc oriandi illis, qua ex numeris propositis deducti fuerunt, congrui esse debent; quod nisi eneniat, vitium in calcuIum irrepsisse concluditur.

Sed qumm haer hisqne similia abunde sint nota, diutius is immorari superfluum foret. 
SECTIO SECVNDA

DE

CONGRVENTIIS PRIMI GRADVS.

13. Theonema. Productume éduobus nume. ris positizis numero primo dato minoribus per huno priminn diztidi sequit.

Sit $p$ prinius, et $a$ positious $<p$ : tum nullus numerus positiuus $b$ ipso $p$ minor dabitur, ita vt sit $a b \equiv 0(\bmod . p)$.

Dem. Si quis negret, supponamus dari numeros $b, c, d$, etc. omnes $<p$, ita vt $a b \equiv 0$; $a c \equiv 0 ; a d \equiv 0$ etc. $(\bmod . p)$. Sit omnium minimus $b$, ita vt omnes numeri ipso $b$ minores hac proprietate sint destituti. Manifesto erit $b .>1$ : si enim $b=1$, foret $a b=a<p$ (hyp.), adeouque per $p$ non diuisibilis. Quare $p$ tamquarm prinus per $b$ diuidi non poterit, sed inter duo ipsius $b$ multipla proxima mb et $(m+1) b$ cadet. Sit $p-m b=b^{\text {I }}$, eritque $b^{\mathbf{I}}$ numerus positiuus et $<b$. Iam quia supposuimus, $a b \equiv 0$ (mod, $p$ ), habebitur quoque $m a b$ $\equiv 0$ (art. 7), et hinc, subtrahendo ab \& $p \equiv 0$, erit $a(p-m b)=a b^{\mathrm{I}} \equiv 0$; $\dot{x}_{0} e_{0} b^{\mathrm{x}}$ inter nuw 
meros $b, \hat{c}$, $a$, etc. referendus, licet minimo eorum bit minor. Q. E. $A$.

i's. Si nec a nec b per nemeram primuin $p$ ditidi potest: etion produstzm a b per $p$ diatdi non poterit.

Sint numerorum $a, b$, secundum modulitm p residua minima posilita es quorum neatrum erit o (hyp.). Iam si esset $a b \equiv$ o (mod. $p)$, foret quoque, propter $a b \equiv a b, a b \equiv 0$, quod oum theoremate praec. consistere nequit.

Huius theorematis demonstratio iam ab Euclide tradita, El. VIT. 32. Nos tamen omittere eap noluimus, tum quod recentioram compluresseu ratiocinia vaga pro demonstratione venditauerunt, seu theorema omnino praeterisrunt, tum quod indoles methodi hic adhibitae, qua infra ad multo reconditiona enodanda vteraur, e casu simpliciori facilius deprehendi poterit.

15. Si nullius wumerorzsm $a, b, c, d$ etc. per

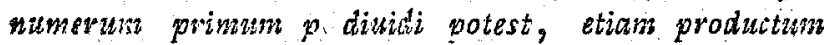
abcd etc. per p dituidi non poterit.

Secundum artic. praec $a b$ per $p$ diuidi ne. quit; ergo etian $a b c$; hinc $a b c d$, etc.

r6. THEOREMA. Numerus compositus quicunque vnico tantum modo in factores primos resolui potest.

Dem. Quemuis numerum compositum in factores primos resolui passe, ex elementis constat, sed pluribus modis diuersis fieri hoc non posse perperam plerumque supponitur tacite. Fingamus numerum compositum $A$, qui sit = $a^{*} b^{6} c^{\gamma}$ etc., designantibus $a, b, c$ etc. numeros primos inaequales, alio adhuc modo in factores primos esse resolubilem. Primo manifea 
stum est, in secundum hoc factorum systema alios primos quam $a, b, c$ etc. ingredi non posse, quum quicunque alius primus numerum $A$ ex his compositum metiri nequear. Similiter. etiam in secundo hoc factorum systemate nullis primorum $a, b, c$ etc. deesse potest, quippe qui alias ipsum $A$ non metiretur (art. praec.). Quare hae binae in factores resolutiones in eo tnatummodo differre possunt, quod in altera aliquis primus pluries quam in altera habeatur. Sit talis primus $p$, qui in altera resolutione $m$, in altera vero $n$ vicibus occurrat, site que $m>n$ : Iam deleatur ex utroque systemiate factor $p, n$ vicibus, quo fiet vt in altero adhuc $m-n$ vicibus remaneat, ex altero vero ommino abierit. Io e numeri $\frac{A}{p^{n}}$ duae in facrores resolutiones habentur, qrarum altera a factore $p$ prorsus libera, altera vero $m-n$ vicibus eum continet, contra ea quae modo demonstrauimus.

17. Si itaque numerus compositus $A$ est productum ex $B, C, D$ etc., patet, inter factores primos numerorum $B, C, D$ etc. alios esse non posse, gram qui etiam sint inter factoress numeri $A$, et quemuis horum factorum toties in $B, C, D$ coniunctim occurrere debe. re, quoties in $A$. Hinc colligitur criterium, vtrum numerus $B$ alium $A$ metiatur, necne. Illud eueniet, si $B$ neque alios factores primos, neque vllum pluries inuoluit, quam $A$; quarum conditionum si aliqua deficit, $B$ ipsum $A$ non metietur.

Facile hinc calculi combinationum auxilio deribari potest, si $A=a^{*} b^{b} c^{\gamma}$ ete. designanti- 


\section{if}

bus vt supra $a, b, c$ etc. numeros primos dituer. sos: $A$ habere $(\alpha+1)(b+1)(\gamma+1)$ etc. dilaisores diuersos, inclusis etiam I et $A$.

8. Si igitur $A=a^{\alpha} b^{b} c^{\gamma}$ etc, $\quad K=k^{\alpha} b^{\lambda}$ mp etc., atque primi $a, b, c$ etc., $k, b$, n etc. omnes diuersi, patet $A$ et $K$ diuisorem communem praeter I non habere, siue inter se esse primos.

Pluribus numeris $A, B, C$ etc. propositis maximc ontuibus caminunis menstiva ita determinatur. Resoluantur omnes in suos factores primos, atque ex his excerpantur ii, qui omnibus numeris $A, B, C$ etc. sunt communes, (si tales non adsunt, nullus diuisor erit omnibus communis). Tun quoties quisque horum factorum primorum in singutis $A, B, C$ etc. contineatur, siue quot dimnensiones in singulis $A, B, C$ quisque habeat, adnotetur. Tandem singulis factoribus primis tribuantur dimensiones cmnizm quas in $A, B, C$ etc habent minimae, componaturque productum ex iis, quod erit mensuca communis quaesita.

Quando vero numerorum $A, B, C$ ete. mini mus comminis diuidus desideratur, ita procedendum. Colligantur omnes numeri primi, qui numerorum $A, B, C$ etc. aliquem metiuntur, tribuatut cuiuis dimensio omnium quas in numeris $A, B, C$ etc. habet maxima, sicque ex omuibus productum confletur, quod erit diuz duus quaesitus.

Ex. Sit $A=504=2337 ; \quad B=2880=$ $26325 ; C=864=2535$.ro inueniendo diui- 
sore communi maximo habentur factores pri. mi 2, 3, quibus dimensiones 3,2 tribuendi; vide fiet $=2^{3} \overline{3}^{2}=72$; dividuus vero communis minimus erit $2^{6} 3,5.7=60480$.

Demonstrationes propter facilitatem omittimus. Ceterum quomodo haec problemata sol. uenda sint, quando numerorum $A, B, C$ etc. in factores resolutio non detur, ex elementis notum.

19. Si numeri $a, b, c$ etc. äd alium $k$ sunt primi, etiam productum ex illis a bc etco ad k primum est.

Quia enim nulli numerorum $a, b, c$ etc. factor primus cum $k$ est communis productumque $a b$ etc. alios factores primos habere ne. quit, quam qui sunt factores alicuius numerosum $a, b, c$ etc., productum abc etc. etiam cum $k$ factorem primum communem non habebit. Quare ex arto praec $k$ ad abe etc. primus,

Si numeri $a, b$, $c$ etc. intgr se sunt prini, atium que $k$ singuli metiuntur: etiam productusn ex illis unmerum $k$ metietur.

Hoc aeque facile ex artt. 17, 8 deriuatur. Sit enim quicunque producti abcetc diuisor primus quem contineat $x$ vicibus, manifestumque est, aliquem nimerorum $a, b, c$ etc. eundem huno diuisorem vicibus continere debere. Quare etiam quern hic numerus metitur, $\pi$ vicibus diuisoren $p$ continet. Similiter de religquis producti abc etc. diuisoribus.

Hinc si äto numeri $m$, $n$ secunduni plures modulos inter se grimios $a, b, c$ etc. santicongrai, etian se- 
cundum productum ex his congrui eromt. Qumm enin $m$ - n per singulos o, $b$, etc sit diuisibilis, etiain per eorum productum diuidi poterit.

Denigue si a ad b primus et ak per $b$ dinisibilis. trit etian her $b$ diussibilis. Namque quoniam ab tam per a quam per b diuisibilis, etiam per ab dimili poterit, i. e $\frac{a k}{a b}=\frac{k}{b}$ erit integer.

20. Qiscando $A=a^{*} b^{6} c^{\gamma}$ etc., designantibus $a, b, c$ etc. numeros primos incequales, est potestas ciliquan, puta $=h_{n}$; ownes exponentes $\alpha, 6, \gamma$ etco per $n$ erint diuisibites:

Numerus enim $k$ alios factores primos quam $a, b, t$ etc. non inuoluit. Contineat lactorem $a, a^{\mathrm{T}}$ vicibus, continebitque $k^{\mathrm{a}}$ situe $A$ hunc factorem $n a^{x}$ vicibus; quare $x_{x}^{x}=\alpha$, et $\frac{\alpha}{n}$ integer. Similiter $\frac{6}{n}$ etco integros esse demonstratur.

2. Quando $a, b, c$ etc, sunt inter se privini, et productum a b. etc. potestas aliqua, puta $=k^{\mathrm{n}} \div$ singuli nuneri $a, b, c$ etc. similes potestetes erint.

Sit $a=l^{\lambda} m^{k} \cdot p^{\pi}$ etc., designantibus $1, m$, D etc. numeros primos diuersos, quorum nil. lus per hyp. est factor numerorum $b, c$ ete. Quare productum a bc etc. factorem $l$ implica. jit $\lambda$ vicibus, factorem $m$ vero vicibus etc. hine (art.praec) $\lambda, \mu, \pi$ etc. per $n$ diuisibiles adzoque $\sqrt{ } a=l_{n}^{\lambda} m_{n}^{\mu} p_{n}^{\pi}$ etc. integer. Similiter le reliquis $b, c$ etc.

Haec de numeris primis praemittenda erant; am ad ea quae finen nobis proposicum prolius atlinent conuertimur. 


\section{- ris -}

22. Si numeri $a_{j} b$ pgr alizm $k$ diuisibiles sècun.

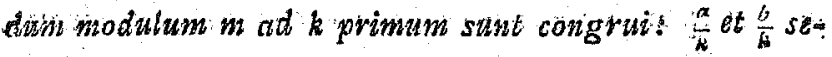
cundisin eundem modulum congrui erint.

Patet enint $a-b$ per $k$ diuisibilem fore, nec mînus per $n$ (hyp ); quare (art. Ig) $\frac{\alpha_{-} b}{k}$ per m diusibilis erit, $i$. e. erit $\frac{a}{k}=\frac{b}{k}(\bmod . m$ ).

Si auten reliquis manentibus ot $h$ habent diuisorem communem maximum e, eric $a=\frac{b}{a}\left(\bmod \frac{m}{s}\right)$. Namque $\frac{b}{\varepsilon}$ et $\frac{m}{e}$ inter se primi. At $a-b$ tam per $k$ quam per m diuisibi lis adeoque etiam $\frac{a=b}{\rightarrow}$ tain per $\frac{k}{b}$ quam per $\frac{m}{a}$ hincque per $\frac{k m}{b a}$ i. e. $\frac{a-b}{k}$ per $\frac{m^{2}}{b}$ siue $\frac{a}{k}=\frac{b}{k}$ $\left(\bmod , \frac{m}{6}\right)$

23. Si ad an printsis, et $e_{j} f$ numeri secundum modulzun wh intongmit erwnt etian a $e$, af iwcongrui secunidizm $m$.

Hoc est tanum conuersio theor art prasc.

Hinc vero manifestum est, si a per omnes numeros integros a $o$,sque ad m- I multiplicetur productaque secundum modulum ad residua stia minima xeducantur, haec omnia fore inaequalia. Et quum horum residuorurn, quorum nullum $>m_{\text {, }}$ numerus sit $m$, totidemque dentur numeri a o usque ad $m-1$, pater, nullum horum numerorum inter illa residua deesse posse.

24k Fxpressio $a x+b$, denotantibus $a, b$ numeros datos, $x$ numeram indeterminatum sou vatiabilem, sectindum inodutim $n$; ac a

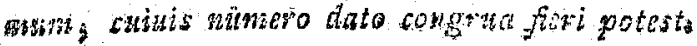


Sit numerus, cui congrua fietí debet; $c$ et residuum minimuni positiuum ipsius $c-5$ secundum modulum $m, e_{0}$ Ex art. praec. necessario datur valor ipsius $x<m$, talis, vt producti $a x$ secundum modulum in residuun minimum fiat $e$; esto hic valor $v$, eritque $a v \equiv$ $c \equiv c-b ;$ vnde $a v+b \equiv c$ (mod. m) Q.E.F. 25. Expressionem duas quantitates con. gruas exhibentem ad instar aequationum, congrutentian vocamus; quae si incognitarn implicat, resolui dicitir, quando pro hac valor Inuenitur congruentiae satisfaciens (radir). Hinc porro intelligitur, guid sit congruentia resolutilis. et congruentia irresolubilis. Tandem facile perspicitur similes distincriones locum hic habere posse vti in aequationibus. Congruentiarnm tronsscendentium inlra exempla occurrent; älğebraicat vero secundum dimensionem maximam incognitae in congruentias primi, secundi altiorumque gradum distribuuntur. Nec minus congruentiae plures proponi possunt plures incognitas inuoluentes, de quarum elinituatione disquirendum.

26. Congruentia itaque primi gradus, $d x$ $+b \equiv c$ ex art 24 semper resolubilis, quando modulus ad a est primus. Quodsi vero $v$ fue. rit valor idoneus ipsius $x$, siue radix congruen tiae, palam est, omnes numeros, ipsi $v$ secundum congruentiae propositae modulum congruos, etiam radices fore (art. $9_{1}$ ) Neque minus facile perspicitur, omnes radices ipsi $y$ congruos esse debere: si enim alia radix fuerit $t$, erit $a v+b \equiv a t+b$, vnde $a v \equiv a t$, et hinc $\dot{v} \equiv$ (art, 22), Hinc colligitus congruentiama $x+t$ 


\section{$-6 \quad-$}

(mod m.) exhibere resolutionem conipletam congruentiae $a x+b \equiv c$.

Quia resolutiones congruentiae per valores ipsius $x$ congruos per se sunt obuiae, itque, hoc respectu, numeri congrui tamquanu aecquiualentes considerandi, tales congruentiae resolutiones pro vna eademque habebimus. Quamobrem quum nostra congruentia $a x+b \equiv c$ alias resolutiones non admittat, pronunciabimus, vrico tantum modo eam esse resolíbilem seu vnam tantum radicem habere. Ita e. g. congruentia $6 x+5 \equiv x 3$ (mod. I I) alias radices non admittit, quam quae sunt $\equiv 5$ (mod. 11$)$. Haud perinde res se habet in congruentiis altiorum graduum, siue etiam in congruentiis primi gradus, vbi incognita per numerum est multiplicata, ad quem modulus non est primus.

97. Superest, vt de inuenienda resolutio. ne ipsa congruentiae huiusmodi, quaedam adiiciamus. Primo obseruamus, congruentiann formae $a x+t \equiv u$, cuius modulum ad a primum supponimus, ab hac, $a x \equiv \pm$ is pendere: si exim huic satisfacit $x \equiv r$, illi satisfaciet $x \equiv \pm(u-t) r_{0}$. At congruentiale $a x \equiv$ +5 , modulo per $b$ designato, aequiualet aequa tio indeterminata $a x=b y \neq \mathrm{r}$, quae quomodo sit soluenda hoc quidem tempore abunde est notrm; quare nobis sufficiet, calculi algorithInam huc transscripsisse.

Si quantitates $A, B, C, D, E$ etc. ita ab his $\alpha, b, \gamma$, etc. pendent, ve habeatur $A=s, B=$ $6 A+1, C=\gamma B+A, D=\delta C+B, B=D$ 
+ $C$ etc. breuitatis gratia ita ens designamus, $A=[\alpha] ; B=[\omega, 6] ; C=[\omega, 6, \gamma], D=[\alpha, 6, \gamma, d]$ etc. \%. Iam proposita sit aequatio indeterminata $a x=b y+1, v b i a, b$ positiui. Supponamus, id quod licet, a esse non $<b$. Tun ad instar algorithmi noti, secundum quem dnorum numerorum diuisor communis maximus inuestigatur, formentur per diuisionem vulga. rem aequationes,

$a=b+c$

$b=6 c+d$

$c=\gamma d+$ etc.

ita vt $a, b, \gamma$ etc. $\varepsilon, d, e$ etc. sint integri positiu et $b, c, d, e$ continuo decrescentes, donec perueniatur ad

$m=\mu n+1, q u o d$ tandem euenire debere con stat. Erit itaque $a=[n, \mu, \ldots, \gamma, b,] ; b=[n$, $\mu \ldots \gamma, 6]$. Tum fiat $x=\left[\mu_{1}, \gamma_{,} 6\right], y=[\mu, \ldots$ $\gamma 6, \alpha]$, eritque $a x=b y+1$, quando numero. rum $\alpha, 6, \gamma \ldots \ldots, n$ multituda est pars aut $a x=$ $b y$ - I; quando est impär. Q.E. $F_{\phi}$

23. Resolutionem generalem huiusmodi aequationum indeterminatarum ill. Euler pria

-) Multo generalius haecce relatio considerari potest, quod negotiuna alia forsan occasione suscipiemus. Hic duas tantum propositiones mdicimus, quae vsura suum in praesenti inuestigatione habent; scilicet,

${ }_{10}^{0}[\alpha, 6, \gamma \ldots \ldots, \lambda, \mu],[6, \gamma \ldots \ldots \lambda]-[\alpha, 6, \gamma, \ldots \lambda]$ $[\ell, \gamma, \ldots \lambda, \mu]= \pm I$, vbi signum superius accipiendum quando numerorum $\alpha, 6, \gamma \ldots, \lambda, \mu$ multitudo par, inferius quando impur.

$2^{\circ}$. Numerorum $\alpha, b, \gamma$ etc. ordo intuerti potest, $\left[\alpha, b, \gamma, b_{i}\right.$ $\lambda, \mu]=[\mu, \lambda \ldots, \gamma, 6, \infty] . \quad$ Demonstrationes quae non sun difficiles his supprimimus. 
mus docuit, Comment. Petrop. T. VIT. p. 46. Met hodus qua vsus est consistit in substitutione aliarum incognitarum loco ipsarum $x, y$, atque hoc quidem tempore satis est nota. Ill. Ia Grange paullo aliter rem aggressus est: scilicet ex theoria fractionum continuarum constat si fractio in fractionem continuam

$$
\begin{aligned}
& \frac{1}{\frac{+1}{6+x}} \frac{1+\text { etc. }}{y+1} \\
& \frac{4 \pi}{x-1}
\end{aligned}
$$

conuertatur, haecque deleta vitima sui parte $\underset{n}{\not}$ in fractionem communen $\frac{x}{y}$ restituatur, fore $a x$ $=b y \pm 1$, siquidem fuerit $a$ ad $b$ primus. Ceterum ex vtraque methodo idem algorithmus deriuatur. Inuestigationes ill. la Grange exstant Hist. de 1 Ac. de Berlin Anné 1767 p. 175 . et cum aliis in Supplementis versioni gallicae Alge orae Euberianae adiectis.

29. Congruentia a $x+t \equiv u$ cuius modurIus ad a non primus, facile ad casum praecedentem reducitur. Sit modulus maximusque numerorum a, $m$ diuisor communis $d$ Primo patet quemuis valorem ipsius $x$ congruentiae secundum modulum $m$ satisfacientem eidem etiam secundum modulum \& satisfacere (art. 5). At semper $a x \equiv 0(\bmod . \delta)$ quoniam ipsum a metitur. Quare, nisi $t \equiv \varkappa$ (mod. $\delta)$ i. e. $t$ - per d diuisibilis, congruentia propo. sita non est resolubilis. 
Ponamas itaque $a=\delta e, m=\$ f, t-u=$ dk, eritque $e$ ad $f$ primus. Tum vero congruentiae propositae $\delta e x+\delta k \equiv 0(\bmod$. $f f)$ aequiualebit haec $e x+k \equiv 0(\bmod f)$, i. e. quictuque ipsius $x$ valor huic satisfaciat, etiam illi satisfaciet et vice versa. Manifesto enim $e x+k$ per $f$ diuidi poterit, quando $\delta e x+\delta k$ per $\delta f$ diuidi potest, et vice versa. At congrtuentiam $e x+\hat{k} \equiv 0$ (mod. $f$ ) supra soluere docuimus; vinde simul patet, si $v$ sit vnus ex valoribus ipsius $x, x \equiv v$ (mod. $f$ ) exhibere resolutionem completam congruentiae propositae.

3o. Quando modulus est compositus, nonnumquarn praestat sequenti methodo vti.

Sit modulus $=m n$; atque congruentia proposita $a x \equiv b$ Soluatur primo congraentia haec secundum modulum $m$, ponamusque ei satisfieri, si $x \equiv v$ (mod. $\frac{m}{\gamma}$ ), designante $\delta$ di. uisorem communem maximum numerorum $m, \omega_{\mathrm{b}}$ Iám manifestum est, quemuis valorem ipsius $x$ congruentiae $a x \equiv b$ secundum modulum $m$ satisfacientem eidem etiam secundum modulum satisfacere debere: adeoque in forma $y+\frac{m}{\delta} x$ contineri, designante $x$ numerum in determinatum, quamuis non vice versa omnes numeri in forma $v+\frac{m}{\delta} x^{\prime}$ contenti congruen tiae secundum mod. $m$ n satisfaciant. Quomodo autem $x^{\prime}$ determinari debeat, vt $v+x^{*}$ fiat radix congruentiae $a x=b(\bmod , m n)$, ex solutione congruentiae $\frac{a m s}{\delta} x^{\prime}+a v \equiv b(\bmod . m n)$ deduci potest, cui aequiualet haec $\frac{g}{\delta} x^{\gamma} \doteq \frac{b-a n}{m}$ (mod $n$ ). Hinc colligitur solutionem congruentiae cuiuscunque primi gradus secundum modulum mi B 2 
reduci posse ad solutionem duarum congruentiarum secundum modulum $m$ et $n_{*}$. Facile aútem perspicietur, si m iterum sit productum e duobus factoribus, solutionem congruentiae secundum modulum $n$ pendere a solutione duarum congruentiarum quarum moduli sint illi factores. Generaliter solutio congruentiae secundum modulum compositum quemcumque pen. det a solutione aliarum congruentiarum, quarum moduli sunt factores illius numeri; hi autem, si commodum esse videtur, ita semper accipi possunt ${ }_{\text {g }}$ vt sint numeri primi.

Ex. Si congruentia I9 $x \equiv 1$ (mod. 140) proponitur: soluatur primo secundum modulum 2, eritque $x \equiv 1$ (mod, 2$)$. Ponatur $x$ $=\mathrm{I}+2 x^{\prime}$, fietque $39 x^{\prime} \equiv-18$ (mod. I 40 ) cui aequitalet $19 x^{\prime} \equiv 9$ (mod.70). Si haec iterum secunidum modulum 2 soluitur, fit $x^{\prime} \equiv \mathrm{r}$ (mod. 2) positoque $x^{\prime}=1+2 x^{\prime \prime}$, fit $38 x^{\prime \prime}$ $\equiv-28$ (mod. 70 ) sine $19 x^{1 / 4} \equiv-14$ (mod. 35). Haec secundum 5 soluta dat $x^{\prime \prime} \equiv 4$ (mod. 5), substitutoque $x^{\prime \prime}=4+5 x^{\prime \prime \prime}$, fit $9^{5} x^{\prime \prime \prime} \equiv-9^{\circ}$ (mod. 35) siue $: 9 x^{\prime \prime \prime} \equiv-18$ (mod. 7 ). Ex hac tánden sequitur, $x^{\prime \prime \prime} \equiv 2$ (mod. 7), positoque $x^{\prime \prime \prime}=2+7 x^{\prime \prime}$ colligitur $x=59+140 x^{1 V} ;$ quare $x \equiv 59$ (mod. 140 ) est solutio completa congruentiae propositae.

31. Simili modo vt aequationis $a x=b$ radix per $\frac{b}{a}$ exprimitur, etiam congruentiae $a x$ $\equiv b$ radicem. quamcunque per ${ }_{a}^{b}$ designabimus, congruentiae modulum, distinctionis gratia, ap- 
ponentes. Ita e.g. $\frac{x g}{17}(\bmod .12)$ denotat quemuis numerum, qui est $x_{1}$ (mod. 12$\left.){ }^{*}\right)$. Generaliter ex praecedentibus patet, $\frac{b}{a}$ (mod.c) mihil reale significare (aut si quis malit aliquid ima ginarii), si $a, c$ habeant diuisorem communem, qui ipsum o non metiatur: At hoc casu excepto, - expressio $\frac{b}{a}$ (mod.c) semper valores reales habebit, et quidem infinitos: hi vero ompes secumdum $c$ erunt congrui quando $a$ ad $c$ primus, aut secundum $\frac{c}{d}$, quando f numerorum $c$, diussor communis maximus.

Hae expressiones similem fere habent algorithmum vt fractiones vulgares. Aliquot pro. prietates quae facile ex praecedentibus deduci possunt hic apponimus.

x. Si secundum modulum $c, a \equiv \alpha, b \equiv b$ ex pressiones $\frac{a}{b}(\bmod . c)$ et $\frac{\alpha}{b}(\bmod . c)$ sunt aequiualentes.

2. $\frac{a}{b j}(\bmod , d)$ et $\frac{a}{b}(\bmod , c)$ sunt aequiua lentes.

5. $\frac{a k}{b k}(\bmod c)$ et $\frac{a}{b}(\bmod . c)$ sunt aequiualentes quando $k$ ad $c$ est primus.

Multae aliae similes propositiones afferri possent: at quum nulli difficultai sint obnoxiae, neque ad sequentia adeo necessariae, ad alia properamus.

32. Problema quod magnum in sequentibus rsum habebit, inuenire omnes numeros, qui sem cundum modulos quotcunque datos residuc data praebent, facile ex praecedentibus solui poteșt. Sint pri-

9) id quod ex analogia per $\frac{\pi}{I}$ (cod. I2) designari potest

B 3 
mo duo moduli, $A, B$, secundum quos numerus quaesitus, $z$, numeris $a, b$ respectiue congruus esse deheat. Ommes itaque valores ipsius z sub forma $d x+a$ continentar, ybi $x$ est indeterminatus sed talis vit fiat $A x+a \equiv b$ (mod. $B$ ). Quodsi iam numerornm $A, B$ diuisor communis maximus est i) resolutio completa huius congruentiae hano habebit formam $x \equiv v\left(\bmod \frac{B}{\delta}\right)$ siue quod eodem redit, $x=v+\frac{k B}{\delta}$, denotante numerum intege um arbitrarium. Hinc formula $A v+\frac{k A B}{\delta}$ omnes ipsius $z$ valores comprehendet, i. e. $z$ $A v(\bmod . A)$ erit resolutio completa problematis. $S i$ ad modulos $A, B$, tertius accedit, $C$, secundum quem numerus quaesitus $x$, debet esse $=c$, manifesto eodem modo procedendum, quum binae priores conditiones in vnicam iam sint conflatae. Scilicet si numerorum $\frac{A B}{\delta}, C$ diuifor communis maximus $=s$, atque congruentiae $\frac{A B}{\partial} x+A v \equiv c(\bmod . C)$ resolutio: $x \equiv v\left(\bmod . \frac{G}{d}\right)$, problema per congruentiam $z \equiv \frac{A B v}{\delta}+A v$ (mod, $\angle B C$ ) complete erit resolutum. Similiter pracedendum, quotcunque moduli proponan. tur. Obseruari conuenit $\frac{A B}{\delta}$, $\frac{A C C}{\delta}$ esse numerorum $A, B$; et $A, B, C$ respectiue minimos communes diuiduos, facileque inde perspicitur, quotcunque habeantur moduli $A, B, C$ etc., si eorum minimus communis diuiduus sit $M$, resolutionem completam hanc formam habere, $z \equiv r$ (mod. $M$ ). Ceterum quando vlla congruentiarum auxiliarum est irresolubilis, problema impossibilitatem inuoluere concludendum est. Perspicuum vero, hoc euenire non posse, quando omnes numeri $A, B, C$ etc. inter se sint primi, 


\section{$-23-$}

Ex. Sint numeri $A, B, C ; a, b, c, 504,55$, $16 ; 17,-4,53 ;$, hic duae conditiones vt $z$, sit $\equiv 1.7(\bmod .504)$ et $\equiv-4(\bmod .35)$ vnicae, vt sit $\equiv 52$ r (mod. 2520) aequiualent; ex qua cum hac: $z \equiv 33$ (mod, 16$)$ coniuncta, promanat $z=304 \mathrm{I}(\bmod .5040)$.

- 35. Quando omnes numeri $A, B, C$ eto inter se sunt primi, constat, productum ex ipsis esse minimum omnibus communem diuiduum. In quo casu manifestum est, omnes congruentias $z \equiv a(\bmod . A) ; z \equiv b(\bmod B)$ etc. vnicae $z \equiv r$ (mod. $\mathbb{R}$ ) prorsus aequiualere, denotante $R$ numerorum $A, B, C$ etc. productum. Hinc' vero vicissim: sequitur, vnicam conditionem $z=r$ (mod: $R$ ) in plures dissolui posse: scilicet si $R$ quomodocunque in factores inter se primos $A, B, C$ etc. resoluitur, conditioness $z \equiv r(\bmod . A), z \equiv r(\bmod . B), z \equiv r(\bmod$. $C)$, etc. propositam exhaurient. Haec obseruatio methodum nobis aperit non modo imposa sibilitatem, si quam forte conditiones propositae implicent, statim detegendi, sed etiam calo culun commodius atque concinmius instituendi.

34. Sint vt supra conditiones propositae; vt sit $z \equiv a(\bmod . A) z \equiv b$ (mod. $B), z \equiv b$ (mod. C). Resoluantur omnes moduli in factores inter se primos, $A$ in $A^{\prime \prime} A^{\prime \prime} A^{\prime \prime \prime}$ etc.; $B$ in $B^{\prime}$ $B^{\prime \prime} B^{\prime \prime \prime}$ etc. etc. et quidem ita vt numeri $A^{\prime \prime}$, $A^{\prime \prime}$ etc. $B^{\prime}, B^{\prime \prime}$ etc. etc. sint aut primi, aut prímorum potestates. Si vero aliquis numerorum $A, B ; C$ etc. iam per se est primus, aut primi potestas, nulla resolutione in factores pro hocce opus esta Tum vero ex praecedentibus pa- 


\section{$-24 \quad-$}

tescit, pro conditionibus propositis hasce sub. stitui posse: $z \equiv a$ (mod. $A^{\prime}, z \equiv a\left(\bmod . A^{\prime \prime}\right)$, $z \equiv a$ (mod. $\left.A^{\prime \prime \prime}\right)$ etc., $z \equiv b$ (mod. $B^{\prime}, z \equiv b$ (mod, $B^{\prime \prime}$ ) etc. etc." Iam nisi omnes numeri $A$, $B, C$ etc. fuerint inter se primi, ex. gr. si $A$ ad $B$ non primus, manifestum est, omnes diuisores primos ipsorum $A, B$ diuersos esse non posse, sed inter factores $A^{\prime}, A^{\prime \prime}, A^{\prime \prime \prime}$ etc. vnum aut alterum esse debere, qui inter $B^{\prime}, B^{\prime \prime}, B^{\prime \prime \prime}$ etc, aut aequalem aut multiplum aut submultiplam babeat. Si primo $A^{\circ}=B^{\prime}$, conditiones $z=a$ $\left(\bmod A^{\prime}\right), z \equiv b\left(\bmod B^{\prime}\right)$ identicae esse de. bent, siue $\equiv b$ (mod. $A^{\prime}$ vel $B^{\prime}$ ), quare alterutra reiici poterit. Si vero pon $a \equiv b\left(\bmod . A^{\prime}\right)$, problema impossibilitatem implicat. Si secundo $B^{\circ}$ multiplum ipsius $A^{\prime}$, conditio $z \equiv a\left(m o d^{\prime} A^{\prime}\right.$ in hac $z=b\left(\bmod B^{\prime}\right)$ contenta esse debet, siue haec $x \equiv b(\bmod$. A) quat ex posteriori deducitur cum priori identica esse debet. Vncie sequitur conditionem $z \equiv a\left(\bmod . A^{4}\right)$, nisi alteri repugnet (in quo casu problema impossibile) reiici posse. Quando omnes conditiones superfluae ita reiectae sunt, patet, omnes modulos ex his $A^{\prime}, A^{\prime \prime}, A^{\prime \prime \prime}$ etc. $B^{\prime}, B^{\prime \prime}, B^{\prime \prime}$ etc etc. Temanentes inter se primos fore: tum igitur de problematis possibilitate certi esse et secundum praecepta ante data procedere possumus.

35, Ex. Si ut supra esse debet $z=17$ $(\bmod .504) ; \equiv-4(\bmod .35)$, et $\equiv 33$ (mod. I6); hae conditiones in sequentes resolui pos. sunt, $z=17(\bmod 8), \equiv 17(\bmod .9), \equiv 17$ $(\bmod 7) ; \equiv-4(\bmod .5), \equiv-4(\bmod 7)$ $\equiv 33$ (mod. 6 ). $\quad \mathrm{Ex}_{x}$ his conditiones $z=7$ (mod. 8$), z \equiv 7$ (mod. 7 ) reiici possunt, 


\section{- $25-$}

quum prior in conditione $z \equiv 33$ (mod. I6) contineatur, posterior vero cum hac $z \equiv-4$ (mod, 7$)$ sit identica; remanent itaque

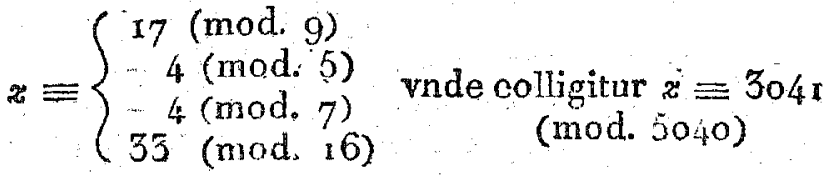

Ceterum palam est, plerumque commodias fore, si de conditionibus remanenribus eaequae ex vna eademque conditione euolutae erant seorsim recolligantur, quum hoc nullo negotio fieri pos. sit; e.g. quando ex conditionibis $z=a\left(\bmod A^{\prime}\right.$ ) $z \equiv a\left(\bmod . A^{\prime \prime}\right)$ etc. aliquae abierunt: quae ex reliquis restituitur, haec erit, $z \equiv a$ secundum modulum qui est productum omnium mo. dulorum ex $A^{\prime}, A^{\prime \prime}, A^{\prime \prime \prime}$ etc. remanentium. Ita in nostro exemplo ex conditionibus $a=-.4$ $(\bmod , 5) z \equiv 4(\bmod .7)$ ea ex qua ortae erant $z \equiv-4(\bmod , 35)$ sponte restituitur. Porro hine sequitur haud prorsus perinde esse, quaeniam ex conditionibus superfinis reliciantur, quantum ad calculi breuitatem: sed haec aliaque artificia practica, quae ex vsu mullo facilius quam ex praeceptis ediscuntur hic tradere non est instituti nostri.

36. Quando omnes moduli $A, B, C, D$ etc. inter se sunt primi, sequenti methodo saepius praestat vii. Determinetur numerus' a secundum $A$ vnitati, secundum reliquorum modulorum productum vero cifrae congruus, siue sit a valor quicunque (plerumque praestat minimum accipere) expressionis $\frac{x}{B C(j \mathrm{Jec}}$ (mod. $\left.A\right)$, per $B C D$ etc, multiplicatae (vid. art. 32 ); similiter sit 
$6 \equiv$ I $(\bmod . B)$ et $\equiv 0(\bmod . A C D$ etc. $), y \equiv$ $I(\bmod . C)$ et $\equiv \mathbf{o}\left(\bmod _{1} A B D\right.$ etc.), etc. Tunc si numerus a desideratur, qui secundum modulus $A, B, C, D$ etc. numeris $a, b, c, d$ etc. respectiue sit 'congruus, poni poterit

$z=\alpha a+c b+\gamma c+\delta d$ etc. (mod. $A B C D$ etc.). Manifesto enim, $a \equiv a$ (mod. $A$.$) ; reliqua au-$ tem membra $\& b, \gamma c$ etc. omnia $\equiv 0(\bmod . A)$; quare $z \equiv a$ (mod. A). Similiter de reliquis modulis demonstratio adornatur. Haec solutio priori praeferenda, quando plura huiusmodi problemata sune soluenda, pro quibus maduli $A, B, C$ etc, valores suos retinent; tunc enim numeri $\omega_{6}, \%$ etc. valores constantes nanciscuntur. Hoc vsu venit in problemate chronologico vbi quaeritur, quatus in periodo Jaliana sit annus, cuius indictio, numerus aureus, et cyclus solaris dantur. Hic $A=\mathrm{i}, 5$, $B=19, C=28$; quare, quum valor expressionis $\frac{x}{x .28}(\bmod 15)$, siue $\frac{x}{532}$ (mod. 15$)$, sit 13 , erit ${ }_{\alpha}=6916$. Similiter pro $\&$ inuenitur 4200 , et pro $r$ 4845, quare numerus quaesitus erit residnum minimum numeri $6966 a+4200$ b +4845 , denotantibus a indictionem, b numerum aureum, c cyclupn solarem.

3\%. Haec de congruentiis primi gradus. snicam incognitam continentibus sufficiant. Su. perest vt de congruentiis agamụs, in. quibus plures incognitae sunt permixtae. At quoniam hoc caput, si omni rigore singula exponere velimus, sine prolixitate absolui non potest, propositumque hoc loco nobis non est, omnia exhaurire, sed ea tantum tradęre, quae atten- 


\section{- 27}

tione digniora videantur: hic ad paucas obseruationes inuestigationem restringimus, vberiorem huius rei expositionem ad aliam occasionem nobis reseruantes.

1) Simili modo, vt in aequationibus, perspicitur, etiam hic totidem congruentias haberi debere, quot sint incognitae determinandae

a) Propositae sint igitur congruentiae

$$
\begin{aligned}
& a x+b y+c z \ldots \equiv f(\text { mod. } m) . . . . .(A) \text {. } \\
& a^{\prime} x+b^{\prime} y+c^{\prime} z \ldots \equiv f^{\prime} \\
& u^{\prime \prime} x+b^{\prime \prime} y+c^{\prime \prime} z \ldots \equiv f^{\prime \prime}
\end{aligned}
$$

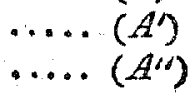

etc.

totidem numero, quot sunt incognitae $z, y, z$ etc。

Iam determinentur numeri $\xi$, $\xi^{\prime}, \xi^{\prime \prime}$ etc ita vt sit

$b z+b^{\prime} z^{\prime}+b^{\prime \prime} z^{\prime \prime}+$ etco $=0$

$c \xi+c^{\prime} \xi^{2}+c^{\prime \prime} \xi^{\prime \prime}+$ etc. $=0$.

etc.

et quidem ita vt omnes sint integri nullumque factorem communem habeant, quod fieri posse ex theoria aequationum linearium constat.

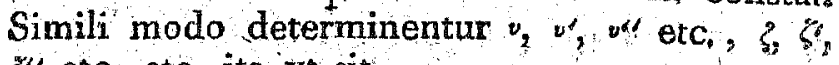
$\xi^{\prime \prime \prime}$ etc, etc. ita vt sit

$a_{v}+a^{\prime} v^{\prime}+a^{\prime \prime} v^{\prime \prime}+$ etc. $=0$.

$c_{v}+c^{\prime \prime} v^{\prime}+c^{\prime \prime} u^{\prime \prime}+$ etc. $=0_{0}$

elc.

$a \zeta+a^{2} \xi^{\prime}+a^{\prime \prime \prime} \xi^{\prime \prime}+e^{t} c_{0}=0$,

$b^{2} \xi^{2}+b^{\prime} \xi^{\prime}+b^{\prime \prime} \xi^{\prime \prime}+$ etc $=0$.

etc. etc.

3) Marifestum est si congruentiae $A, A_{\pi}$.

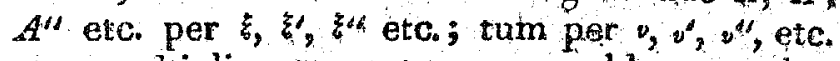
etca multiplicentur, tuncque addantior, has congruentias prouenturas esse: 
$\left(a z+a^{\prime} z^{\prime}+a^{\prime \prime} z^{\prime \prime}+\right.$ etc $) x \equiv f z+f^{\prime} \xi^{\prime}+f^{\prime \prime} z^{\prime \prime}+$ etc. $\left(b v+b^{\prime} v^{\prime}+b^{\prime \prime} v^{\prime \prime}+\right.$ etc. $) y=f v+f^{\prime} y^{\prime}+f^{\prime \prime} v^{\prime \prime}+$ etc. $\left(c \zeta^{\prime}+c^{\prime} \xi^{\prime}+c^{\prime \prime} \xi^{\prime \prime}+\right.$ etc. $) z \equiv f^{\prime} \zeta+f^{\prime} \zeta^{\prime}+f^{\prime \prime} \xi^{\prime \prime}+$ etc. etc.

quas brenitatis gratia ita exhibemus:

$\Sigma(a \xi) x \equiv \Sigma(f \xi)$

$\Sigma(b v) y \equiv \Sigma(f v)$

$\Sigma(c) z \equiv \Sigma(f \dot{\zeta})$ etc.

4) Iam plures casus sunt distinguendi, Primo quando omnes incognitarum coefficientes, $\Sigma(a \xi), \Sigma(a t u)$ etc. ad congruentiarum.modulum $m$ sunt primi, hae congruentiae secundum praecepta ante tradita solui possunt, problematis. que solutio completa per congruentias formae $x \equiv p(\bmod m), y \equiv q(\bmod m)$ etc. exhibebitur * $)$. E. g. Si proponurtur congruentiae $x+3 y+z \equiv \mathrm{I} ; 4 x+y+5 z=7 ; z x$ $+2 y+z \equiv 3(\bmod 8)$, inuenietur $z=9$, $\xi^{\prime}=1, z^{\prime \prime}=-14$, vnde fit $-15 x \equiv-26$; quare $x \equiv 6$ (mod. 8 ); eodem modo inuenitur $15 y \equiv-4,15 z \equiv 1$, et hinc $y \equiv 4, z \equiv 7$ (mod. 8 ).

5) Secwndo quando non omnes coefficientes, $\Sigma$ (a $), \Sigma(b y)$ etc. ad modulum sunt primi,

- Obserpare conuenit hancce conclusionem demonstratione egere, quam atıtem hic supprimimus. Proprie enim nihil aliud ex analysi nostra sequitur, quam quod congruentiąe propositae per alios incognitarum $x, y$ etc. valores splui nequeant: hỏs vero satisfacere non sequitur. Fieri enim posset vt nulla omnino solutio daretur. Similis paralogismus etiam in aquationum linearium explicatione plerumque committitur. 
sint $\alpha, \zeta$, , etc. diuisores communes maximi ipsius $m$ cum $z(a \xi, \quad(b v), 2(c)$ etc. resp., patetque problema impossibile esse, nisi illi numeros $\Sigma(f), 2\left(f_{i} ;, \Sigma(f)\right.$ etc. resp. metiantur. Quando vero hae conditiones locum habent, congruentiae in (3) complete resoluentur per tales $x \equiv$ $p\left(\bmod \cdot \frac{m}{n}\right), y \equiv q\left(\bmod . \frac{m}{6}\right), z \equiv r\left(\bmod \frac{m}{\gamma}\right)$ etc., aut si mauis dahuntur \& yalores dinersi ipsius $x$ (i. e. secundum $m$ incongrui puta $p, p+$ $\frac{m}{\alpha} \ldots p+\frac{(\alpha-I) m}{\alpha}, 6$ valores diuersi ipsius $y$ etc., illis congruentitis satisfacientes: manifestoque omnes solutiones congruentiarum propositárum (si quae omnino dantur) inter illas reperientur. Attamen hanc conclusionem conuertere non licet; nam plerumque non omnes combinationes omnium * valorum ipsius $x$ cum omnibus ipsius $y$ cum omnilbus ipsius $z$ etc. problemati satisfaciunt, sed quaedam tantum, quarum nexum per vnam pluresue çongruentias conditionales exhibere licêtt. At quum completa hutius problematis resolutio ad sequentia non sit necessaria, hoc argumentum fusius hoc loco non exsequimur, exemploque ideam qualemcunque de eo dedisse sat habemus.

Propositae sint congruentiae $3^{x}+5 y+z$ $\equiv 4,2^{x}+3^{y}+2 z \equiv 7,5^{x}+y+3 z \equiv$

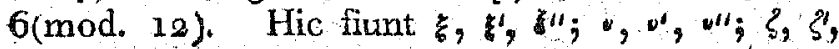
$s^{\prime \prime}$ resp. $=1,-2, x ; 1, x,-1 ;-13,22$ $-I$, vide $4^{x} \equiv-4,7 y \equiv 5,28 z=9^{6}$. Hinc prodeunt quatuor valores ipsius $x$ puta $\equiv$ $2,5,8, \mathrm{II}$; vnus valor ipsius y puta $\equiv \mathrm{II}$; quatuor valores ipsius $z$ puta $\equiv 0,3,6,9$ (mod. I2). Iam yt sciamus, quasmam combina- 


\section{$-50$}

tiones valorum ipsius $x$ cum valoribus ipsius $z$ adhibere liceat, substituimus in congruentiis propp. pro $x, y, z$ resp. $2+3^{t}, \mathbf{I}, 3^{u}$, vnde transeunt in has $57+9 t+3 u \equiv 0,30+6 t+$ $6 u \equiv 0,15+15 t+9 u \equiv 0(\bmod 12)$, quibus facile intelligitur aequiualere has $19+3^{t}+$ $u \equiv 0,10+2 t+2 u \equiv 0,5+5 t+3 u \equiv$ - (mod. 4). Prima manifesto requirit vt sit $u$ $\equiv t+\mathrm{t}$ (mod. 4 ), quo valore in reliquis substituto etiam his satisfieri inuenitur. Hinc colligitur, valores ipsius $x$ hos $2,5,8, x \mathbf{x}$ (qui prodeunt statuendo $t=0,1,2,3$ ) necessario combinandos esse cum valoribus ipsius $z$ his $z$ $\equiv 3,6,9$, o resp., ita vt omnino quatuor solutiones habeantur :

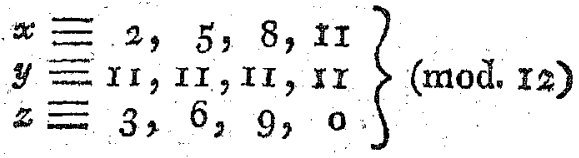

$$
\begin{aligned}
& \text { * * * * }
\end{aligned}
$$

His disquisitionibus, per quas sectionis propositum iam absolutum est, adhac quasdam propositiones similibus principiis innixas adiunginus, quibus in sequentibus frequenter opus erit.

38. Problema. Inuenire, quot numeri po: sitiui dentur numero positiuo dato $A$ minores simulque ad ipsum primi.

Designemus breuitatis gratia multitudinem numerorum posititiorum ad numerum datum primorum ipsoque minorum per praefixum characterem $\phi_{0}$ Quaeritur itaque $\varphi A$. 


\section{- 3.}

I. Quando $A$ est primus, manifestum est omnes numeros ab $\mathrm{I}$ vsque ad $A-\mathrm{I}$ ad $A$ primos esse; quare in hoc casu erit $\varphi A=$ A - I.

II. Quando $A$ est numeri primi potestas puta $=p \mathrm{~m}$, omnes numeri per $p$ diuisibiles ad $A$ non erunt primi, reliqui erunt, Quamobrem de $p^{m}-1$ numeris hi sunt reiiciendi: $p, 2 p_{k}$ $3 p \ldots . .\left(p^{\mathrm{m}-1}-1\right) p ;$ remanent igitur $p^{\mathrm{m}}-1-$ $\left(p^{\mathrm{m}-\mathrm{l}}-1\right)$ siue $\mathrm{p}^{\mathrm{m}-\mathrm{s}}(p-1)$. Hinc $p^{\mathrm{m}}=$ $p^{\mathrm{m}-\mathrm{x}}(p-1)$.

III. Reliqui casus facile ad hos reducuntur ope sequentis propositionis: $S_{i} A$ in factores $M, N, P$ etc. inter se primos est resolutus, erit $\phi A=\phi M_{0} \phi N . \phi P$ etco, quae ita demonstratur. Sint numeri ad $M$ primi ipsoque minores, $m, m^{\prime}, m^{\prime \prime}$ etc. quorum itaque multitudo $=\Phi M$. Similiter sint numeri ad $N, P$ etco respectiue primi ipsisque minores, $n, h^{\prime}, n^{\prime \prime}$ etc. $p, p^{\prime}, p^{\prime \prime}$ etc. etc, quorum multitudo $\phi N \phi P$ etc. Iam constat omnes numeros ad productum $A$ primos etiam ad factores singulos $M, N, P$ etc. primos fore et vice versa (art. 19); porro omnes numeros qui liorum $m, m^{2}, m^{\prime \prime}$ etc. alicui sint congrui secundum modulum $M$ ad $M$ primos fore et vice versa, similiterque de $N, P$ etc. Quaestio itaque huc reducta est: determinare quot dentur numeri infra $A$, qui secundum modalum $M$, alicui numeroruns $m, m^{\prime}, m^{\prime \prime}$ etc. secundkm mos dulum $N$, alicui ex his $n, n^{\prime}, n^{\prime \prime}$ etc. etc. sint congrui. Sed ex art. $5_{2}$ sequitur, omnes nus meros, secundum singulos modulos $M, N, P$ etc. residua determinata dantes, congruos secun- 


\section{$-\quad 52$}

chom eorum productum $A$ fore, adeoçue infra $A$ viicum tantum dari, secundum sinendos $M, N, P$ etc. residuis datis congrutm, Q a are ntmerus quaesitus aequalis erit numero combinationum singulorum numerorum $m, n^{\prime}, n^{\prime \prime}$ cum singulis $n, n, n^{\prime \prime}$ atque $p, p^{\prime}, p^{\prime \prime}$ atcen ato Hunc vero esse $=\varnothing M . \Phi N, \phi P$ etc. ex theoria combinationum constat. Q. E. D.

IV. Iam quomodo hoc ad casum de quo aginus applicandum sit facile intligitur. Kesoluatur $A$ in factores suns primos sine reducatur ad formam $a^{z} b^{6} c^{7}$ etc. designantibus $a$, $6, c$ etc numeros prinos diuersos. 'I'um erit $\phi A=\phi a^{*}, \phi b^{s}, \phi b^{*}$ elc $=a^{x}(a-1) b^{*} t$ $(b-1) c^{y-x}(c-1)$ etc. seu concinutus $\varphi A=$ $A \frac{a-x}{4}+\frac{b= \pm}{b}, \frac{b y}{6}$ etc.

Exempl. Sit $A=60=2^{2} .5 .5$, adeoque

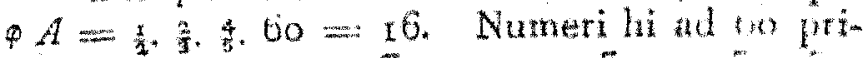
mi sunt $1,7,11,13,17,19,23,29,51,5 \%$ $4 \mathrm{r}, 43,47,49,55,59$.

Solutio prima huius probematis expat in

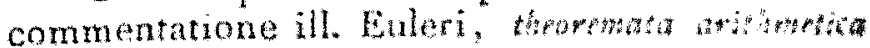
nona methodo denonstrata, Comm sun. 1. Pretrop.

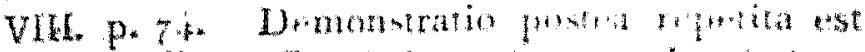

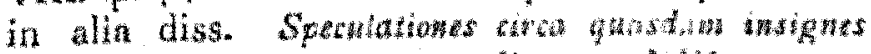
proprietabes numerortim, Acta betrop. 114 p. ${ }^{7} 7$.

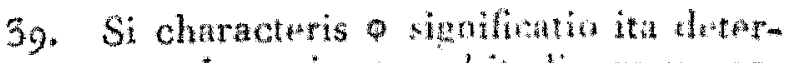
minutur, vt $A$ expinat muhitulinwn mane-

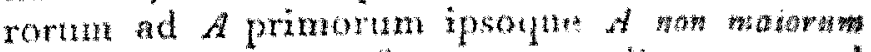
perspicuum est o r fore non amplins:=0, sed De $\mathrm{g}$; in omnibus relitus cakilus whil hine imfinutari. Hince defititionem adoptantes sequen habebimus theorema, 
Si $a, a^{\prime}, a^{\prime \prime}$ etc. swnt onnes diussores ipsius $A$, (vritate et ipso $A$ non cxclusis) erit $\phi a+\varphi a^{\prime}+$ $Q a^{\prime \prime}+e t i_{n}=A$.

Ex. sit $A=30 ;$ tum erit $\phi x+\phi 2+3 \phi$ $+\varphi 5+\varphi 6+410+\varphi 15+\varphi 50=1+$ $1+2+4+2+4+8+8=30$.

Demonstr. Multiplicentur omnes numeri ad a primi ipsoque a non maiores per $\frac{A}{a}$, similiter onines ad $a^{\prime}$ primi per ${ }^{*}$ etc., habebunturque $\varphi a+\varphi a^{\prime}+\varphi a^{\prime \prime}+$ etc. numeri, omnes ipso $A$ non maiores. At

1) omnes hi numeri erunt inaequales. Omnes enim eos qui ex eodem ipsius $A$ diuisore sint generati, inaequales fore, per se clarum. Si vero e diuisoribus diuersis $M, N$ numerisque $\mu, y$ ad istos respectiue primis aequales prodiissent, ${ }^{2}$. si esset $A \mu=\frac{A}{N}$, sequeretur $\mu N$ - M. Ponatur $M>N$ (id quod licet). Quoniam $M$ ad $\mu$ est primus, atque numerum $\mu N$ metitur, etiam ipsum $N$ metietur, maior minorem. Q. E. $A$.

2) inter hos numeros, omnes $h i x, 2,3 \ldots . A$ inuenientur. Sit numerus quicunque ipsum $\mathscr{A}$ non superans $t$, maxima numerorum $A$, $t$ communis mensura $\delta$ eritque $\frac{A}{\delta}$ diuisor ipsius $A$ ad quem $\&$ primus. Manifesto hinc numerus $t$ inter eos inuenietur qui ex diuisore $\frac{\&}{\delta}$ prodierunt: 3) Hinc colligitur horum numerorum multitudinem esse $A$, quare $\phi a+\varphi \sigma^{\prime}+\phi a^{\prime \prime}+$ etc. $=$ A. Q. E. D.

40. Si maximus qunterorum $A, B, C, D$ etc. diuisor commonis $=\mu:$ numeri $a, b, c, d$ etc. ita deter. minari possuts, vt sit $a A+b B+c C+e t c=k$. 


\section{- 34 -}

Dem. Consideremus primo duos tantmm numeros $A, B$, sitque horum diuisor maximus communis $=\lambda$, Tum congruextia $A x \equiv \lambda$ (mod. B) erit resolubilis (art. 30). Sit radix $\equiv *$, ponaturque $\frac{\lambda-A \alpha}{B}=6.6$. Tum erit $a A+$ $G B=\lambda$, vti desiderabatur.

Accedente numero terito $C$, sit maximus divisor communis numerorum $\lambda, C,=\lambda$, eritque hic simul maximus divisor communis numerorum $A, B, C^{*}$ ). Determinentur numeri $k$, $y^{\prime}$ ita vt sit $k \lambda+{ }_{\gamma} C=\lambda^{\prime}$; , eritque $k a A+k b B$ $+\gamma C=\lambda^{\prime}$.

Accedente numero quarto $D$, ponatur maximus dinisor communis numerorum $\lambda^{\prime}, D$ 'quem simul esse maximum diuisorem communem numerorum $A, B, C, D$ facile perspicitur) $=\lambda^{\prime \prime}$, fiatque $k^{\prime} \lambda^{\prime}+\delta D=\lambda^{\prime \prime}$. Tum erit $k k^{\prime} \alpha A+$ $k^{\prime} \cdot{ }^{6} B+k^{\prime} \gamma C+\delta D=\lambda^{\prime \prime}$

Simili modo proced ipotest, guotcunque alii numeri accedant.

Si itaque numeri $A, B, C, D$ etc. diuisorem communem non habent, patet fieri posse a $A$ $+b B+c C+$ etc. $=\mathrm{I}_{0}$

4r. Si $p$ est mumerus primus atque habentur p res, inter quas quotcunque aequales esse possunt, modo non onnes sint aequales: numerus permutationam ha. Fum rersum per perit diuisibilis.

4) Metietur enim manifesto $\lambda l$ omnes $A . B, C$. Si vero non esset diusol commus maximus: maximus forte maior quam $\lambda^{\prime}$ lam quoniam hic diutsor maximis metur ipsos $A$. $B$; metietur etian

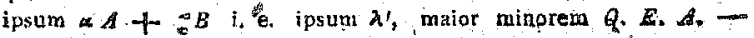
Facilius adauc hoc ex art 18 deduci potest. 
Exempl. Quinque res $A, A, A, B, B$, decem modis diuersis possunt transponi.

Demonstratio huius theorematis facile quidem ex nota permutationum theoria peti potest. Si enim inter has res sunt primo a aequales nempe $=A$, tum $\theta$ aequales nempe $=B$, tum $c$ aequales nempe $=C$ etc. (vbi numeri $a, b, c$ etc. etiam unitatem designare possunt), ita vt habeatur $a+b+c+$ etc. $=p$, numerus

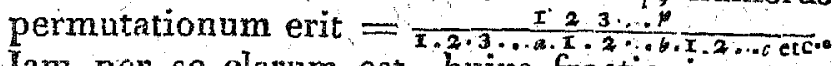
Lam per se clarum est, huius fractionis nume. ratorem per denominatorem diuisibilem esse, quoniam numerus permutationum debet esse integer: at numerator per $p$ diuisibilis est, denominator vero, qui ex factoribus ipso $p$ minoribus est compositus, per $p$ non diuisibilis (art. 15). Quare numerus permutationum per $p$ erit diuisibilis (art. 19).

Speramus tamen fore quibus etiam sequens demonstratio haud ingrata sit futura.

Quando in duabus permutationibus rerum - quibus compositae sunt ordo in eo tantum discrepat, vt ea res quae in altera primum locum occupat, aliam sedem in altera teneat, reliquae autem eodem in vtraque ordine progrediuntur, eamque quae in altera vltima est, ea quae est prima, in altera excipit; jermutationes similes vocemus $\left.{ }^{*}\right)$. Ita in ex. nostro permutationes $A B A A B$ et $A B A B A$ similes, erunt, $\mathrm{C} 2$

- Si permutationes similes in circulum scriptae esse concipiluntur ita vt vltima res prizase fiat contigua, nulla ompino erit discrepantiaz quoniam nullus locus priknus aut vitimus pocari poterit. 


\section{$-36-$}

quoniam res quae in priori primum secundum etc. locum occupant, in posteriori loco tertio quarto etc. eodem ordine sunt collocatae.

Iam quoniam quaeque permutatio ex $p$ re; bus constat, patet cuiuis $p-1$ similes adinueniri posse, si ea res quae prima fuerat, ad secundum, tertium etc. locum promoueatur. Quarum si nullae identicae esse possunt manifestum est, omnium permutationum numerum per $p$ diuisibilem euadere, quippe qui $p$ vicibus maior sit quam numerus omnium permuta. tionum dissimilium. Supponamus igitur duas permutationes $P Q \ldots T V \ldots F Z ; V \ldots$ $T Z P Q \ldots T$, quarum altera ex altera per terminorum promotionem orta sit, identicas esse situe $P=V$ etc. Sit terminus $P$ qui in priori est primus, $n+r$ tus in posteriori. Erit igitur in serie posteriori terminus $n+\mathrm{I}$ tus aequalis primo, $n+2$ tus secundo etc. vnde $2 n+1$ ins rursus primo aequalis euadet, ea. demque ratione $3 u+x$ tus etc.; generaliterque terminus $k n+m$ tus $m$ to (vbi quando $k n+m b$ ipsum $p$ superat, aut series $V \ldots Y Z P Q \ldots T$ semper ab initio repeti concipienda est, aut a $n n+m$ multiplum ipsius $p$ proxime minus rescindendum). Quamobrem si $k$ ita determinatur, vt fiat $k n \equiv \mathrm{I}(\bmod p)$, quod fieri potest quia $p$ primus, sequitur generaliter terminum m tam $m+1$ to aequalem esse, siue quemuis terminum sequenti, i. e. omnes terminos aequales esse contra hypothesin.

42. Si coefficientes $A, B, C \ldots . . N ; a, b, c \ldots$. is duarum functionum formae 
$x^{\mathrm{m}}-1 A x^{\mathrm{m}-\mathrm{r}}+B x^{\mathrm{m}-2}+C x^{\mathrm{m}-3} \ldots \ldots+N \ldots(P)$ $x^{\mu}+a x^{\mu-1}+b x^{\mu-z}+c x^{\mu-3} \ldots . .+n+\ldots . .(Q)$ omes sunt rationales, neque vero omnes integri, proa ductumque ex $(P)$ et $(Q)=$ $x^{m+\mu}+9 x^{m+u-1}+3 x^{m+\mu-2}+e t c_{0}+3:$ omnes coefficizntes $\mathfrak{H}, \mathfrak{B} \ldots .3$ integri esse nequeunt.

Demonstr. Exprimantur omnes fractiones in coefficientibus $A, B$ etc. $a, b$ etc. per numeros quam minimos, eligaturque ad libitum numerus primus $p$, qui aliquem aut plures ex denominatoribus harum fractionum metiatur. Ponamus, id quod licet, $p$ metiri denominatorem alicuius coefficientis fracti in $(P)$, patetque si (Q) per $p$ diuidatur, etiam in $\frac{(Q)}{n}$ dari ad minimum vaum coefficientem fractum cuius deno: minator implicet factorem $p$ (puta coefficientem (primum $\frac{T}{*}$ ). Iam facile perspicitur, in $(P)$ dae tum iri terminum vnum, fractum, cuius denominator inuoluat ptures dimensiones ipsius quam denominatores omnium sinilium praecedentium, et non pauciores quam denominatores omnium sequentium, sit hic terminus $=G x$, et multituda dimensionum ipsius $p$ in denominatore ipsius $G,=t_{0}$ Similis terminus dabitur in $\frac{(Q)}{r}$ qui sit $=\mathrm{r} x^{y}$ et multitudo dimensionum ipsius $p$ in denominatore ipsius $F,=\tau_{\text {。 }}$ Manifesto hic erit $t+\tau$ ad minimum $=2$. His ita praeparatis, terminus $x^{g}+\gamma$ producti ex $(P)$ et $(Q)$ coefficientem habebit fractum, cuius denominator $t+\tau-1$ dimensiones ipsius $p$ inuoluet, id quod ita demonstratur.

Sint termini qui in $(P)$ terminum $G x s$ praecedunt, 'G $x^{\xi+1}, "{ }^{G} x^{8+2}$, etc. sequentes vero $G^{\prime} x^{t-1}, G^{\prime \prime} x^{g-2}$ etc.; similiterque in $\left(\frac{Q}{p^{2}}\right)$ 


\section{$-38-$}

praecedant terminum $\Gamma x^{\gamma}$ termini $\Gamma x^{\gamma+1}$, $" \Gamma x \gamma+2$ etc. sequantur autem termini $\Gamma^{\prime} x \gamma-x$, $\Gamma^{\prime} x^{\gamma-2}$ etc. Tum constat in producto ex $(P)$, $\frac{(Q)}{y^{2}}$ coefficientem termini $x^{g+\gamma}$ fore $=G \Gamma$

$$
\begin{aligned}
& +{ }^{\prime} G \Gamma^{\prime}+" G{ }^{\prime \prime}+\text { etc. } \\
& +\int G^{\prime}+" \Gamma G " \text { etc. }
\end{aligned}
$$

Pars $G$ r erit fractio quae si per numeros quam minimos exprimitur in denominatore $t+\tau$ dimensiones ipsius $p$ inuoluit, reliquae autem partes si sunt fractae, in dominatore pauciores dimensiones numeri p implicabunt, quoniam omnes sunt producta e binis factoribus quorum alter non plures quan $t$, alter vero panciores quam $r$ dimensiones ipsius $p$ implicat; vel alter non plures quam $z$; alterque pauciores quam to Hinc $G r$ erit formae $\frac{f^{t}}{f p^{t}{ }^{T}}$, reliquarum vero summa formae $\frac{\theta^{\prime}}{f_{p} t-i-\delta-\delta}$, vbi $\delta$ posizus et $e, f, f^{\prime}$ a factore $p$ liberi: quare omnium summa erit $=\frac{e f^{\prime}+e^{\prime} f p^{\delta}}{f^{f} p^{t}+\frac{p_{y}}{r}}$ cuius numerator per $p$ non dinisibilis, adeoque denominator per nullam reductionem pauciores dimensiones quam. $t+*$ obtinere potest. Hinc coefficiens termini

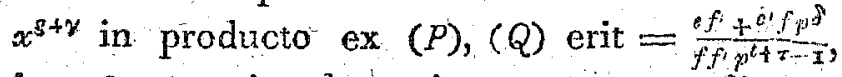
i. e fractio cuius denominator $t+\tau-1$ dimensiones ipsius o implicat. Q. E. D.

43. Congruentia anti gradus, $A x^{\mathrm{m}}+B x^{\mathrm{m}-\mathbf{1}}$ $+C x^{\mathrm{m}-2}+e t c .+M x+N \equiv 0$, cuins modulus est sumerns primus $p$, ipsum $A$ non meitens, pluribus quan modis dizersis solwi non potest, sine pluves quam $n$ radices secundum $p$ incongruos non habet (Vid. artt. 25, a6) 


\section{$-39-$}

Si quis neget, ponamus dari congruentias dinersocum graduum $m$, $n$, etc. quae plures quam $m, n$ etc. radices habeant, sitque minimus gradus, $m$, ira vt omnes similes congruentiae inferiorum graduum theoremati nosiro sint consentaneae Orod quum de primo grado iam supra sit demonstratum (art. 26), manifestum est, $m$ fore aut $=2$ aut maiorem. Admitret itaque congruentia $A x^{\mathrm{m}}+B x^{\mathrm{m}-\mathrm{I}}+$ etc. $+M x+N \equiv 0$ saltem $m+\mathrm{I}$ radices, quae sint $x$ $\equiv{ }^{\infty}, x \equiv b, x \equiv \gamma$ eto, ponamusque id quod licet omnes numeros $x, 6, \gamma$ etc. esse positiuos et minores quarn $p$, omniumque minimum $\propto$ Iam in congruentia proposita substituatur pro $*, y+\alpha$, transeatque inde in hanc $A^{\prime} y^{\text {in }}+B^{\prime} y^{\text {m-1 }}+C^{\prime} y^{m-2} \ldots+M^{\prime} y+N^{\prime \prime} \equiv 0 。$ T'um manifestum est, huic congruentiae satis. fieri deberi, si ponatur $y \equiv 0$, aut $\equiv-\infty$, aut $\equiv \gamma-\infty$ etc., quae radices omnes erunt diuersae, numerusque earum $=m+\mathrm{r}$, At ex eo quod $y \equiv a$ est radix, sequitur, $N^{s}$ per $p$ diuisibilem fore. Quare etirm haec expressio, $y\left(A^{\prime} y^{\mathrm{m}-1}+B^{2} y^{\mathrm{m}-2}+\right.$ etc $\left.+M^{\prime}\right)$ Get $=0$ (mod. $p$ ), si ipsi y vnus ex m valoribus, 6-as $\gamma-$ etc. tribuitur, quiomres sunt $>0$ et $<p$, adeoque in ombibus hisce casibus etian $A^{\prime} y^{\mathrm{m}-\mathrm{r}}+$ $B^{\prime} y^{\text {ma }}+$ +etc. $+M M^{\prime}$ fiet $\equiv 0$ art. 22 ; i. e congruentia $A^{\prime} y^{\mathrm{m}-\mathrm{I}}+B^{\prime} y^{\mathrm{m}-\mathrm{z}}+$ etc. $+W^{\prime} \equiv 0$, quae est gradus $m-i$ ti, $m$ radices habet et proin theoremati nostro aduersatur (patet enim facile, $A$ fore $=A$, adeoque per $p$ non diuisibilem, vti requiritur) licet supposuerimus, omnes congruentias, inferioris gradus guam $n{ }^{t}$, theoremati consentire. Q.E.A. 


\section{$-40-$}

44. Quamuis hic supposuerimus, modu$\operatorname{lum} p$ non metiri coefficientem termini summi, tamen theorema ad hunc casum non restringitur. Si enim primus coefficiens siue etiam aliqui sequentium per $\varphi$ diuisibiles essent, hi termini tuto reiici possent, congruentiaque tandem ad inferiorem gradum deprimeretur, vbi coefficiens primus per $p$ non amplius foret diuisibilis, siquidem non omnes coefficientes per $p$ dividi possunt; in quo casu, congruentia foret, identica atque incognita prorsus indeterminata.

Theorema hoc primum ab ill. La Grange propositum atque demonstratum est (Nem. de "Ac, de Berlin, Année 1768 p. 192.). Exstat etjam in dissert. ill. Le Gendre, Recherches d" Analyse icdeterminée, Hist. de l'Acado do Paris 1785 . 9. 406. Ill. Euler in Nou, Comm. Ac. Petr. XVIII. p. 93 demonstrauit congruentiam $x^{\mathrm{n}}-1 \equiv 0$ plures quam $n$ radices diuersas habere non posse. Quae quamuis sit particularis, tamen methodus qua vir summus vsus est omnibus congruentiis facile adaptari potest. Casum adhuc magis limitatum iam antea absoluerat, Comm. Ac. Petr. V. p. 6, sed haec methodus generaliter adhiberi nequit. Infra Sect. VIII, alio adhuc modo theorema demonstrabimus; at quantumuis diuersae primo aspectu omnes hae methodi videri possint, periti qui comparare eas voluerint facile certiores fient omnes eidem principio superstructas esse. Ceterum qaum hoc theorema hic tantum tamquam lemma sit considerandum, neque completa expositio huc pertineat: de modulis compositis seorsim agere supersedemus. 


\section{$-4 \mathrm{r}-$}

Segtro Tertia

DE

RESIDVIS POTESTATVM.

45. Theorema. $I_{12}$ omeni progressione geonuetrica, $x, a, a a, a^{3}$ etc proteter primum $\mathrm{r}$, alius adhas datur terminus, at, secundun modulum $p$ ad a. primum vnitati congruss, cuizus exponens $t<p$.

Demonstr. Quoniam modulus $p$ ad $a$, adeoque ad quamuis ipsius a potestatem est primus, nullus progressionis terminus erit $\equiv 0$ (mod. p.), sed quiuis alicui ex his numeris $1,2,3 \ldots p-x$ congruas, Quorum multitudo quum sit $p-1$, manifestum est, si plures quan $p-1$ progressionis termini consilerentur, omnes residua minima diuersa habera non posse. Quocirca inter terminos $1, a, d a$, $a^{3} \ldots a^{p-r}$ bini ad minimum congrui inue. nientur. Sit itaque $a^{m} \equiv a^{n}$ et $m \triangleright n$, fietque diuidendo per $a^{n}, a^{m-n} \equiv 1$ (art.22) vbi $n-$ $q<p$, et $>0 . Q$ Q. E. D.

$E x$. In progressione $\mathrm{r}, 2,4,8$ etc. terminus primus qui secundum modulum is vnitatí C 5 
est congruus, invenitur $2^{12}=4096$. At sem cundum modulum 23 in eadem progressione fit $2^{I I}=20+8 \equiv 1$. Similiter numeri 5 potestas sexta, 15625, vnitati congrua secundum modulum 7 , quinta vero, 3125 , secundum ir. In alis igitur casibus potestas expsnentis minoris gram $p-x$ vnitati congrua euadit, in alis contra vsque ad potestatem $p-$ I tam ascendere necesse est.

46. Quando progressio vltra terminum qui rnitati est congruus contiriuatur, endem quae ab initio habebantur residua prodeunt iterum. Scilicet si $a^{t} \equiv \mathrm{x}$, erit $a^{\mathrm{t}+\mathrm{I}} \equiv a^{a}, a^{\mathrm{t}+2} \equiv$ ac erc. donec ad terminum a perueniatur, cuius residuum minimum iterum erit $\mathrm{x}$, atque residuorum periodum denuo inchoat. Habetur itaque periodus $t$ residua comprehendens, quae simulac finita est ab initio semper repetitur; neque alia residua quam quae in hac periodo continentur in tota progressione occurrere pos. sunt. Generaliter erit $a^{\mathrm{mt}} \equiv 1$, et $a^{\mathrm{nt}+\mathrm{n}} \equiv a^{\mathrm{n}}$, id quod per designationem nostram ita exhibetur:

Si $r \equiv \rho($ modo $t)$ erit

$$
a^{r} \equiv a^{m}(\bmod , p)
$$

47. Petitur ex hoc theoremate compendium potestatum quantumuis magxo exponente affectarum residua expedite inueniendi, simulac potestas vnitati congroa innotescat. Si ex. gro residum e divisione potestatis $3^{1000}$ per 03 oriundum ghaeritur, erit propter $3^{3} \equiv$ (mod. :5), $t \equiv 3$; quare quum sit $1000 \equiv \mathbf{I}$ mod. 3.), erit $3^{2000} \equiv 3$ (mod. 13).

48. Quando at est infina potestas vnitati congerua (praeter $a^{\circ}=1$, ad quem casuin hic 


\section{$-43 \quad$}

non respicimus), illi $t$ termini, residuorum periodum conerimentes omnes erunt diuersi, vit ex demonstratione art. 45 nullo negotio perspicitur. Tum autem propositio art. 46 conuerti potest; scilicet si $a^{\mathrm{m}} \equiv a^{\mathrm{n}}$ (mod. p) erit $n \equiv n$ (mod. $t$ ). Si enim $m, n$ secundurn modulim $t$ incongrui essent, residua eorum minima, $\mu$, diuersa forento At $a^{k} \equiv a^{m}, a^{y} \equiv a^{\mathrm{n}}$, cquare $a^{k}$

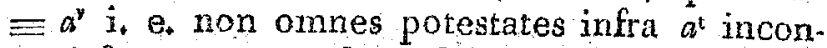
grui forent contra hypoth.

Si itaque $a^{k} \equiv \mathrm{r}$, (mod. p), erit $k \equiv 0$ (mod. $t) i_{0} e_{0}{ }^{k}$ per $t$ diuisibilis.

Hactenus de modulis quibrascunque si modo ad a sint primi diximus. Inm modulos qui sunt numeri absolute primi seorsim considere. mus atque huic fundamento inuestigationem generaliorem postea superstruamus.

49. Theorema si $p$ est numerts primus ipsum a uon metiens, atgue a infina ipsits a potestas secundwin modulum $p$ vnitati congrual, exponeris $t$ aut erit $=p-x$ aut pars aliquota huius numeri.

Conferantur exempla art. praec.

Demorstr. Quum ian ostensum sit, $t$ esse aut $=y-\mathrm{x}$, aut $\langle p-\mathrm{x}$, superest, vt in poi steriori casu $t$ semper ipsius $p-1$ partem aliquotam esse euincatur.

I. Colligantur residua minima positita omnium horum terminorum, $1, a, a a, \ldots a^{t-7}$, quae per $\alpha, \alpha, \alpha^{\prime \prime}$ etc. designentur, ita vt sit " ${ }_{\mathrm{I}}, \alpha^{\prime} \equiv a, \alpha^{\prime \prime} \equiv a a$ etc. Perspicuum est, haee omnia fore diuersa, si enim duo termini $\mathrm{a}^{\mathrm{m}}, \mathrm{an}^{\mathrm{m}}$ 
eadem praeberent, foret (supponendo $n>n$ ), $a^{m-n}=1$ atque $m-n<t, Q . E . A$. quum nulla inferior potestas quam $a^{t}$ vnitati sit congrua, inp. Porro omnes $\alpha^{\prime} \alpha^{\prime \prime}$ etc. in serie numerortum $1,2,3 \ldots p-x$ continentur, quam lamen non exhaurient, quum $t<p-x$. Comploxum omnium $\alpha, \alpha^{\prime}, \alpha^{\prime \prime}$ etc. per $(A)$ designa. bimus. Comprehendet igitur $(A)$ terminos $t$.

II. Accipiatur numerus quicunque $6 \mathrm{ex}$ his $x, 2,5 \ldots p-x$, qui in $(A)$ desit. Multiplicelur : per omnes $\alpha, \alpha^{\prime}, \alpha^{\prime \prime}$ etc., sintque residua minima inde oriunda $6 \%$, etc., quorum numerus etiam erit $t$. At haec residua tum inter se quam ab omnibus $*$, " $"$ "ll ete erunt diuersa. Si enim prio assertio falsa esset, haberetur $: a^{m}=a^{\mathrm{n}}$ adeoque diuidendo per $6, a^{\text {th }}$ $a^{n}$, contra ea quae nodo demonstramimus; si voro posierior, haberetur $6 a^{2 n} a^{n}$, wnde, quando $n<n, 6=a^{n-m}$ i. e. 6 alicut ex his $x x^{\prime}, w^{n}$ tet. congrums contra hyp.; quancio vero $m>n$, sequilur multiplicando per $a^{-m}$, fas a a $a^{2}$ siun propter $a x=1,6 a^{2}+1-m$, quae est androm absurditas. Designetur complexus omnium 5, etc. quorum multitudo $=t$, per $(B)$, habe. bunturque iam $2 t$ numeri ex his $x, x^{\circ}, 5 .$. -1. Quodsi igitur $(A)$ et $(B)$ ommes hos numeros complectuntur, fit $z^{*}=1$ adeoque theorema demonstratum.

III. Si vero aliqui adhuc deficiunt, sit ho* rum aliquis $\%$ Per lune multiphicentur mmes ${ }_{\alpha},{ }^{\prime \prime}$ etc., productorumgue residua minima sint $\gamma=y^{\prime} y^{\prime \prime}$ etci omnum complexus per (C) designetur. (C) igitur comprehendet numeros 
ex his $\mathrm{r}, 2,3 \ldots p-\mathrm{r}$, quae omnes tum inter se quam a numeris in $(A)$ et $(B)$ contentis erunt diuersi. Assertiones priores eodem modo demonstrantur vt in II, tertia ita. Si esset $y a^{\mathrm{m}} \equiv 6_{a n}$, fieret $y \equiv \sigma^{\mathrm{n}-\mathrm{m}}$, aut $\equiv 6 a^{\mathrm{t}+\mathrm{n}-\mathrm{m}}$ prout $m<n$, aut $>n$, in vtroque casu $\gamma$ alicui ex $(B)$ congrua contra hyp. Habentur igitur 3 t numeri ex his $x, 2,3 \ldots p-x$, atque si nulli amplius desunt, fiet $t=\frac{p-x}{3}$ adeoque theorema erit demonstratum.

IV. Si vero etiamnum aliqui desunt eodem modo ad quartum numerorum complexum, $(D)$, progrediendum erit etc. Patet vero quoniam numerorum $x, 2,3 \ldots p-1$ multitudo est finita, tandem eam exhaustum iri, adeoque multiplum ipsius fore: quare $t$ erit pars aliquota numeri $p-$ I. Q. E. $D$.

5o. Quum igitur $\frac{x}{t}$ sit integer, sequitur euehendo vtramque partem congruentiae $a_{t} \equiv \mathrm{r}$ ad potestatem exponentis $\frac{x_{-1}-x}{r}, a^{p-x} \equiv 1$, siue $a p-1$ - I semper pey $p$ diusibibilis est, quando $p$ est primus ipsum a non metietss.

Theorema hoc quod tum propter elegantiam tum propter eximiam vtilitatem omni attentione dignum, ab inuentore theorema Fermatianums appellari solet. Vid. Fermatii Opera Mathem. Tom tosat 1679 fol. p. 163. Demonstrationem inuentor non adiecit, quam tamen in potestate sua esse professus est. IIl. Euler primus demonstrationem publici iuris fecit, in diss. cui titulus Thearrosatum quorundam ad thumeros primos spectantium demonstratio, Comm. Acad. Petrop. T. 
VIII $)^{*}$. Innititur ista euolutioni potestatis $(a+1)$, vbi ex coefficientium forma facillime deducitur $(a+x)^{p}-a^{p}-\mathbf{r}$ semper per p fore diuisibilem, adeoque $(a+1)^{p}-(a+1)$ per $p$ dinisibilem fore, quando $a^{\mathrm{p}}-a$ per $p$ sit diuisibilis. Iani quia $1 p-1$ semper per $p$ diuisibilis est, etiam $2^{p}-2$ semper erit; hinc etiam $3 p$ -3 etc. generaliterque $a^{p}-a$ Quodsi itaque $p$ ipsum $a$ non metitur, etiam $a^{p-1}-1$ per $p$ diuísibilis erit. Haec sufficient ad methodi indolem declarandam. Clar. Lambert similem demonstrationem tradidit in Actis Eruditt. 1769. p. Iog. Quia vero euolutio potestatis binomii a theoria numerorum satis aliena esse videbatur, aliam demonstrationem ill. Euler imuestiganit quae exstat Comment. nou. Petr. T. VII p. 70, atque cum ea quấm nos art. praec. exposuimus prorsus conuenit. In sequentibus adhuc aliae quaedam se nobis offerent. Hoc loco vnam superaddere liceat, quae similibus principiis innititur, vti prima ill. Euleri. Propositio sequens, cuius casus tantum particularis est theorema nostrum, etiam ad alias inuestigationes infra adhibebitur.

Polynomii $a+b+c+$ stc. potestas p ta secun dum modulaim $p$ est $\equiv a^{\mathrm{P}}+b^{\mathrm{p}}+\mathrm{c}^{\mathrm{P}}+$ etc, siquider $p$ est numerus qrimus.

-) In colument. anteriore vir summus ad scopum nondum peruenerat; Com. Peít. T. Vl. p. IOG. - In controuersia famosa inter Maumpertuís et König, a principio actionis minimae orta, sed mox ad res heterogeneas egressa, König in maubus se habere dixit antographum Leibnitiantu, in quo demonstratio hutus theormatis cum Euleriana prorsus conspirans contipeatur. Appel au public. p. 'IO6. Licet vero fitem huic testimonio denegare noliwns, certe Leibuitius inuentum sum numquam publicauit. Conf. Hist, do $l$ 'Aci de Prusse. d. 7750.7350 . 
Demonstr. Constat potęstatem $p^{\text {tam }}$ polynomii $a+b+c+$ etc. esse compositam e partibus formae $\times a^{x} b^{6} c^{y}$ etc. vbi $\alpha+6+\gamma$ etc. $=$ $p$, et $*$ designat, quot modis $p$ res, quarum $\alpha$, $\epsilon, \gamma$ etc. respectiue sunt $=a, b, c$ etc. permutari possint. At supra art. $4 \mathrm{i}$ ostendimus, hunc numerum semper esse per $p$ diuisibilem, nisi omnes res sint aequales, i, e. nisi aliquis mo merorum $\alpha, 6, \gamma$ etc. sit $=p$ reliqui vero $=0$ Vnde sequitur omnes ipsius $(a+b+c+$ etc.) partes, praeter has $a^{\mathrm{p}}, b \mathrm{p}, \epsilon^{\mathrm{p}}$ etc., per $p$ diuisibiles esse; quae igitur quando de congruen. tia secundum modulum $p$ agitur, tuto omitti poterunt, fietque $(a+b+c+\text { etc. })^{p} \equiv a^{p}+b^{p}+$ CP + etc. Q. E. D.

Quodsi iam omnes quantitates $a, b, c$ etc. $=1$ ponuntur, numerusque earum $=k$, fiet ap $\equiv k$ vti in art praec.

52. Quoniam igitur alii numeri quam qui cunt divisores ipsias $p-x$ nequeunt esse exponentes potestatum infimarum ad quas" euecti numeri aliqui vnitati congrui fiunt, quaestio sese offert, num omnes ipsius $p-1$ diuisores ad hoc sint idonei, atque, quando omnes numeri per $p$ non diuisibiles secundum exponen. tem infimae suae potestatis vnitati congruae classificentur, quot ad singulos exponentes sint peruenturi. Vbi statim observare conuenit, sufficere, si omnes numeri positiui ab \& vsque ad $p-x$ considerentur; manifestum ${ }^{*}$ enim est, $n u$. meros congruos ad eandem potestatem eleuari debere, quo vnitati fiant congruae, adeoque numerum quemcunque ad eundem exponentem esse referendum ad quem residuum sum mi- 


\section{$-48 \quad-$}

nimum positiuum. Quocira in id nobis erit incumbendum, vt quomodo hoc respectu numaxi $f, 2,3 \ldots \ldots-1$ inter singulos $p-1$ factores distribuendi sint eruamus. Breuitatis gratia, si $d$ est vnus e diuisoribus numeri $p-1$ (ad quos etiam $I$ et $p-1$ referendi), per $\psi d$ designabimus multitudinem numerorum positiuorum ipso $p$ minorum quorum potestas dite est infima vnitati congrua.

53. Quo facilius haec disquisitio intelligi possit, exemplum apponimus. Pro $p=19$ distribuentur numeri $1,2,3 \ldots 18$ inter diuiso. res numeri 18 hoc modo:

$$
\begin{array}{l|l}
11 & \text { I. } \\
3 & 8 . \\
5 & 7,11 . \\
6 & 8,12 . \\
9 & 4,5,6,9,16,17 . \\
18 & 2,5,10,13,14,15 .
\end{array}
$$

In hoc igitur casu fit $\psi \mathrm{I}=\mathrm{x}, \psi_{2}=\mathrm{x}, \psi 3=2$, $\psi 6=2, \psi_{9}=6, \psi 18=6$. Vbi exigua attentio docet, totidem ad quemuis exponentem pertinere, quot dentur numeri hoc non inaiores ad ipsumque primi, siue esse in hoo certe casu, retento signo art. $40, \psi d=\phi d$. Hanc autem obseruationem generaliter veram esse ita demonstramus.

I. Si numerus aliquis habetur, $a$, ad exponentem $d$. pertinens (i. $\boldsymbol{\varepsilon}$. cuius potestas $d^{\text {th }}$ vnirati congrua, omnes inferiores incongruae), omines huius potestates, $a b, c^{3}, a^{4} \ldots \ldots a^{\mathrm{d}}$ siue ipssarum residua minima proprietatem priorem etiam possidebunt (vt potestas ipsarum d $d^{\text {ta }}$ vnitati sit congrua) et quum hoc ita etiam expri- 


\section{- $49-$}

mi possit, residua minima numerorum $a_{3} a a_{9}$ $a^{3}$..... $a^{\mathrm{d}}$ (quae omnia sunt diuersa) esse radices congruentiae $x^{\mathrm{y}} \equiv \mathrm{I}$, haec autem plures quam $d$ radices diuersas habere nequeat, manifestum est, praeter numerorum $a, a a, a^{3} \ldots$ $a^{d}$ residua minim alios numeros inter $i$ et $p-1$ incl, non dari quorum potestates exponentis do congruae sint vnitati. Hinc patet omnes numeros ad exponentem $\boldsymbol{d}$ pertinentes inter residua minima numerorum $a, a a, a^{3} \ldots \ldots d^{d}$ reperiri. Quales vero sint, quantaque eorum multitudo ita definitur. $S i$ d primus, omnes potestates ipsius $a^{\mathrm{k}}$, quarum exponentes $\langle d$, vnitati non erunt congrui : esto enim $\frac{x}{k}(\bmod d) \equiv m$ (vid. art. $\left.3_{r}\right)$ eritque $a^{\mathrm{km}} \equiv a$; quare si potestas $e^{\text {ta }}$ ipsius $a^{\mathrm{k}}$ vnitati esset congrua atque $e<d$, foret etiam $a^{\mathrm{kmi}} \equiv \mathrm{r}$ et hinc $a^{e} \equiv \mathrm{I}$ contra hyp. Hinc manifestum est, residuum minimum ipsius $a^{k}$ ad exponentem $d$ pertinere. Si vero $k$ diuisorem aliquem, d) cum d communem habet, ipsius $a^{k}$ residuum minimum ad exponentem $d$ non pertinet quoniam tum potestas sta iam vnitati fit congrua (erit enim $\frac{k d}{d}$ per $d$ diuisibilis, siue $\equiv$ - (mod. $d$ ) adeoque $a^{\frac{k d}{d}} \equiv \mathrm{I}$ ). Hinc colligitur, totidem numeros ad exponentem \& pertiner quot numerorum $\mathrm{r}, 2,3 \ldots d$ ad $d$ sint primi. At memorem esse oportet, hanc conclusionem innixam esse suppositioni, vnum, numerum $a$ iam haberi ad exponentem $d$ pertinentem. Quamobrem dubium remanet, fierine possit vt ad aliquem exponentem nullus omnino numerus pertineat; conclusioque eo liahitatur vt $\psi d$ sit vel $=0 \mathrm{vel}=\phi d$ 。 


\section{- 50}

54. II. Iam sintomnes diuisores numeri $p-$ I hi: $d, d^{\prime}, d^{\prime \prime}$, etc. eritque, quia onnes numeri $x, 2,3 \ldots p-x$ inter hos sunt distributi, $\dot{\psi} d$ $+\psi d^{\prime}+\psi d^{\prime \prime}+$ etc. $=p-x$. At in art. 40 demonstrauimus esse $\varphi d^{\prime}+\phi d^{\prime}+\phi d^{\prime \prime}+$ etc. $=p,-\mathrm{x}$, atque ex art. praec. sequitur $\psi d d$ ipsi $d^{d}$ aut aequalem aut ipso minorem esse, majorem esse non posse, similiterque de $\psi d^{\prime}$ er $\phi d$, etc. Si itaque aliquis terminus $e x$ his $\psi d, \psi d^{d}, \psi d^{\prime \prime}$ etc. termino respondente ex his $\varphi d, \phi d^{\prime}, \varphi d^{\prime \prime}$, esset minor (sive etiem plures) illorum summa summa horum aequalis esse non posset. Vnde tandem conclidimus $\$$ i ipsi od semper esse aequdem, adeoque a magnitudine ipsius $p-1$ non pendere.

55. Maximam autem attentionem meretur casus particularis propositionis praecedentis scilicet semper dari numeros quorum nutla potestas inferior quam $q$ - I to vaitati congrat, et quidem totidem inter $x$ et $p-x$ quot infra $p-1$ sint numeri ad $p-z$ primi. Cuxus theorematis demonstratio quum minime tam obuia sit quam primo aspectu videri possit, propter theorema* tis dignitatem liceat alian adhuc adiicere a praecedente aliquantmm diuersam, quandoquidem methodotum diuersitas ad res obseuriores illustrandas plurimum conferre solet, Resol. uatur $p-x$ in factores suos primos fintque $p-s$ $=a^{*} b^{*} c^{\gamma}$ etc., designantibus $a, b$, c etc. nut meros primos inaequales. Tum theorematis demonstrationem per sequentia absoluemus:

I. Semper inueniri posse numerum $A_{\text {, }}$ (aut plures), ad exponentem a pertinentem, 


\section{$-58$}

similiterque numeros $B, C$ etc. ad exponentes $b_{b}, c^{\gamma}$ etc. respective pertinentes.

II. Productum ex omnibus numeris $A_{\text {o }}$ $B, C$ etc. (sine huius producti residuum minimum) ad exponentem $p-I$ pertinere. Haec autem ita demonstramus.

I. Sit $g$ numerus aliquis ex his $I, 2,5 \ldots$ $p-I$, congruentiae $x^{x-\underline{x}} \equiv 1$ (mod. $p$ ) non sam tisfaciens, omres enim hi numeri congruentiae huic, cuius gradus $<p-I$, satisfacere nequeunt. Tum dico si potestas $\frac{p-I}{n-1}$ ta ipsius $g$ ponatur $\equiv h$, hunc numerum, siue eius residuum mininum ad exponentem $a^{\alpha}$ pertinere.

Namque patet potestatem $a^{x}$ tam ipsius congruam fore potestati $p-x$ tre ipsius $g$. $\varepsilon_{0}$ vnitati, potestas vero $a^{\alpha-x t a ~ i p s i u s ~}$ a congrua erit potestati $\frac{w-x}{a t}$ tae ipsius $g, i$. $\ell$. vnitati erit incongrua, multoque minus potestates $a^{\alpha-2}$, $a^{\alpha-3}$ the etc. ipsius $h$ vnitati congruae esse possunt. At exponens infimae potestatis ipsius $h_{8}$ vnitati congruae, siue exponens ad quem per-. tinet $h$, numerum $a^{*}$ metiri debet (art. 48). Quare quum $a^{\text {s }}$ per alios numeros diuisibilis non sit quam per se ipsum, atque per inferio. res ipsius a potestates, necessario $a^{\alpha}$ erit exponens ad quem $h$ pertinet. Q.E.D. Per similem methodum dernonstratur, dari numeros ad exponentes $b^{\vec{b}}, c^{y}$ etc. pertinentes.

II. Si supponimus, productum ex omnibus $A, B, C$ etc. non ad exponentem $p-x$, sed ad minorem pertinere, $i$ ipsum $p-1$ metietur (art. 48), sine erit $\frac{p_{-x}}{t}$ integer vnitate maior. Facile autem perspicitur, humc quotientem vel

D. $\&$ 


\section{$-52-$}

esse vnum e numeris primis $a, b, c$ etc. vel saltem per aliquem eorum diuisibilem (art. 17 \%, ex. gr. per $a$, de reliquis enim simile est ratiocinium. Metietur itaque $t$ ipsum $\frac{p-x}{x} ;$ quare productum $A B C$ etc. etiam ad potestatem $\frac{p-x}{\alpha}$ tan eleuatum vnitati erit congruum (art. 45) " Sed perspicuum est singulos $B, C$, etc. (exemto ipso $A$ ) ad potestatem $\frac{x-I}{a}$ tam eleuatos vnitati congruos feri, quum exponentes $b^{b}, c^{\nu}$, etc. ad quos singuli pertinent ipsum metiantur. Hinc erit $A^{\frac{v-x}{a}} B^{\frac{p-I}{a}} C^{\frac{p-x}{a}}$ etc $\equiv A^{\frac{p-\pi}{a}} \equiv \mathbf{I}$. Vnde sequitur exponentem ad quem $A$ pertinet ipsum $\frac{p-I}{a}$ metiri debere (art. 48), i. e.

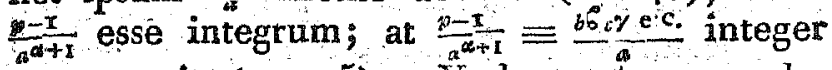
esse nequit (art. 15). Vnde tandem conclu. dere oportet, suppositionem nostram consistere non posse, i. eo productum $A B C$ etc. reuers ad exponentem $p-I$ pertinere, Q. E. $D$.

Demonstratio posterior priori aliquantulum prolixior esse videtur, prior contra posterioni minus directa.

56. Hoc theorema insigne exemplumi suppeditat, quanta circumspectione in theoria numerorum saepenumero opus sit, ne, quae non sunt, pro certis assumamus. Celeb. Lambert in diss. jam supra laudata Acta Erudit. 1769 p. 127 huius propositionis mentionem facit seddemonstrationis ne necessitatem quidern attigit. Nemo vero demonstra: tionem tentauit praeter summum Eulerum Comsmento sous. At, Pstrop. T. XVIII ad annum 
1773 Demonstrationes circe residua ex dinisione potem statum per numeros primos restaltantia p. 85 seqq. vid. imprimis art. 37 vbi de demonstrationis necessitate fusius locutus est. At demonstratio quam Vir sagacissimus exhibuit duos defectus habet. Alterum quod art. 3 I et sqq. tacite supponit, congruentiam $x^{\mathrm{n}} \equiv \mathrm{I}$ (translatis ratiociniis illic adhibitis in nostra signa) reuera * radices diuersas. habere, quam quam ante nihil aliud fuerit demonstratum quam quod plures habere nequeat; alterum, quod fornulam art 34 per inductionem tantummodo deduxit.

57. Numeros ad exponentem $p-1$ per. tinentes radices primitiuas cum ill. Eulero voca. bimus, Si igitur $a$ est radix primitiua, potestatum $a, a a_{1} a^{3} \ldots a^{p-r}$ residua minima omnia erunt diuersa; vnde facile deducitur, inter haec omnes numeras. $1,2,3, \ldots p-1$, qui totidem sunt multitudine quot illa, residua minima, re periri debere, i. e. quemuis numerum per $p$ non diaisibílem potestati alicui ipsius a congruum esse. Insignis haec proprietas permagnae est vtilitatis, operationesque arithmeticas ad congruentias pertinentes, haud parum sublevare potest, simili fere modo, vt logarithmorum introductio operationes arithmeticae vulgaris. Radicem aliquam primitiuam, $a$, ad lubitum pro basi adoptabimus, ad quam omnes numeros per $p$ non diuisibiles referemus, et si fuerit $a^{e} \equiv b(\bmod . p)$, e ipsius $b$ indicem vocabimus. Ex. gr. si pro modulo 19, radix primitiua 2 pro basi assumatur respondebunt

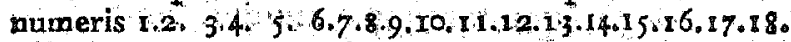

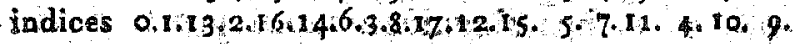

D 3 


\section{$-5 \%-$}

Ceterum patet, manente basi, cuique mumero piures indices conuenire, sed hos omnes secundum modulum $p-$ I fore congruos; quamobrem quoties de indicibus sermo erit, qui secundum modulum $p-$ I sunt congrui pro aequiualentibus habebuntur, simili modo vti numeri ipsi, quando secundum modulum $p$ sunt congrui, tamquam aequiualentes spectantur.

58. Theoremata ad indices pertinentia prorsus analoga sunt iis quae ad logarithmos spectant.

Index producti o quotcunque factoribus conflati congruns est stumial indicum singuloriom factorum se= cxudum modulum $p-I$.

Index potestatis numeri alicuius congruus esti producto ex indice numeri dati in exponentem potestasis, secundum mods $p-\mathrm{r}$.

Demonstrationes propter facilitatem omit timus.

Hinc perspicitur si tabulam construere velimus ex qua omnium numerorum indices pro modulis diuersis desumi possint, ex hac tum omnes numeros modulo maiores, tum omnes compositos omitti posse. Specimen huius modi tabulae ad calcem operis huius adiectum est, Tak, 1 , vbi in prima columna verticali positi sunt numeri primi primorumque potestates a 3 rsque ad 97 , qui tamquam moduli sunt spectandi, iuxta hos singulos numeri pro basi as- 


\section{$-55-$}

sumti; tum sequuntur indices numerorum primorum successiuorum, quorum quini semper per paruulum interuallum sunt disiuncti, eo. demque ordine supra dispositi sunt numeri primi; ita $v t$ quis index numero primo dato secúndum modulum datum respondeat, facile tu. toque inueniri possit.

Ita ex. gr. si $p=67$ index numeri 6o, asa sumto 12 pro basi erit $\equiv 2$ Ind. $2+$ Ind. $3+$ Ind. 5 (mod, 66$) \equiv 58+9+39 \equiv 40$.

59. Index valoris cuizuscunque expressionis : (mod. p.), (art. 31) congruus est secundum modulums p- I differential indicuns numeratoris a et denominatoris $b$, siquidem numeri $a, b$ per pons sunt dinisibiles.

Sit enim valor quicunque $c$; eritque $b c \equiv a$ (mod. $p$ ); hinc Ind. $\vec{b}+$ Ind. $c \equiv$ Ind. $a$ (mod. $p-1)$ adeoque

Ind. $\varepsilon_{*} \equiv$ Ind. $a-$ Ind. $b_{\text {}}$

Si itaque tabula habetur, ex qua index cuique numero respondens pro quouis modulo primo, aliaque ex qua numerus ad indicem datum pertinens deriuari possit, omnes congruentiae primi gradus facillimo negotio solui poterunt, quoniam omnes reduci posswnt ad tales, quarum modulus est numerus primus (art. 30). E. g. proposita congruentia $29 x+$ $7 \equiv 0(\bmod .47)$ erit $x=\frac{-7}{29}(\bmod .47)$.

Hinc Ind. $x \equiv$ Ind. -7 - Ind. $29 \equiv$ Ind. 40 - Ind. $2 g \equiv 15-43 \equiv 8(\bmod .46)$. At numerus cuins index 8 inmenițur 3. Quar D. 4 
$-56=$

$x \equiv 3(\bmod 47)$ - Tabulam secundam quidem non adiecimus: at huius vice alia defungi poterit vti Sect. VI ostendemus.

6o. Simili modo vt art. $3 x$ radices congruentiarum primi gradus designauimus, in sequentibus etiam congruentiarum purarum altio. rum graduum radices per signum exhibebimus. Vti scilicet $\mathfrak{n} A$ nibil aliud significat quam radicem aequationis $x^{\mathrm{n}}=A$, ita apposito modulo

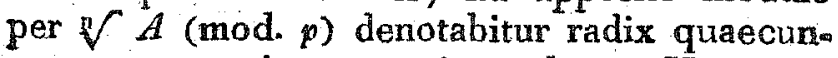
que congruentiae $x^{\mathrm{n}} \equiv A$ (mod. $p$ ). Hanc expressionem $\sqrt[\mathrm{n}]{ } A$ (mod. $p$ ) tot valores habere dicemus, quot habet secundum $p$ incongruos, omnes enim qui secundum $p$ sunt congrui tamquam aequinalentes spectandi (art. 26). Ceterum patet, si $A, B$ secundum $p$ fuerint con. grui, expressiones $\sqrt[n]{ } A, \sqrt[n]{ } B$ (mod. $p$ ) aequiualentes fore.

Iam si ponitur $\sqrt{n} A=x(\bmod . p)$, erit Ind. $x \equiv$ Ind $A$ (mod, $p$ r). Ex hac congruentia deducuntur ad praecepta sectionis praec. valores ipsius Ind, $x$ atque ex his valo. res respondentes.ipsius $x_{*}$ Facile vero perspicitur $x$ habere totidem valores, quot radices congruentia $n$ Ind, $x \equiv A$ (mod. $p-1$ ). Manifesto igitur $\sqrt[n]{ } A$ vnum tantummodo valorem habebir quando $n$ ad $p$ est primus; quando vero numeri $n, p$... diuisorem communem habent $\delta$, atque hicest maximus, Ind. $x$ habebit $\rho$. valores incongruos secundum $p-1$, adeoque $\sqrt[n]{ } A$ totidem valores incongruos secundum $p$, siquidem Ind. $A$ per $d$ est diuisibilis, Qua con- 
ditione deficiente $\sqrt[n]{A}$ nullum valorem realem habebit.

Exemplum. Quaeruntur valores expressionis $5 \sqrt[5]{1}$ i (mod. 19). Solui itaque debet congruentia 15 Ind. $x \equiv$ Ind. $11 \equiv 6$ (mod, 18 ), inuenienturque tres valores ipsius Ind $x \equiv 4$, Io, 16 (mod. 18). His vero respondent valores ipsius $x, 6,9,4$.

6r. Quantumuis expedita sit methodus haec quando tabulae necessariae adsunt, debemus tamen non obliuisci, indirectam eam esse. Operae igitur pretium erit inquirere quantum methodi directae polleant: trademusque hic ea quae ex praecedentibus hauriri possunt: alia, quae considerationes reconditiores postulant, ad sectionem VIII reseruantes. Initium facimus a casu simplicissimo, vbi $A=1$, siue vbi radices congruentiae $x^{\mathrm{n}} \equiv \mathrm{s}$ (mod. p) quaeruntur. Hic itaque, assumta radice quacunque primitiua pro basi, debet esse * Ind $x \equiv o(\bmod p-1)$. Quae congruentia, quando $n$ ad $p-I$ est primus, vnam tantummodo radicem habebit, scilicet Ind. $x \equiv 0$ (mod. $p-1)$ : quare in hocce casu $\sqrt[n]{1}(\bmod p)$ vnicum valorem habet, scilicet $\equiv \mathrm{I}$. Quando autem numeri $n, p-\ldots$ r habent diuisorem communem (maximum) $\delta$, congruentiae $n$ Ind. $x \equiv$ o $(\bmod , p-1)$ solutio completa erit Ind. $x \equiv 0\left(\bmod . \frac{\left.-\frac{x}{8}\right)}{8}\right.$ V. art. 3o., i. e. Ind. $x$ secundum modulum $p-\mathrm{I}$ alicui ex his numeris, o, $\frac{p-I}{\delta}, \frac{z(p-1)}{\delta}, \frac{3(p-1)}{b} \ldots \frac{(\delta-1)(p-1)}{\delta}$ congruus esse debebit, sive \& valores secundum modu- 
lum $p-1$ incongruos habebit; quare etiam $x$ in hocce casu \& valores diuersos (secundum mo. dulum $p$ incongruos) habebit. Hinc perspicitur, expressionem $\sqrt{ } \mathrm{I}$ etiam $\delta$ valores diuer sos habere, quorum indices cum ante allatis prorsus conueniant. Quocirca expressio $\mathcal{S}_{t}$ (mod: $p)$ huic $\sqrt[v]{ } r(\bmod , p)$ omnino aequiualet, i. e. congruentia $x_{f} \equiv x$ (mod. $p$ ) easdem radices habet quas haec, $x^{n} \equiv I(\bmod , p)$. Prior autem inferioris erit gradus siquidem $\delta$ et $n$ sunt inaequales.

Ex. $\sqrt[45]{ }$ (mod. 19 ) tres habet valores; quia 3 maxima numerorum 15,18 mensura communis, hique simul erunt valores expressionis $\sqrt[3]{1}$ (mod. 19 ). Sunt autem hi, I, 7, x r。

62. Per hanc igitur reductionem id lucra. mur vt alias congruentias formae $x^{n} \equiv \mathrm{I}$ soluere non sit opus, quam vbi $n$ moduli est dìuisor, Infra vero ostendemus, congruentias huius formae semper vlterius adhuc deprim: posse, licet praecedentia ad hoc non sufficiant. Vnum tamen casum iam hic absoluere possum mus scilicet vbi $n=2$. Manifesto enim valores expressionis $\sqrt[2]{x}$ erunt $+\mathrm{I}$ et $-\mathrm{x}$ quia plures quam duos habere nequit, hique $+\mathrm{r}$ et $-\mathrm{x}$ semper sunt incongrui nisi modulus sit $=2$, in quo casu $\sqrt[7]{1}$ vnam tantum valorem habere posse, per se clarum. Hino sequitur, +1 et -1 etiam fore valores expressionis $\sqrt[2 m]{\mathrm{x}}$ quando $m$ ad $\frac{k-x}{2}$ sit primus. Hoc semper eueniet, quoties modulus est eius indolis vt $\frac{p-x}{2}$ fiat numerus absolute primus . (nisi forte $p-1=2 m$ in 
quo casu omnes numeri $x, 2,3 \ldots \ldots-1$ sunt radices) ex. gr. quando $p=3,5,7$, r r, 23, 47, $59,83, \mathbb{1 0}_{7}$ etc. Tamquam corollarium hic annotetur, indicem ipsius - I semper esse $=\frac{n-1}{2}(\bmod . p-\mathrm{I})$, quaecunque radix primitiua pro basi accipiatur. Namque 2 Ind. $(-1)$ $=0:(\bmod , p-1)$. Quare Ind. $(-1)$ erit vel $\equiv 0$, vel $\equiv \frac{-x}{2}$ (mod. $\left.p-I\right)$ : o vero semper index ipsius $+I$, atque $+I$, et $-I$ semper indices diuersos habere debent (praeter casum $p=2$ ad quem hic respicere operae non est pre. tium).

63. Ostendimus art. 60 expressionem $\sqrt{ } A$ (mod. $p$ ) habere $\&$ valores diuersos, aut omnino nullum, si fuerit \& diuisor communis maximus numerorum $n, p-x$. Iam vti modo docuimus $\sqrt[n]{ } A$ et $\sqrt{ } A$ aequiualentes esse, si fuerit $A \equiv \mathrm{I}$, generalius probabinus, expressionem $\sqrt[n]{ } A$ semper ad aliam $\& B$ reduci posse cui aequiualeat. Illius enim valore quocunque denotato per $x$ erit $x^{\mathfrak{n}} \equiv A$; iam sit $t$ valor quicunque expressionis $\delta(\bmod , p-r)$; quamvalores reales habere ex art. 3 r perspicuum; eritque $x^{\mathrm{tn}} \equiv A^{\text {th }}$ at $x^{t n} \equiv x^{\prime \prime}$ propter $t n=\delta$ mod. $(p-1)$. Quare $x^{*} \equiv A$ adeoque quicunque ipsius $\sqrt[n]{ } A$ valor erit etiam valor ipsius $\& A$ t. Quoties igitur $\sqrt[n]{ } A$ valores reales habet, expressioni $4 t$ prorsus aequiualens erit, quoniam illa neque alios habet quam haec neque pauciores, licet quando $\sqrt[1]{A}$ nullum, valorem realem habet, feri tamen possit vt $f(A$ valores reales habeat.

Ex. Si valores expressionis $\sqrt[2]{2} / 2(\bmod .31)$ quaeruntur, erit numerorum 21 et 50 diuisor 
communis maximus 3 , expressionisque ix (mod. 30) valor aliquis 5, quare si $\sqrt[x]{2}$ valores reales habet, huic expressioni $\sqrt[3]{ } a^{3}$ siue $\sqrt[3]{8}$ aequinalebit, inuenieturque retera, posterior s expressionis valores qui sunt 2,10 , t9 etiam priori satisfacere.

64. Ne autem hanc operationem incas sum stiscepisse periclitemur, regulam inuestigare oportet, per quam statim dindicari possit verum $\ddot{C} A$ valores reales admittat neene. Quodsi tabula indicum habetur, res in prontu est; num rute ex art. 60 manifestum est, valo. res reales dari, si ipsins $A$ index, radice quacuntue primitiua pro basi accepta, per sit diaisibilis, sin vero minus, non tari. Attamen hoc etiam absque tali tabula inueniri pom test Posito enim indice ipsius $A=k$, si hic fuerit per o diuisibilis, erit $k y_{y}^{y}$ per $p-I$ diuisibilis et vice versa. Atqui numeri $A_{d}^{*}$ in dex erit $\frac{k(x)}{*}$. Quare si $\sqrt{ } A$ (mod. $p$ ) habet valores reales, $A^{2} y$ vnitati congruus erit, sin minus, incongruus. Ita in exemplo art. praec. habetur $2^{00}=1024= \pm$ (mod, 31$)$, vnte con. cluditur $2 \sqrt{3}(\bmod , 3 n)$ valores reales habare. Similiter enrtiores hine fimus, $\sqrt[P]{-t}$ (mad.p) semper valores binos realas habere, quando $p$ sit formas $4+1$, nullum vero, quando $p$ sit forma $4 m+5 ;$ propter $(-1)^{2 m}=1$ et $(-1)^{2} a_{1}=-1$. Flegans hoo theorema, quod

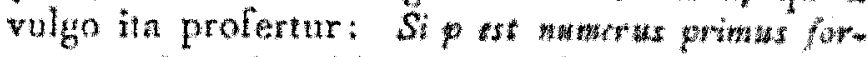

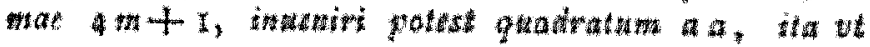

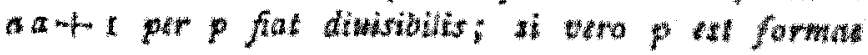




\section{- 6r -}

4m-I, tale quadratum non datur, hoc modo demonstratum est ab ill. Eulero, Conm nous. Acado Petrop. T. XVIII. p. I 2 ad annum 1773 . Demonstrationem aliam iam multo ante dederat, Comm. nou. T. V. p. 5 qui prodiit a. 1760 . In dissert. priori, Comm. nou. T. IV, p. 25 , rem nondum perfecerat. Postea etiam ill. La Grange theorematis demónstrationem trádidit, Nouveaux Mem. de l'Ac. de Berlin A. ${ }^{7} 77^{5}$ p. 342. Aliam adhuc demonstrationem in sectione sequenti vbi proprie de hoc argumento agendum erit, dabimus.

65. Postquam omnes expressiones $\mathfrak{p} A$ (mod. $\eta^{\prime)}$ ad tales reducere docuimus, vbi diuisor numeri $p-\mathbf{I}$, criteriumque nacti sumus ytrum valores reales admittat, necine, tales expressiones $\sqrt{ } A(\bmod , p)$ vbi $n$ ipsius $p-I$ est diuisor accuratius considerabimas. Primo ostendemus, quam relationem valores singuli expressionis inter se habeant, tum artificia quaedum trademus, quorum auxilio vnus valor expressionis saepenumero inueniri possit.

Primo, quando $A \equiv \mathrm{r}$, atque $r$ aliquis ex * valoribus expressionis $\sqrt[n]{ } \times(\bmod . p)$, siue $m^{n} \equiv I$ (mod, $p$ ), omnes etiam ipsius $r$ potestam tes erunt vilores istius expressionis; horum autem totidem erunt diuer'si quot vnitates habet exponens ad quem, pertinet (art. 48). Quodsi igitur $r$ est valor ad exponentem $n$ pertinens, potestates ipsius $r$ hae $r, r^{2}, r^{3} \ldots .$. ra (vbi loco vltimae vnitas substitui potest) omnes expressiopis $\sqrt[y]{ } \times$ mod.p) valores inuoluent. Qualia 


\section{$-62-$}

autem subsidia exstent ad tales valores inueniendos qui ad exponentem $n$ pertineant, in sect. VIII fusius explicabimus.

Secusdo. Quando $A$ vnitati est incongruus, Fvalor musque Texpressionis $\sqrt{ } A s$ (mod. $p)$ nötus, qui sit $z$; reliqui hoc modo inde deducuntur. Sint valores expressionis $\sqrt[n]{1} 1 / p, r^{2} \ldots p^{n-x}$ (vti modo ostendimus), eruntque omnes expr. $\sqrt[n]{A}$ valores hi: $z, z r, z r^{2} \ldots z z^{n-I}$; namque omnes hos congruentiae $x^{\mathrm{n}} \equiv A$ satisfaçere inde manifestum quod, posito quocunque eorum $\equiv z r^{\text {h }}$ potestas ipsius $n^{t_{x}}, z^{\mathrm{n}} \boldsymbol{r}^{\mathrm{nk}}$, propter $r^{\mathrm{n}} \equiv \mathrm{I}^{\prime}$ et ipsi $A$, $z^{\mathrm{h}} \equiv A$, fit congrua : omnes diuersos esse ex art. 23 facile intelligitur; plures autem valores quam hos quorum numerus est n, expressio $\sqrt[\pi]{ } A$. habere nequit. Ita ex. gr. si alter expressionis $\sqrt[3]{A}$ valor est $z$, alter erit $-z$. Denique hinc concludendum omnes valores expr. $\sqrt[n]{A}$ inuenire non posse, nisi simul omnes valores expr. $\sqrt[n]{ } \mathrm{I}$ constent.

66. Secundum quod nobis proposuere. mus fuit, docere, in quo casu vnus expressionis $\sqrt[n]{ } A$ (mod. $p$ ) valor (vbi i supponitur esse diuisor ipsius $p-I$ ) directe inueniri possit. Hoc euenit quando aliquis valor potestati alicue ipsius $A$ congruus euadit, qui casus quum haud raro occurrat, aliquantum huic rei immorari non superfluum erit. Sit talis valor, si quis datur $z$, siue $z \equiv A^{\mathrm{k}}$ et $A \equiv z^{\mathrm{n}}$ (mod. $p$ ). Hinc colligitur $A \equiv A^{\mathrm{kn}}$; quare si numerus $k$ habetur, ita vt sit $A \equiv A^{\mathrm{kn}}, A^{\mathrm{k}}$ erit valor quaesitus. At huic conditioni aequiualet ista, vit sit $I=k n(m o d, t)$, 
designante $t$ exponentem ad quem pertinet $A$ (art. 46, 48). Vt vero haec congruentia possibilis sit, requiritur, vt sit $n$ ad $t$ primus. Hoc in casu erit $k \cong \frac{x}{n}$ (mod. $\left.t\right)$; si vero $t$ et $n$ diuisorem communem habent, nullus valor $z$ po. testati ipsius $A$ congruus esse potest.

67. Quum autem ad hanc solutionem ipsum $t$ nouisse oporteat, videamus quomodo procedere possimus, si hunc numerum ignoremus. Primo facile intelligitur, $t$ ipsum $\frac{i-\pi}{i}$ metiri debere, siquidem $\sqrt{ } A(\bmod , p)$ valores reales habeat, vii hic semper supponimus. Sit enim quicunque valor $y$, eritque tum $y^{p-x} \equiv r$, tum $y^{n} \equiv A(\bmod . p)$; quare eleuando partes posterioris congruentiae ad potestatem $\frac{p-x}{n}$ tam, fiet $A^{\frac{p-x}{n}} \equiv 1$; adeoque $\frac{x-x}{n}$ per $t$ divisibilis (art 48). Iam si $\frac{p-\underline{x}}{n}$ ad $n$ est primus, con. gruentia art. praec. $k n \equiv$ r. etiam secundum mo. dulum $\frac{p-I}{n}$ solui poterit, manifestoque valor ipsius $k$ congruentiae secundum modulum hunc satisfaciens eidem etiam secundum modulum $t_{\text {, }}$ qui ipsum $\frac{n-x}{n}$ metitur, satisfaciet, (art 5). Tum igitur quod quaerebatur inuentum. Si vero $\frac{k-x}{n}$ ad $n$ non est primus, ommes ipsius $\frac{x-1}{n}$ factores primi qui simul ipsum m metiuntur ex $\frac{p-x}{n}$ eiiciantur. Finc nanciscemur numerum $p-\underline{n}$, ad $n$ primum, designante $q$ produ cturn ex omnibus illis factoribus primis, quos eiecimus. Quodsi iam conditio ad quam in artic. praec. peruenimus vt $t$ ad $n$ sit primus lo. cum habet, $t$ etiam ad $q$ erit primus adeoque etiam ipsum $\frac{p-t}{m q}$ metietur. Quare sì congruen- 


\section{- 64 -}

entia $k a \equiv I$ (mod. $\left.\frac{-\mathrm{r}}{\bar{m}}\right)$ soluitur (quod fieri potest quia $n$ ad $\frac{p-\underline{x}}{n^{4}}$ primus), valor ipsius $k$ etiam secundum modulum $t$ congruentiae satisfaciet, id quod quaerebatur. Totum hoc artificium in eo versatur, vt numerus eruatur qui ipsius $t$, quem ignoramus, vice fungi possit. Attamen probe meminisse oportet, nos quando $\frac{p-I}{n}$ ad $n$ non est primus, śupposuisse conditio. nem art. praec. locum habere, quae si deficir omnes conclusiones èroneae erunt; atque si regulas ditas temere sequendo pro $z$ valor inuenitur, cuius potestas $n$ ta $\mathrm{ipsi} A$ non sit congrua, indicio hoc est, conditionem deficere adeoque methodum hanc omnino adhiberi non posse.

67. Sed in hocce etiam casu saepe prodesse potest, hunc laborem súscepisse; operaeque pretium est, quomodo hic valor falsus ad veros sese habeat inuestigare. Supponamus itaque numeros $k, z$ rite esse determinatos sed $z^{\mathrm{n}}$ non esse $\equiv A(\bmod p)$. Tum si modo valores expressionis $\mathbb{V} \frac{A}{x^{\natural}}(\bmod , p)$ determinari possint, hos singulos per $x$ multiplicando valores ipsius $\sqrt[V]{ } A$ obtinebimus, Si enim $v$ est valor aliquis ipsius $\sqrt{\frac{A}{z^{n}}}$ : erit $(v z)^{\mathrm{n}} \equiv A$. Sed expressio $\sqrt{ } \frac{A}{z^{n}}$ eatenus hac $\sqrt[n]{A}$ simplicior, quod $\frac{A}{z^{n}}$ (mod. $p$ ) ad exponentem minorem plerumque pertinet quam $A$. Scilicet si numerorum $t, q$ divisor communis maximus est $d, \frac{d}{z^{n}}$ (mod, $\varphi$ ) ad exponentem $d$ pertinebit, id quod ata demonstratur. Substituto pro $z$ valore, fit

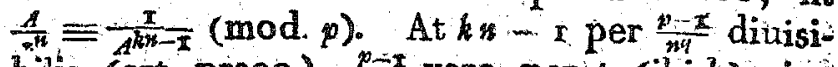

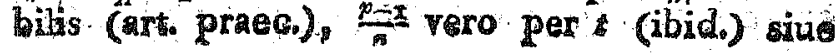




\section{$-65-$}

siue $\frac{p-1}{n d}$ per $\frac{t}{d t^{\circ}}$ Atqui $\frac{t}{d}$ ad $\frac{q}{d}$ est primus (hyp, $)_{0}$

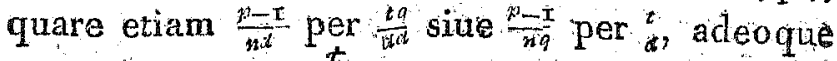
etian $k n-x$ per $\frac{t}{d}$ et $(k n-1) d$ per $t$ erit di. uisibilis. Hinc $A(k n-I) \stackrel{d}{=}$ (mod. $p)$. Vnde facile deducitur, $\frac{A}{z^{\mathrm{x}}}$ ad potestatem $t^{\mathrm{a}}$ tam euectun vinitati congrum fieri, Quod vero $\frac{A}{z^{i}}$ ad exponentem minorem quam $d$ pertinere non pos. sit facile quidem demonstraxi potest, sed quo niam ad finem nostrum non requiritur, huic rei non immoramur, Certi igitur esse possu. mus, $\frac{A}{* w^{10}}$ (mod. $p$ ) semper ad minorem exponen. tem pertinere, quam $A$, rnico excepto casu, scilicet quando $z$ ipsum q metitur, adeoque $d=t$

Sed quid hutat; quod $\frac{d}{z^{n}}$ ad minorem exo ponentem pertinet, qriam $A$ ? Plures numeri dantur qui possunt esse $A$ quam qui possunt esse $\frac{A}{z h i}$ et quando secundum eundem moduo lum plures huiusmodi expressiones $\sqrt[n]{ } A$ euol. uere occasio est, id lucramtir vt plures ex eo. dem fonte haurire possimus, Ità ex, $\mathrm{gr}_{\text {; }}$ sémper vnicum saltent valorem expressionis $\sqrt[2]{ } \boldsymbol{A}$ (mod. 29) determinare in potestate erit, si modo expressionis $\sqrt[3]{-1}$ (mod. 29 ) valores (qui sunt \pm 12) innotierint. Facile enim ex art. praec. perspicitur, huiusmodi expressionum vnum valorem semper directe determinari posse, quando $t$ impar, et $d$ fieri $=2$ quando $t$ par; praeter $-I$ autem nullus nus: merus ad exponentem 2 pertinet. 


\section{$-66-$}

68. Exemplat.

Quaeritur $\sqrt[3]{3}(\bmod , 37)$. Hic $p-1=36$, $n=3, \frac{n-x}{3}=12$, adeoque $q=3:$ debet igitur esse $3 k \equiv x$ (mod. 4) quod obtinetur ponendo $=3$. Hinc $z \equiv 3 \mathrm{I}^{3}\left(\bmod .3_{7}\right) \equiv 6$, inueniturque reuera $6^{3} \equiv 3 \mathrm{r}\left(\bmod , 3_{7}\right)$. Si valores ex: pressionis $\sqrt[3]{\mathrm{x}}(\bmod .37)$ sunt notae, etiam re. liqui expr. $\sqrt[3]{6}$ valores determinari possunt. Sunt vero illi $r$, ro, 26 , per quos multiplicando ipsum 6 , prodeunt reliqui $\equiv 23$ et 8 .

Si autem quaeritur valor expr. $\sqrt[2]{5}$ (mod. 37 ), exit $n=2, \frac{p-x}{n}=18$; adeoque $q=2$. Hinc debet esse $2 k \equiv \mathbf{I}(\bmod .9)$, vade fit $k \equiv 5$ (mod. 9). Quare $z \equiv 3^{5} \equiv 21 \quad(\bmod .37)$; at $2 x^{2}$ non $\equiv 3$, sed $\equiv 34$; est autem $\frac{3}{34}(\bmod .37)$ $\equiv-\pi$, atque $\sqrt[2]{-1}(\bmod .37) \equiv \pm 6 ;$ vnde obtinentur valores veri $\pm 6.21 \equiv \pm 15$.

Haec fere sunt, quae hic de talium expressionum euolutione tradere licuit. Palam est methodos directas satis prolixas saepe euasuras: at hoc incommodum tantum non omnibus methodis directis in numerorum theoria incumbit neque ideo negligendum censuimus, quantum hic praestare valeant ostendere. Etiam hic obseruare convenit, artificia particularia quae exercitato haud raro se offerunt sigillatim explicare, non esse instituti nostri.

69. Reuertimur nunc ad radices quias di: ximus primitiuas. Ostendimus, radice primitiua quacunque pro basi assumta ommes numeros, quorum indices ad $p$ - I primi, etiain lo. 
re radices primitiuas, nullosque praeter hos: vnde simul radicum primitiuarum multitudo sponte innotescit. V. art. 53. Quamnam autem radicem primitiuam pro basi adoptare velimus, in genere arbitrio nostro relinquitur; vn. de intelligitur, etiam hic, vt in calculo loga. rithmico, plura quasi systemata dari posse ${ }^{*}$ ), quae quo vinculo connexa sint videamus. Sint $a, b$, duae radices primitiuae, aliusque numerus $m$, atque, quando a pro basi assumitur, in. dex numeri $b \equiv 6$, numeri $m$ vero index $\equiv k$ (mod. $p-x$ ); quando autem $b$ pro basi assumitur, index numeri $a \equiv \alpha$, nunneri im vero (mod. $p-1)$. Tum erit $\alpha 6 \equiv \mathrm{I}(\bmod , p-1)$; namque $a^{b} \equiv b$, quare $a^{\alpha^{b}} \equiv b^{a} \equiv a(\bmod . p)$, (hyp), hinc $\propto \mathscr{b} \equiv \mathrm{r}(\bmod , p-\mathrm{I})$. Per simile ratiocinium inuenitur $\nu \equiv \alpha \mu$, atque $\mu \equiv b_{v}$ (mod. $p-1$ ). Si igitur tabella indicum pro basi a constructa habetur, facile in aliam conuerti potest, vbi $b$ basis. Si enim pro basi $a$ ipsius $b$ index est $\equiv \mathscr{b}$, pro basi $b$ ipsius $a$ index erit $\equiv \mathbf{E}(\bmod , p-I)$, multiplicandoque per hunc numerum omnes tabellae indices, habebuntur omnes indices pro basi $b$.

70. Quamuis autem plures indices numero dato contingere possint, aliis aliisque radicibus primitiuis pro basi acceptis, omnes tamen in eo conuenient, quod omnes eundem diuisorem maximum cum $p-I$ communem has E 2

- In eo autem differunt, quod in logarithmis, systemntum numerus est infinitus, hic vero tantus, quantus numerus radicum primitiun rum, Manifesto enim bases congruae idem syatema generant. 
bebunt. Si enim pro basi $a$, index nimeri dati est $m$, pro basi $b$ vero $n$, atque diuișores ma. ximi his cum $p-i$ communes, $\mu_{2}, v_{2}$ supponun. tur esse inaequales, alter erit maior, ex. gr. $p>$, adeoque $\mu$ ipsum $n$ non metietur. At designato indice ipsius a, quando 6 pro basi as: sumitur, per $\alpha$, erit (art. praec*) $n \equiv \alpha m$ (mod, $p-1)$ adeoque $\mu$ etiam ipsum $n$ metietur. Q. $E$. $A_{0}$

Hunc diuisorem maximum indicibus nù meri dati, ipsique $p-\mathrm{I}$ communem, a basi non pendere, etiam inde perspicuum, quod aequalis est ipsi $\frac{x-i}{t}$, designante $t$ exponentem ad quem numerus; de cuius indicibus agitur; pertinet. Si enim index pro basi quacunque est $k$, erit $t$ minimus numerus per quem $k$ multiplicatus ipsius $p-I$ multiplum euadit, (excepta cifra) vidd. artt. 48,58 , siue minimus valor expressionis $\frac{o}{k}\left(\bmod _{0} \not p-x\right)$ praeter cifram; hunc autem aequalem esse diuisori max ximo communi numerorum $k$ et $p-i$ ex art. 29 nullo negotio deriuatur.

77. Porro facile demonstratur; basin ita semper accipere licere, vt numerus ad exponentem $t$ pertinens indicem quemlibet datum nanciscatur, cuius quidem maximus diuisor cum $p-I$ communis $=\frac{p-x}{t}$. Designemus hune breuitatis gratia per $d$, sitque index propositus $\equiv d m$, numerique propositi, quando quaelibet radix prima a pro basi accipitur, index $\equiv d n_{\text {, }}$ eruntque $m, n$ ad $\frac{p-I}{d}$ siue ad $t$ primi. Tum si. - est valor expressionis $\frac{d w}{d m}(\bmod , p-1)$, simul- 
que ad $p-x$ primus, erit $a^{q}$ radix primitiua, qua pro basi accepta numerus propositus indicem $d m$ adipiscetur (erit enim $a^{\mathrm{ddm}} \equiv a^{\mathrm{dn}} \equiv$ numero proposito), id quod desiderabatur, Sed expressionem $\frac{d n}{d m}(\bmod . p-1)$ valores ad $p-r$ primos admittere, ita probatur. Aequiualet illa expressio huic: $\frac{n}{m}\left(\bmod , \frac{p-\underline{x}}{\alpha}\right)$ sine $\frac{p}{m}(\bmod . t)$ vid. art. $3_{1}, 2$, eruntque omnes eius valores ad t primi; si enim aliquis valor $e$ diuisorem cum $t$ communem haberet, hic diuisor etiam ipsum me metiri deberet, adeoque etiam ipsum n, cui me secundum $t$ congruus, contra hypoth., ex qua $n$ ad $t$ primus. Quando igitur omnes diuisores primi ipsius $p-I$ etiam ipsum $t$ me. tiuntur, omres expr. $\frac{m}{m}$ (mod. $t$ ) valores ad $i-x$ primi erunt multitudoque eorum $=d$; quando autem $p-1$ alios adhuc diuisores primos, $f$, $g, h$ etc. implicat, ipsum $t$ non metientes, ponatur valor quicunque expr. $\frac{n}{n}(\bmod t)$. Tum autem quia omnes $t, f, g, k$ etc. inter se primi, inueniri potest numerus ? qui secundum $b$ ipsi $e$, secundum $f, g, h$ etc. vero numeris quibuscunque ád hos respectiue primos fiat congruus. (art, 32) Talis itaque numerus per nulium factorem primum ipsius $p-1$ diujsibilis adeoque ad $p-1$ primus erit, vti desiderabatur. Tandem haud difficile ex combinationum theoria deducitur, talium valorum multitu-

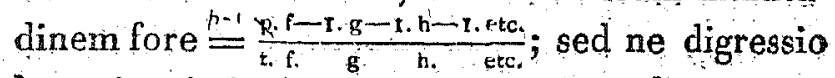
haec in nimian molem excrescat, demonstrationem, quum ad institulum nostrum non sit adeo necessaria, omittimus. 
72: Quamuis in genere prorsus arbitra. rum sit, quaenam radix primitiua pro bas: adoptetur, interdum tamen bases aliae prat aliis commoda quaedam peculiaria praeber possunt. In tabula I semper numerum to pro basi assumsimus, quando fuit radix primitiua; alioquin basin ita semper determinauimus vl numeri 10 index euaserit quam minimus; i. e $=\frac{k-x}{t}$ denotante $t$ exponentem ad quem so per. tinuit. Quid vero hinc lucremur, in Sect. VI, ostendemus vbi eadem tabula ad alios adhuc vsus adhibebitur. Sed quoniam etiam hic aliquid arbitrarii remanere potest, vt ex art. praec. apparet: vt aliquid certi statueremus, ex omnibus radicibus primitiuis quaesitum praestantibus minimam semper pro basi eleximus. Ita pro $p=73$, vbi $t=8$ atque $d=9$, habet $\frac{72}{8.3}$ i. e. 6 valores, qui sunt 5 , I4, 20, $28,3 \mathrm{~g}$, 40. Assurnsimus itaque mininum 5 pro basi.

73. Methodi radices primitiuas inueniendi maximam partem tentando innituntur. Si quis ea quae art. 55 docuimus cum is quae infra de solutione congruentiae $x \equiv$ trademus confert omnia fere, quae per methodos directas effici possunt, habebit. ,Ill. Euler confitetur, Opusc. Analyt T. I. p. I52, maxime difficile videri, hos numeros assignare, eorumque indolem ad pro: fundissima numerorum mysteria esse referen. dam. At tentando satis expedite sequenti modo determinari posssunt. Exercitatus operationis prolixitati per multifaria artificia particularia succurrere sciet: haec vero per vsum multo citius quam per praecepta ediscuntur 


\section{- $7 !-$}

1. Assumatur ad libitum numferus ad (ita semper modulum designamus) primus, $a_{s}$ (plerumque ad calculi breuitatem conducit, si quam minimum accipimus, $\mathrm{ex}^{\mathrm{e}}$ gr. numerum 2) determineturque eius periodus (art. 46 ), i. e. residua minima ipsius potestatum, donec ad potestatem $a^{t}$ perueniatur, cuius residuum minimum sit $\mathrm{F}$ ). Iam si fuerit $t=p-1, a$ est radix primitiua,

$2^{\circ}$. Si vero $t<p-I$, accipiatur alius nu. merus $b$ in periodo ipsius $a$ non contentus, inuestigeturque simili modo huius periodus. Designato exponente ad quem $b$ pertinet per $u$ facile perspicitur $u$ neque ipsi $t$ aequalem neque ipsius partem aliquotam esse posse, in vtroque enim casu feret $b^{t} \equiv \mathbf{r}$, quod esse nequit, quum periodus ipsius a omnes numeros amplectatur, quorum potestas exponentis $t$ vnitati congrua (art. 53). Quodsi is fuerit $=p-r$, erit $b$ radix primitiua; si vero is non quidem $=p-r$, sed tamen multiplum ipsius $t$, id lucratì sumus, vt numerus constet ad exponentem maiorem pertinens, adeoque scopo nostro, qui est inuenire numerum ad exponentem mo: xinumin pertinentem, propiores iam simus: Si vero $u$ neque $=p-\mathbf{1}$, neque ipsius $p$ multiplum, tamen numerum inuenire possumus ad expo. nentem ipsis $t, u$ maiorem pertinentem, nempe ad exponentem minimo' diuiduo communi nu. merorum $t$, $u$, aequalem. Sit hic $\doteq y$, resoluaE 4 .

-) Quisquis sponte perspiciet, non opus esse has potestakes ipsas nouisse, quum cuiusuis residuum minimum facile ex residuo minimo. potestatis praecedentis obtineri possit 
turque y ita in duos factores inter se primos, $m, n$, vt alter ipsum $t$, alter ipsum $u$ metia. tur $\left.{ }^{*}\right)$ Tum fia potestas $\frac{t}{m}$ ta ipsius $a_{2} \equiv A$, po. testas $\frac{u t a}{n}$ ipsius $b, \equiv B\left(\bmod _{t} p\right)$, eritque pro. ductum $A B$ numerus ad exponentem $y$ pertinens; facile enim intelligitur, $A$ ad exponen. tem $m, B$ ad exponentem $n$ pertinere; adeoque productum $A B$ ad $m n$ pertinebit, quia $m, n$ inter se sunt primi, id quod prorsus eodem mo. do vti in art 55, II processimus probari pots erit.

30. Iam si $y=\mu-\pi, A B$ erit radix pri mitiua; sin minus, simili modo vt antea alius numerus adhibendus erit, in periodo ipsius $A B$ non occurrens; eritque hic aut radix primitiaa, at pertinebit ad exponentem ipso y maiorem, aut certe ipsius auxilio (vti ante) numerus ad exponentem ipso y maiorem pertinens inueniri poterit, Quum igitur numeri qui per repetitionem huius operationis prodeunt, ad exponentes continuo crescentes pertineant, manife. stum est tandem numerum inuentum iri, qui ad exponentem maximum pertineat, $i_{i}$ e radicem primam, $q_{0}, e_{0} f_{\diamond}$

-) Quomodo hoc fiers possit ex art. I8 haud difficulter deriuatur, Re. soluatur $y$ in factores tales, qui sint aut numeri primi dịuersi aut numerorum primorum diuersorum poteskates, Horum quisque aiterutrum numerarum $t, u$ metietur (siue etiam vtrumque). Adscriban. tur singuli aut numero aut sumero $u$, prout illum aut bune me. tiuntur: quaando aliquis vtrumqque metitur, arbitrarium est, cui adscribatur ; productum ex is qui ipsi adscripti sunt, sit $=m$, prom ductum e reliquis $\equiv n$, facileque perspicietur $m$ ipsum $t \cdot n$ ipsum * metiri, nqque esse $m n=y$. 


\section{$-7^{3}-$}

74. Per cxemplum praecepta haec cla: riora fient. Sit $p=73$, pro quo radix primitiua quaeratur. Tentemus primo numerum 2, cuius periodus prodit haec:

I. 2. 4. 8, 16. 32. 64. 55. 57. I. etc。

о. т. 2. 3. 4. 5. 6, 7. 8. 9. etc.

Quum igitur iam potestas exponentis 9 vnitati congrua fiat, 2 non est radix primitiua. Tentetur. alius numerus in periodo ipsius a non occurrens ex. gr. 3, cuius periodus est haec:

x. 3. $9,27.8,24,72,70,64,46,65,-49, x$ etc. o. 1. 2. 3.4. 5. 6. 7. 8. 9. 10. 11. 12 etc. Quare neque 3 est radix primitiua, Exponentium autem ad quos 2,3 pertinent, (i.s. e. numerorum 9, I2) diuiduus communis minimus est 36, qui in factores 9 et 4 ad praecepta art. praec. resoluitur. Euehendus, itaque 2 ad potestatem exponentis $\frac{9}{g}, i_{\text {, e. numerus } 2}$ ipse retinendus; 3 autem ad potestatem exponentis 5 : productum ex his est 54, quod itaque ad exponentem 36 pertinebit, Si denique ipsius 54 periodus computatur numerusque in hac non conteritus ex. gr. 5 denuo tentatur, hunc esse radicem primitiuam, reperietur.

75. Antequam hoc argumentum deseramus, propositiones quasdam trademus, quae ob simplicitatem suam attentione haud indignae videntur,

Productum ex omnibus terminis periodi numeri cuiusuis est $\equiv \mathrm{I}$, quando ipsorum muittitudo, sine ex. qonens ad quem mumerus pertinet, esi impar, ${ }_{2}=\mathrm{m}$, quando ille exponens est par. 


\section{- $74=$}

Ex:" Pro modulo $\mathrm{x}$, periodus numeri 5 constat ex his terminis, $x, 5,12,8$ quorum productum $480 \equiv-I$ (mod. 13$)$.

Secundum eundem modulum periodus numeri 3 constat e terminis s, 3, 9 quorum pro. ductum $27 \equiv \mathrm{L}(\bmod \cdot \mathrm{r} 3)$.

Demonstr. Sit exponens, ad quem numerus pertinet, $t$, atque index numeri, $\frac{p-\tau}{t}$, id quod si basis rite determinatur semper fieri potest (art. 71 ). Tum index producti ex omnibus periodi terminis erit $(x+2+3+$ ete $+t-x) \frac{p-x}{t}=\frac{(t-x)(p-x)}{2}$ i. $e . \equiv 0($ (nod. $p-1)$ quando $t$ impar, et $\equiv$ $\frac{2-T}{2}$, quando $t$ par; hine in priori casu productum illud $\equiv \mathrm{I}$ (mod. $p)$; in posteriori vero E-I (mod.p), (art. 62). Q. E. D.

76. Si numerus iste in theor. praecedente est radix primitiua, eius periodus omnes numeros $x, 2,3 \ldots \ldots p-1$ comprehendet, quorum productum itaque semper $\equiv-I$ (namque $p-1$ semper par, vnico casu $p=2$ excepto in .quo -1 et +1 aequiualent). Theorema hoc elegans quod ita enunciari solet: productum ex ommibus numeris anmero primo dato minoribus, vnitote cuctum per hunc primum est dintisibiles, primum a cel. Waring est prolatum arrnigeroque Wilson adscriptum, Meditt. algebr. Ed. 3, p. 380. Sed neuter demonstrare potuit, et cel. Waring fatetur demonstrationem eo difficiliorem videri, quod nulla notatio fingi possit, quae numerum primum exprimat. - At nostro quidem iudi- 


\section{$-7^{5}-$}

cio huiusmodi veritates ex notionibus potius quam ex notationibus hauriri debebant. Postea ill. La Grange demonstrationem dedit, Nouv. Mem, de l'Ac. de Berlin, $\times 77 \mathrm{x}$. Innititur ea considerationi coefficientium ex euolutione producti $x+1 . x+2 x+3 \ldots x+p-x$ oriundirum. Scilicet posito hoc producto $=x^{\mathrm{p}-\mathrm{r}}+A x^{\mathrm{p}-2}+B x^{\mathrm{p}-3}+\mathrm{etc}_{\mathrm{r}}+M x+N$, coefficientes $A, B$, etc., $M$ per $p$ erunt diuisibiles, $N$ vero erit $=$ I. $2.3 \ldots p-1$. Iam pro $x=x$, productum per $p$ diuisibile; tuno autem erit $\equiv x+N$ (mod. $p$ ); quare necessario $\mathrm{x}+N$ per $p$ diuidi poterit.

Denique ill. Euler in Opusc, analyt. T. I. p. 329 demonstrationem dedit, cum ea quam nos hic exposuimus conspirantem. Quodsi ta. les viri theorema hoc meditationibus suis non indignum censuerunt, non improbatum iri speramus, si aliam adhuc demonstrationem apponimus.

77. Quando secundum modulum $p$, productum duorum numerorum $a, b$ vnitati est congruum, numeros $a, b$ cum ill. Euler, sacios vocemus. Túm secundum sect, praec. quiuis numerus positiuus ipso p minor socium habebit positiuum ipso $p$ minorem et quidem vnicum. Facile autem probari potest ex numeris $1,2,3 \ldots p-1 ;$ et $p-1$ esse vnicos qui sibi ipsis sint socii : numeri enim sibi ipsis socii, radices erunt congruentiae $x x \equiv 1$; quae quoniam est secundi gradus plures quam duas radices; $i_{0}$ e, alias quam i et $p-x$ babo. 
re nequit. Abiectis itaque his numerorum re. liquorum $2,3 \ldots . . p-2$ bini semper erunt associati; quare productum ex ipsis erit $\equiv \mathrm{I}$ adeoque productum ex omnibus $1,2,3 \ldots p-I$, $\equiv p-\mathrm{r}$ siue $\equiv-\mathrm{I}$. Q.E. D.

Ex. gr. pro $p=13$ numeri $2,3,4 \ldots$ r ita asscciantur: 2 cum $7 ; 3$ cum $9 ; 4$ cum $_{-10}$; 5 cum $8 ; 6$ cum $11 ;$ scilicet $2.7 \equiv 1 ; 3.9 \equiv \mathrm{r}$ etc. Hinc 2. 3. $4 \ldots . \mathrm{xI} \equiv 1$; adeoque $x$. 2 . $3 \ldots+2 \equiv-1$.

78. Potest autem theorema Wilsonianum generalins sic proponi. Productum ex omnibus numeris, numero q $\mathrm{u}$ o $\mathrm{c}$ un $\mathrm{q}$ ue dato $A$ minoribus simulque ad ipsum primis, congrum est secundum $A$, vanitati vel negatizu vel positive sumtae. Negatiue sumenda est. vnitas, quando $A$ est formae $p^{\mathrm{m}}$, aut huiusce, $2 p^{\text {th }}$, designante $p$ numerum primum a 2 diuersum, instiperque quando $A=4$; positive autem in omnibus casibus relicuiss. Thearema, quale a cel. Wilson est prolatum, sub casu priori continetur. - Ex.gr, pro $A=15$ productum e numeris $\mathrm{r}, 2,4,7,8,1 \mathrm{r}$, 13, 14 est $=1$ (mod. 15). Demonstrationem breuitatis gratia non adiungimus: obseruamus tantum, eam simili modo perfici posse vt in art. praec., excepto quod congruentia $x x \equiv$ r plures quam duas radices habere potest, quae considerationes quasdam peculiares postulant. Posset etiam demonstratio ex consideratione indicum peti, similiter vt in art. $7^{5}$, si ea quae mox de modulis non primis trademus conferantur, 
79. Reuertimur ad enumerationem aliarum propositionum (art. $7^{5}$ ).

Summi omnium terminorum periodi numeri cuinsuis est $\equiv 0$, vti in ex art. $75,1+5+12+8$ $=25 \equiv 0(\bmod 13)$

Dem. Numerus de cuius periodo agitur, sit $=a$, atque exponens ad quem pertinet, $=t$, eritque. summa terminorum omnium periodi; $\equiv \mathbf{1}+a+a a+a^{3}+$ etc $+a^{t-1} \equiv \frac{a^{t}-x}{a-x}$ (mod. $p)$. At $a^{t-1} \equiv 0$ : quare summa haec semper erit $\cong 0$ (art. 22), nisi forte $a-1$ per $p$ sit diuisibilis, siue $a \equiv \mathrm{I}$; hunc igitur casum excipere oportet, si vel vnum terminum, periodum vocare velimus.

80. Productun ex omnibus radicibus primitius est 1 , excepto vnico casu, $p=3$; tum enim vna tantum datur radix prima, 2 ,

Demonstr. Si radix primitiua quaecunque pro basí assumitur, indíces radicum omnium primitiuarum erunt numeri ad $p-1$ primi simulque ipso minores. At horum numerorum summa, i. e. index producti ex omnibus radicibus primitiuis, est $\equiv$ o (mod. $p-1$ ) adeoque productum $\equiv I(\bmod . p) ;$ facile enim perspicitur, si $k$ fuerit numerus ad $p-$ I primus, etlam $p-I-k$ ad $p-r$ primum fore adeoque binos numeros ad $p-1$ prinos summam constituere per $p-i$ diuisibilem; ( $k$ autem ipsi $p-1-k$ numquam aequalis esse potest, prae. ter casum, $p-1=2$, siue $p=3$, quem exce. 


\section{$-7^{8}-$}

pimus; manifesto enim $\frac{p-x}{\bar{t}}$ in omnibus reliquis casibus ad $p-I$ non est primus).

81. Summa omnium radicum primitinarum est awt $\equiv 0$ (quando $p-\mathrm{p}$ per quadratum aliquod est diuisibilis), aut $\equiv \pm$ I (mod. $p$ ), (quando $p-1$ est productum e numeris primis inaequalibus; quorum multitudo si est par signum positiuam, si vero impar, negatiuum sumendum).

Ex. Io pro $p=13$, habentur radices primitiuae $3,6,7,1 \mathrm{r}$, quarun summa $26=0$ $(\bmod , 13) .22^{\circ}$ pro $p=11$, radices primitiuae sunt $2,6,7,8$ quarum summa $23 \equiv+$ I (mod. I I). $3^{\circ}$ pro $p=5 \mathrm{I}$, radices primitiuae sunt $3, \mathrm{II}_{3}$ 12, $13,17,21,22,24$, quarum summa, 123 $\equiv-I(\bmod .3 \mathrm{r})$.

Demonsty. Supra demonstranimus (art.

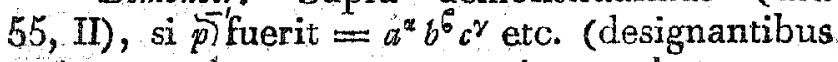
$a, b, c$ etc. numeros primos inaequales), atque $A, B, C$ numeri quicunque ad exponentes $a^{\alpha}$, $b_{5}, c^{\gamma}$ etc. respectiue pertinentes, omnia producta $A B C$ etc. exhibere radices primitiuas. Facile vero etiam demonstrari potest, quamuis radicem primitiuam per huiusmodi produ. ctum exhiberi posse et quidem vnico tantum modo * $\%$

-) Determinentur scilicet numeri $a_{f} b_{1} c$ etc., ita, vt sit $a \equiv \mathbf{I}$ (mod, $\left.a^{\alpha}\right)$ et $\equiv 0 \quad\left(\bmod , 66 c^{\circ}\right.$ etc.) $6 \equiv 1(\bmod , 66)$ et $\equiv 0$ (mod. $a^{\alpha} c \gamma$ etc.) etc. (vid. art 32 ), vnde fiet $a+c \pm$ etc. $\equiv \mathrm{I}(\bmod , p-I)$, (art Ig): lam si radix primitiua quaecunque. - per productum $A B C$ etc. exbiberi debet accipiatur $A \equiv r a_{0}$ $B \equiv r \mathfrak{E}, C=r c$ etc., atque pertinebunt $A$ ad exponentem $a *, B$ ad exporientem 66 etc.; productumque ex omnibus $A, B, C$ etc. erit Er (mod. $p$ ): denique facile perspicitur $A, B, C$ etc. "alio" modo Letermizari non posse. 


\section{$-79-$}

Vnde sequitur haec producta loco ipsarum radicum primitiuarum accipi posse. At quoniam in his productis omnes valores ipsius $A$ cum omnibus ipsius $B$ etc combinari oportet, omniurn horum productorum summa aequalis est producto ex summa omnium valo. rum ipsius $A$, in summam omnium valorum ipsius $B$, in summam omnium valorum ipsius $\mathcal{C}$ etc. vti ex doctrina combinationum notum est. Designentur omnes valores ipsorum $A$; $B$ etc., per $A, A^{\prime}, A^{\prime \prime}$ etc. ; $B, B^{\prime}, B^{\prime \prime}$ etc. etc., eritcque summa omnium radicum primitiuarum. $\equiv\left(A+A^{\prime}+\right.$ etc. $)\left(B+B^{\prime}+\right.$ etc. $)$ etc. Iam dico, si exponens fuerit $=I$, summam $A+$ $A^{\prime}+A^{\prime \prime}+$ etc. fóre $\equiv-1(\bmod p)$, si vero fuerit $>1$, summam hanc fore $\equiv 0$. similiterque de reliquis $6, \gamma$, etc. Simulac haec erunt demonstrata, theorematis nostri veritas maniFesta erit. Quando enim $p-I$ per quadratum. aliquod diuisibilis est, aliquis exponentium a, 6 , etc vnitatem superabit, adeoque aliquis factorum, quorum producto congrua est summa omnium radicum primitiuarum, erit $\equiv 0$, et proin etiam productum ipsum: quando vero p... I per nullum quadratum diuidi potest, omnes exponentes $* 6, r$ etc. erunt $=1$, vnde summa omnium radicum primitiuarum congrua erit producto ex tot factoribus, quorum quìsque $\equiv-1$, quot habentur numeri $a, b, c$ étc, adeoque erit $\equiv \pm$, prout horum numerorum multitudo par vel impar. Illa autem ita probantur.

I0. Quando $\rightleftharpoons x$ atque $A$ numerus ad ex ponentem a pertinens, reliqui numeri ad hume 
exponentem pertinentes erunt $A^{2}, A^{3} \ldots \ldots A^{\mathrm{a}-\mathrm{t}}$. $\mathrm{At}$ i $+A+A^{2}+A^{3} \ldots+A^{a-1}$ est summa periodi completae, adeoque $\equiv 0$ (art. 79), quare $A+A^{2}+A^{3} \ldots . .+A^{2}-1 \equiv-\mathrm{I}$.

$2^{\circ}$. Quando autem $a>x$, atque $A$ numerus ad exponentem $a^{*}$ pertinens, reliqui numeri ad hunc exponentem pertinentes habebuntur, si ex his $A^{2}, A^{3}, A^{4} \ldots A^{\alpha}-\mathrm{x}$ reiliciuntur $A^{\mathrm{a}}$, $A^{2 a} ; A^{3 a}$ etc., vid, art. 53 ; quare summa eorum erit $\equiv \mathrm{I}+A+A^{2} \ldots .+A^{a^{\alpha}-\mathrm{r}}-\mathrm{CI}+$ $\left.A^{\mathrm{a}}+A^{\mathrm{x}} \ldots \ldots+A^{\mathrm{a}-\mathrm{a}}\right) \mathrm{i}_{\text {o }}$ e. congrua differentiae duarum periodorum, adeoque $\equiv$ O. Q.E.D.

82. Onnia quae hactenus exposuimus innituntur suppositioni, modulum esse numerum primum. Superest vt eum quoque casurn consideremus, vbi pro modulo assumitur numerus compositus. Attamen quum hic neque proprietates tam elegantes eniteant, quäm in casu priori, neque ad eas inueniendas artificiis subtilibus sit opus, sed potius omnia fere per solam principiorum praecedentium applicationem erui possint, omnes minutias hic exhaurire superfluum atque taediosum foret. Breuiter itaque quae huic casui cum priori sint communia quaeque propria exponemus.

83. Propositiones art. $45-48$ generaliter iam sunt demonstratae. At prop. art. 49 ita immutari debet:

Si $f$ designat; quot numeri dentur ad mi prisi simulipso m minores, i. e. si $f=\phi m$ (art. 38 ) : exponens, $t$, infinae potestatis numeri dati, a, ad mo primi, quae sectudam modutum unitati est congrua, wet orit $=f$ vel pars aliquota huius numer 


\section{$-\quad 81$}

Demonstratio prop. art. 49 etiam pro hoc casu valere potest, si modo vbicue loco ipsius $p, m$, loco ipsius $p-I, f$, et loco numerorum $1,2,3, \ldots . p-1$, numeri ad $m$ primi simulque ipso $m$ minores substituantur. Huc itaque lectorem ablegamus. Ceterum demanstrationes reliquae de quibus illic locuti sumus (art. 5o, 5I) non sine multis ambagibus ad hunc casum applicari possunt. - At respectu propositionum sequentium, art. 52 sqq. magna difterentia incipit inter modulos, qui numerorum primorum sunt potestates, eosçue, qui per plures numeros primos diuidi possunt. Seorsim itaque modulos prioris generis com. templabimur.

84. Si modulus $m=p^{\mathrm{n}}$, designante p numerum primum, erit $f=p^{\mathrm{D}-\mathrm{I}}(p-1)$ (art. 38.) Iam si disquisitiones in artt. $5 \mathrm{I}, 55$ contentae ad hunc casum applicantur, mutatis mutandis vti in art. praec. praescripsimus, inuenietrar, omnia quae ibi demonstrata sunt etiam pro hoc casu locum habere, si modo ante probatum esset, congruentiam, formae $x^{t}-1 \equiv 0$ (mod. $\left.p^{n}\right)$ plures quam $t$ radices diuersas habere non posse. Pro modulo primo hanc veritatern ex propositione generaliori art. 43 deduximus, quae autem in omni. sua extensione de modulis primis tantummodo valet, neque adeo ad hunc casum applicanda. Attamen proposi. tionem pro hoc casu particulari veram esse per methodum singularem demonstrabimus. Infra (sect. VIII.) idem facilius inuenire docebimuLS. 


\section{$-82-$}

Demonstrandum proponimus nobis hoc

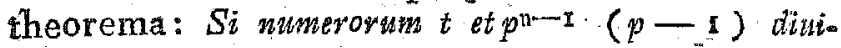
sor communis maximus est e, congruentia $x x^{t} \equiv I$ (mod. $y^{\mathrm{n}}$ ) habebit e rodices diwersas.

Sit $e=k p p$, ita vt $k$ factorem $p$ non inuol. uat, adeoque numerum $p-1$ metiatur. Tum congruentia $x^{t} \equiv 1$ secundum modulum $p$ habebit $h$ radices diuersas, quibus per $A, B, C$ etc. designatis, radix quaecunque eiusdem congruentiae secundum modulum $p^{\mathrm{n}}$, congrua esse debet secundum modulum $p$ alicui numerorum $A, B, C$ etc. Iam demonstrabimus, congruentiam $x \equiv x$ (mod, $\left.p^{\mathrm{n}}\right)$ habere ' $p$ " radices ipsi $A$, totidem ipsi $B$ etc. congruas se ${ }^{g}$ cundum modulum p. Quo facto ornnium ra. dicum numerus erit kp siue $e$, vii diximus. Illam vero demonstrationem ita adornabimus, vt primo ostendamus, si * fuerit radix ipsi. $A$ secundum modulum $p$ congrua, etiam $\alpha+p^{\mathrm{n} \rightarrow}$, $\alpha+2 p^{n-b}, a+3 p^{n-y}, \ldots \ldots \alpha+\left(p^{n-1}\right) p^{n-0}$ fore radices; secundo, numerosipsi $A$ secundum modulum $p$ congruos alios quam qui in forma $\alpha+h p^{n \rightarrow}$ sint comprehensi (deno. tante $h$ integrum quemcunque, , radices esse non posse: vrde manifesto $p^{n}$ radices diuersae liabebuntur, et non plures: atque idem etiam de radicibus, quae singulis $B, C$ etc. sunt congruae, locum habebit: tertio docebimus, 'quomodo sempêr radix, ipsi $A$ secundum $p$ congrua, inueniri possit.

86. Theorema. Si vit in art. praec. $t$ est mumerus per pp, neque vero per $p^{y, k x}$ diwisibilis, erit $(a+$ 


\section{$-83-$}

$\left.h p^{u}\right) t-\alpha^{t} \equiv o\left(\bmod _{0} p^{\mu+\nu}\right)$, at $\equiv \alpha^{t-x} h p^{u t}$ (moil. $p^{k+1+1+1)}$ Theorematis pars posterior locum non habet, quando $p=2$ simulque $\mu=\mathbf{r}$.

Demonstratio huius theorematis ex euolutione potestatis binomili peti posset, si ostendere. tur omnes terminos post secundum per $p^{p+p+r}$ diuisibiles esse. Sed quonian consideratio de. nominatorum coefficientium in aliquot ambages deducit, methodum sequentem praeferimus.

Ponamus primo $\mu>I$ atque $v=I$, enitque, propter $x^{t}-y^{t}=(x-y)\left(x^{t-1}+x^{t-2}\right) y+$ $x^{t-3} y^{2}+$ etci $\left.+y^{t-1}\right),\left(\alpha+h p^{k}\right)^{k}-\alpha^{t}=h p^{k}$ $\left(\left(\alpha+h p^{\alpha}\right)^{t-1}+\left(\alpha+h p^{\alpha}\right)^{t-2} a\right.$ etc. $\left.+\alpha^{t-1}\right) \cdot$ At est $\alpha+h p^{\mu}=\alpha\left(\bmod p^{2}\right)$, quare quis. que termunus $(\alpha+h p h)^{t-1},\left(\alpha+h^{\mu}\right)^{-2}$ etc. erit $\equiv a^{t-1},\left(\bmod . p^{2}\right)$ adeoque omnium summa $\equiv t_{x^{t}-x}\left(\bmod , p^{2}\right)$ sine formae $t a^{t-1}+V p^{2}$ denotate $V$ numerum quemcunque. Hinc $\left(+h p^{\mu}\right)^{t}-\alpha^{t}$ erit formse $e^{t-1} h p^{\mu} t+\nu h p^{k+2}$, i. e. $\equiv \alpha^{t-1} h_{p}^{k} t$ (mod. $g^{k+)}$ et $\equiv \circ$ (mod. $p^{\mu+1}$ ) Pro hoc itaque casu theorema est demonstratum.

Iam si theorema pro aliis ipsius , valoribus verum non esset, manente etiamnum $\mu>x$, limes aliquis necessario daretur, vsque ad quem theorema semper verum foret, vltra vero falsum. Sit minimus valor ipsius ", pro quo falsum est $=\phi$, vnde facile perspicitur, si $t$ per $p^{\varphi-1}$ non autem per $p^{\phi}$ fuerit diuisibi- 


\section{$-84-$}

lis, theorema adhuc verum .esse, at si loco ipsius $t$ substituatur $t p$, falsum. Habemus itaque

$$
\left(a+h p^{\mu}\right)^{t} \equiv a^{t}+a^{k-x} h p^{\mu t}\left(\bmod \cdot p^{\mu+\phi}\right)
$$

siue $=a^{t}+\alpha^{t-s}$ hpkt $+u p^{\mu}{ }^{k} \phi$ denotante $u$ numerum indeterminatur. At quia pro' $=I$ theorema iam est demonstratum, erit $\left(a^{t}+\right.$

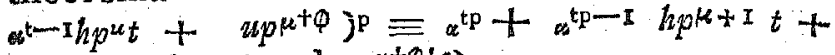
atp $-\mathrm{t} u p^{\mu+\phi+1}$ (mod. $p^{\mu+\varphi+1}$ ),

adeoque etiam

$\alpha+h p \mu)$ tp $\equiv \alpha^{t p}+\alpha^{t p-I} h p^{\mu t p}\left(\bmod , p^{\mu+p t r}\right)$ i. e. theorema etiam verum, si loco ipsius $t$ substituitur $t p$, i. e. etiam pro $y=\phi+r$, contra hypothesin. Vnde manifestum pro omnibus ipsius " valoribus theorema verum esse.

87. Superest casus vbi $\mu=$ r. Per methodum prorsus similem ei qua in art. praec. vsi sumus, sine adiumento theorematis binomialis demonstrari potest, esse

$$
(\alpha+h p)^{t-1} \equiv \alpha^{t-1}+\alpha^{t-2}(t-1) h p\left(\bmod p^{2}\right)
$$

- $(\alpha+h p)^{t-2} \equiv \alpha^{t-1}+\alpha^{t-2}(t-2) h p$

$\omega(\alpha+h p)^{t-3} \equiv a^{t-1}+a^{t-2}(t-3) h p$

etc.

vide aggregatum erit (quia partium multitudo $=t)$

$$
\equiv t a^{t-\mathrm{r}}+\frac{(1-1) t}{2} \cdot a^{t}-2 h p\left(\bmod \cdot p^{2}\right)
$$

At quoniam $t$ per $p$ diuisibilis, etiam $\frac{(t-s) t}{2}$ per $p$ diuisibilis erit in omnibus: casibus excepto eo ybi $p=2$ de quo. iam in art. 


\section{$-85-$}

praec. monuimus. In reliquis autem casibus erit $\frac{(t-1) t}{2} \alpha^{t-2} h p \equiv 0\left(\bmod p^{2}\right)$, adeoque ete iam illud aggregatum $\equiv \operatorname{tat} a^{t-2}\left(\bmod . p^{2}\right)$ vt in art. praec. In reliquis demonstratio hic eodem modo procedit vt istic.

Colligimus igitur generaliter vnico casu. $p=2$ excepto, esse.

$\left.\left(\alpha+h_{i p k}\right)^{r} \equiv \alpha^{t}\left(\bmod . p^{\mu t}\right)^{t}\right)$ et

$\left(\alpha+h p^{k}\right)^{t}$ non $\equiv \alpha^{t}$ pro quouis modulo qui sit altior potestas ipsius $p$, quam haec, pkt", quoties quidem $h$ per $p$ non est diuisibilis, atcque $p^{y}$ potestas suprema ipsius $p$ quae numerum $t$ diuidit.

Hinc protinus deriuantur propositiones I. et 2. quas art. 85 demonstrandas nobis proposueramus: scilicet

primo, si $\alpha^{t} \equiv \mathrm{I}$, erit etiam $\left(\alpha+h p^{n-1}\right)^{\mathfrak{t}} \equiv \mathrm{x}$ (mod. $\left.p^{n}\right)$;

secundo si numerus aliquis $\alpha^{\prime}$ ipsi $A$ adeoque etiam ipsi \& secundum modulum $p$ congruns, neque vero huic secundum modulum $p^{\text {p.-." }}$, congruentiae $x^{t} \equiv 1\left(\bmod , p^{\mathrm{n}}\right)$ satisfaceret, ponamus $\alpha^{\prime}$ esse $=\alpha+i p^{\lambda}$, ita vt $i$ per $p$ non sit diuisibilis, eritque $\lambda<\mu-n$, tunc autem $\left(\alpha+l p^{\lambda}\right)^{k}$ secundum modulum $p^{\lambda t y}$ ipsi $a^{t}$ congruus erit, non autem secundum modulum $p^{k}$, quae est altior potestas, quare $\alpha^{\prime}$ radix congruentiae $x^{t} \equiv$ I esse nequit.

88. - Tertium vero fuit radicem aliquam congruentiae $x^{t} \equiv \mathrm{I}\left(\bmod . p_{i}\right)$, ipsi $A$ con- 
gruam, ininenire. Ostendemus hic tantummo: do quomodo hoc fieri possit, si iam radix eiusdem congruentiae secundum modulum $p^{\mathrm{n}-\mathrm{I}}$ innotuerit; manifesto hoc sufficit, quum a moduio $p$ pro quo $A$ est radix, ad modulum $p^{2}$, sicque dinceps ad omnes potestates consecu. tiuas progredi possimus.

Esto itaque a radix congruentiae $x^{t} \equiv$ I $\left(\bmod p^{\mathrm{n}-1}\right)$ quaeriturque radix eiusdem con. gruentiae secundum modulum $p^{\mathrm{n}}$, ponatur haec $=a+h p^{n-s-x}$, quam formam eam habere debere ex art. praec. sequitur (casum vbi $=n$ - I postea seorsim considerabimus: maior vero quam $n-1$, esse nequit). Debet ita-

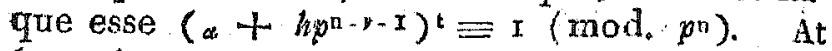

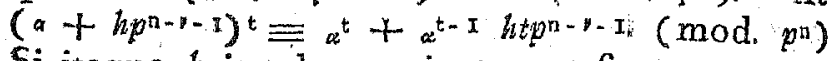
Si itaque $h$ ita demerminatur, vt fiat $I \equiv \alpha^{t}+$ $a^{k-1} h t^{n-y-1}\left(\bmod\right.$. $\left.p^{\mathrm{n}}\right)$; sive (quia per hyp."I $\equiv \alpha^{t}\left(\bmod _{0} p^{12-1}\right)$ atque $t$ per $p^{\prime \prime}$ diuisibilis $)$ ita vt hiat $\frac{x^{t}-\frac{1}{p^{0}-1}+x^{t-x}}{h p^{\prime}}$ per $p$ diuisibilis, quaesito satisfactum erit. Hoc auten semper fieri posse ex Sect. praec. manifestum, quum $t$ per altiorem ipsius $p$ potestatem quam $p^{\prime}$ diuidi non posse hic supponamus, adeoque $\alpha^{t+1} \frac{t}{p^{p}}$ ad $p$ sit primus.

Si vero $=n-1 j_{\text {, }}$ e $t$ per $p^{n-1}$ silue etiam per altiorem ipsius $p$ potestatem diuisibilis quituis valor $A$, congruentiae $x^{t} \equiv \mathrm{I}$ secundum modulum $p$ satisfaciens eidem etiam secundum modulum $p^{n}$ satisfaciet. Sit enim 
$t=p^{\mathrm{n}-1} t$, eritque $t \equiv x(\bmod p-x)$ : quare quoniam $A \mathrm{t} \equiv \mathrm{r}(\bmod . p)$ erit etiam $A^{\tau} \equiv I$ (mod. $\left.p\right)$. Ponatur itaque $A^{r}=I-1$ $h p$ eritque $A^{k}=(\mathrm{I}+h p)^{p^{n-1}} \equiv \mathrm{I}\left(\bmod , p^{n}\right)$ art. 87 .

89. Omnia quae art. 57 sqq adiumento therematis, congruentian $x^{\mathrm{t}} \equiv$ I plures quam $t$ radices cinersas non habere eruimus, etiam pro modulo qui est numeri primi potestas locum habent, et si radices primitimae vocantur nume$r i$ qui ad exponentem $p^{\mathrm{M}-\mathrm{I}}(p-1)$ pertinent, siue in quorum periodis omnes numeri per $p$ ron diuisiuiles inuerintur, etiam hic radices primitiuae exstabunt: Omnia autem quae supra de indicibus eorumque vsu tradidimus, nec non de solutione congruentiae $x^{t} \equiv \mathbf{I}$, ad hunc quoque cesum applicari possunt. Quas quum nulli difficultat obnoxia sint omnia ex integro repetere superflum foret. Praeterea radices congruentiae $x^{\mathrm{t}} \equiv$ I secundum modulum $p^{n}$ e radicibus eiusdem congruentiae sècundum $p$ deducere docuimus. Sed de eo casu, ubi potestas aliqua numeri 2 est modulus quia supra exceptus fuit, aliqua adhuc sunt adicienda.

90. \$i potestas aliqua numeri 2 , altior quam secanda, puta $2^{11}$ pro modulo accipitur, numeri cuiusuis imparis potestas exponentis $2^{\mathrm{n}-2}$, vnitati est congrua.

Ex. gr. $5^{\mathrm{s}}=656_{\mathrm{I}} \equiv \mathrm{x}(\bmod 32)$.

Quiuis enim numerus impar vel sub forma $14 h$, vel sub hae $-I+4 h$ compre F 4 
benditur: vnde propositio protinus sequitur (theor. art. 85).

Quoniam igitur exponens ad quem quicunque numerus impar secundum modulum $2^{n}$ pertinet, diuisor ipsius $2^{n-2}$ esse debet, quiuis ad aliquem horum numerum pertinebit 1 , $2,4,8 . \ldots 2^{2 n-2}$, ad quemnam vero pertineat ita facile diiudicatur. Sit numerus propositus $=4$ te $\mathrm{I}$, atque exponens maximae potestatis numeri 2 , quae ipsum $h$ metitur, $=m$ ( qui et$\mathrm{iam}=0$ esse potest, quando scilicet h est impar); tum exponens ad quem numerus propositus pertinet, erit $=2^{\mathrm{n}-\mathrm{m}-2}$, siquidem $n>m$ +2 ; si autem $n=$ vel $<m+2$, numerus propositus est $\equiv \pm$ adeoque vel ad exponentem $\mathrm{I}$ vel ad exponentern 2 pertinebit. Numerum enim formae $\pm 1+2^{\text {n+x }} k$, (quae huic aequiualet, 4 in \pm 1 ) ad potestatem exponentis $2^{\mathrm{n}-\mathrm{m}-2}$ eleuatum vnitati secundum modulum $2^{\mathrm{n}}$ congruum fieri, ad potestatem autem exponentis, qui est inferior numeri 2 potestas, incongruum, ex art. 86 nullo negotio deducitur. Numerus itaque quicunque formae $8 k+3$ vel $8 k+5$ ad exponentem $2^{n-2}$ pertinebit.

9r. Hinc patet eo sensu quo supra expressionem accepimus, radices primitiuas hic non dari, nullos scilicet numeros, quorum periodus omnes numeros modulo minores ad ipsumque primos amplectatur. Attamen facile perspicitur, analogon hic haberi. Inuenitur enim, numeri formae $8 k+5$ pofestatem expo. nentis imparis semper esse formae $8 k+3$, 
potestatem autem exponentis paris, semper formae $8 k+1$; nulla igitur potestas formae $8 k$ +7 esse potest. Quare quum periodus numeri formae $8 k+3$, ex $2^{n-2}$ terminis diversit coistet, quorum quisque aut formae $8 k+3$ aut huius, $8 k+1$, neque plures huiusmodi numeri modulo minores dentur quam $2^{\mathrm{n}-2}$, manifesto, quiuis numerus formae $8 k+1+\mathrm{r}$ vel $s k$ +3 congruus est secundum modulum $2^{\mathrm{n}}$ potestati alicui numeri cuinscunque formae $8 k$ + 3. Simili modo ostendi potest periodum numeri formae $8 k+5$ comprehendere omnes numeros formarum $8 k+1$ et $8 k+5 . \quad \mathrm{Si}$ igitur numerus formae $8 k+5$ pro basi assumitur, omnes numeri formae $8 k+1$ et $8 k+5$, positine, omnesque formae $8 k+3$ et $8 k+7$, negatiue sumbi, indices reales nanciscentur, et quidem hic indices secundum $2^{\mathrm{n}-2}$ congrui pro aequiualentibus sunt habendi. Hoc modo tabula nostra I intelligenda, vbi pro modulis I 6 ; 32 et 64 (namque pro modulo 8 nulla tax bula necessaria erit) semper numerum 5 pro basi accepimus. Ex.gr. numero 19 qui est formae $8 n+3$ adeoque negatiue sumendus, respondet pro modulo 64 index 17 , id quod significat esse $5^{z} \equiv-19$ (mod. 64). Numeris autem formarum $8 n+x, 8 n+5$ negatiue, atque numeris formarum $8 n+3,8 n+7$ positine acceptis, indices quasi imaginarii tribuendi forent. Quos introducendo calculus indicum ad algorithmum perquam simplicem reduci potest. 'Sed quoniam, si haeo ad omnem rigorem exponere vellemus, nimis longe euagari oporteret, hoc negotium ad aliam occasionem 


\section{- $9^{\circ}-$}

nobis reseruamus, quando forsan fusius quan. titatum imaginarium theoriam, quae nostro quidem iudicio a nemine hactenus ad notiones claras est reducta, pertractare suscipiemuis. $\mathrm{Pe}$ riti hunc algorithmum facile ipsi eruent: qui minus sunt excercitati, perinde tamen tobula hac.vti poterunt, vt ii qui recentiorum commenta de logarithmis imaginariis ignorant, logarithmis vtuntur, si quidem principia supra stabilita probe tenuerint.

92. Secundum modulum e pluribus primis compositum tantum non omnia quae ad residua potestatum pertinent ex theoria congruentiarum generali deduci possunt; quia vero infra congruentias quascunque secundum modizlum e pluribus primis compositum ad congruentias, quarum modulus est primus aut primi potestas, reducere fusius docebimus, non est quod huic rei nultum hic inmoremur. Obseruamus tantum, bellissimam proprietatem, quae pro reliquis modulis locum habeat, quod seilicet semper exstent numeri quorum periodus omnes numeros ad modulum primos complectaturr, hic deficere-, excepto vnico casu, quando scilicet modulus est dupplum numexi primi, ant potestatis numeri primi. Si enim modulus nn redigitur ad formam $A^{a} B^{b} C$ c etc. designantibus $A, B, C$ etc. numeros primos diuersos, praeterea $A^{\mathrm{a}-\mathrm{x}}(A-1)$ disignatur per $\alpha_{9} B^{\mathrm{b}-\mathrm{I}}$ $(B-I)$ per 6 etc. denique $z$ est numerus ad $m$ primus; erit $z^{\alpha} \equiv \mathrm{I}\left(\bmod . A^{\mathrm{a}}\right) z^{b} \equiv \mathrm{I}$ $\left(\bmod . A^{b}\right)$ etc. Quodsi igitur $\mu$ est minimus numerorum $\alpha, 6, \gamma$ etc. diuiduus communis, erit 
$z^{\mu} \equiv \mathrm{x}$ secundum omnes modulos $A^{\mathrm{a}}, B^{\mathrm{b}}$ etc. adeoque etiam secundum $m$, cui illorum productum est aequale. At excepto casu vibi est duplum numeri primi ant potestatis nume. ri primi, numerorum $a, b, y$ etc, diniduus com muxis minimus, ipsorum producto est minor (quoniam numeri $\alpha, \bar{b}, \gamma$ etc. inter se primi esse nequeunt sed certe diuisorem a communem habent), Nullius itaque numeri periodas tot terminos comprehendere potest, quot dantur numeri ad modulum primi ipsoque minores, quia horum numerus producto ex $\alpha,:$, y etc. est aequalis, Ita ex. gr. pro $m=$ roo I cuiusuis numeri ad $m$ primi potestas exponeutis 60 vnitati est congrua, quia 60 est dividuus communis numerorum 6, 10, 12. - Casus autem vbi modulus est duplum numeri primi aut diplum. potestatis numeri primi illi vbi est primus aut: primi potestas prorsus est similis.

93, Scriptorum in quibus alii geometrae de argumento in hac sectione pertractato egerunt, fam passim mentio est facta. Eos tamen qui quaedam fusius, quam nobis breuitas permisit, explicata desiderant, ablegamus inprimis ad sequentes ill. Euleri commentationes, ob perspicuitatem qua vir stummus prae omnibus semper excelluit, maxime commendabiles.

Theorenata circe residua ex dizuisione potestatum rea Licta. Com. nou. Pesr. T. VII, p. 49. sqq.

Demonstrationes circa residua ex diwisione potestatum per numeros primos resultantic. Ibid,T, XVIII. p. 85 sqq. Adiungi his possunt Opusculorum analyt, T. I, dissert. 5 et 8 . 


\section{Sectio Qrarta}

Dx

CONGRVENTIIS SECVNDT GRADVS.

94. Theorema, Numero quocunque, $m$, pro modulo acceppo, ex nusneris $o, I, 2,3 \ldots m-I$, plures quam $\frac{1}{2} m+I$ quando $m$ est par, size plures quam $\frac{\mathrm{T}}{2} m+\frac{\mathrm{r}}{2}$, quando m est impar quadrato conm grui fieri non possunt.

Dem. Quoniam numerorum congruorum quadrata sunt congrua: quiuis numerus, qui vlli quadrato congruus fieri potest, etiam quadrato alicui cuius radix $<m$ congruus erit. Sufficit itaque residua minima quadratorum 0 , $x, 4,9 \ldots(m-1)^{2}$ considerare ${ }^{*}$ At facile perspicitur, ésse $(m-I)^{2} \equiv \mathbf{I},(m-2)^{2} \equiv$ $2^{2},(m-3)^{2} \equiv 3^{2}$ etc. Hinc etiam, quando $m$ est par, quadratorum $\frac{\pi}{2}(m-1)^{2}$ et $\left(\frac{T}{2} m+1\right)^{2}$, $\left(\frac{\pi}{2} m-2\right)^{2}$ et $\left(\frac{\pi}{2} m+2\right)^{2}$ etc residua minima eadem erunt: quando vero $m$ est impar, quadrata $\left(\frac{\pi}{2} m-\frac{\pi}{2}\right)^{2}$ et $\left(\frac{T}{2} m+\frac{x}{2}\right)^{2} ;\left(\frac{\pi}{2} m-\frac{3}{2}\right)^{2}$ et $\left(\frac{\pi}{2} m+\frac{3}{2}\right)^{2}$ etc. erunt congrua. Vnde palam est, alios numeros, quam qui alicui ex quadratis $o, I$, 


\section{$-9^{3}-$}

4, 9... ( $\left.\frac{\mathrm{r}}{2} m\right)^{2}$ congrui sint, quadrato congruos fieri non posse, quando $m$ par; quando vero impar, quemuis numerum, qui vlli quadrato sit congruus, alicui ex his $o, x, 4,9 \ldots$ $\left(\frac{\pi}{2} m-\frac{\pi}{2}\right)^{2}$ necessario congruum esse. Quare dabuntur ad summum in priori casu $\frac{r}{2} m+x$ residua minima diverșa, in posterioni $\frac{x}{2} m+\frac{x}{3}$ Q. E. D.

95. Exemplum. Secundum modulum $: 3$ quadratorum numerorum $0, x, 2,3 \ldots .6$ residua minima inueniuntur, $0,1,4,9,3,12,10$, post haec , vero eadem ordine inuerso recurrunt $10, x_{2}, 3$, etc. Quare numeris quisque, nulli ex istis residuis congruus, siue qui alicui ex his est congruus, $2,5,6,7,8, \mathrm{Ir}$, nulli quadrato congruus esse potest.

Secundum modulum 5 haec inueniuntur residua, $0,1,4,9,1$, Io, 6, if post quae èa. dem ordine inuerso recurrunt. Hic igitur numerus residuuorum, quae quadrato congrua $\mathrm{f}$ eri possunt, minor adhuc est, quam $\frac{\pi}{2} n+\frac{\pi}{2}$, quum sint $0,1,4,6,9$, ro. Numeri autem $2,3,5,7,8,11,12,13,14$, et qui horum alicui sunt congrui, nulli quadrato secundum

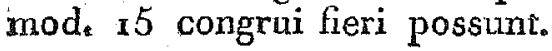

96. Hinc colligitur, pro quouis modulo omnes numeros in duas classes distingui posse, quarum altera contineat numeros, qui quadrato alicui congrui fieri possint, altera eos qui non possint. Illos appellabimus residua quadratica 
numeri istius quem pro modulo accepimus $)^{3}$, hos vero ipsius non-residus quadratica, siue etiam, quoties ambiguitas nulla inde oriri potest, sim. pliciter residua et non-residzc. Ceterum palam est sufficere, si omnes numeri o, $r, 2 \ldots m-c$ in classes redacti sint: numeri enim congrui ad eandem classem erunt referendi.

Etiam in hac disquisitione a modulis primis initium faciemus, quod itaque subintelligendum erit, etiamsi expressis verbis non mo. neatur. Numerus primus 2 autem excludendus, siue numeri primi impares tantum considerandi.

96. Numpro primo pe pro modulo accepto nismerowum $x, 2,3 \ldots q-$ I semissis erunt residua quadratica, reliqui non residwa, i. e. dabuntur $\frac{\tau}{2}(p$ -I) residua, totidemque non-residua.

Facile enim probatur, omnia quadrata $x$, 4, $9 . \ldots \frac{\pi}{2}(p-1)^{2}$ esse incongrua. Scilicet si heri posset $r r \equiv r^{\prime} r^{\prime}$ ( $\bmod . p$ ) atque numeri $r ; r^{\prime}$ inaequales et non maiores quam $\frac{1}{2}(p-r)$ posito $r>r^{\prime}$ i. $q$ licet, feret $\left(r+r^{\prime}\right)(r$ $\left.+r^{\prime}\right)$ positiuus et per $p$ diuisibitis. At vter-

*) Proprie quidem hic casu secundo alio sensu vtimur, quan hucusque fecimus. Dicere scilicet oporteret, $r$ esse residuum quadrati cia secundum modulum, $m$ quando $r \equiv a d(\bmod m)$; at brenitatis gratia in hac sectione semper $\gamma$ ipsius $n$ residuum quadraticum vocamus neque hinc vlla ambiguitas metuenda Expressionem enim, residum, quando idem significat quod numerus congruus, abhinc non adhibebimus, nisi forte de residuus mivinis sermo sit vi nulluin dubiun esse potest. 


\section{$-9^{5} \quad-$}

que factor $r-r^{\prime}$, et $r+r$ ipso $p$ est minor, quare suppositio consistere nequit (art. I3). Habentur itaque $\frac{x}{2}(p-1)$ residua quadratiça, inter hos numeros $x, 2,3 \ldots p-x$ contenta; plura vero inter ipsos esse nequeunt quia accedente residuo oprodeunt $\frac{x}{2}(p+r)$, quem numerum omnium residuorum multitudo superare nequit. Quare reliqui numeri erunt non residua horumque multitudo $=\frac{T}{2}(p-I)$,

Quum cifra semper sit residuum, hanc numerosque per modulum diuisibilesab inuestigationibus his excludimus, quia hic casus per se est clarus, theoreniatumque concinnitatem tantum turbaret. Ex eadem caussa etiam modulum 2 exclusimus.

97. Quum plura quae in hac Sect. exponemus etiam ex principiis Sect. praec. deriuari possint, neque inutile sit, eandem veritatem per methodos diuersas perscratari, hunc nexum ostendemus. Facile vero intelligitur, omnes numeros quadrato congruos, indices pares habere, eos contra, qui quadrato nullo modo congrui fieri possint, impares. Quia vero $p-1$ est numerus par, tot indices pares erunt quot impares, scilicet $\frac{\pi}{2}(p-1)$, totidemque tum residua tum non residua dabuntur.

\section{Exempla.}

Pro modulis sunt residua

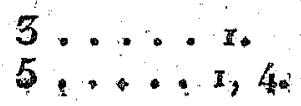




$$
\begin{gathered}
-9^{6}- \\
7 \ldots \ldots 1,2,40 \\
11 \cdots 1,3,4,5,9 . \\
13 \ldots \ldots 1,3,4,9,10,12 . \\
17 \ldots \ldots 1,2,4,8,9,13,15,16 . \\
\text { etc. }
\end{gathered}
$$

reliqui vero numeri, his modulis minores non residua.

98. Theonema. Productum e duobus residuis quadraticis numeri primi $p$, est residunm; productum e residno in non residuum, est non residuntm; denique productum e drobus non-residuis, residizum.

Demoustr. I. Sint $A, B$ residua e quadratis aa, $b b$ oriunda siue $A \equiv a a, B \equiv b b$, eritque productum $A B$ quadrato numeri ab congruum i. e. residuum.

II. Quando $A$ est residuum, puta $\equiv a a$, $B$ vero non residuum, $A B$ erit non-residuum. Ponatur enim si fieri potest $A B \equiv k k$, sitque valor expressionis $\frac{k}{a}$ (mod. $\left.p\right) \equiv b$; erit itaque $a a B \equiv a a b b$, vnde $B \equiv b b, \dot{i}$. e. $B$ residuum contra hyp.

Aliter. Multiplicentur omnes numeri qui inter hos $x, 2,3 . \ldots p-$ I sunt residua (quo. rum multiudo $=\frac{\pi}{2}(p-\mathrm{I})$, per $A$ omniaque producta erunt residua quadratica, et quidem erunt omnia incongrua. Iam si non-residuum $B$ per $A$ multiplicatur, productum nulli pro. ductorum quae iam habentur congruum erit; quare si residuum esset, haberentur $\frac{\pi}{2}(p+x)$ residua incongrua inter quae nondum est residuum o, contra art 96. 
III. Sint $A, B$, non-residua. Multiplicentur omnes numeri qui inter los $1,2,3 \ldots$ $p-1$ sunt residua per $A$, habebunturque $\frac{r}{2}(p-1)$ non-residua inter se incongrua (II); iam productum $A B$ nulli illorum congrum esse potest; quodsi igitur esset nor-residuum, haberentur $\frac{\pi}{2}(p+1)$ non-residua inter se incongrua, contra art. 95. Quare productum etc. Q. E. D.

Facilius adhuc haec theoremata e principiis sect. praec. deriuantur. Quia enim residuorum indices semper sunt pares, non-resi. duorum vero impares, index producti e duobus residuir vel non-residuis erit par, adeoque productum ipsum, residuum. Contra index producti e residuo in non-residuum erit impar adeoque productum ipsum non-residuum.

Vtraque demonstrandi methodus, etiam pro his theorematibus adhiberi potest: Expressionis $\frac{a}{b}(\bmod . p)$ valor exit residium, quando numeri $\sigma_{2}$ $b$ simeal sunt residua, vel simal non residia; contra autem erit non residuam, quando numororum $a, b$ altep est residum alter nontresiduinu. Possunt etiam ex conuersione theorr. art. praec, obtineri.

99. Generaliter, productum ex quotcunque Lactoribus est residum tum quando omnes sunt residua, tum quando non residriorum, quae inter eos occurrunt, multitudo est par; quando. vero multitudo non residuorum quae inter fa $\mathrm{E}$ ctores reperiuntur est impar, productun erit non-residuum, Facile itaque diudicari potest, 


\section{$-98-$}

trum numerus compositus sit residuum, necne, si modo quid sint singuli ipsius factores constet. Quamobrem in tabula II numeros primos tautummodo recepimus. Oeconomia huius tabulae haec est. In margine positi sunt moduli, ${ }^{*}$ ) in facie vero numeri primi successini; quando ex his alquis fuit residuum moduli alicuius, in spatio vtrique respondente lineola collocata est, quando vero numerus pri. mus fuit non residuum moduli, spatium respondens vacuum mansit.

I00. Antequam ad difficiliora progredianur, quaedam de modulis non primis adiicienda sunt.

Si numeri primi $p$, potestas aliqua $p^{\mathrm{n}}$ pro modulo assumitur ( vbi pon esse 2 supponimus), omnium numerorum per $p$ non diuisibiliun moduloque minorum altera semissis erunt residua, altera non residua, i. e. vtrorumque multitudo $=\frac{\pi}{2}(p-I) p^{a-1-x_{0}}$

Si enim $*$ est residuum: quadrato alicui ${ }^{*}$ congruus erit, cuius radix moduli dimidium non superat, vid. art. 94. Iam facile perspicitur, dari $\frac{x}{2}(p-1) p^{\mathrm{u}-1}$ numeros per $p^{\text {non }}$ diuisibiles modulique semisse minoribus; superest itaque vt démonstretur, omnium horum numerorum quadrata incongrua esse, siue residua quadratica diuersa suppeditare. Quodsi duo rum numerorum $c_{p} b$ per $p$ non diuisibilium

$\therefore$ Quomodo etiam modalis compositis carere possimus mox docebimus. 


\section{- $99 \quad-$}

modulique semisse minorum quadrata essent congrua, foret $a a-b b$ siue $(a-b)(a+b)$ per $p^{n}$ diuisibilis (posito i. q. licet $a>b$ ) Hoc vero fieri non potest, nisi vel alter numerorum $a-b, a+b$ per $p^{\text {n }}$ fuerit dinisibilis, quod heri nequit, quoniam vterque $<p^{\mathrm{n}}$, vel alter per $p^{\mathrm{m}}$ alter vero per $p^{\mathrm{n}-\mu}$, i. e. vterque per p. Sed etiam hoc fieri nequit. Manifesto enim etiam summa et differentia $2 a$ et $2 b$ per $p$ foret diuisibilis adeoque etiam $a$ et $b$ contra hyp. - Hinc tandem colligitur inter numeros per $p$ non diuisibiles moduloque minores $\frac{I}{2}(p-I) p^{\mathrm{n}}$ residua dari, reliquos quorum multitudo aeque magna, esse non-residua $Q$. D. - Potest etiam theorema hoc ex conside. ratione indicum deriuari simili modo vt art. 97

I 0 . Quinis numeress per $p$ non diuisibitis, qui ipsius p est residuum, erit residursm etiam ipsius pon ${ }_{0}$ qua vero ipsiss $p$ est son-sesidwum, etiams ipsius. pn nonse residun erit.

Pars posterior huitus propositionis per se est manifesta. Si itaque prior falsa esset, inter numerosipso $p^{\mathrm{n}}$ minores simulque per $p$ mor diuisibiles plures forent residua ipsius $p$, quam spsius $p^{\mathrm{n}}$, i. e. plures quam $\frac{\pi}{2} p^{\mathrm{n}-\mathrm{x}} \cdot(p-1)$. Nullo vero negotio perspici poterit, multitudinem residuorum numeri $p$ inter illos numeros esse praecise $=p^{\mathrm{n}-\mathrm{I}}(p-1)$.

Aeque facile est, quadratum reipsa inue: nire, quod secundum modulum $p^{\text {n }}$ residuo da to sit congruum, si quadratum huic residuo. secundum modulum congrutum habetux. 
Sailicet si quadratum habetur, an, quod residuo dato $A$ secundum modulum $p^{k}$ est congram, deducitur inde quadratum ipsi $A$ secundum modulum $p^{p}$ congraum ( vbi $\nu_{\mu}>_{\mu}$ et $=$ vel $\angle 2 \mu$ supponitur) sequenti modo. $P_{0}$. natur radix quadrati quaesiti $= \pm a+x p \mu$, quam formam eam habere debere facile per. spicitur; debetque esse aa $\pm 2 a x p^{\mu}+x \times p^{2 \mu}$ $\equiv A\left(\bmod . p^{*}\right)$ sine propter $2^{\mu}>\forall, A-a$ $\equiv+2 a x p\left(\bmod p^{\nu}\right)$ Sit $A-a \omega=p \alpha d$, erit. que, $x$ valor expressionis $+\frac{d}{2 u}$ (mod. $p^{p}-\dot{p}$ ) quae huic $+\frac{A}{2 \sin ^{4}{ }^{4}}\left(\bmod _{0} p^{\eta}\right.$ aequiualet.

Dato igitur quadrato ipsi $A$ secundum $p$ congruo, deducitur inde quadratum ispi $A$ secundum modulum $p^{2}$ congruum; hinc ad mo. dulam $p^{4}$, hinc ad $p^{8}$ etc. ascendi poterit.

Ex. Proposito residuo 6, quod secundum modulum 5 quadrato I congruum, inuenitur quadratum $9^{2}$ cui secundum 25 est congruum, $16^{2}$ cui secundum 125 congruum etc.

roz. Quod vero attinet ad numeros per $p$ diuisibiles, patet, eoruin quadrata per pp fore diuisibilia, adeoque omnes numeros per $p$ qui. dem diuisibiles, nequevero per $p p$, ipsitis po. re non residua. Generaliter vero, si proponitưr numerus $p^{k} A$ vbi $A$ per $p$ non est diuisi bilis, hi casus erunt distinguendi:

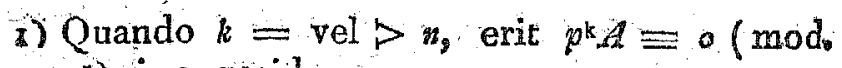
$\left.p^{n}\right)$, i. e residuum.

2) Quando $<4$ atque impar, erit pl $A$ non residuum. 
Si enim esset $p^{k} A=p^{2 x+\pi} A \equiv s s$ (mod. $\left.p^{n}\right)$, ss per $p^{2^{x}+1}$ diuisibilis esset, id quod. aliter fieri nequit, $q$ uam si fuerit $s$ per $p^{2 k+1}$ diuisibilis. 'Tunc veross etiam per $p^{2 x+2}$ diuisibilis, adeoque etiam (quia $2^{*}+2$ certo non maior quam. v) $p \mathrm{k} A$, i. e. $p^{2 x+1} A$; siue $A$ per $p$, contra hyp。

5) Quando $k<n$ atque par. Tum $p^{k} A$ erit residum vel non residuum ipsius $p^{\mathrm{n}}$, prout $A$ est residuum vel non-residuum ipsius $p_{\alpha}$ Quando enim $A$ est residuum ipsius $p$, erit etiam residuum ipsius $p^{n-k}$. Posito autem $A$ $\equiv a a\left(\bmod . p^{\mathrm{n}-\mathrm{k}}\right)$ erit $A p^{\mathrm{k}} \equiv \operatorname{aapk}\left(\bmod \cdot p^{\mathrm{k}}\right)$ aapk vero est quadratum. Quando autem $A$ est non residuum ipsius $p, p^{\mathrm{k}} A$ residuum ipsius $p^{\mathrm{u}}$ esse nequit. Ponatur enim $p^{\mathrm{k}} A \equiv a \equiv$ (mod. $\left.p^{\mathrm{n}}\right)$, eritque necessario aa per $p^{k}$ diuisibilis. Quotiens erit quadratum cui $A$ secundum modulum $p^{n-k}$ adeoque etiam secundum modulum $p$ congruus, i. e. $A$ erit residuum ipsius p contra hyp.

103. Quoniam casum $p=2$ exclusimus, de hoc adhuc quaedam dicenda. Quando numerus 2 est modulus, numerus quicunque erit residuum, non-residua nulla erunt. Quando vero 4 est modulus, omnes numeri impares formae $4 k+\mathrm{r}$ erunt residua, omnes vero formae $4 k+3$ non residua. Tandem quando 8 aut altior potestas numeria estmodulus, omnes numeri impares formae $8 k+1$ erunt residua, reliqui vero, seu ii qui sunt formarum $8 k+5$, $8 k+5,8 k+7$, erunt non residua. Pars posterior huius propositionis inde clara, quod qua. dratum cuiusuis numeri imparis, siue sit for mae $4 k+r$, siue formae $4 k-1$, fit formae $8 k$ + I. Priorem ita probamus. 
I) Si duorum numerorum vel summa vel diffe. rentia per $2^{\mathrm{n}-\mathrm{I}}$ est diuisibilis, numerorum quadrata erunt congrua secundum modulum $2^{\star}$. Si enim alter ponitur $=a$, erit alter formae $2^{n-1} h \pm a$, cuius quadratum inuenitur $\equiv a$ $\left(\bmod , 2^{n}\right)$.

2) Quiuis numerus impar, qui ipsius $2^{n}$ est residuum quadraticum, congruus erit quadrato alicui, cuius radix est numerus impar et $<2^{n-2}$ Sit enim quadratum quodcunque cui numerus ille congruus, at atque numerus $a \equiv \pm \alpha\left(\bmod .2^{n-1}\right)$ ita vt a modu. li semissem non superet (art. 4), eritque a $\doteq$ Qu. Quare etiam numerus propositus erit $\equiv \alpha$. Manifesto vero tum $a$ tum $*$ eruntim. pares atque $\alpha<2^{\mathrm{n}-2}$.

5) Omnium numerorum imparium ipso $2^{\mathrm{n}-2}$ minorum quadrata secundum modulum $2^{\text {n }}$ incongrua erunt, Sint enim duo tales numeri $r$ et $s$, quorum quadrata si secundum $2^{\text {" }}$ essent congrua, foret $(r-s)(r+s)$ per $2^{\mathrm{n}}$ diuisibilis (posito $r s s$ ). Facile vero perspicitur numeros $r-s, r+s$, simul per 4 diuisibiles esse non posse, quare si alter tantummodo per 2 est diuisibilis, alter vt productum per $2^{\text {n }}$ diuisibilis fieret, per $2^{\mathrm{n}-1}$ diuisibilis esse deberet, Q.E.A. quoniam vterque $<_{1} 2^{n-x_{*}}$

4) Quodsi denique haec quadrata ad residuo sua minima positina reducuntur, habebun. tur $2^{n-3}$ residua quadratica diuersa modulo ninora, quorum quoduis erit formae $8 k+x$ 


\section{- $103-$}

Sed quum praecise $2^{\mathrm{n}-3}$ numeri- formae $8 k+I$ modulo minores exstent, necessario hi omnes inter illa residua reperientur. Q. E. D.

Vt quadratum numero dato formae $8 k+r$ secundum modulum $2^{\mathrm{n}}$ congruum inueniatur, methodus similis adhiberi potest, vt in art 102 ; vid. etiam art. 88. - Denique de numeris paribus eadem valent, quae art. 102 generaliter exposuimus.

T04. Circa multitudinem valorum diuersorum (i. e. secundum modulum incongruorum), quos expressio talis $V=\sqrt{ } A\left(\bmod . p^{n}\right)$ admittit, siquidem $A$ est residuram ipsius $p^{\mathrm{n}}$, facile e praecc。 colliguntur haec. (Numerum p. supponimus esse primum, vt ante, et breuitatis caussa casum $n$ $=\mathrm{I}$ statim includimus). I. Si $A$ per $p$ non est diuisibilis, $D$ vnum valgrem habet pro $p=2, n=$ $x$, puta $V \equiv \mathrm{I}$; duos, quando $p$ est impar, nec non pro $p=2, n=2$, puta ponendo vnum $\equiv v$, alter erit $\equiv-v$; quatuor pro $p=2, n>2$, scilicet ponendo vnum $\equiv v$, reliqui erunt $\equiv-v$, $\dot{2}^{n-2}+v, 2^{n-2}-v$. II. Si $A$ per $p$ diuisibilis est, neque vero per $p^{\mathrm{n}}$, sit potestas altissima ipsius $p$ ipsum $A$ metiens $p^{2 w}$ (manifesto enim ipsius exponens par esse debebit) atque $A=$ $a p^{2 / \mu}$. Tunc patet, omnes valores ipsius $\nu$ per $p$ diuisibiles esse, et quotientes e diuisione ortos fieri valores expr. $n=\sqrt{ } a\left(\bmod . p^{n-2 \mu}\right)$; hinc omnes valores diuersi ipsius $\nu$ prodibunt, multiplicando omnes valores expr. $\nu^{\prime \prime}$ inter o et $p^{n-\mu_{0}}$ sitos per $p^{\text {ic }}$, quare illi exhibebuntur per $v p^{4}$, $v p_{-}^{\mu}+p^{n-\mu}, v p^{\mu}+2 p^{n}-\mu \nu p^{\mu}+\left(p^{\mu}-I\right) p^{n-\mu}$ 
si $v$ indefinite omnes valores diuersos expr. In exprimit, jta vt illorum multitudo fiat $p^{\mu}, 2 p^{\text {se }}$ vel $4 p^{\mu}$, prout multitudo horum (per casum I) est I, 2 vel 4 . III. Si $A$ per $p^{n}$ diusibibilis est, facile perspicietur, statuendo $n=2 m$ vel $=2 m$ - I, prout par est vel impar, omnes numeros per $p^{m}$ diuisibiles, neque vllos alios, esse valores ipsius $V$; quare omines valores diuersi hi erunt $\underset{p^{n-m}}{0, p^{m}}, 2 p^{m} \ldots\left(p^{n-m}-1\right) p^{m}$, quorum multitudo

105. Superest casus, vbi modulus $m$ pluribus numeris primis compositus est Sit $m=$ $a b c \ldots$, designantibus $a, b, c$ etc. numeros primos diuersos aut primorum diuersorum potestates, patetque statim, si $n$ sit residuum ipsius $m$, fore etiam $n$ residuum singulorum: $a, b, c$ eic., adeoque $n$ certo nonresicuum ipsius $m$ esse, si fuerit NR. vllius e rumeris $a, b, c$ etc. Vice versa autem, si $n$ singulorum $a, b, c$ etc. residuum est, etiam residuum producti $m$ erit. Supponendo enim, $n=A^{2}, B^{2}, C^{2}$ etc. sec. mod. $a, b, c$ etc. resp., patet, si numerus $N$ ipsis $A, B, C$ etc. sec. mod. $a, b, c$ etc resp. congruus eruatur (art. 32 ), fore $n \equiv N N$ secundum ommes hos modulos adeoque etiam secundum productum $m_{1}$ - Quum facile perspiciatur, hoc modo e combinatione $c u-$ iusuis valoris ipsius $A$ siue expr. $\sqrt{ } n(\bmod , a)$ cum quouis valore ipsius $B$ cum quouis valore ipsius $C$ etc oriri valorem ipsius $N$ siue expr. $\sqrt{n}(\bmod m)$, nec non e combinationibus diuersis produci diuersos $N$, et e cunctis cunctos: multitudo ommum valorum diuersorum ipsius $N$ aequalis 


\section{$-105-$}

erit producto e multitudinibus valorum ipsorum $A, B, C$ etc. quas determinare in art. praec. docuimus. - Porro manifestum est, si vnus valor expressionis $\sqrt{ } n$ (mod. $m$ ) siue ipsius $N$ fuerit notus, hunc simul fore valorem omniun $A, B, C$ etc, ; et quum hinc per art. praec. omnes reliqui valores harum quantitatum deduci possint, facile sequitur, ex vno valore ipsius $N$ omnes reliquos obtineri posse.

Ex. Sit modulus 3 ,5 cuius residuum an non residuum sit 46 , quaeritur. Diuisores primi numeri 3 r5 sunt $3,5,7$, atque numerts ${ }_{3} 6$ residuum cuiusuis eorum quare etiam ipsins $3 \mathrm{r} 5$ erit residurm. Porro, quia $46 \equiv \mathrm{r}$, et $\equiv 64(\bmod ).) ; \equiv 1$ et $\equiv 16(\bmod .5) ; \equiv 4$ et $\equiv 25$ (mod. 7 ), inueniuntur radices qua. dratorum, quibus 46 secundum modulum 3.5 congruus, r9, 26, 44, $89,226,27 \mathrm{r}, 289,296$.

106. Ex praecedentibus colligitur, sitantummodo semper dignosci possit vtrum numerus primus datus numeri primi dati residuum sit an nonresiduum, omnes reliquos casus ad hune reduci posse. Pro illo itaque casu criteria certa omni studio nobis erunt indaganda. Antequam autem hanc perquisitionem aggrediamur, criterium quoddam exhibemus ex Sect. petitum quod quamuis in praxi nullum fere vsum habeat tamen propter simplicitatem atque generalitatem memoratu dignum est.

Numerus quicunque $A$ per numerum primum 2 $n+1$ non diuisibilis, huizs primi residunm est vel non-tesidum, prout $A^{\mathrm{w}} \equiv+\mathrm{I} \mathrm{et}^{\mathrm{t}} \equiv-\mathrm{I}$ (mot. $2 m+$ I)

\section{G 5}




\section{- $106-$}

Sit enim pro modulo $2 m+r$ in systemate quocunque numeri $A$ index, $a$, eritque $a$ par, quando $A$ est residuum ipsius $2 m+\mathrm{x}$, impar vero quando $A$ non-residuum. At numeri $A^{\mathrm{m}}$ index erit $m a$, i. $e . \equiv 0$ vel $\equiv m(\bmod .2 m)$, prout a par vel impar. Hinc denique $A^{\mathrm{m}}$ in priori casu erit $\equiv+1$, in posteriori vero $=-$ I (mod. $2 m+1$ ). V. artt. 57,62 .

Ex. 3 ipsius 13 est residuum quia $3^{6} \equiv 1$ (mod. 13), 2 vero ipsius 13 non-residuum, quo. niam $2^{6} \equiv-i$ (mod. 13).

At quoties numeri examinandi mediocriter sunt magni, hoc criterium ob calculi immensitatem prorsus inuitile erit.

107. Facillimum quidem est, proposito modulo, omnes assignare numeros, qui ipsius residua sunt vel non residua. Scilicet si ille numerus ponitur $=$ m, determinari debent qua. drata, quorum radices semissem ipsius $m$ non superant, siue etiam numeri his quadratis secundum $m$ congrui (ad praxin methodi adhuc expeditiores dantur), turicque omnes numeri horum alicui secundum $m$ congrui, erunt residua ipsius $m$, omnes autem numeri nulli istorum congrui erunt non-residua. - At quaestio inuersa, proposito nunuero atiquo, assignare ommes numeros quartum ille sit residitum vel non-residuum. multo altioris est indaginis, Hoc itaque pro. blema, a cuius solutione illud quod in art. praee nobis proposuimus pendet, in sequentibus perserutabimur, a casibus simplicissimis inchoantes. 


\section{$-107-$}

108. Theorema. Ommizm numerorum formae $4^{n}+\mathrm{I},-\mathrm{I}$ est residurum quadraticam, omnizms vero sumerertum primorum format $4 n+3$, non-reo sidusum.

$E x_{0}-1$ est residuum numerorum $5, \mathrm{r} 3$, $17,29,37,4 \mathrm{I}, 53,61,73,89,97$ etc., e quadratis numerorum $2,5,4,12,6,9,23,11,27$, 54,22 etc. respêtiue oriundum; contra non. residuum est numerorum $3,7, \mathrm{x} x, \mathrm{r} 9,23,5 \mathrm{r}$, $43,47,59,67,7 x, 79 ; 83$ etc.

Mentionem huius theor. iam in art. $64 \mathrm{fe}$ cimus. Demonstratio vero facile ex art. 106 petitur. Etenim pro numero primo formae $4 \mathrm{n}$ $+\mathrm{x}$ est $(-1)^{2 \mathrm{n}} \equiv \mathrm{I}$, pro numero autem formae $4 n+3$ habetur $(-\mathrm{r})^{20,1} \equiv-\mathrm{T}$. Conuenit haec demonstratio cum ea quam l. c.tradidimus. Sed propter theorematis elegantiam atque vtilitatem non superfluum erit, alio adhuc modo idem ostendisse.

Iog. Designemus complexum omnium residuorum numeri primi $p$, quae ipso $p$ suntminora, excluso residuo o, per literam $C$, et quoniam horum residuorum multitudo semper $=\frac{p-z}{2}$, manifestum est, eam fore parem, quoties p sit formae $4 n+\mathrm{r}$, imparem vero, quoties $p$ sit formae $4 n+3$. Dicantur, ad instar art. 77 , vbi de numeris in genere agebatur, residua socia talia, quorum productum $\equiv \mathbf{x}(\bmod p)$; manifesto enim sir est residuum, etiam $\frac{I}{r}$ (mod. p) residuum erit. Et quoniam idem residuum plura socia inter residua $C$ habere nequit, patet omnia residua $C$ in classes distribui posse, 


\section{- $108-$}

quarum quaeuis bina residua socia contineat. Iam perspicuum est, si nullum residuum daretur, quod sibi ipsi esset socium, i. e. si quaeuis classis bina residuae inaequalia contineret, omnium residuorum numerum fore duplum nameri ornium classium; quódsi vero aliqua dantur residua sibi ipsis socia, i. e. aliquae classes quae vnicum tantum residum aut, si quis malit, idem residuun bis continent, posita harum classium multitudine $=a$, reliquarumque multitudine $=b$; erit omnium residuorum $C$ numerus $=a+2 b$. Quare quando $p$ est formae $4 n+1$, erit a numeros par; quando autem $p$ est formae $4 n+3$, erit a impar. At numeri ipso $p$ minores. alii, quam $\Sigma$ et $p$ - 1 , sibi ipsis socii esse nequeunt (vid art. 77); priorque ' certo inter residua occurrit; vnde in priori casu $p-1$ (seu quod hic idem valet, -1 ) debet esse residuum, in posteriori vero non-residuum; alias enim in illo casu foret $a=1$, in hoc autem $=2$, quod fieri nequit。

I10. Etiam haec demonstratio ill. Fule. ro debetur, qui et priorem primus inuenit. $V$. Opuse. Anal. T. T. p 135. - Facile quisquis videbit eam similibus principiis innixam esse, vt demonstratio nostra secunda theor. Wilsoniani art. 77. Si vero hoc theorema supponere velimus, facilius adhuc demonstratio exhiberi poterit. Scilicet inter numeros $1,2,3$... $p-I$ erunt $\frac{p-x}{2}$ residua quadratich ipsius $p$ totidemque non-residua; quare non-residuorum multitudo erit par, quando $p$ est formae $4 n+5$. Hinc productum ex omnibus numeris 
I, 2, 3...p $-\mathbf{x}$ in priori casu erit residuam, in posteriori non-residuum (art. 99). At productum hoc semper $\equiv-1(\bmod , p)$; adeoque eriam - I in priori casu residuum, in posteriori non-residunm erit,

III. Si itaque $r$ est residuum numeri alicuius primi formae $4 n+1$, etiam $\cdots r$ huius primi residuum erit, omnia autem talis numeri non-residua, etiam signo contrario sumta nonresidua manebunt. Contrarium enenit pro numeris primis formae $4 n+3$, quorum residua quando signum mutatur, non-residua fiunt et vice versa, vid. art. 98 .

Ceterum facile ex praecadentibus deriua. tur regula generalis: - I residuum omnium numerorum qui neque per 4 neque per ullum numerum primum formae $4 n+5$, diuidi possunt; omnium reliquorum non-residuum. $V$. artt rob et 105.

I12. Progredimurad residua $+2 e t-2$.

Si ex tabula II colligimus omnes numeros primos quorum residuum est +2 , hos habebimus: $7,17,23,31,41,47,7 \mathrm{I}, 73,79,89,97$. Facile autem animaduertitur, inter hos numeros nullos inueniri formarnm $8 n+3$ et $8 n+5$.

Videamus itaque, num haec inductio ad certitudinem enehi possit.

Primum obseruamus quemuis numerum compositum formae $8 n+3$ vel $8 n+5$ neces-

-) Quando igitur de numero quocunque loquemur guatenus nutwerl formae $4 \mathrm{rt}+\mathrm{I}$ residnum vel non-resiaum est, ipsius signum omnino negligere sine etiam signum auceps to ipsi tribuere poteo rimus. 
sario factorem primum alterutrius formae $\$ n$ +3 vel $8 n+5$, inuoluere; manifesto enim solis numeris primis formarum $8 n+1,8 n$ +7 , alii numeri quam qui sunt formae $8 n+$ I vel $8 n+7$, componi nequeunt. Quodsi itaque inducto nostra generaliter est vera, nullus omnino numerus formae $8 n+3,8 n+5 \mathrm{da}$ bitur, cuins residuum +2 ; sicque nullus certe numerus huius formae infra 100 exstat, cuius residuum sit -2 . Si autem vltra hunc limitem tales numeri repirirentur, ponamus mimimum omnium $=t$. Erit itaque $t$ vel formae $8 n+3$ vel $8 n+5 ;+2$ ipsius residuum erit, omnium autem numerorum similium minorum non-residuum. Ponatur $2 \equiv a a(\bmod . t)$ poteritque a ita semper accipi vt sit impar simulque $<$, $t$, (habebit enim a ad minimum duos valores positiuos ipso $t$ minores quorum summa $=t$, quorumque adeo alter par alter impar vo art. 104. 105). Quo facto sit aa=2 $+t u$, siue $t u=a a-2$, eritque aa formae $8 n+x$, tu igitur formae $8 n-1$, adeoque $n$ formae $8 n+3$ vel $8 n+5$, prout $t$ est for. mae posterioris vel prioris. At ex aequatione $a a=2+t u$ sequitur, etiam $2 \equiv a$ (mod. $u$ ) i. e. 2 etiam ipsius residuum fore Facile vero perspicitur, esse $u<t$; quare $t$ non est minimus numerus inductioni nostrae contrarius contra hyp. Vrde manifesto sequitur id quod per inductionem inueneramus generali: ter verum esse.

Combinando haec cum prop. arti xII. sequentia theoremata nanciscimur. 


\section{III}

I. Numerorum omnium primorum forma $8 n+3$, + 2 erit non-residuru, - vero residunm.

II. Numerornm omnitum primorum fornas 8 n +5 , tum +2 tum -2 erunt non-residua.

I 3, Per similem inductionem ex tab. II inueniuntur numeri primi quorum residumm est -2 hi: $3,11,17,19,41,59,67,73,83$, $89,97^{*} \%$. Inter quos quum nulli inueniantur formaruni $8 n+5,8 n+7$, num etiam haec inductio theorematis generalis vim adipisci possit inuestigemus. Ostenditur simili modo vt in art. praec. quemuis numerum compositum formae $8 n+5$ vel $8 n+7$, factorem primum inuoluere formae $8 n+5$ vel formae $8 u$ * 7 , ita $v t$, si inductio nostra generalitervera, - 2 nullius omnino numeri formae $8 n+5$ vel $8 n+7$ residuum esse possit. Si autem rales numeri darentur, ponatur omnium minimus $=t$, fiatque $-2=a t-t u$. Vbi si vti supra a impar ipsoque $t$ minor accipitur, as erit formae $8 n+5$ vel $8 n+7$, prout $t$ formae $8 n$ +7 vel $8 n+5$. At ex eo quod an $+2=t u$ atque $a<t$; quisquis facile deriuari poterit, etiam $u$ ipso $t$ minorem fore. Denique -2 etiam ipsius residuum erit; $i_{0}$ e. $t$ non erit minimus numerus qui inductioni nostrae aduersatur, contrahyp. Quare necessario - 2 omnium numerorum formarum $8 n+5,8 n+7$ non residuum.

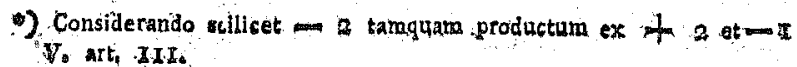




\section{2}

Combinando haec cam propp. art i in, pro. deunt theoremata haec:

I. Omnism numerorssm primorum formae $8 n+5$, tum - 2, tum +2 sunt non-residua, vit iam in art. praec. inuenimus,

II. Omnium numerorum primorumm formas 8 n $+7,-2$ est non - residursm, +2 vero residum.

Ceterum in vtraque demonstratione pro a etiam valorem parem accipere potuissemus; tunc autem 'casum vbi a fuisset formae $4 n+2$, ab eo distinguere oportuisset, vbi $a$ formae $4 n$. Euolutio autem perinde procedit vti supra, nullique difficultati est obnoxia.

I 14. Vus adhuc superest casus, scilicet vbi numerus primus est formae $8 n+$ I. Hic vero methodum praecedentem eludit, artificia. que prorsus peculiaria postulat.

Sit pro modulo primo $S n+x$, radix quae. cunque primitiua, a, eritque (art. 62 ) $a^{4 \mathrm{n}} \equiv$ - I (mod. $8 n+I$ ), quae congruentia ita etiam exhiberi potest, $\left(a^{2 n^{2}}+I\right)^{2} \equiv 2 a^{2 n}$ (mod. Sn+1), sine etiam ita, $\left(v^{2 n}-1\right)^{2} \equiv-2 a^{2 n}$. Vnde sequitur tum $2 a^{2 \mathrm{n}}$, tum $-2 a^{2 \mathrm{n}}$ ipsius $8 n+1$ esse residuum at quia $a^{2 n}$ est quadratum per modulum non diuisibile, manifesto etiam tum +2, tum -2 residua erunt (art. 98.)

I5. Haud inutile erit, adhuc aliam huius theorematis demonstrationem adiicere, quae similem relationem ad praecedentem habet, vt theorematis art. ro 8 demonstratio secunda 


\section{$-\quad 13-$}

(art. 109 ) ad primam (art. 108). Periti facilius tunc perspicient, binas demonstrationes tam-illas quam has non adeo heterogeneas esse, quam primo forsan aspectu videantur.

I. Pro modulo quocunque primo formae $4 m+r$, inter numeros ipso minores $\mathrm{I}, 2,3 \ldots$ $4 \mathrm{~m}$, reperientur im qui biquadrato congrui esse possunt, reliqui vero $3 m$ non poterunt.

Facile quidem hoc ex principiis sect. praec: derinatur, sed etiam absque his demonstratio haud difficilis. Demonstrauimus enim pro tali modulo - $x$ semper esse residuum quadraticum. Sit itaque $f f \equiv-x$ patetque, si $z$ fuerit numerus quicanque per. modulum non diuisibilis, quaternorum numerorum $+z$, $-z,+f z,-f z$ (quos incongruos esse facile perspicitur) biquadrata inter se congrua fore; porro manifestum est biquadratum numeri cuiuscunque, qui nulli ex his quatuor congruus, illorum biquadratis congruum fieri non posse, (alias enim congruentia $x^{4} \equiv z^{4}$ quae est quarti gradus plures quam 4 radices haberet, 'contra art. 43). Hinc facile colligitur, omnes numeros $1,2,3, \ldots 4 m$, tantummodo $m$ biquadrata incongrua praebere, quibus inter eosdem numeros $m$ congrui reperientur, reliqui antem nulli biquadrato congrui esse poterunt.

II. Secundum modulum primum formae $8 n+I,-I$ biquadrato congruus fieri poterit $(-\mathrm{I}$ erit residum biquadraticum huius numeri primi ). 


\section{$-114-$}

Omnum enim residuorum biquadraticorum ipso $8 n+$ I minorum (cifra exclusa) multitudo erit $=2 n$ i. e. par. Porro facile probatur, si $r$ fuerit residum biquadraticum ipsius

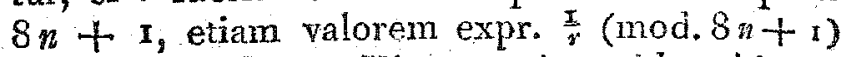
fore tale residum. Hinc omnia residua biqua dratica in classes simili modo distribui poterunt, vti in art. 109 residua quadratica distri. birnus: nec non reliqua demonstrationis pars. prorsus eodem modo procedit vt illic.

III. Iam sit $g^{4} \equiv-\mathrm{r}$, et $h$ valor expr. $\frac{\pi}{8}(\bmod .8 n+I)$. Tunc erit $(g \pm h)^{2}=$ $g^{2}+h^{2} \pm 2 g h \equiv g^{2}+h^{2} \pm 2$ (propter $g h$ $\equiv$ I). At $g^{4} \equiv$ - I, adeoque $-h^{2} \equiv g^{4} h^{2}$ $\equiv g^{2}$, vnde tandem $g^{2}+h^{2} \equiv 0$, atcue $(g$ $\Rightarrow h)^{2} \equiv \pm 2$ i. e. tum +2 , tum - 2 resi. duun quadraticum ipsius $8 n+$ r. Q. E. D.

116. Ceterum ex praec. facile regula se. quens generalis deducitur: +2 est rosidum smeri cuizusuis, qui neque per 4, neque per vllum prinium formae $8 n+3$ vei $8 n+5$ dinidi potest, reliquorum antem ( $\mathrm{ex} . \mathrm{gr}_{+}$omnium numerorum formarum $8 n+3,8 n+5$, siue sint primi, siue compositi) non-residusum.

- 2 est residuum numeri cuizusuis, qui neque por 4, neque per vilum primun formae $8 n+5$ vel $8 n$ +7 chuidi potest, omnizm antem reliquorum non-re. sidunm.

Theoremata haec elegantia iam sagaci Fermatio innotuerunt, $O_{\dot{p}}$. Mathem. p. 168. 


\section{- II5}

Demonstrationem vero quam se habere profes: sus est, nusquam communicauit. Postea ab i1l. Euler frustra semper est inuesrigata: at ill. La Grange primus demonstrationem rigorosam reperit, Nouv. Mem. de l'Aca de Berlin 1775 . p. 349, 35s. Quod ill. Eulerum adhuc latuisse videtur, quando scripsit diss, in opusc. Analyt. conseruatam, I. I. p. $25 \mathrm{~g}$.

I 77. Pergimus ad residua +3 et -3 . A posteriori initium faciamus.

Reperiuntur ex tab. II. numeri primi quod rum residuum est -3 , hi: $3,7,13,19,3 \mathrm{r}$, $37,43,6 x, 67,73,79,97$, inter quos nullus inuenitur formae $6 n+5$. Quod vero etiam vlira tabulae limites nulli primi huius formae dintur quorum residuum -3 , ita demonstramus: Primo patet quemuis numerum compositum formae $6 n+5$ necessario factorem primuin alicuem einsdem formae inuoluere. Quousque igitur nulli numeri primi formae $6 n+5$ dantur, quorum residuum -3 , eousque tales etiam compositi non dabuntur. Quodsi vero vltra tabulae nostrae limites tales numeri daw rentur, sit omnium mininus $=t$, ponaturque $-3=a a-t u$. Tunc erit, si acceperis a parem ipsoque $t$ minorem; $u<t$, atque -3 residuum ipsius th. Sed quando a formae $6 n$ \pm 2 , tu erit formae $6 n+1$, adeoque $u$ formae $6 u+5, Q . E . A$. quia $t$ minimum esse numerum inductioni nostrae aduersantem supposuimus. Quando vero a formae 6n, erit tu formae $36 n+3$ adeoque $\frac{x}{3} t u$ formae $12 n+I_{3}$ 


\section{$-116-$}

quare $\frac{\text { a }}{3} u$ erit formae $6 n+5$; patet antem - 3 etiam ipsius $\frac{x}{3} u$ residuum fore, arque esse $\frac{x}{3} u<t$, Q. E. A. Manifestum inque, -5 nullius numeri formae $6 n+5$ residuum esse posse.

Quoniam quisque numerus formae $6 n+5$ necessario vel sub forma i $2 n+5$, vel sub hac $12 n+$ I I continetur, prior autem forma sub hac $4 n+1$, posterior sub hac $4 n+5$, haec habentur theoremata:

I. Cuinsuis numeri primi formate $12 n+5$, tun -3 tum +3 non-residuun est.

II. Cuinsuis numeri primi formas $12 n+\mathrm{I},-3$ est non-residunn, + 3 vero residuinus.

18. Numeri quorum residuum est +3 ex tabula 11. inueniuntur hi: 3 , I $x, 13,23,57$, $47,-59,6 x, 7 x, 7^{5}, 83,97$, inter quos nulli sunt formae $12 n+5$, vel $12 n+7$. Nullos autem omnino numeros formarum $12 n+5$, I2n +7 dari quorum +3 sit residuum, eodem prorsus modo, vt in artt. $112, \times 13,117$, comprobari potest, quare hoc negotio supersedemus. Habemus itaque collato art. ix i theoremata:

I. Numeri cuiusuis primi formae I2n +5 , son-residua sunt tum +3 tum -3 , (vti iam in art. praec. inuenimus).

II. Numeri cuinsuis primi formad I $2 n+7$ nonoresidurum est $+32-3$ vero resicitum. 


\section{- $\operatorname{xr}-$}

I19. Nihil autem per hanc methodum pro numeris formae $r a n+I$ inueniri potest, qui proin artificia singularia requirunt. Ex inductione quidem facile colligitur, omnium numerorum primorum huius formae residua esse + 3 et - 3, Manifesto autem demonstrari tantummodo debet, numerorum talium residuam esse - 3, quia tunc necessario etiam +3 residuum esse debet (art. I I I). Ostendemus autem generalius, - 3 esse residuum numeri cuiusuis primi formae $3 n+I$.

Sit $p$ huiusmodi primus atque $c$ numerus pro modulo $y$ ad exponentem 3 pertinens (quales dari ex art. 55 manifestum, quia 3 submultiplum ipsius $p-1$ ). Erit itaque $a^{3} \equiv \mathbf{I}$ $(\bmod p)$ i. e. $a^{3}-1$ sive $\left(a^{2}+a+1\right)$ (a - I) per $p$ diuisibilis. Sed patet a esse non passe $\equiv{ }^{1}$ (mod. $p$ ), quia $\mathrm{I}$ ad exponentem I pertinet, quare $a-1$ per $p$ diuisibilis non erit; sed $a^{2}+a+1$ erit, hincque etian $4 a b+4 a+4 ;$ i. e. erit $(2 a+1)^{2} \equiv-3$ (mod. $p)$ siue -3 residuam ipsiusp. Q. E. D.

Ceterum patet, hanc demonstrationem (quae a praecedentibus est independens) etiam numeros primos formae $12 n+7$ complecti quos iầm in art. praec. absoluimus,

Obseruare adhuc conuenit, hanc analysin ad instar methodi in artt. $x 09$, 15 vsitatae exhiberi posse, at breuitatis gratia huic rei non immoramur.

120. Colliguntur facile ex praec, theore mata haec (vid, artt, 102, 103, 105). 


\section{- IIS:-}

1. -5 est residum omnizm numerorum, quineque per 8, neque per 9, nezue per villws numertum primum formae $6 n+5$ disidi possunt, non.resi. dunn autem omnizn reliquorum.

II, + 3 est jesiduim omnium mumeromum, qui neque per 4 , neque per 9, neque per vllum prinuzn format I $2 n+5$ vel $12 n+7$ dissidi possunt, omninn reliw quorum non-residunm.

Teneatur imprimis casus particularis hic:

- 3 est residurm omnium numerorum primorum formae $3 n+1$, seu, quod idem est omnium, qui ipsins 3 sunt residua, non-residum vero omnium numerorum primorum formae $6 s$ +5 , seu, excluso numero 2 , omnium fomae $3 n+2$, i. e omnizm qui ipsius 3 szut non, residutu Facile vero perspicitur omnes reliquos casus ex hoc sponte sequi.

Propositiones ad residua +3 et -5 perti: nentia iam Fermatio notae fuerunt, oppra Wallisii T. II. p. 857. At ill. Euler primus demonstrationes tradidit, Comm. nou. Petr. T. VIII. p. 105 sqq. Eo magis est mirandum, demonstrationen propositionum ad residua +2 et -2 pertinentium, prorsus similibus artificiis innixas, semper ipsius sagacitatem fugisse. Vid. etiam comment. ill. La Grange, Nouv. Men. de l'Ac. de Berlin, ${ }^{2} 77^{5}$ p. 352 .

121. Per inductionem deprehenditur, +5 nullius numeri imparis formae $5 n+2$, vel $5 n$ \& 3 residuum esse, i. e. nullius numeri impa- 
ris qui ipsius non-residuum sit. Hanc vero regulam nullam exceptionem pati, ita demonstratur. Sit numerus minimus, si quis datur, ab hac regula excipiendus $=t$, qui itaque numeri 5 est non-residuam, 5 autem ipsius $t$ residumm. Sit $a c=5+t u$, ita vt $a$ sit par ipsoque $t$ minor. Erit igitur $u$ impar ipsocue $t$ minor, +5 autem ipsius $u$ residuum erit. Quodsi iam a per 5 non est diuisibilis, etiam us non erit; manifesto autem $t u$ ipsius 5 est residuum, quare quam $t$ ipsius 5 sit nonresiduum, etiam non-residuum erit; i. e. da tur non-residuum numeri 5 cuius residuum est +5 , ipso $t$ minus, contra hyp. Si vero a per 5 est diuisibilis, ponatux $a=5 b$, atque $u=5 v$, vnde $t v \equiv-\mathrm{I} \equiv 4_{4}(\bmod , 5), i_{0} e_{0}$ tv erit residuum numeri 5. In reliquis demonstratio perinde procedit vt in casu priori.

122. Onnium igitur numerorum primorum, qui simul sunt ipsius 5 non-residua simulque formae $4 n+i$, i. e. omnium numerom rum primorum formae $20 n+13$ vel $20 n+x 7$, tum +5 quam -5 non residua erunt; omnium autem nunerorum primorum formas $20 n+3$ vel $20 n+7$, non residuam erit $+5,-5$ re siduam.

Potest vero prorsus simili modo demon strari, -5 esise non-residuum omnium numerorum primorum formarum $20 n+I I_{2}$, $20 n t$ $13,20 n+17,20 n+19$, facileque perspicitur hinc sequi, +5 esse residun omnium numerorum primorum formae $20 n+x \mathrm{H}$, vel $20 n+19$, non H 4 
residuum autem omnium formae $20 n+13$, vel $20 n+17 . \quad$ Et quoniam quiuis numerus primus, praeter 2 et 5 (quorum residuum \pm 5 ), in aliqua harum formarum continetur $20 n+1$, $3,7,9, \mathbf{1}, 13,17, \mathbf{9} 9$, patet, de omnibus iam iudicium ferri posse, exceptis iis qui sint formae $20 n+I$, vel formae $20 n+9$.

123. Ex inductione facile deprehenditur, +5 et -5 esse residua omnium numerorum primorum formae $20 n+1$, vel $20+9$. Quodsi hoc generaliter verum est, lex elegans habebitur, +5 esse residunum omniwn numeromm primorum qui ipsius 5 sint residua, (hi enim in alterutra formarum $5 n+1$ vel $5 n+4$ siue in aliqua harum, $20 n+1,9$, II, 19 , continentur, de quarum tertia et quarta illud iam ostensum est) non-residusum vero onnizm numerorum qui ipsius 5 sint non-residua, vt iam supra demonstrauimus. Clarum autem est, hoc theorema sufficere, "ad diiudicandum, vtrum +5 (eoque ipso, -5 , si tamquam productum ex +5 et -1 consideretur) numeri cuiuscunque dati residuum sit an non-residuum. Denique obseruetur huius theorematis cum illo quod art. 120 de residuo - 3 exposuimus analogia.

At verificatio illius inductionis non adeo facilis. Quando numerus primus formae $20 n$ + I, siue generalius formae $5 n+I$ propos nitur, res simili modo absolui potest, vt in artt. I I 4, I Ig. Sit scilicet numerus quicunque pro modulo $5 n+1$ ad exponentem 5 pertinens , quales dari ex sect. praec. manifestum, erit- 
que $a^{5} \equiv 1$, siue $(a-1)\left(a^{4}+a^{3}+a^{2}+\right.$ $a+i) \equiv 0(\bmod .5 n+1)$. At quia nequit esse $a \equiv \mathbb{I}$, neque adeo $a-1 \equiv 0$; necessario erit $a^{4}+a^{3}+a^{2}+a+1 \equiv 0$. Quare etiam $4\left(a^{4}+a^{3}+a^{2}+a+1\right)=(2 a a$ $+a+2)^{2}-5 a^{2}$ erit $\equiv 0$ i. e. $5 a^{2}$ erit residuum ipsius $5 n+I$, adeoque etiam 5 , quia $a^{2}$ est residuum per $5 n+1$ non diuisibile ( $a$ enim per $5 n+1$ non diuisibilis propter $a^{5} \equiv$ I). Q. E. D.

At casus, vbi numerus primus formae 5 s + 4 proponitur, subtiliora artificia postulat. Quoniam vero propositiories quarum ope negotium absoluitur in sequentibus generalius tractabuntur, hic breuiter tantum eas attingimus.

I. Si $p$ est numerus primus atque $b$ nonresiduum quadraticum datum ipsius $p$, valor expressionis $(A) \ldots \frac{(x+\sqrt{b})^{p+1}-(x-\sqrt{b})^{\mathrm{p}+\mathrm{I}}}{\sqrt{b}}$ (ex qua euoluta irrationalitatem abire facile perspicitur), semper per $p$ diuisibilis erit, quicunque numerus pro $x$ assumatur. Patet enim ex inspectione coefficientitm qui ex etrolutione ipsius $A$ obtinentur, omnes terminos a secundo vsque ad penultimum (incl.) per $p$ dnuisibiles fore, adeoque esse $A \equiv 2(p+1)(x p$ $\left.+x b^{\frac{p-1}{2}}\right),(\bmod p)$. At quoniam $b$ ipsius $p$ non-residuum est, erit $b^{\frac{p-1}{2}} \equiv-1$ (mod. $\left.p\right)$, (art. 106); $x$ p autem semper est $\equiv x$ (sect. praec.) vnde fit $A \equiv 0, Q . E, D$.

II. In congruentia $A=0(\bmod p)$, in. determinata $x$ habet $p$ dimensiones, omnesque H 5 
numeri $0, r, 2 . . . p-I$ illius radices emne. Iam ponatur $\varepsilon$ esse diuisorem ipsius $p+1$, eritcue expressio $\frac{(x+\sqrt{b})^{\mathrm{e}}-(x-\sqrt{ } b)^{\mathrm{e}}}{\sqrt{ } b}$ (quam per $B$ designamus) si euoluifur, abirrationalitate libera, indeterminata $x$ in ipsa $z-1$ dimensiones habebit, constatque ex analyseos primis elementis, $A$ per $B$ (indefinite) esse diuisibilem. Iam dicó $e-I$ valores ipsins $x^{4}$ dari, quibus in $B$-substitutis, $B$ per $p$ diuisibi. lis euadat. Ponatur enim $A=B C$, habebit. que $x$ in $C$ dimensiones $p-e+x$, adeocrie congruentia $C \equiv o$ (mod. $p$ ) non plures cruara $p-e+\mathrm{r}$ radices. Vnde facile patet, onmes reliquos numeros ex his $0, x, 2,5 \ldots p . x$, quorum multitudo $=\epsilon-\mathrm{I}$, congruentiae $B \equiv 0$ radices fore.

III. Iam ponatur $p$ esse formae $5 n+4$ $-5, b$ non-residuúm ipsius $p$, atque numerum a ita deterninatum, vt sit $\frac{(a+\sqrt{b})^{3}-(a-\sqrt{b})^{5}}{\sqrt{b}}$ perp diuisibilis. At illa expressio $\mathrm{at}=10 a^{4}+20 a a b+2 b b=$ $2\left((b+5 a a)^{2}-20 a^{4}\right)$. Erit igitur etiam $(b+5 a a)^{2}-20 a^{4}$ per $p$ diuisibilis i. e, 20a* residuum ipsius $p$; at quoniam $4 a^{4}$ residuum est per $p$ non diuisibile (facile enim intelligitur, a per $p$ diuidi non posse), etiam 5 residuum ipsius $p$ erit. Q. $E . D$.

Hine patet theorema in initio huius articuli prolatum generaliter verum esse. - 


\section{$-123-$}

Obseruamus adhuc, demonstrationes pro vtroque casu ill. La Grange deberi, MIsm. de l'Ac. de Berlin ${ }_{17} 77^{5}$, p. $35 \mathrm{2}$ sqq.

I24. Per similem methodum demon tratur,

-7 esse non residunm cuiusuis numeri prini ipsius 7 sit non residurum.

Ex inductione vero concludi potest,

-7 esse residuun cuitusuis numeri qui ipsitus 7 sit residuun.

At hoc a nemine hactenus rigorose demonstraturn. Pro is quidem residuis ipsius 7 , qui sunt formae 4n- 1 , facilis est demonstratio; etenim per methodum ex praec, abunde notam ostendi potest, +7 semper esse talium numerorum primoram non-residuum, adeoque -7 residuum. Sed parum binc lucramur : reliqui enim casus per hanc methodum. tractari nequeunt. Vnum quidern adhuc casum simili mod. vt artt. i 9 , i23 absoluere possumas." Scilicet si $p$ est numerus primus formae $7^{n}+1$, atque a pro modulo $p$ ad exponentem 7 pertinens, facile perspicitur $\frac{4\left(a^{2}-1\right)}{a \cdots I}$ $=\left(2 a^{3}+a^{2}-a-2\right)^{2}+7\left(a^{2}-a\right)^{2}$ per $p$ diuisibilem, adeoque $-7\left(a^{2}-a\right)^{2}$ ipsius $p$ ressiduum fore. At $\left(a^{2}-a\right)^{2}$, tamquram quadratum, ipsius $p$ residuum est, insuperque per $p$ non diuisibile; qquum enim $a$ ad exponentem 7 pertinere supponatur, neque $\equiv 0$, neque $\equiv I(\bmod , p)$ esse potest, i. e. neque 


\section{- $124 \quad-$}

neque $a-I$ per $p$ diuisibilis erit, adeoque etiam quadratum $(a-1)^{2} a^{2}$. Vnde manisto etiam 7 ipsius $p$ residuum erito Q. E. D. At primi numeri formae $7^{n}+2$ vel $7^{n}+4$ omnes methodos hucusque traditas eludunt. Ceterum etiam haec demonstratio ab ill, La Grange primum est detecta $t_{0} . c_{0}$ - Infra sect. VII. docebimus generaliter, expressionem $\frac{4\left(x^{p}-1\right)}{x-I}$ semper ad formam $X^{2}$-F $p x^{2}$ reduci possé, (vbi signum superius est accipiendum quando $p$ est numerus primus formae $4 n$ $+\mathrm{I}$, inferius quando est formae $4 n+3 y$, de. notantibus $X, x$ functiones rationales ipsius $x$, a fractionibus liberas. Hanc discerptionem ill. La Grange vltra casum $p=7$ non perfecit v. l. a. p. 352.

125. Quoniam igitur methodi praecedentes ad demonstrationes generales stabiliendas non sufficiunt, iam tempus est, aliam ab hoc defectu liberam exponere. Initium facimus a theoremate, cuius demonstratio satis diu operam nostram elusit, quamuis primo aspectu tam obuium videatur, vt quidam ne necessita. tem quidem demonstrationis intellexerint. Est vero hoc: Quemuis numerum, praiter quadra ta positiue sumta aliquorum numerorum primortim nonresidurum esse. Quia vero hoc theoremate tan. tummodo tamquam auxiliari ad alia demon. stranda vsuri sumus, alios casus hic non explicamus quam quibus ad hunc finem indigemus. De reliquis casibus postea sponte idem consta- 


\section{$-125$}

bit. Ostendemus itaque, quemuis numerum primum formae $4 n+\mathrm{I}$, size positiue siue negatiue accipiatur ${ }^{*}$ ), non-residun esse aliquorum numerorum primorum, et quidem talium qui ipso sint minores.

Primo, quando numerus primus $p$, forma $4 n+1$, negatiue sumendus proponitur, sit $2 a$ numerus par proxime maior quam $\sqrt{ } p$; tum facile perspicitur, $4 a a$ semper fore $\angle 2 p$ sine $4 a \pi$ $-p<p$. At $4 a a-p$ est formae $4 n+3$, $+p$ autem residuum quadraticum ipsius $4 a 6$ - $p$, (quoniam $p \equiv 4 a a(m o d .4 a c-p)$ ); quodsi igitur $4 a a-p$ est numerus primus, $p$ ipsius non-residaum erit; sin minus, necessario factor aliquis ipsius $4 a a-p$ formne $4 n+$ 3 erit; et quum $+p$ etiam huius residuum esse debeat, - $p$ ipsius non-residuum erit. Q. $E, D$.

Pro numeris primis positive sumendis duos casus distinguimus. Primo sit p numerus primus formae $8 n+5$. Sit a numerus quicunque positiuus $<\sqrt{ } \frac{1}{2}$. Tum $8 n+5-2 a a$ exit numerus positiuus formae $8 n+5$ vel $8 n+3$, prouta par vel impar) adeoque necessario per nume. rum aliquem primum formae $8 n-5$ vel $8 n$ +f 5 diuisibilis, productum enim ex quotcunque numeris formae $8 n+$ r et $8 n+7$ neque formam $8 n+3$ neque hanc $8 n+5$ habere potest. Sit hic $q$, eritque $8 n+5 \equiv 2 a^{2}$ (mod. $q$ ). At 2

7) I I autem excipi oportere per se manifestum est. 


\section{$-\quad 126=$}

ipsius $q$ non-residuum erit (art. I I2), adeoque etian $2 a^{2}{ }^{*}$ ) et $8 n+5$. Q. E. D. D.

126. Sed numerum quemuis primum formae $8 n+I$ positiue acceptum semper alicuius mumeri primi ipso minoris non residuum esse, per artificia tam obuia demonstrari nequit. Qunm antem haec verilas maximi sit momenti, demonstrationem rigorosam, quamuis aliquantum prolixa sit, praeterire non possumus. Fraetermittus sequens

LeMra. Si habontur duse series numerorsm, $A$, $B, C$, etc. .... (I), $A^{\prime}, B^{\prime}, C^{\prime}$, etc . . . (II), (vtrum terminorum multitudo in vtraque idem sit necne nihil interesi) ita comparata, vt, derio.

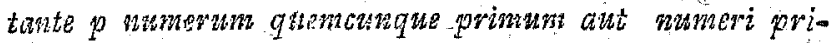
oni potestatem, terminum aliquen seinndae seriei (siue eiton piturs) metienteni, totiden ad miniman temini in serie prima sint per $p$ dinzisibiles, quot sunt in secundia: Eum dico produtuns ex onnibus numeris $(I)$ dinibile fore ger prodiuctum ex onatibus numeris (II).

Exempl. Constet (I) e numeris 12, I $S$, $45 ;$ (II) ex his $3,4,5,6,9$. Tum diuisibiles erunt per $2,4,5,9,5$ in (I) $2,1,3,2$, stermini, in (II) $2, I, 3, I$, I termini, respeciue; producum autem onnium terminorum $(\mathrm{C})=9720$ diuisibile est per productum o. misium terminorum (II), 32/0.

* Art 98 . Putet enim $a^{2}$ esse residun ipsius $q$ fer $q$ non diulsibile, nam allas etiam numeras wrimus $y$ per a foret diuisibilis. Q. E. A. 
Demonstr. Sit productum ex omnibus terminis $(I)=Q$, productum omniam terminorum seriei (II), $=Q^{\prime}$. Patel quemais numerum primum qui sit diuisor ipsius $Q^{\prime}$ etiam ipsius $Q$ diuisorem fore. Iam ostendemus quemuis factorem primum ipsius $Q$, in $Q$ totidem ad minimum dimensiones habere quot habent in $Q^{\prime}$. Esto talis diuisor $p$, ponaturque, in serie (I) terminos esse per $p$, diuisibiles neque vero per $p^{2}$, $b$ terminos per $p^{2}$ non autem per $p^{3}$ diuisibiles, $c$ terminos per $p^{3}$ non autem per $p^{4}$ etc. similia denotent literae $a^{\prime}, b^{\prime}, c^{\prime}$ etc pro serie (II), perspicieturque facile, $p$ in $Q$ habere $+b+c+$ etc. dimensiones, in $Q^{\prime}$ vero $a^{\prime}+$ $b^{\prime}+c^{\prime}+$ etc. At $a^{\prime}$ certe non maior quam $a$, $b^{\prime}$ non maior quam $b$ etc. (hyp.); quare $a^{\prime}+$ $b^{\prime}+c^{\prime}$ etc. certo non eit $>a+b+c$ etc. - Quum itaque nullus numerus primus in $Q^{\prime}$ plures dimensiones habere possit, quam in $Q, Q$ per $Q^{\prime}$ diuisibilis erit (art. I 7 ) $Q . E . D$.

127. Lemita. In yrogressione $\mathrm{I}, 2,3,4 . \ldots$ n, phures termini esse nequeant per numertm quenumaque $h$ diuisibiles quam in hac $a, a+1, a+2 \ldots \ldots$ $a+n-I$ ex totiden terminis constante.

Nullo enim negotio perspicitur si $n$ fuerit multiplum ipsius $h$, in vtraque progressione $\frac{z}{h}$ terminos fore per $h$ diuisibiles; sin minus, po. natur $n=h+f$, ita vt $f$ sit $<h$, eruntque in priori serie e termini per $h$ diuisibiles, a posteriori autem vel totidem vel $e+\mathrm{I}$. 
Hinc tamquam Coroll. sequitur propositio ex numerorum figuratorum theoria nota, sed a nemine, ni fallimur, hactenus directe demonstrata, $\frac{a \ldots a+r a+2 \ldots a+n}{2 \ldots}$ semper esse numerum integrum.

Denique Lemma hoc generalius ita proponi potuisset:

In progressione $a, a+1, a+1, \ldots a$ $+n-I$ totidem ad minimum dantur termini secundum modulum $h$ numero cuicunque dato, $r$, congrui, quot in hac, $1,2,3 . \ldots n$ termini per $h$ diuisibiles.

128. Theonema. Sit a numerus quicunque formae $8 n+1$, p numeris quicunque ad a prinus, cuius residunn $+a$, tandem $m$ numbrus arbitrarius: tums dico, in progressione $a, \frac{\pi}{2}(a-I), 2(a-4)$, $\frac{T}{2}$ $(a-9), 2(a-10), \ldots 2\left(a-m^{2}\right), v_{e}$ $\frac{1}{2}\left(a-m^{2}\right)$, prout m par vel impar, totidem ad minimum dari terminos per $p$ diuisibiles quot denturin hac $x_{1} 2,3 \ldots 2 m+\mathfrak{r}$. Priorem progressionem designamus per (I) posteriorem per(II).

Demonsir. I. Quando $p=2$, in (I) omnes termini praeter primum, i. e. $m$ termini diuisibiles erunt; totidem autem erunt in (II).

II. Sit $p$ numerus impar, vel numeri imparis duplum vel quadruplum, atque $a \equiv r$ $(\bmod , p)$. Tum in progressione, $-m,-c m$ $-I),-(m-2) \ldots .+m$ (quae terminorum multitudine cum (I) et (II) conuenit et per 


\section{$129-$}

(III) designabitur) totidem ad minimum termini erunt secundum modulum $p$ ipsi $r$ congrui, quot in serie (II) per $p$ diuisibiles (art praec.). Inter ilos autem, bini, qui signo tantum, non magnitudine, discrepent, occurrere nequeunt *). Tandem quisque eorum correspondentem ha. bebit in serie (I), qui per $p$ erit diuisibilis. Scilicet si fuerit $\pm b$ aliquis terminus seriei (III) ipsi $r$ secundum $p$ congruus, erit $a-b b$ per $p$ diuisibilis, Quodsi igitur $b$ est par, terminus seriei $(1), 2(a-b b)$, per $p$ diuisibilis erit. Si vero $b$ impar, terminus $\frac{x}{2}(a-b b)$ per $p$ diuisibilis erit: namque manifesto $\frac{a-b b}{p}$ erit integer par, quoniam $a-b b$ per 8 , p autem ad summuin per 4 divisibilis (a enim per hyp. est formae $8 n+1 ; b b$ autem ideo quod est numeri imparis quadratum eiusdem formae erit, quare differentia erit formae 8n). Hinc tandem concluditur, in serie (I) totidem terminos esse per $p$ diuisibiles, quot in (III) sint ipsi secundum $p$ congrai, $i$. e totidem aut plures quam in (II) sint per $p$ diuisibiles, $Q . E . D$.

III. Sit $p$ formae $8 n$, atque $a \equiv r$ (mod. 2p). Facile enim perspicitur, a, quum ex hyp. ipsius $p$ sit residuum, etiam ipsius $2 p$ residuum

4). Si enim esset $r \equiv-f \equiv+f$ (mod $p$ ), feret $2 f$ per diuisibilis, adeoque etiam $2 a$ (propter $f f \equiv$ (mod. $p$ )) Hoc autem aliter feri nequit, quam si $p=2$, quum, per bypa $a$ ad $p$ sit primus. Sed de hoc casu iam seorsim diximus 


\section{$-250-$}

fore. Tum in serie (III) totidem ad minimum termini erunt ipsi $\boldsymbol{r}$ secundum $\boldsymbol{p}$ congrui quot in (II) sunt per $p$ diuisibiles, illique omnes matgnitudine erunt inaequales. At cuique eorum respondebit aliquis in (I) per $p$ diuisibilis. Si enim $+b$ vel $-b \equiv r$ (mod. $p$ ), erit $b b \equiv$ $\left.r(\bmod .2 p),{ }^{*}\right)$ adeoque terminus $\frac{1}{2}(a-r)$ per $p$ diuisibilis, multoque magis $2(a-r r)$. Quare in (1)'totidem ad minimum termini erunt per $p$ diuisibiles quam in (II). Q. E. D.

129. Tréonems, Si a est numerus primus formae $8 n-1$, necessario infra $2 \sqrt{ }$ a dabitur aliquis numerus primus cuirs non-residum sit a.

Demonstr. Esto, si fieri potest, a residuum omnium primorum ipso $2 \sqrt{ }$ a minorum.-Tum Facile perspicietur, a etiam omnium numerorum compositorum ipso $2 \sqrt{a}$ minorum residuum fore (conferantur praecepta per quae diiudicare docuimus, vtrum numerus propositus sit numeri compositi residuum necne; art. z 05$)$. Sit numerus proxime minor quam $\sqrt{ }$ $=m . \quad \operatorname{Tum}$ in serie (I)..... $a, \frac{1}{\approx}(a-I)$, $2(a-4), 2(a-9) \ldots .2(a-m m)$, vel $\frac{x}{3}(a-m m)$, totidem aut plures termini erunt per namerum quemcunque ipso $2 \sqrt{ } a$ mino. rem diuisibiles, quam in hac (II).... $1,2,5$, 4.... $2^{m}+$ I (art. praec.), Hinc vero sequitur, productum ex omnibus terminis (I) per productum omnium terminorum (II) divisibile

a) Erit scilicet $b b-w=(b-r)(b+r)$ e duobus factoribus compositus, quorum alter per $p$ diustibilis '(hyp.), alter per 2 (quia tum $b$ tum $\gamma$ sunt impares); adeoque $b 6-i b$ per $2 p$ diuisibilis. 


\section{$-\pi \sigma^{2}=$}

esse, (art. 126). At illud est aut $=a(a-\pi)$ $(a-4) \ldots .(a-m m)$ aut semissis huius producti (prout $m$ aut par aut impar). Quare productum $a(a-1)(a-4) \ldots(a-m m)$ certo per prom ductum omnium terminorum (II) diuidi pote. rit, et, quia omnes hi termini ad sunt primi, etiam productum illud omisso factore $\omega_{0}$ Sed productum ex omnibus terminis (II) ira etiam exhiberi potest, $(m+1) \cdot\left((m+1)^{2}-1\right)$. $\left((m+1)^{2}-4\right) \cdots\left((m+1)^{2}-m^{2}\right)$. Fietigitur $\frac{1}{(m+1)^{2}} \cdot \frac{a-1}{(m+1)^{2}-1} \cdot \frac{a-4}{(m+1)^{2}-4}$ $a-m^{2}$

$\overline{(m+x)^{2}-m^{2}}$ numerus integer, quamquam sit productum ex fractionibus vnitate minoribus: quia enim necessario $\sqrt{ } a$ irrationalis esse debet; erit $m+x p \sqrt{a}$, adeoque $(m+1)^{*}$ $>a_{\text {. Hinc tandem concluditur suppositionem }}$ nostram locum habere non posse. $Q$ E. $D$.

Iam quia $a$ certo $>4$, erit $2 \sqrt{a}<a$, dabitur que adeo aliquis primus $<a$ cuius non - residuum $a$. 130. Postquam rigorose demonstrauimus quemuis numerum primum formae $4 n+I_{2}$ et positive et negatiue acceptum, alicuiuis numeri primi ipso minoris non-residuum esse, ad comparationem exactiorem et generaliorem numerorum primorum quatenus vnus alterius residuum vel non-residuum est, statim transimus,

Omni rigore supra demonstrauimus, -3 et +5 esse residua vel non-residua omnium numerorum primorum, qui ipsorum 5,5 respectiue sint residua vel non-residua 


\section{$-\quad 132$}

Per inductionem autem circa numeros equentes institutam, inuenitur:

$-7,-11,+13,+17-19,-23$, $+29,-3 x,+37,+4 x,-43,-47,+$ $53,-59$ etc. esse residua vel non-residua omnium numerorum primorum, qui, positiui sumti, illorum prinorum respectiue sint residua vel non-residua. Inductio haec perfacile adinmento tabulae II confici potest.

Quiuis autem leui attentione adhibita obseruabit, ex his numeris primis signo positiuo affectos esse eos, qui sint formae $4 n+1$, negatiuo autem eos, qui sint formae $4 n+3$.

35. Quod hic per inductionem deteximus, generaliter locam habere mox demonstrabimus, Antequam autem hoc negotium adeamus, necesse erit, omnia quae ex theoremate, si verum esse supponitur, sequuntur, eruere. Theorema ipsum ita enunciamus.

Si $p$ est numerus prinus formde $a^{n}+\mathrm{I}$, erit $4 p$, si vero $p$ formae $4 n+3$, erit $-p$ residisum vel non residunm cuinsuis numeri primi qui positiue cicceptus ipsizis $p$ est residun vel non-residuum.

Quia omnia fere quae de residuis quadraticis dici possunt, huic theoremati innituntur, denominatio theorematis fundamentatis, qua in sequentibus vtemur, haud absona erit.

Vt ratiocinia nostra quam breuissime exhiberi possint, per $a, a^{\prime}$, $a^{\prime \prime}$ etc, numeros primos 


\section{$\quad 133 \longrightarrow$}

formae $4 n+\mathbb{1}$, per $b, b^{\prime}, b^{\prime \prime}$ ètc. numeros primos formae $4 n+3$ denotabimus; per $A, A r$ $A^{\prime \prime}$ etc. numeros quoscunque formae $4 n+\mathrm{I}$, per $B, B^{\prime}, B^{\prime \prime}$ etc. autem numeros quoscunque formae $4 n+3$; tandem litera $R$ duabus quantitatibus interposita indicabit, priorem sequentis esse residuum, sicuti litera $N$ significationen contrariam habebit. Ex. gr. $+5 R$ I I, $\pm 2 N 5$, indicabit +5 ipsius I I esse residurum, +2 yel - 2 esse ipsius 5 non-residuum. Iam collato theoremate fundamentali cum theorematibus art. I1 , sequentes propositiones facile deducentur.

$$
\begin{aligned}
& \text { Si erit } \\
& \text { ז. } \pm a R a^{\prime} \ldots \ldots \ldots \pm a^{\prime} R a \\
& \text { 2. } \pm a N a^{\prime} \ldots \ldots \ldots \pm a^{\prime} N a \\
& \text { 3. }\left[\begin{array}{l}
+a R b \\
-a N b
\end{array}\right] \cdots \cdots+b R a \\
& \text { 4. }\left[\begin{array}{l}
+a N b \\
-a R b
\end{array}\right] \cdots \cdots+b N d \\
& \text { 5. } \pm b R a_{0} \ldots \ldots\left(\begin{array}{l}
+a R b \\
-a N b
\end{array}\right. \\
& \text { 6. } \pm b N a \ldots \ldots\left[\begin{array}{l}
+a N b \\
-a R b
\end{array}\right. \\
& \text { 7. }\left[\begin{array}{l}
+b R b^{\prime} \\
-b N b^{\prime}
\end{array}\right] \cdots \cdots\left[\begin{array}{l}
+b^{\prime} N b \\
-b^{\prime} R b
\end{array}\right. \\
& \text { 8. }\left[\begin{array}{l}
+b N b^{\prime} \\
-b R b^{\prime}
\end{array}\right] \cdots \cdots\left[\begin{array}{l}
+b^{\prime} R b \\
-b N a
\end{array}\right.
\end{aligned}
$$




\section{$-134=$}

152. In his omnes casus, qui, duos numeros primos comparindo, occurrere possunt, continentur: quae sequuntur, ad numeros quoscunque pertinent: sed harum demonstrationes minus sunt obuiae.

$$
\begin{aligned}
& \text { Si erit } \\
& \text { 9. } \pm a R A \ldots \ldots \pm A R \\
& \text { Io. } \pm b R A \ldots \ldots\left[\begin{array}{l}
+A R b \\
-A N G
\end{array}\right. \\
& \text { II. + } a R B \ldots \ldots \pm B R \\
& \text { 12. }-a R B \ldots \ldots \pm B N G \\
& \text { 13. }+b R B . \ldots\left[\begin{array}{l}
-B R b \\
+B N b
\end{array}\right. \\
& \text { 14. - } b R B \ldots \ldots\left[\begin{array}{l}
B R b \\
+B N_{1} b
\end{array}\right.
\end{aligned}
$$

Quum omnium harum propositionum de: monstrationes ex iisdem principiis sint petendae, necesse non erit omnes euoluere: demon. stratio prop. 9, quan apponimus tamquam exemplum inseruire potest. Ante onnia autem obseruetur, quemuis numerum formae $4 n+1$ aut nullom tactorem formae $4 n+3$ habere, aut duos, aut quatuor etc. i. e multitudinem talium factoram (inter quos etiam aequales esse possunt) semper fore parem: quemuis vero formae $4 n+3$ multitudinem imparem factorum formae $4 n+3$ (i. e. aut vnum aut tres aut 
quinque etc.) implicare. Multitudo factorum formae $4 n+1$ indeterminata manet.

Prop. 9 ita demonstratur. Sit $A$ prodictam e factoribus primis $a^{\prime}, a^{\prime \prime}, a^{\prime \prime \prime}$ etc., $b, b^{\prime}, b^{\prime \prime}$ etc.; eritque factorum $b, b^{\prime}, b^{\prime \prime}$ multitudo par (possunt etiam nulli adesse, quod eodem redit). Iam si $a$ est residutum ipsius $A$, erit residuum etiam omnium factorum $a^{\prime}, a^{\prime \prime}, a^{\prime \prime \prime}$ etc. $b, b^{\prime}, b^{\prime \prime}$ etc. quare per propp. 1,3 art. praec. singuli hi factores erunt residua ipsius a, adeoque etiam productum $A$. $-A$ vero idem esse debet. Quodsi vero - $a$ est residuum ipsius $A_{2}$ eoque ipso omnium factorum $a^{\prime} a^{\prime \prime}$ etc. $b, b^{\prime}$ etc.; singuli $a^{\prime} a^{\prime \prime}$ etc. erant ipsius a residua, sirguli $b, b^{\prime}$ etc. autem non residua. Sed quum posteriorum multitudo sit par, productum ex omnibus, $2 . A$, ipsius a residuum erit hince que etiam - $A$

133. Inuestigationem adhuc generalius instituanus. Contemplemur duos numeros quos. cunque impares inter se primos, signis quibuscunque affectos, $P$ et $Q . \quad$ Concipiatur $P$ sine respectu signi sui in factores suos primos resolutus, designeturque per $p$, quot inter hos reperiantur quorum non-residuum sit $Q$. Si vero aliquis numerus primus, cuius non-residuum est $Q$, pluries inter factores ipsius $P$ occurrit, pluries etiam numerandins erit. Similiter sit $q$ multitudo factorum primorim ipsius Q. quorum-non-residaum est $P$ : Tum numeri $p$, q certam relationem nutuam habebunt ab 
indole numerorum $P, Q$ pendentem. Scilicet si alter numerorum $p$, est par vel impar, numerorum $P, Q$ forma docebit, vtrum alter par sit vel impar. Haec relatio in sequenti tabula exhibetur.

Erunt $p, q$ simul pares vel simul impares; quando numeri $P, Q$ habent formas:
I. $+A_{1}+A^{\circ}$
2. $+A,-A^{\circ}$
3. $+A+B$
4. $+A,-B$
5. $-A_{1}-A^{\prime}$
6. $+B_{9}-B^{\prime}$

Contra numerorum $p, q$ alter erit par, alter impar, quando numeri $P, Q$ habent formas :

$$
\begin{aligned}
& \text { 7. }-A,+B \\
& \text { 8. }-A,-B \\
& \text { 9. }+B,+B^{\prime} \\
& \text { I0. }-B ;-B^{\circ}
\end{aligned}
$$

Ex Sint numeri propositi -55 et + II97, qui ad casum quartum erunt referendi. Est autem Irg7 non-reşiduum vnius factoris primi ipsius 55 , scilicet numeri $5,-55$ au. tem non residuum trium factorum primorum ipsius 1:97, scilicet numerorum 3,3 , 19 .

Si $P$ et $Q$ numeros primos designant, propositiones hae abeunt in eas quas art. $13 \mathrm{r}$ tra- 


\section{- $\quad 337$ -}

didimus. Hic scilicet $p$ et $q$ maiores quam I fieri nequeunt, quare quando $p$ ponitur esse par necessario erit $=0 \dot{i}$, e, $Q$ erit residuum ipsius $P$, quando vero $p$ est impar, $Q$ ipsius $P$ non-residuum erit, Et vice versa. Ita scriptis $a, b$ loco ipsorum $A, B$, ex 8 sequitur, si - a fuerit residuum vel non-residuum ipsius $b$, fo$r e-b$ non-residuum vel residuum ipsius $a$, quod cam 3 et 4 art. $13 x$ conuenit:

Generaliter vero patet, $Q$ residuum ipsins $P$ esse non posse nisi fuerit $p=0$; si igitur $p$ impar, $Q$ certo ipsius $P$ non-residuum erit.

Hinc etiam propp. art. praec. sine diffcultate deriuari possunt.

Ceterum mox patebit, hanc repraesentationem generalem plus esse quam speculatio. nem sterilem, quum theorematis fundamentalis demonstratio completa absque ea vix perfici possit.

134. Aggrediamur nunc deductionem harum propositionum.

I. Concipiatur, vt ante, $P$ in factores suos primos resolutus, signis neglectis, insuperque etiam $Q$ in factores quomodocunque resoluatur. ita tamen vt signi ipsius $Q$ ratio habeatur. Combinentur illi singuli cum singulis his. Tum si $s$ designat multitudinem omnium combinationum, in quibus factor ipsius Q est non-residuum factoris ipsius $P, p$ et $s$ vel simul pares vel. 


\section{$-138-$}

simul impares erunt. Sint enim factores primi ipsius $P$, hi $f, f^{\prime} f^{\prime \prime}$ etc. et inter factores in quibus $Q$ est resolutus, sint $m$ qui ipsius $f$ sint non-residua, $m^{\prime}$ non-residua ipsius $f^{\prime}, m^{\prime \prime}$ nonresidua ipsius $f^{\prime \prime}$ etc Tum facile quisquis perspiciet, fore $s=m+m^{\prime}+m^{\prime \prime}+$ etc., $p$ autem exprimere quot numeri inter ipsos $m$ m', " etc. sint impares. Vnde sponte patet, $s$ fore parem, quando $p$ sit par, imparem quando p sit impar.

II. Haec generaliter valent, quomodocunque $Q$ in factores sit resolutus. Descendamus ad casus particulares. Contemplemur primo casus, vbi alter numerorum, $P$, est positiuus, aiter vero, $Q$, vel forma $+A$ vel formae $-B$. Resoluantur $P, Q$ in factores suos primos, attribuatur singulis factoribus ipsius $P$ signum positium, singulis autem factoribus ipsius $Q$ signum positiuum vel negatiuum, prout sunt formae $a$ vel $b$; 'tunc autem manifesto $Q$ fiet vel formae $+A$ vel $-B$ vti requiritur. Combinentur factores singuli ipsius $P$ cum j'singulis factoribus ipsius $Q$, designetque vt ante $s \mathrm{mul}$ titudinem combinationum in quibus factor ipsius $Q$ est non residuum factoris ipsius $P$, similiterque $t$ multitudinem combinationmm in quibus factor ipsius $P$ est nonaresiduum factoris ipsius $Q$ : At ex theoremate fundamentali sequitur illas combinationes indenticas fore cum his adeoque $s=t$. Tandem ex is quae modo demonstrauimus sequitur esse $p \equiv s$ (mod. 2 ); $g \equiv t(\bmod 2)$, vade fit $p \equiv q(\bmod 2)$ 


\section{- $\quad 139 \quad-$}

Habentur itaque propp. $x, 3,4$ et 6 art. 135.

Propositiones reliquae per methodum similem directe erui possunt, sed vna consideratione noua indigent; facilius autem ex praecedentibus sequenti modo deriuantur.

III. Denotent rursus $P, Q$, numerus quoscunque impares inter se primos, $p, q$ multitudinem factorum primorum ipsorum $P, Q$, quorum non-residua $Q, P$ respectiue. Tandem sit $p^{\prime}$ multitudo factorum primorum ipsius $P$, quorum non - residuum est $-Q$ (quando $Q$ per se est negatiuus, manifesto $-Q$ numerum positiuum indicabit). Iam omnes factores primi ipsius $P$ in quatuor classes distribuantur. est $Q$.

1) in factores formae $a$, quorum residuum.

2) factores formae $b$, quorum residuum $Q$. Horum multitudo sit $x$.

3) factores formae $a_{\text {, }}$ quorum non-residuum est Q. Horum multitudo sit $\psi$ :

4) factores formae $b$, quorum non-residuam Q. Quorum multirudo $=\omega_{0}$

Tum facile perspicitur fore $p=\psi+\cdots$, $p=x+\psi$

Iam quando $P$ est formae $\pm A$, erit $x+$. adeoque etiain $x-\propto$ numerus par: quare fiet $p^{\prime}=p+x-\ldots p(\bmod 2)$; quando vero $P$ est formae $\pm B$, per simile ratiocinium in 


\section{$-\quad \times 40 \quad-$}

uenitur, numeros $p, p^{\prime}$ sec mod. 2 incongruos fore.

IV. Applicemus haec ad casus singulos. Sit primo tum $P$, tum $Q$ formae $+A$, eritque $\mathrm{ex}$ prop r. $p \equiv q(\bmod , 2)$; at erit $p^{\prime} \equiv p$ (mod. 2); quare etiam $p^{\prime} \equiv q$ (mod. 2 ). Quod conuenit cum prop. 2. - Simili modo si $P$ est formae $+A, Q$ torma $-A$, erit $p \equiv q$ (mod. 2) ex prop 2 quam modo demonstrauimus; hinc, ob $p^{\prime} \equiv p$, erit $p^{\prime} \equiv q$. Est itaque etiam prop. 5 demonstrata.

Eodem modo prop. 7 ex 3 ; prop. 8 vel ex 4 vel ex 7 ; prop. 9 ex 6 ; ex eademque prop. ro deriuantur.

135. Per art. praec. propositiones art. I35 non quidem sunt demonstratae, sed tamen earum veritas a veritate theoremitis furdamentalis quam aliquantisper supposuimus pendere ostensa est. At ex ipsa deductionis methodo manifestum est, illas valere pro numeris $P, Q$, si modo theorema fundamentale pro omnibus factoribus primis horum numerorum inter se comparatis locum habeat, etiamsi generaliter verum non sit. Nunc igitur ipsius theorematis fundamentalis demonstrationem aggrediamur. Cui praemittimus sequentem explicationem.

Theorena fundamentale vsque ad numerum atiquen $M$ verum esse dicenus, si valet pro duobus numeris primis quibuscunque, quorum neuter ipsum $M$ superat. 


\section{I4.I}

Simili modo intelligi debet, si theoremata artt. $131,132,133$ vsque ad aliquem terminum vera esse dicemus. Facile vero perspicitur, si de veritate theorematis fundamenlalis vsque ad aliquem terminum constet, has propositiones vsque ad eundum terminum lo. cum esse habituras.

136. Theorema fundamentale pro numeris paruis verum esse per inductionem facile confirmari, atque sic limes determinari potest vsque ad quem certo loco teneat. Hanc, in. ductionem institutam esse postulamus: prorsus autem indifferens est quousque eam persequuti simus; sufficeret adeo, si tantummodo vaque ad numerum 5 eam confirmauissemus, hoc autem per vnicam obseruationem absoluitur, quod est $+5 N 3, \pm 3 N 5$.

Iam si theorema fundamentale generaliter verum non est, dabitur limes aliquis, $T$, vsque ad quem valebit, ita tamen vt vsque ad numerum proxime maiorem, $T+x$, non amplicis valeat. Hoc autem idem est ac si dicamus, dari duos numeros primos quorum maior sit $T+1$, et qui inter se comparati theoremati fundamentali repugnent, binos autem alios numeros primos quoscunque, si modo ambo ipso $T+r$ sint minores, huic theoremati esse consentaneos. Vnde sequitur, propositionês arti. 151,132, i 33 vique ad $T$ etiam locum habituras. Hanc vero suppositionem consistere non posse numc ostendemus. Erunt autem secundum formas diuersas, quas tum 


\section{- $44_{2}-$}

$T+\mathrm{I}$, tum numerus primus ipso $T \Psi$ I minor, quem cum $T+\&$ comparatum theoremati repugnare supposuimus, habere possunt casus sequentes distinguendi. Numerum istum primum per $p$ designamus.

Quando tum $r+\mathrm{r}$ tum $y$ sunt formae $4 n+I$, theorema fundamentale duobus modis falsum esse posset, scilicet si simul esset, $v e l \pm v R(T+\mathrm{I})$ et $\pm(T+\mathrm{I}) N_{p}$, vel simul $\pm p N(T+1)$ et $\pm(T+1) R p$.

Quando tum $T+\mathrm{I}$ tum $p$ sunt formae $4 n+3$, theor. fund. Falsum erit, si simul fuerit vel $+p R(T+\mathrm{r})$, et $\ldots(T+\mathrm{x}) N p$ (siue quod eoden redit $-p N(T+\mathrm{s})$ et $+(T+\mathrm{r})$ $R p) ; v a l+p N(T+\mathrm{r})$ et $-(T+\mathrm{I}) R p$ $($ sine $-p R(T+r)$ et $+(T+I) N p)$.

Quando $T+\mathrm{r}$ est formae $4 n+\mathrm{r}, p$ ve: ro formae $4^{n}+3$, theor. fund. falsum erit, si fuerit $v e t \pm p \cdot R(T+I)$ et $+(T+1) N p$ $($ sine $-(T+1) R p) ;$ vel $\pm p N(T+1)$ et $-(T+1) N p($ siue $+(\bar{T}+1) R p)$.

Quando $T+1$ est formae $4 n+3, p$ vero formae $4 n+x$, theor. fund. falsum erit, si fuerit vel $+p R(T+\mathrm{r})$, (siue $p \bar{N}(T+\mathrm{r})$ ) et $\pm(T+l) N p, v e l+p N(T+\mathrm{r})($ sine $-p R$ $(T+I))$, et $\pm(T+x) R p$.

Si demonstrari poterit, nullum horum octo casuum locum habere posse, simul certum erit, theorematis fundamentalis veritatem nul. 


\section{$-143$.}

lis limitibus circumscriptam esse. Hoc itaque negotium nunc aggredimur: at quoniam alii horum casuum $a b$ alins sunt dependentes, eunduni ordinem, quo eos hic enumeratumus ser. uare non licebit.

137. Casus primus. Quando $T+I$ est for mae $4 n+1,(=a)$, atque $p$ eiusdem formae; instuper vero $\pm p R a$, nan potest esse $\pm a N p$. Hic casus supra fuit primus.

Sit $+p \equiv e^{2}(\bmod . a)$, atque $e$ par et $<a$, (quod semper obtineri potest). Iam duo casus sunt distinguendi.

I. Quando eper $p$ non est diuaisibilis. Pónatur $e^{2}=p+a f$, eritque $f$ positiuns, formae $4 n+3$ (siue formae $B$ ), $<a$, et per $p$ non diuisibilis. Porro erit $e^{2} \equiv p$ (mod. $\left.f\right)$, i. e. $p R f$ adeoque ex prop is art. $13_{2} \div f R p$ (quia ening $p, f$ $<a$, pro his propositiones istae valebunt). At est etiam af $R p$, quare fiet quoque $\pm a^{\prime} R_{p}$.

II. quando $e$ per $p$ est diuisibilis, ponatur $e=g p$, atque $e^{2} \equiv p+a p h$, sine $p g^{2}=1+$ ah. Tum erit $h$ formae $4 n+3(B)$, atque ad $p$ et $g^{2}$ primus. Porro erit $p g^{2} R \bar{h}$, adeoque etiam $p R h$, hinc (prop. II art. 132 ), $\geq h R$. At est etiam - ah Rp,"quia - ah $=x$ (nod. p); quare fiet etian $\mp a R p$.

158. Casts secundus. Quando $T+I$ est formae $4^{n}+\mathrm{I},\{=a)$, p formal $4^{n}+3$, stat 


\section{$-\quad 144$}

$\div p(T+I)$, non potest esse $+(T+I) N p$, siue $-(T+1) R p$. Hic casus supra fuit quintus.

$<a$.

Sit vt supra $e^{n}=p+f a$ atque $e$ par et

I. Quando per $p$ non est diuisibilis, erit etiam $f$ per $p$ non diuisibilis. Praeterea autem erit $f$ positiuus, formae $4 n+\mathrm{r}$ (siue $A$ ), at que $\langle a ;+p R f$, adeoque (prop. ro art. 132) $+f R p$. Sed est etiam + faRp, quare fiet + $a R p$, siue - aNp.

II. Quando e per $p$ est diuisibilis, sit $\varepsilon=p g$, atque $f=p h$. Erit itaque $g^{2} p=I$ I ha. Tum $h$ erit positiuus, formae $4 n+3$ ( $B$ ), et ad $p$ et $g^{2}$ primus. Porro $+g^{2} p R h$, adeoque $+p R h$; hinc fit (prop. 13 art 132 ) $h k p$. At est - ha $R p$, vnde fit + $R p$ atque $a N p$.

139. Casus tertius. Quando $T+1$ est for" mde $4^{n}+\mathrm{I},(=a), p$ eiusdem formae, atque \pm $p N a$ : non potest esso to aNp. (Supra casus secundus ).

Capiatur aliquis numerus primus ipso $a$ minor, cuius non-residuum sic $+a$, quales dari supra demonstrauimus ( arti $^{\prime}$ r 25, 129). Sed. hic duos cabus seorsim considerare oportet, prout hic numerus primus fuerit formae $4 n+1$ vel $4 n+3$; non enim demonstratum fuit, dari tales numeros primos vtrizsque formae.

I. Sit iste numerus primus formae $4 n+a$ et $=a^{\prime}$. Tum erit \pm a $a n\left(\right.$ art. $\left.{ }^{13} 7\right)$ adeoqua 


\section{$-145-$}

$\pm a^{\prime} p R a . \quad$ Sit igitur $\varepsilon^{2} \equiv a^{\prime} p\left(\bmod _{*} a\right)$ atque e par, $\angle a$ a Tunc iterum quatuor casus erunt distinguendi.

1) Quando e neque per $\phi$ neque per $a^{\prime}$ est diuisibilis. Ponatur $e^{z}=a^{\prime} p \pm a f$, signis ita acceptis vt $f$ fiat positiuus, Tum erit $f<a$, ad $a^{\prime}$ et $p$ primus atque pro signo superiori formae $4 n+3$, pro inferiori formae $4 n+1$. Designemus breuitatis gratia per $[x, y]$ multitudinem factorum primorum numeri $y$ quorum non-residuum est $x$. Tum erit $a^{\prime} p K f$ adeoque $\left[a^{\prime} p, f\right]=0$. Hinc erit $\left[f, a^{\prime} p\right]$ numerus par, (prop. 1, 3, art。 I33.), i. e. aut $=0$ aut $=2$. Quare erit $f$ aut residuum vtriusque numerorum $a^{\prime}, p$, aut neutrius. Illud autem est impossibile, quum \pm af sit residuum ipsius $a^{\prime}$, atque $\pm a N a^{\prime}$ (hyp.); vnde fit $\pm f N a^{\prime}$. Hinc $f$ debet esse vtriusque numerorum $a^{\prime}, p$ non-re. siduum. At propter $\pm a f R p$ erit $\pm a . N p$. Q. E. D.

2) Quando e per neque vero per á est diuisibilis, sit $e=g p$, atque $g^{2} p=a^{\prime} \pm a \bar{h}$, signo ita determinato, tt $h$ fiat positiuus. Tum erit $h<a$, ad $a^{\prime}, g$, et $p$ primus, atque pro signo superiori formae $4 n+3$, pro inferiori vero formae $4 n+i$. Ex aequatione $g^{2} p=a^{\prime}$ I ah si per $p$, et $a^{\prime}$ multiplicatur, nullo negotio deduci potest; $p a^{\prime} k h . . . .(\alpha) ;$ \#ahp $R a^{\prime} \ldots$ (6) a a $h R_{p} \ldots . . .(y)$. Ex (k) sequitur $\left[p a^{\prime}, h\right]=0$, adeoque (prop, i, 3 , art. 53 ) $\left[h, p^{\prime}\right]$ par, i. e. erit $h$ non-residum vel vtriusque $\xi^{\prime}$, vel K. 
neutrius. Prioni incasu ex (6) sequitur, Iap $N a$, et quum per hyp. sit $\pm a N a^{\prime}$, erit $\pm p R^{\prime} a^{\prime}$, Hinc per theor. lundam. quod pro numeris m?, $a^{\prime}$ ipso $T+\mathrm{r}$ minoribus valet, $a^{\prime} R p$. Hinc et ex eo quod $h N p$, fit per (y) $a N p$ Q. $E . D$. Posírioni casu ex (6) sequitur \pm apka', hinc $\pm p N a^{\prime}$ I $a^{\prime} N p$ hincque tandem et ex $h R_{p}$ fit ex $(\gamma) \pm a N p$. Q. E. D.

3) Quando e per $a^{\prime}$ non auten per p. est diuisibilis. Pro hoc casu demonstratio tantum non eodem modo procedit vt in proec, ne minemque qui hanc penetrauit poterit morari.

4) Quando e tum per a' tum per p est diuisibilis adeoque etiam per productum a $a^{\prime} p$ (numeros a',p enim inarquales fesse supponimus, quia alias id quod demonstrare operam damus, esse $a N a^{\prime}$ iam in hypothesi $a N p$ contentum foret), sit $\varepsilon=$ ga'patgue $g^{2} \alpha s^{\prime} p=1$ tah. Tum erit $h<a$, ad $a^{\prime}$ et $p$ primus atque pro signo superiori formae $43+5$, pro inferiori formae $4^{n}+x$. Facie vero perspicitur, ex ista aequatione deduci posse haec $a^{\prime} p h h,+a h p R a^{\prime}, \pm$ $a n h R p$, quae cum iis quae in (2) inuenimus conueniunt. In reliquis autem demonstratio est eadem.

II. Quando iste numerus primus est formae $4 n+3$, demonstratio praecedenti tam similis est, vt eam apponere superflum nobis visum sit. In eorum gratiam qui per se eam enoluere gestiunt (quod maxime commenda- 


\section{$-x_{47}-$}

mus), id tantam obseruamus, postcuam ad ta. lem aequationem $e^{2}=b p$ af (designante $b$ illum numerum primum) peruentum fuerit, ad perspicuitarem profuturum, si vtrumque siguum seorsim consideretur.

140. Casus quartus. Quando $T+1$ est formar $4 n+\mathrm{I},(=a), p$ formae $n+3$, atque \# $p a$, non poterit esse $+a R p$, siue - $a N p$. (Casus sextus supra).

Etiam huius casus demonstrationem quum prorsus similis sit demonstrationi casus tertii breuitatis gratia omittimus:

14. Casus quintus. Qnando $T+I$ est formae $4 n+3,(=\dot{b}), p$ eiusdiem format, atque $+p R b$ $($ siue - $p N b)$, nequit esse $+b R p$, siue $-b N p$. (Casus tertius supra).

Sit $p \equiv e^{2}(\bmod \cdot b)$, atque $\varepsilon$ par et $<b$.

I. Quando $e$ per $p$ non est diuisibilis. Ponatur $e^{2}=p+b f$, eritque $f$ positiuus, formae $4 n+3,<b$ atque ad $p$ primus. Porro erit $p R f$ adeoque per prop. 13 . art $132,-f R p$. Hinc et ex $+b f R p$ fit $-b R p$ adeoque $4 b N p$ Q. $E, D$.

II. Quando $e$ per est diuisibilis, sit $e$ $=p g$, atque $g g p=\mathbf{I}+b h$. Tum erit $h$ formae $4 n+1$ atque ad $p$ primus, $p \equiv g^{2} p^{2}$ (mod, $h$ ), adeoque $p R h$; binc fit $+h R p$ (prop.

K 2 


\section{$-\quad 148$}

xo art 53 ), vnde et ex - bkRp sequitur LRp, situe + $b N p$. Q. E. D.

4. 2. Casus sextias. Quando $T^{*}+$ I est fow.

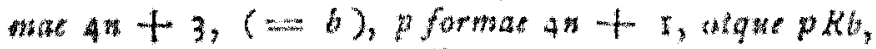
non poterit esse + - $6 N p$. Supra casus septimus.

Demontrationem praecedenti omnino similem, omitimus.

15. Casms seysmus. Quando $T+$ tot for

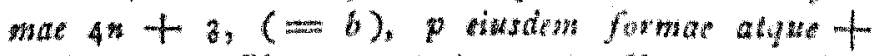

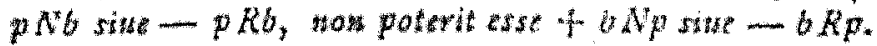
(Casus quartus stupra)

Sit $-p=*^{2}(\bmod . b)$, atque e par et $<b$.

1. Quando per won diuisibilis. Sit $p=t^{2}-b$ eritque $f$ positiuks, forma a $p+t$, ad $p$ primus ipsoque $b$ minor cotanim o ctrto non maior quam $b-1, p+b-1, q$ quarecrit $b=b^{2}+p<b^{2}-b$ i. c. $\left.f+b-1\right)$

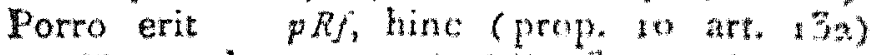

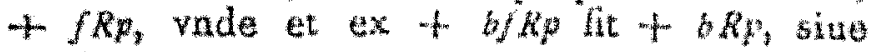
bNp.

I1. Quando per $p$ est diuisibilic, sit $=p g$, atque $g^{*} p=-1+b h_{n}$ ' 1 mum erit posititus, formate $4 n+3$, ad $p$ primus at $<$ b.

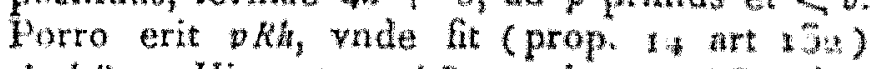
$+h k p$. Hinc et ex $h R_{p}$ equatur $+b k$ siue - BNp. Q. E. D. 


\section{- 49}

144. Casus octanus. Quando $T+\mathrm{r}$ est formate $4 n+3,(=b), p$ format $4 n+\mathbf{I}$, atque $+p N 6$ siue - $p R b$, non poterit esse $\pm b R p$. Casus vlti. mus supra.

Demonstratio perinde procedit vt in casu praecedente.

x 45. In demonstrat. praec. semper pro valorem parem accepimus (art: 137. 144); obseruare conuenit, etiam valorem imparem adhiberi potuisse, sed tum plures adhuc distint. ctiones introducendae fuissent. Qui his disquisitionibus delectantur, haud inutile facient, si vires suas in enolutione horum casuum exercitent. Praeterea theoremata ad residua + 2 et - 2 pertinentia tunc supponi debuissent; quum vero nostra demonstratio absque his theorematibus sit perfecta, nouam hinc inethodum nanciscimur, illa demonstrandi. Quae minime est contemnenda, quum methodi, quibus supra pro demonstratione theorematis, \pm 2 esse residuum cuiusuis numeri primi formae $8 n+x$, vsi sumus, minus directae videri possint. Reliquos casus (qui ad numeros primos formarum $8 n+5,3 n+5,8 n+7$ spectant) per methodus supra traditas demonstratos, illudque theorema tantummodo per inductionem inuentum esse supponemus; hanc autem. inductionem per sequentes reflexiones ad cer: titudinis gradum enehemus.

Si ti a omnium numerorum primorum Cormae $8 *$ f $x$ residuum non esset, ponatur K 5 


\section{$-\quad$ ז50 -}

minimus primus huius formae, cuivs non-residuum $\pm 2,=a$, ita vt pro omnibus prinis ipso a minoribus theorema valeat. Tum accipiatur numerus aliquis primus $<\frac{\mathrm{x}}{2} a$, cuius non: residuum a (qualem dari ex art. I29 facile de. ducitur). Sit. hic $=p$ eritque per theor. fund. $p \mathrm{Na}$ Hinc fit $\pm 2 p R a_{*}-$ Sit itaque $e^{2} \equiv 2 p$ (mod. a) ita vt $e$ sit impar atque $<a . \quad$ Tum duo casus erunt distinguendi.

I. Quando per $p$ non est diuisibilis. Sit $e^{2}=2 p+a q$ eritque $q$ positiuus, formae $8 n+7$ vel formae $8 n+3$, (prout $p$ est formae $4 n+\mathrm{r}$ vel $4 n+3),<a$, atque per $p$ non diuisibilis. Iam omnes factores primi ipsius $q$ in quatuor classes distribuantur, sint scilicet $"$ formae $8 n+r, f$ formae $8 n+3$, $g$ formáe $8 n+5, h$ formae $8 n+7$; productum e factoribus primae classis sit $E$, producta e factoribus secundae, tertiae, quartae classis respectiue, $F, G, H^{*}$ ). His ita factis, conside: remus primo casum vbi $p$ est formae $4^{n}+\mathrm{r}$, siue $q$ formae $S_{n}+7 \cdot$ Tum facile perspicitur fore $2 R E, 2 R H$, vnde $p R E, p R H$, hincque tan. $\operatorname{dem} E R p, H R p$. Porro erit 2 non-residuum cuiusuis factoris formae $8 n+3$ aut $8 n+5$ adeoque etiam $p$; hinc quiuis talis factor nonresiduum ipsius $p$; vnde facile concluditur $F G$ fore ipsius $p$ residuum, si $f+g$ fuerit par, non-residuum, si $f+g$ fuerit impar. At $f+g$ impar esse non potest; facile enim perspicietur omnes casus enumerando, EFGH siue q feri

-) Si ex aliqua classe nulli factores adessent, loco producti ex his t scribere oporteret. 


\section{- ${ }_{15} 5-$}

vel formae $8 n+3$ vel $8 n+5$, si fuerit $f+g$ impar, quidquid sint singuli $e, f, g, h$ contra hyp. Erit igitur FOR hincque tandem, propter aq $R_{p}$, a Rp contra hypo Secundo quando $p$ est formae $4 n+3$, simili modo ostendi potest, fore $p R E$ adeoque $E R p$, - $p R F$ adeoque $F R p$, tandem $g+h$ parem hincque GHRp, vnde tandem sequitur $q R p$, alip contra hyp.

II. Quando e per po disusibilis, demonstratio simili modo adomari, et a peritis (quibus so is hic articulus est scriptus) haurl difficulter enolui poterit. Nos breuitatis gratia eam amittimus.

I46. Per theorema fundamentale atque propositiones ad residua - $i$ et te 2 per: tinentes semper determinari potest vtrum numerus quicunque datus numeri primi dati residunm sit an non residuum. At hand inutile erit, reliqua etiam quee supra tradidimus hic iterum in conspectum producere, vt omria coniuncta habeantur quae sunt necessaria ad solutionem.

Prodiemaris: Propositis atuobus numepis quibuscuacue $P, Q$, inuenive, vinum alter $Q$, alterius $P$ residatum sit an non-residuabz.

Sol. I. Sit $P=\sigma^{e} 6 c^{*} c^{\gamma}$ etc. designantibns $a, b, c$ etc numeros primos inaequales positiue acceptós ( nam $P$ manifesto absolute est sumendus). Breutatis gratia in hoc art. relationem duorum numerorum $x, y$ simpliciter di $\mathrm{K}$ \& 


\section{$-\quad 152$}

cemus eam quatenus prior $x$ posterioris y residuum est vel non-residuum. Pendet igitur relatio ipsorum $Q, P$ a relationibus jpsorum $Q, a^{\text {s }} Q, b:$ etc. (art. 105)。

II. Vt relatio ipsorum $Q, a^{\alpha}$ (de reliquís enim $Q, b_{5}$ etc. idem valet) innotescat, duo casus distinguendi.

I. Quando $Q$ per a est diuisibilis. Pona. tur $Q=Q^{\prime} a^{\mathrm{e}}$, ita vt $Q^{\prime}$ per a non sit diuisibilis. Tunc si $\varepsilon=\alpha^{\prime}$ vel $\ell>\alpha$, erit $Q R a^{\alpha}$; si vero $e<$ a atque impar, erit $Q N \alpha^{\alpha}$ : tandem si $e<*$ atque par, habebit $Q$ ad $\alpha_{*}^{\infty}$ eandem relationem quam habet $Q^{\prime}$ ad $a^{q-e_{*}}$ "Reductus est itaque hic casus ad

2. Quando $Q$ per a non est diuisibilis. Hic denuo duos casus distinguimus.

(A) Quando $a=2$. Tunc semper erit $Q R a^{\alpha}$, quando $\alpha=1$; quando vero $\alpha=2$, requiritur, vt sit $Q$ formae $4 n+1$; denique quando $x=3$ vel $>3$. Q debet esse formae $\$ n+x$ Q Quae conditio si locum habet, erit $Q R a^{*}$;

(B) Quando est alius numerus primus. Tunc $Q$ ad $a^{\alpha}$ eandem relationem habebit quam habet ad $a_{0}$ ( V, art. Io I).

III. Relatio numeri culuscunque $Q$ ad numerum primum a (imparem) ita inuestigatur. 


\section{$-153 \quad-$}

Quando $Q>a$, substituatur loco ipsius $Q$ ipsius residuum minimum positiuum secundum mo. dulum $a^{*}$ ). Hoc ad $a$ eandem relationem habebit quam habet $Q$.

Porro resoluatur $Q$, siue nurnerus ipsius loco assumtus, in factores suos primos $p, p^{\prime}$, $p$ etc., quibus adiungendus factor -1 , quando $Q$ est negatiuus. Tum constat relationem ipsius $Q$ ad a pendere a relationibus singulorum $p, p^{\prime}, p^{\prime \prime}$ etc. ad $a$. Scilicet si inter illos factores sunt $2 m$ non-residua ipsius $a$ erit $Q R a$, si vero $2 m+1$, erit $Q N a$. Facile autem perspicitur, si inter factores $p, p^{\prime}, p^{\prime \prime}$ etc., bini aut quaterni aut seni aut generaliter $2 k$ aequales occurrant hos tuto eiici posse.

IV. Si inter factores $p, p^{\prime}, p^{\prime \prime}$ reperiuntur - I et 2 , horum relatio ad a ex artt. ros; I 12, I13, I14 inueniri potest, Reliquorüm autem relatio ad a pendet a relatione ipsius $a$ ad ipses (theor, fund, atque propp. art. $13_{1}$ ), Sit $p$ vnus ex ipsis, inuenieturque, (tractando numerus $a, p$ eodem modo vt antea $Q$ et $a$ illis respectiue maiores) relationem ipsius $a$ ad $p$ aut per artt. $108-114$ determinari posse (si scilicet residuum minimum ipsius $a(\bmod , p)$ nullos factores primos impares habeat), aut in-

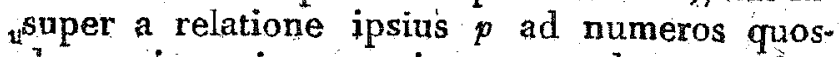
dam primos jpso $p$ minores pendere. Idem valet de reliquis factoribus $p^{\prime}, p^{\prime \prime}$ etc, Facile

*) Residusm in signific art. 4. - Plerninque praestat residuum bsolute minimum accipere, 


\section{$-154=$}

iam perspicitur per continuationem huius ope. rationes tandem ad numeros peruentum iri quorum relationes per propp. artt. io 8- I 4 . determinari possint. Per exemplum haec clariora fient.

Ex. Quaeritur relatio numeri +453 ad I236. Est $1236=4.3 .105 ;+453 R 4$ per 11 . $2(A) ;+453 \cdot R 3$ per II. 1. Superest igitur vt relatio ipsius +453 ad 105 exploretur. Ertdem autem erit quam habet $+4 \mathrm{I} \equiv 453$, mod. 103 ) ad 103 ; eadem ipsius $+103 \mathrm{ad}$ if (theor. fund.), siue ipsius - 20 ad $4 \mathrm{I}$. At est $-20 R 4 \mathrm{I}$; namque $-20=1 \ldots$ r. $_{2}$ 2. $5 ;-1 R 4$, (art. 108 ); atque $+5 R$ is ideo quod $41 \equiv \mathrm{I}$ adeoque ipsius 5 residum est (theor. fund.). Hinc sequitur $+453 R$ ro3, bincque tandem $+453 R$ 1236. Est autem reuera $453 \equiv 297^{2}(\bmod .1236)$.

147. Proposito numero quocunque $A$, formalae certae exhiberi possunt, sub quibus omnes numeri ad $A$ primi quorum residnam. est $A$ continentur, siue omnes qui esse possunt diuisones numerorum formae $x x-A$ (designante $x x$ quadratum indeterminatum ) Sed breuitatis gratia ad eos tantum diuisores respiciemus, qui sunt impares atque ad $A$ prim mi, quum ad hos casus reliqui facile reduci possint.

6) Huinsmodi numeros simpliciter dinisores insius $x x-A$ dicemus pude sponte patet quid sint ron-dinisorts. 


\section{$-\quad \times 55-$}

Sit primo $A$ aut numerus primus positiuus formae $2 n+1$, aut negatiuus formae $4 n-\mathrm{I}$. Tum secundum theorema fundamentale o. mnes numeri primi, qui, positive sumti, sunt residua ipsius $A$, erunt diuisores ipsius $x: t$ $A$ : omnes autem numeri primi (excepto numero 2 qui semper est diuisor) qui ipsius $A$ sunt non residua erunt non duisores ipsius $x x$ - $A$. Sint omnia residua ipsins $A$ ipso $A$ minora (exclusa cifra), $r, r^{\prime \prime}$ etc. omnia nonresidua vero $n, n^{\prime} n^{\prime \prime}$ etc. Tum quinis numerus primus, in aliqua formarum $A k+r, A k+r$, $A k+r^{\prime \prime}$ etc. contentus, erit diuisor ipsius $x x$ - $A$, quiuis antem primus in aliqua forma$\operatorname{rum} A k+n, A k+n^{t}$ etc. contentus non-diuisor erit, designante $k$ numerum integrum indeterminatum. Illas formas dicimus formas ditisorum ipsitss $x x-A$, has vero formas noib-ditisorumb Vtroramqne multitudo erit $\frac{x}{2}(A-1)$, Porro si $B$ est numerus compositus impar at. que $A R B$, omnes factores primi ipsius $B$ in aliqua formarum primorum continentur adeoque etiam $B$. Quare quists numerus inpar in forma non diuisorum contentus, erit non-dinisor formae $x x-A$. Sed hoo theorema cunuertere non licet; nam si $B$ est nond diuisor compositus impar formae $x: x-A$, inter faotores primos ipsius $B$ aliqui non-diuisores $\mathrm{e}$. runt, quorum multitudo ši est por, $B$ nihilominus in aliqua forma divisorum reperietur, V. art. 99 .

Ex Hoc modo pro $A=-1$ formae diuisorum ipsius $x x+$ II inueniuntur hae; 


\section{$-\quad \$ 56-$}

In $+\pi, 3,4,5,9$, formae non diuisorum au. tem erunt in $+2,6,7,8$, Io. Erit itaque - Ir non-residuum omnium numerorum imparium, qui in aliqua posteriorum formarum continentur, residuum autem omnium primorum ad aliquam priorum pertinentium.

Similes formae dantur pro diuisoribus atque non-diuisoribus ipsius $x x-A$, quemcunque numerum designet $A$. Sed facile perspicitur, eos ipsius $A$ valores tantummodo considerari oportere, qui per nullum quadratum sint divisibiles; patet enim si fuerit $A=a^{2} \cdot A^{\prime}$, omnes diuisores *) ipsius $x x-A$ etiam fore diuisores ipsius $x x-A^{\prime}$, similiterque non-diuisores. - Distinguemus autem tres casus, I) quando $A$ est formae $+(4 n+I)$ vel $(4 n-1)$. 2) quando, $A$ est formae $-(4 n+x)$ vel $+(4 n-I) \cdot 3)$ quando $A$ est par siue formae $\pm\left(4^{n}+2\right)$

148. Casus primus, quando $A$ est formae $+(4 n+1)$ vel $-(4 n-1)$. Resoluatur $A$ in factores suos primos, tribuaturque iis qui sunt formae $4^{n}+1$ signum positiuum, is vero qui sunt formae $4 n-1$ signum negatiuum (vnde fiet productum ex ipsis $=A$ ). Sint hi factoreg $a, b, c, d$ etc. Distribuantur omnes numeri ipso $A$ minores et ad $A$ primi in duas classes, et quidem in primam classem omnes nu-

7) Nempe qui șint pritri $2 d A$ 


\section{$-\quad{ }^{15} 7 \quad-$}

meri qui sunt nullius ex numeris $a, b, c$, d etc. non-residua, aut duorum, aut quatuor aut generaliter multitudinis paris; in secundam vero ii, qui sunt non residua vnius ex numeris $a, b$, cetc. aut trium etc، aut generaliter multitudinis imporis. Designentur priores per $r, r^{\prime} r^{\prime \prime}$, etc. posteriores per $n, n$; $n^{\prime \prime}$ etc. Tum formae $A k+\%, A k+r^{\prime}$ $A k+r^{\prime \prime}$ etc. erunt formae diuisorum ipsius $x x-A$, formae vero $A k-n, A k-n^{2}$ etc. erunt formae non-diuisorum ipsius $x x-A$ (i. c. numerus quicunque primus, praeter 2 , erit diuisor aut non diuisor ipsius $x x-A$ prout in aniqua formarum priorum aut posteriorum continetur). $\mathrm{Si}$ enim $p$ est numerus primus positiuus atque alicuius ex numeris $a, b, c$ etc. residuum vel non-residuum, hic ipse numerus ipsius $p$ residuum vel non-residuum erit (theor. fund.). Quare si inter numeros $a, b, c$ etc. sunt $m$, quorum non-residuum est $p$; totidem erunt non-residua ipsius $p$, adeoque si $p$ in aliqua formarum priorum continetur, erit $m$ par et $A R_{p}$, si vero in aliqua posteriorum, erit $n$ impar atque $A N p$.

Ex. Sit $A=+\mathrm{ro} 5=-3 x+5 x$ -7 . Tum numeri $r, r^{\prime}, r^{\prime \prime}$, etc. erunt hi: $x, 4,16,46,64,79$, (qui sunt non-residua nullius numerorum $3,5,7) ; 2,8,23,32$, $53,9^{2}$ (qui sunt non-residua numerorum 3,5 ; $26,4 \mathrm{I}, 59,89$, 101, 104 (qui sunt non resi. dua numerorum 3,7$), 13,52,73,82,97$, jo3 (qui sunt non-residua numerorum 5,7 ). Numeri autem $n, w^{\prime}, n^{\prime \prime}$ etc. erunt hi: Ir, 29, $44,71,74,86 ; 22,37,43,58,67,88$ $19,51,34,61,76,94 ; 17,38,47,62,68$, 


\section{- ${ }_{158}-$}

83. Seni primi sunt non-residua ipsius 3 , se: ni posteriores non-residua ipsius 5 , tum sequuntur non-residua ipsitis 7 , tandem ii qui sunt non-residua omnium trium simul.

Facile ex combinationum theoria atque artt. $32,9^{6}$ deducitur, numerorum $r_{4} r^{\prime}, r^{\prime \prime}$ etc. multitudinem fore $=t\left(x+\frac{l \cdot l-1}{x \cdot 2}+\right.$ $\left.\frac{1.1-1 . t-2 . t-3}{3.2 .} \cdot 3\right)$, numerorum $n, n^{\prime}$ s " $"$ etc. multitudinem $=t\left(l+\frac{l \cdot t \cdot \mathrm{r} \cdot t-2}{\mathrm{r} \cdot 2 \cdot 3}+\right.$ l. $\left.t-x_{0} \ldots b-4, \ldots\right)$, vbi $l$ designat multitudinem numerorum $a, b, c$ etc.; $t=2-1(a-1)$ $(b-I)(c-1)$ etc., et vtraque series continitanda donec abrumpatur. (Dabuntur scilicet $t$ numeri, cqui sunt residua omnium $a, b, c$ etc. t. $. .1-\mathrm{I}$, qui sunt non-residua duorum etc. 1. 2.

sed demonstrationem hanc fusius explicare breuitas non permittit). Vtriusque autem seriei summa ${ }^{*}$ ) est $=2^{-1} \boldsymbol{1}_{0}$. Scilicet posterior prodit ex hac I $+(t-\mathrm{I})+\frac{t-\mathrm{I} \cdot \frac{1-2}{\mathrm{I}}}{2}$ etc. iungendo terminum secundum et tertium, quartum et quintum etc. posterior vero ex eadem ungendo terminum primum atque secundum, tertium et quartum etc. Dabuntur

4) Neglecto factore $\boldsymbol{t}$. 


\section{$-159$}

itaque tot formae diuisorum ipsius $x x-A$, quot dantur formae non diuisorum, scilicet $\frac{1}{2}(a-1)(b-1)(c-1)$ etc.

149. Casum secundum et tritin hic simul contemplari possumus. Poterit scilicet $A$ sem. per hic poni $=(-$ I $) \cdot Q$ aut $=\left(+_{2}\right) Q$. ant $=(-2) Q$, designante $Q$ numerorum formae $+(4 n+x)$, aut - $(4 n, \quad I)$, quales in art praec. consideramus. Sit generaliter $A=\alpha Q$. ita vt sit $\alpha$ aut $=-\mathrm{r}$, aut $= \pm 2$. Tum erit $A$ residuum omnium numerorum, quorum residuum est aut vterque a et $Q$, aut neater; non-residuum autem omnium, quorum non residunm alteruter tantum numerorum $\alpha, Q$. Hinc formae diuisorum ac non-diuisorum ipsius $x x-A$ facile deriuantur. Si $a=\ldots$ distribuantur omnes numeri ipso $4 A$ minores ad ipsumque primi in duas classes, in priorem ii, qui sunt in aliqua forma diuisorum ipsias $x x-Q$, simulque in forme $4 n+1$, ique, qui sunt in aliqua forma non-dicisorum ipsius $x x$ Q simulque in forma $4 n+3$; in posteriorem reliqui. Sint priores $r, r, r^{\prime \prime}$ etc., posteriores $n, n^{\prime}, n^{\prime \prime}$ etc, eritque $A$ residuum omnium numerorum primorum in aliqua formarum $4 A k+r, 4 A k+r^{\prime}, 4 A k+r^{\prime \prime}$ etc. contentorum, non residuum autem omnium primorum in aliqua formarum $4 A k+n, 4 A k+n^{n}$ etc. contentorum, $\mathrm{Si} \alpha= \pm 2$, distribuantur omnes numeri ipso $8 Q$ minores ad ipsumque primi in duas classes, in primam ii, qui continentur in aliqua forma diuisorum ipsius $x x-Q$ simulque in aliquaformarum $8 n+x, 8 n$ 
+7 pro signo superiori, vel formarum $8 n+r_{s}$ $8 n+3$ pro inferiori, iique qui contenti sunt in aliqua forma non diuisorum ipsius $x x-Q$ simulque in aliqua harum $8 n+3,8 n+5$ pro signo superiori, vel harum $8 n+5,8 n+7$ pro inferiori, - in secundam reliqui. Tum designatis numeris classis prioris per $r, r, r^{\prime \prime}$ etc, numerisque classis posterioris per $n, n^{\prime}, n^{\prime \prime}$ etc. $\pm 2 Q$ erit residuum omnium numerornm primorum in aliqua formarum $8 Q^{k}+r, 8 Q^{k}$ $+r, 8 Q k+q^{\prime \prime}$ etc contentorum, omnium autem primorum in aliqua formarum $8 Q k+n_{s}$ $8 Q^{k}+n^{d}, 8 Q^{k}+n^{\prime \prime}$ etc. non-residuum. Cey terum facile demonstrari potest, etiam hic totidem formas diuisorum ipsius $x x-A$ datum iri ac non-diuisorum.

Ex. Hoc modo inuenitur + to esse residuum omnium numerorum primorum in aliqua formarum $40 k+1,5,9,13,27,31,37,39$, contentorum, non-residuum vero omnium primorum, qui sub aliqua formarum $40 k+7,11$, $17,29,2 r, 23,29,33$ continentur.

150. Formae hae plures habent proprietates satis memorabiles, quarum tamen vnam tantummodo apponimus. $\mathrm{Si} B$ est numerus compositus ad $A$, primus, inter cuius factores primos occurrunt $2 m$, qui in aliqua forma nondiuisorum ipsius $x x-A$ continentur, $B$ in aliqua forma diuisorum ipsius $x x-A$ contentus erit; si vero multitudo factorum primorum ipsius $B$ in aliqua forma non diuisorum ipsius 
$x x-A$ contentus erit. . Demonstrationem quae non est difficilis omittimus. Hinc vero sequitur, non modo quemuis numerum primum sed etiam quemuis compositum imparem ad $A$ primum, qui in aliqua forma non-diuisorum contineatur; non diaisorem fore; necessario enim aliquis factor primus talis numeri debet esse non-diuisor.

I5r. Theorema fundamentale, quod sa ne inter elegantissima in hoc genere est referendum, in eadem forma simplici, in qua supra propositum est, a nemine hucusque fuit prolatum. Quod eo magis est mirandum, cuum aliae quaedam propositiones illi superstruendate ex quibus ad illud facile reveniri potnisset, ill. Eulero iam innoluerint. Formas certas dari, in quibus omnes diuisores primi numerorum formae $x x-A$ contineantur, aliasque in quibus omnes non-diuisores primi numerorun eiusdem formae sint comprehensi, ita vt hae illas excludant, nouerat, methodumque illas for mas inueniendi eruerat: sed omnes ipsius co. natus ad demonstrationem perueniendi semper irriti fuerunt, veritatique illi per inductionem. inuentae maiorem tantummodo verisimilitudinem conciliauerunt. In aliqua quidem tracta tione, Noune demonstrationes circa diusores numeronum formae $x+x y y$, quae in Acad. Petrop. recitata est $177^{5}$ Nou. 20 , et post mortem viri summi in T. I. Nou. Act huius Ac. p. 47 sqq esr conseruata, voti se compotem credidisse videtur: sed hic error irrepsit, scilicet p. 65. tacite supposuit, formas tales diuisorum 
et non-diuisorum exstare $)$, vnde non difficile erat quales esse debeant deriuare: methodus autem qua vsus est ad comprobationem illius suppositionis haud idonea videtur. In alio schediasmate, De criteriis aequationis $f x x+g y y=h z z$ vitrumque resolationem cadmittat necne, $O p$ us $c_{0}$ A $A$ a $l_{\text {. }}$ T. 1. (vbi $f, g ; h$ sunt dati, $x, y, z$ indeterminati) per inductionem inuenit, si aequatio pro aliquo valore ipsius $h=s$ solubilis sit, eandem pro quouis alio valore ipsi $s$ secundum mod. $4 f g$ congruo, siquidem sit numerus primus, soluoilem fore, ex qua propositione suppositio de qua diximus haud difficile demonstrari potest. Sed etiam huius theorematis demonstratio omnes ipsius labores elusit ${ }^{*}$ ), quod non est mirandum, quia nostro iudicio a theoremate fundamentali erat proficiscendum. Cetertim veritas huits propositionis ex is quae in sect. sequenti docebimus sponte demanabit.

Post Eulerum, clar. Le Gendre eidem argumento operam nauauit, in egregia tract. Re-

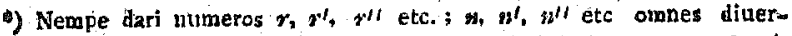
sos et $<4 A$ tales vt omnes diuisores primi ipsius $x x-A$ sub aliqua formarum $4 A k+r, 4 A k+r^{\prime}$ etc contineatur, omnesque non-diuisores primi sub aliqua harum $4 A k+3,4 A k+$ is! etc. (designante $k$ numerum indétecminatum);

*) Vti ipse fatetar, 1. c. p. $216^{\prime}$.Huius elegantissimi theorematis demonstratio adhuc desideratur, postquaio a piuribus iamdudum fro. stra est ihuestigata.... Quocirca plurimum is praestitisse censendus erit, cui successerit demonstrationem huits theorematis inuenire." - Quanto ardore vir inmortal's demonstrationem huius th orematis aliorumque, quae taneummodo csus speciales theor. fundam. sunt, desiderauerit, videre licet ex multis atiis locis Opusc. Anall Conf diditamestum ad diss VIII, T. I. et diss. XIII, T. II. pluresque dics, in Connent, Petrop., jam passim laudatae. 
cherches d'analyse indeterminé, Hist. de PAc. des Sc. 1785 p. 465 sqq., bi peruenit ad theorema, quod si rem ipsam spectas cum th. fund. idem est, scilicet designantibus $p, q$ duos numeros primos positiuos, fore residua absolute minima potestatum $p \frac{q-1}{2}, q \frac{p-I}{2}$ sec. mod. $q, p$ resp. aut ambo $+\mathrm{I}$, aut ambo. $-\mathrm{I}$, quando aut $p$ aut $q$ sit formae $4 n+1$; quando vero tum $p$ tum $q$ sit formae $4 n+3$, alterum res. min fore +1 , alterum - I, p 5,6 , ex quo sec. art, 106. deriuatur, relationem (in signif. art. I 46 acceptam) ipsius $p$ ad $q$ ipsiusque $q$ ad $p$ eandem esse, quando aut $p$ aut $q$ sit formae $4^{n}+$ I, oppositam, quando tum $p$ tum $q$ sit formae $4 n+3$. Propos. haecinter propp art. 13 I est contenta, sequitur etiam ex I, 3,9 , art I 33 ; vicissim autem theor. fund. ex ipsa deriuari potest. Clar. Le Gendre etiam demonstrationem tentauit, de qua quum perquam ingeniosa sit in Sect. seq. fusius loquemur. Sed quoniam in ea plura sine de. monstratione supposuit (vti ipse fatetur p. 520 . Nous avons supposé seulement etc.), quae partim a nemine hucusque sunt demonstrata, partim nostro quidem iudicio sine theor. fund. ipso demonstrari nequeunt: via quam ingressus est, ad scopum deducere non posse videtur; nostraque demonstratio pro prima erit habenda. - Ceterum infra duas alias demonstrationes eiusdem grauissimi theorematis trademus, a praec. et inter se toto coelo diuersas.

152. Hactenus congruentiam puram $\equiv A(\bmod . m)$ tractanimus, ipsiusque resolubilitatem dignoscere docuimus, Radicum ipsarum 


\section{$-\quad{ }^{64} \cdot$}

inuestigatio per art. ro5 ad eum casum est reducta, vbi $m$ est aut primus aut primi potestas, posterior vero per art. $10 \mathrm{l}$ ad eum vbi m est primus. Pro hoc autem casu ea quae in art. 6I sqq. tradidinus vna cum iis quae in sect. V et VIII docebimus, omnia fere complectuntur quae per mothodos directas erui possunt. Sed hae vbi sunt applicabiles plerumque infinities prolixiores sunt quam indirectae quas in sect. VI. docebimus, adeoque non tam propter vtilitatem suam in praxi quam propter pulcritudinem memorabiles. - Congruentiae secsndi gradus non-purae ad puras facile reduci possunt. Proposita congruentia $a x x+b x$ $+c \equiv 0$ secundum mod. $m$ soluenda, hưic aequiualebir congruentia $4 a a x x+4 a b x+4 a c$ $=o(\bmod .4 a m), i$, e. quiuis numerus alteri satisfaciens etiam alteri satisfaciet. Haec vero ita exhiberi potest $(2 a x+b)^{2} \equiv b b-4 a c$ (mod. $4 a m$ ), vnde omnes valores ipsius $2 a x+b$ minores quam 4am si qui dantur inueniri possunt. Quibus per $r$, $r^{\prime}$, s' etc. designatis, omnes solutiones congr. prop. deducentur ex solutionibus congruentiarum $2 d x \equiv r-b$, $a t x \equiv r^{\prime}-b$ etc (mod. 4am) etc.; quas in sect. II inuerire docuimus. Ceterum obseruamus, solutionem plerumque per varia artificia contrahi posse, ex gr. loco congr. prop. aliam inueniri posse $a^{\prime} x x+2 b^{\prime} x+c^{\prime} \equiv o$, illi aequipollentem, et in qna $a^{\prime}$ ipsum $m$ metiatur; haec vero de quibus Sect, vltima conferri potest, hic explicare brentas non permittit. 


\section{$-{ }_{165}-$}

\section{Sectio Quinta}

DE

FORMIS AEQVATIONIBVSSQVE INDETERMINATIS

SECVNDT GRADVS,

153. In hac sectione imprimis de functionibus duarum indeterminatarum $x, y$, huius formae, $a x x$ $+2 b x y+c y y$; vbi $a, b, c$ sunt integri dati tractabimus, quas formas secundi gradius, siue simpliciter fornas dicemus: Huic disquisitioni superstruetur solutio problematis famosi, inuenire omnes solutiones aequationis cuinscunque indeterminatae secundi gradus duas incognitas implicantis, siue hae incognitae valores integros siue rationales tantum nancisci debeant. Problema hoc quiden iam ab-ill. La Grange in omni generalitate est, solutum, multaque insuper ad naturam formarum pertinentia tum ab hoc ipso magno geortietra, tum ab ill Eulero partim primum inuenta, partim, a Fermatio olim inrienta, demonstrationibus munita. Sed nobis acriter formarum perquisitioni insistentibus tam siulta noua se obtulerunt, vt totum argumen:

L 3 
tum $\mathrm{ab}$ integro resumere operae pretium duxerimus, eo magis, quod Vurorum illorum inuenta, multis locis sparsa, paucis innotuisse comperti sumus; porro quod methodus per quam haec tractabimus nobis ad maximam partem est propria; tandem quod nostra sine no. ua illorum expositione ne intelligi quidem possent Nullum vero dubium nobis esse videtur, quin multa eaque egregia in hoc genere adhuc lateant in quibus alii vires suas exercere possint. Ceterum quae ad veritatum insignium historiam pertinent, loco suo semper trademus.

Formam $a x x+2 b x y+c y y$, quando de indeterminatis $x, y$ non agitur, ita designabimus, $(a, b, c)$ Haec itaque expressio denotabit indefinite summam trium partium, producti numeri dati $a$ in quadratum indeterminatae cuiuscunque; producti duplicati numer $b$ in hanc indeterminatam in aliam indetermina. tam; producti numeri $c$ in quadratum huius secundae indeterminatae Ex. gr. $(r, 0,2)$ exprimet summam quadrati et quadrati duplicati. Ceterum, quamuis formae $(a, b, c)$ et $(c, b, a)$ idem designent, si ad partes ipsas tantum respicimus, tamen different si insuper ad partium ordinem attendimus; quare sedulo eas in posterum distinguemus; quid vero inde lucremur in sequentibus sufficienter patebit.

154. Numerum aliquem datum per formam datam repraesentari dicemus, si formae indeterminatis tales valores, integri tribuuntur, 
vt ipsius valor numero dato frat aequalis. Hic habebimus fequens

Theonema. Si numerus $M$ ita per formam $(a, b, c)$ repraesenturi potest, $v t$ indeterminatarum valores, per quos hoc efficitur, inter se sint primi : etit $b 5$ - ac residusm quadraticum numeri $M_{\text {a }}$.

Dem. Sint valores indeterminatarum $m_{n} n_{m}$ scilicet $a m m+2 b m n+c m n=M$, accipianturque numeri $\mu$, , ita vt sit $\mu m+m=1$ (art, 40). Tum per euolutionem facile probatur esse, $(a m m+2 b m n+c n n)\left(a_{n}-2 b k y+c \mu\right)=$ $(\mu(m b+n c)-n(m a+n b))^{2}-(b b-a c)$ $(m \mu+n)^{2}$, siue $M(a w n-2 b \mu \nu+c \mu \mu)=(\mu$ $(m b+n c)-(m a+n b))^{2}-(b b-a c)$ Quare erit $b b \quad a c \equiv(\mu(m b+n c)-,(m a+n b))^{2}(\bmod$. $M)$, i. e. bb - ac residum quadraticum ipsius $M 2$.

Numeruml $b b-a c$, a cuius indole prom prietates formae $(a, b, c)$ imprimis pendere, in sequentibus docebimus, determinantem huius formae vocabimus.

155. Erit itaque $\times(m b+n c)-2(m a+n b)$ valor expressionis $\sqrt{(b b}-a c)$ (mod. $M)$. Constat autem, numeros $\mu$, infinitis modis ita determinari posse vt sit $\mu m+n=1$, vnde alii aliique valores illius expressionis prodibunt, qui quem nexum inter se habuant videamus. Sit non modo $\mu m+m=1$, sed etaim $\mu^{2} m+$ $n^{\prime} n=\mathrm{I}$, ponaturque $\mu(m b+n c)-v(m a+n b)$ $=v, \mu^{\prime}(m b+n c)-v(m a+n b)=v^{\prime}$. Multiplicando aequationem $\mathrm{km}+\mu_{2}=1$ per $\mu_{2}$ als L. 4 


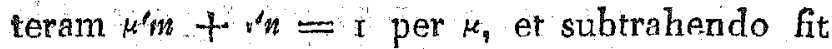
$\mu^{\prime}-\mu=n\left(\mu^{\prime} v-\mu^{\prime}\right)$ similiterque multiplicando illam per hanc per " fit subtrahendo $v^{\prime}-v=n\left(\omega^{\prime}-\mu^{\prime}{ }^{\prime}\right)$. Hinc statim prodit $v^{\prime}-v=\left(\mu v^{\prime}-\mu^{\prime}\right)(a m m+2 b m n+c n n)=$ $\left(\mu^{\prime} y-\mu^{\prime}\right) M$, sile $v^{\prime} \equiv v(\bmod M)$. Quomodocunque igitur $\mu$, determinentur, formula $\mu(m b+n c) \cdots(m a+n b)$ valores diuersos (i. e. incongruos) expressionis " $(b b-a c)$ $(\bmod M)$ dare nequit. Si itaque $v$ est valor quicunque illius formulae: repraesentationem numeri $M$ per formam $a x x+2 b x y+c y y$ eam vbi $x=m, y=n$, pertinere dicemus ad valo.

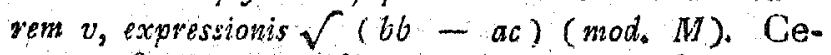
terum facile ostendi potest, si valor formulae illius aliquis sit $v$ atque $v^{\prime} \equiv v(\bmod M)$, loco numerorum $\mu$, "qui dant $v$, alios $k^{\prime}, v^{\prime}$ ac cipi posse, qui dent $v^{\prime}$. Scilicet faciendo $\mu^{\prime}=$ $\mu+\frac{n(u-v)}{M}, v^{\prime}=-\frac{m(u-v)}{M}$, fiet $\mu^{\prime} m+$ $i^{\prime} n=\mu m+n=x$, valor autem formulae ex $\mu$, " prodiens sup erabit valoren ex $\mu, "$ prodeuntem qaantitate $\left(\mu^{\prime} \nu-\mu \nu^{\prime}\right) M$, quae fit $=$ $(\mu m+v n)\left(v^{\prime}-v\right)=v^{\prime}-v$, siue valor ille erit $=v^{\prime}$

156. Si duae repraesentiones eiusdem numeri $M$ per eandem formam $\left(a, b_{2} c\right)$ habentur, in quibus indeterminatae valores inter se primos habent: hae vel ad eundem valorem expr. $\sqrt{ }(b b-a c)(\bmod . M)$ pertinere possunt vel ad diuersos. Sit m $=a m+2 b m$ $+c n n=a m^{\prime} m^{\prime}+2 b m^{\prime} n^{\prime}+c^{\prime} n^{\prime}$, atque $n n+$ $m=1, \mu m+1 n^{\prime \prime}=1$, patetque si fuerie 


\section{$-{ }^{6} 69-$}

$\mu(m b+n c)-,(m a+n b) \equiv \mu^{\prime}\left(m^{\prime} b+n^{\prime} c\right)-\nu^{\prime}\left(m^{\prime} a\right.$ $+n, b)(\bmod , M)$, congruentiam semper manere; qaicunque alii valores idonei pro $\mu, " ; \mu^{\prime}, n^{\prime}$ accipjantur, in quo casu vtramque repraesentationem ad eundem valorem expr. $\sqrt{ }(b b-a c)(\bmod . M)$ pertinere dicemus; si vero congruentia pro vllis valoribus ipsorum $\mu, \eta ; \mu^{\prime}$, "locum non habet, pro nullis locum habebit, repraesentationesque ad valores diusr'sos pertinebunt. Si vero $\mu(m b+w c)-v(m a+n b) \equiv-\left(\mu^{\prime}\left(m^{\prime} b\right.\right.$ $\left.\left.+n^{\prime} c\right)-\cdots\left(m^{\prime} a+n^{\prime} b\right)\right):$ repraesentationes ad valores oppositos expr. $\sqrt{ }(b b-a c)$ pertinere dicentur. Omnibus hisce denominationibus etiam vtemur, quando de pluribus repraeséntationibus eiusdem numeri per formas diitiersas, sed quae eundem determinantem habent, agitur.

Ex. Sit forma proposita haec $(3,7,-8)$ cuius determinans $=73$. Per hanc formam habentur repraesentationes numeri 57 hae: $3.13^{2}+14.13 .25-8.25^{2} ; 3.5^{2}+14.5 .9-8.9^{2}$. Pro prima poni potest $\mu=2, r=-1$, vnde prodit valor expr. $\sqrt{73}$ (mod. 57 ) ad quam repr. pertinet $=2(13.7-25.8)+($ i3. $3+$ $25.7)=-4$. Simili modo repraesentatio secunda pertinere inuenitur, faciendo $\mu=2, v=$ - I, ad valorem + 4. Quare ambae reprae. sentationes ad valores oppositos pertinent.

Antequam vlterius progredimur, obserua. mus, formas quarum determinans $=0$ ab inuestigationibus sequentibus prorsus exclusas esse, quippe quae theorematum concinnitatem tantummado turbarent, adeaque tractationem. peculiarem postulent. 


\section{$\rightarrow 170-$}

15\%. Si forma, $F$, cuius indeterminatae sunt $x, y$ in aliam $F$, cuius indeterminatae sunt $x^{\prime}, y^{\prime}$ per substitutiones tales $x=x^{4}+$ $6 y^{\prime}, x=\gamma x^{\prime}+\partial y^{\prime}$ transmutari potest, ita vt $*, 6, \gamma, \delta$ sint integri: priorem implicare posteriorem, siue posteriorem sub priori contentam esse dicemus. Sit forma $F$ haec $a x x+2 b x y+$ cyy, forma $F^{\prime}$ vero haec $a^{\prime} x^{\prime} x^{\prime}+b^{\prime} x^{\prime} y^{\prime}+c^{\prime} y^{\prime} y^{\prime}$, habebunturque sequentes tres aequationes:

$$
\begin{aligned}
& a^{\prime}=a_{a b}+2 b \times y+c \gamma \\
& b^{\prime}=a_{x b}+b(a b+b y)+c x b \\
& b^{\prime}=a b b+2 b 6 \delta+c 8 c
\end{aligned}
$$

Multiplicando aequationem secundam per se ipsam, primam per tertiam, et subtrahendo fit deletis partibus se destruentibus $b^{\prime} b^{\prime}-a^{\prime} c^{d}$ $=(b b-a c)(a d-b y)^{2}$. Vnde sequitur determinantem formae $F^{\prime}$ per determinantem formae $F$ diuisibilem et quotientem esse quadra tum; manifesto igitur hi determinantes eadem signa habebunt. Quodsi itaque insuper forma $F^{\prime}$ per similem substitutionem in formam $F$ transmutari potest, i. e. si tum $F^{\prime}$ sub $F$, tum $F$ sub $F$ contenta est, formarum determinantes erunt aequales ${ }^{*}$ ) atque $(\alpha \delta-6 y)^{2}$ $=1$. In hoc casu formas arquizalentes dicemus. Quare ad formarnm aequiualentiam aequalitas determinantium est conditio nécessaria, licet illa ex hac sola minime sequatur. -

4) Manifestun est ex analysi praecedente hanc propositionem etiana ad formas qquarum determinans $=0$, patere. Sed aequatio $(\alpha)$ $6 y=1$ ad hunc casum nop est extendenda 
Substitutionem $x=\mu x^{\prime}+6 y^{\prime}, y=\gamma x^{\prime}+2 y^{\prime}$, yo. cabimus transformationem propriam, si $\delta^{j}-6 y$ est numerus positiuus, impropriam, si ad - er est negatiuus; formam $F^{\prime}$ proprie aut improprie sub forma $F$ contentam esse dicemus, si $F$ per transformationem propriam aut impropriam in formam $F^{\prime}$ transmutari potest. $\mathrm{Si}^{\prime}$ itaque formae $F, F^{\prime}$ sunt aequiualentes, erit $(\alpha \delta-6 y)^{2}$ $=I_{2}$, adeoque si transformatio est propria, a :y $=+1$, si est impropria, $=-1$. - Si plures transformationes simul sunt propriae, aut simul impropriae, siniles eas dicemus; pro. priam contra et impropriam dissimiles.

158. Si formarum $F, F^{\prime}$ determinontes sisnt aequales atque $F$ 'sub $F$ contenta: etiam $F$ sub $F^{\prime}$ contenta eyit et quidem proprie vel improprie prout $F^{\prime}$ sub $F$ proprie vel improparie continetur. Transeat $F$ in $F^{\prime}$ ponendo $x=\alpha x^{\prime}+6 y^{\prime}, y=x x^{\prime}+d y^{\prime}$ transibitque $F^{\prime}$ in $F$ ponendo $x^{\prime}=8 x-6 y, y^{\prime}=$ $-\gamma x+y$. Patet enim per hanc substitutio. nem ex $F$ fieri idem, quod flat ex $F$ ponendo $x=\alpha(\delta x-6 y)+6(-\gamma x+\alpha y) ; y=\gamma(\delta x-6 y)$ $+\delta(-\gamma x+\alpha y)$ siue $x=(\alpha \delta-6 y) x, y=$ $(\alpha \delta-6 y) y$. Hinc vero manifesto ex $F$ fit $\left(x 0^{\circ}-6 y\right)^{2} F$ i. e. rursus $F$ (art. praec.). Perspicuum autem est, transformationem posterio. rem esse propriam vel impropriam, prout prior sit propria vel impropria.

Si tum $F^{\prime}$ sub $F$, tum $F$ sub $F^{\prime}$ propric continetur, formas propuie aequinalentes, si illae sub inuicem improprie, vocabimus improprie ae 
quiuatentes. - Ceterum vsus harum distinctio. num mox innotescet.

Exmpl. Forma $2 x x-8 x y+3 y y$ per substitutiones $x=2 x^{\prime}+y^{\prime}, y=5 x+2 y^{\prime}$ transit in formam - $15 x x-12 x y-2 y y$, haec vero in illam factis $x^{\prime}=2 x-y, y^{\prime}=$ $-3 x+2 y$. Quare formae $(2,-4,3),(-$ $13,-6,-2)$ erunt proprie aequizsalentes.

Problemata quae tractare iam aggrediemur sunt haec: I Propositis duabus formis quibus cunque eundem determinantem habentibus inuestigare vtrum sint a equiualentes necne, vtrum proprie aut improprie aut vtroque modo, nam etiam hoc fieri potest. Quando vero determinantes inaequales habent, annon saltem altera alteram implicet, proprie vel improprie vel vtroque modo. Denique inuenire omnes trans formationes alterius in alteriam, tam proprias quam improprias. II. Proposita forma quacunque, inuenire vtrum numerus datus peream repraesentari possit omnesque repraesentationes assignare. Sed quoniam formae determinantis negatiui hic aliam methodum requirunt quam formae determinantis positiui, primo trademus ea quae vtrisque sunt communia, tum vero formas cuiusuis generis seorsim conside. rabimus.

\$59. Si forma $F$ formin $F^{\prime}$ implicat, haec vero forman $F^{\prime \prime}$, forma $F$ eliam forman F" itmplicabito 


\section{- $17^{3}-$}

Sint indeterminatae formarum $F, F^{\prime}, F^{\prime \prime}$ respectiue $x, y ; x^{\prime}, y^{\prime} ; x^{\prime \prime}, y^{\prime \prime}$ transeatque $F$ in $F^{\prime}$ ponendo $x={ }_{a}^{\prime}+6 y^{\prime}, y=x^{\prime}+\delta y^{\prime} ; F^{\prime}$ in $F^{\prime \prime}$ ponendo $x^{\prime}=a^{\prime} x^{\prime \prime}+{ }^{\prime} y^{\prime \prime},=y^{\prime} x^{\prime \prime}+\delta^{\prime \prime} y^{\prime \prime}$ patetque, $F$ in $F^{\prime \prime}$ transmutatum iri ponendo $x=x\left(x^{\prime} x^{\prime \prime}+b^{\prime} y^{\prime \prime}\right)+b\left(x^{\prime} x^{\prime \prime}+d^{\prime} y^{\prime \prime}\right), y=$ $y\left(\alpha^{\prime} x^{\prime \prime}+b^{\prime} y^{\prime \prime}\right)+b\left(\gamma^{\prime} x^{\prime \prime}+d^{\prime \prime} y^{\prime \prime}\right)$, siue $x=\left(\alpha \alpha^{\prime}+\right.$ $\left.6^{\prime}\right) x^{\prime \prime}+\left(\alpha 0^{\prime \prime}+68\right) y^{\prime \prime}, y=\left(y^{\prime}+\partial y^{\prime}\right) x^{\prime \prime}+$ $\left(y^{\prime \prime}+80^{4}\right) y^{\prime \prime} \cdot$ Quare $F$ ipsam Fr implicabit.

Quia $\left(\alpha x^{\prime}+6 \gamma^{\prime}\right)\left(y \sigma^{\prime}+8 \delta^{\prime}\right)-\left(\alpha 6^{\prime}+6 \delta^{\prime}\right)$ $\left(\gamma \alpha^{\prime}+\delta \gamma^{\prime}\right)=\left(\alpha^{\prime}-\sigma_{y}\right)\left(\alpha^{\prime} \partial^{\prime}-\sigma^{\prime} \gamma^{\prime}\right)$, adeo-

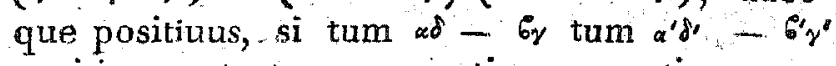
positiuus aut vterque negatiuus, negatiuus vero si alter horum numerorum positiuus alter negatiuus: forma $F$ formam $F^{\prime \prime}$ proprie implicabit, si $F$ ipsam $F^{\prime}$ et $F^{\prime \prime}$ ipsam $F^{\prime \prime}$ eodem modo implicant, improprie si diuerso.

Hinc sequitur, si quotcunque formae habeantur $F, F^{\prime}, F$, F" etc., quarum quaeuis sequentem implicet, primam implicaturam es. se vlimam, et quidem proprie, si multitudo formarum, quae sequentem suam improprie implicant, fuerit par, improprie si multitudo haec impar.

Si forma $F$ formae $F^{\prime}$ est aequinalens, formaque

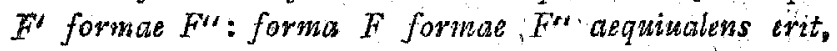
et quidem proprie, si forma $F$ formate F' eodem modo aequitualet vt forma $F^{\prime}$ forma $F^{\prime \prime}$, improprie, si diuerso.

Quia enim formae $F, F^{\prime}$, his $F^{\prime}, F^{\prime \prime}$, respectiue, sunt aequiualentes, tum illie has resp. 


\section{$-874-$}

implicabunt, adeoque $F$ ipsam $F^{\prime \prime}$, tum hae illas. Quare $F, F^{\prime \prime}$ aequiualentes ernnt. Ex praec. vero sequitur, $F$ ipsam $F^{\prime \prime}$ proprie vel improprie implicare, prout $F$ ipsi $F^{\prime}$ et $F^{\prime}$ ipsi $F^{\prime \prime}$ eodem modo vel diuerso sint aequiualentes, vt et $F^{\prime \prime}$ ipsam $F$ : quare in priori casu $F, F^{\prime \prime}$ proprie, in posteriori improprie aequiualentes. erunt.

$$
\text { Formate }(a,-b, c),(c, b, a),(c,-b, a)
$$
formae $(a, b, c)$ aequiuatent, et quidem duat priores, improprie; vitima, proprie.

Nam $a x x+2 b x y+c y y$, transit in $a x^{\prime} x^{\prime}$ $-2 b x^{\prime} y^{\prime}+c y^{\prime} y^{\prime}$, ponendo $x=x^{\prime}+o y^{\prime}, y=$ $o x^{\prime}+y^{\prime}$, quae transformatio est impropria propter $1 x-1-00=-1$; in forman $c x^{\prime} x^{\prime}+2 b x^{\prime} y^{\prime}+a y^{\prime} y^{\prime}$ vero per transformationem impropriam $x=0 x^{\prime}+y^{\prime}, y=x^{\prime}+0 . y$; et in formam $c x^{\prime} x^{\prime}-2 b x^{\prime} y^{\prime}+a y^{\prime} y^{\prime}$ per propriam $x=0 . x-y^{\prime}, y=x^{d}+0 . y^{\prime}$ 。

Hinc manifestum est, quamuis formam, formae $(a, b, c)$ aequiualentem, vel ipsi, vel formae $(a,-b, c)$ proprie aequitualere; similim terque, si qua forma formam $(a, b, c)$ implicet aut sub ipsa contineatur, eam vel formam $(a, b, c)$ vel formam $(a,-b, c)$ proprie implicare, aut sub alterutra proprie contineri. Formas $(a, b, c),(a-b, c)$ oppositas rocabimus.

160. Si formae $(a, b, c),\left(a^{\prime}, b^{\prime}, c^{\prime}\right)$ eundem determinantem habent, insuperque est $c=a^{\prime}$ et $\dot{b} \equiv-b^{\prime}$ (mod. $c$ ), siue $b+b^{\prime} \equiv 0$ 


\section{$-\quad 175$}

(mod. c), formas has contiguas dicemus, et quidem, quando determinatione accuratiori opus est, priorem posteriori a parte prima, posteriorem priori a parte vitima contiguam dicemus,

Ita ex gr, forma $(7,5,2)$, formae $(3,4,7)$ - parte vltima contigua, forma $(3,1,3)$ oppositae suae $(3,-1,5)$ ab vtraque parte.

Formae contiguae semper sunt proprie dequiuslentes. Nam forma $a x x+2 b x y+c y y$ transit in formam contiguam $c x^{\prime} x^{\prime}+2 b^{\prime} x^{\prime} y^{\prime}+c^{\prime} y^{\prime} y^{\prime}$ per substiturionem $x=-y^{\prime}, y=x^{\prime}+\frac{b+b^{\prime}}{b} y^{\prime}$ (quae est propria ob $o \times \frac{b+b}{b}-I \times-I=1$ ), vti per euolutionem adiumento aequationis $b b$ - $a c=b^{\prime} b^{\prime}-c c^{\prime}$ facile probatur; $\frac{b+b^{b}}{c}$ vero per hyp. est integer. - Ceterum hae difinitiones et conclusiones locum non habent, si $c=a^{\prime}=0$. Hic vero casus occurrere nequit, nisi in formis quarum determinans est numerus quadratus.

Formae $(a, b, c),\left(a^{\prime}, b^{\prime}, c^{\prime}\right)$ proprie aequi. ualentes sunt, si $a=a^{\prime}, b \equiv b^{\prime}(\bmod a)$. For. ma enim $(a, b, c)$ formae $(c,-b, a)$ proprie arequiualet (art praec.), haec vero formae $\left(a^{\prime} b^{\prime}, c^{\prime}\right)$ a parte prima contigua erit.

I6r. Si forma $(a, b, c)$ formam $\left(a^{\prime} b^{d}, c^{\prime}\right)$ im. plicat, quis diuisor communis numerarism $a, b$, cetiam maneros $a^{\prime}, b^{\prime}, c^{\prime}$ metietur, et quimis diuisor commanis numerorum $a, 2 b, c$ ipsos $a^{\prime}, b^{\prime}, c^{\prime}$. 


\section{- $7^{6}-$}

Si enim forma $a x x+2 b x y+c y y$ per sul stitutiones $x=\alpha x^{\prime}+6 y^{\prime}, y=\gamma x^{\prime}+\delta y^{\prime}$ in fo mam. $a^{\prime} x^{\prime} x^{\prime}+2 b^{\prime} x^{\prime} y^{\prime}+c^{\prime} y^{\prime} y^{\prime}$ transit: habebur tur hae aequationes:

$a_{\alpha u}+2 b \alpha \gamma+a \gamma=a^{\gamma}$

$a_{a b}+b\left(\alpha \delta+b^{\prime}\right)+c=b^{\prime}$

$a_{b 0}^{\circ}+2 b_{0 d}^{\circ}+c \delta \delta=c^{\prime}$

vnde propositio statim sequitur (pro parte se cunda propos loco aequationis secundae han adhibendo $\left.2 a * b^{2}+2 b(\alpha \delta+6 y)+2 c \gamma \delta^{\prime}=2 b^{\prime}\right)$.

Hinc sequitur maximum diuisorem communem numerorum $a, b(2 b), c$ simul metiri diuisorem communem maximum numerorum $a^{\prime}, b^{\prime}\left(2 b^{\prime}\right), c^{\prime}$; Quodsi igitur insuper forma $\left(a^{\prime}, b^{\prime}, c^{\prime}\right)$ formam $(a, b, c)$ implicat, $\dot{j}_{0}$ e. formae sunt aequiualentes, diuisor communis maximus numerorum $a$, $b(2 b), c$, diuisori communi maximo numerorum $a^{\prime}, b^{\prime}\left(2 b^{\prime}\right), c^{\prime}$ aequalis erit, quoniam tum ille hunc metiri debet, tum hic illum. Si itaque, in hoc casu, $a, b(2 b), c$ diuisorem communem non habent, i. e. si naximus $=1$, etiam $a^{\prime}, b^{\prime}\left(2 b^{\prime}\right)$, $c^{\prime}$ diuisorem communem non habebunt.

162. Promlema. Si forma $A X X+2 B X V^{\circ}$ $+C r x . . F$, formom axx $+2 b x y+c y y . \ldots f$, inplicat, atque transformatio aliqua illius, in hanc est. - data: ex hac omnes reliquas transformationes ipsi similes deducere.

Solutio. Sit transformatio data haec $X=$ $x+b_{y} \cdot x=\gamma x+\delta y_{1}$ ponamusque primo 


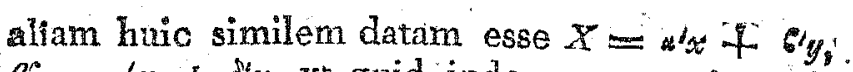
$x^{\circ}=\gamma^{\prime} x+d^{y} y$, vt quid inde sequatur inuestigemus. Tum positis determinantibus formaIum $F, f,=D, d$, atque $2 d-6 y=e, \infty 18$ - $6^{\prime} \gamma^{\prime}=e^{\prime}$, erit (art. 157 ), $d=D e=D e^{\prime} \varepsilon^{\prime}$ et quum ex hyp. $e$, eadem signa habeant, $e=e^{\prime}$, Habebuntur autem sequentes sex ae quationes:

$A_{\alpha \alpha}+2 B \alpha \gamma+C_{\gamma \gamma}=a . \ldots \ldots \ldots$ [i]

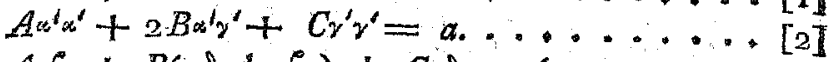
$A \cdots 6+B(a \delta+6 y)+C \gamma \delta=b \ldots[3]$

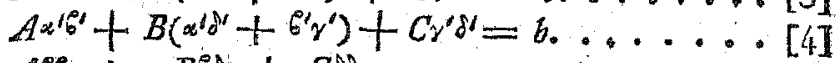
$A B E+2 B D+C B D=c \ldots \ldots[5]$

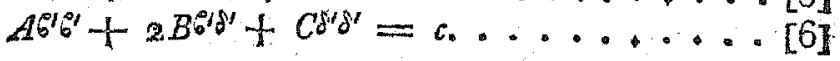

Si breuitatis gratia numeros $A \alpha \alpha^{*}+B\left(\alpha \gamma^{\circ}\right.$ $\left.+y \alpha^{\prime}\right)+C \gamma x^{\prime}, A\left(\alpha b^{\prime}+6 x^{\prime}\right)+B\left(\alpha \delta^{\prime \prime}+6 y^{\prime}+y b^{\prime \prime}\right.$ $\left.+\delta x^{\prime}\right)+C\left(\gamma \delta^{\prime}+\gamma^{\prime}\right), A b^{\prime}+B\left(60^{\prime \prime}+\delta 0^{\prime \prime}\right)+$ $C \$ \delta^{\prime}$ per $a^{r}, 2 b^{\prime}, c^{\prime}$ designamus, ex aequ. praecc.: sequentes nouas deducemus*):

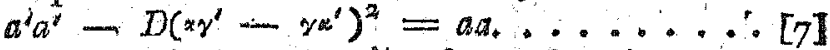
$2 a^{\prime} b^{\prime}-D\left(\alpha \gamma^{\prime}-\gamma \alpha^{\prime}\right)\left(\alpha \delta+6 \gamma^{\prime}-2 b^{\prime}-\delta x^{\prime}\right)=2 a b[8]$ $4 b^{\prime} b^{\prime}-D\left(\left(a d^{\prime \prime}+6 y^{\prime}-y^{\prime}-8 a^{\prime}\right)^{2}+24 c^{\prime}\right)=2 b b+2 a c_{3}$ vnde fit, addendo $2 D e e^{\circ}=2 d=2 b b-2 a c$, $4 b^{\prime} b^{\prime}-\left(x \delta^{\prime \prime}+6 \gamma^{\prime}-\gamma b^{\prime}-\delta a^{\prime}\right)^{2}=4 b 6 \ldots[9]$ $a^{\prime} c^{y}-D\left(\alpha d^{\prime}-y^{\prime}\right)\left(6 y^{\prime}-d \alpha^{2}\right)=66$,

*) Origo harum aequationum haec est: $7^{\prime \prime}$ fit ex I. 2 (i e. si aequa tio (1) in aequationem (2) multiplicatur, siue potius, si, illius pass prior in partem priorem huius multiplicatur, illiusque pars postesior in posteriorem huins, productaque aequalia ponuntur); 8 ex I. $4+2$. 3 i sequens quae non est numerata ex $1.6+25$ $+3.4-3.4$ sequens non numerata ex 3. 4; II ex 3. 6 4 4. 5;, 32 ex 5, 6. Simili designatione etian in sequentibus * emper ptemur. Euolutionem vero lectoribus relinquere debemass 


\section{- ${ }_{1} 7^{8}-$}

vnde subtrahendo $D(\alpha d-b y)\left(\alpha^{\prime} y^{\prime}-b^{\prime} y^{\prime}\right)=b b-a$ fit

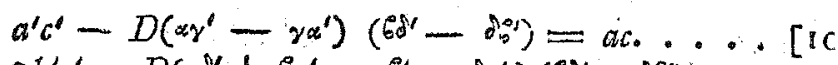
$2 b^{\prime} c^{\prime}-D\left(\alpha d^{\prime \prime}+6 \gamma^{\prime}-\gamma^{\prime} b^{\prime}-\delta a^{\prime}\right)\left(6 \partial^{\prime}-\delta \sigma^{\prime}\right)=2 b c[11$

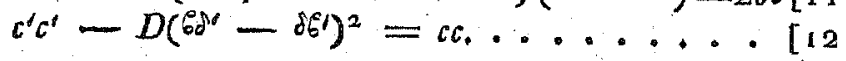

Ponamus iam, diuisorem communem ma ximum numerornm $a, 2 b, c$ esse $m$ numerosqu $\mathfrak{A}, \mathfrak{B}, \mathfrak{C}$ ita determinatos vt fiat $\mathfrak{2} a+2 \mathfrak{B} b+\mathfrak{C}_{c}=$. (art. 40); multiplicentur aequationes $7,8,9$

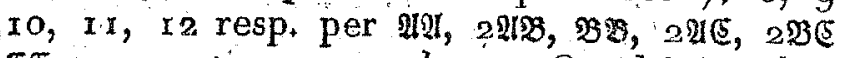
EE summenturque producta. Quodsi iam bre uitatis caussa ponimus

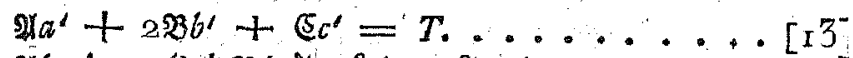
$\mathscr{I}\left(\alpha \gamma^{\prime}-\gamma \omega^{\prime}\right)+\mathscr{B}\left(\alpha \delta^{\prime}+6 \gamma^{\prime}-\gamma b^{\prime}-\delta \alpha^{\prime}\right)+\mathfrak{C}\left(\delta\left(\delta \delta^{\prime}-\delta b^{\prime}\right)=\bar{L}\right.$

$\ldots \ldots \ldots \ldots \ldots \ldots \ldots$ [14] vbi $T, U$ manifesto erunt integri, prodibit: $T T-D U U=m m$.

Deducti itaque sumus ad hanc conclusionem elegantem, ex binis quibuscunque transformationibus si. milibus formae $F$ in $f$ sequi solutionems aequationis indeterminatae ${ }_{t}-D u n=m m$, in integris, scilicet $t=T, w=U$. Ceterum quum in ratiociniis nostris non supposuerimus, transformationes esse dinersas: vna adeo transformatio bis considerata solutionem praebere debet. Tum vero fit propter $\alpha^{\prime}={ }^{\prime}, b^{\prime}=b$ etc. $a^{\prime}=a, b^{\prime}=b$, $c^{\prime}=c$, adeoque $T=m, U=0$, quae solutio per se est obuia.

Iam primam transformationem solutionemque aequationis indeterminatae tamquam cognitas consideremus, et quomodo hinc altera transformatio deduci possit, siue quomodo $\alpha^{\prime}, \varepsilon^{i}, \gamma^{\prime}, \mathrm{J}^{\prime}$, 


\section{$-179$}

ab his $\alpha, b, \gamma, \delta, T, U$ pendeant, inuestigemus. Ad hune finem multiplicamus primo aequationem [1] per $\delta x^{\prime}-6 y^{\prime},[2]$ per $a \delta^{\prime}-y^{6}$, [3] per $\alpha \gamma^{\prime}-\gamma^{\prime},[4]$ per $\gamma \alpha^{\prime}-\alpha \gamma^{\prime}$, addimusque producta, vnde prodibit:

$\left(\varepsilon+e^{\prime}\right) a^{\prime}=\left(\alpha \delta^{\prime}-\because y^{\prime}+\delta x^{\prime}\right) a \ldots[15]$ Simili modo fit ex $\left(d 6^{\prime}-6 d^{\prime}\right)([x]-[2])+$ $\left(\alpha \delta y-6 y^{\prime}-26^{\prime}+\delta x^{\prime}\right)([3]+[4])+\left(\alpha y^{\prime}-\gamma \alpha^{\prime}\right)$ $([5]-[6]):$

$2\left(e+e^{\prime}\right) b^{\prime}=2\left(\alpha d^{\prime \prime}-62^{\circ}-2 b^{\prime}+\delta_{\alpha^{\prime}}\right) b . .[\mathrm{I} 6]$ Denique ex $\left(\mathrm{dg}^{\mathrm{C}}-\mathrm{Ed}^{\prime}\right)([3]-[4])+$ $\left(\alpha \delta^{\prime}-26^{\prime}\right)[5]+\left(\delta \alpha^{\prime}-6 \gamma^{\prime}\right)[6]$ prodit: $\left(e+e^{\prime}\right) c^{\prime}=\left(\alpha \delta^{\prime}-6 \gamma^{\prime}-2 b^{\prime}+\delta \alpha^{\prime}\right) c_{0} \cdot[17]$ fit:

Substituendo hos valores $(15,16,17)$ in 13

$\left(e+e^{\prime}\right) T=\left(\alpha \delta^{\prime}-6 \gamma^{\prime}-2 a^{\prime}+\delta \alpha^{\prime}\right)(2 a+23 b b$

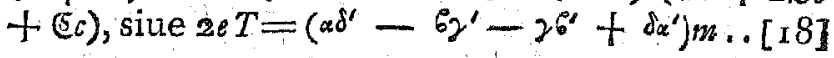
vnde $T$ multo facilius deduci potest, quam ex [13]. - Combinando hanc aequationem cum $15,16,17$ obtinetur $m a^{\prime}=T a, 2 m b^{\prime}=$ $2 T b ; m c^{\prime}=T_{c}$. Quos valores ipsorum $a^{\prime}, 2 b^{\prime}, c^{\prime}$ in aequ. 7 - 2 substituendo et loco ipsius $T T$ scribendo $m m+D U U$, transeunt illae post mutationes debitas in has:

$\left(\alpha \partial^{\prime}-2 \alpha^{\prime}\right)^{2} m=a a U U$

$\left(a \gamma^{\prime}-2 \alpha^{\prime}\right)\left(\alpha d^{\prime \prime}+6 \gamma^{\prime}-26^{\prime}-8 \alpha^{\prime}\right) m m=2 a b U U$ $\left(a \delta^{\prime}+6 \gamma^{\prime}-26^{\prime}-\delta x^{\prime}\right)^{2} m m=46 b U \hat{U} U$ $\left(a \gamma^{\prime}-\gamma \alpha^{\prime}\right)\left(6 \delta^{\prime}-\delta 6\right) m m=a c U V^{\circ}$

$\left(\alpha^{\prime}+6 y^{\prime}-j b^{\prime}-\delta x^{\prime}\right)\left(\delta \delta^{\prime}-\delta^{\prime \prime}\right) m m=2 b c U U$ $\left(6 \delta^{\prime \prime}-\delta 6^{\prime}\right)^{2} m m=c c U U$

$\mathrm{M} 2$ 


\section{$-180-$}

Hinc adiumento aequationis [ $\mathrm{I} 4 \mathrm{f}]$ et huius $202 a b+\mathfrak{C}_{c}=m$, facile deducitur (mulaiplicando primam, secundam, quartans; secun. dam, tertiam, quintam; quarkam, quintam, sextam, resp. per $\mathfrak{U}, \mathfrak{B}$, (E addendoque producta): $\left(\omega^{\prime}-2 \alpha^{\prime}\right) U w m=m a U U,\left(\alpha d^{\prime \prime}+\epsilon_{2}^{\prime}-26^{\prime}\right.$ $\left.-\delta a^{\prime}\right) U m m=2 m b U U,\left(6 \delta^{\prime}-\delta 6^{\circ}\right) U m m=m c U U^{\dagger}$ atque hinc, dinidendo per $\left.m U^{*}\right), a U=\left(\alpha \gamma^{\prime}-\right.$ $\left.2 \alpha^{\prime}\right) m_{*} \ldots[\mathrm{rg}] ; 26 U=\left(u \delta^{\prime}+6 \gamma^{\prime}-26^{\prime} \ldots \delta x^{\prime}\right) m$ ..$[20] ; c U=\left(b^{\prime}-\partial 6^{\prime}\right) m_{*} . .[21]$, ex quarum aequationum alliqua $U$ multo facilius quam ex [44] deduci potest. - Simul hinc colligitur,

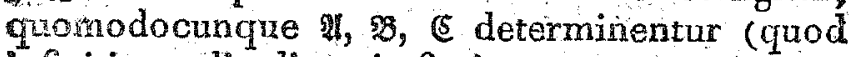
inf hinitis modis diuersis fieri potest ), tum $T$ tum it eundem valorem adipisci.

Iam sî aequatio 18 multiplicatur per $\alpha$ a 9 per 26, 20 per - 4 , fit per additionem $2 a t$ $2(6 a-\alpha b) U=2\left(a \delta-b_{2}\right) \alpha^{\prime} m=2 e^{\prime} m$.

Simili modo fit ex $6[18]+6[20]-2 \alpha[21]$, $2 b c T+2(b b-\alpha c) U=2\left(\alpha \delta^{j}-b_{2}\right) b^{\prime} m=2 e^{6} m_{0}$

Porro ex $2[18]+2 \delta[19]-2[20]$ fit

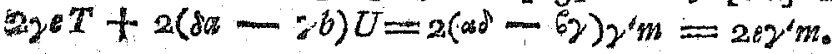

Tandem ex $d[18]+\delta[20]-22[21]$ prodit $28 T+2(86-2 c) U=2(20)-6 \gamma) \delta^{\prime} m=$ aeding

1) Hoo non liceret, si esset $y=0$ : tunc vero aequationum 'I 9 .' 20, 2 I veritas statim ex priraa, tertia et sexta praecedentium ssqueretur. 


\section{$-8 \pi$}

In quibus formulis, si pro $a_{3} b_{j} \bar{c}$ valores ex $I, 3,5$ substituuntur, fit

$g^{\prime} m=a T-(\alpha B+2 C) U$
$\sigma^{\prime}=6 T-(6 B+\delta C) U$
$\gamma^{\prime} n=2 T+(a A+2 B) U$
$s_{m}=\delta T+(E A+\delta B) U$

Ex analysi praec, sequitur, nullam transe formationem formae $F$ in $f$ propositae similem dari, quae non sit contenta sub formula $X=$ $\frac{\pi}{m}(\alpha t-(\alpha B+2 C) u) x+\frac{x}{m}(6 t-(6 B+\delta C) u) y ;$ $I=\frac{x}{m}(2 t+(\alpha A+2 B) u) x+\frac{x}{m}(\delta t+(6 A+\delta B)$ u) $x)$... (I), designantibus $t, u$ indefinite omnes numeros integros aequationi $t i-D w n=m$ satisfacientes. Hinc vero concludere nondum possumus, omnes valores ipsorum $t, u$, aequa: tioni ill satisfasientes, in formula (I) substitus tos, transformationes idoneas praebere. At

x. Formam $F$ per substitutionem, e quia busuis ipsorum $t$, valoribus ortam, semper in formam $f$ transmutari, per euolutionem confrmari facile potest adiumento aequationem $r$, 3,5 et huius $t t-D u=m m$. Calculum prolixiorem quam difficiliorem breuitatis gratia sup: primimus.

2. Quaeuis transformatio ex formula de ducta propositae erit similis. Namque $\frac{\underline{I}(a t-}{m}$ ( $(a B+2 C) u) \times \frac{I}{m}(\delta t+(6 A+\delta B) w)-I_{m}(6 t-$ $(G B+\delta C) u) \times \frac{I}{m}(\gamma t+(\alpha A+\gamma B) u)=\frac{x}{m b i z}(\alpha \delta$ $\left.G_{y}\right)(t t-D w w)=d-6 y$. 


\section{$-182-$}

3. Si formae $F, f$ determinantes inaequales habeut, fieri potest, vt formula (I) pro quibusdam valoribus ipsorum $t$, th praebeat substitutiones, quae fractionss implicent, adeoque reiici debeant, Omnes vero reliquae erunt transformationes idoneae, aliaeque praeter ipsas non dabuntur.

4. Si vero formae $F, f$ eundem determinantem habent adeoque sunt aequiualentes, for mula (I) nullas transformationes quae fractiones implicent praebebit, adeoque in hoc casu solutionem completam problematis exhibebit. Illud vero ita demonstramus.

Ex theoremate art. praec. sequitur in hocce casit, $m$ simul fore diaisorem communem numerorum $A, 2 B, C$. Quoniam $t t-D i u=$ $m m$, fit $t t-B$ Bun $=m m-A C u n$, quare' $t t$ - $B$ Bu per min diuisibilis erit: hinc etiam a potiori $4 t t-4 B B u n$ adeoque (quia $2 B$ per $m$ diuisibilis) etiam 4 tt per $m m$ et proin $2 t$ per $m$. Hinc $\frac{2}{m}(t+B u), \frac{2}{m}(t-B u)$ erunt integri, et quidem, quoniam differentia inter ipsos, $\frac{4}{m} B$ est par), aut vterque par, aut vterque impar. Si vterque impar esset, etiam productum impar foret, quod tamquam quadruplum numeri $\frac{I}{m m}(t t-B B u u), \quad$ quem integrum esse modo ostendimus, necessario par: quare hic casus est impossibilis, adeoque $\frac{2}{m}(t+B u), \frac{2}{m}(t-B u)$ semper pares, vnde $\frac{I}{m}(t+B u), \underset{m}{m}(t-B u) e-$ runt integri. Hinc vero nullo negotio deducitur, omnes quatuor coefficientes in (I) semper esse integros. Q, E. D. 


\section{$-183=$}

Ex praecedentibus colligitur, si omnes solutiones aequationis $t t-D w z=m m$ habeantur, omnes transformationes formae $(A B, C)$ in $(a, b, c)$ transf. datae similes inde deriuari. Illas ve. ro in sequentibus inuenire docebimus. Hic tantummodo obseruamus multitudinem solutionum semper esse finitam quando $D$ sit negatiuus, aut positiuus simulque quadratus: quando vero $D$ positiuus non quadratus, infinitam. Quando hic casus locum habet, simulque $D$ non $=a$ (supra $3^{\circ}$ ), disquiri inisuper deberet, quomodo ii valores ipsorum $t$, $t$, qui substitutiones a fractionibus liberas, ab iis, qui fractas producunt, a priori dignosci possint. Sed pro hocce casu infra aliam methodum ab hoc incommodo liberam exponemus (art. 214),

Ex. Forma $x x+2 y y$ per substitutionem propriam $x=2 x^{\prime}+7 y^{\prime}, y=x^{\prime}+5 y^{\prime}$ transit in formam $(6,24,99)$ : desiderantur amnes transformationes propriae formae illius in hanc. Hic $D=-2, m=3$, adeoque aequatio soluenda baec: $t t+2 i s t=9$. Huic sex modis diuersis satisfit ponendo scilicet $t=3,-3$, I, - I, I, $-1 ; u=0,0,2,2,-2$ - 2, resp. Solutio tertia et sexta dant sub. stitutiones in fractis, adeoque sunt reiiciendae: ex reliquis sequuntur quatuor substitutiones:

$x=\left\{\begin{array}{c}2 x^{\prime}+7 y^{\prime} \\ -2 x^{\prime}+7 y^{\prime} \\ 2 x+9 y^{\prime} \\ -2 x^{\prime}+9 y^{\prime}\end{array}=\left\{\begin{array}{r}x^{\prime}+5 y^{2} \\ x^{\prime}+5 y^{\prime} \\ x^{\prime}+3 y^{\prime} \\ x^{\prime}+3 y^{2}\end{array}\right.\right.$

(quarum prima est proposita).

M 4 


\section{- ISír}

I65. Iam supra obiter diximus feri posse vt forma aliqua, $F$, aliam, $F$ ', tam proprie quarn improprie implicet. Perspicuum est hoc euenire, si inter formas $F$, $F$ alia $G$ interponi possit, ita vt $F$ ipsam $G, G$ ipsarn $F^{\prime}$ implicet, formaque $G$ ita sit comparata, vt sibi ipsa sit improprie aequiualens, $\quad \mathrm{Si}$ enim $F$ ipsam $\vec{G}$ proprie vel improprie implicare supponitur: quum $G$ ipsam $G$ improprie implicet, $F$ ipsam $G$ improprie vel proprie (resp.) implicabit, adeoque, in vtroque casu, tam proprie quam improprie. (art. 159). Eodem modo hinc deducitur, quomodocunque $G$ ipsam $F^{\prime}$ implicare supponatur, $F$-semper ipsam $F^{\prime}$ tum proprie tum improprie implicare debere. - Ta. les vero formas dari, quae sibi ipsae sint improprie aequiualentes, videtux in casu maxime obuio, vbi formae terminus medius $=0$. Talis enim forma sibi ipsa erit opposita (art. 1.59) adeoque improprie aequinalens, Generalius quaeuis forma, $(a, b, c)$, hac proprietate est praedita, in qua $2 b$ per a est dinisibilis. Huic enim forma $(c, b, a)$ a parte prima erit conti. gua (art. 160 ) adeoque proprie aequiualens: sed $(c, b, a)$ per art ${ }^{1} 59$ formae $\left(a, b, b^{a}\right)$ improprie aequiualet: quare $(a, 1 b, c)$ sibi ipsa improprie aequiualebit. Tales formas $(a, b, c)$ in quibus $2 b$ per $a$ est diuisibilis, ancipites yocabimus. Habebimus itaque theorema hoc:

Forma $F$, aliam formam $F^{\prime}$ tum proprie tum imo proprie inplicabit, si forma anceps inuenire potest sub $F$ contenta ipsam $F$ vero implisans. Sed haec pro: positio etiam conuerti potest: scilicet 


\section{$-185=$}

164. Theorema. Si forma $A x x+2 B x y+$ Cyy...(F) formam $A^{\prime} x^{\prime} x^{\prime}+2 B^{\prime} x^{\prime} y^{\prime}+C x^{\prime} y^{\prime} \ldots$ (F) tun propsie tum impropris implicat: forma anceps inuensi potest, sub F contemis formanguge $F$ implicans.

Ponamus, formam $F$ transire in formam $F^{\prime}$ tum per substitionem $x=\alpha x^{\prime}+\varepsilon_{y^{\prime}}, y=$ $2 x^{\prime}+\delta_{y^{\prime}}$, tum per hanc illi dissimilem, $x=$ $x^{\prime} x^{\prime}+{ }^{6} x^{\prime}, y=\gamma^{\prime} x^{\prime}+\delta^{\prime} y^{\prime}$. Tum designatis numeris ad- $6 y$, e $d^{\prime}-b^{\prime} y^{\prime}$ per $2, \varepsilon^{\prime}$, erit $B^{\prime} B^{\prime}-$ $A^{\prime} C^{\prime}=\operatorname{ee}(B B-A C)=e^{d} \varepsilon^{\prime}(B B-A C)$; hinc $\varepsilon s=e^{t} e^{\prime}$, et, quia per hyp. $e, e^{t}$ signa opposita habent, $e=-e^{\prime}$ siue $a+e^{\prime}=0$. Iam patet si in $F^{\prime}$ pro $x^{\prime}$ substituatur $d^{\prime \prime} x^{\prime \prime}-6^{\prime} y^{\prime \prime}$, et pro $y^{\prime},-\gamma^{\prime} x^{\prime \prime}+\alpha^{\prime} y^{\prime \prime}$, eandem formam esse prodituram ac si in $E$ scribatur ant $x$ pro $\left.x,{ }^{\prime \prime} x^{\prime \prime}-6^{\prime} y^{\prime \prime}\right)+6\left(-y^{\prime \prime}+x^{\prime \prime} y^{\prime \prime}\right) i$ e. $\left(\alpha d^{2}-6 y^{\prime}\right)+\left(6 x^{\prime}-46^{\prime}\right) y^{\prime \prime}$, et pro $y, 2\left(\delta^{\prime} x^{\prime \prime}\right.$ $\left.\therefore b^{\prime} y^{\prime \prime}\right)+d\left(-y^{\prime} x^{\prime \prime}+x^{\prime \prime} y^{\prime \prime}\right)$ e. $\left(y^{\prime \prime}-\delta y^{\prime}\right) x^{\prime \prime}$ $+\left(d x^{\prime}-26^{\prime}\right) y^{\prime \prime} ;$ aut 2$)$ pro $x$, a $^{\prime}\left(8^{\prime \prime} x^{\prime \prime}-\right.$ $\left.e^{\prime} y^{\prime \prime}\right)+b^{\prime \prime}\left(-\gamma^{\prime} x^{\prime \prime}+\alpha^{\prime} y^{\prime \prime}\right)$ i. e. $e^{\prime} x^{\prime \prime}$, et pro $y_{0}$ $y^{\prime}\left(d^{\prime} x^{\prime \prime}-6^{\prime} y^{\prime \prime}\right)+y^{\prime \prime}\left(-z^{\prime \prime} x^{\prime \prime}+a^{\prime} y^{\prime \prime}\right)$ i. e. $e^{\prime} y^{\prime \prime}$ Designatis itaque numeris $a \delta^{\prime}-.62,6 \alpha^{\prime}-4 b^{\circ}$ $y \partial^{\prime}-2 y, 2 a-2 b^{b}$ per $a, b, c, d$ : forma $F$ per duas substitutiones $x=a x^{\prime \prime}+b y^{\prime \prime}, y=$ $c x^{\prime \prime}+d y^{\prime \prime} ; x=e^{\prime \prime} x^{\prime \prime}, y=e^{\prime} y^{\prime \prime}$ in eandem formam transmutabitur, vade obtinemus tres aequationes sequentes:

$A a a+2 B a c+C_{c c}=A e^{\prime} e^{\prime} \ldots \ldots . . . .[r]$ $A a b+B(a d+b c)+C c d=B e^{\prime} e^{\prime} \ldots \ldots[2]$ $A b b+2 B b d+C d d=C e^{\prime} e^{\prime} \ldots \ldots \ldots[3]$ Ex valoribus ipsorum $a, b, c$, d autem inuenitur $a d-b u=b^{\prime}=-e t=-e^{\prime} e^{\prime} \ldots \ldots \ldots$ [4] 


\section{$\therefore \quad \mathbf{1} 86$}

Hinc $f t$ ex $d[1]-c[2],(A a+B c)\left(a a^{*}-\right.$ $b c)=(A d-B c) e^{\prime} e^{\prime}$, adeoque $A(a+d)=0$. Porro ex $(a+d)[2]-b[d]-c[3]$ fit $(A b+$ $B(a+d)+C b)(a d+b c)=(-A b+B(a+d)$ - $C c) e^{\prime} e^{d}$, adeoque $B(a+d)=0$. Denique ex $a[3]-b[2]$ fit $(B b+C d)(a d-b c)=(-B b$ $+C(b) \varepsilon^{\prime} \varepsilon^{\prime}$ adeoque $C(a+d)=0$. Quare quum ommes $A, B, C$ nequeant esse $=0$, necessario erit $a+d=0$ siue $a=-d$.

\section{$\operatorname{Ex} a[2]-b[\mathrm{I}]$ fit $(B a+C c)(a d-b c)$ $=(B a-A b) e^{\prime} \varepsilon^{\prime}$, vade $A b-2 B a-C c=0[5]$}

Ex aequationibus $e+e^{\prime}=0, a+d=0$ siue $a b-6 z+a^{\prime} 8^{\prime}-b^{\prime} y^{\prime}=0, a \delta^{\prime}-6 y^{\prime}=2^{6}+$ $d x^{\prime}=0$ sequitur $\left(\alpha+\alpha^{\prime}\right)(\delta+\delta)=(6+$ $6)(2+\gamma)$ siue $(a+\alpha):(2+2)=(64$ $6):(\delta+\delta)$. Sit rationi huic $\left.{ }^{*}\right)$ in numeris minimis aequalis ratio $m: n$, ita vt $m, n$ inter se primi sint, accipianturque $\mu, \nu$ ita vt fiat $\mu \mathrm{m}$ $+m=$ r. Porro sit $r$ diu. comm. max. numerorum $a, b, a$; cuius quadratum propterea metietur ipsum $a c+b c$ siue $b c-a d$ sine $e$; quare $r$ etiam ipsum $e$ metietur. His ita factis, si forma $F$ per substitutionem $=n n t+\frac{1}{r}$ $u, y=n t-\frac{\mu_{t}}{r} u$ in formam $M t t+2 N t u+P_{u} u$ $(G)$ transire supponitur, haec anceps erit formamque $F^{\prime}$ implicabit.

-) Si ombes $\alpha+\alpha, y+\gamma^{\prime}, 6+6^{\prime}, 8, \alpha^{\prime}$ essent $=0$, ratio indeterminata foret, adeoque methodus non applicabilis. Sed exigua attentio docet, hoc cum suppositionibus nostris consistere non posse. Foret enim $4 \delta-6 y=x^{\prime} \delta^{\prime}-b^{\prime} y^{\prime} i$ e. $=e^{\prime}$ adeoque, quia $e=-\varepsilon^{\prime},=0$. Hinc vero etiam $B^{\prime} B^{\prime}$. $A^{\prime} C^{\prime}$ i. $\theta$. determinans formae $\xi^{\prime}$ fieret $=0$, quales formas omnino exclusimus: 


\section{$-{ }_{187}-$}

Dem. I. Quo pateat, formam $G$ esse ancipitem, ostendemus esse $M(b \mu \mu-20 \mu r-c r)=2 N_{r}$ vnde quiaripsos $a, b, c$ metitur, $\frac{x}{b}\left(b \mu \mu-2 a \mu \nu-c_{w \nu}\right)$ integer erit, adeoque $2 N$ multiplum ipsius $M$. Erit autem $M=A m n+2 B m+C_{n n} N r=$ $\left(A m_{v}-B\left(m \mu-m_{v}\right)-C m \mu\right) e$. Porro per euo. Iutionem facile confirmatur esse $2 e+2 a=:$ $-e^{\prime}+a-a=\left(a-a^{\prime}\right)(\delta+\delta)-(b-$ b) $\left(2+z^{\prime}\right), 2 b=\left(a+a^{\prime}\right)\left(b-b^{\prime}\right)-(a-$ $\left.\alpha^{\prime}\right)\left(6+6^{\prime}\right)$ Hinc quoniam $m\left(\nu+\gamma^{\prime}\right)=n(a$ $\left.+a^{\prime}\right), m\left(\delta+d^{\prime \prime}\right)=n(6+6)$, erit $m(2 e+2 a)=-2 n b$ siue $m e+m a+n b=0 \ldots[7]$. Eodem modo erit $2 \varepsilon-2 a=e-e^{\prime}-a+d=(a+$ $\left.\alpha^{\prime}\right)\left(\delta-\delta^{\prime}\right)-\left(6+6^{\prime}\right)\left(2-2^{\prime}\right), 2 c=\left(2-2^{\prime}\right)$ $\left(\delta+\delta^{\prime}\right)-(2+2)(\delta-\delta j)$, atque hinc $n(2 e$ $-2 a)=-2 m c$, siue $m e-m a+m c=0 . .[8]$

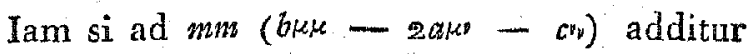
$\left(\mathrm{s}-m \mu-m_{v}\right)(m p(e-a)+(m \mu+1) b)$ $+(m e+m a+n b)(n z \mu v+v)+(n e-m a+m c) m_{w y}$ quod manifesto $=0$, propter $x-\mu m-m=0$, $m e+m a+n b=0, n e-n a+m c=0:$ prodit productis rite euolutis partibusque se destruentibus deletis, $2 m v e+b$. Quare erit $m m\left(b \mu \mu-2 a k v-c_{0}\right)=2 m v e+b \ldots \ldots\left[{ }_{G}\right]$

Eodem modo addendo ad $m n(b \mu \mu-2 a \mu-$ cin) haec:

$\left(\mathrm{I}-m \mu-w^{v}\right)\left((n \mu-m \mu) e-\left(\mathrm{I}+m^{\mu}+n v\right) a\right)$ $-(m \varepsilon+m a+n b) m \mu k+\left(n_{\ell}-n a+m c\right) n n$ inuenitur

$m n(b \mu \mu-2 a \mu v-c w)=\left(n v-m_{\mu}\right) e-a \ldots[10]$

Denique addendo ad $n n(b \mu \mu-2 a \mu v-c v)$ haec: $(m u+z-1)(n u+a)+(n+1) c)$ 


\section{- $388-$}

$-(m e+n a+n b) n \omega t-(n \varepsilon-n a+n c)(n \mu+\mu)$

fit

$m s(b \mu \mu-2 d \mu \nu-c r)=-2 n u e-c \ldots . .[$ [ I ]

Lam ex 9,10, I r, deducitur

$(A m m+2 B m n+C m n)(b k \mu-2 a k v-c v)=$ $2 e(A n v+B(n v-n n u)-C n \mu)+A b-2 B c t$ $-C_{i}$, sine propter [6],

$M(b k \mu-2 a k v-a w)=2 N r \cdot Q \cdot$ E. D.

II. Vt probetur, formam $G$ implicare formam $F^{\prime}$ demonstrabimus, primo $G$ transire in $F^{\prime}$ ponendo $t=(\mu a+g)+w^{\prime}+(\mu b+\delta) y^{\prime}, a=$

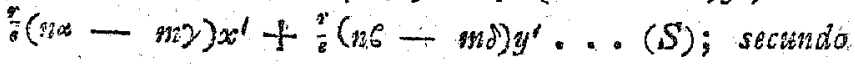
$\%(n \alpha-m) ;\left(n^{b}-m\right)$ esse integros.

I. Quoniam $F$ transir in $G$ ponendo $x$

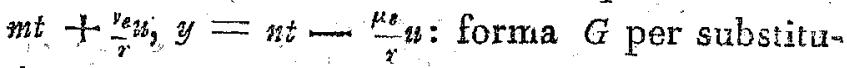
tionem $(S)$ transmutabitur in eandem formann in quami $F$ transformatur ponendo $x=m((\mu a x$ $\left.+n y) x^{\prime}+\left({ }^{\prime} 6+x^{2}\right) y^{\prime}\right)+\left(\left(n \alpha-m_{2}\right) x^{\prime}+(n 6\right.$ - mo) $\left.y^{\prime}\right)$ i. e. $\left.=a\left(m \omega+n_{n}\right) x^{\prime}+\epsilon_{(m i \mu}+m\right) y^{\prime}$ siue $=x^{4}+\epsilon_{y^{\prime}}$; et $y=u\left(\left(\mu \alpha+{ }^{2}\right) x^{\prime}+(\mu t\right.$ $\left.+8) y^{\prime}\right)-\mu\left((n a-m y) x^{\prime}+\left(n^{6}-m \delta\right) y^{\prime}\right) i_{0} e_{0}=$ $2\left(n^{\prime}+m k\right) x^{\prime}+8\left(n n+m y^{\prime} y^{\prime}\right.$ sive $=2 x^{\prime}+\delta y^{\prime}$. Per hanc vero substitutionem $F$ transit in $F^{\prime}$ : quare per substitutionem $(S)$ etiam $G$ transibit in $F^{\prime}$.

2. Ex valoribus îpsorum $e, b, d$ inuenitur $a^{\prime} b_{\text {ne }}+2 b-\alpha d=0$, siue propter $d=-a, n a^{\prime} e$ $+n_{a}+n_{b}=0$; hinc ex $[8], n_{\alpha}{ }^{\prime} e+n \alpha_{a}=$ $m z e+m a$ siue $(n-w z) a=\left(m z-n w^{\prime}\right) e$ [12] 


\section{$189-$}

Porro fit $\alpha m b=-\alpha m(e+a), 2 m b=-$ $m\left(\alpha c_{c}+\alpha a\right)$ adeoque $(n s-m z) b=\left(x^{\prime}-\alpha\right) m e[15]$

Denique fit $\% e-2 a+a c=0$ : hinc multiplicando per n, et pro na substituendo valorem ex [8] fit $\left(n \alpha-m_{2}\right) c=(2-2, n e . . .[I]]$

Simili modo eruitur $b c+b-6 d=0$, site $n 6^{\prime} \varepsilon+n \delta b+n b_{a}=0$, adeoque per $[7] n^{\prime} c+n b_{b}$ $=m \delta e+m \delta a$, sine $(n 6-m \delta) a=\left(m b \delta-n \sigma^{\prime}\right) e[15]$

Porro fit $b_{n b}=-b_{n}(e+a), \delta_{n} b=-$ $m\left(6 e^{\prime}+6 a\right)$ adeoque $(n 6-m s) b=\left(6^{\prime}-6\right) n z[16]$

Tandem $\delta \varepsilon-\delta a+\epsilon_{c}=0$ : hinc multi. plicando per et substituendo pro na valorem ex $[\delta]$ fit $\left(n^{6}-m\right) c=\left(\delta-\delta^{\prime}\right) n e \ldots . .[\mathrm{r} 7]$

Iam quum diuisor communis maximus numerorum $a, b, c$ sit $r$ integri $\$$, $\$$, E ita accipi possunt vt fat $\mathfrak{S a}^{2}+\mathfrak{B} b+\mathfrak{E}_{c}=\% \quad$ Quo facto erit ex $12, " 15,14,15,16,17$

$\mathfrak{L}\left(m \gamma-n^{\prime}\right)+\mathfrak{B}\left(\alpha^{\prime}-\alpha\right) m+\mathfrak{S}\left(\nu-\gamma^{\prime}\right) n=(n \alpha-m)$

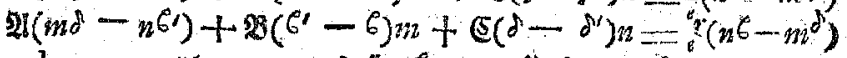
adeoque $(n \alpha-m),(n E-m d)$ integri. Q. E. D.

165. Ex. Forma $3 x x+14 x y-4 y y$ in formam - $2 x^{\prime} x^{\prime}-18 x^{\prime} y^{\prime}+39 y^{\prime} y^{\prime}$ transmutatur, tum proprie, ponendo $x=4 x^{\prime}+$ I I $y^{\prime} ; y=-$ $x^{\prime}-2 y$, tum improprie, ponendo $x=-74 x^{d}$ $+89 y^{\prime}, y=x 5 x^{\prime}-13 y^{\prime}$. Hic igitur $a+\alpha^{\prime}$ $6+6,2+2,8+8$ sunt - 70, 100, 14. 20 ; est autem $-70: 14=100:-20=5$ : - I. Faciemus iraque $m=5, n=-$ I, $\mu$ $0, y=-x$. Numeri autem $a, b, c$ inuenim 


\section{$-190 \quad-$}

tur $-237,-\operatorname{Ir} 70,48$, quorum diuisor com. munis maximus $=3=r$; denique fit $e=3$. Hinc transformatio $(S)$ haec erit: $x=5 t-u$, $y=-t$. Per quam forma $(3,7,-4)$ trans. it in formam ancipitem $t t-16 t u+3 u u$.

Si formae $F, F^{\prime}$ sunt aequiualentes : forma $G$; sub $F^{\prime \prime}$ contenta, etiam sub $F^{\prime}$ contenta erit Sed quoniam eanden formam etiam implicat, ipsi aequiualens erit, et proin etian formae $F$. In hoc igitur casu theorema ita enunciabitur:

Si $F, F^{\prime}$ tam proprie, quan improprie 'sunt atm quiualentes: forma anceps vtrique aequiualens inueniri poterit. - Ceterum in hoc casu $e= \pm 1$, ad. eoque etiam $\%$, ipsum $\varepsilon$ metiens, $=x$ erit.

Haec de formarum transformatione in genere sufficiant: transimus itaque ad considerationem repraesestationum.

166. Si forma $F$ formam $F^{\prime}$ implicat: quicunque numerus per Frepraesentari potest etian per $F$ poterit.

Sint indeterminatae formarum $F, F^{\prime}$ respectiue $x, y ; x^{\prime}, y^{\prime}$, ponamusque numerum $M$ per $F^{\prime}$ rcpraesentari faciendo $x^{\prime}=m, y^{\prime}=n$, formam $F$ vero in $F^{\prime}$ transire per substitutionem $x=a x^{6}$ $+b_{y^{\prime}}, y=2 x^{\prime}+\delta y^{\prime}$. Tum manifestum est si ponatur $x=\alpha n+\epsilon_{n} y=2 n+\delta n, F$ transire in $M$.

Si $M$ pluribus modis per formam $F^{\prime}$ repraesentari potest, e. g. etiam faciendo $x^{\prime}=m^{\prime}$, 
$y^{\prime}=n^{\prime}:$ plures repraesentationes ipsius $M$ per $F$ inde secruentur. Si enim esset tum am + $c_{n}=a n^{\prime}+b_{n^{\prime}}$ tum $2 m+b_{n}=2 m^{\prime}+\delta_{n}^{\prime}$, foret aut ad $-\epsilon_{2}=0$, adeoque etiam determinans formae $F^{\prime}=0$ contra hyp., aut $m=$ $m^{\prime}, n=n^{\prime}$. Hinc sequitur $M$ ad minimum totidem modis diuersis per $F$ repraesentari posse quot per $F^{\prime}$.

Si igitur tum $F$ ipsam $F^{\prime}$,tum $F^{\prime}$ ipsam $F$ impli. cat i. e. si $F, F^{\prime}$ sunt aequiualentes, numerusque $M$ per alterutram repraesentari potest: etiam per alteram repraesentari poterit, et quidem totidem modis diuersis per alteram; quot per alteram.

Denique obseruamus, in hocce casu diuisorem communem maximum numerorum $i n$ aequalem esse diuisori comm. max. nnmerorum $m+{ }^{2} n, 2 m+\delta_{n}$. Sit ille $=\Delta$, nnmerique $\mu$, ita accepti vt fiat $\mu m+n=\Delta$. Tum erit $(\delta n-2 n)(a m+6 n)-(6 k-a n)(2 m+\delta n)=$ $(a \delta-b \gamma)(\mu m+n)= \pm \Delta$. Hinc dit. comm. max, numerorum am $+6 n, 2 m+{ }_{n}$ metietur ipsum $\Delta, \Delta$ vero etiam illum metietur, quia manifesto ipsos $a m+b_{n}, 2 m+{ }_{n} m$ metitur. Quare necessario ille erit $=\Delta_{0}-\quad$ Quando igitur $m_{1} n$ inter se primi sunt, siam $\alpha m+c_{n}, 2 m+$ on inter se primi erunt.

167. Théorema. Si formae axx $+2 b x y+$ cyy ... (F), $a^{\prime} x\left(x^{\prime}+2 b^{\prime} x^{d} y^{\prime}+c^{\prime} y^{\prime} y^{\prime} \ldots .(F)\right.$ sunt aequiualentes, ipsarmon ceterminosus $=D$, posieriorque in prioren transit ponendo $x^{\prime}=\alpha x+6 y, y^{\prime}=2 x+d y$; 


\section{$-192 \quad-$}

porro numcrss $M$ per $F$ repraesentatur, faciendo $x=$ $m_{2} y=n$, adeoque pey $F^{\prime}$ faciendo $x^{\prime}=a m+b_{n}$ $=m^{\prime}, y^{\prime}=2 m+$ on $=n^{*}$ et quidem ita $v n$ ad $n$

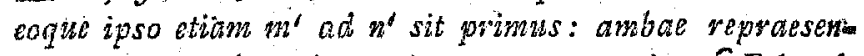
tationes aut ad eundem valorow expressionis $D$ (mod. M) pertinebunt, aut aci oppositos, prout transformatio formele $F^{\prime}$ in $F$ propria est vel impropria.

Dens. Determinentur numeri $\mu$, ita vt fiat

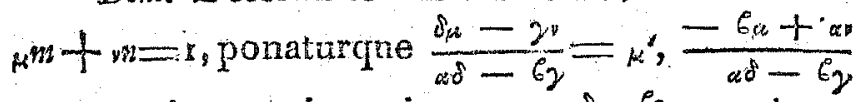
$=$ (qui erunt integri propter $a \delta-C_{2}= \pm \mathbf{m}$ ). Tum erit km $+v^{\prime} n^{\prime}=$ r.(Cf. art praec, frn.). Porro sit $\mu(b m+c n)-v(a n+b n)={ }^{\prime} V_{n} c^{\prime}\left(b^{\prime} m^{\prime}+c^{\prime} n^{\prime}\right)$ $-v^{\prime}\left(a^{\prime} m^{d}+b^{\prime} n^{\prime}\right) \Longrightarrow V^{\prime}$, eruntque $V, V^{\prime}$ valores expr. $\sqrt{ } M_{0}(\bmod , D)$ ad quos repraesentatio prima et secunda pertinent. Si in $V^{\prime}$ pro $\mu, v, m, n$ valores ipsorum substituuntur; in $V$ vero pro $a ; a^{\prime} \alpha a+2 b^{\prime} \times 2+c^{\prime} 2 \gamma$ pro $b, a^{\prime} \alpha b+b^{\prime}(\alpha \delta+6 z)$ $+c 28$; pro $c_{2} a^{\prime 66}+2 b^{\circ}+{ }^{\circ}+c^{480}$ inuenietur euolutione facta $V=V(\alpha \delta-6 y)$. Quare eric aut $V=V^{\prime}$, aut $V=-V^{\prime}$, prout as $-62=$ $\rightarrow$ I aut $=-I$, i. e. repraesentationes pertinebunt ad eundem valoren expr. $\sqrt{ } M(\bmod . D)$ vel ad oppositos, prout transformatio formae $F^{\prime}$ in $F$ est propria vel impropria.Q.E. $D$.

Si itaque plures repraesentationes nuneri $W$ per formam $(c, b, c)$, ope valorum inter se primorum indeterminatarum $x, y$, habentur ad valores diuersos expr. $\sqrt{ } D($ mod. $M)$ pertinentes: repraesentationes respondentes per formam $\left(a^{i}, b^{a}, c^{\prime}\right)$ ad eosder resp, valores pertine. bunt, et si nulla repraesentatio numeri $M$ per 


\section{- 93}

formam aliquam ad valorem quendam deters minatum pertimens datur, nulla quoque dabitur ad hunc valorem pertinens per formam illi aequiualentem.

168. Theonemia. Si mumerzs $M$ per formars $a x x+2 b x y+c y y$ repraesentatur tribuendo ipsis $x, y$ valores inter se primos $m, n$, vulorqua expressionis $\sqrt{D}$ (mod. $M$ ), ad quem haec repraesentatio pertinet, est $N$ : fornate $(a, b, c),\left(M, N, \frac{N N-D}{M}\right)$ proprie oequisialene tes erust.

Demonstr. Ex art. 155 patet, numeros integros $\mu_{\text {, }}$, inuensi posse ita vt sit $m \mu+x_{n}$ $\mu(b m+c n)-v(a m+b n)=N \quad$ Quo facto forma $(a, b, c)$ per substitutionem $x=m x-y y^{\circ}$ $y=n x^{\prime}+\mu y^{\prime}$, quae manifesto est propria transit in formam cuius determinans $=D\left(m_{\mathrm{s}}\right.$ $+w^{2} i_{0}=D$, siue in formam aequiualen tem: quae forma si ponitur $=\left(M, N^{\prime}, \frac{N^{\prime} N-D}{M^{\prime}}\right)$ erit $M^{\prime}=a n m+2 b m n+c n n=M ; N^{\prime}=$ $-m b+(m \mu-n u) b+n \mu c=N$. Quare forma in quam $(a, b, c)$ per transformationem illam mutatur erit $\left(M, N, \frac{N N-D}{M}\right), Q . D_{0}$.

Ceterum ex aequationibus $m+w_{1}=I_{1}$ $\mu(m b+n c)-v(m a+n b)=N$ deducitur $\mu=$ $\frac{n N+m a++n b}{a m m+2 b m n+c n s}=\frac{n N+m a+n b}{M} ; y=\frac{m b+n c-m N}{N}$ qui numeri itaque erunt integri. 
Porro obseruandum, hanc propositionem locum non habere, si $M=0$; tum enim ter minus $\frac{N-D}{M}$ fit indeterminatus ${ }^{*}$ ).

I69. Si plures repraesentationes numeri M, per $(a, b, c)$ habentur, ad eundem valorem expr. $\sqrt{ } D(\bmod . M), N$, pertinentes (vbi valores ipsorum $x, y$ semper inter se primos supponimus): plures etiam transformationes propriae formae $\left(a_{i} b, c\right),(F)$, in $(M, N$. $\left.\frac{N N-D}{M}\right),(G)$ inde deducentur. Scilicet si etiam per hos valores $x=n^{d}, y=n^{\prime}$ talis repraesentatio prouenit, $(F)$ etiam per substitu. tionem $x=m^{\prime} x^{\prime}+\frac{m^{\prime} N-w^{\prime} b-w^{\prime} c}{N} y^{\prime}, y=n^{\prime} x^{\prime}+$

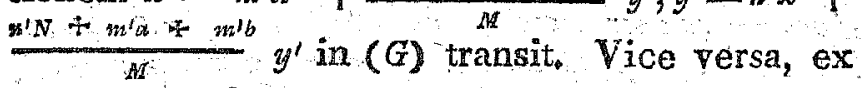
quauis transformatione propria formae $(F)$ in (G) sequetur repraesentatio numeri $M$ per for: mam $(\bar{F})$, ad valorem $N$ pertinens. Seilicet si (F) transit in (G) positis $x=m x^{\prime}-v y^{\prime}, y=$ $m x^{\prime}+y^{\prime}, I M$ repraesentatur per (F) ponendo $x=m, y=n_{,}$et quoniam hic $m_{\mu}+n=1$; valor expr. $\sqrt{D}(\bmod , M)$ ad quem repraesentatio pertinet exit $\mu(b m+c n)-(a m+b n)$ i. e. N. Ex pluribus vero transformationibus propris diuersis, sequentur totidem repraesentationes diuersae ad $N$ pertinentes **). - Hinc

*) In hoc enim casu, si ad ipsum phracin extendere volumus, haec: $N$ esse valorem expr. $\sqrt{ } D(\bmod , M)$, sine $N N \equiv D(\bmod M)$ significabit, $N N-D$ esse multiplum ipsius $M$, adeoque $=0$.

*) Si ex duabus transformationibus proprijs diuersis eadem repraesentatio defluere supponitur, illae ita se habere debebunt: 1) $x=$ $\left.m x^{\prime}-y^{\prime}, y=n x^{\prime}+\mu y^{\prime} ; 2\right) x=m x-x^{\prime} y^{\prime}, y=x x^{\prime}$ 


\section{$-{ }_{19}^{5}-$}

facile colligitur, si omnes transformationes proi priae formae $(F)$ in $(G)$ habeantur, ex his omnes repraesentationes ipsius $M$ per $(F)$ ad valorem $N$ pertinentes sequi. Vnde quaestio de repraesentationibus numeri dati per formam datam (in quibus indeterminatae valores inter se primos nanciscuntur) inuestigandis, reducta est ad quaestionem de inueniendis omnibus transformationibus propriis formae illius in datam ae quiualentem.

Applicanda iam ad haec, ea quae in art. I62 docuimus, facile concluditur: Si repraesentatio aliqua numeri $M$ per formam $(F)$ ad ralorem $N$ pertinens sit haec: $x=\alpha, y=y=$ formulam generalem ommes repraesentationes. eiusdem numeri per formam $(F)$, ad valorem $N_{\text {in }}$ pertinentes, comprehendentem fore hanc: $x=\frac{\alpha t-\left(a b+\gamma c^{\prime} u\right.}{m}, y=\frac{\gamma t+\left(a a+\gamma_{b) u}\right.}{m}$, vi $n$ dinisor communis maximus numerorum $a, 2 b_{y}$ $c$; et $t, u$ omnes numeri, indennite, aequation $t t-D_{t w}=m$ satisfacientes

170, Si forma $(a, b, c)$ ancipiti alicui aequiualens, adeoque formae $\left(M, N, \frac{N N-D}{M}\right)$ tam proprie, quam improprie, siue tam formae $\left(M, N, \frac{N N-D}{M}\right)$, quam huic $\left(M,-N, \frac{N N-D}{N}\right)$

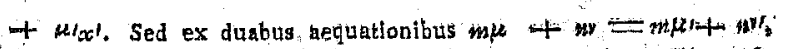
$\mu(m b+n c)-v(m a+m b)=k(m b-n s)-n(m a+1$

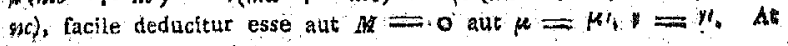
$M=$ o. lam exclusimus. 


\section{$-196-$}

proprie: repraesentationes numeri $M$ habebuntur per formam $(F)$, tamad valorem $N$, quam ad valorem $-N$, pertinentes. Et vice versa si plures repraesentationes numeri $M$ per eandem formam $(F)$, ad valores oppositos expr. $\sqrt{ } D$ (mod. $M, N,-N$, pertinentes habentur: for. ma $(F)$ formae $(G)$ tam proprie quarm improprie aequitualens erit, formaque anceps assigna. ri poterit, cui ( $F$ ) aequiualeat.

Haec generalia de repraesentatíonibus hic sufficiant: de repraesentationibus, in quibus indeterminatae valòres inter se non primos habent, infra dicemus. Respeetu aliarum proprietatum, formae quarum determinans est negatiuus prorsus alio modo sunt tractandae, quam formae determinantis positiui: quare iam vtrasque seorsim considerabimus. Ab illis tamquam facilioribus initium facimus.

I7.1. Problema. Proposita farma quacunque $a, b$, $\left.a^{\prime}\right)$ cuius determinans negatimus, $=-D$, designante $D$ wumerumi positiuusm, inuenire forman inuic proprie atequisatentem, $(A, B, C)$, in qua $A$ non $>\sqrt{\frac{4}{3} D} B$ non $>\frac{\pi}{2} A, C$ non $<A$.

Soiutio. Supponimus in forma proposita non omnes tres conditones simul locum habere; alioquin enim aliam formam quaerere opus non esset. Sit $b^{\prime}$ residuum abs. min. numeri $-b$ secundum modulum $a^{\prime \prime m}$, atque $a^{\prime \prime}=\frac{b^{\prime \prime}+D}{a^{\prime}}$,

* Obseruare conuenit, si formae alicuius $\left(a, b, a^{\prime}\right)$ terminus primus vel vitimus a vel a' sit $=0$, ipsius determinantem esse quadratum positiuum: quare illud in casu praesenti enenire nequit. Ex simili ratione termini exteri $a$, formae deterwal nantis negatiMi, signa opposita habere non possunt. 
qui erit integer quia $b^{\prime} b^{\prime} \equiv b b, b^{\prime} b+\infty \equiv b b+$ $D \equiv a a^{\prime} \equiv$ o (mod. $\left.a^{\prime}\right)$. Iam si $a^{\prime \prime}<a^{\prime}$, fiat denuo $b^{\prime \prime}$ resid. abs. min. ipsius - $b^{\prime}$ secundum mod. $a^{\prime \prime}$, atque $a^{\prime \prime \prime}=\frac{b^{\prime \prime} b^{\prime \prime}+D}{a^{\prime \prime}}$. Si hic iterum $a^{\prime \prime \prime}<a^{\prime \prime}$, sit rursus $b^{\prime \prime \prime}$ res. abs. min. ipsius $b^{\prime \prime}$ secundum mod. $a^{\prime \prime \prime}$ atque $a^{1 v}=\frac{b^{\prime \prime \prime} b^{\prime \prime \prime}+b}{a^{\prime \prime \prime}}$. Haec operatio continuetur donec in progressione $a^{\prime}, a^{\prime \prime}, a^{\prime \prime \prime}, a^{\mathrm{yv}}$ etc. ad terminum $a^{\mathrm{m}+\mathrm{I}}$ perueniatur, qui praecedente suo $a^{\mathrm{m}}$ non sit minor, quod tandem euenire debet, quia alias progressio infinita numerorum integrorum continuo decrescentium haberetur. Tum forma $\left(a^{\mathrm{m}}, b^{\mathrm{m}}, a^{\mathrm{m}+\mathrm{x}}\right)$ omnibus conditionibus satisfaciet.

Dem. I. In progressione formarum $\left(a, b, a^{\prime}\right)$ $\left(a^{\prime}, b^{\prime}, a^{\prime \prime}\right),\left(a^{\prime \prime}, b^{\prime \prime}, a^{\prime \prime \prime}\right)$ etc, quaeuis praecedenti est contigua, quare vltima primae proprie. aequiualens erit (artt. 159,160 ).

II. Quum $b^{\text {m }}$ sit residuum absolute minin mum ipsius - $b^{\mathrm{m}-\mathrm{r}}$ secundum mod. $a^{\mathrm{m}}$ maior quam $\frac{1}{2} a^{m}$ non erit (art. 4).

III. Quia $a^{\mathrm{m}} \cdot a^{\mathrm{m}+1}=D+b^{\mathrm{m}} b^{\mathrm{m}}$, atque $a^{\mathrm{m}+\mathrm{I}}$ non $\left\langle a^{\mathrm{m}}, a^{\mathrm{m}} a^{\mathrm{m}}\right.$ non erit $>D+b^{\mathrm{m}} b^{\mathrm{m}}$, et quum $b^{\mathrm{m}}$ non $>\frac{\mathrm{r}}{2} c^{\mathrm{m}}, a^{\mathrm{m}} a^{\mathrm{m}}$ non erit $>D+$ $\frac{\mathrm{r}}{4} a^{\mathrm{m}} a^{\mathrm{m}}$ et $\frac{3}{a_{1}} a^{\mathrm{m}} a^{\mathrm{m}}$ non $P D$, tandemque $a^{\mathrm{m}}$ non $\rightarrow \sqrt{\frac{4 D}{3}}$.

Exempl. Proposita sit forma (3o4, 217, 155) cuius determinans $=-3 \mathrm{r}$. Hic inuenitur progressio formarum: $(304,217,455),(155,-$ 


\section{- $198-$}

$62,25),(25,12,7),(7,2,5),(5,-2,7)$. Vltima est quaesita. - Eodem modo formae $(121,49,20)$, cuius determinans $=-19$, aequiualentes inueniuntur: $(20,-9,5),(5,-$ $I, 4),(4, I, 5):$ quare $(4, i, 5)$ erit forma quaesita,

Tales formas $(A, B, C)$, quarum determirans est negatiuus et in quibus $A$ non $>\sqrt{\frac{4}{3}} D, B$ non $>\frac{x}{2} A, A$ non $>C$, farmas reductas vocabimus. Quare cuinis formae determinantis negatiui, forma reducta proprie aequiualens inueniri potm erit.

172. Problema, Inuenire conditiones, sub quibus duat formae reductae non iáentical, einsdem determsinastis, $-D,(a, b, c),\left(a^{\prime}, b^{\prime}, c^{\prime}\right)$ proprie cequin valentes esse possint.

Sol. Supponamus, id quod licet, $a^{\prime}$ esse non $>$ $a$, formamque $a x x+2 b x y+c y y$ transire in $a^{\prime} x^{\prime} x^{t}$ $+2 b^{\prime} x^{\prime} y^{\prime}+c^{\prime} y^{\prime} y^{\prime}$ per substitutionem propriam $x=\alpha x^{\prime}+b_{y^{\prime}}, y=\gamma x^{\prime}+\delta y^{\circ}$. Tum_habebun$t^{\text {ur aequationes }}$

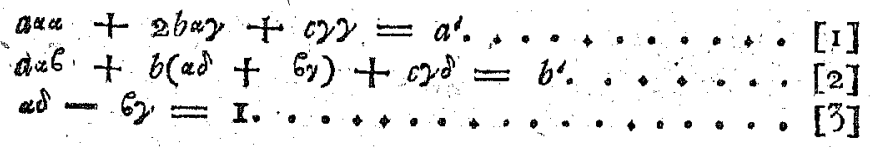

Ex $[x]$ sequitur $a a^{\prime}=(a \alpha-f b \gamma)^{2}+D \gamma \gamma ;$ quare $a a^{\prime}$ erit positiuus; et quuma $a c=D+b b, a^{\prime} c^{\prime}=D-1$ $b^{\prime} b^{\prime}$, etiam $a c, a^{\prime} c^{\prime}$ positiui erunt: quare $a, a^{\prime}, c, c^{\prime}$ omnes eadem signa habebunt. Sęd tum a tum $a^{\prime}$ non $>\sqrt{\frac{4 D}{3}}$, adeoque $a a^{\prime}$ non $>\frac{4 D}{3}$; quare 
multo minus $D_{\gamma \gamma}\left(=a a^{d}-\left(a_{\infty}+b_{\gamma}\right)^{5}\right)$ maion quam $\frac{4}{3} D$ esse poterit. Hine 2 erit aut $=0_{i}$ aut $= \pm 1$.

I. Si $\nu=$ Q, ex [3] sequitur esse aut a $=\mathrm{r}, \delta=\mathrm{r}$, aut $\alpha=-\mathbf{I}, \delta=-\mathbf{I}_{\mathrm{b}}$ In vtroque cast fit ex [r] $a^{\prime}=a$, et ex [2] $b^{\prime}-b= \pm 6 a_{h}$ Sed $b$ noi $>\frac{1}{2} a$, et $b^{\prime}$ non $>\frac{1}{2} a^{\prime}$ proin etiam non $>\frac{x}{2} a$. Quare aequatio $b^{\prime}-b= \pm 6 a^{\prime}$ consistere nequit nisi fuerit

aut $b=b^{\prime}$, vnde sequeretur $c^{i}=\frac{b^{\prime} b t+D}{a^{\prime}}$ $=\frac{b b+D}{a}=c$, quare formae $(a, b, c),\left(a^{\prime}, b^{\prime}, c^{\prime}\right)$ identicae essent contra hyp.

aut $b=-b^{2}= \pm \frac{\mathrm{T}}{2} a_{\text {r }}$ In hoc etiam cad su erit $c^{\prime}=c$ formaque $\left(a^{\prime}, b^{\prime}, c^{\prime}\right)$ erit $(a,-b, c)$ i. e. formae $(a, b, c$,$) opposita. Simul patet for$ mas has esse ancipites propter $2 b= \pm a_{0}$

II. Si $\gamma= \pm \mathrm{x}$, fit ex [I] $\alpha \alpha \alpha+c-a^{\prime}$ $= \pm 2 b \alpha_{0}$. Sed $c$ non minor quam $a$, adeoque non minor quam $a^{\prime}:$ hinc $a * a+c-a^{\prime}$ siue 26. certo non minor quam axa. Quare quum $a b$ non sit maior quam $a$, erit $a$ non minor quam $\alpha \alpha$; vnde necessario aut $\alpha=0$, aut $=x_{\text {. }}$

1) $\mathrm{Si} a=0$, fit ex $[\mathrm{I}], a^{b}=c$, et quoniam $a$ neque maior quam $c$, necque minor quam $a^{\prime}$, erit necessario $a^{\prime}=a=0$. Porro ex [3] fit $b_{y}=$ $-i$, vnde ex [2] $b+b^{\prime}= \pm \delta c= \pm d a_{a^{\prime}}$ Hinc simili modo $v t$ in (I) sequitur esse 
aut $b=b^{\prime}$, in quo casu formae $(a, b, c)$ $\left(a^{\dagger} b^{\prime}, c^{\prime}\right)$ forent identicae, contra byp.

aut $b=-b$, in quo casu formae $(a, b$, c), $\left(a^{d}, b^{\prime}, c^{\prime}\right)$ erunt oppositae.

2) $\mathrm{Si}= \pm \mathrm{x}$, ex $[\mathrm{x}]$ sequitur $\leftarrow 2 b=a$ (4. $c-a^{\prime}$. Quare quum rieque $a$, neque $c$ $<a^{\prime}$, erit $2 b$ non $<a$, et non $<c$. Sed $2 b$ etiam non $>a$, neque $>c$, vnde necessario \pm $2 b=a=c$, et hinc ex aequ. $\mp 2 b=a+c$ $a^{\prime}$ etiam $=a^{\prime}$. Fit igitur ex $[2] b^{\prime}=a(a b$ $+\gamma \delta)+b(\varepsilon \delta+6 y)$, siue, propter as $-62=$ a, $b^{*}-b=a(\alpha b+2 \delta)+2 b y=a(\alpha e+2 b$ $\pm 6 y$ ), quare necessario, vt ante identicae, contra hyp.

ant $b=-b$, adeoque formae illae op positae. Simul in hoc casu propter $a= \pm 2 b$, formae erunt ancipites.

Ex his omnibus colligitur, formas $(a, b, c)$ $\left(a^{\prime}, b^{\prime}, c^{\prime}\right)$ proprie aequiualentes esse non posse nisi fuerint oppositae, simulque aut ancipites, aut $a=c=a^{\prime}=c^{\prime}$. In hisce casibus formas $(a, b, c),\left(a^{\prime}, b^{\prime}, c^{\prime}\right)$ proprie aequinalere, wel a priori facile praeuideri potuit; si enim formae sunt oppositae, improprie, et si insuper ancipites, etiam poprie aequiualentes esse debent; si vero $a=c$, forma $\left(\frac{D+(a-b)^{2}}{a}, a-b, a\right)$ formae $(a, b, c)$ contigua et proin aequiualens erit; sed propter $D+b b=a c=a a \mathrm{fit} \frac{D+(a-b)^{2}}{a}$ 
$=2 a-2 b$, forma vera $(2 a-2 b, a-b, a)$ est anceps; quare $(a, b, c)$ oppositae suae etiam proprie aequiualebit.

Aeque facile iam diudicari potest quando duae formae reductae $(a, b, c),\left(a^{\prime}, b^{\prime}, c^{\prime}\right)$ non oppositae improprie aequiualentes esse possint. Erunt enim impr. aequiualentes, si $(a, b, c)$ $\left(a^{\prime},-b^{\prime}, c^{\prime}\right)$, quae non identicae erunt, proprie sunt aequiualentes, et contra. Hinc patet, conditionem, sub qua illasimproprie sint aequiualentes, esse, vt sint identicae, insuperque aut ancipites aut $a=c_{0}-$ Formae vero reductae quae neque identicae sunt neque oppositae, reque proprie neque improprie aequiualentes esse possunt.

173. Progrema. Propositis drabus formis eiesdem deternimantis negatizi, $F$ et $F^{\prime}$, inuestigare virum sint aequalentes.

Solutio. Quaerantur duae formae reductae $f, f^{\prime}$ formis $F_{,} F^{\prime}$ resp., proprie aequinalerites: si formae $f, f$ sunt proprie, vel improprie vel vtroque modo aequiualentes, etiam $F, F^{\prime}$ erunt; si vero $f, f^{\prime}$ nullo modo aequiualentes sunt, etiam $F ; F^{\prime}$ non erunt.

Ex art. praec. dari possunt quatuor casus:

I) Si $f, f^{\prime}$ neque identicae neque oppositae, $F, F^{\prime}$ nullo modo aequipalentes erunt.

2) Si $f, f$ sunt primo vel identicae vel oppositae, et sacundo vel ancipites, yejeterninitos N5 
suos extremos aequales habent: $F, F$ tum proprie, tum improprie aequiualentes erunt.

3) Si $f, f^{\prime}$ sunt identicae, neque vero ancipites neque terminos extremos aequales habent: $F, F^{\prime}$ proprie tantum aequiualebunt.

4) Si $f, f^{\prime}$ sunt oppositae, neque vero ancipites, neque terminos extremos aequales habent: $F, F^{\prime}$ proprie tantum aequiualentes erunt.

Ex. Formis $(4 \mathrm{x}, 35,30),(7,18,47)$ quarum determinans $=-5$, reductae $(1,0,5)$, $(2,8,3)$ aequiualentes inueniuntur, quare illae nullo modo aequiualentes erunt. - Formis vero $(23,38,63),(15,20,27)$ aequinalet eadem reducta $(3,1,3)$, quae quum simul sit anceps, formae $(23,38,63),(15,20,27)$ tum proprie tum improprie aequiualebunt. - Formis $(37,53,78),(53,73,102)$, aequiualent reductae $(9,2,9),(9,-2,9)$ quae quum sint oppositae, ipsarumque termini extremi aequales: formae propositae tam proprie quam im. proprie erunt aequiualentes.

174. Multitudo omnium formarum reductarum, determinantem datum - $D$ habentium, semper est fínita, et, respectu numeri $D$, satis modica: formae hae ipsae vero duplici modo inueniri possunt. Designemus formas reductas leterminantis - $D$ indefinite per $(a, b, c)$, vbi 


\section{- $203-$}

itaque omnes valores ipsorum $a, b, c$ determina: ri debent.

Methodus prima. Accipiantur pro a omnes numeri, tum positiui tum negatiui non maiores quam $\sqrt{ } \frac{4}{3} D$, quorum residuum quadraticum $-D$, et pro singulis $a$, fat $b$ succes. siue aequalis omnibus valoribus expr. $\sqrt{ }-D$ (mod. a), non maioribus quam $\frac{x}{2} a$, tum positiue tum negatiue acceptis; $c$ vero pro singulis valoribus determinatis ipsorum $a, b$, ponatur $=\frac{D+b b}{a}$. Si quae formae hoc modo oriuntur in quibus c $<a$, hae erunt reiiciendae, reliquae autem manifesto erunt reductae.

Methodus sesunda. Accipiantur pro $b$ omnes numeri, tum positiui tum negatiui, non maiores quam $\frac{\pi}{2} \sqrt{ } \frac{4}{3} D$, siue $\sqrt{\frac{1}{3}} D$; pro singulis 6 resoluatur $b 6+D$ omnibus quibus fieri potest modis in binos factores (etiam signorum diuersitatis ratione habita) ambos ipso $2 b$ non mino. res ponaturque alter factor, et quidem, quando factores sunt inaequales, minor, $=a$, alter $=c$. Si quae formae hoc modo prodeunt, in quibus a $>\sqrt{3} D$, erunt reiiciendae, reliquae vero omnes manifesto erunt reductae. - De. nique patet, nullam formam reductam dari posse quae non per-vtramque methodum inueniatur.

Ex. Sit $D=85$. Hic limes valorum ipsius $a$ est $\sqrt{\frac{340}{3}}$ qui iacet inter to et I 1. Nou meri vero inter t et yo (incl.) quorum residuum -85 , sunt $1,2,5$, ro. Vnde habentur formae duodecim: $(1,0,85),(2,1,43),\left(2,-r_{3}\right.$ $43),(5,0,17),(10,5, x) ;(10-5,18) ;(-$ 


\section{$-204$}

$x, 0,-85)(-2, x,-43),(-2,-1,-43)$ $(-5,0,-17),(-10,5,-$ II $),(-10,-5$, - II).

Per methodum alteram limes valorum ipsius $b$ habetur $\sqrt{\frac{85}{3}}$, qui situs est inter 5 et 6 . Pro $b=0$, prodeunt formae $\left(1,0,7^{5}\right),(-1$, $0,-85),(5,0, i 7),(-5,0,-17)$, pro $b= \pm 1$ hae: $(2, \pm 1,43)(-2, \pm 1,-$ 43). Pro $b= \pm 2$ nullae habentur, quia 89 in duos factores, qui ambo non $>4$, resolui nequit. Idem valet de $\pm 3, \pm 4$. Tandem pro $b= \pm 5$, proueniunt $(10, \pm 5$, iI $),(-$ I0, $\pm 5,-11$.

175. Si ex omnibus formis reductis determinantis dati, formarum binarum, quae, licet non identicae, tamen proprie sunt aequiualentes, alterutra reiicitur: formae remanentes hac insigni proprietate erunt praeditae, vt, quaeuis forma eiusdem determinantis alicui ex ipsis proprie sit aequiualens, et quidem vnicae tantum (alias enim inter ipsas aliquae proprie aequiualentes forent). Vnde patet, omnes formas einsdem deternimantis in totidem classes distribui posse quot formas remanserint, referendo scilicet formas eidem reductae proprie aequiualentes in eandem classem. Ita pro $D=85$, remanent formae $(\mathrm{x}, 0,85),(2, \mathrm{x}, 43),(5,0, \mathrm{r} 7),($ ro, 5, r 1$),(-\mathrm{I}$, $0,-84),(-2,1,-43),(-5,0,-17),(-10$, 5 - I 1) ; quare omnes formae determinantis 85 in octo classes distribui poterunt, prout formae primae, aut secundae etc. proprie aequivalent. Perspicuum vero est, formas in eadem 


\section{$-205-$}

classe locatas proprie aequiualentes fore, farmas ex diuersis classibus proprie, aequiualentes esse non posse. Sed hoc argumentum de classificatione formarum infra multo fusius exsequemur. Hic vnicam obseruationem adjicimus. Iam supra ostendimus, si determinans formae $(a, b, c)$ fuerit negatiuus $=-D, a$ et $c$ eadem signa habere (quia scilicet $a c=b b+$ $D$ adeoque positiuus); ; eadem ratione facile perspicitur, si formae $(a, b, c),\left(a^{\prime}, b^{i}, c^{\prime}\right)$ sint aequiualentes, omnes $a, c, a^{\prime}, c^{\prime}$ eadem signa habituros. Si enim prior in posteriorem per substitut: $x=\alpha x^{\prime}+b y^{\prime}, y=2 x^{\alpha}+\delta y^{\prime}$ transit: erit $a \alpha \omega+2 b a \gamma+c 22=a^{\prime}$, hinc $a a^{\prime}=\left(a^{\alpha}\right.$ $+b b^{2}+D 2 \gamma$, adeoque certo non negatiuus; quoniam vero neque $a$, neque $a^{\prime}=0$ esse potest, erit $a a^{\prime}$ positiuus et proin signa ipsorum $a$, $a^{\prime}$ eadem. Hinc manifestum est, formas quarum termini exteri sint positiui, ab iis quarum termini exteri sint negatiui, prorsus esse separatas sufficitque ex formis reductis eas tantum considerare quae terminos suos exteros positiuos habent, nam reliquae totidem sunt multitudine, et ex illis oriuntur, tribuendo terminis exteris signa opposita; idemque valet de formis ex reductis reiciendis et remanentibus.

176. Ecce itaque pro determinantibus quibusdam negatiuis tabulam formarum, secundum quas omnes reliquae eiusdem determinantis in classes distingui possunt; apponimus autem, ad annotat. art. praec., semissem tantum, scilicet eas quarum termini exteri positiui. 


\section{$206-$}

$D$

$$
\begin{array}{r|l}
1 & (1,0,1) \\
2 & (1,0,2) \\
3 & (1,0,3),(2,1,2) \\
4 & (1,0,4),(2,0,2) \\
5 & (1,0,5),(2,1,3) . \\
6 & (1,0,6),(2,0,3) . \\
7 & (1,0,7),(2,1,4) \\
8 & (1,0,8),(2,0,4),(3,1,3) \\
9 & (1,0,9),(2,1,5),(3,0,3) . \\
10 & (1,0,10),(2,0,5) . \\
11 & (1,0,11),(2,1,6),(3,1,4),(3-1,4) . \\
\text { I2 } & (1,0,12),(2,0,6),(3,0,4),(4,2,4) .
\end{array}
$$

Superflum foret hanc tabulam hic vlterius continuare, quippe quam infra multo aptias disponeme docebimus.

Patet itaque, quamuis formam determinan: tis $-i$, formae $x x+y y$ proprie aequiualere, si ipsius termini exteri sint positiai, vel huic - $x$ - y y si sint negatiui quamuis formam determinantis - 2, cuius termini exteri positiui, formae $x x+2 y y$ etc $;$ quamuis formam determinantis $=$ II, cuius termini exteri positiui, alicui ex his $x x+11 y y, 2 x x+2 x y+6 y y$, $3 x x+2 x y+4 y y, 3 x x-2 x y+4 y y$ etc.

I77. Promenas. Habetur series formarum, quarism quaeuis praecdenti a parte posteriori contigua : desideratur transformatio aliquat propria primate in formam quamcunque seriei.

Solutio. Sint formae $\left(a, b, a^{\prime}\right)=F ;\left(a^{\prime}, b^{\prime}, a^{\prime \prime}\right)$ $=F^{\prime} ;\left(a^{\prime \prime}, b^{\prime \prime}, a^{\prime \prime \prime}\right)=F^{\prime \prime} ;\left(a^{\prime \prime \prime}, b^{\prime \prime \prime}, a^{\mathrm{ly}}\right)=F^{\prime \prime \prime}$ 


$$
207, \square
$$

etc. Designentur $\frac{b+b^{\prime}}{a}, \frac{b^{\prime}+b^{\prime \prime}}{a^{\prime}}, \frac{b^{\prime \prime}+b^{\prime \prime \prime}}{a^{\prime \prime}}$ etc. respectiue per $h$, $h^{\prime \prime}, h^{\prime \prime}$ etc. Sint indeterminatae formarum $F, F^{\prime}, F^{\prime \prime}$ etc. $x, y ; x^{\prime \prime}, y^{\prime} ; x^{\prime \prime}$, $y^{\prime \prime}$ etc. Ponatur $F$ transmutari

in. $F^{\prime}$ positis $x=\alpha^{\prime} x^{\prime}+6^{\prime} y^{\prime}, y=y^{\prime} x+y^{\prime} y^{\prime}$ $F^{\prime \prime}+{ }^{\prime}+x=x^{\prime \prime} x^{\prime \prime}+e^{\prime \prime} y^{\prime \prime}, y=\gamma^{\prime \prime} x^{\prime \prime}+\delta^{\prime \prime} y / d$ F'. . . $x=x^{\prime \prime \prime} x^{\prime \prime \prime}+{ }^{6 \prime \prime} y^{\prime \prime \prime}, y=\gamma^{\prime \prime \prime} x^{\prime \prime \prime}+{ }^{\prime \prime} y^{\prime \prime \prime}$

etc.

Tum quia $F$ transit in $F^{\prime}$ positis $x=-y^{\prime}$, $y=x^{\prime}+h^{\prime} y^{\prime} ; F^{\prime}$ in $F^{\prime \prime}$ positis $x^{\prime}=-y^{\prime \prime}, y^{\prime}$ $=x^{\prime \prime}+h^{\prime \prime} y^{\prime \prime} F^{\prime \prime}$ in $F^{\prime \prime \prime}$ positis $x^{\prime \prime}=-y^{\prime \prime \prime}$, $y^{\prime \prime}=x^{\prime \prime \prime}+h^{\prime \prime \prime} y^{\prime \prime \prime}$ etc. (art. 160), facile eruetur sequens algorithmus (art, 159):

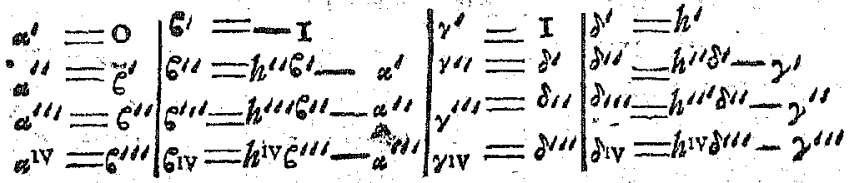
etc.

- siue

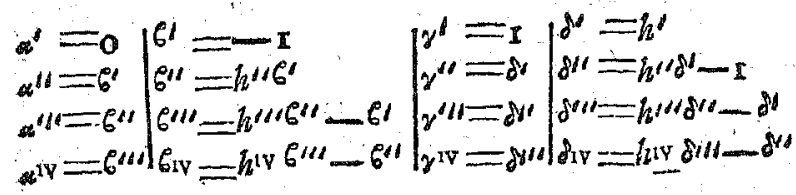

etc.

Omnes has transformationes esse proprias tum ex ipsarum formatione tum ex art, $15 \mathrm{~g}$ nullo negotio deduci potest 


\section{$-208-$}

A.lgorithmus hic perquam simplex et ad calculum expeditus, algorithmo in art 27 exposito est analogus, ad quem etiam reduci potest $\left.{ }^{*}\right)$. Ceterum solutio haec ad formas deter: minantis negatiui non est restricta; sed ad o. mnes casus patet, si modo nullus numerorum $a^{\prime}, a^{\prime \prime}, a^{\prime \prime \prime}$ etc $=0$.

178. Problema. Propositis duabus formis $F, f$, eivsdem deterninantis negatiut $t_{9}$ proprie aequiuatentibus: inuenire traqsformationem aliquam propriam alterius in altering.

Sol. Supponamus formam $F$ esse $\left(A, B, A^{\prime}\right)$ et per methodum art 17 I inuentam esse progressionem formarum $\left(A^{\prime}, B^{\prime}, A^{\prime}\right),\left(A^{\prime \prime}, B^{\prime \prime}, A^{\prime \prime \prime}\right)$ etc. vsque ad $\left(A^{\mathrm{m}}, B^{\mathrm{m}}, A^{\mathrm{m}+\mathrm{I}}\right)$ quae sit reducta: similiterqun $f$ esse $(a, b, a)$ et per ean: dem methodum inuentam seriem $\left(a^{\prime}, b^{\prime}, a^{\prime \prime}\right)$; $\left(a^{\prime \prime}, b^{\prime \prime}, a^{\prime \prime \prime}\right)$ vsque ad $\left(a, b n, a^{n+1}\right)$, quae sit reducta. Tum duo casus locum habere possunt.

I. Si formae $\left(A^{\mathrm{m}}, B^{\mathrm{m}}, A^{\mathrm{m}} \mathrm{x}\right),\left(a^{\mathrm{n}}, b^{\mathrm{n}}\right.$, $a^{\text {n+ }}$ ) sunt ant identicae, aut oppositae simulque ancipites. Tum formae $\left(A^{\mathrm{m}-\mathrm{x}}, B^{\mathrm{n}-\mathrm{r}}, A^{\mathrm{m}}\right)$, $\left(a^{\mathrm{n}},-b^{\mathrm{n}-\mathrm{x}}, a^{\mathrm{n}-\mathrm{x}}\right)$ erunt contiguae (designante $A^{\mathrm{m}-\mathrm{x}}$ terminum progressionis $A_{g} A^{\prime}, A^{\prime \prime} \ldots A^{\mathrm{m}}$ penultimum, similiaque $B^{\mathrm{m}-\mathrm{r}}, a^{\mathrm{n}-\mathrm{r}}, b^{\mathrm{n}-\mathrm{I}}$ ). Nam

$\Rightarrow$ Erit scilicet in signis axt. $27,6^{\mathrm{n}}=4\left[-b_{3}\left\|, b_{3}\right\|,-h_{1} v\right.$.

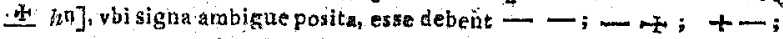
$++i$ prout s formale $4 k+0 ; 102 ; 3-$ et $\delta n= \pm$ $\left[h^{\prime}-h^{\prime \prime}, h^{\prime \prime \prime} \ldots \ldots+h^{\mathrm{n}}\right]$, vbi signa ambigua esse debent + -; +1- -; - + prout s form2e $4 k+0 ; 1 ; 2 ; 3$. Sed hoc, quod quiujs facile ipge confirmare poterit, fusius ex'sequi, nobis brenitas non permittit. 


\section{- 209. -}

$A^{\mathrm{m}}=a^{\mathrm{n}}, B^{\mathrm{m}-\mathrm{I}} \equiv-B^{\mathrm{m}}\left(\bmod \cdot A^{\mathrm{m}}\right), b^{\mathrm{n}-\mathrm{I}} \equiv$ - $b^{\mathrm{n}}\left(\bmod a^{\mathrm{n}}\right.$ siue $\left.A^{\mathrm{m}}\right)$, vnde $B^{\mathrm{m}-I}-b^{\mathrm{n}-\mathrm{s}}$ $\equiv b^{\mathrm{n}}-B^{\mathrm{m}}$ adeoque $\equiv \mathrm{o}$, si formae $\left(A^{\mathrm{m}}, B^{\mathrm{m}}, A^{\mathrm{n}+\mathrm{t}}\right)$, $\left(a_{\mathrm{n}}, b_{\mathrm{n}}, c^{\mathrm{n}}+1\right)$ sunt identicae et $\equiv 2 b^{\mathrm{n}}$ adeoque $\equiv 0$, si sunt oppositae et ancipites. Quare in progressione formarum $\left(A, B, A^{\prime}\right),\left(A^{\prime}, B^{\prime}, A^{\prime \prime}\right)$ $\cdots\left(A^{\mathrm{m}-\mathrm{r}}, B^{\mathrm{m}-\mathrm{r}}, A^{\mathrm{m}}\right),\left(a^{\mathrm{n}},-b^{\mathrm{n}-\mathrm{r}}, a^{\mathrm{n}-\mathrm{l}}\right),\left(a^{\mathrm{n}-\mathrm{r}}\right.$, $-b^{\left.\mathrm{n}-2, a^{\mathrm{n}}-2\right)} . .\left(a^{\prime},-b, a\right),\left(a, b, a^{\prime}\right)$ quaeuis forma praecedenti contigua erit, adeoque per art praec. transformatio propria primae $F$ in vitimam $f$ inueniri poterit.

II. Si formae $\left(A^{\mathrm{m}}, B^{\mathrm{in}}, A^{\mathrm{m}+\mathrm{r}}\right),\left(a^{\mathrm{n}}, b^{\mathrm{n}}\right.$, $a^{a+1}$ ) non identicae, sed oppositae simulque $A^{\mathrm{m}}=A^{\mathrm{n}+\mathrm{I}}=a^{\mathrm{n}}=a^{\mathrm{n}+\mathrm{r}}$. Tum progressio formarum $\left(A, B, A^{\prime}\right),\left(A^{\prime}, B^{\prime}, A^{\prime \prime}\right) \ldots .\left(A^{A^{\mathrm{m}}}, B^{\mathrm{m}}\right.$, $\left.A_{\mathrm{n}}+1\right),\left(a^{\mathrm{n}},-b^{\mathrm{n}-1}, a^{\mathrm{n}-1}\right),\left(a^{\mathrm{n}-1},-b^{\mathrm{n}-2}\right.$, $\left.a^{0}-2\right), \ldots\left(a^{\prime},-b, a\right),\left(a, b, a^{\prime}\right)$ eadem proprietate erit praedita. Nam $A^{\mathrm{m}+\mathrm{r}}=a^{\mathrm{n}}$, et $-B^{\mathrm{n}}$ $-b^{n-1}=-\left(b^{n}+b^{n-1}\right)$ per $a^{\text {n }}$ diuisibilis. Vnde per art. praec. inuenietur transformatio propria formae primae $F$ in vltimam $f$.

Ex. Ita pro formis $(23,38,63)$, (15, 20, 27) habetur progressio $(23,38,63),(63,25$, 10), $(10,5,3),(3, x, 2),(2,-7,27)(27,-$ $20,15),(15,20,27)$, quare $h^{\prime}=1, h^{\prime \prime}=3, h^{\prime \prime \prime}$ $=2, h^{\mathrm{v}}=-3, h^{\mathrm{v}}=-\mathrm{x}, h^{\mathrm{vi}}=0$. Hinc deducitur transformatio formae $23 x x+76 x y$ $+63 y y$ in $x 5 t t+40 t u+27 u m$ haec: $x=$ $13 t-18 u, y=8 t+15 u_{0}$ 
Ex solutione hac nullo negotio sequitur solutio problematis: Si formae $F, j$ improprie sunt aequinalentes, inuenive transformationem impropriam formas $F$ in $f$. Sit enim $f=a t t+2 b t u+a^{\prime} u u$ eritque forma opposita app - $2 b p q+a^{\prime} q q$ formae $\bar{B}$ proprie aequiualens. Quaeratur transformatio propria formae $F$ in illam, $x=\alpha p+b_{q}, y=$ $\partial p+\delta q$, patetque $F$ transire in $f$ positis $x=$ $t-i q, y=2 t-\delta q$, hancque transformationem fore impropriam.

Quodsi igitur formae $F, f$ tam proprie quam improprie sunt aequiualentes: inueniri poterit tam transformatio propria aliqua quam impropria.

179. Problema. Si forma $F, f$ sunt aequiztalestes: inuenise omnes transformationes format $F$ in $f_{0}$

Sol. Si formae $F, f$ vnico tantum modo sunt aequiualentes $i_{.}$e proprie tantum vel im. proprie tantum: quaeratur per art. praec. transformatio val formae $F$ in $f$, patetque alias quam quae huic sint similes dari non posse. Si vero formae $F, f$ tam proprie quam improprie aequiualent, quaerantur duae transformationes, altera propria, altera impropria, Iam sit forma $F=(A, B, C), B B-A C=-D$, numerorumque $A, 2 B, C$ diuisor communis mas ximus $=m_{*}$ Tum ex art 162 patet, in priori casu omnes transformationes formae $F$ in $f$ ex vna transformatione, in posteriori omnes proprias ex propria omnesque improprias ex impropria deduci posse, si modo omnes solutio. 


\section{$-2 I I$}

nes aequationist $+D u=m m$ habeantur. His igitur inuentis problema erit solutum.

Habetur autem $D=A C-B B, 4 D \Rightarrow$ $4 A C-4 B B$, quare $\frac{4 D}{m m}=4 \frac{A}{m} \frac{C}{m}-\left(\frac{2 B}{m}\right)^{2}$ erit integer. Iam si

1) $\frac{4 D}{m m}>4$, erit $D \gg m m$ : quare in $t t+$ $D u=m m$, necessario debebit esse $=0$, adeoque $t$ alios valores quam $+m$, et $-m$ habe. re nequit. Hinc si $F, f$ vrico tantum modo aequiualentes sunt et transformatio aliqua $x=$ ${ }^{2} x^{\prime}+6 y^{\prime}, y=\gamma x^{\prime}+\delta y^{\prime}$ : praeter hanc ipsam quae prodit ex $t=m$ (art. 162 ), et hanc $x$ $=\alpha x^{\prime}-\epsilon_{y^{\prime}}, y=-2 x^{\prime}-\delta y^{\prime}$ aliae locum ha. bere non possunt. Si vero $F, f$ tum proprie tum improprie aequinalent, atque propria aliqua transformatio habetur $x=\alpha x^{\prime}+6 y^{\prime},=2 \dot{x}^{\prime}$ $+\delta y^{\prime}$; impropriaque $x=x^{\prime} x^{\prime}+6 y^{\prime}, y=y^{\prime} x^{\prime}$ If $\delta^{\prime} y^{\prime}$ ', praeter illam (ex $t=m$ ) et hancce $x=$ $-\alpha^{\prime} x^{\prime}-b^{\prime} y^{\prime}, y=-\gamma^{\prime} x^{\prime}-\delta y^{\prime}(e x t=$ 9n) alia propria non dabitur; similiterque nulla impropria praeter $x=\alpha^{\prime} x^{\prime}+\delta^{\prime} y^{\prime}, y=\gamma^{\prime} x^{\prime}+\delta^{\prime} y^{\prime}$ et $x=-\alpha^{\prime} x^{\prime}-6^{\prime} y^{\prime}, y^{\prime}=-2 x^{\prime}-\delta^{\prime} y^{\prime}$.

2) Si $\frac{4 D}{m m}=4$, siue $D=m m$, aequatio $+D u=m m$ quatuor solutiones admittet $t, u$ $=m, 0 ;-m, o ; 0, k ; 0,-$ r. Hinc si $E, f$ vnico tantum modo sunt aequiualentes et trans. formatio aliqua $x=\alpha x^{\prime}+6 y^{\prime}, y=2 x^{\prime}+{ }^{\prime} y^{\prime}$ : quatuor omnino transformationes dabuntur, $x=$ $\pm \alpha x^{\prime}+6 y^{\prime}, y= \pm 2 x^{\prime} \pm \delta y^{\prime} ; x=\mp \frac{\alpha B+\gamma C}{m} x^{\prime}$ $\mp \frac{B^{B} S C}{m} y^{\prime}, y= \pm \frac{\alpha A+y B}{m} x^{\prime}$ I $\frac{6 A^{\top} \partial^{B}}{m} y^{\prime} \quad$ Si Ye 


\section{$-212$}

ro $F, f$ duobus modis aequiualent, siue praeter transformationem illam datam alia ipsi dissimilis habetur: haec quoque suppeditabit quatuor illis dissimiles, ita vt octo transformationes habeantur. - Ceterum facile demonstrari potest in hoc casu $F, f$ semper reuera duobus modis aequiualere. Nam quum $D=$ mm $=A C-B B, m$ etiam ipsum $B$ metietur. Formae $\left(\frac{A}{m}, \frac{B}{m}, \frac{C}{m}\right)$ determinans erit $=-\mathrm{r}$, quare formae $(1,0,1)$ vel huic $(-1,0,-1)$ erit aequiualens. Facile vero perspicitur, per eandem zransformationem per quam $\left({ }_{m}^{A}, \frac{B}{m},{ }_{m}^{C}\right)$ transeat in $( \pm \mathrm{r}, 0, \pm \mathrm{l}$ ) formam $(A, B, C)$ transire in ( $m$, $0, \pm m$ ), ancipitem. Quare forma $(A, B, C)$, ancipiti aequiualens, cuivis formae, cui aequiualet, tum proprie tum improprie aequiualebit.

3) $\mathrm{Si} \frac{4 D}{m m}=3$, siue $4 D=3 m$. Tum $m$ erit par omnesque solutiones aequationis $t t+$ Duu $=m m$ erunt sex, $t, u=m, 0 ;-m, 0 ; \frac{T}{2} m$, $x ;-\frac{1}{2} m,-I ; \frac{\pi}{2} m,-1 ;-\frac{\pi}{2} m, I$, Si itaque duae transformationes dissimiles formae $F$ in $f$ habentur, $x=x^{\prime}+6 y^{\prime}, y=2 x^{\prime}+\delta y^{\prime} ; x=$ ${ }^{\prime} x^{\prime}+b^{\prime} y^{\prime}, y=y^{\prime} x^{\prime}+d^{\prime} y y^{\prime}$ : habebuntur duodecim transformationes, scilicet sex priori similes

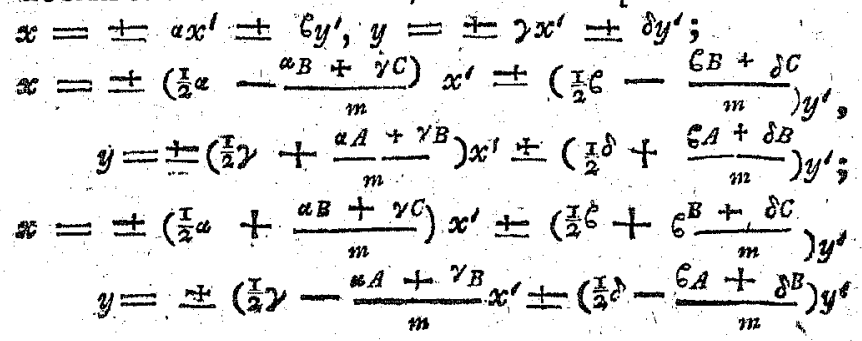




\section{$-213$}

et sex posteriori similes, quae ex his nascund tur ponendo pro $\alpha, b, \gamma$, dhos $\alpha^{\prime}, b^{\prime}, \gamma^{\prime}, \delta^{\prime}$ 。

Quod vero in hoc casu semper $F, f$ vtroque modo aequiualent, ita demonstramus. Formae $\left(\frac{2 A}{m}, \frac{2 B}{m}, \frac{2 C}{m}\right)$ determinans erit $=-\frac{4 D}{m m}$ $=-3$, adeoque (art. 176 ) aut formae $( \pm 1,0$, \pm 3 ) aut huic $(2,1,2)$ aequiualens. Vnde facile perspicitar, formam $(A, B, C)$ aut formae $\left(+\frac{\mathrm{r}}{2} \mathrm{~m}\right.$, $\left.o, \pm \frac{3}{2} m\right)$ aut huic $\left.\left( \pm m, \frac{1}{2} m, \pm m\right)^{\star}\right)$ quae ambae sunt ancipites, aequiualere adeoque, cuivis aequiualenti, vtroque modo.

4) Si supponitur $\frac{4 D}{n m m}=I_{2}$ fit $\left(\frac{2 B}{m m}\right)^{2}=4 \frac{A C}{m m}$ -2, adeoque $\equiv 2(\bmod .4)$. Sed quam nullum quadratum esse possit $\equiv 2(\bmod .4)$ hic casus Iocum habere nequit-

5) Supponendo $\frac{4 D}{m m}=\mathrm{I}$, fit $\left(\frac{2 B}{m}\right)^{2}=4 \frac{A C}{m m}-$ $I \equiv-I(\bmod .4)$. Quod quum impossibile sit, etiam hic casus nequit locum habere.

Ceterum quum $D$ neque $=0$, neque ne. gatiuus sit, alii casus praeter enumeratos darî non possunt.

1So. Probiema. Inuenire omses repraesentatioszes numeri dati $M$ per formam axx $+2 b x y+c y y$ $\ldots F$, determinantis negatiui $-D$, in quibus $x, y$ valores inter se primos nanciscuntur.

) Demonstrari potest, forman $(A, B, O)$ uecessario posteriori aequi snlere: sed hoc bis non necessarium.

0.3 


\section{$-\quad 214$}

Sol. Ex art. 154 patet, $M$ eo quo requiritur modo repraesentari non posse, nisi - $D$ sit resid, quadr, ipsius $M$. Inuestigentur itaque primo ormes valores dinersi $(i, \varepsilon$. incongrui) expr. $\sqrt{ }-D(\bmod . M)$, qui sint $N,-N, N^{\prime} N^{\prime}$, $N^{\prime \prime},-N^{\prime \prime}$ etc.; quo simplicior euadat calculus, omnes $N, N^{\prime}$ etc. ita determinari possunt, vt non sint $\gg \frac{r}{2} M$. Iarn quoniam quaeuis re. praesentatio ad aliquem horum valorum pertinere debet singuli seorsim considerentur.

Si formae $F,\left(M, N, \frac{\Delta+N N}{M}\right)$, non sunt proprie aequiualentes, nulla repraesentatio ipsius $M$ ad valorem $N$ pertinens dari potest (art. I58). Si vero sunt, inuestigetur transformatio propria formae $F$ in $M x^{\prime} x^{\prime}+2 N x^{\prime} y^{\prime}+\frac{D+N N^{\prime} y^{\prime} y^{\prime}}{M}$ quae sit $x=\alpha x^{\prime}+6 y^{\prime}, y=2 x^{\prime}+\delta y^{\prime}$, eritque $x=$ * $y=2$ repraesentatio numeri $N$ per $F$ ad $N$ pertinens. Sit diu. comm, max. numerorum $A$, $2 B, C,=m$ distinguanturque tres casus (art. praec.):

1) Si $\frac{4 D}{m m}>4$, aliae repraesentationes ad $N$ pertinentes quam hae duae $x=\alpha, y=y ; x$ $=-\alpha, y=-2$ non dabuntir (artt. 169, I 80 ).

2) Si $\frac{4 D}{m m}=4$, habebuntur quatuor reprae. sentationes $x= \pm \infty, y= \pm \gamma ; x=\mp$ $\frac{\alpha B+\gamma C}{\sin }, y= \pm \frac{\alpha A+\gamma B}{m}$ 
3) Si $\frac{\Delta D}{m m}=3$, habebuntur sex repraesen: tationes, $x= \pm \alpha, y= \pm 2 ; x= \pm\left(\frac{x}{2} \alpha-\right.$ $\left.\frac{\alpha B+\gamma C}{m}\right), y= \pm\left(\frac{\bar{x}}{2} \gamma+\frac{\alpha A+\gamma B}{m}\right) ; x= \pm\left(\frac{\frac{X}{2} \alpha}{2}\right.$ 女 $\left.\frac{x_{B}+\gamma C}{m}\right), y= \pm\left(\frac{\pi}{2} \gamma-\frac{\alpha_{A}+\gamma^{B}}{m}\right)$.

Eodem modo quaerendae sunt repraesen: sentationes ad valores $-N, N_{1}^{\prime}-N^{\prime}$ etc. pertinentes.

I8 I. Inuestigatio repraesentationum numeri $M$ per formam $F$ in quibus $x, y$ valores inter se non primos habent, ad casum iam cone sideratuin facile reduci potest. Fiat talis re. praesentatio ponendo $x=\mu e, y=\mu f$, ita vt $\mu$ sit diu. comm. max. ipsorum $\mu \ell, \mu f$, siue $e, f$ inter se primi. Tum erit $M=\mu k(A e e+2 B e f$ $+(C f)$ ) adeoque per $\mu \mu$ diuisibilis ; substitutio vero $x=e, y=f$ erit repraesentatio numeri $\frac{M T}{\mu_{\mu}}$ per formam $F$ in qua $x, y$ valores inter se primos habent. Si itaque $M$ per nullum quadratum (praeter $r$ ) dinisibilis est, e. g. si est numerus prinus: tales repraesentationes ipsius $M$ non dabuntur. Si vero $A$ diuisores quadraticos implicat, sint hi $\mu \mu, n, \pi \pi$ etc. Quaerantur primo omnes repraesentationes numeri $\frac{M A}{K_{\mu}}$ per formam $(A, B, C)$, in quibus $x$, $y$ valo$x_{e}$ inter se primos habent, qui valores si per $\mu$ multiplicantur praebebunt omnes repraesentationes ipsius $M$, in quibus diu. comm. max numerorum $x, y$ est $\mu_{0}$. Simili modo omnes repraesentationes ipsits $\frac{M}{w}$ in quibus valores 


\section{$-216-$}

ipsorum $x, y$ inter se primi sunt, praebebunt onmes repraesentationes ipsius $I I$ in quibus dia. comm, max, valorum ipsorum $x, y$ est, etc.

Palam igitur est, per praecepta praecelentia omnes repraesentationes numeri diti per formam datam determinantis negatiui inueniri posse.

182. Descendimus ad cuosdam casus particulares, tum propter insignem ipsorum elegantiam, tum propter assiduam operamab ill. Fixlero ipsis impensan, vnde classican quasi dignitatem sunt nacti。

1. Per formam $x x+y y$ ita repraesentari vt $x$ ad $y$ sit primus, (siue in duo quadrata inter se prima discerpi), nullus numerus potest nisi cuius residuum quadraticum est - $x$, tales vero numeri, positiue accepti, omnes poterunt. Sit $M$ talis mumerus, omnesque valores expr.

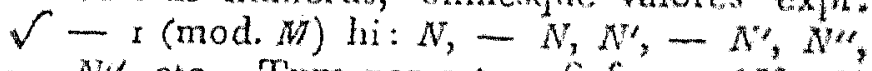
- $N^{\prime \prime}$ etc. Tum per art. 176 forma $\left(n, N^{\prime}\right.$, $\left.\frac{N N+x}{M}\right)$ formae $(x, o, x)$ proprie aequinatens crit. Sit transformatio aliqua propria huius in illim, $x=a x^{\prime}+6 y^{\prime}, y=2 x^{\prime}+8 y^{\prime}$, eruntque repraesentationes numeri $M$ per formam $x x^{2}+$ y $y$ ad $N$ pertinentes hi quatuor $*: x= \pm \alpha_{\text {; }}$ $y= \pm 2 ; x=\mp y, y= \pm$.

9) Patet cuim, hunc casum sub (2) ask. $x 80$ contenturn ossen, 


\section{- $217-$}

Quum forma $(1,0, i)$ sit anceps, patet, etiam formam ( $\left.M,-N, \frac{N N+1}{H}\right)$ ipsi proprie nequiualentem fore, illamque proprie in hanc transmurari positis $x=\alpha x^{\prime \prime}-\varepsilon_{y^{\prime}}, y=-\gamma x^{\prime}$ $+i y^{*}$. Hinc deriuantur quatuor repraesentationes ipsius $M$ ad $-N$ pertinentes, $x= \pm \infty$, $y=\mp y ; x= \pm 2, y=\mp a$. Manifestum itaque est, octo repraesentationes ipsins $M$ da$r i$, quarum semissis altera ad $N$, altera ad $-N$ pertincat; sed hae ornnes vwitam tantummodo discerptionem numeri $\pi$ in duo quadrata exhibent, $M=+2 \%$, siquidem ad quadrata ipsa tantum, neque vero ad ordinem ra. dicumue signa spectamus.

Quodsi itarque alii valores expr. $\sqrt{ }-\mathbb{}$ (mod. $M$ ) praeter $N$ et $-N$ non dantur, quod e. $g$. euenit, quando $M$ est numerus primus, $M$ vnico tantum modo in duo quadrata inter se prima resolui poterit. fam quum - $x$ sit residuum guadraticum cuinsuis numeri primi formae $4 \%+1$ (art. 108), manifestoque numerus primus in duo quadrata inter se non prima dis. cerpi nequeat, habemus theorema:

Quinis numerus primus formae $4 n+\mathrm{I}$ in duo quadrata decomponi potest, ct quidem vnico tantum modo. $1=0+x, 5=1+4,13=4+9$ $x 7=x+x 6,29=4+25,57=x+36$, $4 x=16+25,53=4+49,6 x=25+$ $36,75=9+64,89=25+64,97=16$ +81 ctc. 


\section{$-218$}

Theorema hoc elegantissimum iam Ferma: tio notum fuit, sed ab ill. Eulero primo demonstratum est, Connm, nout Petr. T. $V_{k}$ ad ans= nos 1754,1755, p. a sqq. In $T$. $1 V$, diss, exstat ad idem argumentum pertinens, $p$ s sqq.; sed tum rem penitus nondum absoluerat, vid. imprimis art. 27.

Si igitur numerus aliquis formae $4 n+\mathrm{I}$ aut pluribus modis aut nullo modo in duo quadrata resolui potest, certo non erit primus.

Vice versa autem, si expr. $\sqrt{ }-\mathbf{I}$ (mod. M) praeter' $N$ et $-N$ alios adhuc valores habet, aliae adhuc repraesentationes ipsius $M \mathrm{da}$. buntur, ad hos pertinentes. In hoc itaque casu II. pluribus modis in duo quadrata resolui poterit e. g: $65=\mathrm{x}+64=\mathrm{x} 6+49,22 \mathrm{x}=$ $25+196=100+121$.

Repraesentationes reliquae, in quibus $x, y$ valores obtinent non primos inter se, per methodum nostram generalem facile inceniri possunt. Obseruamus tantummodo, si numerus. aliquis factores formae $4 n+3$ inuoluens, per nullam diuisionem per quadratum ab his liberari possit (quod fiet, si aliquis aut plures talium factorum dimensionem imparem habet), hunc nullo nodo in duo quadrata resolui posse *\%.

๑) Si numerus $M=2^{k} S a^{*} b_{c} c_{4} \ldots$ ita $v t \mu, b_{1}$, etc. sint numert primi inaequales formae $4 n+1$, atque $S$ productum ex omnibus factoribus primis jpsius $M$ formae $4-3$ (ad quam formam 
II. Per formam $x x+2 y y$ nullus numerus, cuius non residuum,- 2 , ita repraesentas ri potest vt $x$ ad $y$ sit primus, reliqui omnes poterunt. Sit $\ldots 2$ residuum numeri $M$, atque $N$ valor aliquis expr. $\sqrt{ }-2(\bmod , M)$. Tum per art. I76 formae $(1,0,2),(M, N$, $\left.\frac{N N+2}{M}\right)$ proprie aequiualentes erunt. Transeat illa proprie in hanc ponendo $x=\alpha x^{\prime}+6 y^{\prime}$, $y=2 x^{\prime}+\partial y^{\prime}$, eritque $x=\alpha, y=\nu$ repraesentatio numeri $M$ ad $N$ pertinens. Praeter quam et hanc $x=-\alpha_{3}=-2$ aliae ad $N$ non pertinebunt (art. 180).

Simili modo, vt supra, perspicitur, reprae. sentationes $x= \pm \alpha y=\mp \gamma$ ad valorem - $N$ pertinere. Omnes vero hae quatuor repraesentationes vnicam tantum discerptionem ipsius $M$ in quadratum et quadratum duplex exhibent, et si praeter $N$ et $-N$ alii valores expr. $\sqrt{-2}$ (mod. $M$ ) non dantur, aliāe discerptiones non dabuntur. Hinc adiumento proposs. art. I 6 facile deducitur theorema:

quinis uumerus positinus reduci potest, faciendo $\mu \mathrm{m}=0$ quando $M$ est irpar, et $S=$ I quando $M$ nulles cactores formae $4 n+$. 3 implicat): $M$ nullo modo in duo quadrata resoluit potetit, si $S$ est, non-quadratus; si vero $S$ est quadratus, dabutntur $\frac{T}{2}(x+1)(6+1)$ $(\gamma+1)$ etc discerptiones ipsius $M$, quando allquis wumeroum \%. 6. $\gamma$ ete. est impar, aut $\frac{1}{2}(\alpha-1)(6-1)(\gamma+1)$ etc. $+\frac{1}{2}$ quando omnes $a, 6, y$ etc. sunt pares (siquidem ad quadrata ipsa tautum respicitur), Qui in calculo combinationam allquatum sunt versati, demonstrationem, huius theorematis (cui, perinde ve aliis gavticularibus, immorari nobis non licel) ex theoria nostra tenerali hand difficulter eruere poterunt, $\mathrm{Cf}$ art. 105. 
Quinis sumerus primus format $8 n+x$ vel $8 \mathrm{n}$ +5 in quadratum et quadratum duplex deconpon potest et quidens vnica tantum moda. I $=1+0$, $5=\mathrm{I}+2, \mathrm{x} \mathrm{r}=9+2, \mathrm{x} 7=9+8, \mathrm{x} 9$ $=\mathrm{x}+18,4 \mathrm{r}=9+32,43=25+18$. $59=9+50,67=49+18,73=x+$ $72,85=81+2,89=81+8,97=45$ +72 etc.

Etiam hoc theorema, vti plura similia, Fermatio innotuit: sed ill. La Grange primus demonstrationem dedit, Suite des recherhs d Atrithmetique, Nouv. Mem. de $l A c$. de Berlin 1775 . p. 523 sqq. Multa ad idem argumentum pertinentia iam ill. Euler absoluerat, Specimen de vsto obseruationum in mathesi pura Comm. nou petr. T. IY p. 185 sqq. Sed demonstratio completa theorematis semper ipsius industriam elusit, $p$. 220. Conf. etiam diss. in T. VIII (ad annos

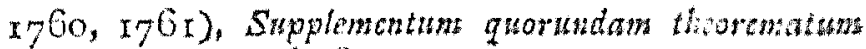
arithmeticoram, $6 \mathrm{ub} \mathrm{fin}$.

III. Per methodum similem demonstratur, quemuis numerum curus residum quadr. it - 5 repraesentari posse aut per formam $x x+$ $3 y y$, aut per hane $2 x x+2 x y+2 y y$, ita ve valos ipsius $x$ ad yalorem ipsius sit primus. Quare qum -3 sit residum omnium numerornm primorum formae $3 n+ \pm$ (art. 199) manifestoque per formam $2 x x+2 x y+2 y y$ numer parts tatum repraesentari possint: eodem mo: do ve supra habetur theorema: 
Qubits numerts primus formae $3 n+1$ in quadratunt at audroitum triplex decomponi potest, et quiden vniso tanium modo. 1 .... I $+0,7=4+3$, $15-1+12,19=16+3,51=4+27$, $572.5+12,43=16+27,6 x=49 \pm$ I2, $67=64+3,7^{3}=\mathbf{1}+7^{2}$ etc.

Demonstrationem huins theorematis ill. Euler primus tradidit in commentatione modo laudata, Comm. now. Petr." T. VIII, p. 105 sqq.

Simili modo vlterius progredi et $\varepsilon$. $g$. ostendere possemus, quemuis numerum primum formae $20 n+1$, vel $20 x+3$, vel $20 \%+7$, vel son +9 (quppe quorum residuum - 5) per alterutran formam $x x+5 y y, 2 x x+2 x y+3 y y$ reproesentari posse, et quidem numeros primosformae $20 n+1$ et $20 n+9$ per priorem, primos format wn $+5,20 n+7$, per posteriorem, neo non dupla primorum formae $20 n+r, 20 n+9$ per formam $2 x x+2 x y+3 y y$, dupla primorum formae $20 y+3,20 n+7$, per formam $x x+5 y y$ : sed hanc propositionem infinitasque alias particulares quiuis proprio marte ex praecedentibus et infra tradendis deriuare poterit. - Transimus itaque ad formas determinentis positui, et quum harum indoles prorsus alia sit, quando determinans est quadratus, alia, quando non-quadratus: formas determimantis quadrati hic primo excludimus posteatue seorsim considerabimus.

183. Proulema. Proposita forma quactuque $\left(a, b, a^{\prime}\right)$, cutus deteminans positiunes non. nutadratus $D$ : inwewire formam hatic proprie atguthatutem, $(A$. 
$B, C$ ), in qu $B$ sit posititus et $\Leftrightarrow \sqrt{D} ; A$ vero si est positinus, vel $-A$, si $A$ negatiuus, inter $\sqrt{ } D$ at $\sqrt{ } D-B$ situs.

Sol. Supponimus in forma proposita vtramque conditionem nondum locum habere; alio. quin enim aliam formam quaerere opus non esset. Porro obseruamus, in forma determi. nantis non-quadrati terminum primum vel vltimum $=o$ esse non posse (art. 17r ann.). Sit $b^{\prime} \equiv-b\left(\bmod . a^{\prime}\right)$ atque intra limites $\sqrt{ } D$ et $\sqrt{ } D \mp a$ situs (accepto signo superiori, quan. do $a^{\prime}$ positiuus, inferiori, quando est negatiuus) quod fieri posse simili ratione vt art. 3 , facile demonstratur, ponaturque $\frac{b^{\prime} b^{\prime}-D}{a^{\prime}}=a^{\prime \prime}$, qui erit integer, quia $b^{\prime} b^{\prime}-D \equiv b b-D \equiv a a^{\prime} \equiv$ - (mod. a'). Iam si $a^{\prime \prime}<a^{\prime}$, fiat denuo $b^{\prime \prime} \equiv$ - $b^{\prime}$ (mod. $\left.a^{\prime \prime}\right)$ et inter $\sqrt{ } D$ et $\sqrt{ } D \mp a^{\prime \prime}$ situs (prout $a^{j d}$ positiuus vel negatiaus) et $\frac{b^{\prime \prime} b^{\prime \prime}-D}{a^{\prime \prime}}$ $=a^{\prime \prime \prime}$. Si hic iterum $a^{\prime \prime \prime}<a^{\prime \prime}$, sit rursus $b^{\prime \prime \prime}$ $\equiv-b^{\prime \prime}\left(\bmod . a^{\prime \prime \prime}\right)$, etinter $\sqrt{ } D$ et $\sqrt{ } D \div a^{\prime \prime \prime}$ situs atque $\frac{b^{\prime \prime \prime} b^{\prime \prime \prime}-D}{a^{\prime \prime \prime}}=a^{\mathrm{iv}}$. Haec operatio continuetur, donec in progressione $a^{\prime}, a^{\prime \prime}, a^{\prime \prime \prime}, a^{\mathrm{w}}$ etc. ad terminum $a^{\text {m+r }}$ perueniatur, praecedente a non minorem, quod tandem euenire debet, quia alioquin progressio infinita numerorum integrorum continuo decrescentium haberetur. Tum positis $a^{\mathrm{m}}=A, b^{\mathrm{m}}=B, a^{\mathrm{m}+\mathrm{t}}=C$, forma $(A, B, C)$ omnibus conditionibus satisfaciet. 


\section{$-223-$}

Dem. I. Quoniam in progressione forma. rum $\left(a, b, a^{\prime}\right),\left(a^{\prime}, b^{\prime}, a^{\prime \prime}\right),\left(a^{\prime \prime}, b^{\prime \prime}, a^{\prime \prime \prime}\right)$ etc. quaeuis praecedenti est contigua: vltima ( $A$, $B, C)$ primae $\left(a, b, a^{\prime}\right)$ proprie aequiualens erit。

II. Quia $B$ inter $\sqrt{ } D$ et $\sqrt{ } D \mp A$ situs est (accipiendo semper signum superius quando. $A$ est posituzus, inferius quando $A$ est negatiugus): patet, si ponatur $\sqrt{ } D-B=p, B$ - $(\sqrt{ } D \mp A)=q$, hos $p, q$ fore positiuos. Iam facile confirmatur, fore $q q+2 p q+2 p \sqrt{ } D$ $=D+A A-B B ;$ quare $D+A A-B B$ erit numerus positiuus, quem ponemus $=r$. Hinc propter $D=B B-A C$, fit $r=A A-$ $A C$, adeoque $A A-A C$ numerus positius : quia vero per hyp. $A$ non est maior quam $C$, manifesto illud aliter fieri nequit, quam si $A C$ est negatiuus, adeoque signa ipsorum $A, C$ opposita. Hinc $B B=D+A C<D$ adeoque $B<$ ir $D$

III. Porro quia $-A C=D-B B$, erit $A C$ $\angle D$, et hinc (quia $A$ non $<C$ ), $A<\sqrt{ } D$. Quare $\sqrt{ } D \mp A$ erit positiuus, adeoque etiam $B$, qui inter limites $\sqrt{D}$ et $\sqrt{ } D A$, est situs.

IV. Hinc a potiori $\sqrt{ } D+B \mp A$ positiuus, et quia $\sqrt{ } D-B \pm A=-q$, est negatiuus, $\neq A$ situs erit inter $\sqrt{ } D+B$ et $\sqrt{ } D$ -B. Q.E. D.

Ex. Proposita sit forma $(67,97,140)$, cuius determinans $=29 . \quad$ Hic inuenitur pro- 


\section{- $224-$}

gressio formarum $(67,97, \mathbf{1 4 0}),(\mathbf{r} 40,-9 \%, 67)$ $(67,-37,20)(20,-3,-x),(-1,5,4)$. Vltima erit quaesita.

Tales formas $(A, B, C)$ determinantis positiui non-quadrati $D$, in quibus $A$ positiue acceptus iacet inter $\sqrt{ } D+B$ et $\sqrt{ } D-B, B$ vero positiuus est atque $\langle\sqrt{ } D$, formas reothstas vocabimus. Formae itaque reductae determinantis positiui non-quadrati aliqua ntum differunt a formis reductis determinantis negatiu; sed propter magnam analogiam inter has et illas, denominationes diuersas introd ucere noluimus.

384. Si aequiualentia duarum formarum seductarin determinantis positiui aeclue facilo dignosci posset, vt in formis determi intartis negatiui (art. 172), aequiualentiam dia arum formarum quarunicunque eitsdem determinantis positiui nullo negotio diindicare poss $t$ mus. Sed hic res longe aliter se habet, fierique? potest vt permultie formae reductie inter se aequiualentes sint. Antequam itaque jroble nna bocisy grediamur, profundius in naturam for nuarmm reductarum (deterninantis positini nora quatdrati. quod semper hic subintelligendum) intuirere necesse erit.

3) Si $(a, b, c)$ est forma redircta, a et a signa opposita habebunt. Nam posizodeterninante formae $=D$, exit $a c=b b-D$, adeonue, propter $b<\sqrt{ } D$, negatiuus. 


\section{$-205$}

2) Numerus c perinde vt $a$, positive acceptus, inter $\sqrt{ } D+b$ et $\sqrt{ } D-b$ situs erit. Nam $-c=\frac{b-6}{t}$; quare, ahstractione Cacta a signo, c incebit inter $\frac{D-b i}{\sqrt{D}+b}$ et $\frac{D-b b}{\sqrt{D-b}}$ i. e. inter $\sqrt{ } D-b$ et $D+b$.

5) Hinc patet, etian $(c, b, a)$ fore formam reductam,

1) Itum a tum $c$ erunt $<2 \sqrt{D} D$. Vtergue enim est $<\sqrt{D} D+D$, adeoque a potiori $<\sqrt{ } D$.

5) Numerus $b$ situs erit inter $\sqrt{ } D$ et $\sqrt{ } D$ - a laccepto signo superiori quando a positiuns, inforioni quando est negatius). Quia enim $-a$ incet inter $\sqrt{ } n+b$ et $\sqrt{ } D-b$, erit $+a-(\sqrt{ } D-b)$, siue $b-(\sqrt{D}+a)$ positiuss; $b-\sqrt{D}$ autem est negatiuus; Ifumobrem o iuter $\sqrt{ } D$ et $\sqrt{ } D=$ a erit situs. - Prorsus codem modo demonstratur, $b$ inter $\sqrt{D}$ at $\sqrt{D} \neq c$ iacere (prout $c$ pos. vel neg.).

6) Cainis formae reductae $(a, b, c)$ ab vtraque parte contigua est rectucta una, et an plures.

Fiat $a^{\prime}=c, b^{\prime} \equiv-b$ (mod. $\left.a^{\prime}\right)$ et inter $\sqrt{D}$ et $\sqrt{D} ; a^{*}$ situs $\left.{ }^{*}\right), c^{\prime}=\frac{b b^{\prime}-D}{a^{\prime}}$, eritque forma $\left(a^{\prime}, b^{\prime}, c^{\prime}\right)$ formae $(a, b, c)$ ab vltima

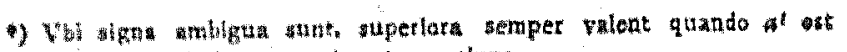

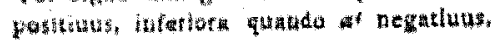




\section{- $226-$}

parte contigua, simulque manifestam est, si vlla forma reducta formae $(a, b, c)$ ab vltima parte contigua detur, eam ab hac $\left(a^{\prime} b^{\prime}, c^{\prime}\right)$ diuersam esse non posse. Hanc vero reuera es. se reductam, ita demonstramus.

A) Si ponitur $\sqrt{ } D+b=a^{\prime}=p, \pm a^{\prime}-$ $(\sqrt{ } D-b)=q, \sqrt{ } D-b=r$, hi $p, q_{0} v \operatorname{ex}(2)$ supra et defin. formae reductae erunt positiui. Porro ponatur $b^{\prime}-\left(\sqrt{ } D F a^{\prime}\right)=q^{\prime}, \sqrt{ } D-b^{\prime}=$ $\sigma^{\prime}$ eruntque $q^{\prime},{ }^{\prime \prime}$ positiui, quia $b^{\prime}$ iacet inter $\sqrt{ } D$ et $\sqrt{D} \mp a^{\prime}$. Denique sit $b+b^{\prime}= \pm$ $m a^{\prime}$ eritque $m$ integer. Iam patet esse $p+q^{\prime}$ $=b+b^{\prime}$, adeoque $b+b^{\prime}$ siue $\pm m a^{\prime}$ positiuum, et proin etiam $m$; vnde sequitur $m-\mathbf{r}$ certe non esse negatiuum. Porro fit $r+q^{\prime}$ $\pm m a^{\prime}=2 b^{\prime} \pm a^{\prime}$, siue $2 b^{\prime}=r+q^{\prime} \pm(m s$ - I) $a^{\prime}$, vnde $2 b^{\prime}$ et $b^{\prime}$ necessario erunt positiui. Et quoniam $b^{2}+d^{d}=\sqrt{ } D$, erit $b^{2}<\sqrt{ } D$.

B) Porro fit $r \pm m a^{\prime}=\sqrt{D}+b^{\prime}$, siue $\pm(m \quad x) a^{\prime}=\sqrt{ } D+b^{b} \mp a^{\prime} ;$ quare $\sqrt{ } D$ $+b^{\prime} \mp a^{\prime}$ erit positiuus. Hinc et quoniam $\pm a^{\prime}-\left(\sqrt{ }-b^{\prime}\right)=q^{\prime}$, adeoque positiuus, $\mp a^{\prime}$ iacebit inter $\sqrt{ } D+b^{\prime}$ et $\sqrt{ } D-b^{\prime}-$ Quocirca $\left(a^{\prime}, b^{b}, c^{\prime}\right)$ erit forma reducta.

Eodem modo demonstratur, si fiat ${ }^{\prime} c=a$, $" b \equiv-b$ (mod. $c$ ) et inter $\sqrt{ } D$ et $\sqrt{ } D \pm t c$ situs, ${ }^{\prime} a=\frac{w^{\prime} b-D}{c}$, formam $\left({ }^{\prime} a, b, c c\right)$ fore reductam. Manifesto autem forma haec formae $(a, b, c)$ a parte prima est contigua, aliaque 
reducta praeter $(c, b, c)$ hac proprietate prae. dita esse non poterit.

Ex. Formae reductae $(5$, i -14$)$, cuius determinans $=\mathrm{rg} \mathrm{r}$, a parte vltima contigua reducta ( $-14,3,13)$, a parte prima vero haec $(-22,9,5)$.

7) Si formae reductae $(a, b, c)$ a parte vltima contigua est reducta $\left(a^{\prime}, b^{\prime}, c^{\prime}\right)$ : reductae $(c, b, a)$ contigua erit a prima parte forma $\left(c^{\prime}, b^{\prime}, a\right)$; et si reductae $(a, b, c)$ a prima parte contigua est forma $\left({ }^{\prime} a,{ }^{\prime} b,{ }^{\prime} c\right)$; reductae $(c, b, a)$ reducta $\left({ }^{\prime} a,{ }^{\prime} b,{ }^{\prime} a\right)$ contigua erit ab vltima parte. Porro etiam formae $\left(-a, b,-{ }^{\prime} c\right),(-a, b,-c)$, $\left(-a^{\prime}, b^{\prime},-c^{\prime}\right)$ reductae erunt, et secunda primae; tertia secundae ab vitima parte contiguae, siue prima secundae, secundaque tertiae a parte prima; similiterque tres formae $\left(-c^{\prime}, b^{\prime}\right.$ $\left.-a^{\prime}\right),(-c, b, a),\left(-{ }^{\prime} c, b,-a\right)$. Haec tam obuia sunt rt explicatione non egeant.

185. Multitudo omnium formarum re. ductarum determinantis dati $D$ semper est finita, ipsae vero duplici modo inueniri pos. sunt, Designemus indefinite omnes formas re. ductas determinantis $D$ per $(a, b, c)$, ita vt omnes valores ipsorum $a, b, c$ determinare oporteat.

Methodus prima. Accipiantur pro a omnes numeri (tum positiue, tum negatiue) minores quam $2 \sqrt{ } I$ quorum residuum quadraticum $D_{3}$, et pro singulis of ponatur $b$ aequalis omn:P 2 
bus valoribus positiuis expr. $\sqrt{ } D$ (mod. $a$ ) inter $\sqrt{ } D$ et $\sqrt{ } D=a$ iacentibus, $c$ vero pro singulis valoribus determinatis ipsorum $a, b$, ponatur $=\frac{b b-D}{a}$. Si quae formae hoc modo oriuntur, in quibus $\pm a \operatorname{extra} \sqrt{ } D+b$ et $\sqrt{D}-b$ situs est, reiiciendae sunt.

Methodus secunda. Accipiantur pro b omnes numeri pósitiui minores quam $\sqrt{ } D$, pro singulis $b$ resoluatur $b b-D$ omnibus quibus fieri potest modis in binos factores qui neglecto signo inter $\sqrt{ } D+b$ et $\sqrt{ } D-b$ iaceant, ponaturque alter $=a$, alter $=c$. Manifestum est, singulas resolutiones in factores praebere binas formas, quia vterque factor tum $=$ $a$, tum $=c$ poni debet.

Ex. Sit $D=79$ ertintque valores ipsius $a$ viginti duo $F \mathrm{r}, 2,3,5,6,7,9,10,13,10,15$. Vnde inueniuntur formae vndeuiginti: $(x, 8,-$ I5), $(2,7,-15),(3,8,-5),(3,7,-10),(5$, $8,-3),(5,7,-6),(6,7,-5),(6,5,-9)$, $(7,4,-9) ;(7,3,-10),(9,5,-6),(9,40$ $-7),(10,7,-3),(10,3,-7),(13,1,-$ $6),(14,3,-5),(15,8,-1),(15,7,-2)$, $(15,2,-5)$, totidemque aliae quae fint ex his si terminorum exterorum signa commutantur, puta $(-1,8,25),(-2,7,15)$ etc. ita vt omnes triginta octo sint. Sed ex his reiiciendae sex $( \pm 13,1, \mp 6),( \pm 14,3, \pm 5),(\mp 15,2$, \pm 5 ); reliquae triginta duae omnes reductas amplectuntur. Per methodum secundam eae- 
dem forma prodeunt sequenti ordine $\left.{ }^{*}\right):( \pm 7$; $3, \mp 10),( \pm 10,3, \mp 7),( \pm 7,4, \mp 9)$, ( $\pm 9,4, \mp 7),( \pm 6,5, \mp 9),( \pm 9$, $5, \mp 6),( \pm 2,7, \mp 15),( \pm 3,7, \mp 10)$, ( $5,7, \mp 6),( \pm 6,7, \mp 5),( \pm 10,7, \mp 3)$ $( \pm 15,7, \mp 2),( \pm 1,8, \mp 15),( \pm 3,8, \mp$ 5), $( \pm 5,8, \mp 3),( \pm 15,8, \mp 1)$.

186. Sit $F$ forma reducta determinantis $D$, ipsique ab vltimo parte contigua forma reducta $F^{\prime}$; huic iterum ab vltima parte contigua reducta $F^{\prime \prime}$, reducta $F^{\prime \prime}$ ipsi $F^{\prime \prime}$ contigua ab vltima parte etc. Turn patet, omnes formas $F^{\prime}, \vec{F}^{\prime \prime}, F^{\prime \prime \prime}$ etc. esse prorsus determinatas, et tum inter se tum formae $F$ proprie aequiualentes. Quoniam vero multitudo omnium for marum reductarum determinantis dati est finita, manifestum est, omnes formas in progressione infinita $F, F^{\prime}, F^{\prime \prime}$ etc. diuersas esse non posse, Ponamns $F^{\mathrm{m}}$ et $F^{\mathrm{m}+{ }^{\mathrm{n}} \mathrm{n}}$ esse identicas, eruntque $F-x, F+n-I$ reductae, eidem for mae reductae a parte prima contiguae, adeoque identicae; hinc eodem modo $F^{\mathrm{m}-2}$ et $F^{\mathrm{m}}+\mathrm{n}-2$ etc. tandenque $F$ et $F^{\text {n }}$ identicae erunt. Quare in progressione $F, F^{\prime}, F^{\prime \prime}$ etc., si modo sa. tis longe continuatur, necessario tandem forma prima $F$ recurret; et si supponimus $F^{\mathrm{n}}$ esse primam identicam cum $F$, siue omnes $F^{\prime}, F^{\prime \prime}$... $F^{\mathrm{n}}-\mathrm{I}$ a forma $F$ diuersas: facile perspicitur, omnes formas $F, F^{\prime}, F^{\prime \prime} \ldots F^{n-1}$ diuersas fore.

7) Pro $b=$ I, -28 in duos facteres qui neglecto signo inter $\sqrt{ } 79+1$ et $\sqrt{ } 79-1$ iaceant, resolui nequit; quare hic valor est pratereundus, ex eademque ratione valores 2 et 6 .

$$
\text { P } 3
$$


Complexum harum formarum vocabimus perio. dum formae $\vec{F}$. Si igitur progressio vltra vltimam periodi formam producitur, eaedem formae $E, F^{\prime}, F^{\prime \prime}$ etc. iterum prodibunt, progressioque tota infinita $F, F^{\prime}, F^{\prime \prime}$ etc. constituta erit ex hac periodo formae $F$ infinities repetita.

Progressio $F, F^{\prime}, F^{\prime \prime}$ etc. etiam retro continuari potest, praeponendo formae $F$ reductam ' $F$, quae ipsi a parte prima est contigua; huic iterum reductam " $F$, quae ipsi a prima parte contigua etc. Hoc modo habebitur pro: gressio formarum vtrimgque infinita

$$
\ldots .{ }^{\prime \prime} F_{9} \| F, ' F, F, F^{\prime \prime}, F^{\prime \prime}, F^{\prime \prime \prime} \circ . .
$$

perspicieturque facile, $T$ identicam fore cum $F^{\mathrm{n}-\mathrm{I}}$, $F$ cum $F^{\mathrm{n}-2}$ etc adeoque progressionem etiam a laeua parte e periodo formae $F_{\text {, }}$ infinities repetitä, esse conslitutam.

Si formis $\dot{F}, F$ ', $F$ ", etc. ' $F, " F$, etc. aribuuntur indices $0, \mathrm{I}, 2$ etc., $-\mathrm{I},-2$ etc. generaliterque formae $F_{\mathrm{m}}$ index $m$, formae $m F$ index $-i n$, patet, formas quascunque seriế identicas fore vel diutersas, prout ipsarum indices congrui sint vel incongrui secundum modulum $\%$.

Ex. Periodus formae $(3,8,-5)$ cuius determinans $=79$, inuenitur haec: $(3,8-5)$ $(-5,7,6),(6,5,-9),(-9,4,7),(7,3$, - IC), $(-10,7,3)$. Post vltimam iterum prodit $(3,8,-5)$. Hic itaque $n=6$.

187. Ecce quasdam obseruationes generales circa has periodos. 


\section{$-23 x-$}

I) Si formae $F, F$; $F{ }^{\prime \prime}$ etc.; $F, " F$, d $F$ etc. ita exhibetur: $\left(a, b,-a^{\prime}\right),\left(-a^{\prime}, b^{\prime}, a^{\prime \prime}\right),\left(a^{\prime \prime}\right.$, $\left.b^{\prime \prime},-a^{\prime \prime \prime}\right)$ etc.; ( $\left.{ }^{\prime} a, " b, a\right)$, (" $\left.a, " b,-{ }^{\prime} a\right)$, $(-" \prime a, " b, " a)$ etc: omnes $a, a^{\prime}, a^{\prime \prime}, a^{\prime \prime \prime}$ etc. "a, " $a$, "'a etc, eadem signa habebunt (art. I84, I), omnes vero $b, b^{\prime}, b^{\prime \prime}$ etc. $' b_{2}$. positiui.

2) Hinc manifestum est, numerum $n$ (muld tirudinem formarum ex quibus periodus formae $F$ constat) semper esse parem. Etenim terminus primus formae cuiusuis $F^{\mathrm{m}}$ ex hao periodo manifesto idem signum habebit vti terminus primus formae $F$, si m est par, oppositum, si est impar. Quare quum $F_{\text {n }}$ et $F$ identicae sint, n necessario erit par.

3) Algorithmüs per quem numeri $b^{\prime}$, $b^{\prime \prime}$, $b^{\prime \prime \prime}$ etc., $a^{\prime \prime}, a^{\prime \prime \prime}$ etc. inueniuntur, ex art. 184, 6 est hic:

inter limites:

$$
\begin{aligned}
& \sqrt{D} \text { et }
\end{aligned}
$$

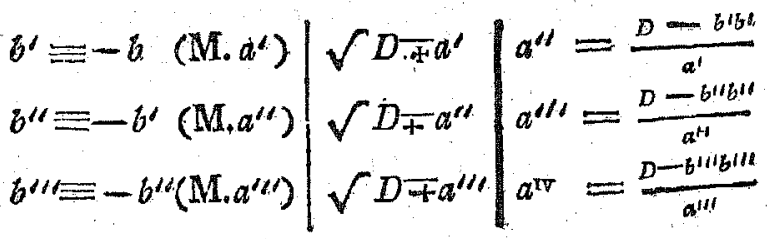

vbi in columna secunda signa superiora vel inferiora sunt accipienda, prout $a, a^{\prime}, a^{\prime \prime}$ etc. sunt positiui vel negatiui. Loco formularum in coP. 


\section{- $232-$}

lumna tertia etiam sequentes adhiberi possunt, quae commodiores euadunt, quando $D$ est numerus magnus:

$$
\begin{aligned}
& a^{\prime \prime}=\frac{b+b^{\prime \prime}}{a^{\prime}}\left(b-b^{\prime}\right)+a \\
& a^{\prime \prime \prime}=\frac{b^{\prime}+b^{\prime \prime}}{a^{\prime \prime}}\left(b^{b}-b^{\prime \prime}\right)+a^{b} \\
& a^{\mathrm{iv}}=\frac{b^{\prime \prime}+b^{\prime \prime}}{a^{\prime \prime \prime}}\left(b^{\prime \prime \prime}-b^{\prime \prime \prime}\right)+a^{\prime \prime} \text { etc. }
\end{aligned}
$$

4) Forma quaecunque $F^{m}$, in periodo formae $F$ contenta, proprie eandem periodum habet vt $F$. Scilicet periodus illa erit. $F \mathrm{~m}, F \mathrm{~m}+\mathrm{x}$ .... $F^{n-1}, F, F^{\prime} \ldots F^{\mathrm{m}-\mathrm{r}}$, in qua eaedem formae esdemclue ordine occurrunt, vt in perio. do formae $F$, et quae ab hac tantummodo respectu initii et finis discrepat.

5) Hinc patet, omnes formas reductas eiusdem delerminantis $D$ in periodos distribui posse. Accipiatur aliqua harum formarum, $F$, ad libitum inuestigeturque ipsius periodus, $F, F^{\prime}, F^{\prime \prime}$ $\ldots F^{n-1}$, quam designemus per $P . \quad S i$ haec omnes formas reductas determinantis $D$ nondum amplectitur, sit aliqua in ipsa non contenta $G$ huiusque periodus $Q$. Tum patet $P$ et $Q$ nullam formam communem habere posse; alioquin enim etiam $G$ in $P$ contenta esse deberet periodique oninino coinciderent. Si $P$ et $Q$ omnes formas reductas nondum exhauriunt, aliquáz ex deficientibus, $B$, periodum tertiam, $R$, suppeditabir, quae neque cum $P$ neque cum $Q$ fornam communem habebit. Hoc modo continuare possumns, vsquedum omnes formae re- 


\section{$-\quad 233$}

ductae sint exhaustae. Ita $\ell_{\text {. }} g$. omnes formae reductae determinantis 79 in sex periodos distribuuntur:

I. $(1,7,-15),(-15,7,2),(2,7,-15),(-15,8,1)$.

II. $(-1,8$, I 5$),(15,7,-2),(-2,7,15),(15,8,-$ I). III. $(3,8,-5),(-5,7,6),(6,5,-9),(-9$, $4,7),(7,3,-10),(-10,7,3)$.

IV. $(-3,8,5),(5,7,-6),(-6,5,9)$, $(9,4,-7),(-7,3,10),(-10,7,-3)$.

V. $(5,8,-3),(-3,7$, 10), $(10,3,-7),(-$ $7,4,9),(9,5,-6),(-6,7,5)$.

VI. $(-5,8,3),(3,7,-10),(-10,3,7)$, $(7,4,-9),(-9,5,6),(6,7,-5)$.

6) Vocemus formas socias, quae ex iisdem terminis constant, sed ordine inuerso positis; vt $\left(a, b,-a^{\prime}\right),\left(-a^{2}, b, a\right)$. Tum facile perspicitur ex art. 184, 7 , si periodus formae reducrae $F$ sit $F, F^{\prime}, F^{\prime \prime} \ldots \ldots F^{2 n-\text { x }}$, formae $F$ so. cia $f$ formisque $F^{2 \mathrm{n}-1}, F^{2 \mathrm{n}-2} \ldots F^{\prime \prime}, F^{\prime}$ resp. sociae sint formae $f^{\prime}, f^{\prime \prime}, \ldots f^{\mathrm{n}-2}, f^{\mathrm{n}-\mathrm{x}}$ : periodum formae $f$ fore $f, f^{f}, f^{\prime}, \ldots f^{n-2}, f^{n-x}$, adeoque ex totidern formis constare, vt perio. dum formae $F$. Periodos formarum sociarum vocabinus periodos socias. Ita in exemplo nostro sociae sunt periodi III et VI; IV et $\mathrm{V}$.

7) Sed fieri etiam potest, vt forma $f$ ipsa in periodo sociae suae $F$ occurrat, vti in ex. nostro in periodo I et $I$, adeoque periodus. P 5 


\section{- $234-$}

formae $F$ cum periodo formae $f$ conneniat, siue vt periodus format $F$ sibi ipsi sit socia. Quoties hoc euenit, in hac periodo duat: fimmats ancipites inuenientur Ponamus enim juriodum formae $F$ constare e su formis sine $F$ ot $F^{2}$ esse identicas; porro sit $2 m+1$ index formae $f$ in periodo formae $F^{*}$ ), siue $F a m+1$ et $F$ sociae. Tum patet etiam $F^{\prime}$ et $F^{2 m}$ fore socias nec non $F^{\prime \prime}$ et Frm-1 etc, adeoque etiam Fra et $F^{\mathrm{n}} \mathrm{t}^{+}$. Sit $F^{\mathrm{m}}=\left(a^{\mathrm{m}}, b^{\mathrm{m}},-a^{\mathrm{m}+1}\right), F^{m+1}=$ $\left(-a^{m+1}, b^{\mathrm{m}-m}, a^{\mathrm{m}+}+3\right)$. Tum erit $b^{\mathrm{m}}+b^{\mathrm{m}+\mathrm{s}}=0$, (mod. $a^{m+1}$; ex dofin. formarum sociarum vero erit $b^{m}=b^{m+1}$ atque hinc $2 b^{\mathrm{m}}+1=0$ (mod. $\left.a^{m+1}\right)$, sine formae $F^{n+}$ anceps. - Eodem modo $F^{2 m+1}$ et $F^{2 n}$ erunt sociae; hine $F^{2 m+2}$ et $F^{2 n-1} ; F^{2 m-3}$ et $F^{2 n-2}$ etc. tandernque $F^{m-n}$ et $F^{m}+n x^{x} x$, quarum posterior erit anceps, vti per simile ratiocinium facile prohatur. Quia vero $m+\mathrm{r}$ et $m+n+1$ sechur dum mod. $2 n$ sunt incongrui, lormae $F^{\text {wa }}+\mathrm{t}$ et

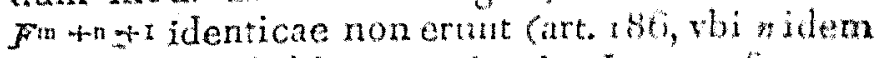
denotat, quod hic 2n). Ita in I sumt forma ancipites $\left(1,8,-x^{5}\right),(9,7,-15)$, in ll vero $(-1,8,15),(-2,7,15)$.

3) Vice versa, quatuis periadus, in quat forma anceps occurnit, sibi ipsi sotia est. Facile exim perspicitur, si $F^{\mathrm{m}}$ sit. forma redurata anceps: formam "ipsi sociam, (quae etiam est reducta), simul ipsi a parte prima contiguam esse, i. e. $F^{n-z}$ et fom socias. Tum vero to-

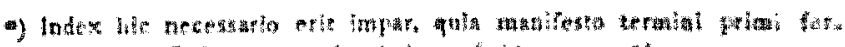

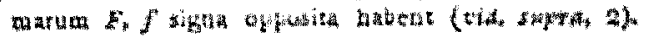




\section{$-235$}

ta periodus sibi ipsi socia erit. - Hinc patet, fieris mon posse, vt unica tantum forma anceps in periodo aliqua contenta sit.

9) Sed etiam ptures quam duas in eadem periodo isse nequetrt. Ponamus enim in periodo formate $F$, ex an formis constante, tres formas. ancipites dari $F^{\lambda}, F^{\mu}, F^{\prime}$, ad indices $\lambda, \mu, v$ reepectiue pertinentes, ita vt $\lambda, \mu, v$ sint numeri inaciuales inter limites $o$ et $2 n-x$ (incl.) siti. Tum formae $F^{\lambda-1}$ et $F^{\lambda}$ erunt sociae; similiterque $F^{\lambda-2}$ et $F^{\lambda+1}$ etc. tandemque $F$ et $F_{2} \lambda-\mathrm{r}$. Ex eadem ratione $F$ et $F^{2 \mu-1}$ sociae erunt, nec non $F$ et $F^{2}-1$; quare $F^{2 \lambda}-1, F^{2 k-x}$, $F^{2}{ }^{2}-1$ identicae, indicesque $2 \lambda-r, 2 \mu-i r$, $2 s$ - I secundum modultm $2 n$ congrui erunt, et proin etiam $\lambda \equiv \mu \equiv{ }(\bmod , n)$. Q. E. A. quia manifesto inter limites o et $2 n-\mathrm{r}$ tres numeri dixersi secundum modulum $*$ congrui iacere nequennt.

x88. Quum oxnes formae ex eadern periodo proprie sint aequilualentes: quaestio oritur, annon etiam formae e periodis diuersis proprie aequiualentes esse possint. Sed ante: quam ostendamus, hoc esse impossibile, quaedam de transformatione formarum reductarum sunt exponenda.

Quoniam in sequentibus de formartm iransformationibus persaepe agendum erit; vt prolixitatem quantum fieri potest euitemus, sequenti scribendi compendio abhinc semper vtemur. Si forma aliqua $L X X+2 M X Y+$ 
NY $X$ per substitutionem $x=\alpha x+b y, x=$ $2 x+b y$ in formam $l x x+2 m x y+n y y$ transformatur: simpliciter dicemus, $(L, M, N)$ transformari in $(i, m, n)$ per substitutionem $\alpha, C_{,}, \delta_{0}$. Hoc modo opus non erit, indeterminatas formarum singularum, de quibus agitur, per signa propria denotare. - Palam vero est, indeterminatam primam a secunda in quauis forma probe distingui debere.

Proposita sit forma reducta $\left(a, b,-a^{\prime}\right) \ldots$ $f$, determinantis $D$. Formetur simili modo vt in art. $\mathrm{x} 66$ progressio formarum reductarum vtrimque infinita, .... ${ }^{\circ} f, f^{\prime} f, f, f^{\prime}, f^{\prime \prime}, \ldots$, et quidem sit $f^{\prime}=\left(-a^{\prime}, b^{\prime}, a^{\prime}\right), f^{\prime \prime}=\left(a^{\prime \prime}, b^{\prime \prime}\right.$, - $\left.a^{\prime \prime \prime}\right)$ etc.; $f=(-a, " b, a), "{ }^{\prime} f=\left({ }^{\prime \prime} b\right.$ $" b,-a)$ etc Ponatur $\frac{b+b^{\prime}}{-a^{\prime}}=h^{\prime}, \frac{b^{\prime}+b^{\prime \prime}}{a^{\prime \prime}}=h^{\prime \prime}$,

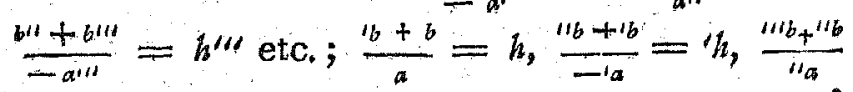
${ }^{\prime \prime h}$ etc. Tum patet, si ( $\mathrm{vt}$ in art. 177) numeri $\alpha^{\prime}, a^{\prime \prime}, a^{\prime \prime \prime}$ etc. $6^{\prime}$, $b^{\prime \prime \prime}$, $b^{\prime \prime \prime}$ etco etc. formentur secundum algorithmum sequentem

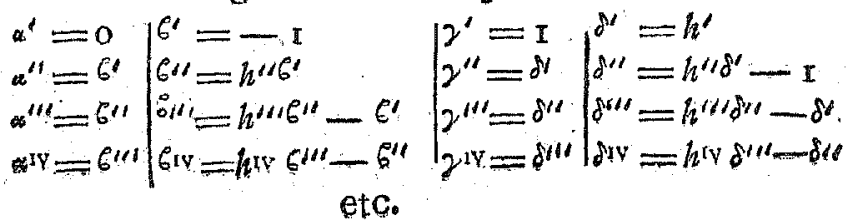

$f$ transformatum iri

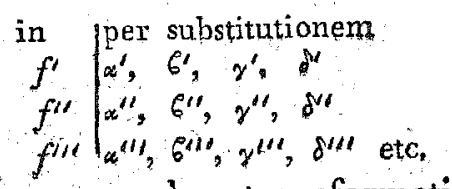

omnesque has transformationes fore proprias. 


\section{$-\quad 237 \quad-$}

Quum of transeat in $f$ per substitutionem. propriam $0,1,1, h$ (art. 161 ) : $f$ transibit in of per subst. propr. $h, I,-\mathrm{I}$, o. Ex simili ratione 'f transibit in "f per subst. propr. ' $h, r$, $-1,0$; "f $f$ in " $f$ per subst. pr. " $h, 1,-1, o$ etc. Hinc per art. 159 eodem modo vt art:

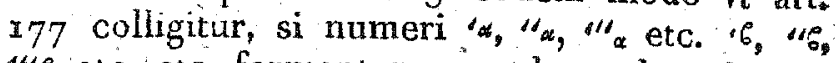
"G etc. etc. formentur secundum algorithmum sequentem

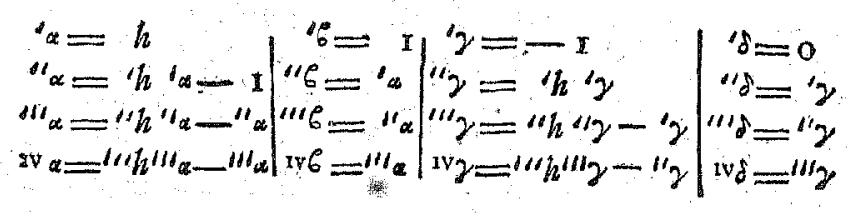

etc.

$f$ transformatum siri

$$
\begin{aligned}
& \text { in per substitutioneme } \\
& \text { of } \begin{array}{l}
\text { of } \\
\text { off }
\end{array}
\end{aligned}
$$

omnesque has transformationes fore proprias.

Si ponitur $a=1, b=0, y=0, \delta=1$ : hi numeri eandem relationem habebunt ad formam $f$, quam habent $a^{\prime}, b^{\prime}, 2^{\prime}, \nu^{\prime}$ ad $f^{\prime} ; a^{\prime \prime}, b^{\prime \prime}$,

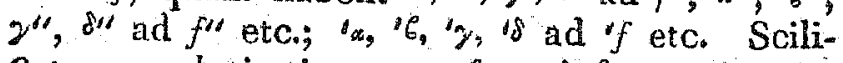
Cet per substitutionem $\alpha, \epsilon, 2, \delta$ forma $f$ transibit in $f_{0}$ Tum vero progressiones infinitae $\alpha^{\prime}$, $\alpha^{\prime \prime}, \alpha^{\prime \prime \prime}$ etc., ' $\alpha,{ }^{\prime} \alpha_{\alpha},{ }^{\prime \prime}{ }_{\alpha}$ etc., per intercalationem terminis $\alpha$, concinne iungentur ita vt tram continuam vtrimque infinitam constituere concipi possint secundum eandem legem vbique pro- 


\section{$-238 \quad-$}

gredientem ... $\|^{\prime \prime}, "{ }_{\alpha,},{ }^{\alpha} x, \alpha_{1} \alpha^{\prime}, \alpha^{\prime \prime}, \alpha^{\prime \prime \prime}, \ldots$ Lex progressionis haec est: $" \|_{\alpha}+\alpha_{\infty}=" h{ }^{\prime} \infty,{ }_{\infty}$ $+\alpha=h^{\prime} \alpha, \alpha_{\alpha}+\alpha^{\prime}=h \alpha, \alpha+\alpha^{\prime \prime}=h^{\prime} \alpha^{\prime} \alpha^{\prime}+$ $\alpha^{\prime \prime \prime}=h^{\prime \prime} \alpha^{\prime \prime}$ etc., siue generaliter (si indicem negatiuum a dextra scriptum idem designare supponimus, vt positiuum a laeua) $a^{m-x}+a^{m+1}=$

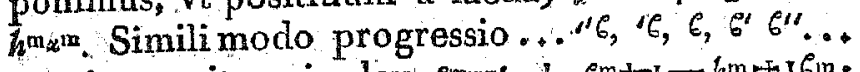
continua erit, cuius lex $6^{\mathrm{m}-1}+6^{\mathrm{m}+\mathrm{x}}=h^{\mathrm{m}+16 \mathrm{~h}^{\mathrm{n}}}$; et proprie cum praecedente identica, omnibus terminis vmo loco promotis, $6^{\prime \prime}={ }^{\circ} *{ }^{\circ} 6=\alpha, 6$. $=\alpha^{\circ}$ etc, Lex progressionis continuae ...." $\gamma, \gamma, \gamma^{\prime}, \gamma^{\prime \prime} \ldots \ldots$ erit haec $2^{m-x}+\gamma^{m+3}=$ $h^{\mathrm{m}} \mathrm{gm}^{\mathrm{m}}$, et lex huius... " $\delta, \&, \delta, \delta, \delta \mu_{0} .$. erit $\delta^{\mathrm{m}-\mathrm{r}}$ $+\delta^{m+1}=h^{m+r o m}$ insuperque generaliter $\delta^{m}=$ $2^{m+1}$

Ex. Sit forma proposita $f$ haec $(3,8,-5)$ quae transformabitur

in formam per substitutionem

wi $f(-10,7,3) \mid-805,-152,+145,+27$

$\operatorname{vrf}(3,8,-5)-52,+45,+27,-S$

$|v f|(-5,7,6) \mid+45,+17,-8,-3$

Ivf $(6,5,-9)+17,-11,-3,+2$

"ff $(-9,4,7)-11,-6,+2,+\pi$

"f $f(7,3,-10)-6,+5,+1,-\tau$

$f \mid(-10,7,3)+5,+I_{1}-I_{0} \quad 0$

$f^{\prime}|(3,8,-5)|+1,-0,0,+1, \quad 0,-1,-3$

$f^{\prime \prime}(6,5,-9) \mid-\mathrm{I},-2,-3,-7$

$f^{\prime \prime \prime}(-9,4,7)-2,+3,-7,+10$

fiv $(7,3,-10)+3,+5,+10,+17$

$f_{0}(-10,7,3+5,-8,+17,-27$

$f \times 1(38-5)-8,-45,-27,-152$ $f_{\mathrm{vu}}(-5,7,6) \mathrm{f}-45,+45,-152,+483$

etc. 


\section{$-\quad 239$}

189. Circa hunc algorithmum sequentia sunt annotanda,

I) Omnes $a$, $a^{\prime} a^{i t}$ etc, ' $a$, "a etc. eadèm signa habebunt; omnes $b, b^{\prime}, b^{\prime \prime}$ etc. ' $b$, " $b$ btc. erunt positiui ; in progressione .... $/ h, h, h$, $h^{\prime}, h^{\prime \prime} \ldots$... signa alternabunt, scilicet si omnes $a, a^{\prime}$ etc. sunt positiui, $h^{\mathrm{m}}$ vel $\mathrm{m} h$ erit positiuus quando $m$ est par, negatiuus quando $m$ impar ; si vero $a, a^{\prime}$ etc. sunt negatini, $h^{\mathrm{m}}$ vel $\mathrm{m} h$ pro on pari erit negatiuus, pro impari positiuus.

2) Si $a$ est positiuus adeoque $h^{\prime}$ negatiuus, $h^{\prime \prime}$ positiuus etc, erit $a^{\prime \prime}=-1$ neg., $\alpha^{\prime \prime l}=$ $h^{\prime \prime} \alpha^{\prime \prime}$ neg. et $>\alpha^{\prime \prime}\left(\mathrm{vel}=\alpha^{\prime \prime}\right.$ si $\left.h^{\prime \prime}=x\right)$; $\alpha^{\mathrm{IV}}$ $=h^{\prime \prime \prime \alpha^{\prime \prime \prime}}$ - " pos et $>\alpha^{\prime \prime \prime \prime}$ (quia $h^{\prime \prime \prime} \alpha^{\prime \prime \prime}$ pos., $\alpha^{\prime \prime}$ neg.) $\alpha^{\mathrm{v}}=h^{\text {2v }} \alpha^{\text {av }}-\alpha^{\prime \prime \prime}$ pos, et $>a^{\text {IV }}$ (quia hiv alv pos) etc. Hinc facile concluditur, pro. gressionem $\alpha^{\prime}, \alpha^{\prime \prime}, \alpha^{\prime \prime \prime}$ etc. in infinitum crescere duoque signa positiva semper duo negatiua excipere ita $\mathrm{vt}$ am habeat signum,,++- , - prout $m \equiv 0,1,2,3(\bmod \cdot 4)$ - Si a est negatiuus, per simile ratiocinium inuenitur $a^{\prime \prime}$ neg., $\alpha^{\prime \prime \prime}$ pos. et vel $>$ vel $=\alpha^{\prime \prime} ; \alpha^{10}$ pos. $>\alpha^{\prime \prime \prime}$;

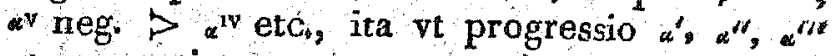
etc. continuo crescat, signumque termini m sit t,,,--+ prout $m \equiv 0,1,2$, 5 (mod, 4)

3) Hoc modo inuenitur, omnes quatuor progressiones infinitas $a^{\prime}, a^{\prime \prime}, a^{\prime \prime \prime}$ etc. $\gamma_{2}^{\prime} \gamma^{\prime} \gamma^{\prime \prime}$

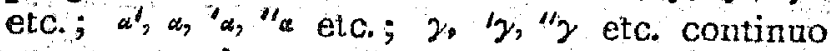
crescere, adeoque etiam sequentes cum illis identicas : $6,6^{\prime}, 6^{\prime \prime}$ etc. $; \delta, \delta, \delta^{\prime \prime} \delta^{\prime \prime}$ etc.; $6,6_{t} 6_{t}^{\prime \prime} 6$ etc.; $d$, "d'etco; et, prout $m \equiv 0,1,2,3(\bmod$ i), 
signum ipsius $a^{\mathrm{m}}+ \pm- \pm$; ipsius $6 \mathrm{~m}, \pm$ $-\mp+$ ipsius $\mathrm{rm}^{\mathrm{m}}, \pm+\mp-$; ipsius $\mathrm{sm}$. $+\mp- \pm$ ipsius $m_{\alpha}+ \pm-F$; ipsius $m b, F$ $+ \pm-;$ ipsius $\mathrm{mg}_{\mathrm{g}} \mp- \pm+$; ipsius $\mathrm{ng}_{\mathrm{g}}$ $+\mp- \pm$ valentibus superioribus quando a est positiuus, inferioribus quando a negatiuus: Teneatur imprimis haec proprietas: Designante $m$ indicem quemcunque positiuum, $\alpha^{\mathrm{m}}$ et $\gamma^{\mathrm{m}}$ habebunt eadem signa quando a positiuus, opposita quando $\dot{a}$ negatiuus, similiterque $f^{m}$ et $\delta^{m}$ contra; $\mathrm{m}_{a b}$ et $\mathrm{m}_{\gamma}$, vel me et mó habebunt eadem signa quando a negatiuus, opposita quando a positiuus.

4) In signis art. 32 magnitudo ipsorum etc. concinne ita exhiberi potest, ponendo $\mp h^{\prime}=k^{\prime}, \pm h^{\prime \prime}=k^{\prime \prime}, \mp h^{\prime \prime \prime}=k^{\prime \prime \prime}$

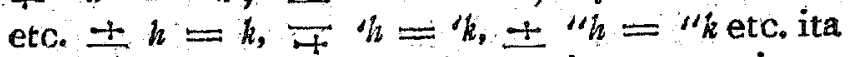
vt omnes $k^{\prime}, k^{\prime \prime}$ etc. $k_{g} \cdot k$ etc. sint numeri positiui: $a^{\mathrm{m}}= \pm\left[k^{\prime \prime}, k^{\prime \prime \prime}, k^{\mathrm{wv}} \ldots k^{\mathrm{m}-\mathrm{x}}\right] ; b_{\mathrm{m}}= \pm$ $\left[k^{\prime \prime} k^{\prime \prime \prime}, k^{1 \mathrm{v}} \ldots . k^{\mathrm{m}}\right] ; 2^{\mathrm{m}}= \pm\left[k^{\prime}, k^{\prime \prime}, k^{\prime \prime} \ldots \ldots\right.$ $\left.k^{\mathrm{m}-1}\right] ; \delta \mathrm{m}= \pm\left[k^{\prime}, k^{\prime \prime}, k^{\prime \prime} \ldots \ldots k^{\mathrm{m}}\right] ; \mathrm{m}_{\alpha}=\mp[k$, $\left.k_{k}, " k, \ldots \mathrm{m}-1 k\right] ; \mathrm{m} \varphi= \pm[k, \alpha, " k, \ldots \mathrm{m}-2 k]$;

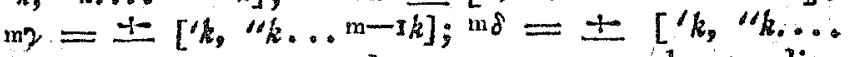
$m-2 k]$; signa vero ad praecepta modo tradita determinari debent. Secundum has formulas, quarum demonstrationem propter facilitatem omittimus, calculus semper expeditissime $a b$ solui poterit.

I 9o. Lemma, Designantibus $m, \mu, n^{\prime}, n_{3}, n^{\prime}$ numeros integros quoscunque, ita tamen vt trium poo

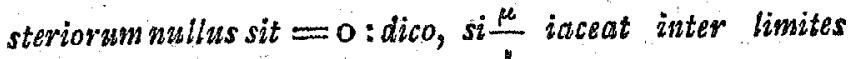
$\frac{m}{n}$ et $\frac{m^{\prime}}{s^{\prime}}$ exclusiue, atque sit $m n^{\prime}-n m^{\prime}=F_{1}, d \ell$ nominatorem, fore maiorem quam net $n^{\prime}$ 。 
Dem. Manifesto $\mu n n^{\prime}$ iacebit inter imn' et sim', adeoque ab vtroque limite minus differet quam limes alter $a b$ altero, i. e. erit mn' - mms $>\mu n n^{\prime}$ - imn et $>\mu n n^{\prime}-v^{\prime} m^{\prime}$, siue , $>n^{*}(\mu n-m)$ et $>n\left(\mu^{\prime}-v m^{j}\right)$. Hinc sequitur, quoniam $\mu n-$ m certe non $=0$ (alioquin enim foret $\frac{\mu}{\nu}=\frac{m}{m}$ contra hyp.), neque $\mu n^{\prime}-m^{\prime}=0$ (ex simili raticne); sed vterque ad minimum $=I$; fore $v>n^{\prime}$ et $\gg$. Q. E. $D$.

Perspicuum itaque est; "non posse esse $=i$, i. e. si fuerit $m n^{\prime}-n m^{\prime}= \pm i$; inter fractiones $\frac{m}{m}, \frac{n^{\prime}}{n^{\prime}}$ nullum numerum integrum iacere posse: Quare etiam cifra inter ipsas iacere nequit, $i_{\text {. }}$. fractiones istae signa oppo . sita habere nequeunt:

ìt. Thronema, $S i$ forma reducta $\left(\vec{a}, \dot{b},-\hat{a}^{\prime}\right)$ determinantis $D$ per substitutionem $\alpha, 6, n, \delta$ transit in reductam $\left(A, B,-A^{\prime}\right)$ eiusdem determinantis i ia cebit, primo, $\frac{ \pm V}{a}$ inter $\frac{\infty}{\gamma}$ et $\frac{6}{d}$ (siquidem neque $\gamma$ neque $\delta=0, i_{0}$. si vterque limes est finitus), ac cepto signo supperiori, quando neuter horum limitum ha: bet signum signo ipisius a opposituni (siue clarius, quando ont vterque idem habet, aut alter idem, alter est $=0$ ), inferiori quaindo neuter habet idem vt $a$; secundo $\pm \frac{V D+b}{a^{\prime}}$ inter $\frac{\dot{y}}{a}$ et $\frac{\delta}{!}$ (siquideni ineque a neque $6=0$ ), sigño suiperioni accepto quando limes neuter signum signo ipsius $a^{\prime}$ (vet a) oppositum habet, inferiori quaria neidter habet iden vt $a^{\prime *}$ ).

क) Manifestum est, alios casus locuni habere non posse, quum ex art. praec. propter $\alpha-6 \gamma= \pm 1$, limites bini neque signi op posita habere, neque simul =o esse possint. 


\section{$-242-$}

Dem. Habentur aequationes aat $+2 b_{a y}-$ $a^{\prime} y_{2}=A . \ldots[1] ; a^{66}+2 b 68-a^{288}=-A^{\prime} \ldots$ [2]. Vnde deducitur

$$
\begin{aligned}
& \frac{a}{2}=\frac{ \pm \sqrt{\left(D+\frac{a A}{2 \gamma}\right)-b}}{a} \ldots \ldots[3] \\
& \frac{6}{b}=\frac{ \pm \sqrt{ }\left(D-\frac{a A^{\prime}}{\delta_{0}}\right)-b}{a} \ldots \ldots[4] \\
& \underline{\gamma}=\frac{ \pm \sqrt{\left(D-\frac{a A}{\alpha \alpha}\right)+b}}{a^{\prime}} \cdots[5] \\
& 5=\frac{\sqrt{c}\left(D+\frac{a^{d}}{6 b}\right)+b}{a^{2}} \ldots[6] .
\end{aligned}
$$

Aequatio $3,4,5,6$ reiicienda erit, si $2, \delta, \infty$, 6 resp. $=0 .-$ Sed dubium hic manet, quae signa quantitatibus radicalibus tribui debeant; hoc sequenti modo decidemus.

Statim patet in [3] et [4] necessario signa superiora accipi debere, quando neque $\frac{x}{2}$ neque $\frac{6}{\delta}$ signum habeat signo ipsius a oppositum; quoniam accepto signo inferiori $\frac{a_{a}}{2}$ et $\frac{a b}{\delta}$. fierent quantitates negatiuae. Quia vero $A$ et $A^{\prime}$ signa eadem habent, $\sqrt{ } D$ cadet inter $\sqrt{ }\left(D+\frac{a A}{\gamma \gamma}\right)$ et $\sqrt{ }\left(D-\frac{a A^{\prime}}{d \delta}\right)$ adeoque in hocce casu $\frac{\sqrt{D-b}}{}$ 
inter $\frac{1}{2}$ et $\frac{6}{8}$ Quare pars prima theorematis pro casu priori est demonstrata.

Eodem modo perspiciur, in [5] et [6] necessario signa inferiora accipi debere, quando neque $\frac{\gamma}{\alpha}$ neque $\frac{\delta}{6}$ signum idem habeant vt $a^{\prime}$ siue $a$, quia accepto superiori $\frac{a y}{\alpha}, \frac{a^{\prime} \delta}{6}$ necessario fierent quantitates positiuae. Vnde proti. nus sequitur $\frac{-\sqrt{D+b}}{a^{l}}$ pro hocce casu iacere inter $\frac{\gamma}{a}$ et $\frac{\delta}{b}$. Demonstrata est itaque etiam pars secunda theorematis pro casu posteriori. Quodsi aeque facile ostendi posset, in [3] et [4] signa inferiora accipi debere, quando neutra quantitatum $\frac{a}{2}, \frac{6}{\delta}$ signum idem habeat vt et in [5] et [6] superiora, quando neque $\frac{2}{a}$ neque $\frac{d}{6}$ signum oppositum : habeat: hinc simili modo sequeretur, pro illo casu $\frac{-V D-6}{}$ iacere inter $\frac{w}{2}$ et $\frac{6}{j}$, pro hoc $\frac{V D+b}{a}$ inter $\frac{\gamma}{a}$ et $\frac{\delta}{6}$, siue pars prima theorematis etiam pro casu posteriori, et secunda pro casu priori demonstratae forent. Sed quum illud difficile quidem non sit, atta. men sire quibusdam ambagibus fieri nequeat, methodum sequentem praeferimus.

Quando nullus numerorum $*, 6,2, \delta=0$, - et $\frac{6}{\delta}$ eadem signa habebunt $y \frac{\partial}{6}, \frac{\delta}{6}$ Quan. Q 2 


\section{- 216}

do itaque neutra larum quantitatum signum idem habet $\mathrm{vt} a^{\prime}$ siue $a$, adeoque $=\frac{\sqrt{D}+t}{a^{\prime}}$ inter

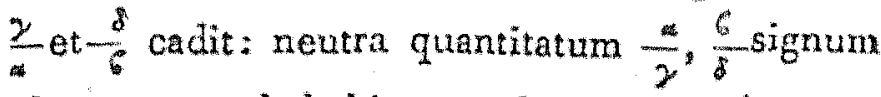
idem vt a habebit, cadetque $=\frac{1}{\sqrt{b+b}}=$ $=\sqrt{ } D-b \quad\left(\right.$ propter $\left.a a^{\prime}=D-b b\right)$ inter $\frac{x}{2}$ et $\frac{C}{b}$. Quare pro eo casu vbi neque nequé $6=0$, pars prima theor. etiam pro casu secundo est demonstrata (nam conditio vt neque 2 neque $\delta=0$, iam in theor. ipso est adiecta). Simili modo, quando nullus numerorum $\neq, 6, \delta=0$, et neque $\frac{\alpha}{\gamma}$ neque $\frac{6}{\delta}$ signum signo ipsius a vel $a^{\prime}$ oppositum habet, adeoque $\frac{\sqrt{b-b}}{a^{\prime}}$ inter $\frac{*}{\gamma}$ et $\frac{6}{y}$ iacet : etiam $\frac{\gamma}{6}$ et signum oppositum signo ipsius a' non habebit, cadetque $\frac{a}{\sqrt{D-b}}=$ $\frac{\sqrt{D+}}{a^{\prime}}$ inter $\frac{\gamma}{2}$ et $\frac{\partial}{6}$. In eo igitur casu vbi neque 2 neque $\delta=0$ pars secunda theor. etiam pro casu secundo est demonstrata.

Nihil itaque superesset quam ve demonstretur, partem primam theor. etiam pro casu secundo locumhabere sialteruter numerorum 6 sit $=0$, et partem secundam pro casu primo si aut $\gamma$ aut $\gamma=0$, At omms hi casus sunt impossibites. Supponamus enim, pro parte prima theor., esse neque $\gamma$ neque $t=0, \frac{*}{2}, \frac{6}{t}$ non habere signum idem vt a atque esse $x$ ).

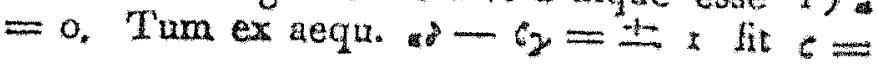


$\pm \mathrm{I}, 2= \pm \mathrm{x}$. Hinc ex $[1] A=-a^{\prime}$, quare $A$ et $a^{\prime}$, adeoque etiom $a$ et $A^{\prime}$ signa opposita habent, vnde fit $\sqrt{ }\left(D-\frac{a A^{2}}{\delta \delta}\right)>\sqrt{ } D>b$. Hinc patet in [4] necessario signum inferius accipi debere, quia accepto superiori $\frac{6}{\delta}$ ma. nifesto signum idem obtineret vt $a_{0}$. Fit itaque $\frac{6}{\delta}>=\sqrt{D-b}>I$ (propter $a<\sqrt{ } D+b$ ex def. formae reductae), Q. E. A. quum $6= \pm$ I, et $\delta$ non $=0 .-2$ ). Sit $6=0$. Tum ex aeqqu. $a \delta-C_{\gamma}= \pm \mathrm{x}$ fit $a= \pm \mathrm{x}, \delta= \pm \mathrm{x}$. Hinc ex [2] $-A^{\prime}=-a^{\prime}$, quare $a$ et $a$ et $A$ signa eadem habebunt, vnde fit $\sqrt{ }(D$ $\left.+\frac{a d}{\alpha x}\right)=\sqrt{ } D p b$. Hinc patet in [3] signum inferius accipi debere, quia accepto superiori $\frac{2}{2}$ signum idem obtineret vt a. Fit itaque $\frac{a}{2}$ $>-\frac{\sqrt{ } D-b}{a}>\mathrm{x}$, Q. E. $A_{\mathrm{a}}$ eadem ratione vt ante. - Pro parte secunda si supponimus neque $\alpha$, neque $6=0 ; \frac{y}{\alpha}, \frac{\partial}{6}$ non habere signum signo ipsius $a^{\prime}$ oppositum atque r) $\gamma=0$ : ex aequ. as $-6 y= \pm$ fit $\alpha= \pm x, \delta= \pm$ s. Hinc ex $[\mathrm{r}] A=a$, quare $a^{\prime}$ et $A^{\prime}$ signa eadem habebunt, wnde fit $\sqrt{ }\left(D+\frac{a^{t} A_{1}}{66}\right)>\sqrt{ } D$ $>b$. Quocirca in [6] signum superius erit accipiendum, quia accepto inferiori, $\frac{8}{6}$ obtineret signum oppositum signo ipsius $a^{\prime}$. Fit igitur

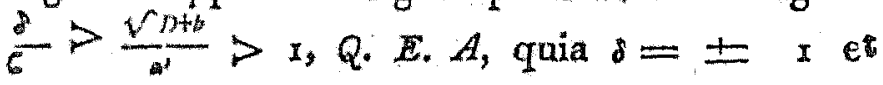
Q 3 


\section{$-266$}

son $=0 . \quad$ Tandem 2) si esset $g=0$, ex $-6 y= \pm$ I fit $6= \pm x, 2= \pm x_{2}$ ad. eoque ex [2]- $A^{+}=a_{0}$. Hinc $\sqrt{\left(D-\frac{a^{\prime} A}{\alpha a}\right)}$ $>\sqrt{ }>b b$, quare in [5] signum superius accipiendum. Hinc $\frac{\partial}{a}>\frac{V^{D+b}}{a^{\prime}}>I$, Q.E.A. Quare theorema in ornni sua extensione est demonstratum;

Quum differentia inter $\frac{a}{2}$ et $\frac{6}{\delta}$ sit $=-\frac{1}{2 \delta^{2}}$ differentia inter $\frac{ \pm D D-b}{\gamma}$ et $\frac{a}{2}$ vel $\frac{b}{\gamma}$ erit $<$ $\frac{x}{2 q}$; inter $\stackrel{ \pm V D}{a}=b$ autem et $\frac{a}{\gamma}$, vel inter ila lam quantitatem et $\frac{6}{\delta}$ nulla fractio iacere poterit, cuius denominator non sit maior quam $\gamma$ aut d (lemma praqc. . . - Eodem modo differentia quantitatis $\frac{ \pm \sqrt{ } D+b}{a}$ a fractione, $\frac{2}{a}$ vel hac $\frac{\delta}{6}$ erit minor quam $\frac{1}{a b}$, et inter illam quantitatem et neutram harum fractionum iacere potest fractio cuius denominator non sit maior quam $\&$ et $\zeta_{0}$

rg2. Ex applicatione theor praec. ad algorithmum art. 188 sequitur, quantitatem $\frac{V D-b}{a}$ quam per $L$ designabimús, iacere inter $\frac{\alpha^{\prime}}{\gamma^{\prime}}$ et $\frac{6^{\prime \prime}}{\delta^{\prime \prime}}$; inter $\frac{m^{\prime \prime}}{\gamma^{\prime \prime}}$ et $\frac{6^{\prime \prime}}{\delta^{\prime \prime}}$; inter $\frac{\alpha^{\prime \prime \prime}}{\gamma^{\prime \prime \prime}}$ et $\frac{611 \prime}{8 \prime^{\prime \prime}}$ etc。 (facile enim ex art. 189,3 fin. deducitur, nul. lum horum limitum habere signum oppositum 


\section{7.}

signo ipsius $a$; quare quantitati radicali $V D$ signum positiuum tribui debet) siue inter $\frac{\alpha^{\prime}}{\gamma^{\prime}}$ et $\frac{\alpha^{\prime \prime}}{\gamma^{\prime \prime}} ;$ inter $\frac{a^{\prime \prime}}{z^{\prime \prime}}$ et $\frac{e^{\prime \prime \prime}}{y^{\prime \prime \prime}}$ etc. Omnes itaque fractiones $\frac{\alpha^{\prime}}{\gamma^{\gamma}}, \frac{\alpha^{\prime \prime \prime}}{\gamma^{\prime \prime \prime \prime \prime}} \frac{\alpha^{v}}{\gamma^{v}}$ etc. ipsi $L$ ab eadem parte iacebunt, omnesque $\frac{\alpha^{\prime \prime}}{\gamma^{\prime \prime \prime}} \frac{\alpha^{\mathrm{tV}}}{\gamma^{\mathrm{iv}}}, \frac{a^{\mathrm{v}}}{\gamma^{\mathrm{vt}}}$ etco a par: te altera. Quoniam vero $g^{\prime}<g^{\prime \prime \prime}$, $\frac{a^{\prime}}{\gamma^{\prime}}$ iacebit extra $\frac{a^{\prime \prime \prime}}{\gamma^{\prime \prime \prime}}$ et $L$, similique ratione $\frac{\alpha^{\prime \prime}}{\gamma^{\prime \prime \prime}}$ extra $L$ et $\frac{\alpha^{\mathrm{IV}}}{\alpha^{\mathrm{V} V}} ; \frac{\alpha^{\prime \prime \prime}}{\gamma^{\prime \prime \prime}}$ extra $I$ et $\frac{\mathrm{wv}^{\mathrm{v}}}{\gamma^{\mathrm{V}}}$ etc Vude manifestum est, has quantitates iacere sequenti ordine:

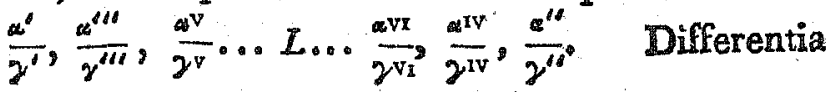
autem inter $\frac{a^{\prime}}{\gamma^{\prime}}$ et $L$ exit minor quam differen tia inter $\frac{a^{\prime}}{\gamma^{\prime}}$ et $\frac{a^{\prime \prime}}{y^{\prime \prime}}$ i. $\theta_{0}<\frac{x}{\gamma^{\prime} \gamma^{\prime \prime}}$, similique ratione differentia inter $\frac{x^{10}}{\gamma^{\prime \prime}}$ et $L$ exit $<\frac{x}{\gamma^{\prime \prime \prime} \gamma^{\prime \prime \prime}}$ etc. Quamobrem fractiones $\frac{\alpha^{\prime}}{\gamma^{\prime}}, \frac{\alpha^{\prime \prime}}{\gamma^{\prime \prime}}, \frac{\alpha^{\prime \prime \prime}}{\gamma^{\prime \prime \prime}}$ etc continuo proprius ad limitem $L$ accedunt, et quoniam $x^{\prime}, y^{\prime \prime}, y^{\prime \prime \prime}$ continuo in infinitum crescunt, differentia fractionum a limite quauis quantitate data minor fieri potesto

Ex art. 189 nulla quantitatum $\frac{\gamma}{2}, \frac{y}{\alpha}, \frac{1 / y}{\sigma_{\infty}}$ etc. signum idem habebit $\nabla t a$; hinc per ratio. cinia praecedentibus omnino similia sequitur, 


\section{$-248=$}

illas et hanc $\frac{-\sqrt{D}+1 \cdot b}{a^{\prime}}$ quam per $L$ designabi,

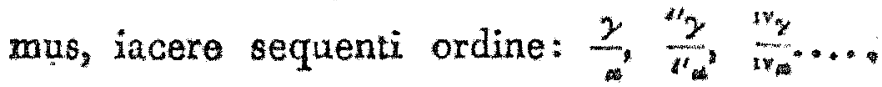

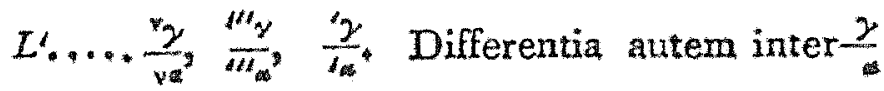
et $L^{\prime}$ minor erit quam $\frac{r}{\alpha_{\alpha \sigma a}}$, differentia inter $\frac{y}{\alpha}$ et $L$ minor quam $\frac{x}{\|_{\alpha}^{\prime} \alpha}$ etc, Quare fractio. nes $\frac{\gamma}{\alpha}$, ' $\gamma$ etc. continua proprius ad $L$ ' accedent, et differentia quauis quantitate data minor fieri poterit.

In ex. art. I 88 , fit $L=\frac{\sqrt{79-8}}{3}=0,2960648$ et fractiones appropinquantes $\frac{8}{1}, \frac{x}{3}, \frac{3}{7}, \frac{3}{6},{ }_{2}^{8} ;$ ${ }_{\pi 52}^{45},{ }_{483}^{x 43}$ etc. Est autem ${ }_{483}^{x 43}=0,3960662$. Ibidem fit $L^{\prime}=\frac{-\sqrt{79}+8}{5}=-0,1776388$. fractionesque approximantes $\frac{0}{x}-\frac{x}{5}-\frac{x}{2}-\frac{2}{x^{2}}$

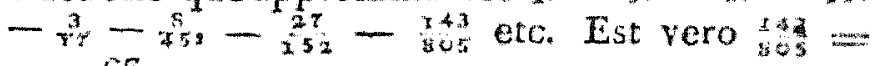
$0, \mathrm{I}_{77} 63_{97}$

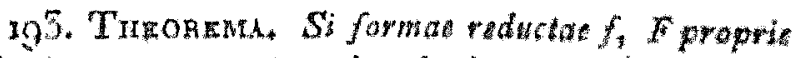
arquinotentes surt: altera in alterius periodo fontarta erit.

$$
\text { Sit } f=\left(a, b,-a^{\prime}\right), F=(A, B,-A) \text {, }
$$
determinans harum formarum $D$, transeatque illa in hanc per substitutionem propriam $2 t, B$, $\mathbb{D}$ D. Tum dico, si periodus formae $f$ quaeratur progressioque vtrimque infinita formarum reductarum atque transformationum for- 


\section{- $249-$}

mae $f$ in ipsas eruatur, eodem modo vt art. 188 : vel + भf fore aequalem termino alicui progressionis... " $\alpha,{ }^{\prime} \alpha, \alpha, \alpha^{\prime}, \alpha^{\prime \prime} \ldots$, hocque posito $=\alpha^{\text {th }}$, $+\mathfrak{B}$ fore $=\mathfrak{b}^{\mathrm{m}},+\mathfrak{C}=2^{\mathrm{m}},+\mathfrak{D}=\delta^{\mathrm{m}} ;$ vet - I fore aequalem termino alicui $\alpha^{\text {an }}$, et $-\mathfrak{B},-\left(\mathrm{s},-\mathcal{D}\right.$ resp. $=\mathrm{G}^{\mathrm{m}}, 2^{\mathrm{m}}, \delta^{\mathrm{m}}(\mathrm{vbi} m$ etiam indicem negatiuum designare potest). In vtroque casu $F$ manifesto identica erit cum $f m_{\text {, }}$

Dem. I. Habentur quatuor aequationes,

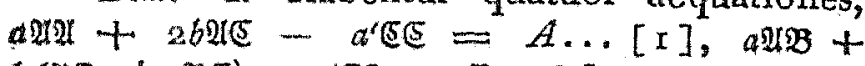
$b(21 D+\mathfrak{B C})-a^{\prime} \mathbb{D}=B_{*},[2], a \mathfrak{B P}+2 b \mathfrak{B D}$ $-a^{\prime} \mathfrak{D D}=-A^{\prime} \ldots[3] ; \mathfrak{I D}-\mathfrak{B} \mathfrak{S}=1 . .$. [4]. Consideramus autem primo casum, vbi aliquis numerorum $\mathfrak{Y}, \mathfrak{B}, \mathfrak{E}, \mathfrak{D}=0$.

$x^{\circ}$ Si $\mathfrak{I}=0$, fit ex [4] $\mathfrak{B}=-\mathbf{r}$, adeoque $\mathfrak{B}= \pm \mathrm{x}, \mathfrak{b}=\mp$. $\mathrm{x}$. Hinc ex $[\mathrm{r}],-$ $a^{\prime}=A ;$ ex $[2],-b \pm a^{\prime} b=B$ siue $B \equiv-b$ (mod, $a^{\prime}$ vel $A$ ); vnde sequitur formam $(A, B$, - $\left.A^{\prime}\right)$ formae $\left(a, b,-a^{\prime}\right)$ ab vltima parte contiguam esse, Quoniam vero illa est reducta, mecessario cum $f^{\prime}$ identica erit. Ergo $B=b^{\prime}$, adeoque ex[2] $b+b^{\prime}=-a^{\prime}\left(\mathbb{C} D= \pm a^{\prime} \mathfrak{D} ;\right.$ hinc propter $\stackrel{b+b^{\prime}}{-a^{\prime}}=h^{\prime}$, fir $\mathfrak{D}=\mp h^{\prime}$. Vnde colligitur, $F \mathfrak{l}, \mp \mathfrak{B}, \mp \mathfrak{c}, \mp \mathfrak{D}$ esse resp: $=0$,

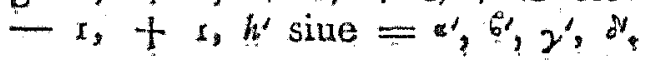

$n^{\circ}$ Si $B=0$, fit ex [4] $20 \pm x, D=$ $\pm x$; ex [5] $a^{\prime}=A^{\prime} ;$ ex [2] $b \mp a^{\prime} \in=B$, si $\overrightarrow{\text { ue }} b=B$ (mod. $a)$. Quoniam vero tum $f$ tum $F$ sunt formae reductae; tum $b$ tum $B$ ia-

Q 5 


\section{- $250 \quad-$}

cebunt inter $\sqrt{ } D$ et $\sqrt{ } D \pm a^{\prime}$ (Prout $a^{\prime}$ pos. vel neg., art. 185,5 ). Quare exit necessario $b=B$, et $\mathfrak{S}=0$. Hinc formae $f, F$ sunt identicae atque $\pm \mathfrak{A}, \pm \mathfrak{B}, \pm \mathbb{E}, \pm \mathbb{D}=1,0,0$, $x=a_{0}, y_{0}, d$ (resp.).

$3^{\circ}$ Si $\mathfrak{C}=0$, fit ex [4] $\mathfrak{A}= \pm \mathrm{x}, D=$ $\neq \mathrm{x} ; \operatorname{ex}[\mathrm{r}] a=A ; \operatorname{ex}[2]+a b+b=B$ siue $b \equiv B$ (mod. $a)$. Quia vero tum b tum $B$ iacent inter $\sqrt{ } D$ et $\sqrt{ } D \mp a:$ erit necessario $B=b$ et $=0$. Quare casus hic a praecedente non differt.

$4^{\circ}$ Si $D=0$, fit ex [4] B $= \pm 1, c=$

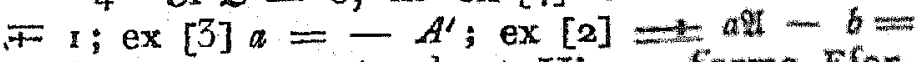
$B$ siue $B \equiv-b$ (mod. $a$ ). Hinc forma Flormae $f$ a parte prima contigua er $\bar{x}$, et proin cum forma of identica. Quare propter ${ }^{b+b}$ $=h$, et $B=\mathfrak{b}$, erit $\pm \mathfrak{a}=h$. Vnde colligitur $\pm \mathfrak{A}_{1} \pm \mathfrak{B}, \frac{-1}{e_{1}} \mathfrak{C}_{1} \pm D$ resp. esse $=\mathfrak{h}_{2}$

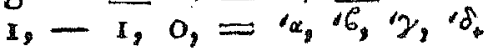

Superest itaque casus vbi nullus numerorum $\mathfrak{A}, \mathfrak{B}, \mathfrak{E}, \mathbb{D}=0$. Hic per Lemma art. rga quantitates $\frac{\mathfrak{U}}{\mathbb{E}}, \frac{5}{D}, \frac{E}{\mathbb{H}}, \frac{D}{D}$ idem signum habebunt, oriunturque inde duo casus, quum signum hoo vel cum signo ipsorum as, $a^{t}$ conuenire vel ipsi oppositum esse possisit.

11. Si $\frac{\mathfrak{H}}{\mathfrak{C}}, \frac{\mathfrak{B}}{D}$ idem sìgnum habent vt $a:$ quantitas $\frac{V D-b}{}$ (quam designabionus per $L$ ) 


\section{$-25 x=$}

inter has fractiones sita erit (art. $\mathrm{Ig} \mathrm{I}$ ). De-

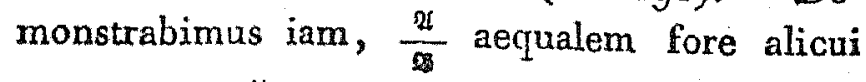
fractionum $\frac{\alpha^{\prime \prime}}{\gamma^{\prime \prime}}, \frac{\alpha^{\prime \prime \prime}}{\gamma^{\prime \prime \prime}}, \frac{\alpha^{1 V}}{\gamma^{10}}$ etc, atque $\frac{B}{D}$ proxime sequenti, scilicet si $\frac{\mathscr{d f}}{\sigma}$ fuerit $=\frac{\alpha^{\mathrm{m}}}{\gamma^{\mathrm{m}}}, \frac{\mathscr{D}}{D}$ fore $=\frac{\alpha^{\mathrm{nn}+\mathrm{x}}}{\gamma^{\mathrm{m}}+\mathrm{x}}$. In art, praec. ostendimus, quantitates $\frac{\alpha^{\prime}}{\gamma^{\prime}}, \frac{\alpha^{\prime \prime}}{\gamma^{\prime \prime}}, \frac{\alpha^{\prime \prime \prime}}{\gamma^{\prime \prime \prime}}$ etc., (quas breuitatis gratia per (1), (2), (3) etc. denotabimus) atque $L$, hunc ordinem (I): obseruare $(\mathrm{r}),(5),(5) \ldots L_{2} . .(6)$, (4), (2); prima harum quantitatum est $=$ o (propter $\boldsymbol{*}^{\prime}=0$ ), reliquae omnes idem signum habent vt $L$ siue a. Quoniam vero per hyp. $\frac{\mathscr{L}}{\mathbb{S}}, \frac{\mathfrak{B}}{\mathfrak{D}}$ (pro quibus scribemus $\mathfrak{M}, \mathfrak{M}$ ) idem signum habent: patet has quantitates ipsi (1) a dextra iacere (aut si mauis ab eadem parte a qua $L$ ), et quidem, quum $I$ iaceat inter ipsas, alteram ipsi $L$ a dextra, alteram a laeua, $\mathrm{Fa}$ cile vero ostendi potest, $\mathfrak{M}$ ipsi ( $(\Omega)$ a dextra iacere non posse alioquin enim $\mathfrak{N}$ iaceret inter ( $(x)$ et $L$, vnde sequeretur prino (2) iacere inter $\mathfrak{M}$ et $\mathfrak{R}$, adeoque denominatorem fractionis (2) maiorem esse denominatore fractionis 92 (art. 190 ), secundo $\mathfrak{N}$ iacere inter ( 1 ) et (2), adeoque denom. fractionis $\mathfrak{R}$ esse majorem quam denom. fractionis (2), Q. $E . A_{0}$

Supponamus $\mathfrak{M}$ nulli fractionum (a), (3), (4) etc. aequalem esse, vt, quid inde sequatur, videamus. 'Tum manifestum est, si fractio $\mathfrak{M}$ ips 


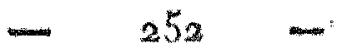

$I$ a laeua iaceat, necessario eam sitam esse aut inter (1) et (5), aur inter (3) et (5), aut inter (5) et ( 7 ) etc. (quoniam $L$ est irrationalis, adeoque ipsi $\mathfrak{M}$ certo inaequalis, fractionesque $(x),(3),(5)$ etc quauis quantitate data, ipsi $x$. inaequali, propius ad $L$ accedere possumt). Si vero $\mathfrak{R}$ ipsi $L$ a dextra iacet: necessario iacebit aut inter (2) et (4), aut inter (4) et (6) aut inter (6) et (8) etc. Ponamus itaque $\mathfrak{M}$ iacere inter $(m)$ et $(m+2)$, patetque quantita. tes $\mathfrak{R}_{1}(m),(m+1),(m+2), L$ iacere sequenti ordine, (II) $):(m),\left(\right.$ Im) $,(m+n), L_{n}$ $(m+1)$. Tum erit necessario $\Re=(m+1)$. Iacebit enim $\mathfrak{R}$ ipsi $L$ a dextra; si vero etiam ipsi $(n+5)$ a dextra iaceret, $(n+1)$ iaceret inter

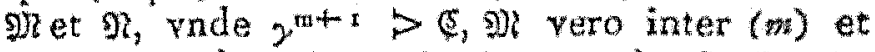
$(n+1)$ vnde $(5) 2^{\text {m+t }}($ art. 190$), Q$. E. A.; si vero $g^{2}$ ipsi $(m+x)$ a laena iaceret, sine inter $(n+2)$ et $(m+1)$, foret $D>2^{n+2}$, et quia $(n+2)$ inter $\mathfrak{N}$ et $\mathfrak{R}$, foret $y^{m+2}>\mathbb{D}$, Q. E. $A$ - Erit itaque $\Omega=(m+x)$, siue

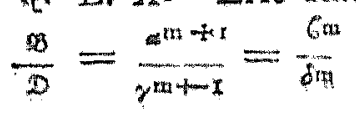

Quia $10-B(E=r$ erit primus ad $D$ et ex simili ratione primus ad * Vade facile perspicitur aequationem $\frac{D}{D}=$ conststere non posse, nisi fuerit aut $=6^{\mathrm{mn}}, D$ $=\delta^{\mathrm{n}}$, aut $B=-6^{\mathrm{m}}, 0=-\delta^{\mathrm{sia}}, \quad$ Iarm

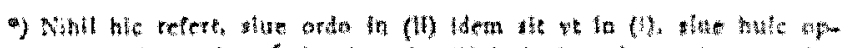

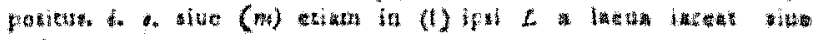
actira. 


\section{- $253-$}

quum forma $f$ per substitutionem propriam $a^{\mathrm{m}}, 6^{\mathrm{m}}, \gamma^{\mathrm{m}}, 8^{\mathrm{m}}$ in formam $f^{\mathrm{m}}$ transmutetur, quae est $\left( \pm a^{\mathrm{m}}, b^{\mathrm{m}}, \mp a^{\mathrm{m}+1-1}\right)$ : habebuntur aed

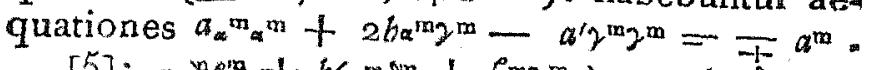

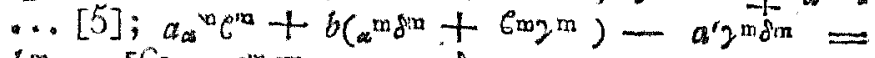
$b^{m} . . .[G] ; a 6^{m} b^{m}+a b^{a n d m}-a^{\prime} g^{m} g^{m}=\mp a^{m}+x$

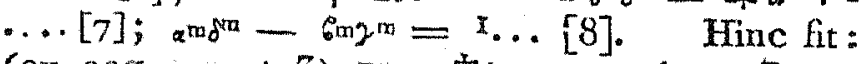
(ex aecqu. 7 et 3), $\mp a^{\mathrm{m}}=-A^{\prime}$. Porro multiplicando aequationem [2] per $\alpha^{\operatorname{mdm}}-6 \operatorname{mon}_{\gamma} \mathrm{m}$, aequationem $[6]$ per $2 \mathbb{D}$ - $\mathfrak{B} \mathfrak{S}$ et subtrahendo facile per euolutionem confirmatur esse

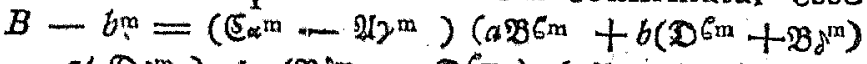

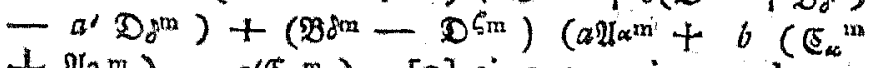
$\left.\left.+\mathfrak{l}_{2} \mathrm{~m}\right)-a^{\prime} \mathfrak{E}_{\gamma^{\mathrm{m}}}\right) \ldots[\mathrm{g}]$ siue quoniam vel $6^{\mathrm{m}}$ $=\mathfrak{B}, \delta^{\mathrm{m}}=\mathfrak{D}$ vel $\left.6^{\mathrm{m}}=-\mathfrak{B}, \mathrm{d}^{\mathrm{m}}=-\mathfrak{D}\right), B$ $-b_{\mathrm{m}}^{\prime}= \pm\left({ }^{\prime} a^{\mathrm{m}}-21 \gamma^{\mathrm{m}}\right)\left(a \mathfrak{B O}+2 b \mathbb{B D}-a^{\prime} \mathfrak{D D}\right)$ $=\mp\left(\mathrm{Sa}^{\mathrm{m}}-\mathrm{Q}^{\mathrm{m}} \mathrm{m}^{\mathrm{m}}\right) A^{\prime}$. Hinc $B \equiv b^{\mathrm{m}}$ (mod. $\left.A^{\prime}\right)$; quia vero tum $B$ tum $b^{\text {ma }}$, inter $\sqrt{ } D$ et $\sqrt{ } D \neq A^{\prime}$ iacent, necessario erit $B=b^{\mathrm{m}}$ ad-

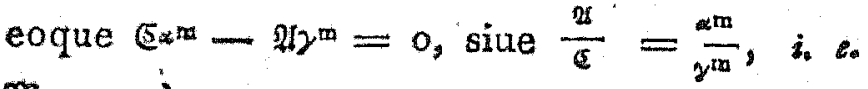
$\mathfrak{m}=(m)$

Hoc modo itaque ex suppositione, $\mathfrak{R}$ nulli quantitatum (2), (3), (4) etc. aequalem esse, deduximus, eam reuera alicui aequalem esse. Quodsi vero ab initio supponimus, esse $\mathfrak{R}=(m)$, manifesto erit vel $\mathfrak{H}=x^{\mathrm{m}}$, है $=2^{\mathrm{m}}$, vel $-\mathfrak{A}=\mathrm{a}^{\mathrm{m}},-\mathfrak{E}=2^{\mathrm{m}}$. In vtroque casu fit ex [x] et [5] $A= \pm a^{\mathrm{m}}$, et ex [9] $B-b^{\mathrm{m}}= \pm\left(B^{8 \mathrm{~m}}-D^{\mathrm{min}}\right) A$, size $B \equiv$ $b^{\mathrm{m}}(\bmod$. $A)$. Hinc simili moda vi supra concluditur $B=b^{m}$, et hinc $\mathfrak{B}^{8 m}=D^{\mathrm{m}}$; quare 


\section{$-254-$}

quum 28 ad $D$ primus sit et $6 \mathrm{~m}$ ad jom.? erit aut $\mathfrak{B}=6^{\mathrm{m}}, \mathfrak{D}=\delta^{\mathrm{m}}$ aut $-\mathfrak{B}=6^{\mathrm{m}}, \cdots D$ $=\delta \mathrm{m}$, et proin ex [7] $A^{\prime}=\mp a^{\mathrm{m}+\mathrm{r}}$. Quamobrem formae $F$, fin identicae erunt. Adiumento aequationis $2 \mathbb{D}-\mathfrak{S E}=a^{\mathrm{m}} \delta^{\mathrm{m}}-$ $6^{6 m} y^{m}$ autem nullo negotio probatur, poni debere $+\mathfrak{B}={ }^{\mathfrak{m}},+\mathfrak{D}=\mathrm{j}_{\mathrm{m}}$, quando $+\mathscr{A}$ $=\alpha^{\mathrm{m}},+\mathfrak{E}=2^{\mathrm{m}} ;$ contra $-\mathfrak{B}=6 \mathrm{~m},-\mathbb{D}$ $=-\operatorname{din}^{\mathrm{m}}$, quando $-\mathscr{H}=\mathrm{m}^{\mathrm{m}},-\mathfrak{C}=2^{\mathrm{m}}$ 。 Q. E. D.

III. Si signum quantitatum $\frac{21}{6}$ signo ipsius a oppositum: demonstratio praecedenti tam similis est, vt praecipua tantum momenta addigitauisse sufficiat. Iacebit $\frac{-\sqrt{ } D+b}{a^{\prime}}$ inter

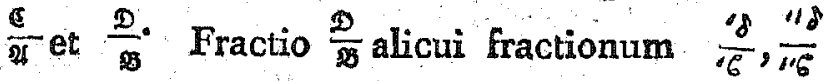

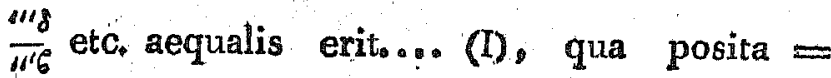
$\frac{m \delta}{m b}, \frac{E}{q b}$ erit $=\frac{m y}{m_{g}} \ldots$. (II)。 Demonstratur aus tem (I) ita: Si $\frac{D}{B}$ nulli illarum fractionum aequalis esse supponitur: inter duas tales $\frac{m \delta}{m 6}$ et $\frac{m+2 \delta}{m+26}$ iacere debebit. Hinc vero eodem modo vt supra deducitur, necessario esse $\frac{\mathfrak{E}}{\mathscr{I}}=\frac{\mathrm{m}+\mathrm{d}}{\mathrm{w}+16}=\frac{\mathrm{m}_{\mathrm{q}}}{\mathrm{m}_{\mathrm{q}}}$, atque rel $\mathscr{2}=$

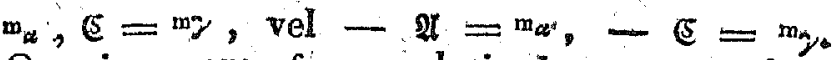
Quoniam vero $f$ per substitutionem propriam $\mathrm{m}_{\alpha}, \mathrm{mb}, \mathrm{m} \gamma$, in $\delta$ in formam $\mathrm{m}_{f}=\left(\longleftarrow \mathrm{m}_{\mathfrak{a}}, \mathrm{m} b_{\text {, }}\right.$ $\pm{ }^{m-x_{a}}$ ) transit: hinc emergunt tres aequa: 


\section{$-255$}

tiones, ex quibus coniunctis cum aequ. I, 2, 3, 4f atque hac, $\mathrm{m}_{\alpha} \mathrm{m} \delta-\mathrm{mbm}_{\mathrm{g}}=\mathrm{r}$ deducitur eodem modo vt supra, terminum primum $A$, formae $F$, termino primo formae $\mathrm{m}_{f}$ aequalem esse, illinsque terminum medium medio huius congruum secundum modulum $A$, vnde sequitur, quia vtraque forma est reducta, adeoque vtriusque terminus medius inter $\sqrt{ } D$ et $\sqrt{ } D$ $\pm A$ situs, hos terminos medios aequales esse: hinc vero deducitur $\frac{m_{\delta} \delta}{m \zeta}=\frac{D}{D}$. Veritas itaque assertionis (I) deriuata hic est ex suppositione illam esse falsam.

Supponendo autem $\frac{m_{d}}{m 6}=\frac{D}{m}$, prorsus simili modo et per easdem aequationes demonstratur, esse etiam $\frac{m_{y}}{m_{w}}=\frac{\mathfrak{s}}{\mathfrak{U}}$, quod erat secundum (II). Hinc vero adiumento aequationum $\mathscr{I D}$ $-\mathfrak{B E}=I, m_{\alpha m_{\delta}}-\operatorname{mim}_{\boldsymbol{g}}=1$ deducitur esse vel $\mathfrak{A}=\mathrm{m}_{\boldsymbol{\alpha}, \mathfrak{B}}=\mathrm{m}_{\ell}, \mathfrak{C}=\mathrm{m}_{2}, \mathfrak{D}=\mathrm{m}_{\phi}$, vel $-\mathfrak{A}=\mathrm{m}_{\alpha}, \mathfrak{B}=\mathrm{m}_{\boldsymbol{\theta}}-\mathfrak{C}=\mathrm{m}_{\gamma_{2}}-\mathfrak{D}=\mathrm{m}_{\delta_{1}}$ formasque $F$, mf identicas. Q. $E, D$,

194. Quum formae quas supra socias vocauimus (art. 187,6) semper sint improprie aequiualentes (art. 160), perspicuum est, si formae reductae $F, f$ improprie aequiualentes sint, formaeque $F$ socia forma $G$, formas $f, G$ proprie aequiualentes fore adeoque formam $G$ in periodo formae $f$ contentam. Quodsi, itaque formae $F, f$ tum proprie tum improprie aequiualentes sunt, patet, tum $F$ tum $G$ in pe- 


\section{$-256 \quad-$}

riodo formae $f$ reperini debere. Quare perio. dus haec sibi ipsi socia erit, duasque formas ancipites continebit (art. 187,7 ). Vnde theorema art. 165 egregie confirmatur ex quo iam poteramus esse certi, formam aliquam ancipitem dari formis $F, f$ aequiualentem.

195. Prowlema Propositis duabus formis quibuscunque $\phi, \Phi$ einsdem determinantis : diudicare utrum acquinalentes sint, annon.

Sol. Quaerantur duae formae reductae $f$, propositis $\Phi, \phi$ resp. proprie aequiualentes (art. I83). Quae prout aut proprie tantum aequiualent, aut improprie tantum, aut vtroque modo, aut neutro; etiam propositae aut proprie tantum aequiualentes erunt, aut improprie tantum, aut vtroque aut neutro modo. Euoluatur periodus alterutrius formae reductae $\varepsilon_{\text {s }} g$, periodus formae $f_{0}$. Si forma $F$ in hac periodo occurrit neque vero simul forma ipsi $F$ socia, manifesto casus primus locum habebit; contra si socia haec adest neque vero $F$ ipsa, secundius si vtraque, tegtius; si neutra, quartws.

Ex. Propositae sint formae ( $129,92,65$ ) (42, 59,81 ) determinantis $790^{\circ}$ His proprie aequiualentes inueniuntur reductae (10, $7,-$ $3),(5,8,-3)$. Periodus formae prioris haec est: $(10,7,-3),(-3,8,5),(5,7,-6),(-$ $6,5,9),(9,4,-7)(-7,3,10)$. In qua quum forma $(5,8,-3)$ ipsa non reperiatur, sed tamen socia $(-3,8,5)$ : formas propositas improprie tantumi aequiualere concludimus. 
Si omnes formae reductae determinantis dati codem modo vt supra (art. 187,5 ) in periodos $P$, $Q, R$ etc. distribuuntur, atque e quauis periodo forma aliqua ad libitum eligitur, ex $P, F$; ex $Q, G$; ex $R, H$ etc.: inter has formas $F, G, H$ etc. duae quae proprie atquiualeant esse non poterunt. Quaeuis autem alia forma eiusdem determinartis alicui ex istis proprie aequiualens erit et quidem vnicae tantum. Hinc manifestum est, onnes formas huins determinantis in totidem classes distribut posse, quot habeantur pprodi, scilicet referendo eas quae formae $F$ proprie aequiualent in primam classem, eas quae formae $G$ proprie aequiualent in secundam etc. Hoc modo omnes formae in eadem classe contentae proprie aequiualentes erunt, formae vero e classibus diuersis non poterunt proprie aequiualere. Sed hic huic argumento intra fusius explicando non immoramur,

196. Problema. Propositis duabuis formis proprie aequinalentibus $\$, \phi$ : inmenire transformationem propriam altertus in alteram.

Sol. Per methodum art. 183 inueniri poterunt

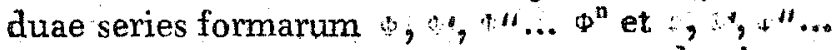
"tales vt quaeuis forma sequens praecedenti proprie aequiualeat, vltimaeque $\varphi^{\mathrm{n}}, \varphi^{3}$ sint formae reductae; et quum $\$$, proprie aequiualentes esse supponantur, necessario $t^{i 1}$ in periodo formae ${ }^{*}$ contenta erit. Sit $\phi^{r}=f$ ipsiusque periodus vsque ad formam $q^{\mathrm{n}}$ haec $f, f^{\prime}, f^{\prime \prime} \ldots f^{\mathrm{n}}-1$, $w^{n}$, ita vt in hac periodo index formae $\Phi^{\text {il }}$ sit $m$; designenturque formae quae oppositae sunt sociis formarum 
$\Phi, \phi, \Phi_{1} \ldots \Phi^{n}$ per $\Psi, \Psi, \Psi \mu \ldots \Psi^{n}$ resp. $*$ ) Tum in progressione $\phi, \phi^{\prime}, \phi^{\prime \prime} \ldots f, f^{\prime}, f^{\prime \prime} \ldots f^{m}-I, \Psi^{m}-I_{3}$ $\Psi-2 \ldots \Psi, \Phi$ quaeuis forma praecedenti ab vitima parte contigua ert, vnde per art. 177 inueniri poterit transformatio propria primae $\phi$ in vltimam Q. Illud autem de formis reliquis progressionis nullo negotio perspicitur; de his $f^{i n}-r, \Psi^{n}-1$ sic probatur: Sit $f^{\mathrm{m}}-\mathrm{r}=(g, h, i) ; f^{\mathrm{n}}$ siue $\Phi^{\mathrm{n}}=$ $\left(g^{\prime}, h^{\prime}, i^{\prime}\right) ; \phi-\mathbf{D}=\left(g^{\prime \prime}, h^{\prime \prime}, i^{\prime \prime}\right)$. Forma $\left(g^{\prime}, h^{4}, i^{\prime}\right)$ tum formae $(g, h, i)$ tum formae $\left(g^{\prime \prime}, h^{\prime \prime}, i^{\prime \prime}\right)$ ab vltima parte contigua erit; hinc $i=g^{\prime}=i^{\prime \prime}$, et $h \equiv h^{i} \equiv-h^{\prime \prime}\left(\bmod . i\right.$ vel $g^{i}$ vel $\left.i^{\prime \prime}\right)$. Vnde manifestum est formam $\left(i^{\prime \prime},-h^{\prime \prime}, g^{\prime \prime}\right)$, i. e. formam $\Psi n-1$ formae $(g, h, \dot{i}), i$ e. formae $f^{\mathrm{n}}-\mathrm{I}$ ab vltima parte contiguam esse.

Si formae $\phi, \phi$ improprie aequiualentes sunt: forma $\Phi$ proprie aequiualebit formae cui opposita est $\Phi$. Inueniri poterit itaque transformatio propria formae $\phi$ in formam cui $\Phi$ est opposita; quae si" supponitur fieri per substitutionem $a, b, \gamma, \delta$, facile perspicitur, $\phi$ improprie transformari in ipsam. $\Phi$ per substitutionem $\alpha,-\epsilon, \gamma,-\delta$.

Hinc etiam perspicuum est, si formae $\Phi, \phi$ tum proprie tum improprie aequiualentes sint, inueniri posse duas transformationes, propriam et impropriam.

$E x$. Quaeritur transformatio impropria formae $(129,92,65)$ in formam $(42,59,81)$, quam

* Ita ve $\Psi$ oriatur ex $\$$ commutando terminum primum et vitimum tribuendoque medio signum oppositum, sinuliterque de seliquist. 


\section{$-259=$}

illi improprie aequiualere in art praec. inuenimus. Inuestiganda erit itaque primo transformatio propria formae $(129,92,65)$ in formam $(42,-59$, 81). Ad hunc finem euoluitur progressio formarum haec: $(129,92,65),(65,-27,10) ;(10$, $7,-3),(-3,8,5),(5,22,81),(81,59,42)$, $(42,-59,81)$. Hinc deducitur transformatio propria $-47,56,73,-87$, per quam (129, 92 , 65 ) transit in $(42,-59,81)$; quare per impropriam $-47,-56,73,87$ transibit in $(42,59,81)$.

197. Si transformatio wna formae alicuius ( $a$, $b, c) \ldots \Phi$ in aequiualentem o habetur: ex hac omnes transformationes similes formae in deduci poterunt, si modo omnes solutiones aequationis indeterminatae $t t-D u u=m m$ assignari pos. sunt, designante $D$ determinantem formarum $\Phi, \phi ;$ $m$ diuisorem comnunem maximum numerorum $a, 2 \dot{b}, c($ art. 162). Hoc igitur problema, quod. pro valore negatiuo ipsius $\mathcal{D}$ iam supra soluimusg. nunc pro positiuo aggrediemur. Quia vero manifesto quiuis valor ipsius $t$ aequationi satisfaciens etiamnum mutato signo satisfacit; similiterque quiuis valor ipsius $u$ : sufficiet si omnes valores positiuos ipsorum $t, u$ assignare possimus, fungeturque quaelibet solutio per valores positiuos, quatuor solutionum vice. Hoc negotium ita absoluemus, it primo valores minimos ipsorum $t, u$ (praeter hos perse obuios $t=m, u=0$ ) inuenire, tum ex his omnes reliquios deriuare doceamus.

i98. Probiema. Inuenire numeros mininos $t$, of aequationi indeteminatae $t t-D_{u} u=m m$ satisfacientes, siquidem forma aliqua $(M, N ; P)$ datur, chins de: R 


\section{$-260-$}

Eorminans est $D$, numerorumque $M, 2 N, P$ diuisor communis maximus $m$.

Sol. Accipiatur ad lubitum forma reducta ( $a$, $\left.b,-a^{\prime}\right) \ldots f$, determinantis $D$, vbi diuisor communis maximus numerorum $a, 2 b, a^{\prime}$ sit $m$, qualem dari vel inde manifestum est, quod forma reducta formae $(M, N, P)$ aequiualens inueniri potest, quae per art. 161 hac proprietate erit praedita : sed ad propositum praesens quaeuis forma reducta in qua conditio haec locum habet poterit adhiberi. Euoluatur periodus formae $f$, quam ex $n$ formis constare supponemus. Retentis oninibus signis quibus in art. i 88 vși sumus, $f^{\text {n }}$ erit $\left(+a^{\mathrm{n}}\right.$, $b^{n},-a^{n+1}$ ), quia $n$ par, et in hanc formam transibit $f$ per substitutionem propriam $\alpha^{\mathrm{n}}, 6^{\mathrm{n}}, \gamma^{\mathrm{n}}, \delta^{\mathrm{n}}$. Quia vero $f$ et $f^{\text {n }}$ sunt identicae: $f$ transibit in $f^{\text {n }}$ etiarn per substitutionem propriam $1,0,0,1$. Ex his duabus transformationibus similibus formae $f$. in $f^{\mathrm{a}}$ per art. 162 deduci poterit solutio aequationis $t t-D u t=m m$ in integris, scilicet $t=$ $\frac{x}{2}\left(x^{\mathrm{n}}+\delta^{00}\right) m$ (aequ, 18 art. ${ }^{262}$ ), $u=\frac{\gamma^{\mathrm{n}} m}{a}$ (aequ. 19) *). Designentur hi valores positiue accepti si forte nondum sunt per $T, U$, eruntque hi $T, U$ valores minimi ipsorum $t, u$, praeter hos $t=m$, $u=0$ (a quibus necessario erunt diuersi, quia manifesto $\gamma^{\mathrm{n}}$ non poterit esse $=0$ ).

Supponamus enim dari adhuc minores valores ipsorum $t, u$ puta $t, \mathfrak{u}$ qui sint positiui et $u$

7) Quae in art. 162 erant $a, 6, \gamma, \delta ; \alpha^{\prime}, b^{\prime}, \gamma^{\prime}, \delta^{\prime} ; A, B, C$; $A^{\prime}, B^{\prime}, C^{\prime} ; \varepsilon$, hic sunt $\mathrm{I}, 0,0,1 ; \mathrm{am}^{\mathrm{m}}, \mathrm{mm}^{\mathrm{m}}, \mathrm{r}^{\mathrm{m}}, \mathrm{dm}^{\mathrm{m}}$; $a, b,-a_{1}^{\prime}, a, b,-a_{;}^{\prime} ;$. 


\section{$-261$}

non $=0$. Tum per art. 162 forma $f$ per substitutionem propriam $\frac{\mathrm{I}}{m}(\mathfrak{t}-b \mathfrak{u}), \frac{\mathrm{I}}{n_{2}} a^{\prime} \mathrm{u}, \frac{\mathbf{I}}{m} a \mathrm{u}$, $\frac{I}{s z}(t+b \mathfrak{u})$ transformabitur in formam cum ipsa identicam. Iam ex art. 193, II sequitur, aut $\frac{1}{m}(\mathfrak{t}-b \mathfrak{u})$ aut $-\frac{\mathrm{I}}{m}(\mathfrak{t}-b \mathfrak{u})$ alicui nume. rorum $\alpha^{\mathrm{II}}, \alpha^{\mathrm{III}}, \alpha^{\mathrm{IV}}$ etc. aequalem esse debere, puta $=\alpha^{\mu}$ (quia enim $\mathfrak{t} \mathbf{t}=D \mathfrak{u} \mathfrak{u}+m m=b b \mathfrak{u} u$ $+a^{\prime} \mathfrak{u} \mathfrak{u}+m m$, erit $\mathfrak{t} \rightarrow b b \mathfrak{n}$, adeoque $t-b \mathfrak{u}$ positiuus; hinc fractio $\frac{t-b \mathfrak{u}}{a \mathfrak{u}}$; quae respondet fractioni $\frac{\mathfrak{U}}{\mathfrak{S}}$ in art. 193 , idem signum habebit vt $a$ vel $a^{\prime}$ ); atque in casu priori $\frac{\mathbf{I}}{m} a^{\prime} \mathfrak{u}_{3}$ $\frac{\mathbb{Z}}{m} a \mathfrak{u}, \frac{\mathbf{I}}{m}(t+b u)$, in posteriori easdem quanvitates mutatis signis, resp, $=\epsilon^{\mu}, \gamma^{\mu}, \mathrm{f}^{\mathrm{m}}$. Sed quum sit $4<U$ i. e. $u \& \frac{\gamma^{n} m}{a}$ et $>0$ : erit $\gamma^{k} \triangleleft \gamma^{n}$ et $>0$; quocirca quum progressio $\gamma, \gamma^{\prime}, \gamma^{\prime \prime}$ etc. continuo crescat, necessario $\mu$ iacebit inter $o$ et $n$ excl. Forma vero respondens, $f^{\mu}$, identica erit cum forma $f, Q . E . A$, quum omnes formae $f, f^{\prime}$, $f^{\prime \prime}$, etc. vsque ad $f^{n}-1$ diuersae esse supponantur. Ex his colligitur, minimos valores ipsorum $t, u$ (exceptis valoribus $m, 0$ ) esse $T, U$.

Ex. Si $D=79, m=1$ : adhiberi poterit forma $(3,8,-5)$, pro qua $n=6$, atque $\alpha^{\mathrm{n}}=$ $-8,2^{n}=-27,8^{n}=-15^{2}$ (art 188). Hinc $T=80, U=9$, qui sunt valores minimi numerorum $t, u$, aequationi $t t-79 u u=1$ satisfacientes. 
199. Ad praxin formulae adhuc commodio. res erui possunt. Erit nimirum $2 b y^{n}=-a\left(a^{n}\right.$ - s), quod facile ex art. 162 deducitur, muliplicando aequ. [19] per $2 b$, [20] per $a$ et mutando characteres illic adhibitos in praesentes, Hinc fit $a^{n}+\delta^{n}=2 \delta^{n}-\frac{2 b}{a} \chi^{n}$ adeoque

$$
\pm T=m\left(s^{n}-\frac{b}{a} \cdot \gamma^{n}\right), U=\frac{\gamma^{n} m}{a}
$$

Per similem methodum hos, valores obtinemus;

$$
\pm T=m\left(*^{\mathrm{n}}+\frac{b}{a^{\prime}} b^{\mathrm{n}}\right)_{2} \pm U=\frac{m^{n}}{a^{\prime}}
$$

Tum hae tum illae formulae perquam commodae. euadunt, propter $\gamma^{n}=f^{n}-r, \alpha^{n}=6-1$, ita vt si hac vteris, solam progressionem $6 \%, 6 \%, 6 \mathrm{~m} .$.

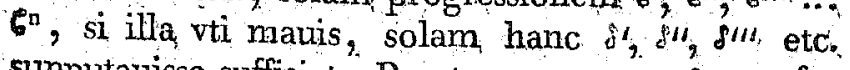
supputauisse sufficiat. Praeterea ex art. 189,3 facile deducitur, quum $n$ necessario sit par, $\alpha^{\mathrm{n}}$ et $\frac{b}{a^{\prime}}$, eadem signa habere ineque minus of et $\frac{b}{a} 2^{n}$, ita ot in formula priori pro, $T$ differentia absoluta, in posteriori summa absoluta accipi debeat, neque adeo ad signa respicere omnino opus sit. Receptis signis in art, 189,4 adhibitis erit ex formula priori

$$
\begin{gathered}
T=m\left[k^{\prime}, k^{\prime \prime}, k^{\prime \prime \prime} \ldots, k^{\mathrm{n}}\right]-\frac{m b}{a}\left[k^{\prime}, k^{\prime \prime}, k^{\prime \prime \prime} \ldots\right. \\
\left.k^{\mathrm{n}}-\mathrm{I}\right] ; \quad U=\frac{m}{a^{\prime} !}\left[k^{\prime}, k^{\prime \prime}, k^{\prime \prime \prime} \ldots ., k^{\mathrm{n}}-\mathrm{I}\right]
\end{gathered}
$$

ex posteriori

$$
\begin{gathered}
T=m\left[k^{\prime \prime}, k^{\prime \prime \prime} \ldots k^{n}-\mathrm{I}\right]+\frac{m b}{a^{\prime \prime}}\left[k^{\prime \prime}, k^{\prime \prime \prime} \ldots \ldots k^{n}\right] \\
U=\frac{m}{a}\left[k_{, \prime \prime}^{\prime \prime} k^{\prime \prime \prime} \ldots \ldots k^{n}\right]
\end{gathered}
$$




\section{$-263-$}

vbi pro valore ipsius $T$ etiam $m\left[k^{\prime \prime}, k^{\prime \prime \prime} \ldots . . k^{n}, \frac{b}{a^{\prime}}\right]$ scribi poterit.

Ex. Pro $D=61, m=2$ adhiberi potest forma $(2,7,-6)$, pro qua eruitur $n=6 ; k \mathbf{I}$, $k^{\mathrm{II}}, k^{\mathrm{III}}, k^{\mathrm{IV}}, k^{\mathrm{V}}, k^{\mathrm{VI}}$ resp. $=2,2,7,2,2,7$. Hinc fit $T=2[2,2,7,2,2,7]-7[2,2,7$, $2,2]=2888-13^{65}=15^{2} 3$, ex formula prima; idem prouenit ex secunda $T=2[2,7,2,2]$ $+\frac{7}{3}[2,7,2,2,7] . \quad U$ vero fit $=\frac{1}{3}[2,2,7,2,2]$ $=[2,7,2,2,7]=195$.

Ceterum plura artificia adhuc dantur, per quae calculus contrahi potest, sed de his fusius hic loqui breuitas non permittit.

200. Vt ex valoribus minimis ipsorum $t, z$ omnes obtineamus, aequationem $T T-D U U=$ $m m$ ita exhibemus $\left(\frac{T}{m}+\frac{U}{m} \vee D\right)\left(\frac{T}{m}-\frac{U}{m} \vee D\right)=$ 1 , vnde etiam erit $\left(\frac{T}{m}+\frac{U}{m} \sim D\right)^{\mathrm{e}}\left(\frac{T}{m}-\frac{U}{m} \sim D\right) \mathrm{e}$ $=1 \ldots .[1$,$] , denotante e$ numerum quemcinque. Iam designabimus breuitatis caussa valores quan. titatum

$$
\frac{m}{2}\left(\frac{T}{m}+\frac{U}{m} \vee D\right)^{\mathrm{e}}+\frac{m}{2}\left(\frac{T}{m}-\frac{U}{m} \vee D\right) \mathrm{e}
$$

$\left.\frac{m}{2 \vee D}\left(\frac{T}{m}+\frac{U}{m} \vee D\right)^{\mathrm{e}}-\frac{m}{2 r D}\left(T-\frac{U}{m} \vee D\right)^{\mathrm{c} *}\right)$ generaliter per $t^{e}, u^{c}$ resp. i.e. illarum valores pro

*) In his solis quatuot expressionibus et in aequ. [ I ] e denotat exposentem potestatis; in reliquis literae apici adscriptae semper indsicing designant. 
$e=0$, per $t^{\circ}, u^{\circ}$ (qui erunt $m, o$ ); pro $e=1$ per $t^{\prime}, u^{\prime}$ (qui fiunt $T, U$ ); pro $e=2$ per $t^{\prime \prime}, u^{\prime \prime}$; pro $t=3$ per $t^{\prime \prime \prime}, z^{\prime \prime \prime}$ etc. - demorstrabimusque, si pro $e$ accipiantur omnes numeri integri non negatiui i. e. 0 , omnesque positiui ab 1 vsque ad $\infty$, expressiones illas, exhibere omnes valores positiuos ipsorum $t, u$ : scilicet I) omnes valores illarum expressionum esse reuera valores ipsorum $t$, $u$; II) omnes valores illos esse numeros integros; III) nullos valores positiuos ipsorum $t$; $u$ dari qui sub formulis illis non contineantur.

I. 'Substitutis pro $t^{e}, u^{e}$ valoribus suis nullo negotio aduimento aequ. [1] confirmatur, esse $\left(t^{e}+u^{t} \sqrt{ } D\right)\left(t^{\mathrm{e}}-u^{\mathrm{e}} \sqrt{ } D\right)=m m ;$ i, e. $t^{e} t^{t}-D u^{e} u^{e}=m m$.

II. Eodem modo facile confirmatur, esse generaliter $t^{\mathrm{t}+\mathrm{r}}+t^{\mathrm{e}}-\mathrm{1}=\frac{2 T}{m} t^{\mathrm{e}}, u^{t}+1+u^{\mathrm{e}-\mathrm{r}}=\frac{2 T}{m} u^{\mathrm{e}}$. Hinc manifestum est duas progressiones $t^{3}, t^{\prime}$, $t^{\prime \prime}, t^{\prime \prime \prime}$ etc., $u^{n}, u^{\prime}, u^{\prime \prime}, u^{\prime \prime \prime}$ etc. esse recurrentes, et vtriusque scalam relationis $\frac{2 T}{m},-1$, scilicet $t^{\prime \prime}=\frac{2 T}{m} t^{\prime}-t^{\prime}, t^{\prime \prime \prime}=\frac{2 T}{m} t^{\prime \prime}-t^{\prime}$ etc. $u^{\prime \prime}=$ $\frac{2 T}{m} u^{\prime}$ etc.

Iam quoniam per hyp. forma aliqua datur, $(M, N, P)$, determinantis $D$, in qua $M, 2 N, P$ per $m$ sunt diuisibiles: habebitur $T^{\prime} T=(N N-$ $M P) ~ U U+m m$, eritque adeo manifesto $4 T T$ per $\mathrm{mm}$ diuisibilis. Hinc $\frac{2 T}{m}$ erit numerus integer 


\section{$-265 \div$}

quidem positiuus. Quia vero $t^{\circ}=m, t^{\prime}=T, u^{\circ}$ $=o, u^{\prime}=U$, adeoque integri: omnes $t^{\prime \prime}$, $t^{\prime \prime \prime}$ etc. $u^{\prime \prime}, u^{\prime \prime \prime}$ etc. etiam integri erunt. Porro perspicuum est, quia $T T>m m$, omnes $t^{\circ}, t^{t}, t^{\prime \prime}, t^{\prime \prime \prime}$ etc. poșitiuos et continuo in infinitum crescentes esse, nec non omnes $u^{\circ}, u^{\prime}, u^{\prime \prime}$, $u^{\prime \prime \prime}$ etc.

III. Supponamus, dari adhuc alios valores positiuos ipsorum $t, u$ qui in progressione $t^{\circ}$, $z^{\prime}, t^{u}$ etc. $u^{\circ}, u^{\prime}, u^{\prime \prime}$ etc. non contenti sint, puta

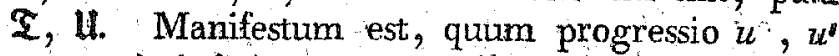
etc. a o in infinitum crescat, $I l$ necessario inter duos terminos proximos, $u^{n}$ et $u^{\mathrm{n}}+\mathbf{I}$ situm fore, ita vt sit $\boldsymbol{U}>u^{n}$ et $\mathbb{U}<u^{n}+\mathbf{I}$. Vt absurditatem huxus suppositionis demonstremus, obseruamus

$1^{\circ}$ Aequationi $t t-D u u=m m$ satisfactum iri etiam ponendo $t=\frac{\mathrm{I}}{m}\left(\mathfrak{T} t^{\mathrm{n}}-D \| u^{\mathrm{n}}\right), u$ $=\frac{I}{m}\left(\boldsymbol{U} t^{\mathrm{n}}-\mathfrak{\Sigma} u^{\mathrm{n}}\right)$. Hoc quidem nullo negotio per substitutionem confirmaturs quod vero hi valores quos ponemus breuitatis gratia $=7,2$, semper sunt numeri integri ita ostendimus. Si $(M$, $N, P$ ) est forma determinantis $D$, atque $m$ diuisor communis numerorum $M, 2 N, P$ : erit tum $\Sigma+N U$ tum $t^{\text {n }}+N u^{\text {n }}$ per $m$ diuisibilis adeoque etiam $\mathbb{U}\left(t^{n}+N u^{\mathrm{u}}\right)-u^{\mathrm{a}}(\mathfrak{T}+N \mathbb{U})$ siue $\mathbb{U} t^{\mathrm{a}}$ - $\mathfrak{T} u^{\mathrm{n}}$. Quare $v$ erit integer et proin etiam ?, quia $17=D v_{\nu}+m m$.

2 Patet 2 non posse esse $=0$; hinc enim sequeretur $\mathfrak{H} \| t^{\mathrm{n}} t^{\mathrm{a}}=\mathbb{T} u^{\mathrm{n}} u^{\mathrm{n}}$ siue $\mathfrak{U} \mathfrak{U}\left(D u^{\mathrm{n}} u^{\mathrm{n}}\right.$ $+m m)=u^{\mathrm{n}} u^{\mathrm{n}}(D \mathfrak{U}+m m)$ siue $\boldsymbol{U} \amalg=$ R 5 
$u^{\mathrm{n}} u^{\mathrm{n}}$, contra hyp. ex qua $\| \mathbf{l}>u^{\mathrm{n}}$. Quum igitur praeter valorem $o$, minimus valor ipsius $u$ sit $U$, erit $v$ certe non minor quam $U$.

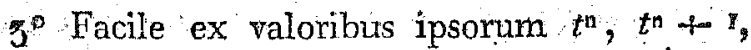
$u^{\mathrm{n}}, u^{\mathrm{n}}+1$ confirmari potest esse $m U=u^{\mathrm{n}}+1 t^{\mathrm{n}}$ $-t^{n}+1 u^{\mathrm{n}}$. Quare $\| t^{\mathrm{n}}-\mathfrak{T} u^{\mathrm{n}}$ certe non erit minor quam $u^{\mathrm{n}}+1 t^{\mathrm{n}}-t^{\mathrm{n}}+\mathrm{I} u^{\mathrm{n}}$.

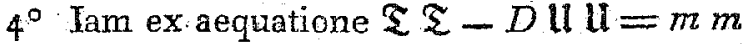
habetur $\frac{\mathfrak{I}}{\mathfrak{u}}=\sqrt{ }\left(D+\frac{m}{\mathfrak{l}} \mathfrak{u}\right)$ et similiter $\frac{t n-1-\mathbb{r}}{\mathfrak{n} n-\mathbf{r}}$

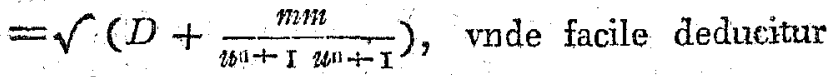
esse $\frac{\mathfrak{I}}{\mathfrak{n}}>\frac{t^{n}+1}{u^{n-1-1}}$. Hinc vero et ex conclusione in $3^{\circ}$ sequitur $\left(\mathbb{l} t^{\mathrm{n}}-u^{\mathrm{n}}\right)\left(t^{\mathrm{n}}+u^{\mathrm{n}} \frac{\mathfrak{I}}{\mathfrak{n}}\right)>$ $\left(t^{n+I}-t^{n}-t^{n+I} u^{n}\right)\left(t^{n}+u^{n} \frac{t^{n-1} I}{u^{n}+I}\right)$, siue, euolutione facta, et loco ipsorum $\mathfrak{Z}, t^{\text {n }} t^{\text {n }}$, $e^{n+I} t^{n+I}$ substitutis valoribus suis $D \mathfrak{U} \mathbf{U}+m m$, $D u^{\mathrm{n}} u^{\mathrm{n}}+m m, D u^{\mathrm{n}+\mathrm{I}} u^{\mathrm{n}-1 \mathrm{I}}+m m$;

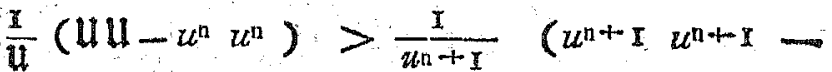

$$
\begin{aligned}
& \left.u^{\mathrm{n}} \quad x u^{\mathrm{n}}\right) \text {, }
\end{aligned}
$$

vnde, quoniam vtraque quantitas manifesto positiua, fit transponendo $\mathbb{l}+\frac{u u^{\mathrm{n}}}{u^{\mathrm{n}} \mathrm{n}+\mathrm{I}}>u^{\mathrm{n}+\mathrm{n}}+\frac{u \mathrm{n}}{\mathfrak{u}}$, Q. E. A., quia quantitatis prioris pars prima minor est quam pars prima quantitatis secundae, nec non illias secunda minor quam secunda bu- 
ius. Quamobrem suppositio consistere nequie et progressiones $t^{*}, t^{i}, t^{\prime \prime}$, etc. $u, u^{\prime}, u^{\prime \prime}$, etc. omnes valores positivos ipsorum, $t, u$ exhibebunt.

Ex. Pro $D=61, m=2$ valores minimos positiuos ipsorum $t, u$ inuenimus 1523,195 : quare omnes valores positiui exhibebuntur per has formulas $t=\left(\frac{1523}{2}+\frac{195}{2} \sqrt{61}\right)^{\mathrm{e}}+\left(\frac{15^{23}}{2}\right.$ $\left.-\frac{195}{2} \sqrt{61}\right)^{2}, u=\frac{1}{\sqrt{ } 6 \mathrm{r}}\left(\left(\frac{15^{2} 3}{2}+\frac{195}{2} \sqrt{61}\right)^{2}\right.$ $\left.-\left(\frac{15^{2} 3}{2}-\frac{195}{2} \sqrt{61}\right)^{\mathrm{e}}\right)$. Inuenitur autem $t^{0}=2, t^{\prime}=1523, \quad t^{\prime \prime}=1523 t^{\prime}-t^{\circ}=$ $23195^{27}, t^{\prime \prime \prime}=1523 t^{\prime \prime \prime}-t^{\prime}=3532618098 \mathrm{etc}$; $u^{\prime \prime}=0, u^{\prime}=195, u^{\prime i}=1523 u^{\prime}-u^{\circ}=296985$, $u^{\prime \prime \prime}=1523 u^{\prime \prime}-u^{\prime}=452307960$ etc.

201. Circa problema in artt. praecc. tractatum sequentes obseruationes adhuc adiicimus.

1) Quum aequationem $t t-D u u=m m$ pro omnibus casibus soluere docuerimus, vbi $m$ est diuisor commurris maximus trium numerorum $M, 2 N, P$, talium vt $N N-M P=D$ : operae pretium est omnes numeros qui tales diuisores esse possunt siue omnes valores ipsius $m$ pro valore dato ipsius $D$ assignare. Ponatur $D=$ $n n D^{\prime}$, ita vt $D^{\prime}$ a factoribus quadraticis omnino sit liber, quod obtinetur si pro $n n$ assumitur maximum quadratum ipsum $D$ metiens: $\sin$ vero $D$ 
Sam per se nullum factorem quadraticum implicaret, fieri deberet $n=1$. Tum dico

Primo, si $D^{\prime}$ fuerit formae $4 k+1$, quemuis diuisorem ipsius $2 n$ fore valorem ipsius $m$, et vice versa. $\mathrm{Si}$ enim $\mathrm{g}$ est diuisor ipsius $2 \mathrm{n}$, habebitur forma $\left(g, n, \frac{n n\left(D^{\prime}-x\right)}{g}\right)$, cuius determinans est $D$, et in qua manifesto diuisor communis maximus numerorum $g, 2 n, \frac{n n\left(D^{i}-\mathrm{I}\right)}{g}$ erit $g$ (patet enim $\frac{n n\left(D^{\prime}-\mathrm{I}\right)}{g g}=\frac{4 n n}{g g} \cdot \frac{D^{g}-\mathrm{I}}{4}$ esse numerum integrum ). Si vero, vice versa, g supponitur essé valor ipsius $m$, scilicet diuisor communis maximus numerorum $M, 2 N, P$, atque $N N-M P=D:$ manifesto $4 D$ siue $4 n n D^{\prime}$ diuisibilis erit per $g g$. Hinc vero sequitur, $2 n$ necessario per $g$ diuisibilem esse. $\mathrm{Si}$ enim $g$ ipsum $2 n$ non metiretur, $g$ et $2 n$ haberent diuisorem communem maximum minorem quam $g$, quo posito $=\delta$, atque $2 n=$ $\delta n^{\prime}, g=\varepsilon^{\prime} g^{\prime}$, foret $n^{\prime} n^{\prime} D^{\prime}$ per $g^{\prime} g^{\prime}$ diuisibilis, $n^{\prime}$ ad $g^{\prime}$ adeoque etiam $n^{\prime} n^{\prime}$ ad $g^{\prime} g^{t}$ primus et proin etiam $D^{\prime}$ per $^{\prime} g^{\prime} g^{t}$ diuisibilis, contra hyp. secundum quam $D^{\prime}$ ab omni factore quadratico est liberatus.

Secundo, si $D^{\prime}$ fuerit formae $4 k+2$ vel $4 k+3$, quemuis diuisorem ipsius $n$ fore valorem ipsius $m$, et vice versa quemuis valorem ipsius $m$ metiri ipsum $n$. Si enim $g$ est diuisor ipsius $n$, habebitur forma $\left(g, 0, \frac{n \cdot n D^{\prime}}{g}\right)$, cuins 


\section{$-269-$}

determinans $=D$, et $v$ bi manifesto numerorum $g, o, \frac{n n D^{\prime}}{g}$ diuisor communis maximus exit $g .-$ Si vero $g$ supponitur esse valor ipsius $m$, puta diuisor communis maximus numerorum $M, 2 N$, $P$, atque $N N-M P=D$ : eodem modo vt supra $g$ metietur ipsum $2 n$, siue $\frac{2 n}{g}$ erit integer. Si quotiens hic esset impar: quadratum $\frac{4 n n}{g g}$ foret $\equiv 2(\bmod 4)$, adeoque $\frac{4 m i D^{\prime}}{g^{\prime}}$ aut $\equiv 2$ aut $\equiv 3\left(\bmod _{4}\right) \quad$ At $\frac{4 n n D}{g g}=\frac{4 D}{g g}=\frac{4 N N}{g g}$ $-\frac{4 N P}{g g} \equiv \frac{4 N N}{g g}(\bmod 4)$, et proin $\frac{4 N N}{g g}$ aut $\equiv 2$ aut $\equiv 3$ (mod.4). Q.E.A., quia omne quadratum aut cifrae aut vnitati secundum modulum 4 congruum esse debet. Quare quotiens $\frac{2 n}{g}$ necessario erit par, adeoque $\frac{n}{g}$ integer, siue $g$ diuisor ipsius $n$.

Patet itaque, 1 semper esse valorem ipsius $m$, siue aequationem $t t-D u u=1$ pro quouis valore pasitiuo non quadrato ipsius $D$ per praecedentia resolubilenn esse; 2 tunc tantummodo esse valorem ipsius $m$, si $D$ fuerit aut formae $4 k$, aut formae $4 k+1$.

2) Si $m$ est maior quam 2 , attanen numerus idoneus, solutio aequationis $t t-D u u=$ $m m$ reduci potest ad solutionem similis aequa- 
tionis, vbi $m$ est aut 1 aut 2. Scilicet posito vt ante $D=n n D^{\prime}$, si $m$ ipsum $n$ metitur, metietur $m m$ ipsum $D$. Tum si valores minimi ipsorum $p, q$ in aequatione $p p-\frac{D}{m m} q q=I$ supponuntur. esse $p=P, q=Q$, valores minimi ipsorum $t, u$ in aequatione $t t-D u u=m m$ erunt $t=m P, u=Q$. - Si vero $m$ ipsum $n$ non metitur, metietur saltern ipsum $2 n$ eritque certo par; $\frac{4 D}{m m}$ autem integer. Et si tunc valores minimi ipsorum $p, q$ in aequatione $p p-\frac{4 b}{m m} q q$ $=4$ inuenti sunt $p=p, q=Q$ : valores minimi ipsonum $t, u$ in aequatione $t t-D u u=m m$ erunt $t=\frac{m}{2} P, u=Q$. In vtroque autem casu non solum ex valoribus minimis ipsorum $p$, $q$ valores minimi ipsorum $t, u$, sed ex omnibus valoribus illorum omnes valores horum per hane methodum manifesto deduci poterunt.

3) Designantibus $t^{\circ}, u^{n} ; t^{\prime}, u^{\prime} ; t^{\prime \prime}$, $u^{\prime \prime}$ etc: omnes valores positiuos ipsorum $t, u$ in aequatione $t t-D u t=m m$ (vt in art. praec.), st contingit vt valores quidam ex serie illa, valóribus primis in eadem secundum modulum quemcunque datum $r$, congrui sint, puta $t^{\rho} \equiv t^{\prime}$ (siue $\equiv m), u^{\circ} \equiv u^{\circ}$ siue $\equiv o(\bmod , r)$; simulque valores proxime sequentes valoribus secundis; puta $t^{\rho}+\mathrm{I} \equiv t^{\prime}, u^{\prime-1} \equiv u^{\prime}(\bmod . r$ : erit etiam $t^{p+2} \equiv t^{\prime \prime} u^{p+2} \equiv u^{\prime \prime}, t^{p+3} \equiv t^{\prime \prime}, u^{p+3} \equiv$ 
u'u etc. Hoc facile inde deducitur, quod vtraque series $t^{\prime}, t^{\prime}, t^{\prime \prime}$ etc., $u^{\circ}, u^{\prime}, u^{\prime \prime}$ etc. est ex recurrentium genere, scilicet quoniam $t^{\prime \prime}=\frac{2 T}{n n} t^{4}$ $t^{\circ}, t^{p+2}=\frac{2 T}{m} t^{p+1}-t^{\rho}$ erit $t^{\prime \prime} \equiv t^{\rho+2}$ similiterque de reliquis. - Hinc autem sequitur, fore generaliter $t^{\mathrm{h}+\mathrm{s}} \equiv t^{\mathrm{h}}, u^{\mathrm{h}+\mathrm{g}} \equiv u^{\mathrm{h}}$ (mod. $r)$, denotante $h$ numerum quemcunque, nec non adkuc generalius, si fuerit $\mu \equiv \nu$ (mod. $\rho$ ), fore $u^{\mu} \equiv t^{\prime}, u^{\mu} \equiv u^{\prime}(\bmod , r)$

4) Conditionibus autem in obseru. praec, re quisitis semper satisfieri potest, scilicet semper inueniri potest index $\rho$ (pro modulo quocunque dato $r$ ) pro quo sit $t^{\rho} \equiv t^{\circ}, t^{\rho+1} \equiv t^{t}, u^{\rho} \equiv$ $u^{\circ}, u^{\rho+-1} \equiv u^{d}$ Ad quod demonstrandum $o b-$ seruamus.

primo, conditioni tertiae semper satisfieri posse. Nullo enim negotio per criteria in (1) tradita perspicietur, etiam aequationem $p p-r r D q q=$ $m m$ solubilem fore; et si walores minimi positiu ipsorum $p, q$ (praeter hos $m, o$ ) supponuntur esse $P, Q$ : inter valores ipsorum $t, u$ manifesto erunt etiam $t=P, u=r Q$. Quare $P, r Q$ in progressionibus $t^{\circ}$, $t^{\prime}$ etc.; $u^{\circ}, u^{\prime}$ etc. contenti erunt, et si $P=t^{\lambda}, r Q=u^{\lambda}$, erit $u^{\lambda} \equiv 0 \equiv$ $u^{\circ}(\bmod \cdot r)$. Praeterea facile perspicietur, inter $u^{\circ}$ et $u^{\lambda}$ nullum terminum fore ipsi $u^{\circ}$ secundum modulum $r$ congruum.

Secundo patet, si hic insuper tres reliquae conditiones adimpletae sint, puta si etiam $u^{\lambda+1} \equiv u^{k}$ 
$t \equiv t^{\circ}, t^{\lambda+1} \equiv t^{\prime}$, poni tantummodo debere $\xi=$. Si vero vna aut altera illarum conditionum locum non habet, dico certe statui posse $\xi$ $=2$. Nam ex aequat. [1] formulisque generalibus pro $t^{\mathrm{e}}, u^{\mathrm{e}}$ in art. praec. deducitur $t^{2 \lambda}=$ $\frac{x}{m}\left(t^{\lambda} t^{\lambda}+D u^{\lambda} u^{\lambda}\right)=\frac{x}{m}\left(m m+2 D u^{\lambda} u^{\lambda}\right)$ adeoque $\frac{t^{2 \lambda}-t^{0}}{r}=\frac{2 D u^{\lambda} u^{\lambda}}{m r}$, quae quanti. tas erit numerus integer, quia per hyp $r$ ipsum $u^{n}$ metititur, nec non $m m$ ipsum $4 . D$, adeoque a potiori $m$ ipsum $2 D$. - Porro erit $u^{2 \lambda}=\frac{2}{\bar{m}} t^{\lambda} u^{\lambda}$, et quoniam $4 t^{\lambda} t^{\lambda}=4 D u^{\lambda} u^{\lambda}+4 \mathrm{~mm}$, adeoque per $m m$ diuisibilis, $2 t^{\lambda}$ erit diuisibilis per $m$, et proin $u^{2 \lambda}$ per $r$, siue $u^{2 \lambda} \equiv u$ (mod. r) - Tertio inuenitur $t^{2 \lambda+1}=t^{k}+\frac{2 D u^{\lambda} u^{\lambda+x}}{m}$. et quoniam ex simili ratione $\frac{2 D u^{\lambda}}{m r}$ est integer, erit $t^{2 \lambda+I} \equiv t^{t}(\bmod . r)$. Tandem reperitur $u^{2 \lambda+x}=u^{\lambda}+\frac{2 t^{\lambda+1} u^{\lambda}}{m}$, et quoniam $2 t^{\lambda+\mathrm{T}}$ per $m$ diuisibilis est, $u^{\lambda}$ per $r$ erit $u^{2 \lambda+\mathrm{I}}$ $\equiv u^{\prime}(\bmod r) \cdot$ Q. E. D.

Ceterum vsus posteriorum duarum obseruationum in sequentibus apparebit.

202. Casus particularis problematis, nempe soluere aequationem $t t-D u u=1$, iam a geometris seculi praecedentis fuit agitatus. Sagacissimus Fermatius problema hoc analystis Anglie 
proposuit, Wallisiusque Brounkerum tamquam inuentorem solutionis quam in Alg. Cap. 98, Opp. T. II p. 418 sqq. tradit nominat; Ozanam Fermatium; denique ill. Euler qui de illo egit in Comm. Petr. VI. p. 175, Comm.nou. XI, p. 28*); Algebra P.II. pag. 226, Opiusc. An. I.p.310, Pellinm, vnde problema illud a quibusdam auctoribus Pellianzem vocatum est. Omnies hae solutiones, si essentian spectas, conueniunt cum ea quam obtinemus, si in art. 198 formam reductam eam adoptamus in qua $a$ $=1$ s attamen operationem quam praescribunt tandem necessario finiri, siue problema semper reuera solubile esse, nemo ante ill. La Grange rigorose**) demónstrauit, Melanges de lo Sor. de Turin T. IV. p I9, et concinnius Hist de l'Ac. de Brrlin, $1767, p$. 237. Exstat haec disquisitio etiam in supplementis ad Euleri Atgebran iam saepins laudatis, Ceterum inethodus nostra (ex principiis ommino diuersis petita, neque ad casum $m=1$ restricta) plerumque plures vias ad solutionem perueniendi suppeditat, quoniam in art 198 a quauis alia forma reducta $\left(a, b,-a^{\prime}\right)$ proficisci possumus.

*) In hac comm, algorithmus quem art. 32 exposuimus, per similla signa exhlbetur, quod nos illic annotare negleximus.

*) Quae Wallisius ad hunc finem protulit l. c. p. 427, 428 nibil ponderis habent. Paralogismus in eo consistit, quod p. 428 l. 4, supponit, proposita quantitate $p$ inueniri posse numeros integros $a ; z$ tales vt $\frac{z}{a}$ minor sit quam $p$, deiectus vero assigrato minor. Hoo vtique verum "est quando defectus assignatus est quantitas data, neque vero, quando ab at $z$ peader adeoque variabilis est, vti in casu praesenti onenit. 
205. Problema. Si formate $\phi$ sibnt aequiwalentes, omnes transformationes alterius in aftoran exhibere.

Sol. Quando formae hae vico tantum modo aequiualentes sunt (i. e. aut proprie tantum aut improprie tantum) quaeratux per art. $19^{6}$ transformatio vna formae $\phi$, in $\phi$, quae sit $s$, $E_{j} \gamma$, \& patetque alias quam quae huic sint simi les, dari non posse. Quando vero $\phi, \Phi$ tum proprie tum improprie aequiualent, quaerantur duae transformationes dissimiles, $\mathbf{i}$. e. altera propria altera impropria, puta $\alpha, 6, \gamma$, \& et $x^{\prime}, 6 ;$ $\gamma^{\prime}, \delta$, eritque quaeuis alia transformatio aut hutic aut illi similis. Si itaque forma est $(a, b, c)$ ipsius determinans $=D$, diuisor communis maximus numerorum $a, 2 b, c$ (vi semper in praec.) $m$, atque $t, u$ indefinite omnes numeri aequationi $t t-D u u=m m$ satiffacientes: in cast priori omnes transformationes formae $\phi$ in conttentae erunt sub prima formularum sequentiun $Y$, in posteriori vel sub prima $[\mathrm{rul}$ sub secunda II.

$$
\begin{aligned}
& \text { I... } \frac{1}{m}(a t-(w b+y c) u), \frac{x}{r}(b t-(c b+b) w) \text {. } \\
& \frac{1}{x}(y t+(x a+b) u) \cdot \frac{1}{x a}(d t+(a+b) w)
\end{aligned}
$$

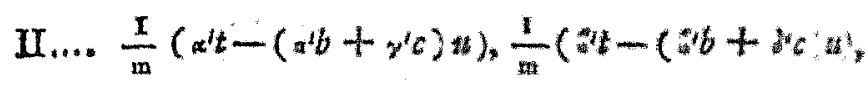

$$
\begin{aligned}
& \frac{x}{m}\left(x^{\prime} t+\left(a^{t} a+x^{t} b\right) w\right), \frac{x}{m}(d t+(b+b) b)
\end{aligned}
$$

Ex. Desiderantur omuts transformativats formae $\left(129,9^{2}, 05\right)$ in forman $(42,39 \%$ 


\section{- $875-$}

81). Ias improprie tantum aequiualentes esse in art. 195 inuenimus et in art. seq. transfornaLonten impropram illius in thane eruimus. .47 , $-56,73,87$. Quamobirm omes transformationes forruat $(129,92,65)$ in $(42,59,81$, exhibebuntur per formulam $\left.\frac{\text { a }}{47} t+421 u\right)$, $-(56 t+503 u), 73 t+653 u, 87 t+730 u$, vbi $t$, us sunt indefinita omuss numberi aequatimit $t t-79 u u=1$ satisfacientes; hi vero exhibentur per formulas

$\pm t={ }_{2}^{x}\left((80+9 \sqrt{79})^{n}+(80-9 \sqrt{79})^{\mathrm{o}}\right)$, $\left.\pm u=\frac{x}{2979}(80+9 \sqrt{79})^{\circ}-(80-9 \sqrt{79})^{\circ}\right)$ vi pro $c$ omnes numexi integri non negatiui sunt accipiendi.

201. Perspicum est, formularz generaln omnes transforthationes exhibratem eo simpiti ciarem etadere, quo simplicior fuert transformatio initialis ex qua lormula est deducta. Iam qum arbitrarum sit, a "qua transionatione proficiscamur, samonumero formula genesalis simplicior

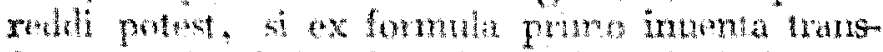

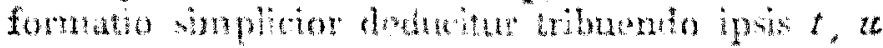

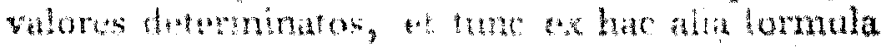
componitr. Ta e. ge positis in formula in ex. art. prate. inument, $t=80, u=-4$, prodit trans-

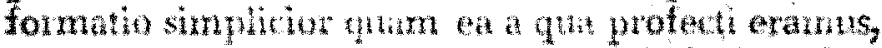
scilice $29,6,-37,-$ tio vnde deducitur for mula gentralis $29 t-26 y, 4 t t-424 t,-$ $37 t+337 u,-60 t+543 u$. Quando itaque per pratecepta pratecedentia formula generalis enuta 


\section{$-276-$}

est, tentari poterit, annon, tribuendo ipsis $u$ valores determinatos $\pm t^{\prime}, \pm u^{\prime} ; \pm t^{\prime \prime}, \pm u^{\prime \prime}$ etc. transformatio obtineatur simplicior quam ea ex qua formula deducta fuit, in quo casu ex illa transformatione formula simplicior deriuari poterit. $-\mathrm{Ce}$ terum in diudicanda simplicitate aliquid arbitrarii remanet, quod si operae pretium esset ad normam fixam reuocare, nec nox in progressione $t^{\prime}$, $u^{\prime} ; t^{\prime \prime}, u^{\prime \prime}$ etc. limites assignare possemus, vltra quos transformationes continuo minus simplices prodeant, ita vt vltra progiedi opus non sit sed intra illos tentamen instituisse sufficiat: attamen quum plerumque per methodos a nobis praescriptas transformatio simplicissima vel statim vel adhibitis pro $t, u$ valoribus $\pm t, \pm u$ prodire soleat, hanc disquisitionem breuitatis gratia supprimimus.

205. Problema. Inuenire onnes repraesentationes ninueri dati $M$ per formulan datam axx + $2 b x y+c y y$, cuius determinans positiuns non - quadratus $=D$.

Sol. Primo obseruamus, inuestigationem repraesentationum per valores ipsorum $x, y$ inter se non primos, hic prorsus eodem modo, vt supra (art. 181) pro formis determinantis negatiui, ad eum casum reduci posse, vbi repraesentationes per valores indeterminatarum inter se primos quaeruntur, quod igitur hic repetere superfluum foret. Ad possibilitatem repraesentationum per valores ipsorum $x, y$ inter se primos autem requiritur, rt $D$ sit residuum quadraticum ipsius $M$, et si omnes valores expressionis $\sqrt{ } D(\bmod M)$ sunt $N$, 
$-N, N^{\prime},-N^{\prime}, N^{\prime \prime},-N^{\prime \prime}$ etc. (quos ita accipere licet vt nullus sit $\left.>\frac{\mathrm{r}}{2} M\right)$, quaeuis repraesentatio numeri $M$ per formam propositam ad aliquem horum valorum pertinebit. Ante ommia itaque valores illi erui debebuit; tunc repraesentationes ad singulos pertinentes deinceps inuestigari. Repraesentationes ad valorem $N$ pertinentes non dabuntur, nisi formae $(a, b, c)$ et $(M, N$, $\frac{N N-D}{M}$ ) proprie aequiualentes sunt; si vero sunt, quaeratur transformatio aliqua propria prioris in posteriorem, quae sit $a, b, \gamma, \delta_{0}$ Tum habebitur repraesentatio numeri $M$ per formam $(a, b, c)$ ad valorem $N$ pertinens haec: $x=$ $\alpha, y=\gamma$, omnesque repraesentationes ad hunc valorem pertinentes exlibebuntar per formulam $x$ $=\frac{1}{\mathrm{~m}}(\alpha t-(\alpha b+\gamma c) u), y=\frac{1}{\mathrm{~m}}(\gamma t+(a a+\gamma b) u)$, designante $m$ diuisorem communem maximum numerorum $a, 2 b, c$; et $t, u$ indefinite omnes numeros aequationi $t t-D u u=m m$ satisfacientes. - Ceterum manifestum est, formulam hanc generalem eo simpliciorem euadere, quo simplicior sit transformatio $\alpha, 6, \gamma, \delta$ ex qua deducta est; quare haud inutile erit, transformationem simplicissimam tormae $(a, b, c)$ in $(M, N$, $\frac{N N-D}{M}$ ) secundum art. praec. antea eruere, et ex hac formulam deducere. - Prorsus eodem. modo repraesentationes ad valores reliquos $-N$, $N^{\prime},-N^{\prime}$ etc. pertinentes (si quae dantur) per formulas generales exhiberi possunt

Ex. Quaeruntur omnes repraesentationes numeri 585 per formulam $42 x x+62 x y+21 y y$. 
Quod ad repraesentationes per valores ipsorum $x, y$ inter se non primos pertinet, statim patets alias huins generis dari non posse, quam in quibus diuisor communis maximus ipsornm $x, y$ sit $3:$ quum 585 per vnicun quadratum 9 diuisibilis sit. Quando itaque omnes repraesentationes numeri $\frac{585}{9}$ i. e. 65 per formam $42 x^{i} x^{2}+$ $6.2 x^{\prime} y^{\prime}+21 y^{\prime} y^{\prime}$ imuentae sunt, in quibus $x^{\prime}$ ad $y^{\prime}$ primus; ornnes repraesentationes numeri 585 per formam $42 x x+62 x y+21 y y$, in quibus $x$ ad $y$ primus, ex illis deniubuntur ponendo $x=$ $3 x^{\prime}, y^{\prime}=3 y^{\prime}$. Valores spressionis $\sqrt{ } 79$ (mod. 65) sunt $\pm 12, \pm 27$. Reprassentatio numeri 65 ad valorem +12 pertinens inuenitur $x^{t}=$ $2, y^{\prime}=-1$; quocirca omnes repraesentationes ipsius $6_{5}$ ad hunc valorem pertinentes exhibebuntur per formulam $x^{\prime}=2 t-4.12, y^{\prime}=$ $-t+53 u$, adeoque omnes repraesmntationes ipsius 585 hine oriundae per formularn $a=6 t-$ $123 u, y=-3 t+169 u$. Simili modo imuenitur formula generalis omnes repratesentationes munteri 65 ad valorem -12 pertinentes whibern $x^{t}=$ $22 t-199 u, y^{\prime}=-9 g t+214 k$; tormula onnes repraesentationes numeri 585 hinc ormulas complectens $x=66 t-597 u, y=-69 t+635 t u$ Ad valores +27 et -27 autern nulls reprotesentatio numeri 65 portinut - Vt repruesertationas numeri 585 per valores ipsonm $x, y$ inter se primos inueniantur, primo valores expressionis $\sqrt{ } 79(\bmod .585)$ eruere oportat, qui sutht $+-7 \%$, $+105, \pm 157, \pm 248$. Ad valores 577, E 109 , I24.8 inuenitur nullam repraesentationem perchesere; ad valorem +157 autem pertinet repraesentav 


\section{$-\quad 279$}

tio $x=3, y=1$, wnde deducitur formula generalis omnes repraesentationes ad hunc valorem pertinentes exhibens $x=3 t-114 u, y$ $=t+157 u ;$ similiterque inuenitur repraesentatio ad -157 pertinens $x=83, y=$ -87 , et formula in tua omnes similes sunt contentae $x=83 t-746 u, y=-87 t+789 k$. Habentur itaque quatuor formulae generales sub quibus omnes repraesentationes numert 585 per forman $4.2 x x+62 x y+2 x y y$ contentae sunt

$$
\begin{array}{ll}
x=6 t-123 u & y=-3 t+159 u \\
x=66 t-597 u & y=-69 t+653 u \\
x=3 t-114 u & y=t+157 u \\
x=83 t-7.46 u & y=-87 t+789 u
\end{array}
$$

vbi $t, u$ indefinite omnes numeros integros denotant, qui aequationi $t t-70 u k=1$ satisfaciunt

Applicationibus specialibus disquisitionum praecedentitm de formis determinantis positiui non-quadrati breuitatis gratia non immoramur, quippe quas simili modo vt artt. 176,182 quisque, sine negotio, proprio marte instituere poterit statimque ad formas determinantis positiui quadrati, quae solae adhuc supersunt, properamus.

206. Probcem. Proposita forma $(a, b, c)$ dethminantis quadrati hh, designante hipasus radicem postuban, anternire formath $(A, B, C)$ illi progrie

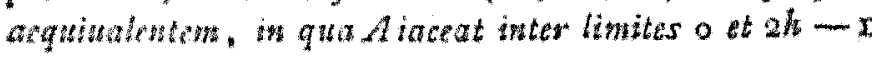
such, $B$ sit $=h, C=0$.

Sol. I. Quoniam $h h=b b-a c$, erit $(h-b): a=c:-(h+b)$. Sit huic rationi aequalis ratio 6 : ita vt 6 ad sit primus, determinenturque $a, \gamma$ ita $\mathrm{nt}$ sit $28-6 \gamma=1$, quae $S_{4}$ 
fieri poterunt. Per substitutionem $\alpha, \epsilon, \gamma, f$ transeat forma $(a, b, c)$ in $\left(a^{t}, b^{t}, c^{\prime}\right.$, quite igitur illi proprie aequiualens erit. Habebitur antem $b$ $=a \omega+b(\omega \delta+b y)+c \delta=(h-b)+$ $b(x+c)-(h+b) b y=h(a \delta-b)$

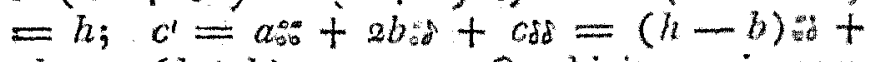
$2 b \%-(h+b)=0$. Quodsi itaque insuper $\boldsymbol{a}^{\prime}$ inter limites o et $2 / 2-1$ iam est silus, forma $\left(a^{\prime}, b^{\prime}, c^{\prime}\right)$ ommibus conditionibus satisfaciet.

II. $S$ i vero $a^{\prime}$ extra limites 0 et $2 h-1$ iacet, sit $A$ residum minimum positiuun ipsius $a^{t}$ secundum modulum $2 h$, quod minifesto inter hos limites situm erit, ponaturque $A-a^{\prime}=2 h k$. Tum forma $\left(a^{\prime}, b^{\prime}, c^{\prime}\right)$ i. e. $\left(a^{\prime}, h, 0\right)$ per stabstitutionem $\mathrm{x}, O, k, I$ transibit in formam $(A, h, o)$, quae formis $\left(a^{\prime}, b^{\prime}, c^{\prime}\right),(a, b, c)$ proprie aequivalens erit omnibusque conditionibus satisfaciet. Ceterum perspicuum est, formam $(a, b, c)$ truns ire in formam $(A, h, 0$ ) per substitutionem + $6 k, 6, y+\delta k, \delta$

Ex. Proposita sit forma $(27,15,8)$ cuins determinans $=9$. I fic $h=r$; rationibus -12 : $27=8:-18$ in numerio minimis aequalis est ratio $4:-9$. Powitis itaque : $=4,+=$ $-9, \alpha=-x, y=2$, form $\left(a, b, b^{\prime}\right)$ it $(-1,3,0)$, quae transit in formam $(5,3,0)$ per subutitutionem $1,0,1,1$. Waec igitur est forma quaesita, transitque in eam proposita per substitutionem propriam $3,4,-7,-9$.

Tales formas $(A, B, C)$ in quibur $C=0$, $B=h, A$ inter limites o et $2 h-1$ situs, form mas reductas vocabimus, quat igitur a formis reductis determinantis negatiu, veI peritui nonquadrati, probe surt distinguendae. 
207. Tuzonera Dute formae ratuctae $(a, b, o)$, (a', h. o), non iduticae propra auqualentes esse won possumt.

Dom. Si enim proprie aequimalero suppontmotur, transwat prior in poskeiorem per substituitonem prophiam $*, \infty, \delta$, habebunturque

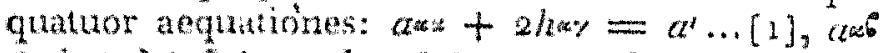
$+h(\ldots+i)=h \ldots[2], a^{*}+2 h=0 \ldots[3]$, * $\%=1 \ldots[4]$ Mluliplicando arguationem secundam per :, tertian per + et subtrahendo

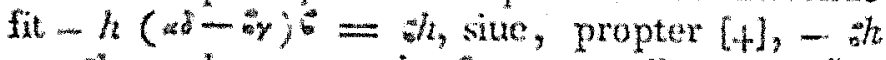
$=a$, vinde necessario $:=0$. Quare ex [4], $* \delta=x$, et $*= \pm x$. Iinc ex [1], $a \pm 2, h$ $=a^{\prime}$, quae awoquatio consi-tere nequit, nisi $\%$ $=0$ (quonian tum a tum $a^{t}$ per hyp. inter 0 et $2 h-1$ iacent) i. $t$ nixi $a=a^{\prime}$, sime formae $(a, h, 0),\left(a^{t}, h, 0\right)$ identicae, contra hyp.

Irine sequentia problemata, quae pro determinantibus non-quadratis multo matorem difficultatem facessebaxt, nullo negotio solui poterunt

I. Propositis duatus formis $F$, Finstem determinantis quadrati, inustigart an proprie acquialeant. Quaterantur duae formae reductae formis $l \%$, $F$ resp. propriv aequiualentes; que si identicae sunt, propositae yroprie aequiualentes erunt, sin minus, non ermal.

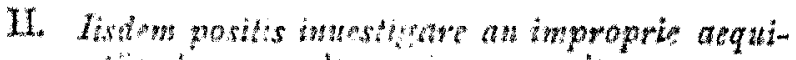

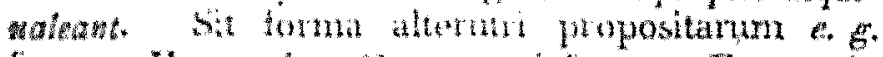

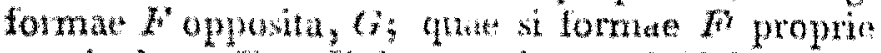
aequitulkt, $f f$ et ${ }^{t}$ inpropre aequixalebunt, et contra. 


\section{$-\quad 282$}

208. Probrema. Propositis duabus formis $F$, F determinantis ha proprie aequinalentibus: intewire transformationem propriam afterius in alterath.

Sol. Formae $F$ proprie aequiualeat forma reducta $\$$, quae itaque per hyp. etiam format $F$ proprie aequiualebit. Quaeratur per art. 206 transformatio propria formae $F$ in $\phi$, quat sit «, $6, \%, \delta$; nec non transformatio propria formas 'F' in $\Phi$, quae sit $\alpha^{\prime}, \phi^{\prime}, \gamma^{\prime}, \delta$. Tunc transtormabitur in $I^{\prime}$ per substitutionem propriam ${ }^{\prime} r_{3}$ $6,-\gamma^{\prime}, \alpha^{\prime}$ et hinc $F$ in $l^{*}$ per substitutionem pro* priam $d^{\prime}-6 x^{\prime}, 6 x^{\prime}-w^{\prime \prime}, y^{\prime \prime}-2 y^{\prime}, d x^{\prime}-y^{\prime \prime \prime}$,

Operae pretium est, alam formulam pro hac transformatione formae $F$ in $F$ euoluere, ad quam formam reductam ipsam nouisse ne opus quidem sit. Ponamus formam $F$ esse $\left(a^{t}, b^{\prime}, c\right)$, $F^{\prime}=\left(a^{\prime}, b^{\prime}, c^{\prime}\right), \Phi=(A, h, 0$ ? Quoniand lationibus $h-b$ : a vel $c:-(h+b)$ in mumeris minimis aequalis est ratio $6: \delta$, facile persincitur $\frac{h-b}{b}=\frac{a}{\delta}$ fore integrum, qui sit $f ;$ nec non $\frac{b}{b}$ $=\frac{-h-b}{\delta}$ integrum fore qui ponatur $=g$. Habebitur autem $A=a_{\text {ata }}+2 b_{2 y}+c y$ adeoque $A$

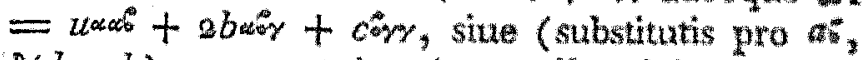
$s(h-b)$, pro $c, g,), A=4 h+b(2, y-b)$ +6 bry sime (propter $b=-h-l g), \quad h=$

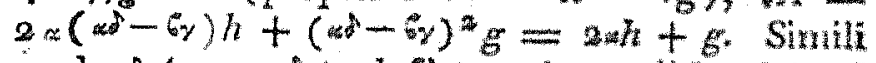

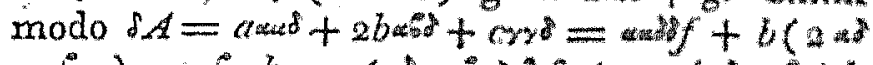

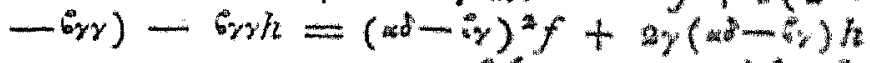
$=2, h+f$. Quare $=\frac{c A-g}{2 h}, y=\frac{d A-j}{2 h}$. 


\section{$-283-$}

Prorsus eodem modo positis $\frac{h-b^{\prime}}{b^{\prime}}=\frac{a^{\prime}}{b^{\prime}}=f^{\prime}, \frac{c^{\prime}}{b^{\prime}}$ $=\frac{-h-b^{\prime}}{\delta^{\prime}}=g^{\prime}$ fit $x^{\prime}=\frac{\delta^{\prime} A-g^{\prime}}{2 h}, \gamma^{\prime}=\frac{\delta^{\prime} A-f}{2 h}$. Quibus valoribus ipsorum $\alpha, \alpha^{\prime} \gamma^{\prime}$ in formula modo tradita pro transformatione formae $F$ in F' substitutis, transit in hanc:

$\frac{g f-d^{\prime} g}{2 h}, \frac{6^{\prime} g-g^{t}}{2 h}, \frac{\delta f^{\prime}-\delta f f}{2 h}, \frac{b^{\prime} f-d g t}{2 h}$, ex qua $A$ omnino abiit.

Si duae formae $F, F^{\prime}$ improprie aequiualentes proponuntur, et transformatio impropria alterius in alteram quaeritur, sit torma $G$ opposita forma $F$, et transformatio presua formae $G$ in $F^{\prime \prime}$

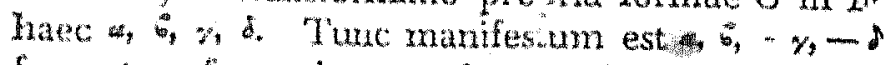
fore transformationem impropriam formae $F$ in $F$.

Denifque patet, si formae propositae et proprie et improprie aequiualentes sint, hoc modo inuenire posse transformationes duas alteram pro. priam alteram impropriam.

209. Nihil itaque jam superest quam vt ex Tha transfonnatione omnes reliquas similes dednceredocemus Hoc vero pentet a solutione aequa-

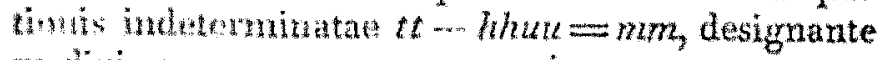
me dusteren communem raximum numerorum $a, 2 b, c$, si $(a, b, c)$ est alterutra formarum aequildentium. Sed hate aequatio semper duobus tatutum modis solui potest, nempe ponendo aut $t$ $=m, u=0$, aut $t=-m, u=0$. Ponamus 
enim dari adhuc aliam solutionem $t=T, u=$ $U$, ita vt $U$ non $=0$. Quia $m m$ ipsum $4 h h$ certo metitur, erit $\frac{4 T T}{m m}=\frac{4 h h U U}{m n}=4$, atque tum $\frac{4 T T}{m m}$ tum $\frac{4 h h L U}{m m}$ quadrata integra. Sed nullo negotio perspicitur, numerum 4 duorum quadratorum integrorum differentiam esse rion posse, nisi quadratum minus sit o i. e. $U=0$, contra hyp. - Si itaque forma $F$ in formam $F$ per substitutionem $\alpha, g_{g}, \delta$ transit, alia transformatio huic similis non dabitur praeter transformationem $-a,-6,-y,-\delta$. Quare si duae formae aut proprie tantum, aut improprie tantum aeçuiuaIent, duae tantum transformationes dabuntur; si vero tam proprie tum improprie, quatuor, nempe duae propriae duaeque impropriae.

210. Theorema. Si duae formae reductae $(a, h, o),\left(a^{l}, h . o\right)$ impropirie sunt aequinatentes, erit $a a^{\prime} \equiv$ mm (mod. $\left.2 m h\right)$, designante m duisorem communen naximutm numerornm a, $2 h$, vel $a^{\prime}$, 2h; et vice versa, si $a, 2 h ; a^{\prime}, 2 h$ entdenn diunsoren conmuneni ma-

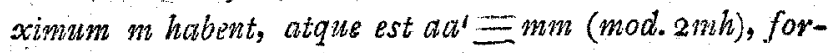
mae $(a, h, o),(a b, h, o)$ improprie aequinalentes enunt.

Dem. I. Transeat forma $(a, h, 0)$ in formam $(\alpha, h, o)$ per substitutionem impropriam $a, b, \gamma, \delta$ ita. vt habeantur quatuor aequationes acos $+2 h \alpha \gamma$ $=a^{\prime} \ldots[1] ; a \alpha^{2}+h(\alpha \delta+b y)=h \ldots[2] ; a^{6} b+$ $2 h \circ=0 \ldots[3] ;$ of $-6 y=-1 \ldots[4]$. Hinc sequitur, multiplicando [4] per $h$ et subtrahendo $a[2]$, quod ita exprimimus $[2]-h[4], \ldots(a *$ $\left.+2 h_{\gamma}\right) 6=2 h \ldots[5] ;$ similiter ex ${ }^{8}[2]-$ 


\section{$-285-$}

yy $[3]-(a+a \nu \gamma+h \gamma d)[4]$ deletis quae sese destruunt $\ldots-a-a \delta=a+2 h y d$, siue $-(a+$ $\left.w_{\gamma}\right) \delta=a \ldots[6] ;$ denique ex $a[1] \ldots a u(a \omega+$ $2 h \gamma)=a a^{\prime}$, siue $\left(a^{2}+2 h \gamma\right)^{2}-a a^{\prime}=2 \hbar \gamma\left(a_{*}\right.$ $+2 h \gamma)$ siue $(a \alpha+2 h \gamma)^{2} \equiv a a^{\prime}(\bmod \cdot 2 h(a \alpha+$ 2hy)) ... [7]: Iam ex [5] et [6] sequitur $a s+$

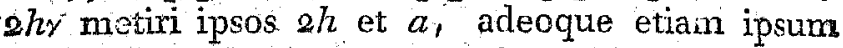
$m$, qui est diuisor communis maximus ipsorum $a, 2 h$; manifesto autem $m$ metietur etiam ipsum $a \omega+2 h \gamma ;$ quare necessario $a \omega+2 h \gamma$ erit aut $=$ $+m$ aut $=-m_{\text {. }}$. Hinc statim sequitur ex $[7]$, $m m=a a^{\prime}(\bmod .2 m h)$. Q. E. P.

II. Si $a, 2 h ; a^{t}, 2 h$ eundem diuisorem communem maximum $m$ habent, insuperque est $\alpha a^{\prime}$ $\equiv m m(\bmod 2 m h), \frac{a}{m}, \frac{2 h}{m}, \frac{a^{\prime}}{m}, \frac{a a^{d}-m i m s}{2 m h}$ erunt integri. Facile vero confirmatir, formam $(a, h, o)$ transire in $(a, h, o)$ per substitutionem $-\frac{a^{\prime}}{m},-\frac{2 h}{m}, \frac{a a^{i}-m m}{2 m h}, \frac{a}{m}$, nec non hanc transformationem esse impropriam. Quare formae illae erunt irnproprie aequiualentes. $Q . E S$.

Hinc etiam statim diiudicari potest, an forma aliqua reducta data $(a, h, 0)$ sibi ipsi improprie aequiualens sit. Scilicet designato diuisore communi maximo numerorum $a, 2 h$ per $m$, esse debebit $a a \equiv m m(\bmod .2 m h)$.

211. Omnes formae reductae determinantis dati $h$ h obtinentur, si in forma indefinita $(A, h, a)$ pro $A$ omnes numeri a $o$ rsque ad $2 h-1$ incl. substituitintur, quarum itaque multitudo erit $2 h$. Perspicuum est omnes formas determinantis $h h$ 
in totidem classes distribui posse, hasque iisdem proprietatibus praeditas fore quas supra (artt. 175, 195) pro classibus formarum determinantis negatiui, et positiui non quadrati attigimus. Ita omnes formae determinantis 25 in decem classes distribuentur, quae per formas reductas in singulis contentas distingui poterunt. Hae formae reductae sunt: $(0,5,0),(1,5,0),(2,5,0),(5,5,0)$, $(8,5,0),(9,5,0)$, quae sibi ipsae simul improprie aequiualent; $(3,5,0)$ cui improprie aequiualet $(7,5,0) ;(4,5,0)$ cui improprie aequiualet $(6,5,0)$.

212. Probiama. Inuenire onnes repraesentationes numeri dati $M$ per formam datam axx $+2 b x y$ + cyy determinantis hh.

Solutio huius problematis ex principiis art. 165 prorsus eodem modo peti potest, vt supra (artt. 180, 181,205) pro formis determinantis negatiui et positiui non quadrati osteridimus; quod, quum nuli difficultati sit obnoxium, hic repetere superfluum esset. Contra haud abs re erit, solutionem ex alio principio quod casui praesenti proprium est deducere.

Positis vt artt 206, 208, $h-b: a=c:-$ $(h+b)=6: \delta ; \frac{h-b}{b}=\frac{a}{\delta}=f ; \frac{c}{b}=$ $\frac{-h-b}{\delta}=g$, nullo negotio probatur, formam propositam esse productum ex factoribus $d x-f y$ et $f x-g y$. Vnde manifestum est; quamuis repraesentationem numeri $M$ per formam propositam praebere resolutionem numeri $M$ in binos fa- 
ctores. Si itaque omnes divisores numeri $M$ sunt $d, d^{n}, d^{n}$ etc. (inclusis etiam 1 , et $M$, et singulis $b$ is sumtis puta tum positive tum negatiue), patet omnes repraesentationes numeri $M$ obtineri; si successiue ponatur $\delta x-b y=d, f x-g y=$ $\frac{M}{d} ; \delta x-b y=d^{\prime}, f x-g y=\frac{M}{d}$ etc., valores ipsorum $x, y$ hinc euoluantur, eaeque repraesentationes eiiciantur vbi $x$ aut $\mathrm{y}$ valores fractos obtinent. Manifesto vero ex duabus primis aequa-

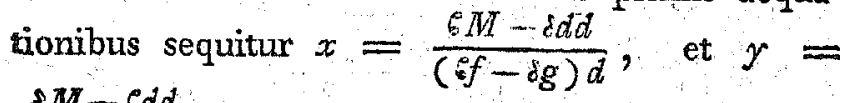
$\frac{\delta M-6 d d}{(6 f-i g) d}$, quos valores semper determinatos fore inde mănifestum quod $f f-l g=2 h$, adeoque numerator certo non $=0 .-$ Ceterum ex eodem principio, puta resolubilitate cuinsuis formae determinantis quadrati in binos factores, etiam reliqua problemata solui potuissent: sed methodo ei quam supra pro formis determinantis non quadrati tradidimus analoga etiam hic vti maluimus.

Ex. Quaeruntur omnes repraesentationes numeri 12 per formam $3 x x+4 x y-7 y y$. Haec resolvitur in factores $x-y$ et $3 x+7 y$. Omnes diuisores numeri 12 sunt $\pm 1,2,3,4,6,12$. Positis $x-y=1,3 x+7 y=12$ fit $x=\frac{19}{10}, y=$

$\frac{9}{10}$, qui valores tamquam fracti sunt reiiciendi. Eodem modo ex diuisoribus $-1, \pm 3, \pm 4, \pm 6$, \pm 12 valores inutiles obtinentur; ex divisore + 2 vero obtinentur valores $x=2, y=0$, et ex diuisore -2 hi $x=-2, y=0$; praeter has duas repraesentationes igitur aliae non dantur. 
Methodus haec adhiberi nequit, si $M=0$. In hoc casu manifestum est onnes valores ipsorum $x, y$ ant aequationi $\delta x-b y=0$, aut huic $f x-$ $g_{y}=0$ satistacere debere. Omries autum solutiones aequationis prioris continentur in formula $x$ $=z, y=\delta z$, designante $z$ indelinite numerum integrain querncunque ( siquidem vit supponitur ;"; dinter se promi sunt ); similiterque ponendo ditisorem comnunem maximun numbronm $f, g=$ $m$, omnes solutiones anquation postroris exhibebuntur per formulam $x=\frac{g z}{m}, y=h z$. Quare hae duap formulae genestales omnes repraesentationes numeri $M$ in hoc casu complectentur.

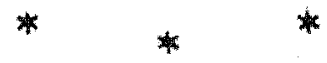

In praecedentibus omnia quae ad cognoscendam aequiulentiam ot ad intumiendas omnes transformationes formarum nec non ad repratenen- tationes omnes numerorm datorum per formas datas indagandas pretinent, ita sunt explicata, ve nihil anplius destiderai posse videatur. Superest itaque tantumuoto, nt propositis daabus formis quae propter detominentim inarequlitatem aequiualeutes esse nequetun, diudicare docearnus, annon altera sub altem contonta wil, et in loc casu omnes transformationes illus in hane inuonire.

213. Suñ artt 157, 1,8 astentimus, si

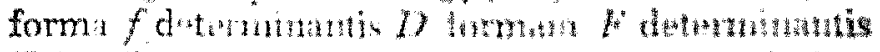

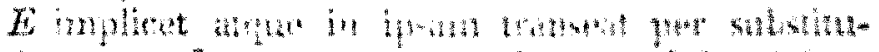

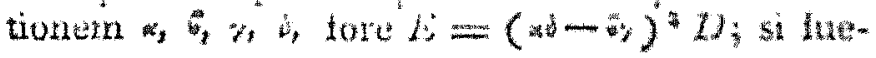




\section{$-\quad 289$}

$w-6 \gamma= \pm 1$, formam $f$ non modo imnlicare formam $F$ sed ipsi aequiuilentem esse et proin si $f$ ipsam $F$ implicet neque vero eidem aequiualeat, quotientem $\frac{E}{D}$ esse integrum maiorem quam 1. Problema itaque hic soluendum erit, cliudicate an forma data f determinantis D formam datam $F$ determinantis Dee impiciet, vil e supponitur esse numerus positilus maior quam a. Hoc negotium ita absolitemus, th multitudinem finitam formarum sub $f$ contentarum assigntape documus quae ita sint comparatate, it $F$ si sub $f$ contenta est necessario alicui ex illis aequiualere debeat.

I. Ponamus ormes dinisores (positiuos) numeri $e$ (inclusis etiam $I$ at $e$ ) esse $m, m^{i}, m^{\text {kt }}$ etc, atque $e^{*}=m n=m^{\prime} n^{\prime}=m^{\prime \prime} n^{\prime \prime}$ etc. De. signemus breuitatis gratia formam in quam $f$ transit per substitutionem propriam $m, 0,0, n$ ita $(m ; 0)$, formam in quarn $f$ transit per substitutionem propriam $m, 1,0, n$ per $(m ; 1 ;$ etc g"weraliterque formam in quam $f$ per subst. proprian, $m k, 0, n$ transmutanur per $(m ; k$ ! simili moto transt at $f$ per subst. propriam $m \cdot 0,0$, $n^{\prime}$ in $\left.m^{\prime} ; 0\right)$; per hanc $m^{\prime}, 1,0, n^{\prime}$ in $\left(m^{\prime} ; 1\right)$; etc, part $m^{n}, 0,0, n^{n \text { in }}\left(n^{n} ; 0\right)$ ete. etc. Ommes hae Worntale wh f proprie conternthe erunt, et ruiusuis detwrminats $=$ Dee Complexum omnium formatum $(m ; 0),(m ; 1,(m ; 2) \ldots(m ; m-1)$

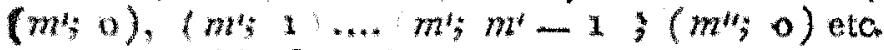
quarum multitudo erit $m+m^{t}+m^{\prime \prime}+$ etc. et quas omnes inter se diuersas fore facile perspicitur, designemus per 1. 


\section{$-200-$}

Si $e . g$. forma $f$ est haec $(2,5,7)$ atque $=5, \Omega$ comprehendet sequentes sex formas $(1 ; 0) ;(5 ; 0),(5 ; 1),(5 ; 2),(5 ; 3),(5 ; 4)$ quae si euoluuntur sunt $(2,25,175),(50,25,7)$, $(50,35,19),(50,45,35),(50,55,55)$, $(50,65,79)$

II. Iam dico, si forma $F$ determinantis Dec sub $f$ proprie contenta sit, necessario eanden alicui formarum $\Omega$ proprie aequiualentem fore. Ponamus formam $f$ transformatri in $f$ per stabstitutionem propriam $\alpha, 6, \%$, eritque $\alpha-* y=e$ Sit numerorum $\gamma$, (qui ambo simul o esse nequeunt) diuisor communis maximus positius acceptus $=n$, atque $\frac{\varepsilon}{n}=m$, qui manifesto erit integer. Accipiantur $g, h$ ita sit $g+h h$ $=n$, denique sit $k$ residuum minimum positiuum numeri ag + th secundum moduhm $m$. Tum forma $(m ; k)$ quae manifesto erit inter formas $\Omega$, formae $f f^{\prime}$ proprie aequiualebit, et quidem in ipsam transfomabitur per substitutionem

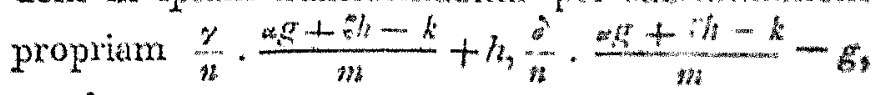
$\frac{\gamma}{n}, \frac{\delta}{n}$. Nam primo perspicuim est hos quatuor ma* meros esse integros; secumlo facile confirmatu" substitutionem esse proprinm; tortio patet, for mam in quam $(m ; k)$ per stabstitutionem illam transeat eandem esse in quam $f^{*}$ ) transent per substitutionem $m\left(\frac{z}{m} \cdot \frac{g+6 h-k}{m}+h\right)+$

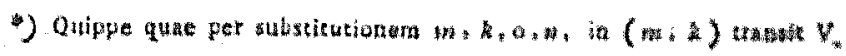
are. 159. 
$\frac{k y}{n}, m\left(\frac{8}{x} \cdot \frac{k g+6 h-k}{m}-g\right)+\frac{k 8}{n}, \gamma, \gamma$ siue quoniam $m n=e=\delta-b_{\gamma}$ adeoque $b_{\gamma}+m n$ $=\alpha^{\prime}, \alpha^{d}-m n=\dot{\phi}$, per hanc $\frac{1}{n}$ (arst $x d h), \frac{x}{n}(6 g y+6 d \hbar), \gamma, \delta$, siue denique quoniam $x \xi+b=n$, per laarc $\alpha, b, \gamma, \delta$ i. a per hyp., in $F$ Quare $(m ; k)$ et $F$ proprie aequiwalentes erunt. $Q . E . D$.

Ex his igitur semper diudicari potest, an forma aliqua data $f$ determinantis $D$ formam $F$ determinantis Dec proprie implicet. Si vero quaeritur an $f$ ipsam $F$ improprie implicet, inuestigari tantummodo debet an forma ipsi $F^{*}$ opposita sub $f$ proprie contenta sit, art 159.

214. PROBLIMA. Propositis duabus formis, $F$, determinatuis $D$, et Fdeterminantis Dee, quarm pritor posteriorem groprie inplicat: exhibere omes sransformationes propriass formate $f$ in $F$.

Sol. Designante $\Omega$ exudem formarum complexurn vt in art praec, excerpantur ex hoc complexu ommes formae quitus $F^{\prime}$ proprie aequiualet, quae sint $\$, \$$, $\$$ in etc. Quatevis harum formatrum sequenti modo suppeditabit transformationes proprias fomae $f$ in $f t^{\prime}$, et quidem aliae alias (b. s. singulae diutsisas), cunctae vero cunctas (i. e. nulla transtormatio propria formae $f$ in $F$ erit quam non vat ex formis $\downarrow$, etc praebeat). Quoniam methodus pro omnibus formis $\Phi$, etc. cadum est, de wa tanturn loquemur. 
Ponamus $\Phi$ esse $(M T ; K)$, atque $=$ MVita vt $f$ in $\Phi$ per substitutionem propriam $M, K, 0$, $N$ transeat. Porro designentur omnes trinsformationes propriae forma $\$$ in $F$ indefinite per $a, b, c, b$. Tum manifesto $f$ transibit in per substitutionem propriam $M \mathrm{o}+K^{*}, M b+K{ }^{\circ}$, $N c, N o$, et hoc modo ex quauis transformatione propria formae $\$$ in $F$ sequetur transformatio propria formae $f$ in $F$ - Eodem modo tractandae sunt formae reliquae $\phi$, कi" etc., quarum singulae transformationes propriae in $F$ transformationem propriam formae $f$ in Fr prabebunt.

Vt appareat hanc solutionem ex omni parte completam esse, ostendendum erit

I. Hoc modo omnes transformationds propherst pussibules formae $f$ in $F$ abtinert. Sit transformatio quaecunque propria formae $f$ in $F$ haec $\alpha, *, \gamma$ atque vt in art. prese. If, $n$ ditisor commuis maximus numerorum $\%$, nuneri $m, k, h, k$ autem eodem modo ve illic detenminati. line forma $(m ; k)$ erit inter furmas on, beth, $a$

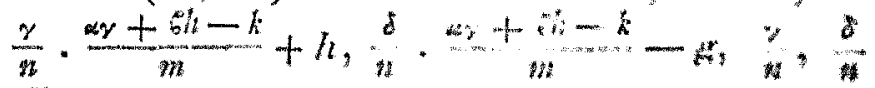
aliqua ex transformationibus prontris latum formae in $F$; ex hac vero per regulam moto tra ditarn obtinetur transtormatio $, y, y$, ; hates ommia in art. praec sunt demonstrata.

II. Onves iransformationes how moll throm

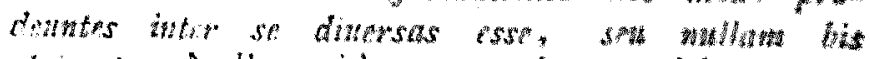

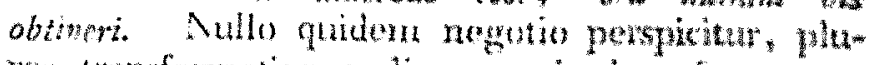
res transfomationes dinersas chusden format. 
vel etc. in $F$ candem transformationem for mae $f$ in $F$ producere non posse; quod vero etiam formae diuersae e. $g$. $\$$ et $\$$ eandem transformationem suppeditare nequeant, ita demonstratur. Supponamus, transformationem propriam $\alpha, b, \gamma, \delta$ formas $f$ in $F$ obtineri tum ex transfomatione propria $a, b, \mathfrak{c}, b$ formac $\phi$ in $F$, tum ex transformatione propria $a^{\prime}, b^{\prime}, \mathfrak{c}^{\prime}, b^{\prime}$ formae in $F$ Sit $\phi=(M ; K), \phi$ $\left(M ; K^{*}\right), \varepsilon=M N=M N^{*}$. Habebuntur itaque aequationes $\alpha=M a+K c=M^{\prime} \mathrm{a}^{r}+$ $K^{\prime} c^{\prime} \ldots[1], G=M b+K^{\prime} b=M M^{\prime}+K^{\prime} b^{\prime} \ldots$ [2], $\gamma=N c=N^{\prime} \ldots[3], \delta=N_{b}=N^{\prime}$ $\ldots[4], a b-b c=a^{\prime}-b^{\prime} c^{\prime}=1 \ldots[j] . \mathrm{Ex}$ $\left.a[4]-b_{3}\right]$ sequitur adimentu aequ. $[j], N=$ $N^{\prime}\left(a b^{\prime}-6 c^{\prime}\right)$, quare $N^{*}$ metietur ipsum $N$; similiter ex a $f]-b \cdot[j]$ fit $N(a b-b c)=$ $N$, çuare $N$ metietur i ipsum $N$, vnde, quia tum V tum $N^{\prime}$ supponumtur esse positiuf, erit necessario $N=N^{i}$, et $M=M^{i}$, et hine ex 5 et $4, c=c^{*}=0$. Porro fit ex a [a]$\left.b^{\prime \prime} 1\right], K=M^{\prime \prime}\left(a b^{\prime}-6 a^{\prime}\right)+K^{\prime}\left(a b^{\prime}-6 c^{\prime}\right)=$ $\left.M T a^{\prime}-6 a^{\prime}\right)+K^{\prime}$, hinc $K \equiv K^{\prime}(\bmod M)$ qrod firri nequit nisi $K^{*}=K^{*}$, quia tum $K$ tum $h^{*}$ iacent inter limites o ot $M I-1$. Quamobun formae $\Phi, \Phi$ non sunt diuersae; contra live.

Cetarum patet, si $D$ fuerit nematiuus vel postinus qualratus, per methodum hane omnes transformationes propurias formae $f$ in $F$ reuera inueniri posse; si vero $D$ posititus non-quadratus, formulae certae generales assignari poterunt in quibus omnes transformationes propriae (quarum multitudo infinita) contentae erunt. 
Denique, si forma $F$ improprie sub forma $f$ contenta est, omnes transformationes impropriae illius in hanc per meihodun tradiam fa-

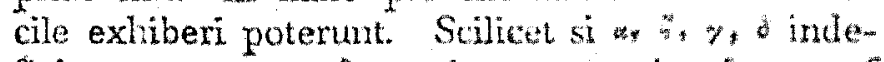
finite omnes transformationes proprias formae $f$ in formam quae formae $F$ opposita est, designare supponitur: ommes transf. impropriae formae $f$ in $F$ exhibebuntur per $\alpha,-6, \gamma,-\AA$

Ex. Desiderantur omnes transformattones formae $(2,5,7)$ in $(275,0-1)$, quat sub illa tum proprie tum inpropire contenta est. Complexum formarum so pro hoc casu latis in art. praec. tradidinus; exanine instituto intzenitur, turn $(5 ; 1)$ tum $(5 ; 4)$ formae $\left(275,0_{3}-1\right)$ proprie aequiualere. Omnes transformationes propriae formae $(5 ; 1)$ i.e. $(50,35,19)$ in $(275$, $0 ;-1)$ per theoriam nostram supra explicutarm inueniuntur contineri sub formula generali $16 t$ $-275 u,-t+16 u,-15 t+2754, t-154$, $v$ bi $t$, $u$ cesignant indefinite ommes numeros integros aequationi $t t-275 u t=1$ satistutientes. quare omnes transformationes promitae fornuat $(2,5,7)$ in $(275,0,-1)$ hint wimble contentae erunt sub formula generali $65 t-1190 t t_{\text {, }}$ $-4 t+65 u,-15 t+2754, t-154$ Simili molo omnes transformations propriat format $(5 ; 4)$ i. e. $(50,65,70)$ in $(205, a,-1)$ contimentur sub formula generali $34+2754$ $t+1.4 u,-15 t-275 u_{2}-t-154$, adnm que omnes transformationes propriate fortane $(2,5,7)$ in $(275,0,-1)$ hine oriumber sta hac $10 t+275 \%, t+10 \%,-15 t-2534,-$ $t-15 t k$ Hae duae furmulat igitur onnes 
transformationes proprias quaesitas amplectmontur. - Eodem vero modo inuenitur omnes transformationes improprias formae $(2,5,7)$ in $(275$, $0,-1)$ sub sequentibus duabus formulis contentas esse: (I) $\ldots 6_{5} t-1100 n, 4 t-65 l h$, $x_{5} t+275 u,-t+15 u$; et (II) ... $10 t+275 u$, $-t-10 u,-15 t-275 u, t+15 u$

215. Hucusque formas determinantis 0 ab omuibus disquisitionibus exclusimus; de his itaque, vi theoria nostra ab ommi parte completa euadat, quaedam adhuc sunt adicienda. Quoniam generaliter demonstratum est, si forma aliqua determinantis $D$ formam deteminantis $D^{\prime}$ implicet, $D^{\prime}$ esse multiplum ipsius $D$, statirn patet forman cuius determinans $=0$ aliam for man quam cums determinans etiam sit $=0$ ime plicare non posse. Quare duo tantummoda problemata soluenda restant, scilicet to propositis duatus formis $f, F$, quarum posterior habet determinaniem 0 , dutudicare utrum prior posteriorem implice necue, et in tho cast otnnes transformationes illius in lianc exhibert. $2^{\circ}$ Inumire onnes reprassentationes unmeri tati par formam datam determinantis o. Problema primm aliam methodum requirit, quando determinans priotis fornae $f$ etiam est $\mathrm{o}_{3}$ alium quando non est o. Haec omnia iam exponemus,

I. Ante omnia obseruamus, quamuis formam $a x x+2 b x y+y y$, cuius determinans $b b-a c=0$, ita exhiberi posse $m(g x+h y)^{2}$, denotantibus $g, h$ numeros inter se primos, $m$ integrum. Sit enim $n$ diuisor communis maxi'T'4 


\section{$-296$}

mus ipsorum $a, c$ eodem signo acceptus quo hi numeri ipsi sunt affecti (hos signa opnosita habere non posse facile perspicitur), eruntque $\frac{a}{n}, \frac{b}{m}$ integri inter se primi non negatiui, produrtumx ex ipsis $=\frac{b b}{m m}$ i. e. quadratum, adeoque illi ipsi quadrata (art. 21). Sit $\frac{a}{m}=g g, \frac{b}{m}=$ $h h$, eruntque etiam $g$, $h$ inter se primi, $g g^{h} /$ $=\frac{b b}{m}$, et $g^{h}= \pm \frac{b}{m}$. Hine patet $m\left(g^{x}\right.$ $\pm h y)^{2}$ fore $=a x x+2 v x y+o y$.

Iam propositae sint duae formae $f, F$, wraque determinantis 0 , et quidem sit $f=n(\mathrm{~g} x$ $+h y), H=M(G X+H Y)^{*}$, ita vo $g$ ad $h$, $G$ ad $H$ sint primi. Tum dico si lorma $f$ impli cet formam $b, m$ aut ipsi $M$ atqualem esse aut saltem ipsum $M$ metiri at quotienten esse quatdratum; et vice versil si $\frac{W}{m}$ sit quadratum inte. grum, fo contentan usse sub f. Si enind f per substitutionem $x=4 x+61, y=3 x+y$ in $F$ transire supponitur, exit $\frac{M}{m}(C+11)=$

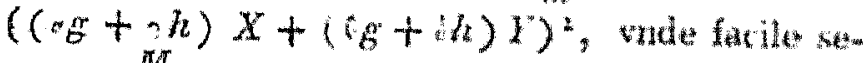
quitur $\frac{M}{m}$ esse quadratum. Ponatur $=4 e$, whim que $e(O X+I I Y)= \pm((g+2 h) X+(k g+$

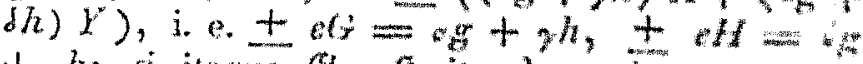

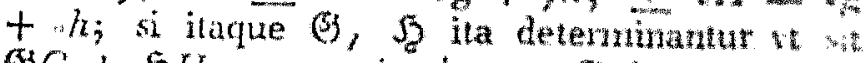

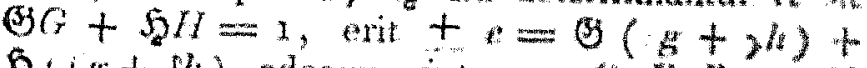
$\mathfrak{S}(b+\delta h)$, adeoyue integer. $Q . E . P-S$ 
rero, vice versa, supponitur, $\frac{M}{m}$ esse quadratum integrum $=c e$, forma $f$ implicabit formam $F$. Scilicet integri $\alpha, \dot{b}, \gamma, \delta$ ita poterunt deternilmari vt fiat $g+, h= \pm e G,+g+d h=$ $\pm c H$. Accipiantur enim integri $8, b$ ita vit fiat $\overline{g g}+\mathfrak{b} h=1$, salisfietque aequationibus illis ponendo $:=+a G g+h z, \mathfrak{g}= \pm e G h-g z$, $\mathrm{c}= \pm \varepsilon \mathrm{Hg}^{-}+h z^{t}, \delta= \pm c \overline{H_{l}}-g z^{t}$, quicumque valores integri ipsis $z, z^{\prime}$ tribuantur; quare $F$ contenta erit sub $f$, Q. E.S. Simul haud dificulter intelligitur, has formulas omnes valores quas $\alpha, 6, \%$ nancisci possunt, i. e. omnes transfurmationes tormat $f$ in $F$ exhibere, si modo $z$, zt indefinite omuses numeros integros exhibere sulponantur,

II. Propositis duabus formis $f=a x x+$ $2 h x y+g y$ cuins detominaus non $=0$, ef $F$ $=M(G X+H Y) \cdot$ cuius determinans $=0$ (desiguantibus vt ante $G, I I$ numeros inter se primos, dico primo, si $f$ implicet ipsam $F$, numexum $M$ per formam $f$ repraesentari posse; sco cinto, si $M$ per $f$ repraesentari possit, $F$ sub $f$ contentam esse; tertio, si in hoc cast omnes reInatesentationes numeri $M$ per forman $f$ indefinite exhibeantur ita $x=y=$ o ommes transformationes lormate $f$ in $F$ exhiberi ita $G \xi, L F$, G., H.. Quate ommia sequenti modo demonstranus.

10 Ponamus $f$ transire in $F$ per substitutionem $\alpha, \mathscr{F}, \%, \delta$, accipianturque numeri $\mathfrak{S}, \mathfrak{K}$ ita

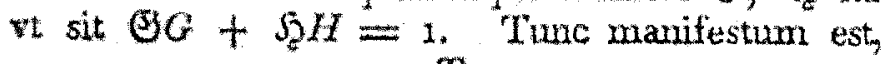
T 5 
si ponatur $x=a \mathbb{S}+6 \mathfrak{H}, y=2 \mathfrak{G}+\delta \mathfrak{S}, \mathrm{va}$ torem formae $f$ fieri $M$, adeoque $M$ repraesenLabilem esse per formam $f$.

$2^{3}$ Si supponitur esse $a^{\xi \xi}+2 b \xi v+c u=$ $M$, manifestum est per substitutionem $G \xi, H \xi$, $G, H$, , formam $f$ transire in $F$. Quod vero

$3^{\circ}$ in hoc casu substitutio $G \xi, H_{\xi}, G_{v}, H_{v}$ omnes transformationes formae $f$ in $F$ exhibeat, si $v$ supponantur exhibere omnes valores ipsorum $x, y$, qui faciunt $f=M_{\text {, }}$, ita perspicitur. Sit $\alpha, \zeta, \gamma, \delta$ transformatio quaecunque formae $f$ in $F$, et vt ante $\mathcal{S G}+\mathfrak{S} H=\mathrm{I}_{2}$ Tum inter valores ipsorum $x, y$ erunt etiam hi, $x=$ aS $+6 \mathfrak{F}, y=2 \mathfrak{G}+\delta \mathfrak{S}$, ex quibus obtimetur substitutio $G(\alpha \mathcal{S}+6 \mathfrak{S}), H(\alpha \mathfrak{S}+6 \mathfrak{g}), G(\gamma \mathbb{S}$ $+\delta \mathfrak{S}), H(\gamma \mathfrak{G}+\mathcal{S} \mathfrak{S})$, siue $\alpha+\mathfrak{S}(\mathscr{G}-\alpha H)$, $6+\mathcal{G}(\alpha H-6 G), \gamma+\mathfrak{S}(\delta G-\gamma H), \delta+$ $\circlearrowleft(\gamma H-\delta G)$. Sed quoniam $a(\alpha X+b Y)^{2}+$ $2 b(\alpha X+6 Y)(x X+\delta Y)+c(\alpha X+\delta Y)^{2}=$ $M(G X+H Y)^{2}$ enit $a(\alpha \delta-6 \gamma)^{2}=M(\delta G-$ $\gamma H)^{2}, c\left(b_{\gamma}-a \delta\right)^{2}=M(G-\alpha H)^{2}$, adeoque (qkum determinans formae $f$ per $(\mu \delta-6 y)^{2}$ multiplicatus, aequalis sit determinanti formae $F$ I e $=0$, adeoque etiam $\alpha \delta-6=0), \delta G-\gamma H$ $=0,6 G-\alpha H=0$. Hinc substitutio illa transit in hanc $\alpha, 6, \gamma, \delta$, vode patet, formulam traditam onnes transformationes formae $f$ in $F$ suppeditare.

III. Súperest vt omnes repraesentationes $\mathrm{nu-}$ meri dati per formam datam determinantis o exhibere doceamus. Sit forma haec $m(g x+$ 
hy. $)^{2}$, patetque statim, numerum illum per $m$ diuisibilem, et quotientem quadratum esse debere. $\quad$ Si itaque numerus propositus statuitur $=$ mee, perspicuum est, pro quibus valoribus ipsorum $x, y$ fiat $m(g x+h y)^{2}=$ mee, pro iisdem fieri $g x+h y$, aut $=+e$, aut $=-e_{0}$. Quare omnes repraesentationes habebuntur, si omnes solutiones aequationum linearium $g x+h y=e$, $g x+h y=-e$ in integris, sunt iruentae. "Has vero solubiles esse constat (siquidem $g, h$ sunt inter se primi vt supponitur). Scilicet si $\mathfrak{g}, \mathfrak{h}$ ita determinantur vt sit $g g+f h^{\prime}=1$, aequationi priori satisfiet ponendo $x=\mathfrak{g} e+\mathfrak{h} z, y=\mathfrak{h} e$ - $\mathfrak{g} z$; posteriori vero faciendo $x=-g e+$ $\mathfrak{h z}, y=-\mathfrak{h} e-\mathrm{g} z$, denotante $z$ integrum quemcunque. Simul vero formulae hae omines valores integros ipsorum $x, y$ exhibent, si $z$ indefinite numerum quemuis integrum designare supponitur.

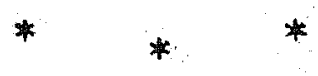

His disquisitionibus coronidis loco apponimus

216. Problema. Intrenire omnes solutiones aequationts generatis *) indeterminatae secundi gradits duas incognitas implicantis

$$
a x x+2 b x y+c y y+2 d x+2 e y+f=0
$$

(vbi $a, b, c$ etc. sunt integri quicunque dati) per numeros integros.

*) St aequatio proponetetur in qua coefficiens secundus, quartus vel quintus non esset par, multiplicata per z eam formam reciperet quam hic supponimus. 


\section{$-500$}

Sol. Introducamus loco incognitarum $x, y$ alias $p=(b b-a c) x+b e-c d$, et $q=$ $(b b-a c) y+b d-a e$, qui manifesto semper erurt integri, quando $x, y$ sunt integri Quo facto habebitur aequatio $a p p+2 b p q+c q q+$ $(b b-a c)(a e e-2 b d e+c d d)=0$, siue posito breuitatis gratia numero $(b b-a c)(a e e-$ $2 b d e+c d d)=-M$, haec $a p p+2 b p q+c q q$ $=M$. Iam omnes solutiones huius aequationis, i. e. omnes repraesentationes numeri $M$ per formam $(a, b, c)$ in praecedertibus inuenire docuimus. $\mathrm{Si}$ vero ex singulis valoribus ipsorum $p, q$; valores respondentes ipsorum $x, y$ adiumento aequationum $x=\frac{p+c d-b e}{b b-a c}, y=\frac{q+a e-b d}{b b-a c}$ determinantur, facile perspicitur, omnes hos valores aequationi propositae satisfacere, et nullos valores integros ipsoruria $x, y$ dari qui hoc modo non obtineantur. Si itaque ex omnibus valoribus ipsorum $x$, $y$ sic prodeuntibus valores fractos eiicimus, omnes solutiones quaesitae remanebunt.

Circa, harc solutionem sequentia sunt $a b-$ seruanda.

10 Si aut $M$ per formam $(a, b, c)$ repraesentari non potest, aut ex nullà repraesentatione valores integri ipsorum $x, y$ sequuntur: aequatio in integris nullo modo solui poterit.

$2^{\circ}$ Quando determinans formae $(a, b, c)_{2}$ i. e. numerus $b b$ - $a c$ est negatiuus, vel positiuus quadratus simulque $M$ non $=0$ : multitudo repraesentationum numeri $M$ per formam $(a, b, c)$ 
erit finita, et proin etiam multitudo omnium solutionum aequationis propositae (si quae omnino dantur) finita erit.

$3^{\circ}$ Quando $b b-a c$ est positiuus non-quadratus, vel quadratus et simul $M=0$ : numerus $M$, si vllo modo, infinitis modis dizcersis per formam $(a, b, c)$ repraesentari poterit; sed quoniam impossibile est, has repraesentationes omnes ipsas inuenire et tentare vtrum valores integros ipsorum $x, y$ praebeant an fractos, necessarium est regulam tradere, per quam, quando forte nulla omnino repraesentatio valores integros ipsorum $x, y$ praebere potest, de hac re certi fieri possimus (nam quotcunque repraesentationes in hot casu tentatae fuerint, absque tali regula ad certitudinem numquam perueńiremus); quando vero. aliae repraesentationes dant valores integros ipso* rum $x, y$, alia fractos: docendum erit quomodo hae $a b$ illis a priori generaliter dignosci possint.

$4^{\circ}$ Quando $b b-a c=0$ : valores ipsorum $x, y$ per formulas praecedentes omnino non possunt determinari; quare pro hoc casu methodus peculiaris inuestigari debebit.

217. Pro eo casu, vbi $b \vec{b}-\alpha c$ est numerus positiuus non-quadratus, supra docuimus, omnes repraesentationes numeri $M$ per formam $a p p+2 b p q+c q q$ (si quae ommino dentur) exhiberi posse, pef vnam vel per plures formulas zales $p=\frac{1}{\mathrm{~m}}(\mathfrak{A} t+\mathbb{B} u), q=\frac{\mathrm{r}}{\mathrm{m}}(\mathbb{S} t+\mathfrak{D} u)$ denotantibus $\mathfrak{A}, \mathfrak{S}, \mathfrak{C}, \mathfrak{D}$ numeros integros da- 
tos, $m$ diuisorem communem maximum numerorum $a, 2 b, c$; denique $t, u$ indefinite ormes mumeros integros aequationi $t t-(b b-a c) k u=$ $m m$ satisfarientes. Quoniam omnes valores ipsorum $t, u$ tum positine tum nergatiue accipi possunt: pro singulis illarum formarum qukternas: alias substituere poterimus, $p=\frac{\mathfrak{I}}{\mathrm{m}}(\mathfrak{U} t+\mathfrak{B} u), q$ $=\frac{1}{n}\left(\mathfrak{S}_{t}+\mathfrak{D} n\right) ; p=\frac{I}{\mathrm{~m}}(\mathfrak{2} t-\mathfrak{B} u), q=$ $\frac{\mathrm{x}}{\mathrm{m}}\left(\mathfrak{C}_{t}-\mathfrak{D} u\right) ; p=\frac{\mathrm{x}}{\mathrm{m}}(-\mathfrak{a} t+\mathfrak{B} u), q=$ $\frac{\mathbf{x}}{\mathrm{n}}\left(-\mathfrak{C} t+\mathfrak{D} u ; ; p=-\frac{\mathbf{x}}{m}(\mathfrak{A} t+\mathfrak{B} u), \eta=-\right.$ $\frac{I}{m}\left(\mathscr{C}_{t}+\mathfrak{D} u\right)$, ita vt multitudo onnium formtlarum nunc quater maior sit quam antea, $t$ to vero non amplius omnes numeros aequationi $t t-$ $(b b-a c) u u=m m$ satisfaciertes exprimant, sed positiuos tantum. Quaeuis harm furmarum itaque scorsin considerari, et qui valores ipsoru:n t, u praebeant valores integros ipsorum $x, y$, inuestigari debebit

$$
\text { Ex formula } p=\frac{\mathrm{r}}{\mathrm{m}}(\mathfrak{I} t+\mathfrak{B} \cdot), \quad q=
$$

$\frac{\mathrm{x}}{\mathrm{ur}}\left(\mathfrak{C}_{t}+\mathfrak{D} u\right) \ldots[1]$ sequantur valores ipsortum $x_{3}$ y hi: $x=\frac{2 t+\$ B u+m a-m i n}{m(b b-a c)}, y=$ $\frac{(\sigma t+\mathfrak{D} u+m a t-m b d}{a t)}$. Supra vero ostendi. mus, omnes valores (positiuos) ipsorum t cunctiture progresionent recurrentem $t^{3}, t^{3}, z^{n}$ etc. similitur valores respondertes ipsius ut thoque seriem recurrentem formare $u, u^{*}, z^{u}$ etc; praeterea assignani posse munerut ta- 
lem, tt secundum modulum quemcunque datum fiat $t^{p} \equiv t^{2}, t^{i+1} \equiv t^{t}, t^{p+1-2} \equiv t^{\prime \prime}$ etc. $u$ $\equiv u^{0}, u^{p}+x \equiv u^{i}$ etc. Pro hoc modulo accipiemus numerum $m(b b-a c)$, designabimusque breuitatis gratia valores ipsorum $x, y$ qui prodeunt ponendo $t=t^{\eta}, u=u^{\top}$, et quibus tribuemus indicem o, per $x^{3}, y$; similiterque eos qui prodemt faciendo $t=t^{\prime}, u=u^{\prime}$, per $x^{\prime}, y^{\prime \prime}$ quibus tribuemus indicem 1 , etc. Tunc nullo negotio perspicietur, si $x^{\mathrm{h}}$, $y^{\mathrm{h}}$ fuerint numeri integri atque $\xi$ rite determinatus, etiam $x^{\mathrm{h}+1-\beta}, y^{h+-\rho}$;

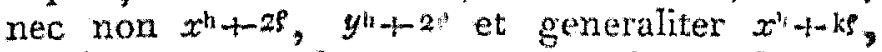
$y^{h}+$ ke, integros fore; et contra si $x^{\text {h }}$ rel $y^{\text {th }}$ sit

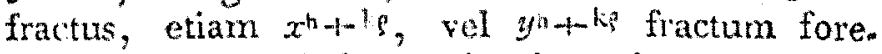
Hinc facile concluditur, si valores ipsorum $x, y$, quibus indices $0,1,2 \ldots, p-1$ competunt, euoluantur, et pro nullo horum indicum tum $x$, tum $y$ integer sit, nullum omnino indicem dari, pro quo tuan $x$, tum $y$ valores integros recipiant, In quo casu ex formula [1] nulli valores integri ipsorum $x, y$ deduci poterumt. Si vero inter illos indices aliqui sunt, puta $\mu, \mu^{\prime}, \mu^{\prime \prime}$ etc. quibus valores integri ipsorum $x, y$ respondent, omnes valores integri ipsorum $x$, $y$, qui quidern ex formula [2] obtineri possunt, ii ermat, quorum indices sub aliqua formularum $\mu+k, \mu^{\prime}+k_{5}, \mu^{\prime \prime}+k^{2}$ atc. sum contenti, denotantae $k$ indefinite omnes numeros integros positiuos, inclusa etiam cifra.

Formulae reliquae sub quibưs valores ipsorum $p, q$ contenti surt, prorsus eodem modo sunt tractandae. Si contingeret, th ex nulla omnin harum formularum valores integri ipsonn $x$ i 
obtineantur, aequatio proposita in interris nullo prorsus modo solui posset; quoties vero reuera est solubilis, omnes solutiones in interris per praecepta in praecc. tradita exhiberi poterunt.

218. Quando $b b-a c$ est numerus quaträtus atque $M=0$, omnes valores ipsorum $N, q$ comprohensi erunt sub duabus hutusmoti iormu-

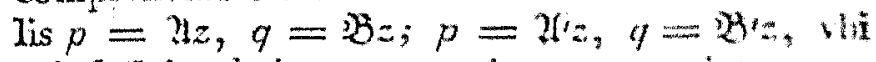
$z$ indefinite designat quemuis numerum intm rum, $21,23,24,23$ vero sunt integri dati, cquorum primus cum secundo, tertius cum quarto diusorem communem non habent (art.212), Omnes itaque valores integri ipsorum $x, y$ ex formula prima oriundi coutenti erunt sub formula [1]

$$
x=\frac{2 x z+c d-h_{c}}{b b-a c}, y=\frac{B z+a c-b d}{b b-a c},
$$

omnesque reliqui ex formula secunda oriundi $s u b$ hac [?]

$$
x=\frac{2 t^{\prime} z+c d-b p}{b b-a t}, y=\frac{5 x^{2}+a b-b d}{b b-b i} .
$$

Sed quoniam vtraqque formula etlam shores fractos. praebere potesst (nisi $b h-a c$ s.se 1 ); opas est vt eos valores ipsius $z$, qui tum ipsum $a$ than inp

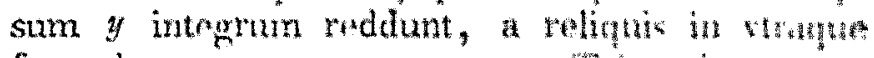
formula separemus; attamen suffi it primunn som lam considerars, quum pro altera prorsus edum methodns aulhibenda sito

Quonian 2C, 8 intese ste primi stun, thass numeros $a$, $b$ ita deturminare licthit it hat $a$ $\mathfrak{G B}=1$. Quo facto lubetur $(a x+b y,(b b-$ 


\section{- $505-$}

$a c)=z+\mathfrak{a}(c d-b e)+\mathfrak{b}(a e-b d)$, mde statim patet, ornnes valores ipsius $z$ qui valores integros ipsorum $x, y$ producere possint, necessario numero a $(b e-c d+b(b d-a e)$ sec. mod. $b b$ - $a c$ congruos, siue sub formula ( $b b$ $-a c) z^{t}+a(b e-c d)+b \cdot b d-a e$ conteritos esse debere, designante $z^{\prime}$ indefinite numerum integrum. Llinc facile loco formulae [1] obtinemus sequentern

$$
\begin{aligned}
& x=\mathfrak{d} z^{t}+b \times \frac{\mathfrak{d}(b d-a e)-B(b e-c d)}{b b \cdot a c} \\
& y=\mathfrak{B} z^{\prime}-a \times \frac{\mathfrak{X}(b d-a t)}{b c-a c}\left(b_{t}-c d\right)
\end{aligned}
$$

quam aut pro ommibus valoritus ipsius $z^{\prime}$ aut pro nulln valores integros ipsonsm $x, y$ praeberr manifestum est, et quilem casus prior semper locum habebit quando 2 (lid ae) et 23 be - $c d$ s spe, mod $b b-a c$ sunt conervi, posterior quanto sunt incongrui. - Prorsus eodem modo twicturda erit formula [2], solutionesque in integris (si quas praebere potest) a reliquis separandue.

210. Quando $b b-a c=0$, forma $a x x$ $+23 x y+g y$ exhiberi poterit ita: $m(\cdot x+$ $\left.0^{\circ}\right)^{2}$, whi $m, \alpha$, f surt integri (art. 215\%). Poztatur $a x+i y=z$, transitque aequatio propositit in hant: $m z z+2 c l x+2 c y+f=0$, vade et $\mathrm{ex} z=\alpha x+\varepsilon^{t} y$, deducitur

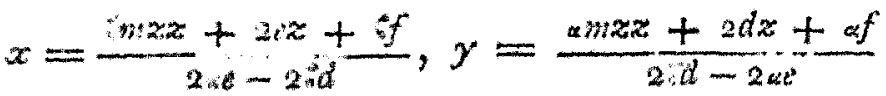

Iani patet, nisi fuerit $\alpha_{\varepsilon}=\epsilon_{c l}$ (quemk casurn 


\section{- $306-$}

statim seorsim considerabimus), valores ipsorum $x, y$, ex his formulis deductos tribuendo ipsi $z$ valorem quemcunque, aequationi propositae satisfacere; quare nithil superest, nisi wt eos valores ipsius $z$ determinare doceamus ex quibus valores integri ipsorum $x, y$ sequantur.

Quoniam $a x+k y=z$, necessario pro $z$ numeri integri tantum accipi possunt; prapterea vero manifestum est, si aliquis valor ipsinus : tum ipsum $x$ tum ipsum $y$ integrum reddat, omnes valores ipsius $z$ illi secundum modulum $2 a e-26 d$ con gruos itidem valores integros producere. Quodsi itaque pro $z$ omnes numeri integri $a$ o vsque ad $2 \alpha e-3 c d-1$ (quando $a c-6 d$ est positiuus) aut ad $26 d$ - see - I (quando - $d$ est negatiuus) incl. substituuntur, et pro mullo horum valorum tum $x$ tum $y$ integri fiunt, nullus omnino valor ipsius $z$ valores integros ipsorum $x, y$ producet, aequatioque proposita in integris nullo modo poterit resolui; si vero quidam ex illis valoribus ipsius $z$ ipsis $x, y$ valores integros conciliant, puta hi per solutionem congrnentiarum secundi grathe ex principiis sect. IV. inuenire licet); omnts st3 lutiones prodibuat ponendo $z=(2 \times t-2 t)$ \% $+\zeta, z=(2 e-2 b)+b^{2}$ etc., designante indefinite omnes numeros integros.

2.20. Pro co quem excluimus cast, whi * $={ }^{\circ}$, methodum peculiarem indagare oportet. Supponamus, \#" : inter se primos toss, quod licere ex art. 215.1 constat, enitque $\frac{d}{2}=$ 
$\frac{\ell}{6}$ numerus integer (art. $19^{\prime}$, quem statuemus $=h$. Tunc aequatio proposita hanc induit formam: $(m \approx x+m y+h)-h l+f=0$, manifestoque adeo ratiomaliter solui nequit, nisi $h h$ - $f$ fieerit mumarus quadratus. Sit $h / 2-f=$ $k h$, patutque aequationi propositae sequentes duas aequnalere: $m \alpha x+m \cdot a y+h+k=0$, et $m a x$ $+m y+h-k=0$, i. e. quamlibet solutionem aequationis proponitae etiam alterutri harum aequationum satisfacere, et vice rersa. Axquatio prior manifesto in integris solui nequit, nisi $h+$ $k$ per $m$ fuerit diuisiblis, similiterque poster ior solutionem in integris non atrnittet, nisi $h \ldots k$ per $m$ fuwrit diusistilis. IYae rero conditiones ad resoluhilitatem vtrinsque -apquationis sufficiunt (quia $\approx:$ inter se primi esee supponuntur ', ommonque solutiones secundurn regulas notas exhiberi poterunt.

221. Casum in art. 217 consideratum (quia omnium difficillimus est) exemplo illustramus. Foposita sit aequatio $x x+8 x y+y y+2 x-$ $4^{y}+1=0$. Ex hac primo per introductionem aliarum incognitartm $p=15 x-9, q=15 y+6$ derimatur a"cquatio $p p+8 p q+q q=-5$ fo. Huius autem solutiones omnes in intergris, contineri inueniuntur sub quatuor formulis sequentibus:

$$
\begin{aligned}
& p=6 t, q=-24 t-90 u \\
& p=t i, q=-24+90 u \\
& p=-6 t, q=24 t-90 u \\
& p=-6 t, q=24 t+90 u
\end{aligned}
$$

denotantibus $t, u$ indefinite omnes numeros integros posituos aequationi $t t-15 k u=1$ satis- 


\section{- $308=$}

facientes, quos complectitur formula $t=\frac{x}{2}((*$ $\left.+\sqrt{15})^{n}+(4-\sqrt{15})^{n}\right), u=\frac{x}{2 \sqrt{15}}((4+$ $\left.\sqrt{ } 15)^{n}-(4-\sqrt{15})^{n}\right)$, si $n$ indefinito omnes numeros integros positiuos (inclusa etiam cifra) designat. Quamobrem omnes valores ipsorum $x, y$ contenti erunt sub formulis his:

$$
\begin{aligned}
& x=x(2 t+3), y=-\frac{x}{5}(8 t+30 u+2) \\
& x=x(2 t+3), y=-\frac{x}{5}(8 t-30 u+2) \\
& x=\frac{x}{5}(-2 t+3), y=\frac{x}{5}(8 t-30 u-2) \\
& x=\frac{x}{5}(-2 t+3), y=(8 t+30 t-2) .
\end{aligned}
$$

Praeceptis autem nostris rite applicatis, reperietur, vt valores integri prodeant, in formula prima et secunda eos valores ipsorum $t, t u$ accipi debere, qui proueniant ex indice $n$ paris in tertia quartaque vero eos, qui ex impari $n$ obtineantur. - Solutiones simplicissimae habentur hae: $x=1,-1,-1 ; y=-2,0,12$ resp.

Ceterum obseruare conuenit, solutionem problematis in artt. preece explicati plerumeque per multifaria antificia ablreniani nosse, pratesen. tim quantum ad exchusonem solutionum inut. lium $i$. e. fractinge implicantim pertinet; sed haec ne nimis longi famus hoc loto pratterine coacti sumus.

222. Qunniam complura ex is quae hucusque pertractatuinus etiatn ab aliis geometris con-

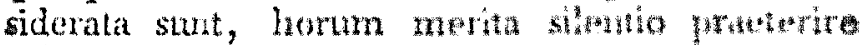

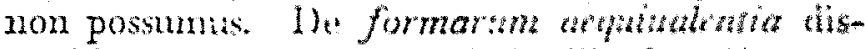
quisitiones pernerates instituit ill. La Grange,

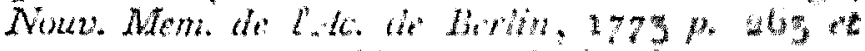
2775 p. 323 . sqg., vil impritnis docuit, pro qua- 


\section{- $309-$}

uis determinante dato multitudinem finitam formarum dari ita comparatarum, vt quaeuis forma illius determinantis alicui ex ipsis aequiualens sit, adeoque omnes formas determinant is dati in classes distribui posse. Postea clar. Le Gendre plures proprictates elegantes huius classificationis, ad maximam partem per inductionem detexit, quas infra trademus demonstrationibusque muniemus. Ceterum distinctionem aequiualentiae propriae et impropriae, cuius vsus maxime in disquisitionibus subtilioribus conspicuus est, nemo hucusque attigerat.

Problema famosum in art. 216 sqq. explicatum ill. La Grange primus complete resoluit, Hist. de CAc. de Berlin ${ }_{17} 67, p .165$, et $1768 p$. $18 \mathrm{r} s q \%$. Exstat solutio (sed minus conpleta) etiam in Sitppl. ad Eultri Algebram iam saepius laukatis. Iam antea ill. Euler idem argumentum aggressus fuerat, Comm. Pctr. T, $/ F I p .175$; Comm. Not T. IX p. 3; Iisid. T. XYIII p. 185 $s q \%$. sed inuestigationem suam eo semper restrinxit, vt ex aliqua solutione, quam iam cognitam esse supponit, aliae deriuentur; praetereaque ipsius methodi in paucis tantummodo casibus omnes solutiones suppeditare valent (vid. La Grange Hist. de l.Ac. de Berlin 1767, p. 237). Qmm vltima harum trium commentt. recentioris dati sit quam solutio La Crrangiana, quae problema ommi generalitate amplectitur nihilque hoc respectu desiderandum relinquit: Euler tuno temporis (Tomus XVuI Commentariorum pertinet ad annum 1775 , et a. 1774 est publicatus) illnn solutionem nondurn nouisse videtur. $\mathrm{Ce}$ terum solutio nostra (perinde vt onulia reliqua U 3 
quae in hac sectione hactemus tradidimus), principiis omnino diuersis est superstructa.

Quae ab aliis, Diophanto, Fermatio etc. huc pertinentia sunt trarita, casus maxime speciales spectunt; quare quum eorum quae praecsetim memoratu dimna visa sunt iam supra mentio facta sit, sigillatim omnia enarrare supersedemus.

* * * *

Quae hactenus de formis secundi gradus exposuimus, pro primis tantum elemetits huis doctrinae sunt habenda: innixius hanc disquisitionem persequentibus campus se aperuit nobis rastissimus, ex quo ea quae attentione imprimis digna videntur in sequentibus excenpemus. Namque algumenturn loc tam fertile tist, vi pormulta alia, quae iam nunc inmenire nobis comatgit, breuitatis gratia silentio practerire opent at: multo vero plura sine dubio adhuc latent motusque conatus expectant. Ceterum in limine haram inuestigationum statim adnotare comuenit, formas determinantis o inde exclusas esse, nisi contuarium moneatur.

223. Iam supra (art. 175, 195,211) ostendimus, proposito numero quocurque integuo $D$ (siue positizo siue negatiuo assignari poske multitudinem finitam formarm $F$, $F$, fit atc steterminantis $D$, ita comparataun, vit quattis forma determinantis $D$ proprie aequitaless sit allicui ex illis et quidem vnicae tantum. Omnes 
igitur formae determinantis $D$ (quarum multitudo est infinita) secundun illas formas classificari poterunt, formando scilicet e complexu omnium formarun formae $F$ proprie aequiualentium classem primam; e formis quae formae F. proprie aequiualent, secundam etc.

Ex singulis classibus formarum determinantis dati $l$ ), forma aliqua eligi, et tamquam forma repratsentans totius classis considerari poterit. Per se quidem prorsis arbitrarium est, quaenam forma ex quaque classe accipiatur, attamen ea semper praeferenda erit, quae reliquas simplicitate suprrare ridetur. Simplicitas formae alicuius $(a, b, c)$ manifesto ex mannitudine numerorum $a, b, c$ aestimanda est, meritoque forma $\left(a^{\prime}, b^{\prime}\right.$, c) minus simplex dicetur quam $(a, b, c)$, si $a^{\prime}>a, b^{\prime}>b, c^{\prime}>c$. Sed hinc res nondum determinatur penitus, arbitrioque nostro relinquitur e. g., vtram ex formis $(17,0,-45)$, $(5,0,-153)$ pro simpliciori habere malimus. Plerumque tanen e re erit, sequentem normam obseruare:

I. Quando determinans $D$ est negatiuus, aloptentur formae reductae in singulis classibus contentae tamquan formae repraesentantes; vbi vero in eadem classe duat formae reductae reperiuntur (quae orunt oppositae, art. 172), recipiatur ea cuius terninus medius positiuus.

\section{Quando determinans $D$ est positius} notr-ukatratus, euoluatur periodus formae alicuius reductae in classe proposita contentae, in qua

$$
U^{4}
$$




\section{$-512-$}

aut duae formae ancipites inuenientur aut nulla (art. $18 z$ ).

1) In casu priori sint formae ancipites hae: $(A, B, C),\left(A, B^{t}, C^{\prime}\right)$; residua minima numerorum $B, B^{\prime}$ secundum modulos $A$, $A^{\prime}$ resp. $M, M$ (quae positiue accipi poterunt nisi sunt

$=0 ;$ denique $\frac{D-M M}{A}=N, \frac{D \cdots M^{*}}{A^{\prime}}$

$=N$. His ita factis, ex formis $(\alpha, M,-N)$, $\left(A, M^{\prime},-N^{\prime}\right)$ ea quae simplicissina videtur pro forina repraesentarte accipiatur. In hoc ialicio forma cuius terminus medius $=0$ praeferatur; quando vero terminus medius aut in vtrique aut in neutra est $o$, ea quas termirum primum minorem habet alteri praehabenda, et quantio termini primi magnitudine sunt aequales signis diuersi, signum negatiuum positiuo postponendum.

2) Quando vero nulla forma anceps in tota periodo habetur, eligatur ex ommilus yoriodi formis ea quae terminum primum sine respectu signi mirimum habet, ita quidem, it si duste tomae in eadem periodo occurrant, in quanm altera idem terminus primus signo posititu wilectus sit in altero negatiuo, posterior priori post ponatur. Sit haec forna $(A, B, C)$, deduce turque ex ipsa eodem modo vt in ctsu prate. forma alia $(A, M,-N)$ (puta, accipiendo pro $M$ residuum absolute minimun ipsine $D$ securn dum mol. $A$, et faciendo $N=\frac{D}{A}$ ): hate demum pro repraesentante adoptetur.

Quodsi vero eueniret, vt idem terminus primus minimus a pluribus periodi formis commu- 


\section{$-313$}

nis sit, omnes hae formae eo quo praescripsimus modo tractandae et ex formis prodeurtibus ea cuius terminus medius quam minimus euadit tamquam forma repraesentans assumenda erit.

Ita e. g. pro $D=305$ habetur periodus inter alias haec: $(17,4,-17),(-\mathrm{r} 7,13,8)$, $(8,11,-25),(-23,12,7),(7,16,-7)$, $(\cdots 7,12,23),(23,11,-8),(-8,13,17)$ ex qua primo eligitur forma $7,16,-7)$, hincque secundo deducitur forma repraesentans $(7,2,-43)$.

III. Quando determinans est positiuus quadratus $=k h$, eruatur forma redurta $(A, k, 0)$ in clases proposita contenta et, si $A<k$ aut $=k$, pro formn reprasentante ips recipiatur; 'si vero $A>k$, assumatur illius Ioco forma $A-2 k$, $k, 0)$, cuius terminus primus erit negatiuus, sed minor quam $k$.

Ex. Ifoc modo omnes formae deterninantis - 255) distribuuntur in classes sedecim, quarum repraesentantes erunt: $(1,0,235),(2,1$; $118,(4,1,59),(4,-1,59),(5,0,47)$, $(10,5,26,13,5,20),(13,-5,20)$, octoqu aliat a praecedentibus in solis signis terminorum externorum diuersae, $(-1,0,-235)$, $(-2,1,-118)$ etc.

Omnes formae determinantis 79 in sex class"s discelunt, quarum repraesentantes $1,0,-$ $79,5,1,-26),(5,-1,-26),(-1,0$, 29), $(-3,1,26),(-3,-1,26)$. 


\section{- $314-$}

224. Per hanc itaque classificationem formae quae proprie aequiualentes sunt a reliquis omnino segregabuntur. Duae formae tiasdem determinantis $D$, si ex eadem classe sunt, proprie aequiualentes erunt; quiuis numerus per vuam repraesentabilis etiam per alteram repraesentari poterit; et si numerus quicunqus $M$ per formam priorem ita repraesentari potest, rt indeterminatae valores inter se primos labetant, iclem numerus per alteram formam eodem molo repraesentari poterit, et quidem ita, it vtratue repraesentatio ad eundem valorem expressionis $\sqrt{ } D(\bmod . M)$ pertineat. Si vero hase formae ad classes diuersas pertinent, proptie arquiualentes non erunt; a repraesentabilitate numeri alicuius dati per vnam ad repraesentabilitatem eiusdem numeri per alteram concludi nequit; contra, si numerus $\boldsymbol{M}$ per alteram repruesentari potest ita $v$ valores indetorminitarum inter se primi sint, statim certi sumus, nullan similem repratestmotionem eiustem numeri per forman alteram dari, quae ad cundem vilorem (xpr. $\sqrt{ }(1)$ mod. $M)$ pertineat. (V.artt. $16 r, 108$ ).

Contra vtique fieri potest, vt formae duae $F, F^{\prime}$, e classibus diuersis $K, K^{\prime \prime}$ improprie aequiualentes sint, in quo casu quatuis forma ex altera classe cuiuis formae ex altera inproprie atequitalebit; quaeuis forma ex $K$ forman sibi oppositam habebit in $K^{\prime}$, classtsque ipsat $K, K^{\prime}$ appositae dicentur. Ita in exemplo primo at. praec. classis tertia formarum det. $-3,35 \mathrm{ght}$ 1:e, septima octaure opjusita est; in exs situmdo classis secunda tertiae, quinta sextae. Propositis 
itaque duabus formis quibuscunque e classibus oppositis, quiuis numerus $M$ qui per alteram repraesentari potest etiam per alteram poterit; quod, si in altera fit per valores indeterminatarum inter se primos, in altera perinde fieri poterit, ita tamen, vt hae duae repratsentationes ad valores oppositos expr. $\sqrt{ } D(\bmod . M)$ pertineant. - Ceterum regulae supra traditae pro electione formarum repraesentantium ita sunt consitutae, vt classes oppositae formas repraesentantes oppositas semper nanciscantur.

Denique dantur etiam classes sibi ipsis oppositre. Scilicet si forma aliqua simul cum forma opposita in eadem classe contin'tur, farile perpicitur, omnes formas huins classis thu proprie tum improprie inter se aequiualentes esse, oppositanque suas secum habere. Hanc indulem quatuis classis habebit, in qua forma anceps contintur, vice versa in quaus classe sibi ipsi opposita necessario forma anceps reperietur (art. 165,165, quamobrem classis anceps nuncupabitur. Ita inter classes formarum determinantis - 235 octo ancipites habentur, quarum repraesentantes sunt $(1,0,235),(8,1,118),(5,0$, $47),(10,5,26),(-1,0,-235),(-2,1$, $-118),(-5,0, \cdots 47,(-10,5,-26)$; inter clisses fornarum determinantis 79 duae, quarum repraestatantes $(1,0,-79),(-1,0$, 79). - Citwum si formae repraesentantes secundum reguhs nostras determinatae sunt, classes ancipites nullo negotio inde cognosei poterunt. Scilicet pro determinante positio non guadrato classis axceps certo formam repraesentantem an- 


\section{- $316-$}

cipitem nanciscitur, (art. 194); pro determiname negatiuo forma repraesentans classis ancipitis aut ipsa anceps erit, aut talis cuius 'termini externi sunt aequales (art. 172); dexique pro determinante positiuo quadrato per art. 210 facile diiudicatur, an forma repraesentans sibi ipsi improprie aequiualens sit adeoque classis quam repraesentat, anceps.

225. Iam supra (art. 1,5.) ostendimus, in forma $(a, b, c)$ detenminantis negatiui termiros externos eadem signa habere tum inter se thm cum terminis externis cuiusuis aliae formae illi aequiualentis. Si $a, c$ sunt positixi, forman ( $a$, $b, c)$ positizam rocabimus, nec non totum classem in qua $(a, b, c)$ continetur et quae e solis formis positiuis constabit, classem positizam dicemus. Contra $(a, b, c)$ exit forma negratiua, et in classe negatiua contenta, si $a, c$ sunt negatini. Per formam posituam nutnei ne ratiu, lat negatiuam positiui repraesentari nequenut. Si forma $(a, b, c)$ est repradenentans alicums chasis positiuae, forma $(-a, b,-c)$ repraesentans classis negatiuae erit, vade schuitur, multitudinem classium positiuanm multiulini nesatiuarum aequalem esse, et, simul ac illae frurint assignatae, etiam has haberi. Quocitca in disquisitionibus super formis determinantis negatiui plerumque sufficit, classes posititus considerare, quippe quarum proprietates ad classes negatiuas facile transferuntur.

Ceterum distinctio haec mice in formis teterminantis negatiui locum habet; per formas 


\section{$-317-$}

determinantis positiui sine discrimine numeri positiui et negatiui repraesentari possunt, quin adeo hand raro duae formae tales $(a, b, c),(-a, b$, $-c$ ) in hoc casu ad eardem classem sunt referendae.

226. Formam quamcunque $(a, b, c)$ primitizarn vocamus, si numeri $a, b, c$ diuisorem communem non habent; alioquin dicetur deriuata, et quidem, posito numeroruus $a, b, c$ diuisore communi maximo $=m$, forma $a, b, c$ ) erit deriuata e forma primitiua ( $\left.\frac{a}{m}, \frac{b}{m}, \frac{c}{m}\right)$. Ex hac definitione statim liquet, omnes formas, quarum determinans per nullum quadratum (prater I) diuisibilis sit, necessurio primitiuas esse. Porro ex art 161 patet, si in aliqua classe data formarum determinantis $D$ forma primitiua inueniatur, omnes formas huius classis primitiuas fore, in quo casu classis ipsa primitiua dicetux: Porro manifestum est, si forma aliqua $F$ determinantis $D$ deriuata sit ex forma primitiua $f$ determinantis $\frac{D}{m m}$, classesque in quibus format $F, f$ resp. contineantur sint $K, k$, omnes formas e classe $K$ deriuatas fore e classe primiuiua $k$; quocirca classem $K$ ipsam ex classe primitiua $k$ deriuatan int hoc casu vocabiunus.

Si $(a, b, c)$ est forma primitiua, neque vero $a, c$ simul pares (i. e. si aut vterque impar aut saltem alteruter, facile intelligitur, non modo $a, b, c$, sed etiam $a, \Delta b, c$ diuisorem communem habere non posse, in quo casu forma 
$(a, b, c)$ dicetur proprie primitua siue simpliciter forma propria. Si vero $(a, b, c)$ est forma primitiua, numeri $a, c$ auten ambo pares, patet, numeros $a, 2 b, c$ diuisorem comminem 2 habere (qui simul erit maximus, vocabuturque $(a, b, c)$ forma improprie primititat, sime simpliciter forma impropria *). In hor cusen b necessario erit impar alioquin enim $(a, b, c)$ non esset forma primitia); quare erit $b b=1$ (mod. 4) adeoque quoniam ac per 4 ditulitilis, duterninans $b b . a c \equiv 1$ (mod. 4 ). Finmue impropriae itaque tantummodo pro determinante formee $4 n+2$, si est positiuse, wel format, $-(4 n+3)$, si est negatiuss, for um habmnt Ex art. 161 auten perspicuum est, si in clusse aliqua data forma proprie primitiua inueniatur, omnes formas huius classis proprie primnitus esse; contra classern quae formarn impropie primitixam implicet ex solis formis impropute primitiuis constare. Quamobrem classis ipsa in casu priori proprie primitiua sen simphicite" propria; in posterioli, improprte mimitiot setu impropria appellabitur. Ita $*$ tr. inter classes puitiuas formarum determinantis m... $25 \%$ sex sunt propriae, puta quarum repraesentautes 1,0 , $235),(4,1,59),(4,-1,59), 5,0,47)$ $(13,5,20), 13,-5,20)$, totidemque intex negatiuas; binae vero inter vtrasque impropriae.

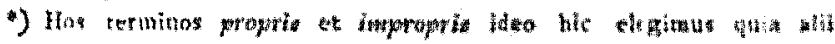

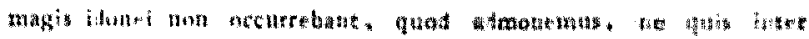

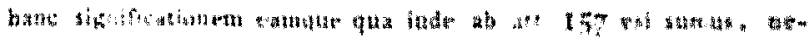

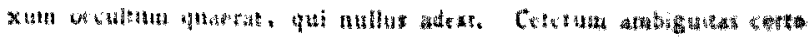
hido non est motheodia, 
- Classes formarum determinantis 79 (ytpote numeri formae $4, r^{i}+3$ ) omnes sunt propriae.

Si forma $(a, b, c)$ est deriuata, et quidem e primitiua $\left(\frac{a}{m}, \frac{b}{m}, \frac{c}{m}\right)$, haec aut proprie primitiua aut improprie esse poterít. In casu priori $m$ exit dinisor communis maximus etiarn numerorum $a, 2 b, c$; in posteriori horum numerorum diu. comm. max. erit an. Hinc intelligitur distinctio inter formam e forma propric primiua deriuatan, es formam ex improprie primitiva deriuatam; nec non (quoniun propter art. 161 omnes formae eiusdem dassis hoc respectu perinde se habent) inter classem drriuntom e classe propric primitiua et classem ex imprepric primitiuk teriuntam.

P'er las distinctiones funtamentum primum nacti sumus, cui distributionem omnium class urn formarum deterninantes dati in varios ordines superstruere possumus, Classes duas, quarurn. repraesentantes sunt formae $(a, b, c),\left(a^{\prime}, b^{\prime}, c^{\prime}\right)$ in eundern ordinem. coniiciemus, tum si numeri $a, b, c$ eundem diusorem communem maximum habent vt $a^{\prime}, b^{\prime}, c^{\prime}$, tum $a, 2 b, c$ eurdem vt $a^{t}, b^{\prime}, c^{\prime}$; si vero aut alterutra aut vtraque harum conditionum locum non habet, classt's ad ortines dinersos reforentur. Ilinc statim patet, omness classes projrie primitiuas vnum ordinem constituere; omnes classes improprie primitiuas, alium; si mm est quadratum determinantern $D$ metiens classes deriuatue e clas. vibus proprie primitiuis determinantis $\frac{D}{n m m}$ for- 
mabunt ordinem peculiarem, aliumque classes deriuatae e classibus improprie primitiuis determinantis $\frac{D}{m m}$ etc. $S i$ forte $D$ per nullum quadratum (praeter 1) diuisibilis est, ordines classium deriuatarum non aderunt adeoque wit vous tantum orda dabitur quando $D=2$ \% secundum mod. 4.) puta ordo classium proprics primitiuarum, aut duo quando $D=1(\bmod 4))$ scilicet $O$. classium proprie primitiuarum et $\mathrm{O}$. $\mathrm{cl}$. impr. primitiuarum. Per principia calculi combinationum haud difficile conditur regitla sequens generalis: $\quad \mathrm{Si}$ suppouitur $D=D^{*} 2^{2 *} a^{2 *}$

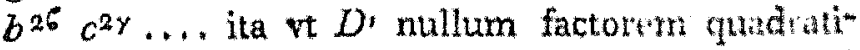
cum implicet, et $a, b, c$ etc. sint numeti minti impares diuersi (ad quam formam quiuis nuxwmrus redigi potest faciendo $\mu=0$ quando $D$ yer 4 non est diuisibilis; et $\alpha, 6, \gamma$ etc. omnes $=0$ siue quod eodem redit omittendo factores $a^{2}$, $b^{25}, c^{2 \gamma}$ etc. quando $D$ per nullma quadratum impar diuidi potest, : habebuntur aut ordines $(\mu+1)(x+1)(x+1)(x+1) \ldots$ nempe quando $D^{\prime} \equiv 2$ vel 5 (mod. 4 ; aut ordines $(\alpha+2)(\alpha+1)(x+1)(\gamma+1) \ldots+$ quando $D^{\prime} \equiv 1$ (mod. 4). Sed demonstrationem huius regulae supprimimus, quoniam neque difficilis neque hic adeo necessaria est.

Ex. I. Pro $D=45=5.5^{2}$ habentur sex classes, quarum repratsentantes $(1,0,-$ $45),(-1,0,45),\left(2,1,-23^{\circ},(2,1\right.$, 22), $(5,0,-15), 6,5,-6)$. I Hat the tribuuntur in quatuor ordines, scilicet 0.1 com- 
prehendet duas classes proprias quarum repr. $(1,0,-45),(-1,0,45) ; 0$. II continebit duas classes improprias, quarum repr. $(2,1,-$ $2.2),(-2, x, 22) ; 0$. IIl coninebit vnam classem deriuatam e propria determinantis 9, puta cuius repr. $(3,0,-15) ; 0$. IV constabit ex vna classe deriuata ex impropria det 9 , puta cuius repr: $(6,3,-6)$.

Ex. 2. Classes positiuae determinantis $99=-11.3^{*}$ inter quatuor ordines distribuen tur: 0.1 complectetur classes proprie primitiuas sequentes $*):(1,0,99),(4,1,25),(4,-$ $x, 25),(5,1,20),(5,-1,20),(9,0,11)$; O. II continebit classes improprias $(2,1,50)$, $(10,1,10) ; 0$. III classes deriuatas e proprís detuminantis - I1, $(3,0,33),(9,3,12)$, $(9,-3,12) ; 0$. IV classem vaicam deriuatam ex impropria det. $-12,(6,5,18)$ - Class.s ntatiatiuae huius determinantis prorsus eodem modo in ordines distribui poterunt.

Obseruamus, classes oppositas semper ad eunclem ordinem referri, cuius theorematis ratio nullo négotio perspicitur.

207. Ex his diuersis ordinibus imprimis ordo clitsium proprie primitiuarum maximam attentionem meretur. Nam singulae classses deriwata centis classibus primitiuis (determinantis minoris) originem trahunt, ex quarum conside.

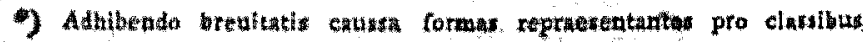

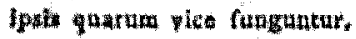




\section{$-322-$}

tatione ea quae ad illas spectant plerumque sponte sequuntur. Infra autem docebimus, quamlibet classem improprie primitiuam simili modo quasi associatam esse aut vnicae classi proprio primitiuae aut tribus (eiusdem determinantis). Porro pro determinantibus negatiuis classes negatiuas praeterire licebit; quippe quibus singulis certae classes positiuae semper respondent. Vt itaque naturarn classium proprie primitiuarum profundius penetremus, ante omnia differentiam certam essentialem explicabimus, secundum quam totus ordo classium propriarum in plura generct subdiuidi potest. Quoniam hoc argumentum gra. nissimum hactenus nondum attigimus, res $a b$ integro nobis exit repetenda.

228. Trrorema, $P e r$ forman quancunque prom prie primititum F repraesentari possunt infinite mithi numeri per numerum primun quemcunque datum " non dizuisibiles.

Derr. Si forma $F=a x x+a b x y+c y$; manifestum est, $p$ omnes tres numeros $a, 2 b, c$ simul metiri non posse. Xam quando a per $p$ mon est divisibilis, patet, si pro $x$ assumatur numerus quicunque per $p$ non diuisibilis, pro $y$ vero numerus per $p$ diuisibilis, valorem forma $F$ fier non diuisibilem per $p$; quando $c$ per $p$ non est diuisibilis, idem obtinetur tribuendo ipsi $x$ valorem dinisibilem ipsique $y$ valorem non diuisibilem; denique quando tum $a$ tum $c$ per $p$ sunt diuisibiles, adeoque 2 b non diuisibilis, forma $F$ valorem per $p$ non diuisibilern induet tribuendo tum ipsi $x$ tum ipsi $y$ valores quoscunque per $p$ non diusibiles, Q.E. D. 


\section{$=325-$}

Manifestum est, theorema etiam pro formis improprie primitiuis locum habere, si modo non fuerit $p=2$.

Quoniam plures huiusmodi conditiones simul consistere possunt, vt idem numerus per quosdam numeros primos datos diuisibilis sit, per alios non diuisibilis ( $v$. art. 32. ): facile perspicitur, numeros $x, y$ infinite multis modis ita determinari posse, vt forma primitiua $a x x+2 b x y$ $+c y y$ valorem per quotcunque numeros primos datos non diuisibilem adipiscatur, a quibus vnice excludendus est 2 quoties forma est improprie primitiua. Ilinc patet, theorema generalius ita proponi posse: Por forman quamcunque prtmitizam reprasscritari possunt infinite milti numen, qui ad namoram quencusqut dotum (tmparem, quando forma sst improprese primituta) sint primi.

229. Treoreda. Sit F forma primitia determinantis $D, p$ sumerus primus ipsun $D$ metiens: tum numeri per ip non diatisubilas quit per formam $F$ repracsentart possuth in co conenient, wt vet onnes sint restdua 'quadratica ipsius $p$, vel omnes non resuduc.

Dem. Sit $F=(a, b, c) ; m, m^{\prime}$ duo numeri quicunque per $p$ non diuisibiles qui per formam $F$ repritesentari possumt, scilicet, $m=a g g$ $+2 b g h+c h h, n^{\prime}=a^{\prime} g^{\prime}+2 b g^{\prime} h^{\prime}+c h h^{\prime}$. Tum erit $m m^{\prime}=\left(a g^{\prime}+b\left(g h^{\prime}+h g^{\prime}\right)+\right.$ $\left.c h h^{t}\right)^{2}-D\left(g h^{\prime}-h g^{t}\right)^{2}$; quare $m m^{t}$ quadrato congruas erit secundum modulum $D$, adeoque etiam secundum $p$, i. e. $\mathrm{mm}^{\prime}$ erit tesiduum quadraticum ipsius $p$, Hinc sequitur, aut vtrumqua 
$m, m^{\prime}$ esse residuum quadraticum ipsius $p$, aut vtrumque non-residuuxa. Q.E. $D$.

Simili modo probatur, quando determinans $D$ per 4 sit diuisibilis, ommes numeros intupares per $F$ repraesentabiles vel esse $\equiv 1$, vel omres $\equiv$ (mod. 4.). Scilicet productum e duobus numeris talibus in hoc casu semper erit residuum quadr. ipsius 4 , adeoque $\equiv I(\bmod 4) ;$ quare vel vterque erit $\equiv x$, vel vterque $\equiv 3$.

Denique quando $D$ per 8 est dinisibilis, productum e duobus numeris quibuscunque imparibus, qui per $F$ repraesentari possunt, erit R. Q. ipsius 8 et proin $\equiv 2$ (mod 8$)$. Quare in hoc casu omnes numeri impares per $F$ repraesentabiles vel erunt $\equiv I$, vel omnes $\equiv 3$, vel omnes $\equiv 5$, rel omnes $\equiv 7$ (mod. 8$)$.

Ita e. g. quum per formam $(10,5,17)$ repraesentari possit numerus to qui est $N$. R. ipsius 7 : omnes numeri per 7 non diuisibiles, qui per formam illam repratesentari possunt, non-residua ipsius 7 ermat. - Qunm - 3 per formam $(-5,1,49)$ repracstutibilis et sec mod. 4. sit $\equiv 1$, omnes numeni impares porr formaro hanc repraesentabiles perinde se habebunt.

Ceterum, si ad proprositum praesens necessarium esset, facile demonstrare possemus, mu* meros per formarn $F$ repraesentabiles ad nullum numerum primum qui ipsum $D$ non metiatur, talem relationem fixam habere, sed promiscue tum residua tum non-residua numeri cuiusuis grimi ipsum $D$ non metientis per formam $F$ re. 


\section{$-325-$}

praesentari posse. Contra respectu numerorum 4 et 8 analogorn quoddam etiam in aliis casibus locum habet, quos praeterire non possumus.

I. Qmando determinars $D$ formae primitiuae $F$ est $\equiv 3$ (mod. 4): omnes numbri impares, per formam $F$ repraesentabites, erunt vel $\equiv \mathrm{x}$, vel omnes $\equiv 3(\bmod .4)$. Si enim $m, m^{\prime}$ sunt duo numeri per $F^{\prime}$ repraesentabiles, productum $\mathrm{mm}^{\prime}$ eodem modo vt supra sub formam $p p-D_{q q}$ redigi poterit. Quando itaque vterque $m, m^{\prime}$ est impar, necessario alter numerorum $p, q$ par erit, alter impar, adeoque alterum quadratorum $p p_{p}$ $q q \equiv 0$, alterum $\equiv 1$ ( $m o d .4)$. Vnde facilo deducitur, $p p-D q q$ certo esse $\equiv 1$ (mod. 4), adeoque aut vtrumque $m, m^{\prime} \equiv \mathrm{I}$, aut vtrumque $\equiv z(\bmod .4)$. Ita $e . g \cdot$ per forman $(10,3,17)$ alii numeri impares quam qui sunt formae $4 n$ + I repraesentari nequeunt.

II. Quando dotuminans $D$ formae primitiucte $F$ est $\equiv 2$ (mod. 8 ) omwes ontmeri impares, per Fe repratesentabules, enunt vel partim $\equiv \mathrm{I}$ partim $\equiv \tau$; vel partin $=3$ partim $\equiv 5$ (mad. 8). Ponamus enim $m, m^{\prime}$ esse duos numeros impares per $F$ repraesentabiles, quorum igitur productum $\mathrm{mm}^{\prime}$ sub formam $p p$ - Dqq redigi poterit. Quando ergo vterque $m, m^{\prime}$ est impar, necessario $p$ impar esse debebit (quia $D$ par), adeoque $p p \equiv$ $\mathbf{x}$ (nod. 8); $q 9$ vero erit vel $\equiv 0$, vel $\equiv \mathbf{x}$, vel $\equiv 4$, et proin $D q q$ vel $\equiv 0$ vel $\equiv{ }_{2}$.

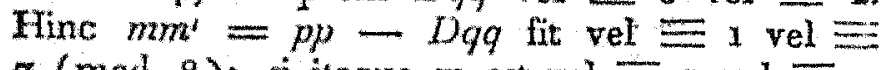
7 (mod. 8 ); si itaque $m$ est vel $\equiv 1$ vel $\equiv 7$, etiam $m^{*}$ erit vel 1 vel $\equiv 7$; si vero $m$ est vel 


\section{$-326-$}

$\equiv 5$, vel $\equiv 5$, etiam $m^{4}$ erit vel $\equiv 5$ vel $\equiv 5$. E. g. omnes numeri impares per formam $(5, x, 5)$ repraesentabiles sunt aut $\equiv 5$, aut $\equiv 5$ (mod. 8), nullique numeri formae $8 n+1$ aut $8 n+7$ per formam illam repraesentari passunt.

III. Quatdo deternitians $D$ fomae prmitivae $F$ est $=6$ (mod. 8): per formam hanc vepracsentart posstunt numeri impares vel taits tantum qui sunt $\equiv$ at 3 (mod.8), vel tales tantum qui sunt $\equiv 5 \mathrm{st}=7$ (nod. $8 \%$ Demonstrationem praecedenti (in II) omnino similem quisque nullo negotio etuoluere poterit. - Ita e. g. per formam $(5,1,7)$ vaice tales numeri impares possunt repraesentari qui sunt aut $\equiv 5$ aut $\equiv 7$ (mod. 8$)$.

230. Omnes igitur numeri qü per formam primitiuam datam $F$ determinantis $D$ repraesentari possunt, relationem fixam labebunt ad singulos diuisores primos ipsius $D$ (per quos quidem ipsi non sunt divisibiles), numeri impares vero qui per $F$ possunt repraesentari, in quibusdam casibus etiam ad numeros 4 ot 8 relation nem fixam lubebunt, scilicet ad 4 , quoties $D$ aut $\equiv 0$ aut $\equiv 3(\bmod .4$, et ad 8 quoties $D$ aut $\equiv 0$, aut $\equiv 2$ aut $\left.\equiv 6(\bmod 8)^{*}\right)$. Th* lem relationem ad sirugulos hos numeros, characterem seu characterem particularem formae $F$ vocabimus sequentique modo exprimemus:

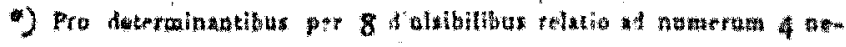

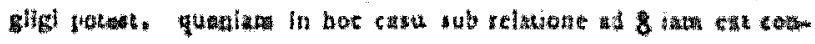
then: 
Quando sola residua quadratica numeri primi $p$ per formam $F$ repraesentari possunt, tribuemus ipsi characterem $R p$, in casu opposito characterem $N p$; similiter scribemus 1,4 , quando alii numeri impares per formam $F$ repraesentari nequeunt nisi qui sunt $\equiv 1(\bmod .4$.$) , rnde sta-$ tim liquet quales characteres exprimantur per signa $3 ; 4 ; 1,8 ; 3,8 ; 5,8 ; 7,8$. Denique formis per quas numeri impares tales soli repraesentari possunt qui sec. mod. 8 sunt vel $\equiv \mathbf{1}$ vel $\equiv 7$, tribuemus characterem 1 et 7,8 ; ex quo significatio characterum 3 et 5,$8 ; 1$ et 3,8 ; 5 et 7,8 sponte sequitur.

Characteres singuli formae primitiuae datae $(a, b, c)$ determinantis $D$ semper ex vuo saltem numerorum $a, c$ (qui manifesto per formam illam ambo surt repraesentabiles) cognosci possunt. Nam quoties $p$ est diuisor primus ipsius $D$, certe vnus numerorum $a, c$ per $p$ non erit diuisibilis; si enim vterque per $p$ diuisibilis esset, $p$ etiam ipsum $b b(=D+a c)$ metiretur, et proin etiam ipsum $b, i$. e. forma $(a, b, c)$ non esset primitiua. Simili modo in iis casibus rbi forma $(a, b, c)$ ad numerum 4 vel 8 relationem fixam habet, certo ad minimum rnus numerorum $a, c$ impar erit, ex quo igitur relatio illa deprehendi poterit. Ita e. fo. character formae $(7,0,25)$ respectu numeri 23 e numero $z$ concluditur $N$ 23, eiusdem formae character respectu numeri 7 habetur ex numero 23 puta $R T$; denique character huius formae respectu numeri 4 , puta 3 , 4 , vel e numero 7 vel e numero 23 colligi potest. 


\section{$-328-$}

Quoniam omnes numeri qui per formam aliquam $F$ in classe $K$ contentam repraesentari possunt, etiam per quamlibet aliam formam huîus classis sunt repraesentabiles: manifesto singuli characteres formae $F$ omnibus reliquis formis huifus classis quoque competent, quapropter illos tamquam characteres totius classis considerare licebit. Singuli itaque characteres formae cuiuslibet primitiuae datae ex ipsius forma repraesentante cognoscuntur. Classes oppositae semper characteres omnes eosdem habebunt.

251. Complexus omnium characterum particularium formae vel classis datae constituet characterem integrum huius formae vel classis. Ita e. g. character integer formae $(10,3,17)$, vel totius classis quam repraesentat erit 1,$4 ; N_{7}$; $N_{2}$, Simili modo character integer formae $(7,1,-17)$ erit 7,$8 ; R 3 ; N_{5}$, nam character particularis 3,4 in hoc casu omititur quia in charactere 7,8 iam est contentus. - Ex hoc fonte petimus subdiuisionem totius ordinis classium proprie primitiuarum (positiuarum quando det. est negatiuus) determinantis dati in plura genera diuersa, referendo omnes classes quae eundem characterem integrum habent ad genus idem; quarumque characteres integri diuersi sunt, ad genera diuersa. Singulis vero generibus eos characteres integros tribuemus quos classes sub ipsis contentae habent Ita e. go pro determinante -161 habentur sedecim classes positiuae proprie primitiuae, quae sequenti modo in quatuor genera distribuuntur: 


\section{- $329-$}

\begin{tabular}{l|l} 
Chaxacter & $\begin{array}{l}\text { Classium formae repraesentantes } \\
1,4 ; R 7 ; R 23\end{array}$ \\
1,$4 ; N_{7} ; N_{23}$ & $\begin{array}{l}1,161),(2,1,81),(9,1,18) \\
(5,2,35),(5,-2,33),(10,3,17) \\
(10,-3,17)\end{array}$ \\
3,$4 ; N_{7} ; R 23$ & $\begin{array}{l}(7,0,23), 11,2,15),(11,-2,15) \\
(14,7,15) \\
(3,1,54) ;(3,-1,54),(6,1,27) \\
(6,-1,27) .\end{array}$
\end{tabular}

De mulltitudine characterum integrorum diuersorum, qui quidem a priori sunt possibiles, teneantur sequentia.

I. Quando determinans $D$ per 8 est diuisibilis, respecti numeri 8 quatuor characteres particulares diuersi sunt possibiles; numerus 4 nullum characterem peculiarem suppeditat (annot. ad art praec.). Praeterea respectu singulorum diuisorum primorum imparium ipsius $D$ bini characteres dantur; quare si illorum multitudo est $m$, dabuntur omnino $2^{m+2}$ characteres integri diuersi (statuendo $m=0$, quoties $D$ est potestas binaria).

II. Quando det. $D$ per 8 non est diuisibilis, sed tamen per 4 , insuperque per $m$ numeros primos impares: omnino habebuntur $2^{m+1}$ characteres integri diuersi.

III. Quando det. $D$ est par neque vero per 4 diuisibilis, erit vel $\equiv 2(\bmod .8)$ vel $\equiv 6$. In casu priori dabuntur duo characteres particulares respectu numeri 8 puta 1 et 7,8 , atque $\mathrm{X}_{5}$ 


\section{- $350^{\circ}-$}

3 et 5,8 ; in casu posteriori totidem. Posita igitur multitudine diuisorum primorum imparium ipsius $D,=m$ : habebuntur ompino $2^{\text {m-t-r }}$ characteres integri diuersi.

IV. Quando $D$ est impar, erit vel $\equiv 1_{\text {, }}$ vel $\equiv 3(\bmod .4)$ In casu posteriori respectu numeri 4 duo characteres diuersi dantur, qualis relatio in casu priori in characterem integrum non ingreditur. Quare designante $m$ idem vt ante, in casu priori dabuntur $2^{m}$, in posteriori $2^{\mathrm{m}+}$ characteres integri diuersi.

Probe vero notandum est, hinc neutiquam sequi, totidem genera reuera dari quòt characteres dinersi a priori sint possibiles. In exemplo quidem nostro harum semissi tantum reuera classes siue genera respondent, nullaeque classes po. sitiuae dantur, quibus tharacteres $1 ; 4 ; R 7$, $N_{23}$ vel $1 ; 4 ; N_{7} ; R 23 ;$ vel 3,$4 ; R 7 ; R 23$ vel 3,$4 ; N_{7} ; N_{23}$ competant. De quo argumento grauissino infro fusius agetur.

Formae $(1,0,-D)$, quae haud dubie inter omnes formas determinantis $D$ pro simplicissima habenda est, nomen formae principalis abhinc tribuemus; classem totam in qua illa reperitur classem principalem vocabimus; denique genus totum in quo classis principalis contenta est, genus principale dicetur. Probe itaque distinguendae sunt forma principalis, forma e classe principali et forma e genere principali; nec non classis principalis et classis e genere principali His denominationibus semper ytemur, etiamsi 


\section{$-331-$}

Torte pro determinante aliquo aliae classes praeter principalem, vel alia genera praeter genus principale non dentur, vti e. g. euenit plerumque quando $D$ est numerus primus positiuus formae $4 n+1$.

232. Quamquam ea quae de formarum characteribus explicata surt proxime eum in finem sunt allata, vt subdiuisio ordinis positiui proprie primitiui inde petatur: tamen nilil impedit quominus eadem etiam ad formas classesque negatiuas aut ad improprie primitiuas applicentur, atque tum ordo improprie primitiuus positiuus, tum ordo proprie primitiuus negatiuus, tum ordo improprie primitiuus negatiuus ex eodem principio in genera subdiuidantur. Ita postquam e. $g$. ordo proprie primitiuus formarum determinantis 14.5 in duo genera sequentia subdiuisus est

$$
\begin{aligned}
& R 5, R 26 \quad(1,0,-145),(5,0,-29) \\
& N_{5}, N_{2} 6(3,1,-48),(3,-1,48)
\end{aligned}
$$

etiam ordo improprie primitiuus perinde in duo genera subdiuidi potest:

$$
\begin{array}{l|l}
R_{5}, R_{29} & \left(4,1,-3^{6}\right),(4,-1,-36) \\
N_{5}, N_{29} & \left(2,1,-7^{2}\right),(10,5,-12)
\end{array}
$$

vel, sicuti classes positiuae formarum determinantis - 129 in quatuor genera distribuuntur:

1,$4 ; R_{3} ; R_{43}(1,0,129),(10,1,13),(10,-1,13)$ 1,$4 ; N 3, N_{43}(2,1,65),(5,1,26),(5,-1,26)$ $3,4, R_{3}, N_{43} \quad(3,0,43),(7,2,79),(7,-2,19)$ 3,$4 ; N_{3} ; R_{43}(7,3,23),(11,5,14),(11,-5,14)$ 
etiam classes negatiuae in quatuor ordines disce. dunt

$$
\begin{aligned}
& 3,4 ; N_{3} ; N_{43} \mid \begin{array}{l}
(-1,0,-129),(-10,1,-13), \\
(-10,-1,-13) \\
(-2,1,-65),(-5,1,-16), \\
(-5,-1,-26)
\end{array} \\
& 3,4 ; R_{3} ; R_{43} \\
& 1,4 ; N_{4} ; R_{43} \mid \begin{array}{l}
(-3,0,-43),(-7,2,-19), \\
(-7,-2,-19) \\
(-6,3,-23),(-11,5,-14), \\
(-11,-5,-14)
\end{array} \\
& 1,4 ; R_{3} ; N_{43},
\end{aligned}
$$

Attamen quum systema classium negatiuarum systemati positiuarum semper tam simile euadat, plerumque superfluum videbitur illud seorsim construere. Ordinem improprie primitiuum autem ad proprie primitiuum reducere infra docebimus.

Tandem quod attinet ad ordines deriuatos: pro horum subdiuisione regulae nouae non sunt necessariae. Quum enim quiuis ordo deriuatus ex aliquo ordine primitiuo (determinantis minoris) originem trahat, illiusque classes singulae ad singulas huius sponte referantur: manifesto subdiuisio ordinis deriuati e subdiuisione ordinis primitiui peti poterit.

233. Si forma (primitiua) $F^{\prime}=(a, b, c)$ ita est comparata, vt inueniri possint duo numexi $g, h$ tales vt fiat $g g \equiv a, g h \equiv b, h h \equiv c$ secundum modulum datum $m$, dicemus formam illam esse residuum quadraticum numeri $m$ atque $g x+h y$ valorem expressionis $\sqrt{ }(a x x+$ $2 b x y+c y)$ (mod. $m)$, siue breuius $(g, h)$ valorem expr. $\checkmark(a, b, c)$ vel $\sqrt{ } F(\bmod m)$. 
Generalius, si multiplicator $M$, ad modulum $m$ primus, eius est indolis vt fieri possit $g g \equiv a M$, $g h \equiv b M, h h \equiv c M(\bmod m)$, dicemus $M$ $\times(a, b, c)$ siue $M F$ esse res. quad. ipsius $m$, atque $(g, h)$ valorem expressionis $\checkmark M(a, b, c)$ vel $\sqrt{ } M F(\bmod m)$. Ita e. g. forma $(3,1,54)$ est res. quadr. ipsius 23 atque $(7,10)$ valor expr. $\sqrt{ }(3,1,54)(\bmod 23) ;$ similiter $(2$, - 4) valor expr. $\sqrt{ } 5(10,3,17)(\bmod 23)$. Vsus harum definitionum infra ostendetur: hic notentur propositiones sequentes:

I. Si $M(a, b, c)$ est R. Q. numeri $m$, hic determinantem formae $(a, b, c)$ metietur. Si euim $(g, h)$ est valor expressiones $\sqrt{ } M(a, b, c)$ $(\bmod . m)$, siue $g g \equiv a M, g h \equiv b M, h h \equiv$ $c M(\bmod . m):$ erit $b b M M-a c M M \equiv$ o, siue $(b b-a c) M M$ per $m$ diuisibilis. Quoniam autem $M$ ad $m$ primus esse supponitur, etiam $b b$ - $a c$ per $m$ diuisibilis erit.

II. Si $M(a, b, c)$ est R. $Q$. ipsius $m$, atque $m$ aut numerns primus aut potestas numeri primi, puta $=p^{\mu}$ : character particularis formae $(a, b, c)$ respectu numeri $p$ erit vel $R p$, vel $N p$, prout $M$ est residuum vel non-residuum ipsius $p$. Hoc station inde sequitur, quod tum $a M$ tum $c M$ est residuum ipsius $m$ siue ipsius $p$, atque ad minimum vaus numerorum $a, c$ per $p$ non dimisibilis (art. 230 ).

Simili modo, si (manentibus reliquis) $m$ $=4$, erit vel 1,4 vel 3,4 character part. formae $(a, b, c)$ prout $M \equiv \mathbf{1}$ vel $\equiv 3$; nec non si 


\section{- $334-$}

$m=8$ vel altior potestas numeri 2 , erit $I, 8$; $3 ; 8 ; 5,8 ; 7,8$ char part. formae $(a, b, c)$ pront $M \equiv 1 ; 3 ; 5 ; 7(\bmod .8) \mathrm{resp}$.

III. Vice versa si $m$ est numerus primus aut numeri primi imparis potestas $=p^{\mu}$, determinantem $b \vec{b}-a c$ metiens, atque $M$ vel residuum vel non-residuum ipsius $p$, prout character formae $(a, b, c)$ respectu ipsius $p$ est $R p$ vel $N p$ resp: erit $M(a, b, c)$ resid. quadr ipsius $m$. Quando enim $a$ per $p$ non est diưîsibilis, $a M$ erit res. ipsius $p$ adeoque etiam ipsius $m$; si itaque $g$ est valor expr. $\sqrt{ } a M(\bmod m$ ), $h$ valor expr. $\frac{b g}{a}(\bmod m)$, erit $g g \equiv a \dot{M} ; a h \equiv b g$ adeoque $a g h \equiv b g g \equiv a b M$ et $g h \equiv b M$; de. nique $a h h \equiv b_{g h} \equiv b b M \equiv b b M-(b b-$ ac) $M \equiv a c M$ adeoque $h h \equiv c M$, i. e. $(g, h)$ valor expr. $\sqrt{ } M(a, b, c)$. Quando vero $a$ per $m$ est diuisibilis, certo $c$ non erit; vade facile perspicitur, eadem resultare, si pro $h$ assumatux valor expr, $\sqrt{ } c M(\bmod m)$, pro $g$ valor expr. $\frac{b h}{c}(\bmod . m)$.

Simili modo demonstratur, si $m$ fuerit $=4$ ipsumque $b b-a c$ metiatur, numerusque $M$ accipiatur vel $\equiv 1$ vel $\equiv 3$ prout 1,4 vel 3,4 fuerit char. part formae $(a, b, c)$ : fore $M(a, b, c)$ res. qu ipsius $m$. Nec non, si $m$ fuerit $=8$ vel altior potestas ipsius 2 per quam $b b-a c$ diuisibilis sit, atque $M$ accipiatur $\equiv 1 ; 3 ; 5 ; \mathrm{T}$ (mod. 8) prout character part. formae $(a, b, c)$ respectu numeri 8 postulet: $M(a, b, c)$ fore res. qu insius $m_{0}$ 


\section{$-335 \longrightarrow$}

IV. Si determinans formae $(a, b, c)$ est $=$ $D$, atque $M(a, b, c)$ res. qu. ipsius $D$, omnes character particulares formae $(a, b, c)$ tum respectu singulorum diuisorum primorum imparium ipsius $D$, tum respectu numeri 4 vel numeri 8 (si ipsum $D$ metiuntur) ex numero $M$ statim cognosci possunt. Ita e.g. quum $3,20,10$, 27) sit resid. qu. ipsius 440 , scilicet $(150,9)$ valor expr. $\sqrt{3}(20,10,27)$ sec. mod. 440 , atque $3 N_{5}, 3 R_{11}$ : characteres formae $(20,10,27)$ sunt 3,$8 ; N_{5} ; R 11$. Soli characteres particulares respectu numerorum 4 et 8 , quoties determinantem non metiuntur, nexum necessarium cum numero $M$ non habent.

V. Vice versa, si numerus $I I$ ad $D$ primus onnes characteres particulares forma $(a, b, c)$ in se complectitur (exceptis characteribus respectu numerorum 4,8 , quando ipsum $D$ non mea tiuntur) : erit $M(a, b, c)$ res. qu. ipsius $D$. Nam ex III patet, si $D$ sub formam $+A^{\mu} B^{\zeta} C^{\gamma} \ldots$ redigatur, ita vt $A, B, C$ etc. sint numeri primi diuersi, fore $M(a, b, c)$ resid. qu singulorum $A^{*}, B^{b}, C^{\gamma}$ etc. Si igitur valor expr. $\sqrt{ } M\left(a_{\text {, }}\right.$ $b, c)$ secundum mod. $A^{* 2}$, est $\left(\mathfrak{H}, \mathfrak{H}^{\prime}\right)$; secundum mod. $B^{6},\left(S B, B^{\prime}\right)$; sec. mod. $C^{\gamma},\left(\mathfrak{C}, \mathfrak{V}^{\prime}\right)$ etc. numerique $g, h$ ita determinantur vt sit $g \equiv$ $\mathfrak{H}, \mathfrak{B}, \mathfrak{C}$ etc; $\mathfrak{h} \equiv \mathfrak{H}, \mathfrak{B}$, $\mathfrak{C}$ etco secundum modulos $A^{\alpha}, B^{\bullet}, C^{\gamma}$ etc resp. (art. $3^{2}$ ): facile perspicietur, fore $g g \equiv a M, g h \equiv b M, h h \equiv$ CM secundum ommes modulos $A^{\alpha}, B^{b}, C^{\gamma}$ etc. adeoque etiam secundum modulum $D$ qui illo. rum est productum. 
VI. Propter has rationes numeri tales vt $M$ vocabuntur numeri characteristici formae $(a, b$, $c)$, poteruntque per $V$. plures huiusmodi numeri nullo negotio inueniri simulac omnes claricteres particulares huius formae sunt eruti; simplicissimi autem tentando plerumque euolumbur facillime. Manifestum est, si $M$ sit numprus tharacteristicus forme primitiuat datate det+m minantis $D$, omnes numeros, ipsi $M$ secundum mmi. $D$ congruos, fore numeres chactacteristicon piusdem formae; formas in eadem classe, siue etham in classibus diuersis ex eodem gertere, conteatlas eosdem numeros characteristicos liabere, quamobrem quiuis numerus chararteristicus fomat datae etiam toti classi et generi tribui potest; dunique 1 semper esse numerum characteristicum formae classis et generis principalis, sue guamlibet formam e genere principali esse residum determinaxitis sui.

VII. Si $(g, h)$ est valor expr. $\sqrt{ } M\left(a, b_{3}\right.$ c) (mod. $m$ ), atque $g^{\prime}=g, h^{\prime}=h($ manl. $m)$ : erit etiam $\left(g^{\prime}, h^{\prime}\right)$ valor eiusdem expressionis. Tales valores pro acquiualentibus haheri possunt; contra si $(g, h),\left(g^{\prime}, h^{\prime}\right)$ sunt valores wiuscem expr. $\sqrt{ } M(a, b, c)$, neque tamen simul $g^{*}=$ $g, h r \equiv h(\bmod m)$, diuersi sunt censendi. Manifesto quoties $(g, h)$ est valor talis expressionis, etiam $(-g,-h)$ erit, facileque demonstratur, hos valores semper esse diuersos nisi $m$ 2. Aeque facile demonstratur, expressionem $\sqrt{ } M(a, b, c)$ (mot. $m)$ plures valores diutersos quam duos tales (oppositos) habere non posse, quando $m$ sit aut numerus primus impar aut nu- 


\section{- $357-$}

meri primi imparis potestas aut $=4$; quardo vero $m$ sit $=8$ aut altior potestas numeri 2 , quam tuor ommino dari. Hinc facils deducitur per VI, si determinans $D$ format $(a, b, c)$ sit $= \pm$ $2^{*} A^{*} B^{\circ} \ldots$, designantibus $A, B$ etc. numeros primos impares diuersos quonm multiudo $=n$, atque $M$ numerus charartertsticus illius formae: dari omnine vel $2^{n}$ wol $2^{n-1-1}$ vel $2^{a+2}$ ralores dimersos expr. $\sqrt{M}(a, b, c)$ (mod. $D)$, prout $\mu \mathrm{vel}<2 \mathrm{vel}=2$ vel $>2$. Ita e. g. haberltur sedecin values expr: $\sqrt{7}(12,6,-17)$ (mod. 240), puta $(+18$, F I1), (士18, 士 so), $( \pm 18,791),( \pm 18, \pm 109)( \pm 78$, $\pm 19),\left( \pm 77^{8}, \pm 59\right),(+78,761),( \pm$ $78,7 x 01)$. Demonnsh ativatem ampliorem quam ad sequentia non sit adeo necessaria brenitatis gratia non apponimus.

VIII. Denique obseruamus, si duarum forInatrum aequitualentium $a, b, c),\left(a^{4}, b^{\prime}, c^{2}\right)$ determinans sit $D$, numertus characteristicus $M$, priorque transeat in posteriorem per substitutionem $4, c, 2$ : ex quouis valore expr $r i m a, b$, c) vt $\left(g, h\right.$, sequi valorem expr. $\sqrt{ } M\left(a^{\prime}, b^{\prime}, c^{\prime}\right)$, puta $\left.x^{2}+h, 6 g+h\right)$. Demurstrationem quisque nulio negotio eruere poterit.

234. Postquam hate de formis in classes genfat ordines distribuendis praemisimus, pro-

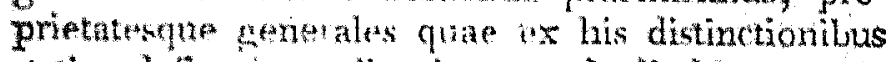
statim delhumit explicauimus, ad aliud argumen. tum grouissimum transimus a nemine hucusque attactum, de formarm compositione. In cuius disquisitionis limine, ne posthac demonstrationum 


\section{$-538-$}

seriem continuam interrumpere oporteat, statim intercalamus

Limma. Habentur quatuor series numerom num integrorum $a, a^{t}, a^{\prime \prime} \ldots a^{n} ; b, b^{2}, b^{n} \ldots b^{n} ; c_{3}$ $c^{\prime}, c^{\prime \prime} \ldots c^{\prime \prime} ; d, d^{\prime \prime}, d^{\prime \prime} \ldots d^{n}$ ex aeque multis (puta $n+x$ ) terminis constantes, atque ita comparatae, $v t c d^{\prime}-d c^{\prime}, c d^{\prime \prime}-d c^{\prime \prime} e t c ., c^{\prime \prime} d^{\prime \prime}-d^{\prime} c_{c}^{\prime \prime} t t c . e t c$. respectiue sint $=k\left(a b^{\prime}-b a^{\prime}\right), k\left(a b^{n}-b a^{\prime \prime}\right)$ etc., $k\left(a^{\prime} b^{\prime \prime}-b^{\prime} a^{\prime \prime}\right)$ etc. etc., siue generaliter $c^{\lambda} d^{\mu}-d^{\lambda} c^{\mu}=k\left(a^{\lambda} b^{k}-b^{\lambda} a^{k}\right)$, denotante $k$ numerum integrum datum; $\lambda,{ }_{\mu}$ integros quoscunque inatquales inter o et n incl. quoram maior $\mu^{*}$ ); praeterea omnes $a^{\star} b^{H}-b^{\lambda} a^{4} d i$ uisorem communem non habent. Tunc intuenirt possunt quatuor numeri integri ${ }_{2}, 6, \gamma$, sales, wit sit $a+b b=c, * a^{\prime}+6 b^{\prime}=c^{i}, a a^{\prime \prime}+b^{\prime \prime}$ $=c^{\prime \prime}$ etc.; $; a+d b=d, j a^{\prime}+\delta b^{\prime}=d^{\prime \prime}$ etc. sime gencraliter $\alpha a^{y}+6 b^{\prime}=c^{\prime}, \quad \gamma a^{y}+s^{\prime} b^{\prime}=a^{*}$ quo facto erit ${ }^{8}-6 y=k$.

Quum per hyp. numeri $a b^{\prime}-b a^{\prime}, a b^{\prime \prime}-$ $\dot{b} a^{\prime \prime}$ etc. $a^{\prime} b^{\prime \prime}-b^{\prime} a^{\prime \prime}$ etc. (quorum multitudo erit $\left.=\frac{5}{2}(n+1) n\right)$ diuisoren commumem non habeant, inuenini poterunt totidem ali numeri integri, per quos illis resp. multiplicatis productorum summa fitt $=1$ (art 4o). Designentur hi multiplicutores per $(0,1),(0,2)$ etc. $(1,0)$ etc., siue generaliter multiplicator ipsius $a^{\lambda} b^{\mu}-$ $b^{\lambda} a^{k}$ per $(\lambda, \infty)$, ita vit $\mathrm{x}(\lambda, \beta)\left(a^{\lambda} b^{k}-\right.$

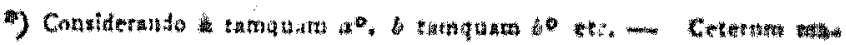

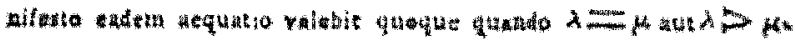




\section{- $539-$}

$\left.b^{\lambda} a^{\mu}\right)=1$. (Per literam $\Sigma$ denotamus aggrega $\alpha$ tum omnium valorum expressionis, cui praefixa est, qui ol iuntur tribuendo ipsis $\lambda, \mu$ omnes valores inaequales inter $o$ et $n$, ita vt sit. $\mu>x)$. Quo facto si statuitur $\Sigma(\lambda, \mu)\left(c^{\lambda} b^{\mu}-b^{\lambda} c^{\mu}\right)$ $=\mu, \Sigma(\lambda, \mu)\left(a^{\lambda} c^{\mu}-c^{\lambda} a^{\mu}\right)=\hat{*}, \Sigma(\lambda, \mu)$ $\left(d^{\lambda} b^{\mu}-b^{\lambda} d^{\mu}\right)=2, \Sigma(\lambda, k)\left(a^{\lambda} d^{\mu}-\right.$ $\left.d^{\lambda} a^{k}\right)=A:$ hi $\alpha, 6, \gamma, \delta$ proprietatibus praescriptis erunt praediti.

Dem. I. Denotante , numerum quemcunque integrum inter o et $n$, érit $\alpha a^{r}+b^{r}=$ $\Sigma(\lambda, \mu)\left(c^{\lambda} b^{\mu} a^{r}-b^{\lambda} c^{\mu} a^{r}+a^{\lambda} c^{\mu} b^{r}-\right.$ $\left.c^{\lambda} a^{\mu} b^{\mu}\right)=\frac{x}{x} \mathrm{x}(\lambda, \mu)\left(c^{\lambda} \cdot d^{\mu} c^{\mu}-a^{\lambda} c^{\mu} c^{r}\right)$ $=\frac{x}{k} c^{\mu} \Sigma(\lambda, \mu)\left(c^{\lambda} d^{\mu \mu}-d^{\lambda} c^{\mu}\right)=c^{r} \Sigma(\lambda, \mu)$ $\left(a^{\lambda} b^{k}-b^{\lambda} a^{k}\right)=c^{r}$. Et per calculum similem eruitur $y a^{r}+. b^{r}=d^{r}$. Q. E. $P$.

II. Quoniam igitur $c^{\lambda}=a a^{\lambda}+6 b^{\lambda}, c^{\lambda}$ $=a^{\mu}+b^{\mu}$, fit $c^{\lambda} b^{\mu}-b^{\lambda} c^{\mu}=a\left(a^{\lambda} b^{\mu}\right.$ $\left.-b^{\lambda} a^{\mu}\right)$, similique modo $a^{\lambda} a^{\mu}-c^{\lambda} a^{\mu}=$ $c\left(a^{\lambda} b^{\alpha}-b^{\lambda} a^{\alpha}\right) ; d^{\lambda} b^{\mu}-b^{\lambda} d^{\mu}=a^{\lambda} a^{\lambda} b^{\mu}$ $\left.-b^{\lambda} a^{\mu}\right) ; a^{\lambda} d^{\mu}-d^{\lambda} a^{\mu}=\delta\left(a^{\lambda} b^{\mu}-b^{\lambda} a^{\mu}\right) ;$ ex quibus formulis valores ipsorum $\alpha, b, \gamma, \delta$ muito facilius erui possunt, si modo $\lambda, \mu$ ita accipiuntur it $a^{\lambda} b^{\mu}-b^{\lambda} a^{\mu}$ non sit $=0$, quod certo fieri poterit, quia ommes $a^{\lambda} b^{\alpha}-b^{\lambda} a^{\mu}$ per hyp. diuisorem communem non habent, adeaque omnes $=0$ esse nequeunt - Ex iisdem aequationibus deducitur, raultiplicando primam $\mathrm{Y} 2$ 


\section{-54.0}

per quartam, secundam per tertiam et subtrahendo, $\left(a^{\lambda}-b^{\alpha}\right)\left(a^{\lambda} b^{\mu}-b^{\lambda} a^{\mu}\right)^{\lambda}=\left(a^{\lambda} b^{\mu}-\right.$ $\left.b^{\lambda} a^{\mu}\right)\left(c^{\lambda} d^{\mu}-d^{\lambda} c^{\mu}\right)=k\left(a^{\lambda} b^{\mu}-b^{\lambda} a^{\mu}\right)^{\lambda}$, made necessario $-\sigma_{y}=$ L. Q. E. $S$.

235. Si forma $A X X+2 B X Y+C Y Y \ldots$ $F$ transit in productum e duabus formis $a x x+$ $2 b x y+c y y \ldots f$, et $a^{t} x^{\prime} x^{\prime}+2 b^{\prime} x^{\prime} y^{\prime}+c^{t} y^{t} y$ $\therefore f^{\prime}$ per substitutionem talem $X=p x x^{\prime}+p^{\prime} x y^{\prime}$ $+p^{\prime \prime} y^{\prime}+p^{\prime \prime \prime} y y^{\prime}, \quad Y=q x x^{\prime}+q^{\prime} x y^{\prime}+q^{\prime \prime} y x^{\prime}$ $+q^{\prime \prime \prime} y y^{\prime}$ (quod breutatis catsa in sequentibus sémper ita exprimernus: Si $F$ transit in $f^{k}$ per substitutionem $\left.p, p^{\prime}, p^{\prime \prime}, p^{\prime \prime \prime} ; q, q^{\prime}, q^{\prime \prime}, q^{\prime \prime}{ }^{*}\right)$ ), dicemus simpliciter, formam $F$ transformabitem esse in $f^{\prime}$; si insuper haec transformatio ita est comparata, vt sex numeri $p q^{\prime}-q p^{t}, p q^{\prime \prime}-q p^{\prime \prime}$,

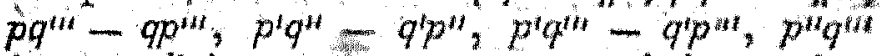
- $q^{\text {up }} p^{\text {in }}$ diưsisorem communern non habeant: formam $F$ e formis $f^{\prime} f^{\prime}$ compositam vocabinus.

Inchoabimus hane disquisitionem a supposi* tione generalissima, formam $F$ in $f f$ transire per substilutionem $p, p^{\prime}, p^{\prime \prime}, p^{\prime \prime \prime} ; q, q^{\prime \prime} q^{\prime \prime}, q^{\prime \prime \prime}$ et quae inde sequantur etuluemus. Manifesto huic suppositioni ex asse aequiualebunt sequentes nouem aequationes ( $i$. e. simulac hae aequationes locum hiabent, $F$ per substitutionem dictam transibit in $f f$, et vice versa :

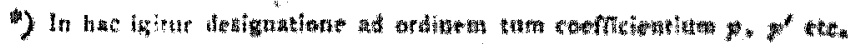

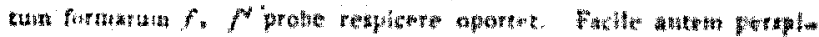

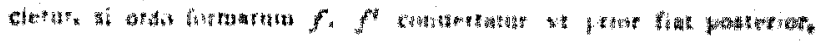

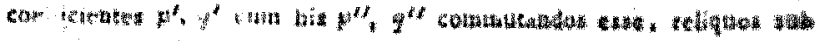

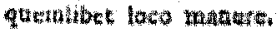


$s p p+2 B p q+C q q=a a^{\prime} \ldots \ldots \ldots[1]$ $A p^{\prime} p^{\prime}+2 B p^{\prime} q^{\prime}+C q^{\prime} q^{\prime}=\alpha c^{\prime} \ldots \ldots \ldots[2]$ $A p^{\prime \prime} p^{\prime \prime}+2 B p^{\prime \prime} q^{\prime \prime}+C q^{\prime \prime} q^{\prime \prime}=c a^{\prime} \ldots \ldots \ldots[\xi]$ $\left.A p^{\prime \prime \prime} p^{\prime \prime \prime}+{ }_{2} B p^{\prime \prime \prime} q^{\prime \prime \prime}+C q^{\prime \prime} q^{\prime \prime \prime}=\dot{c} c^{\prime} \ldots \ldots . . .4\right]$ $A p p^{\prime}+B\left(p q^{\prime}+q p^{\prime}\right)+C q q^{\prime}=a b^{\prime} \ldots \ldots[5]$ $A p p^{\prime \prime}+B\left(p q^{\prime \prime}+q p^{\prime \prime}\right)+C q q^{\prime \prime}=b a^{\prime} \ldots[6]$ $A p^{\prime} p^{\prime \prime \prime}+B\left(p^{\prime} q^{\prime \prime \prime}+q^{\prime} p^{\prime \prime \prime}\right)+C q^{\prime} q^{\prime \prime \prime}=b c^{\prime}[7]$ $A p^{\prime \prime} p^{\prime \prime \prime}+B\left(p^{\prime \prime} q^{\prime \prime \prime}+q^{\prime \prime} p^{\prime \prime \prime}\right)+C q^{\prime \prime} q^{\prime \prime \prime}=c b^{\prime}[8]$ $A\left(p p^{\prime \prime \prime}+p^{\prime \prime} p^{\prime \prime}\right)+B\left(p q^{\prime \prime \prime}+q p^{\prime \prime \prime}+p^{\prime} q^{\prime \prime}\right.$ $\left.+q^{\prime} p^{\prime \prime}\right)+C\left(q q^{\prime \prime \prime}+q^{\prime} q^{\prime \prime}\right)=2 b b^{\prime} \ldots[9]$

Sint determinantes formarum $F, f, f^{\prime}$ resp. $D_{\text {, }}$ $d, d^{\prime} ;$ diuisores communes maximi numerorum $A, 2 B, C ; a, 2 b, c ; a^{\prime}, 2 b^{\prime}, c^{\prime}$ resp. $M, m, m^{\prime}$ (quos omnes positiue accoptos supponimus). Porro determinentu sex numeri integri $\mathfrak{U}, \mathfrak{B}, \mathfrak{E}$, $\mathfrak{Z}^{\prime}, \mathfrak{B}^{\prime}, \mathfrak{C}$, ita vt sit $2 a+2 \mathfrak{B} b+\mathfrak{C}_{C}=m_{3}$ $\mathfrak{2}^{\prime} a^{\prime}+2 \mathfrak{B} b b^{\prime}+\mathfrak{S}^{\prime} a^{\prime}=m^{\prime}$. Denique designennur numeri $p q^{\prime}-q p^{\prime}, p q^{\prime \prime}-q p^{\prime \prime}, p q^{\prime \prime}-q p^{\prime \prime \prime}$ $p^{\prime} q^{\prime \prime}-q^{\prime} p^{\prime \prime}, p^{\prime} q^{\prime \prime \prime}-q^{\prime \prime} p^{\prime \prime \prime}, p^{\prime \prime} q^{\prime \prime \prime}-q^{\prime \prime} p^{\prime \prime \prime}$ respe per $P, Q, R, S, " T, U$, sitque ipsorum diuisor communis maximus positiue acceptus $=k-$ lam ponendo.

$A^{\prime \prime} p p^{\prime \prime \prime}+B\left(p q^{\prime \prime \prime}+q p^{\prime \prime \prime}\right)+C q q^{\prime \prime \prime}=b b^{\prime}+\Delta[10]$

fit ex aequ. 9

$A p^{\prime} p^{\prime \prime}+B\left(p^{\prime} q^{\prime \prime}+q^{\prime} p^{\prime \prime}\right)+C q^{\prime} q^{\prime \prime}=b b^{\prime}-\Delta[1]$

Fx his ndecim aequationibus $1 . . .11$, sequentes nouas enoluimus*):

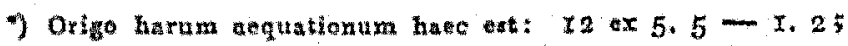
$13 \times 5.9-3.7-2.6 ; 14$ ex 10. $11-6.7 ; 15$ ex $5.8+5.8+10.10+$ IX. Ir $-1.4-2.3-$ $\mathrm{X} 3$ 


\section{$-542-$}

$D P P=d^{\prime} d a \ldots \ldots \ldots \ldots \ldots[12]$ $D P(R-S)=2 d^{\prime} a b \ldots \ldots \ldots \ldots[13]$ $D P U=d^{l} a c-\left(\Delta \Delta-d d^{l}\right) \ldots \ldots .[14]$ $D(R-S)^{2}=4 d^{\prime} b b+2\left(\Delta \Delta-d d^{\prime}\right)[15]$ $D(R-S) U=2 d^{\prime} b c \ldots \ldots \ldots \ldots[16]$ $D U E=d^{\prime} c c \ldots \ldots \ldots \ldots \ldots[17]$ $D Q Q=d a^{\prime} a^{\prime} \ldots \ldots \ldots \ldots \ldots \ldots[18]$ $D Q(R+S)=2 d a^{\prime} b^{\prime} \ldots \ldots \ldots \ldots[19]$ $D Q T=d a^{\prime} c^{l}-\left(\Delta \Delta-d d^{\prime}\right) \ldots \ldots[20]$ $D(R+S)^{2}=4 d b^{\prime} b^{\prime}+2\left(\Delta \Delta-d d^{\prime}\right)$ [21] $D(R+S) T=2 d b^{\prime} c^{\prime} \ldots \ldots \ldots \ldots \ldots[22]$ $D T T=d c^{\prime} c^{\prime} \ldots \ldots \ldots \ldots \ldots \ldots \ldots[23]$

Hinc rursus deducuntur hae duae:

$$
\begin{aligned}
& 0=2 d^{l} a d\left(\Delta \Delta-d d^{\prime}\right) \\
& 0=\left(\Delta \Delta-d d^{\prime}\right)^{2}-2 d^{\prime} a c\left(\Delta \Delta-d d^{\prime}\right)
\end{aligned}
$$

scilicet prior ex 12. $15-13.13$, posterior ex 14. $14-12.17$; vade facile perspioitur, necessario esse $\Delta \Delta-d d^{\prime}=0$, siue sit $a=0$, siue mon sit $=0 *$ ). Supponemus itaque, in aequatt. $14,15,20,21$ ad dextram deleri $\Delta \Delta-d d^{i}$.

Iam statuendo

$$
\begin{aligned}
& 2 P+B(R-S)+C U=m n^{\prime} \\
& 2 U Q+S B(R+S)+C T=m^{\prime} n
\end{aligned}
$$

6. $7-6.7 ;$ I6 ex 8.9-3. $7-4.6 ;$ I 7 eqx 8. 8 - 3. 4. Deductio sex reliquarum eodem modo adornatur, modo aequationes $2,5,7$ cum aequationibus $3,6,8$ resp, commitantur, et reliquae $I, 4,9$, Io, 1 I eodem Ioco deinceps retinentur, puta I8 ex $6,6-\mathrm{I}_{2} 3$ etc,

-) Hacc deriuatio aequationis $\Delta \Delta=d d^{\prime}$ ad institutum praesens sufficit; alioquin analysin elegantiorem sed hic nishio prolixam tradere possemus, directe deducento ex aequationibus $I+\ldots$ I I hane $a=\left(\Delta \Delta-d d^{\prime}\right)^{*}$. 
(vbi $n, n^{\prime}$ etiam fractiones euadere posse probe notandum, etsi $m n^{\prime}, m^{\prime} n$ necessario sint integri): facile ex aequatt. $12 \ldots$ It deducitur

$$
\begin{gathered}
D m m i n^{\prime}=d^{\prime}\left(1 a+2 B b+C_{c}\right)^{2}=d^{\prime} m m \\
\text { simuliterque ex aequ. } 18 \ldots 23
\end{gathered}
$$

$D m^{\prime} m^{\prime} n n=d\left(2{ }^{\prime} a^{\prime}+{ }_{2} B b^{\prime}+\left(\mathfrak{C}^{\prime} c^{\prime}\right)^{2}=d m^{\prime} m^{\prime}\right.$.

Erit igitur $d=D n n, d^{\prime}=D n^{\prime} n^{\prime}$, vnde nanciscimur concLVSIONEM PRIMAM: Determinantes formarum $F, f, f^{\prime}$ necessario inter se habent rationem quadratorum; et secvidaM: $D$ semper metitur numeros $d m^{\prime} m^{\prime}$, $d^{\prime} m i n$. Patet itaque, $D$, $d, d^{\prime}$ eadem signa habere, nullamque formam in productum $f^{\prime}$ transformabilem esse posse, cuius determinans maior sit quam diuisor communis maximus numerorum $d m^{\prime} m^{\prime}, d^{\prime} m m$.

Multiplicentur aequationes $12,13,14$ resp. per $\mathfrak{A}, \mathfrak{B}, \mathfrak{C}$; similiterque per eosdem numeros aequatt. $13,15,16$, et $14,16,17$, addantur terna producta, diuidaturque summa per $D m n^{\prime}$, scripto pro $d^{\prime}, D n^{\prime} n^{\prime}$. Tunc prodit

$$
P=a n^{\prime}, R-s=2 b n^{\prime}, U=c n^{\prime}
$$

Simili modo multiplicatis aequationibus 18 , 19 , 20 nec non $19,21,22$ et $20,22,25$ resp. per $\mathfrak{A}^{\prime}, \mathfrak{B B}^{\prime}, \mathfrak{E}^{\prime}$, obtinetur

$$
Q=a^{\prime} n, R+S=2 b^{\prime} n, T=c^{\prime} n \text {. }
$$

Hinc habetur concursso Tentra: Numeri a, $2 b, c$ proportionales sunt numeris $P, R-$ $\mathbf{I}$ 
$S, U$, positaque illonum ratione ad hos $v t x$ ad $n^{\prime}$, erit $n^{\prime}$ radix quadrata ex $\frac{d^{i}}{D^{j}}$ similiterque numeri $a^{\prime}, 2 b^{\prime}, c^{\prime}$ ad $Q, R+S^{\prime}, T$ eandem rationem habent, quate si pomitur csse vt 1 ad $n_{k}$ erit $n$ radix quadrato ex $\frac{d}{D}$

Ceterum quantitates $n, n^{\prime}$ radices vel posiLinae vel negatiuae $e^{2} \times \frac{d}{d}, \stackrel{d}{D}$ esse possunt, vnde distinctionem petimus, quate primo aspertu sterilis videbitur, sed cuius ssus in sequentibus sufficienter apparebit. Scilicet dicemus, in trausformatione formae $F$ in $/ f^{t}$ formam $f$ arcipt directe quando $n$ est positiua, inuerse quando $n$ negatiua ; similiterque $f^{t}$ accipi directe vel inuerse prout $n^{l}$ pasitiua vel negatiua. Accedente autem conditione vt $k$ sit $=1$, forma $F$ ith ex virtque forma $f, f^{\prime}$ directe composita, inl ex viraque inuerse vel ex $f$ directe et ex f interse, wh ex $f$ inuerse et ex $f$ directe dicentur, prout wh $n, n^{\prime}$ ambae sumt positiuat, rel ambat negatinate, vel prior positiua posterior neatiua, vel prior negatiua posterior positiua. Ceterum quisque facile intelliget, has relationes ab ordine cito forthae $f, f^{\prime}$ collocantur ( vid. annot prima ad art. praes.) non pendere.

Porro obseruamus, diuisorem maxirnum communem numerorum $P, Q, R, S, T, U$ juta $k$ metiri numeros $m n^{\prime}, m^{\prime} n$, vi ex valonitus suptis stabilitts nanitestum est) adeoque yuadra-

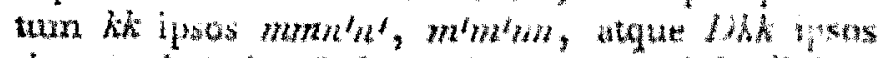
dimn, dm'mt. Sed et vice versa quiuis diuisor, 
communis ipsorum $m n^{\prime}, m^{\prime} n$ metietur ipsum $k_{4}$ Sit enim $e$ talis diuisor qui manifesto etiam numeros $a n^{\prime}, 2 b n^{\prime}, c n^{\prime}, a^{\prime} n, 2 b^{\prime} n, c^{\prime} n$ metietur, $\dot{\mathrm{x}}_{n}$ e. numeros $P, R-S, U, Q, R+S, T$ et proin etiam insos $2 R$ et $2 S$. Iam si $\frac{2 R}{e}$ esset numerus impar, etiam ${ }_{2}^{2} S$ impar esse deberet (quoniam summa et differentia sunt pares) adeoque etiam productum impar. Hoc autem productum fit $=$ $\frac{4}{e e}\left(b^{\prime} b^{\prime} n n-b b n^{\prime} n^{\prime}\right)={ }^{4}\left(d^{\prime} n n+a^{\prime} c^{\prime} n n-d n^{\prime} n^{k}\right.$ $\left.-a c n^{\prime} n^{\prime}\right)={ }_{e e}^{4}\left(a^{\prime} c^{\prime} n n-a c n^{\prime} n^{\prime}\right)$ adeoque par, quia $c$ ipsos $a^{\prime} n_{2} c^{\prime} n_{4} a n^{\prime}, c n^{\prime}$ metitur. Quare $\frac{2 R}{b}$ necessario erit par, et proin $k$ nec non $s$ per diuisibilis. Quoniam igitur $e$ ommes $\operatorname{sex} P, Q, R$, $s, T, U$ metitrx, metietur eliam ipsorum diuisorem communem maximurn k. Q. E. D. - Hinc concluditur $k$ esse diuisorem cornmunerm maxirxum numerorum $m n^{\prime}, m^{\prime} n$; xnde facile perspicietur, Dkk fore diutsorem communem maximum numerorum $d m^{\prime} m^{\prime}, d^{\prime} m m$. Quae est coscursio QVirita. Patet itaque, quoties $F$ ex $f$ et $f^{\prime}$ composita sit, $D$ fore diuisorem communem maximum, numerorum $d m^{\prime} m^{l}, d m^{\prime} m$, et vice versa; quae propritas etiam tamquam defiritio formae compositae aloptari potuisset. Forma igitur composita e formis $f, f^{\prime}$ determinantem maximum yossibilem inter omnes formas in productum off transformabiles habet.

Antequan ylterius progredi possimus, ante omnia valorem ipsius $\Delta$ accuratius definire oportet, 
quem quidem ostendimus esse $=\sqrt{ } d d^{\prime}=$ $\sqrt{ } D D n n n^{\prime} n^{\prime}$, sed cuius signum hinc nondum determinatur. Ad hunc finem ex sequ. fundamentalibus $1-11$ eruimus $D P Q=\Delta a a^{\prime}$ (quae aequ. obtinetur ex $5.6-1.11$ ), adeoque $D a a^{i} n n^{\prime}=$ $\Delta a a^{\prime}$, vnde, nisi aliquis numerorum $a, a^{\prime}$ est $=$ 0 , fit $\Delta=D n n$. Sed prorsus simili modo ex aequatt. fundd. octo aliae deduci possunt in quibus ad laeuam $D_{n n}$ ad dextram $A$ multiplicati habeantur per $2 a b^{\prime}, a c^{\prime}, 2 b a^{\prime}, 4 b b^{\prime}, a b c^{\prime}, c a^{\prime}$, $\left.2 c b^{\prime}, c c^{*}\right)$, vnde facile concluditur propterea quod neque omnes $a, 2 b, c$, neque omnes $a^{\prime}$, $2 b^{\prime}, c^{\prime}$ possunt esse $=0$, in omnibus casibus fieri $\Delta=D n n^{\prime}$, adeoque $\Delta$ idem signum habere vt $D, d, d^{\prime}$ vel oppositum, prout $n, w^{\prime}$ eadem signa habeant vel diuersa.

Porro abseruamus, numeros $a a^{\prime}, 2 a^{b^{\prime}}, a c^{\prime}$, $2 b a^{\prime}, 4 b b^{\prime}, 2 b^{\prime}, c a^{\prime}, 2 c b^{\prime}, c c^{\prime}, 2 b b^{\prime}+2 \Delta, 2 b b^{\prime}-$ $2 \mathrm{~s}$ omnes per $\mathrm{mm}^{\prime}$ diuisibiles esse. De nouem prioribus hoc per se manifestum est, de duolus reliquis autem simili modo demonstruri potest vt antea ostendimus $R$ et $S$ per odiuisibiles esseScilicet patet, $4 b b+4 d$ et $4 b b^{\prime}-43$ per $m m^{\prime}$ diuisibiles esse (quoniam $4.2=\sqrt{26}$ dill $^{\prime}$ atque $4 d$ per $m m$, $4 d^{\prime}$ per $m^{\prime} m^{\prime}$ diuisibilis, aleoque $16 d^{\prime \prime}$ per $\mathrm{mmm} / \mathrm{m}^{\prime}$ et 42 per $\mathrm{mm}^{\prime}$ ) et differentiam quotientium parem; productum ex quotientibus facile demonstratur esse par, rnde vterque quotiens par, et $2 b b^{\prime}+24,2 b b^{\prime}-24$ per mmt diuisibiles.

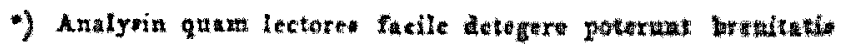
wark aupprimere oportet 
Tam ex vndecim aequationibus fundamentalibus facile deducuntur sex sequentes:

$A P P=a a^{\prime} q^{\prime} q^{\prime}-2 a b^{\prime} q q^{\prime}+a c^{\prime} q q$

$A Q Q=a a^{\prime} q^{\prime \prime} q^{\prime \prime}-2 b a^{\prime} q q^{\prime \prime}+c a^{\prime} q q$

$A R R=a a^{\prime} q^{\prime \prime} q^{\prime \prime \prime}-2\left(b b^{\prime}+\Delta\right) q q^{\prime \prime}+c c^{\prime} q q$

$A S S=a c^{\prime} q^{\prime \prime} q^{\prime \prime}-2\left(b b^{\prime}-\Delta\right) q^{\prime} q^{\prime \prime}+c q^{\prime} q^{\prime} q^{\prime}$

$A T T=a c^{\prime} q^{\prime \prime \prime} q^{\prime \prime \prime}-2 b c^{\prime} q^{\prime} q^{\prime \prime \prime}+c c^{\prime} q^{\prime} q^{\prime}$

$A U U=c a^{\prime} q^{\prime \prime \prime} q^{\prime \prime \prime}-2 \varepsilon^{\prime \prime} q^{\prime \prime} q^{\prime \prime \prime}+c c^{\prime} q^{\prime \prime} q^{\prime \prime}$

Hinc sequitur, omnes $A P P$; $A Q Q$ etc. diuisibiles esse per $\mathrm{mm}^{\prime}$, vade facile deriuatur, quoniam $k k$ diuisor communis maximus numerorum $P P, Q Q, R R$ etc., etiam $A k \bar{k}$ per $m m^{\prime}$ diuisibilem esse. Substitutis autern pro $a, a^{b}, c, a_{2}^{\prime}, 2 b^{\prime}$, $c^{\prime}$ valoribus suis $\frac{p}{n^{\prime}}$ etc. siue $\frac{x}{n^{\prime}}\left(p q^{\prime}-q p^{\prime}\right)$ etc., transibunt in sex alias aequationes, in quibus ad dextram habebuntur producta ex quantitate $\frac{1}{m^{\prime} n^{\prime}}\left(q^{\prime} q^{\prime \prime}-q q^{\prime \prime \prime}\right)$ in $P P, Q Q, R R$ etc. Calculum facillinum lectoribus relinquimus. Hinc sequitur (quoniam omnes $P P, Q Q$ etc. esse $=0$ nequeunt) $A n n^{\prime}=q^{\prime} q^{\prime \prime}-q q^{\prime \prime \prime}$.

Simili modo ex aequationibus fundamentalibus deriuantur sex aliae aequationes, a praecedeutibus in eo tantummodo discrepantes, quod pro $A$ virque habetur $C$ et pro $q, q^{\prime}, q^{\prime \prime}, q^{\prime \prime \prime}$ resp. $p, p_{s}^{\prime} p^{\prime \prime}, p^{\prime \prime \prime}$, quas ipsas breuitatis caussa non adncribinus. Hinc eodern modo sequitur, $C k k$ per $m m^{\prime}$ diuisibilem esse atque $C n n^{\prime}=p^{\prime} p^{\prime \prime}-p p^{\prime \prime \prime}$.

Denique ex eodem fonte petuntur sex aequationes hae: 
$B P P=-a a^{\prime} p^{\prime} q^{\prime}+a b^{\prime}\left(p q^{\prime}+q p^{\prime}\right)-a c p q$ $B \cdot Q=-a a^{\prime} p^{\prime \prime} q^{\prime \prime}+b a^{\prime}\left(f q^{\prime \prime}+q p^{\prime \prime}\right)-c a^{\prime} p q$ $B R R=-a a^{\prime} p^{\prime \prime \prime} q^{\prime \prime \prime}+\left(b b^{\prime}+\Delta\right)\left(p q^{\prime \prime \prime}+q p^{\prime \prime \prime}\right)-c c^{\prime} p q$ $B S S=-a c^{\prime} p^{\prime \prime} q^{\prime \prime}+\left(b b^{\prime}-\Delta\right)\left(p^{\prime} q^{\prime \prime}+q q^{\prime \prime}\right)-c a^{2} p q^{4}$ $B T T=-a c^{\prime \prime \prime} q^{\prime \prime \prime}+b c^{\prime \prime}\left(p^{\prime} q^{\prime \prime \prime}+q^{\prime \prime} q^{\prime \prime \prime}\right)-c q^{\prime \prime} q^{\prime}$ $B U U=-c a^{\prime \prime} p^{\prime \prime \prime} q^{\prime \prime \prime}+\operatorname{cir}^{\prime}\left(p^{\prime \prime} q^{\prime \prime \prime}+q^{\prime \prime} y^{\prime \prime \prime}\right)-c^{2} q^{\prime \prime} q^{\prime \prime}$.

vade perinde vt ante concluditur, $2 \not k k$ diuisibilem esse per $m m^{\prime}$ atque $a b n n^{\prime}=p q^{\prime \prime \prime}+q p^{\text {in }}$ $-p^{\prime} q^{\prime \prime}-q^{\prime} p^{\prime \prime}$

Quoniam itaque $A k k, 2 B k k, C k k$ per $\mathrm{mm} k$ sunt diuisibiles, tacile perspicietur, etiam $M k k$ per $m m^{\prime}$ diuisibilem esse debere. Ex aequationibus fundamentalibus autem colligitur, $M$ metiri ipsos $a a^{\prime}, 2 a b^{\prime}, a c^{\prime}, 2 b a^{\prime}, 4 b b^{\prime}, 2 b c^{\prime}, c a^{\prime}, a c b$; $c c^{\prime}$, adeoque etiam ipsos $a m^{\prime}, \mathrm{abm}^{\prime}, \mathrm{cm}^{\prime}$ (quï sunt diuisores comm. max. thium primorum mediorum et vlitinorum resp.); denique etiam i issum $m h^{\prime}$ qui est horum diu. comm. max. Ithe patet, in eo casta rbi forma $k$ ex formis $f, f$ composita est siue $k=0$, necessario esse $M I=m m m^{\prime}$ Quae est concitisio gronts.

Si diu comm. max, mumerorum $A, B, C$ est $\mathfrak{M}$, hic erit vel $=M$ (quando forma $I^{\circ}$ est proprie primitiua vel ex proprie primitina deriuata) vel $=\frac{x}{2} M$ (quando $f$ est forma improjrie primitiua vel ex improprie pirn. deriuata); similiter designando diuisores comm. max. numerorum $a, b, c ; a, b, c^{z}$ resp. per $m, m^{\prime}$ erit $m$ vel $=m$ vel $m$, et $m^{\prime}$ vel $=m^{\prime}$ vel $=m^{\prime}$. Iam patet, mm metir ipsum $d, \mathrm{~m}^{\prime} \mathrm{n}^{\prime}$ ipsum $d^{\prime}$, adeoque $\mathrm{mmm} \mathrm{m}^{\prime}$ 
ipsum $d d^{\prime}$ siue $\Delta \Delta$, et $\mathrm{mm}$ ipsum $\Delta$. Hinc ex sex vltimis aequationibus pro $B P P$ etc. sequitur, $\mathrm{mm}^{\prime}$ metiri ipsum $B k k$, adeoque : quum etiam ipsos Alk, Ckk metiatur) etiam insum $\mathfrak{n}$ Rkk. Quoties igitur $F$ ex $f, f^{\prime}$ composita est, metietur $\mathrm{mm}^{\prime}$ ipsum 5t. Quando itnque in hoc casu traque $f, f^{\prime}$ est proprie primitiva vel ex proprie primitiua deriuata siue $\mathrm{mm}^{\prime}=m m^{\prime}=M$, erit $\mathfrak{M}=M$, siue $F$ similis' forma. Quando vero, in eadem suppositione, aut vtràque $f$, $f^{\prime}$ aut alterutra saltem est improprie primitiua vel ex improprie primitiua deriata, e. g. forma $f$; ex aequationibus fundamentalibus sequitur, $a a^{\prime}, 2 a b$, $a c^{\prime}, b a^{\prime}, 2 b b^{\prime}, b c^{\prime}, c a^{\prime}, 2 b^{\prime}, c c^{\prime}$ per 90 diuisibiles esse adeoque etiam $\mathrm{cm}^{\prime}, \mathrm{bm}^{\prime} \mathrm{cm}^{\prime}$ et hinc quoque $\mathrm{mm} \mathrm{m}^{\prime}=\frac{r}{2} \mathrm{~mm}^{\prime}=\frac{x}{2} M$; nde necessario in hoc casu erit $59=\frac{\pi}{2} M$, siue etiam for ma $F$ vel impr. prim. vel ex impr. prim. deriuata. Quae elficiunt conclrstowe. sLXTAM.

Tandem obseruamus, si nouem aequationes $a n^{\prime}=P, 2^{i} n^{\prime}=R-s, \mathrm{cn}^{\prime}=U, a^{\prime} n=$ $Q, 2 b^{\prime}=R+s, c_{n}^{\prime}=I, A^{\prime} n^{\prime}=q^{\prime} q^{\prime \prime}-$ $q q^{\prime \prime \prime}, 2 B n n^{\prime}=p q^{\prime \prime \prime}+q p^{\prime \prime \prime}-p^{\prime \prime} q^{\prime \prime}-q q^{\prime \prime}, C m m^{\prime}$ $=p^{\prime} p^{\prime \prime}-p p^{\prime \prime \prime}$ (quas, quoniain in sequentiblis saepius ad ipsas reuenire oportebit, per st designabimus) locum habere stapponantur, spectatis alleo ipsis $n, n^{\prime}$ tanquam incugnitis, quarum tamen neutra $=0$ : per substitutionem facile confirmari, etiam aequationes Jundamentales $1-9$ necessario veras esse siue formam $(A, B, C)$ per substitutionem $p, p^{\prime}, p^{\prime \prime}, p^{\prime \prime \prime} ; q, q^{\prime}, q^{\prime \prime}, q^{\prime \prime \prime}$ in productum e formis $(a, b, c)\left(a^{\prime}, b^{\prime}, c^{\prime}\right)$ transire; praetereaque esse $b b-a c=n t h(B B-\angle A C)$ 
$b^{\prime} b^{\prime}-a^{\prime} c^{\prime}=n^{\prime} n^{\prime}(B B-A C)$. Calculum quem hic apponere nimis prolixum foret lectorum industriae committimus.

236. Problema. Propositis duabus formis quarum determinantes aut aequales sunt aut saltem rationem quadratorum inter se habent: inuenire formaim ex illis compositam.

Sol. Sint formae componendae $(a, b, c)$... $f,\left(a^{\prime}, b^{\prime}, c^{\prime}\right) \ldots f^{\prime}$; harum determinantes $d$ $d^{\prime}$; diuisores communes maximi numerorum $a_{\text {; }}$ $\mathrm{a} b, c ; a^{\prime}, 2 b^{\prime}, c^{\prime}$ resp. $m, m^{\prime}$; diuisor comm. maximus numerorumi $d^{\prime} m^{\prime} m^{\prime}, d^{\prime} m m$ eodem signo vt $d, d^{l}$ affectus $D$. Tunc $\frac{d m^{\prime} m^{\prime}}{D}, \frac{d^{\prime} m m}{D^{-}}$erunt numeri positiui inter se primi ipsorumque productum; quadratum; quare ipsi erunt quadrata art. 21). Hinc $\checkmark \frac{d}{D}, \checkmark \frac{d^{\prime}}{D}$ erunt quantitates rationales quas ponemus $=n, n^{\prime}$, et quidem accipiemus pro $n$ valorem positiuum vel negatiuum, prout forma $f$ in compositionem vel directe vel innuerse ingredi debet, similiterque signum ipsius $n$ ex ratione qua $f$ in compositionem ingredi debet determinabimus. Erunt itaque $m n^{\prime}, m^{\prime} r a$ numeri untegri inter se primi, $n$ et $n^{\prime}$ autem etiam fractiones esse possunt. His ita factis, obseruamus, $a n^{\prime}, c n^{\prime}, a^{\prime} n, c^{\prime} n, b n^{\prime}+b^{\prime} n, b n^{\prime}-b^{\prime} r$ esse integros, quod de quatuor prioribus per se manifestum est (quum $a n^{\prime}=\frac{a}{m} m n^{\prime}$ etc.) $;$ de duobus reliquis eodem modo probatur vt in art. praec. demonstratum fuit $R$ et $S$ per $e$ diuisibiles esse. 


\section{$-351-$}

Iam accipiantur quatuor numeri integri $\mathbb{2}$ $\mathfrak{Q}^{\prime}, \mathfrak{Q}^{\prime \prime}, \mathfrak{Q}^{\prime \prime \prime}$ ad libitum, ea sola conditione vt quatuor quantitates in aequatione sequente (I) ad laeuam positae non omnes simul $=0$ fiant, ponaturque .... (I)

$\mathfrak{D}^{\prime} a n^{\prime}+\mathfrak{D}^{\prime \prime} a^{\prime \prime} n+\mathfrak{D}^{\prime \prime \prime}\left(b n^{\prime}+b^{\prime} n\right)=k q$ $-2 a n^{\prime}+\mathfrak{Q}^{\prime \prime \prime} c^{\prime} n-\mathfrak{Q}^{\prime \prime}\left(b n^{\prime}-b b_{n}\right)={ }^{\prime \prime} q^{\prime}$ $\mathfrak{Q}^{\prime \prime \prime} c n^{\prime}-\mathfrak{D} a^{\prime} n+\mathfrak{D} !\left(b n^{\prime}-b^{\prime} n\right)=\mu q^{\prime \prime}$ $-\mathfrak{Q}^{\prime \prime} c n^{\prime}-\mathfrak{Q}^{\prime} c^{\prime} n-\mathfrak{Q}\left(b n^{\prime}+b^{\prime} n\right)=\mu q^{\prime \prime \prime}$

ita vt' $q, q^{\prime}, q^{\prime \prime}, q^{\prime \prime \prime}$ fiant integri diuisorem communem non habentes, quod obtinetur aceipiendo pro $\mu$ diuisorem communem naximum quatuor numerorum qui in his aequationibus sunt ad laeuam. Tunc igitur per art 40 inueniri poterunt quatuor numeri integri $\mathfrak{Y}, \mathfrak{P}$, $\mathfrak{Y}^{\prime \prime}, \mathfrak{P}$

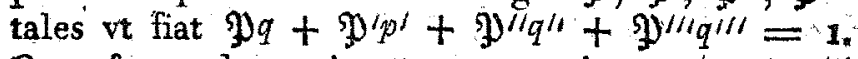
Quo facto determinentur numeri $p, p^{\prime}, p^{\prime \prime}, p^{\prime \prime \prime}$ per aequationes sequentes ... (II):

$\mathfrak{Y}^{\prime} a n^{\prime}+\mathfrak{Y}^{\prime} a^{\prime} n+\mathfrak{y}^{\prime \prime \prime}\left(b n^{\prime}+b^{\prime} n^{\prime}\right)=p$ $-\mathfrak{P} a n^{\prime}+\mathfrak{P}^{\prime \prime \prime} c^{\prime} n-\mathfrak{P}^{\prime \prime \prime}\left(b n^{\prime}-b^{\prime} n\right)=p^{\prime}$ פู ${ }^{\prime \prime} c n^{\prime}-\mathfrak{D} a^{\prime} n+\mathfrak{D}^{\prime}\left(b n^{\prime}-b^{\prime} n\right)=p^{\prime \prime}$ - $\mathfrak{D}^{\prime \prime} c n^{\prime}-\mathfrak{Y}^{\prime} c^{\prime} n-\mathfrak{Y}\left(b n^{\prime}+b^{\prime} n=p^{\prime \prime \prime}\right.$

Tandem ponatur $q^{\prime} q^{\prime \prime}-q q^{\prime \prime \prime}=A n n^{\prime}, p q^{\prime \prime \prime}$ $+q p^{\prime \prime \prime}-p^{\prime} q^{\prime \prime}-q^{\prime \prime} p^{\prime \prime}=2{ }_{2}{ }^{2} n^{\prime}, p^{\prime} p^{\prime \prime}-p p^{\prime \prime \prime}=$ Cnn'. Tunc $A, B, C$ erunt numeri integri formaque $(A, B, C) \ldots F$ ex formis $f, f$ composita.

Dem. I. Ex aequatt. I et II nullo negotio confirmantur sequentes quatuor aequationes... (III):
$0=q^{\prime} c n^{\prime}-q^{\prime \prime} c^{\prime} n-q^{\prime \prime \prime}\left(b n^{l}-b^{\prime} n\right)$
$0 q_{c n^{\prime}}+q^{\prime \prime \prime} a^{\prime} n-q^{\prime \prime}\left(b^{\prime}+b^{\prime} n\right)$ 


$$
\begin{aligned}
& 0=q^{\prime \prime \prime} a n^{\prime}+q_{c}^{\prime} n-q^{\prime}\left(b n^{\prime}+b b^{\prime} n\right) \\
& 0=q^{\prime \prime} a n^{\prime}-q^{\prime} a^{\prime} n-q\left(b n^{\prime}-b^{\prime} n\right)
\end{aligned}
$$

II. Iam ponamus, numeros integros $\mathfrak{Z}, \mathfrak{2}$ $\left.\mathfrak{E}, \mathfrak{U}^{\prime}, \mathfrak{B}^{\prime}, \mathfrak{C}, \mathfrak{N}\right), \mathfrak{N}^{\prime}$ ita determinatos esse vt fat

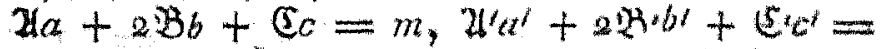
$m^{\prime}, \mathfrak{N} m^{\prime} n+\mathfrak{N}^{\prime} m n^{\prime}=1$, "lunc erit $\mathrm{Ham}^{\prime} \mathrm{n}^{\prime}+$

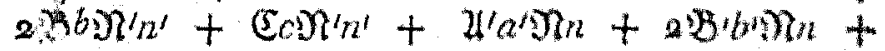
$\mathfrak{C}^{\prime} \mathfrak{N}_{n}=1$. Linc atque ex aequatt. (IIl) ta* cile confirmatur, si statuatur

$$
\begin{aligned}
& -q^{\prime} \mathfrak{M} \mathfrak{M}^{\prime}-q^{\prime} / \mathfrak{M} \mathfrak{M}-q^{\prime \prime \prime} \mathfrak{R N}^{\prime}+\mathfrak{B}(\mathfrak{M})=\mathfrak{q} \\
& \left.q^{2} \mathfrak{M}^{\prime}-q^{\prime \prime}(\mathfrak{C})+q^{\prime \prime}(\mathfrak{B D})^{\prime}-\mathfrak{B} \mathfrak{M}\right)=q^{\prime}
\end{aligned}
$$

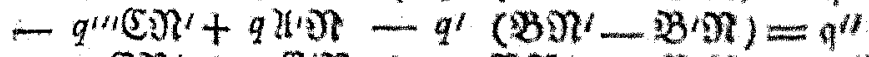

$$
\begin{aligned}
& q^{\prime \prime}\left(\mathbb{N}^{\prime}+q\left(\mathbb{C} \mathfrak{M}+q\left(\mathfrak{D N}+\mathfrak{B N}^{\prime}\right)=q^{\prime 4}\right.\right. \\
& \text { fore .... (IV) }
\end{aligned}
$$$$
\eta^{\prime} a n^{\prime}+q^{\prime \prime} a^{\prime} n+q^{\prime \prime \prime}\left(b n^{\prime}+b h_{n}\right)=q
$$$$
-q a n^{\prime}+q^{\prime \prime \prime} c^{\prime} n-q^{\prime \prime}\left(b_{n}^{\prime}-b_{n}=q^{\prime}\right.
$$$$
q^{\prime \prime \prime} c n^{\prime}-q a^{\prime} n+q^{\prime}\left(b^{\prime}-b n^{\prime} n\right)=q^{\prime \prime}
$$$$
-\mathfrak{q}^{\prime \prime} c n^{\prime}-q^{\prime} c^{\prime n}-\mathfrak{q}\left(b_{n^{\prime}}+b^{\prime} n\right)=q^{\prime \prime \prime}
$$

Qunties $\mu=1$, hae arquationes won sunt necessariat, sad ipsarum loro asquationes (I), qubus omnino analogete sumt, retineri poscunt. Quodsi nume ex aequatt. If, iv valores igsersum

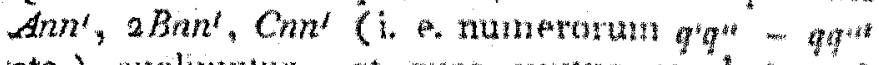
etc.) euoliuntuxi, et quase mutuo se destrunate delentur: imuenietur, singulorum pattes es. se vel producta ex integris in $n n^{\prime}$, vel ex in-

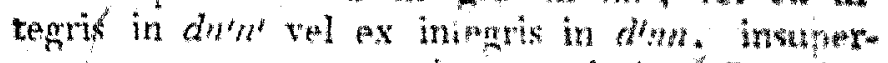

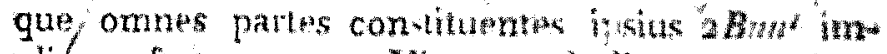
plicare factorem \&. Hline concluditur (quonian 


\section{- $553-$}

$d n^{\prime} n^{\prime}=d^{\prime} n n$, et proin $\frac{d n^{\prime} n^{\prime}}{n n^{\prime}}=\frac{d^{\prime} n^{\circ} n}{n n^{\prime}}=\sqrt{ } d d^{\prime}$ sunt integri), $A, B, C$ esse numeros integros. Q. E. $P$.

III. Substituendo ex aequatt. (II) valores ipsorum $p, p^{\prime}, p^{\prime \prime}, p^{\prime \prime \prime}$, facile comprobatur adiumento aequat. (III) et huius $\mathfrak{P} q+\mathfrak{P}^{\prime} q^{\prime}+\mathfrak{D}^{\prime \prime} q^{\prime \prime}$ $+g^{\prime \prime \prime} q^{\prime \prime \prime}=1$, esse $p q^{\prime}-q p^{\prime}=a n^{\prime}, p q^{\prime \prime \prime}-$ $q p^{\prime \prime \prime}-p^{\prime} q^{\prime \prime}+q^{\prime} p^{\prime \prime}=2 b n^{\prime}, p^{\prime \prime} q^{\prime \prime \prime}-q^{\prime \prime} p^{\prime \prime \prime}=$ $c n^{\prime}, p q^{\prime \prime}-q p^{\prime \prime}=a^{\prime \prime}, p q^{\prime \prime \prime}-q p^{\prime \prime \prime}+p^{\prime \prime} q^{\prime \prime}-$ $q^{\prime} p^{\prime \prime}=2 b^{\prime} n, p^{\prime} q^{\prime \prime \prime}-q^{\prime \prime} p^{\prime \prime}=c^{\prime} n$, quae aequationes identicae sunt cum sex prioribus $(\Omega)$ art. praec.; tres reliquae autem iam per hyp. locum habent. Quare (ifid. sub fin.) forma $F$ transibit in $f^{\prime \prime}$ per substututionem $p, p^{\prime}, p^{\prime \prime}, p^{\prime \prime \prime} ; q, q^{\prime}, q^{\prime \prime}$, $q^{\prime \prime \prime}$; ipsiusque determinans erit $=D$, siue aequalis diuis, comm, max. numerorum $a m^{\prime} m^{\prime}, d^{\prime} m m$, quamobrem per concl. quartam art. praec. $F^{\prime}$ ex $f, f^{\prime}$ composita erit. $Q . E . S$. Denique facile perspicietur, $F$ ex $f, f^{\prime}$ ita compositam esse vt praescriptum sit, quum signa quantitatum $n, n^{\prime}$ iam ab initio rite sint determinata.

23\%. Tinonema. Si forma $F$ in productum e duatus furmis $f$, frest transformabilis, atque forma foforman fumplicat: $F$ atam in productum e formis $f, f^{\prime \prime}$ transformabilis erit.

Dem. Retineantur pro formis $F, f, f$ omnia signa art. 375 ; forma $f^{\prime \prime}$ sit $=\left(a^{\prime \prime}, b^{\prime \prime}, c^{\prime \prime}\right)$, transeatque $f^{\prime}$ in $f^{\prime \prime}$ per stustitutionem $\alpha, \sigma^{2}, \gamma, \delta$. 'Tunc nullo negotio perspicietur, $F$ traxsire in $f^{\prime \prime}$ per substitutioneñ $\alpha p+\gamma p^{\prime}, p+\delta p^{\prime} \alpha p^{\prime \prime}+$ 
op $p^{\prime \prime \prime}, 6 p^{\prime \prime}+\delta p^{\prime \prime \prime} ; \alpha q+\gamma q^{\prime}, q q+\delta q^{\prime}, \alpha q^{\prime \prime}+\gamma q^{\prime \prime \prime}$, Eq" $+\delta q^{\prime \prime \prime}$. Q. E. D.

Positis breuitatis caussa coefficientibus ap +

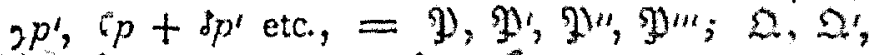

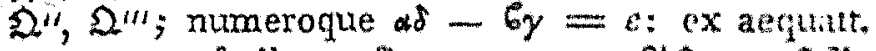

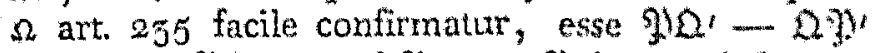

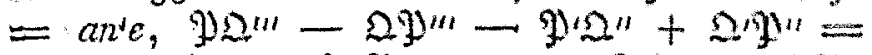

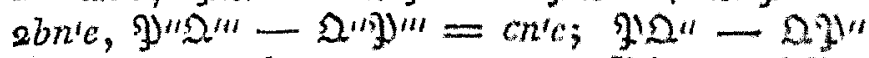
$=\alpha a^{\prime} n+2 \alpha^{\prime} b^{\prime} n+2 y^{\prime} n=a^{\prime \prime} n, 9 \Omega^{\prime \prime}-2 y^{\prime \prime}$

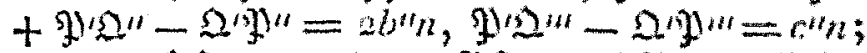

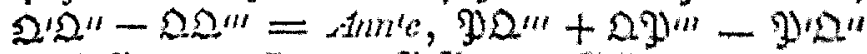

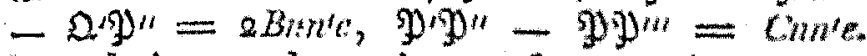
Iam designato determinante formae $f^{\prime \prime}$ per $d "$, erit $e$ radix quadrata ex $\frac{d^{\prime \prime}}{d^{\prime}}$, et quidem positiua vel negatiua, prout forma $f^{\prime}$ formam $f^{\prime \prime}$, vel proprie vel improprie implicat. Quare $n^{\prime} c$ erit radix quadrata ex $\frac{d^{\prime \prime}}{D^{j}}$ vide patet, nouem aequationes praecedentes aequationibus $\Omega$ art. 235 prorus analogas esse, formamque $f$ in transformatione formae $F$ in $f^{\prime \prime}$ eockm modo accipi, it in tratusformatione forme $F$ in $f f f^{\prime}$; forman $f^{\prime \prime}$ tero in illa vel eodem modo vt $f^{\prime}$ in hac, vel opposito, prout $f^{\prime}$ ipsam $f^{\prime \prime}$ proprie implicet vel inproprie.

238. Trrerema, Si forma $f$ swb furmo $F$ est contenta atque in productume formis $f: f^{\prime}$ tratsjoremmabilis: etian forma $F^{2}$ in idem productum transform mabilis erit.

Dem. Retentis pro formis $F, f, f$ isdem signis vt supra et supponendo formam $F$ trans- 
ire in $F$ per substitutionem $\alpha, \sigma, \ddot{y}, d$, facile perspicietur, Fi per substitutionem $\alpha p+6 q_{3}$ $\psi p^{\prime}+6 q^{\prime}, \alpha p^{\prime \prime}+6 q^{\prime \prime}, \alpha p^{\prime \prime \prime}+6 q^{\prime \prime \prime} ; \gamma p+\delta q, \gamma p^{\prime}$ $+\delta q^{\prime}, \gamma p^{\prime \prime}+\delta q^{\prime \prime}, x p^{\prime \prime \prime}+\delta q^{\prime \prime \prime}$, idem fieri quod $F$ per substitutionem $p, p^{\prime}, p^{\prime \prime}, p^{\prime \prime \prime} ; q, q^{\prime}, q^{\prime \prime}, q^{\prime \prime \prime}$, adeoque $F$ per substitutionem illam transire in $f f^{\circ}$. Q. E. D.

Praeterea per similem calculum $v t$ in art. praec facile confirmatur, ff eodem modo in $f f^{t}$ transformabilern fore vt $F$, quando $F$ ipsam $E$ proprie implicet; quando vero $E$ improprie sub $F^{\prime}$ contenta sit, transformationes formae $F$ in $\mathscr{f}^{f}$ et formae $F^{\prime}$ in $f^{\prime}$ oppositas fore respectu vtriusque formae $f, f$, scilicet quae ex his formis in alteram transformationem directe ingrediatur, in altera accipi inuerse.

Ex combinatione theorematis praesentis cum theor. art praec. obtinemus sequens generalius: Si forma $F$ io productum ffi est transformabilis, atque formae $f, f^{\prime}$ resp. inulizant formas $g, g^{\prime}$, forma $F v u-$ ru sub forma $F^{\prime}$ conterta est: $Q$ in productum $g g^{\prime}$ transformabllis erit. Nam per theor. art. praes. $G$, transformabilis exit in $f f$, hinc per theor. art. praec. in $f_{g^{\prime}}$ et per idem theor. etiam in $g g^{*}$. Torro patet, si omnes tres formae $f, f^{\prime}, G$ formas $g, g^{\prime}, F$ proprie inplicent, $G$ eodem modo in $\mathrm{gg}^{*}$ transformabilem fore respectu formarum $g$, $g^{t}$. vt $F^{t}$ in $f^{\prime}$ respectu formarum $f, f^{\prime} s$ idem euenire, si illae tres implicationes omnes sint impropriae; denique aeque facile determinari potexit, quomodo $G$ in $\mathrm{gg}^{-1}$ transformabilis sit, 


\section{- $356-$}

si ex illis implicationibus aliqua duabus reliquis sit dissimilis.

Si formae $F, f, f^{\prime}$ formis $G, g, g^{\prime}$ resp. sunt aequiualentes, hae eosdem determinantes habebunt vt illae, et quod pro formis $f$, $f^{\prime}$ sunt numeri $m, m^{\prime}$, idem erunt pro formis $g, g^{\prime}$ (art. 161 ). Hinc nullo, negotio per conclus. quartam art, 235 deducitur, in hocce casu $G$ ex $g ; g^{\prime}$ compositam fore, si $F$ ex $f, f^{\prime}$ composita sit, et quidem formam $g$ in compositionem illam eodem modo ingredi vt $f$ in hanc, quando $F$ ipsi $G$ eodem modo aequiualeat vt $f$ ipsi $g$, et contra; similiterque $g^{\prime}$ in compositione priori vel eodem modo vel opposito accipiendam vt $f$ in posteriori, prout aequiualentia formarum $f^{\prime} g^{t}$ aequiualentiae formarum $F, G$ similis sit vel dissimilis.

239. THeOREMA. Si forma $F$ ex formis $f, f^{\prime}$ composita est: quatuis alia forma in productum ff' eodem modo transformabilis vt $F$, izsam F proprie impolicabit.

Dem. Retentis pro $F, f, f^{\prime}$ omribus signis art. 235 , aequationes $\Omega$ etiam hic locum habebunt Ponamus formam $F^{\prime}=\left(A^{\prime}, B^{\prime}, C^{\prime}\right), \mathrm{cu}-$ ius determinans $=D$, transire in productum $f f$ per substitutionem $\mathfrak{p}, p^{\prime}, p^{\prime \prime}, p^{\prime \prime} ; q, q^{\prime}, q^{\prime \prime}, q^{\prime \prime}$ designemusque numeros $p q^{\prime}=q p^{\prime}, p q q^{\prime}-q p^{\prime \prime}$, $p^{\prime \prime \prime}-q p^{\prime \prime \prime}, p^{\prime} q^{\prime \prime}-q^{\prime \prime \prime} p^{\prime \prime} q^{\prime \prime \prime}-q^{\prime \prime \prime \prime}, p^{\prime \prime} q^{\prime \prime \prime}$ $q^{\prime \prime} p^{\prime \prime \prime}$ resp. per $P^{\prime}, Q^{\prime}, R^{\prime}, S^{\prime}, T, U$. Tunc habebuntur nouem aequationes ipsis $\Omega$ omnino similes puta $P=a n, R^{\prime}-S^{\prime}=2 b n^{\prime}, U^{\prime}=$ 


\section{- $357-$}

$\mathrm{cn}^{\prime}, Q^{\prime}=a^{\prime} \mathrm{n}, R^{\prime}+S^{\prime}=\mathrm{2}^{\prime} \mathrm{n}, T^{\prime}=c^{\prime} \mathrm{t}, \mathrm{q}^{\prime} \mathrm{q}^{\prime \prime}$ $-q q^{\prime \prime \prime}=A^{\prime n n}, \mathfrak{p q}^{\prime \prime \prime}+q^{\prime \prime \prime}-\mathfrak{p}^{\prime} q^{\prime \prime}-q^{\prime} \mathfrak{p}^{\prime \prime}=$ $2 B^{\prime} n^{\prime}, p^{\prime} p^{\prime \prime}-p^{\prime \prime \prime}=C^{\prime n n}$, quas per $\Omega^{\prime}$ designabimus. Quantitates $\mathfrak{n}, \mathfrak{n}$ hic erunt radices quadratae ex $\frac{d}{D^{\prime}}, \frac{d^{\prime}}{D^{\prime}}$ et quidem iisdem signis resp. affectae wt $n, n^{\prime}$; si igitur radix quadrata ex $\frac{D}{D^{\prime}}$ positiue accepta (quae erit numerus integer) statuitur $=k$, erit $\mathfrak{n}=k n, \mathfrak{n}^{\prime}=k n^{\prime}$. Hinc et ex aequatt. senis prioribus in $\Omega$ et $\Omega^{\prime}$ manifestum est, fore $P^{\prime}=k P, Q^{\prime}=k Q, R^{i}=k R, S^{\prime}$ $=k S, T^{\prime}=k T, U=k U$. Quare per lemma art. 234 determinari poterunt quatuor numeri integri $\alpha, 6, \gamma, \delta$ tales vt fiat $\alpha p+6 q=p$, , $\gamma$ $+\delta q=q^{j} \alpha p^{\prime}+\varepsilon q^{\prime}=p^{\prime}, \gamma p^{\prime}+\delta q^{\prime}=q^{\prime}$ etc. atque $\alpha \delta-6 y=k$. Substitutis his valoribus ipsorum, $\mathfrak{p}, \mathfrak{q}, \mathfrak{p}^{\prime}, \mathfrak{q}^{\prime}$ etc. in aequatt. tribus vltimis $\Omega^{\prime}$, facile confirmatur adiumento aequationum $\mathfrak{n}=$ $k n, n^{\prime}=k n^{\prime}$ triumque vltimarum $\Omega$, fore $A^{\prime} \alpha$ $+2 B_{\alpha y}^{\prime}+C_{\gamma \gamma}^{\prime}=A, A^{\prime \alpha b}+B^{i}(\alpha \delta+6 \gamma)+$ $C_{y d}=B, A^{\prime \prime g s}+2 B^{\prime o d}+C^{\prime} \delta=C$, quapropter forma $F^{l}$ per substitutionem $\alpha, 6, \gamma, \delta$ (quae propria erit, quoniam $\alpha \delta-6 \delta=k$ est positiuus) transibit in $F$, i. e. forman $F$ proprie implicabit. Q. E. D.

Si itaque $F$ e formis $f, f^{\prime}$ etiam composita est (codem modo vt $F$ ex iisdem), formae $F$; $F$ eundem determinantem habebunt, eruntque adeo proprie aequiualentes. Generalius, si forma $G$ e formis $g, g^{\prime}$ eodem modo composita est vt $F$ ex $f, f$ resp. formaeque $g, g$ insis $f, f$ proprie aequiualent: formae $F, G$ propres aequiualebunt.

\section{z 3}


Quum is casus vbi ambae formae componendae compositionem directe ingrediuntur simplicissimus sit, ad ipsumque reliqui facile reducantur, illum solum in sequentibus contemplabimur, ita vt si forma aliqua simpliciter dicatur e duabus aliis composita, semper subintelligere oporteat, ex vtraque illam proprie esse compositam *). Eadem restrictio valebit, quoties forma in productum e duabus aliis transformabilis dicetur.

240. Trmonema. Si e formis $f, f$ composita est forma $F ;$ ex $F$ et fll forma $\mathfrak{F}$; ex $f, f^{\prime \prime}$ forma $F^{\prime} ;$ ex $F^{\prime}$ et $f^{\prime}$ forma $\mathfrak{Z}^{\prime}$ : format $\mathfrak{F}, \mathfrak{F}^{\prime}$ propret atquiwalentes erunt.

Dem. I. Sit $f=a x x+2 b x y+c y y, f$ $=a^{\prime} x^{\prime} x^{\prime}+2^{\prime \prime} x^{\prime} y^{\prime}+c^{\prime} y^{\prime} y^{\prime \prime}, f^{\prime \prime}=a^{\prime \prime} x^{\prime \prime} x^{\prime \prime}+2 b^{\prime \prime} x^{\prime \prime} y^{\prime \prime}$ $+c^{\prime \prime} y^{\prime \prime} y^{\prime \prime}, F=A X X+2 B X Y+C Y Y, \quad F^{\prime}=$ $\left.A^{\prime} X^{\prime} X^{\prime}+2 B^{\prime} X^{\prime} Y^{\prime}+C Y^{\prime} Y^{\prime}, \mathfrak{F}=21 X X+2 \mathfrak{X} X\right)$

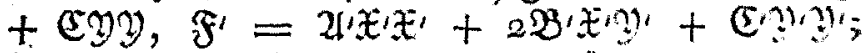
determinantes harum septem formarum resp. $d^{\prime}, d^{\prime}, d^{\prime \prime}, D, D^{\prime}, \mathfrak{D}, D^{\prime}$, qui onues eadem signa et rationem quadratorum inter so habehut. Porro sit $m$ diuisor communis maximus numerorum $a, 3 b, c$, similenque significationem habeant $m^{\prime}, m^{\prime \prime}, M$ relatitu ad formas $f^{\prime \prime} f^{\prime \prime}, \mathrm{F}$. Tum ex concl. 4 art. $235, D$ erit diu. comm. max. numerorum $d m^{\prime} m^{\prime}, d m m$, adeoque $D m^{\prime \prime} m^{\prime \prime}$ diu. comm. max. uumerorum $d n^{\prime} m^{\prime} m^{\prime \prime} m^{\prime \prime}$,

-) Similiter $r$ in compositione tationnm (qune exm compaxs. tione frematum magnam analogiam kaber) aubintehig wolet,

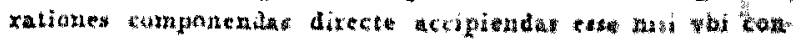
trarium monetur. 
d'mmm ${ }^{\prime \prime} m^{\prime \prime} ; \quad M=m m^{\prime} ;$ D diu. comm, max. num. $D m^{\prime \prime} r^{\prime \prime}, c^{\prime \prime} . M M T$, siue numerorum $D m^{\prime \prime} m^{\prime \prime}$, d" mmm'm'. Iline concluditur, D esse diu, comm.

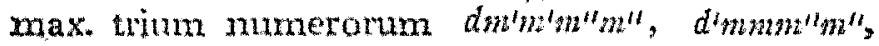
$d^{\prime \prime} m m n^{\prime} n^{\prime} ;$ ex simili autem ratione $\mathbb{D}^{\prime}$ eorundem trium numerorum diuisor communis maximus erit; quare quum $\mathfrak{D}, D^{\prime}$ eadem signa habeant, erit $D=D^{\prime}$, sine formae $\mathscr{F}, \mathscr{F}^{t}$ eundem determinantem habebunt.

II. Iam transeat $F$ in $f f^{\prime}$ per substitutionem $X=p x x^{4}+p^{\prime} x y^{\prime}+p^{\prime \prime} y x^{\prime}+p^{\prime \prime \prime} y y^{\prime}, X=$ $q x x^{t}+q^{\prime} x y^{\prime}+q^{\prime} y x^{\prime}+q^{\prime \prime \prime y y^{\prime},}$ atque $\mathfrak{F}$ in $F f^{\prime \prime}$ per substitutionem $x=x^{\prime \prime}+p^{\prime} X_{y^{\prime \prime}}+p^{\prime \prime} Y_{x^{\prime \prime}}$ $+p^{\prime \prime \prime} Y^{\prime \prime \prime}, y=q X x^{\prime \prime}+q^{\prime \prime} x^{\prime \prime}+q^{\prime \prime} x^{\prime \prime}+$ $q^{\prime \prime \prime} Y^{\prime \prime \prime}$, designenturque radices quadratae posituae ex $\frac{d}{D}, \frac{a^{\prime}}{D}, \frac{D}{D}, \frac{d^{d \prime}}{D}$ per $n, n^{2}, \mathfrak{N}, n^{\prime \prime}$. Tunc per art. 235 habebuntur decem et octo aequationes, quarnm semissjs altera ad transformationem formae $f$ in $l l$ pertinebit, altera ad transformationem formae $\mathcal{F}$ in Ff $^{\prime \prime}$. Prima exit $p q^{l}-q p^{l}=a n^{\prime}$, ad cuins instar facile formaxt poterunt reliquae breuitatis gratia hic omittendae. Ceterum quantitates $n, n^{i}, \mathfrak{n}$, $\mathfrak{n}^{\prime \prime}$ rationales quidem erunt, sed non necessario numeri integri.

III. $\mathrm{Si}$ valores ipsorum $X, Y$ in valoribus ipsoram $\mathcal{X}, Y$ substitumutur, prodit substitutio talis: $\mathfrak{X}=(1) x x^{\prime} x^{\prime \prime}+(2) x x^{\prime} y^{\prime \prime}+(3) x y^{\prime \prime} x^{\prime \prime}$ $+(4) x y^{\prime} y^{\prime \prime}+\left(5 y x^{\prime \prime} x^{\prime \prime}+(6) y x^{\prime} y^{\prime \prime}+(7) y y^{\prime \prime} x^{\prime \prime}\right.$ $+(8) y^{\prime} y^{\prime \prime} ; y=(9) x x^{\prime \prime}+(10) x x^{\prime \prime} y^{\prime \prime}+$ (11) $x y^{\prime \prime} x^{\prime \prime}+(12) x y^{\prime} y^{\prime \prime}+(13) b x^{1} x^{\prime \prime}+(14) y x^{\prime} y^{\prime \prime}$ $+(15) y y^{\prime} x^{\prime \prime}+(16) y y_{z}^{\prime \prime}$, per quam manifesto 
$\left\{\right.$ transibit in productum $f f^{\prime} f^{\prime \prime}$. Coefficiens (1) erit $=p p+q p^{\prime \prime} ;$ valores quindecim reliquorum non apponimus, quippe quos quisque nullo negotio euoluet. Designemus numerun ( 1 ) (10) - (2) (9) per $(1,2)$, xumerum (1) (11) - (3) (9) per $(1,3)$, et generaliter $(g)(8+h)$ - (h) $(8+g)$ per $(g, h)$, supponendo $g, h$ esse integros inaequales intor 1 et 16 quorum maior $h^{*}$ ); hoc. modo omnino viginti et octo signa habehuntur. Iam denotatis radicibus quadratis posisitiuis ex $\frac{d}{\mathfrak{D}}, \frac{d^{\prime}}{\mathfrak{D}}$ per $n, \mathfrak{n}^{\prime}$ (quae erunt $=\mathfrak{n} \mathfrak{N}$, $n(\mathfrak{N})$, eruentur sequentes 28 aequationes: $(x, 2)$ $=a a^{\prime} \mathfrak{n}^{\prime \prime},(1,3)=a a^{\prime \prime} \mathfrak{n}^{\prime},(1,4)^{\prime}=a b^{\prime \prime} \mathfrak{n}^{\prime \prime}+$ $a b^{\prime \prime} \mathfrak{n}^{\prime},(1,5)=a^{\prime} a^{\prime \prime} \mathrm{n},(1,6)=a^{\prime} b m^{\prime \prime}+a^{\prime} b^{\prime \prime} \mathrm{n}$, $(1,7)=a^{\prime \prime} b n^{\prime}+a^{\prime \prime} b^{\prime n},(1,8)=b b^{\prime} n^{\prime \prime}+b b^{\prime \prime} n^{\prime}$ $+b^{\prime} b^{\prime \prime} \mathfrak{n}+D_{m} n^{\prime \prime} \mathfrak{n}^{\prime \prime},(2,3)=a b^{\prime \prime} \mathfrak{n}^{\prime}-a b^{\prime \prime} \mathfrak{n}^{\prime \prime},(2,4)$ $=a c^{\prime \prime} n^{\prime},(2,5)=a^{\prime} b^{\prime \prime}{ }^{*}-a^{\prime} b n^{\prime \prime},(2,6)=$ $a^{\prime} c^{\prime \prime} \mathfrak{n},(2,7)=b b^{\prime \prime} \mathfrak{n}^{\prime}+b^{\prime} b^{\prime \prime} \mathfrak{n}-b b^{\prime} \mathfrak{n}^{\prime \prime}-\mathfrak{D} m m^{\prime} n^{\prime \prime}$, $(2,8)=b c^{\prime \prime} \mathfrak{n}^{\prime}+b^{\prime} c^{\prime \prime n},(5,4)=a c^{\prime \prime} \mathrm{Tt}^{\prime \prime},(5,5)$ $=a^{\prime \prime} b^{\prime} n-a^{\prime \prime} b n^{\prime},(5,6)=b b^{\prime} n^{\prime \prime}+b^{\prime} h^{\prime \prime} n-$

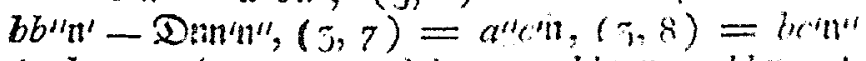
$+b^{\prime \prime} C^{\prime} n,(4,5)=b^{\prime} b^{\prime \prime n}-b^{\prime} n^{\prime \prime}-b b^{\prime \prime} n+$ $\operatorname{Don}^{\prime \prime} n^{\prime \prime},(4,6)=b x^{\prime \prime} n-b x^{\prime \prime n},(4,7)=$ $b^{\prime \prime} c^{\prime} n-b c^{\prime \prime} n^{\prime \prime},(4,8)=c^{\prime} c^{\prime \prime} n,\left(5,6=c t^{\prime} n^{\prime \prime}\right.$, $(5,7)=c a^{\prime \prime} n^{\prime},(5,8)=b^{\prime} n^{\prime \prime}-b^{\prime \prime} c n^{\prime},(6,7)$ $=b^{\prime \prime} c n^{\prime}-b^{\prime} c n^{\prime \prime},(6,8)=c c^{\prime \prime} \|^{\prime},(7,8)=$ $c c^{\prime} \mathfrak{n}^{\prime \prime}$, quas per o designabimus, nouemque aliae: (10) (11) $-(9)(12)=a n^{\prime \prime 2}$, (1) (12) -

-) Yorum signorum signifiratio praesens non ext confurdenda

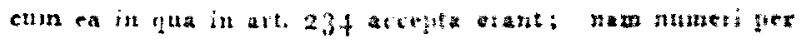

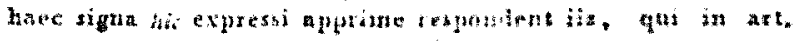

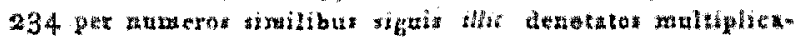
barstur. 
(2) $(11)-(3)(10)+(4)(9)=2 a^{\prime} t^{4 n B},(2)$ (3) $-(1)(4)=a n^{\prime \prime}\left(c_{,}-(9)(16)+(10(15)+\right.$ (11) $(14)-(12)(15)=2 b n(12 a d$, (r) $(16)-$ (2) $(15)-(3)(14)+(4)(13)+(5)(12)-$ (6) $(11)-(7)(10)+(8)(9)=4, b n^{\prime} n^{\prime \prime 3},-$ (1) $(8)+(2)(7)+(3)(6)-(4)(5)=2 b n '$ 'n (14) $(15)-(13)(16)=m^{1114},(5)(16)-$ (6) $(15)-(7)(14)+(8)(13)=2 \mathrm{~cm}^{\prime \prime} \mathrm{u}^{\prime \prime} \mathrm{B}$, (6) $(7)-(5)(8)=\mathrm{cn}^{\prime \prime} \mathrm{C}$, quas designabimus per $\Psi *$ !

IV. Originem omnim harum 37 aequationum deducere nimis prolixum foret: sufficiet quasdam confimauisse, ad quarum instar reliquae haud difficulter demonstrari folerunt.

$$
\begin{aligned}
& \text { I) Habetur }(1,2)=(1)(10)-(2)(9) \\
& =\left(p q^{\prime}-q p^{\prime}\right) p p+\left(p q-q p^{\prime \prime \prime}-p^{\prime \prime}+\right.
\end{aligned}
$$
$\left.q^{\prime \prime} w^{\prime \prime}\right) p q+\left(p^{\prime \prime} q^{\prime \prime \prime}-q^{\prime \prime} p^{\prime \prime \prime}\right) q q=\mathrm{n}^{\prime \prime}\left(A^{\prime \prime} p\right)+$ $2 B p q+C q q)=\mathrm{n}^{\prime \prime}\left(a a^{*}\right.$, quae est aequ. prima.

2) Fit $(i, 5)=(1)(1 i)-(5)(g)=(p q 1$ $\left.-q p^{\prime \prime}\right)\left(p q^{\prime}-q p^{\prime}\right)=a^{u} \mathfrak{W} a n^{t}=a a^{\prime \prime} n^{\prime}$, aequ. secunda.

$$
\text { 3) } \text { Erit }(1,8)=(1)(16)-(8)(9)=
$$
$\left(p q^{\prime}-i p^{\prime}\right) p p^{\prime \prime \prime}+\left(p q^{\prime \prime}-q p^{\prime \prime \prime}\right) p q^{\prime \prime \prime}-\left(p^{\prime \prime} q^{\prime \prime}\right.$ - $\left.q^{\prime \prime} p^{\prime \prime}\right)\left(p^{\prime \prime \prime}+\left(p^{\prime \prime} q^{\prime \prime \prime}-q^{\prime \prime} p^{\prime \prime \prime}\right) q q^{\prime \prime \prime}=n^{\prime \prime}\left(A p p^{\prime \prime \prime}\right.\right.$ $+B\left(p q^{\prime \prime \prime}+q p^{\prime \prime \prime}\right)+C q^{\prime \prime \prime} q^{\prime \prime \prime}+b m\left(m q^{\prime \prime \prime}-\right.$

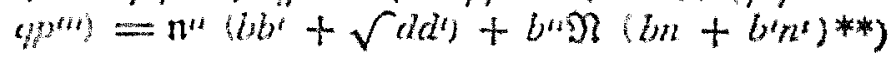

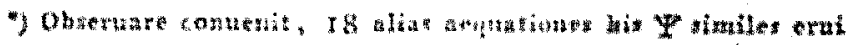

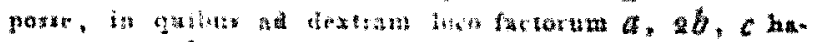

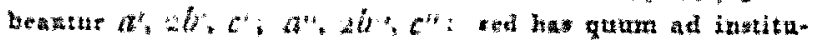

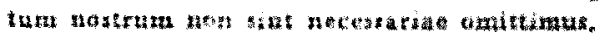

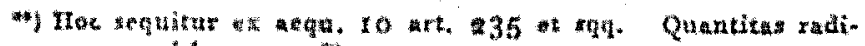

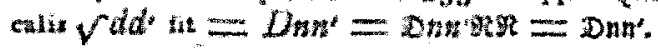




\section{$-\quad 362$}

$=\mathfrak{n}^{\prime \prime} b b^{\prime}+\mathfrak{n}^{\prime} b b^{\prime \prime}+\mathfrak{n} b^{\prime} b^{\prime \prime}+\mathcal{D n} n^{\prime} n^{\prime \prime}$, a equatio octaua in $\Phi$. Aequationes reliquas lectoribus confirmandas linquimus.

V. Ex aequatt. $\Phi$ sequitur, viginti octo numeros $(1,2),(1,3)$ etc. xullum diuisorem communem habere, sequenti moda Primo obseruamus, viginti septem producta e ternis factoribus, quorum primus vel $n$, secundus aliquis numerorum $a^{\prime}, 2 b^{\prime}, c^{\prime}$, tertiusque aliquis umnerorum $a^{\prime \prime}, 2 b^{\prime \prime}, c^{\prime \prime}$; vel primus $n^{\prime}$, secundus aliquis e numeris $a, 2 b, c$, tertius aliqui3 numerorm $a^{\prime \prime}, 2 b^{\prime \prime}, c^{\prime \prime}$; vel denique primus $n^{\prime \prime}$, secundus aliquis mumerorum $a, 2 b, c$ tertiusque aliquis enmeris $a^{\prime}, 2 b^{\prime}, c^{\prime}$ - singula liaec viginti septem producta propter aequatt. $\$$ aequalia esse vel alicui ex viginti octo numeris $(1,2),(1,3)$ etc. vel plurium summae aut differentiae, (e. g. na $a^{\prime \prime}=(1,5)$, $2 n a^{\prime} b^{\prime \prime}=(\mathrm{L}, 6)+(2,5), 4 \mathrm{n} b^{\prime} b^{\prime \prime}=(1,8)+$ $(2,7)+(5,6)+(4,5)$, et sic de reliquis); quamobrem si hi numeri diuisoren communem haberent, hic necessario etiam ommia illa producta metiri deberet. Ilinc vero facile sieducitur adiumento art. 40 et per methodum satepius in praecedentibus adhibitam, omilem diusorem etiam numeros $n m^{\prime} m^{\prime \prime}, n^{\prime} m m^{\prime \prime}, n^{\prime \prime} m m^{\prime}$ metir debre, adeoque horum quadrata quae sunt $\frac{d m^{\prime} m t^{\prime} m m^{\prime \prime} m^{\prime \prime}}{D}, \frac{d^{\prime} m m m^{\prime \prime} m m^{\prime \prime}}{D}, \frac{d^{\prime \prime} m m m^{\prime} m^{\prime}}{D}$ per illius qua. Iratum diuisibilia esse, Q. $E_{.} A_{n}$, quoniam per I trium numeratorum diuisor communis maximus sst $\mathfrak{D}$, adeoque quadrata ipsa diuisorem communem habere nequeunt.

VI. Haec ommia pertinent ad transformationem formae $f$ in $f f^{\prime} f^{\prime \prime} ;$ et ex transformatiouibus 


\section{$-365-$}

formae $F$ in $f f^{\prime}$ formaeque $\gamma$ in $F f^{\prime \prime}$ deducta sunt. Sed prorsus simili modo e transformationibus formae $F^{\prime}$ in $f^{\prime \prime}$ formaeque $F$ in $F f^{\prime}$ deriuabitur transformatio formae $\mathfrak{f}^{\prime}$ in ffifin talis: $\mathfrak{X}^{\prime}=\left(1^{\prime} x x^{\prime} x^{\prime \prime}+(2)^{\prime} x x^{\prime} y^{\prime \prime}+(3)^{\prime} x y^{\prime \prime} x^{\prime \prime}+\right.$ etc, $Y^{\prime}=(0) x x^{\prime} x^{\prime \prime}+(10)^{\prime} x x^{\prime} y^{\prime \prime}+$ etc. (designando omes coefficientes similiter $i t$ in transformatione formae $\mathfrak{F}$ in $f f f^{\prime \prime}$, singulisque distinctionis canssa lineolam affigendo), ex qua perinde $\mathrm{rt}$ ante 28 aequationes ipsis analogae deducentur, quas per designalimus, nouemque aliae ipsis $\Psi$ analogae, quas exprimemus per $\Psi t$. Scilicet denotando $(1)^{\prime}(10)^{\prime}-(2)^{\prime}(9)^{i}$ per $(1,2)^{t},(1)^{\prime}(11)^{k}$ - $(3)^{\prime \prime}(9)^{\prime}$ per $(1,3)^{\prime}$ etc, aequationes $\Phi^{\prime}$ erunt $(1,2)^{\prime}=a a^{\prime} n^{\prime \prime},(1,5)^{\prime}=a b^{\prime} n^{\prime \prime}+a b^{\prime \prime} n^{\prime}$ etc.; aequationes $\Psi^{\prime}$ autem (10)'(II)'-(0)'(ID)t= $a^{\prime} n^{\prime \prime}$ W' etc. (Fuolutionem vberiorem breuitatis gratia lectoribus relinquimus; ceterum periti nowum calculum ne necessarium quidem esse, sed analysin primam per' analogiam facile huc transferri posse inuenient). Quibus ita factis, ex et Q1 statim sequitur $(x, 2)=(1,2)^{x},(1,5)=$ $(1,3)^{\prime},(1,4)^{\prime}=(1,4)^{\prime},(2,3)=(2,3)^{\prime}$ etc.; hinc vero et inde quod omnes $(1,8),(1,5)$, $(s, 3)$ etc. diuisorem communem (per $V$ ) non habent, adiumento lemmatis art 234 concluditur, quatuor numoros integros $\alpha, b, 2, \delta$ ita determinari posse, yt fiat $a(1)^{\prime}+\zeta(9)^{\prime}=$ (1), $a(2)^{4}+6(1)^{\prime \prime}=(2), \alpha(3)^{4}+6(11)^{4}=(3)$ etc; $y(1)^{\prime}+\delta\left(g^{\prime}=(0), \quad g^{\prime}(2)^{\prime}+\delta(10)^{\prime}=(10)\right.$ etc., atque $\alpha \delta-6 y=1$.

VII. ITinc atque substituendo ex tribus aequatt primis $\Psi$ 'valores ipsarum $a \mathfrak{H}, a \mathfrak{B}, a \mathfrak{E}$, 


\section{- $364-$}

et ex tribus aequ. primis $\Psi^{\prime}$ valores ipsorum $a \mathfrak{a}{ }^{\prime}, a^{\mathfrak{B}}{ }^{\prime}, a \mathfrak{C}^{\prime}$ facile confirmatur fore $a\left(\mathfrak{u}_{\alpha \alpha}+\right.$ $\left.2 \mathfrak{B}_{\alpha \gamma}+\mathfrak{E}_{\gamma \gamma}\right)=a \mathfrak{d}, a\left(\mathfrak{U}_{\alpha b}+\mathfrak{B}\left(a \delta+b_{\gamma}\right)+\right.$ $\left.\mathfrak{E}_{\delta^{\prime}}\right)=n \mathcal{H}^{\prime}, a\left(\mathfrak{U}_{6 b}+2 \mathfrak{B}_{b \delta}+\left(\mathfrak{C}_{\delta \delta}\right)=a \mathfrak{S}^{\prime}, \mathrm{vn}-\right.$ de, nisi $a \Longleftarrow 0$, manifesto sequitur, formam $\mathfrak{f}$. transire in $\sigma^{\prime}$ per substitutionem propriam $a, 6$, $r, \delta$ - Adhibendo autem loco trium aequatianum primarum in $\Psi$ et $\Psi$ " tres sequentes, ficile confirmabuntur tres aequationes modo traditis omnino similes, in quibus loco factoris a vbique inuenitur $b$; vnde patet, eandem conclusionem etiamnum valere si modo non sit $b=0$. Denique adhibendo tres vltimas aequationes $\Psi, \Psi$ ' inuenietur eodem modo, conclusionem veram esse nisi $c=0$. Quocirca, quum certo omnes $a, b, c$ simul $=0$ esse nequeant, necessario forma $\mathfrak{F}$ per subst. $\alpha, \varepsilon, \gamma, \delta$ transibit in $\mathfrak{f}$, adeoque huic formae proprie aequiualebit. $Q . E . D$.

241. Talem formam vt $\mathfrak{F}$ vel $\mathfrak{F}$, quae oritur, si vna trium formarum datarum componitur cum ea quae ex compositione duarum reliquarum resultat, ex his tribus formas compositam vocabimus, patetque ex art. praec.; nihil hic interesse quonam ordine tres formae componantur. Simili modo propositis quotcunque formis $f, f^{\prime}, f^{\prime \prime}$; $f^{\prime \prime \prime}$ etc. (quarum determinantes rationem quadratorum inter se habere debent) si forma $f$ componitur cum $f$, resultans cum $f^{\prime \prime}$, quae hinc oritur, cum f'm etc: forma quae ad finem huius operationis prodit ex omnibus formis $f, f^{\prime}, f^{\prime \prime}, f^{\prime \prime \prime}$ etc. composita dicetur. Facile vero demonstratur, etiam hic arbitrarium esse quonam ordine formae componantur; i. e. quocun- 
que ordine hae formae componantur, formas ex compositione oriundas semper proprie aequiualentes esse. - Porro manifestum est, si formis $f, f^{\prime}, f^{\prime \prime}$ etc. proprie aequiualeant formae $g, g^{\prime}, g^{\prime \prime}$ etc. resp., formam compositam ex his proprie aequiualentem fore formae ex illis compositae.

242. Propositiones praecedentes formarum compositonem maxima vniuersalitate complectuntur; progredimur iam ad applicationes magis particulares, per quas illarum ordinem interrumpere noluimus. Ac primo quildem resumemus problema tart. 236 , quod per conditiones sequentes limitabimus: primo vt formáe componendae eundem determinantem habeant, siue sit $d=d^{\prime}$; secundo vt $m, m^{\prime}$ sint inter se primi; tertio vt forma quaesita directe ex vtraque $f$, fi compo sita sit. Hinc etiam $m m, m^{\prime} m^{\prime}$ inter se primi erunt, quare diuisor communis maximus numerorum $d m^{\prime} m^{\prime}, d^{\prime} m m$ i. e. $D$ fiet $=d=d$, atque $n=n^{\prime}=1$. Quatuor quantitates $\mathfrak{N}$, $\mathfrak{L}$, $\mathfrak{2}^{\prime \prime}, \mathfrak{D}^{\prime \prime}$, quae ad libitum assami possunt, statuemus $=-1,0,0,0$ resp., quod semper licebit vnico casu excepto vbi $a, a^{\prime}, b+b^{\prime}$ simul sunt $=0$, ad quem igitur hic non respiciemus; manifesto autem hic casus occurrere nequit nisi in formis determinantis positiui quadrati. Tunc patet, $\mu$ fieri diuisorem communem maximum numerorum $a, a^{\prime}, b+b^{\prime}$; numeros $\mathfrak{9}, \mathfrak{p}, \mathfrak{p}$ ita accipi debere vt fiat $\mathfrak{J}^{\prime} a+\mathfrak{g}^{\prime \prime} a^{\prime}+\mathfrak{j}^{\prime \prime} \mathrm{c} b$ $\left.+b^{\prime}\right)=\mu$; ipsum $\mathfrak{y}$ vero omnino arbitrarium esse. Hinc prouenit, substituendo 1 c. pro $p, q$, $p^{\prime}, g^{r}$ etc valores suos: $A=\frac{c a^{\prime}}{\mu \mu}, B=$ 


\section{$\underline{x}\left(\mathfrak{P} a a^{\prime}+\mathfrak{P}^{\prime} a b^{\prime}+\mathfrak{P}{ }^{\prime \prime} a^{\prime} b+p^{\prime \prime \prime}\left(b b^{\prime}+D\right)\right) ;$} $\stackrel{\mu}{C}$ autem per aequationem $A C=B B-D$ poterit determinari, si modo non simul $a$ et $\dot{a}^{\prime}=0$.

In hac igitur solutione valor ipsius $A$ non pendet a valoribus ipsorum $\mathfrak{P}, \mathfrak{P}^{\prime}, \mathfrak{P}^{\prime \prime}, \mathfrak{P}^{\prime \prime}$ (qui infinitis modis dinersis determinari possunt); $B$ autem alios valores obtinebit tribuendo his numeris alios valores, operaeque pretium est inuestigare, quomodo omnes valores ipsius $B$ inter se connexi sint. Ad hunc finem obseruamus

I. Quomodocunque determinentur $\mathfrak{O}, \mathfrak{Y \prime}$; शु", $\mathfrak{P}^{\prime \prime}$, valores ipsius $B$ inde prodeuntes omnes congruós esse secundum modulum $A$. Ponamus, si statuatur $\mathfrak{P}=\mathfrak{p}, \mathfrak{P}=\mathfrak{p}^{\prime}, \mathfrak{P}^{\prime \prime}=\mathfrak{p}^{\prime \prime}, \mathfrak{P}^{\prime \prime \prime}=$ $\mathfrak{p}^{\prime \prime \prime}$, fieri $B=\mathfrak{B} ;$ faciendo autem $\mathfrak{P}=\mathfrak{p}+\mathfrak{d}$, $\mathfrak{P}^{\prime}=p^{\prime}+\mathfrak{D}^{\prime}, \mathfrak{P}^{\prime \prime}=\mathfrak{p}^{\prime \prime}+\delta^{\prime \prime}, \mathfrak{P}^{\prime \prime \prime}=\mathfrak{p}^{\prime \prime}+\delta^{\prime \prime \prime}$, prodire $B=\mathfrak{B}+\mathfrak{D}$. Tunc igitur erit $a b+$ $a^{\prime} b^{\prime \prime}+\left(b+b^{\prime}\right) b^{\prime \prime \prime}=0, a a^{\prime} b+a b^{\prime} b^{\prime}+a^{\prime} b b^{\prime \prime}$ $+\left(b b^{\prime}+D\right) \quad b=\mu \mathfrak{D}$. Multiplicando aequationis posterioris partem primam per $a p^{\prime}+a^{\prime} p^{\prime \prime}$ $+\left(b+b^{\prime}\right) \mathfrak{p}^{\prime \prime \prime}$ secundam per $\mu$, et subtrahendo a producto primo quantitatem $\left(a b^{\prime} \mathfrak{p}^{\prime}+a^{\prime} b \mathfrak{p}^{\prime \prime}+\right.$ $\left.\left(b b^{\prime}+D\right) p^{\prime \prime \prime}\right)\left(a b^{\prime}+a^{\prime} b^{\prime \prime}+(b+b) b^{\prime \prime}\right)$, quae propter aequationem priorem manifesto erit $=0$, habebitur euolutione facta et sublatis quae se destruunt $a a^{\prime}\left(\mu b+\left(\left(b^{\prime}-b\right) p^{\prime \prime}+c p^{\prime \prime \prime}\right) b^{\prime}+\right.$ $\left.\left(\left(b-b^{\prime}\right) p^{\prime}+c p^{\prime \prime \prime}\right) b^{\prime \prime}-\left(c^{\prime} p^{\prime}+c p^{\prime \prime}\right) b^{\prime \prime \prime}\right)=$ $\mu \mu \mathfrak{D}$, vnde manifesto $\mu \mu \mathfrak{D}$ per $a a^{\prime}$, siue $\mathfrak{D}$ per $\frac{a a^{\prime}}{-\mu \mu} \mathbf{i}_{4}$ e. per $A$ diuisibilis exit, atque $\mathfrak{B} \equiv \mathfrak{B}+\mathfrak{D}$ (mod. $A$ ) 
II. Si valores $\mathfrak{p}, \mathfrak{p}^{\prime}, \mathfrak{p}^{\prime \prime}, \mathfrak{p}^{\prime \prime \prime}$ ipsorum $\mathfrak{Y}, \mathfrak{y}$, Sु", $\mathfrak{P} "$ "reddant $B=\mathfrak{B}$, inueniri posse alios valores horum numerorum ex quibus $B$ ntanciscatur valorem quemcunque datum ipsi $\mathfrak{B}$ secundum mod. $A$ congruum, puta $\mathfrak{B}+k A$. Primo obseruamus, quatuor numeros $\mu, c^{\prime} ; c^{\prime}, b-b^{\prime}$ diuisorem communem habere non posse; nam si quem haberent, hic metiretur sex numeros $a, a^{\prime}$, $b+b^{\prime}, c, c^{\prime}, b-b^{\prime}$ adeoque tum ipsos $a, 2 b$; $c$, thim ipsos $a^{\prime}, 2 b^{\prime}, c^{\prime}$ et proin etiam ipsos $m$, $m^{\prime}$, qui per hyp. inter se sunt primi. 'Quamobrem quatuor numeri integri $h, h^{\prime}, h^{\prime \prime}, h^{\prime \prime \prime}$ poterunt assignari tales vt fiat $h^{\mu}+h^{\prime} c+h^{i} c^{\prime}+$ $h^{\prime \prime}\left(b-b^{\prime}\right)=1 . \quad$ Quo facto si statuitur $k h=$ b, $k\left(h^{\prime \prime}\left(b+b^{\prime}\right)-h^{\prime \prime \prime} a^{\prime}\right)=k^{\prime \prime}, k\left(h^{\prime}\left(b+b^{\prime}\right)\right.$ $\left.+h^{\prime \prime \prime} a\right)=\mu \mathrm{b} \prime,-k\left(h^{\prime} a^{\prime}+h^{\prime \prime} a\right)=\mu \mathrm{u}^{\prime \prime \prime}, \mathrm{pa}$ tet, ipsos $\mathfrak{b}, \mathfrak{b}^{\prime}, \mathfrak{b}^{\prime \prime}, \mathfrak{b}^{\prime \prime \prime}$ esse integros; porro facile confirmatur, fieri $a b^{\prime}+a^{\prime} b^{\prime \prime}+\left(b+b^{\prime}\right) b^{\prime \prime \prime}=0$, $a a^{\prime} b+a^{b} b+a^{\prime} b b^{\prime \prime}+(b b a+D) \cdot d n=$ $\frac{a a^{\prime} k}{\mu}\left(\mu h+c h^{\prime}+c h^{\prime \prime}+\left(b-b^{\prime}\right) h^{\prime \prime \prime}\right)=\mu k_{A}$. Ex aequatione priori patet, etiam $p+b, p^{\prime}+$ $\mathfrak{D}^{\prime}, \mathrm{p}^{\prime \prime}+\mathrm{b}^{\prime \prime}, \mathfrak{p}^{\prime \prime \prime}+\mathfrak{d}^{\prime \prime \prime}$ esse valores, ipsorum $\mathfrak{g}, \mathfrak{D}$, 3ु", yु" ; ex posteriori, hos valores producere $B=\mathfrak{B}+k A$. Q. E. D. - Hinc perspicuum est, $B$ semper ita determinari posse vt laceat inter 0 et $A-1$ incl., siquidem $A$ est positiuus; vel inter o et $-A-1$ si $A$ negatiuus.

243. Ex aequationibus $\mathfrak{P} a+\mathfrak{P}^{a} a^{a}+$ $\mathfrak{P} a{ }^{\prime}\left(b+b^{\prime}\right)=\mu, B=\frac{\mathrm{x}}{\mu}\left(\mathfrak{P} a a^{\prime}+\mathfrak{P}^{\prime} a^{\prime} b^{\prime}+\mathfrak{P}^{\prime \prime} a^{\prime} b\right.$ $+\supseteqq \cdots(b b+D))$ deducitur $B=b+\frac{a}{b}(\$ a+$ 


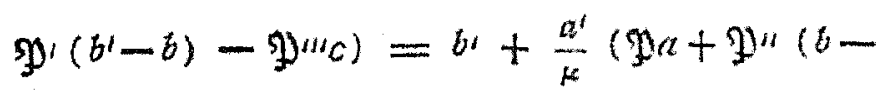
$\left.b^{\prime}\right)-\mathfrak{P}^{\left.\prime \prime c^{\prime}\right)}$; quare $B \equiv b\left(\bmod \frac{a}{\mu}\right)$ et $B \equiv$ $b^{\prime}$ (mod, $\frac{a^{\prime}}{\mu}$ ). Quoties $\frac{a}{\mu}, \frac{a^{\prime}}{\mu}$ inter se prini sunt, inter o et $A-1$ (siue inter o et $-A$ - 1 quando $A$ est negatiuus) wicus tantum numerus iacebit qui secundum mod. $\frac{a}{\beta}$ sit $\equiv b$, et $\equiv b^{\prime}$ sec. mod. $\frac{a^{\prime}}{\mu} ;$ qui si statuitur $=B$ atque $\frac{B B-D}{A}=C$, palam est, $(A, B, C)$ e formis $(a, b, c),\left(a^{\prime}, b^{\prime}, c^{\prime}\right)$ compositam fore. In hoc itaque casu ad inuextionem formae compositae

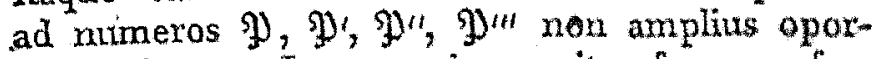
tet respicere. Ita e. g. si quaeritur forma e formis $(10,3,11),(15,2,7)$ composita, erunt $a, a_{2}^{2}$ $b+b$ resp. $=10,15,5 ; \mu=5$; line $A=6 ; B \equiv 3$ (mod. 2.) et $\equiv 2$ (mot. $3)$, vnde $B=5$ atque $(6,5,21)$ forma quiesita. - Ceterum conditio vt $\frac{a}{\alpha}, \frac{a^{\prime}}{\mu}$ inter se primi sint ommino aequiualet huic yt numeri duo $a, a^{\prime}$ diuisorem communem maiorem non habeant quam tres $a, a^{\prime}, b+b^{\prime}$, siue, quad eotem redit, vt diuisor communis maximus numerorum $a_{5} a^{\prime}$ etiam sumerum $b+b$ metiatur. Notenur imprimis sequentes casus particulares:

1) Propositis dualus fornis $\left(a, b, e^{*}\right),\left(a^{*}, b^{4}\right.$ $\left.c^{\prime}\right)$ eiusdem determintutis $D$ ita comparatis te diuisor comm, max. numerorum $a, 2 b, c$ pimus

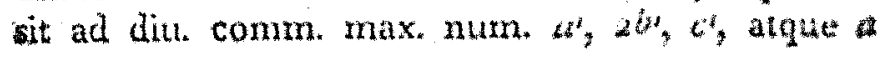




\section{$-369=$}

primus ad $a^{\prime}$ : forma ex his composita $(A, B, C)$ inuenitur faciendo $A=a a^{\prime}, B \equiv b$ (mod. $a$ ) et $\equiv b^{4}$ $\left(\right.$ mod $\left.a a^{\prime}\right), C=\frac{B B \sim D}{A}$. Hic casus semper locum habet, quando allera formarum componendarum est forma principalis, puta $a=1, b=0, c=$ -D. Tunc erit $A=a^{t}, B$ statui poterit $=b$, wnde fiet $C=c^{\prime} ;$ quare ex forma principali et quacuinque alia forma eiusdem determinantis composita est haec forma ipsa.

- Si duae formae oppositae proprie primitiuae sunt componendae, puta $(a, b, c)$ et $\{a$ $-b, c)$, erit $\mu=a$. Hinc facile perspicitur, forman principalem $(i, 0-D$, ex illis esse compositam.

3) Propositis quotcunque formis proprie primitiuis, $a, b, c),\left(a^{\prime}, b^{\prime}, c^{\prime}\right),\left(\alpha^{\prime \prime}, b^{\prime \prime}, c^{\prime \prime}\right)$ etc. eiusdern determinantis $D$, quarum termini antecedentes $a, a^{\prime}, a^{\prime \prime}$ etc sunt numeri inter se pri$\mathrm{mi}$, forma $(\mathcal{A}, B, C)$ ex illis omnibus composita inuenitur, statuendo $A$ aequalem producto ex omnibus $a, a^{\prime}, a^{\prime \prime}$ etc; $B$ congruum ipsis $b$, $b^{\prime}, b^{\prime \prime} z^{\prime \prime}+c$. secundum modulos $a, a^{\prime}$, $a^{\prime \prime}$ etc. resp.; $C=B B \ldots D$ Facile enim perspicietur, ex dualus formis $(a, b, c),\left(a^{\prime}, b^{\prime}, c^{\prime}\right)$ compositam fore forman $\left(a a^{\prime}, B, \frac{B B-D}{a a^{\prime}}\right)$; ex hac atque $\left(a^{\prime \prime}, b^{\prime \prime}, c^{\prime \prime}\right)$ formam $\left(a a^{\prime} a^{\prime \prime}, B, \frac{B B-D}{a a^{\prime} a^{\prime \prime}}\right)$ etc. Vice versa

4) Proposita forma proprie primitiua $(A, B$, C) determinantis $D$, si terminus $A$ in factores 
quoteunque inter se primos $a, a^{\prime}, a^{\prime \prime}$ etc. resolütur; numeri $b, b$, bu etc. ipsi $B$ vel aequales vel saltem sec. mod. $a, a^{t}, a^{\prime \prime}$ etc resp. cougrui accupiuntur, atque fit $a c=b b-D, a^{\prime} c^{\prime}=b b^{\prime}$ $-D, a^{\prime \prime} c^{n}=b^{n} b^{n}-D$ etc: forma $(A, B, C)$ composita erit e formis $(a, b, c),\left(a^{\prime}, b^{\prime}, c^{\prime}\right),\left(a^{\prime \prime}\right.$, $\left.b^{\prime \prime}, c^{\prime \prime}\right)$, sine in has formas resolubilis. Nullo negotio probatur, eandem propositionem athucdum valere; etiamsi forma $(A, B, C)$ sit inmoprie primitiua vel deriuata. Iloc itaque molo quaelibet forma in alias eiusdem deterninumis resolui potes sint vel numeri primi vel numerorum primortum potestates. Talis resolutio saepentumero commode applicari notest, si ex pluributs formis datis con* ponenda est vna. Ita e. $g$ * si quaeritur forma composita e formis $(3,1,234),(10,3,4,1),(15,2$, $27)$, resoluatur secunda in has $(2,1,201),(5 . \ldots$ $2,81)$, teria in has $(3,-1,134),(5,2,81$, pateque, formam ex quinque formis $(3,2,1,3,1,10$, $1,201),(5,-2,81),(3,-1,131),(5,2,8)$ compositam, quocunque ordine accipiantur, etam ex tribus datis compositam fore. At ex complow sitione primae cum quarta oritur forma uincipalis $(1,0,401)$; eadem prouenit ex composititus tertiae cum quinta; quare ex compositione cunstarum conflatur forma $(2,4,201)$.

5) Propter rei vtilitatem operae pretium est, hanc methodum adhuc amplius explicare. Ex obseruatione praecedente manifestum est, problema, quotcumue formas datas proprit patmitiuas eiusden deterninanti componere, redute posse ad compositionem formarum, quarum tex- 
mini initiales sint potestates numerorum primarum nam numerus primus tamquam sui ipsius potestas prima considerari potest). Quamobrem eum imprimis casum contemplari conuenit, vbi duae forma proprie primitiuae $(a, b, c),\left(a^{2}, b^{t}, c^{\prime}\right)$ sunt componendae, in quibus $a$ et $a^{\prime}$ sunt potestates ciusdcm numeri primi. Sit itaque $a=h^{*}$, $a^{\prime}=h^{\lambda}$ designante $h$ numerum primum, supponamusque (quod licet), "non esse minorein quam $\lambda_{*}$ Erit itaque $h^{\wedge}$ diu. comm. max. numerorum $a, a^{t}$, qui si insuper ipsum $b+b^{\prime}$ metitur, habebitur casus initio lhuius art. considr-ratrs, eritque $(A, B, C)$ ex propositis composita si statuitur $A=h^{*-\lambda}, B \equiv b$ (mod. $\left.h^{*-\lambda}\right)$ at $\equiv$ $b^{\prime}$ (mod. 1$)$, quae conditio posterior madifesta omitti potest; $C=\frac{B B}{A} \cdot D \cdot$ Si vero $h^{\lambda}$ ipsum $b+b^{\prime}$ non metitur, necessario dita comm. max. horum numerorum et ipse erit potstas ipsius $h$; sit igitur $=h^{*}$, eritque $<\lambda$ ( statui dubet $"=0$, si forte $h^{\prime \prime}$ et $b+b^{\prime}$ inter se primi sunt). Si itnque $\mathfrak{P}^{\prime}, \mathfrak{P}^{\prime \prime}, \mathfrak{P}^{\prime \prime}$ ita determinantur,

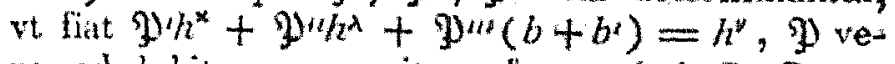
ro ad lubirum assumitur, forma $(A, B, C)$ ex datis erit composita, si statuitur $A=h^{*}+\lambda-2$, $\left.B=b+h^{x-}\left(\mathfrak{P} h^{\lambda}+\mathfrak{D}^{\prime} b-b^{\prime}\right)-\mathfrak{P}^{\prime \prime} c\right)$, $C=\frac{B B D}{A} D$. Sed facile perspicitur, in hoo casu etian IJ $^{\prime}$ ad libitum assumi posse, quare statuendo $\mathfrak{Y}=\mathfrak{W}=0$, fit $B=b-$ $\mathfrak{P}^{\prime \prime} c h^{k \rightarrow}$ sime generaliter $B=k A+b-$ 9l' $\mathrm{Ch}^{*}{ }^{-*}$, designante $k$ numerum arbitrarium (art. praec.). In hanc formulam simplicissimans Aa 
solus $\mathfrak{y}^{\prime \prime}$ ingreditur, qui est valor expr $\frac{b^{*}}{b+b^{*}}$ $\left(\bmod h^{\lambda}\right)$. Si $e$. g. quaeritur forma compositi ex $(16,3,19)$ et $(8,1,37)$, est $h=\$, *=$ $4, \lambda=3, v=2$. Hinc $A=8, \mathfrak{P}^{m}$ valo: expr. $\frac{4}{4}(\bmod .8)$, qualis est $x$, vnde $B=81$ - 73, adeoque faciendo $k=9, B=-1$ atque $C=37$, siue $(8,-1,37)$ forma quae sita.

Propositis itaque formis quotcunque, quarum termini initiales omnes sunt potestates numero. rumo primorum, circumspicientlum grit, num ati. quarum termini antecedentes sint potestates exusitem numeri primi, atque hae inter se respectiue pes regulam modo traditam componendae Hac ra. tione prodibunt formae, quarum termini primi etiamnum erunt potestates numerorum primontam sed ommino diuersorum; forma itaque ex his composita per obseru. tertiam definin pothit E. g. propositis formis $(3,1,47),(4,0,35)$, $(5,0,28),(16,2,9),(9, \pi, 21),(16,6,11)$, ex prima et quinta conflatur forma $(2 \vec{*}, 7,7)$; ex secunda et quarta confit $(16,-6,11$, ax hac et sexta $(x, 0, x 40)$, quae negligi poutest. Supersunt itaque $(5,0,28),(27,7,7)$, ex quibus producitur $(255,-20,4$, cuius luco assumi potest proprie acquiualens $(4,0,35$, Hute itaque est resultans ax compositione sex propositarum.

Ceterum ex hoc fonte plura alia artificia in applicatione vtilia hauriri possunt; sed ne nimit 
Iongi fiamus, vberiorem huius rei tractationem supprimintus, ad alia difficiliora properantes.

24.4. Si per formam aliquam $f$ repraesentari potest numerus $a$, per formam $f^{\prime}$ numerus $a^{t}$, atcque forma $F$ in $f^{\prime}$ est transformabilis: nullo negotio perspicitur, productum $a a^{\prime}$ per formim $F$ repraesentabile fore. Hinc statim sequitur, quatnd o determinantes harum formarum sint negatiui, forman $F^{F}$ positiuam fore si vel vtraque $f$, $f^{\prime}$ sit positiua vel vtraque negatiua; contra $F^{\prime}$ fierí negatiuam si altera formarum $f, f^{\prime}$ sit positiua altera negatiua. Subsistamus in eo imprimis casu, quem in art praect. considerauimus, vi $F$ ex $f, f^{\prime}$ composita est, atque $f, f^{\prime}$ et $F^{\prime}$ eundem deteminantem $D$ habent. Supponamus insuper, repratsentationes numerorum $a$, $a !$ per formas $f, f$ fieri per valores indeterminatarum inter se primos, atque priorem pertinere ad valorem $b$ expressionis $\sqrt{D}$ (mud. $a$ ), posteriorem ad valorem $b^{\prime}$ expr. $\sqrt{D}$ (mod. $a^{\prime}$ ), ponaturque $b b-$ $D=a c, b^{\prime} b^{\prime}-D=a^{\prime} c^{\prime}$. Tunc per art 168 formae $a, b, c),\left(a^{\prime}, b^{\prime}, b^{\prime}\right)$ proprie aequiualebunt formis $f, f ;$ quare $F$ etiam ex illis duabus formis composita erit. Sed ex iisdem formis compositir exit forma $(A ; B, C)$, si, posito rumerorum $a, a^{2}, b+b^{\prime}$ diuisore communi maximo $=\mu$, statuitur $A=\frac{a a^{\prime}}{\mu \mu}, B \equiv b$ et $b r \mathrm{sec}$. modulos $\frac{a}{\mu}, \frac{a^{4}}{\mu}$ resp., $A C=B B-D_{;}$quare haec forma proprie aequiualebit formae $F$. Ium per formam $A x x+2 B x y+C y$ repraesentatur numerus $a a^{2}$, faciendo $x=\mu, y=0$, quorum valorum diuisor comm. max. est $\mu_{j}$ quare aa Aa 3 


\section{- $374-$}

etiam per formam $F$ repraesentari poterit ita vt valores indeterminatarum habeant diuisorem communem maximum $\mu$ (art. 166). Quoties igitur euadit $"=1$, $a a^{\prime}$ per formam $F$ repraesentar poterit tribuendo indeterminatis valores inter se primos, repraesentatioque haec pertinebit ad valorem $B$ expr. $\sqrt{ } D\left(\bmod a a^{3}\right)$, ipsis $b, \quad b^{\prime}$ securidum modulos $a, a^{\prime}$ resp. congruum. Conditio $\mu=1$ semper locum habet, quando $a, a^{t}$ inter se primi sunt; generaliter autem, quando diu. comm. max. ipsorum $a, a^{\prime}$ ad $b+b^{\prime}$ est primus.

24.5. Tinerema. Si forma fad eutem ordin em referenta est $v t \mathrm{~g}$, similiterque $f$ est $2 x$ eodem ordine vt $g^{\prime}$ : forma $F$ ex $f, f$ composita eundem datermunastenu habebit ex eodemque ordine exit vt forma $G$ ex $g$, $\mathrm{g}^{\prime}$ composita.

Dem. Sint formae $f, f^{\prime}, F=(a, b, c),\left(a^{\prime}\right.$, $\left.b^{\prime}, C^{\prime}\right),(A, B, C)$ resp., ipsarmmque deterninantes $=d, d, D$. Porro sit numerorm $a, b, c$ diu. comm. max. $=m$; numerorum $a, b, c$ diu. comm. max. $=m$; similesque significationes baw beant $m^{\prime}, m^{\prime}$ respectu formar $f$, et $h$, Th respectu formae $F$. Tunc ordo formae $f$ determinabitur per numeros $d, m, \mathrm{~m}$, vnle fidern numeri etiam pro forma $g$ valebunt; eatem ratione numeri $d^{\prime}, m^{\prime}, \mathrm{m}^{\prime}$ idem erunt pro forma $\sigma^{*}$ quod sunt pro forma $f^{\prime}$. lam per art. 235 , numeri $D, M, \mathfrak{M}$ determinati sunt per $d, d, m$, $m^{\prime}, \mathrm{m}, \mathrm{m}^{\prime}$; scilicet erit $D$ diuisor communis $\mathrm{mna}^{-}$ ximus ipsorum $\mathrm{d}^{\prime} \mathrm{m}^{\prime} \mathrm{m}^{\prime}, \mathrm{d}^{\prime} \mathrm{m} \mathrm{m} ; \mathrm{M}=\mathrm{mm}^{\prime}$; atque $\mathfrak{m}=\mathrm{mm}^{*}$ (si simnl $\left.m=\mathrm{m}, m^{*}=\mathrm{m}^{\prime}\right)$, vel $=2 \mathrm{~mm}^{2}$ (si $m=2 \mathrm{~m}$, aut $\left.m^{\prime}=2 \mathrm{~m}^{\prime}\right)$. Qua* 
proprietates ipsorum $D, M, \mathfrak{M}$, quum inde sequantur, quod $F$ ex $f$, fo composita est: facile perspicitur, $D, M$ et 5 etiam pro forma $G$ vaIere, adeoque $G$ esse ex eodem ordine vt $F$. Q. E. D.

Ex hac ratione ordinem in quo est forma $F$ compositum dicemus ex ordinibus in quilus sunt formas $f_{2} f_{0}$. Ita e. $\mathrm{g}$. ex duobus ordinibus proprie primitiuis sermper compositus est similis ordo; ex proprie primitiuo et improprie primitiuo, improprie primitius, - Simili modo intelligendum est, si ordo aliquis ex pluribus alis ordinibuts compositus vocabitur.

2.46. Problema. Propositis duabus formis promituss quibuscungue $f, f$, ex quarth compositone

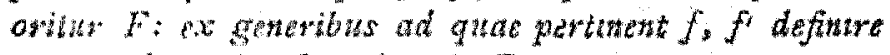
gentus ad quod referenda erit $F$.

Sol. I. Consideremus primo eum casum vbi al minimum rna formarum $f, f \cdot c . g$. prior est prowie primitiua, designemusque determinantes formarum $f, f, f$ per $d, d^{*}, D$. "Tunc $D$ erit dinisor communis maximus numerorum. d $m m^{\prime} m^{\prime}$, $d^{\prime}$, vit $m^{\prime}$ est aut 1 aut $a_{2}$, prout forma $f^{\prime}$ est proprie aut improprie primitiua; $F$ autem. in casu illo pertinebit ad ordinem proprie primitiuum, in hoc ad improprie primitiuum. lam genus formae $F$ definetur per ipsius characteres particulares, nempe tum respectu singulorum diuisorum primorum imparium ipsius $D$, tum, pro quibusdam casibus, respectu numerorum 4 aut 8. Hos igitur singulos determinare. oportebit. 


\section{- $37^{6}-$}

I․ Si $p$ est diuisor quicunque primus impar ipsius $D$, necessario etiam ipsos $d, d^{\prime}$ metietur, adtoque etiam inter characteres formarum $f$, $f^{\prime}$ occurrent ipsanum relationes ad $p$. Iam si per $f$ repraesentari potest nunierus $a$, perfinumerus $z^{\prime}$ : produrtum $a a^{\prime}$ repraesentari poterit per $F$. Si itaque tum per $f$, tum per $f$ repraesentari possunt: residua quadratica ipsius $p$ (per $p$ non diuisibilia), etiam per $F$ residua quadratica ipsius $p$ repraesentari poterunt, i. e. si vtraque $f, f^{t}$ habet characterem $R p$, forma $F$ eundem characterem habebit. Simili ratione $F$ habebit characterem $R p$, si vtraque $f, f^{\prime}$ habet characterem $N p$; contra $F$ habebit char. $N p$, si altera formarum $f, f$ habet $R p$, altera $N_{p}$.

$2{ }^{\circ}$. Si in characterem integrum formae $F$ ingreditur relatio ad numerum 4 , talis rêlatio etiam in characteres formarum $f, f^{\prime}$ ingredi dehet. Nam illud tunc tanturnmodo euenit, quanto $D$ est $\equiv 0$ aut $\equiv 5(\bmod .4)$ Qnande $D$ per 4 est diuisibilis, etiam $d m^{\prime} m^{\prime}$ et $d^{\prime}$ for of dinisibiles erunt, nde statim patet, fi non punse ease improprie primitiuam, adeoque esse $m^{\prime}=1$; hinc tum $d$ tum $d^{\prime}$ per 4 diuisibiles ertunt, et in vtriusque characterem ingredietur relatio at 4 . Quando $D \equiv 5$ (mod. 4, metietur $D$ iprus $d$, $d^{\prime} ;$ quotientes erunt quadrata, adeoque tham $d$, $d^{\prime}$ necessario vel $\equiv 0$ vel $\equiv 5$ inud, t) ${ }_{*}$ et inter characteres ipsarum $f, f$ relatio an $\&$. Hinc eodem modo vt in (1) sequitur, charatelewn formae $F$ fore 1,4 , si vel vtraque $f, f$ labeat 1 , 4 vel viraque $\pi, 4 ;$ contra characterein formae $F$ fore 3,4 , si altera tormarum $f, f$ habeat 1 , 4, altera 3,4 
$3^{\circ}$. Quando $D$ per 8 est diuisibilis, etiam $d^{\prime \prime}$ erit; hirc $f^{\prime}$ certo proprie primitiua, $m^{\prime}=\mathbf{I}$ atque etiam $d$ per 8 diuisibilis; quare inter characteres formae $F$ aliquis e characterilsus 1,8 ; 5,$8 ; 5,8 ; 7,8$ tunc tantum locum habere potest, si etiam in charactere tum formae $f$, tum formate $f^{\prime}$ talis relatio ad 8 adest. Facile autern confirmatur eodem modo vt ante, characterem formae $F$ fore 1,8 , si $f$ et $f$ respectu ipsius 8 eundem habeant; characterem formae $F$ fore 3,8 , si altera formorum $f, f^{\prime}$ habeat 1,8 altera 3,8 , vel altera 5,8 altera 7,$8 ; F$ habere 5,8 , si $f, f$ habeant 1,8 et 5,8 vel 3,8 et 7,$8 ; F$ habere 7,8 , si $f$ et $f$ habeant vel 1,8 et 7,8 , vel 3,8 et 5,8 .

$4^{\circ}$. Quando est $D \equiv 2(\bmod , 8)$, erit $d^{\prime}$ vel $\equiv$ a vel $\equiv 2(\bmod .8)$; hinc $m=1$, adeoque etiam $d$ vel $\equiv 0$ vel $\equiv 2(\bmod .8)$; attamen vterque $d, d^{t}$ per 8 diuisibilis esse nequit, quoniam $D$ est diuisor communis maximus ipsorum. Quare in eo tantum casu alteruter characterum 1 et 7,$8 ; 3$ et 5,8 , formae $D$ tribui debebit, vbi vel vtraque forma $f, f$ aliquem ex illis habet, vel altera aliquem ex illis, altera aliquem horum 1,$8 ; 3,8 ; 5,8 ; 7,8$. Trro facile deducitur, characterem foma $F$ detarminari per talulam sequentem, si character in margine positus pertineat ad alteram formarum $f, f^{\prime}$, ad alteram vero character in facie:

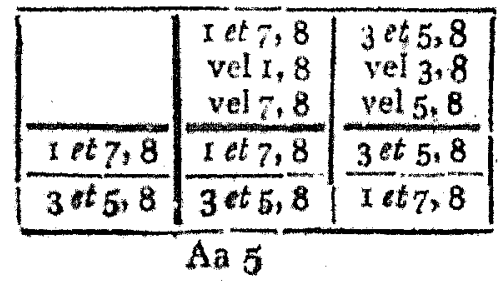




\section{$-\quad 378-$}

$5^{\circ}$. Eodem modo probatur, ipsi $F$ tribui non posse alterutrum characterum 1 et 3,$8 ; 5$ et 7,8 , nisi etiam aliquis ex iisdem saltem vni formarum $f, f^{\prime}$ competat, alterique vel aliquis ex iisdem, vel aliquis ex his 1,$8 ; 3,8 ; 5 ; 8$; 7,8 . Et quidem character formae $F$ determinabitur per hanc tabulam, in cuius margine et facie sunt characteres formarum $f, f^{\circ}$

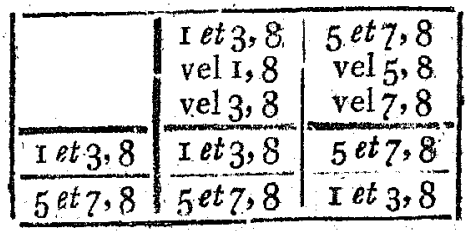

II. Si vtraque forma $f, f$ est improprie primitiua, erit $D$ diuisor communis maximus numerorum $4 d, 4 d$, siue $\frac{\pi}{4} D$ dia. comm. maximus numerorum $d$, $d^{\prime}$. Hinc facile sequitur, tum $d$, tum $d$, tum $\frac{x}{4} D$ fore $\equiv 1$ (mod. 4$)$. Ponendo autem $F=(A, B, C)$, diu. comm. nax. numerorum $A, B, C$ erit $=2$, et diu. comm. max. numerorum $A, 2 B, C$ erit 4. Quare $E$ erit forma deriuata ex improprie primitiua $\left(\frac{x}{2} A, \frac{x}{2} B, \frac{\pi}{2} C\right)$, cuius determinans erit $\frac{\pi}{4} D$, et cuius genus determinabit genus formae $F$. Character autem illiu's formae, tamquam improprie primitiuae, relationes ad 4 vel 8 non implicabit, sed tantummodo relationes ad singulos diuisores primos impares ipsius $\frac{x}{4} D$. Iam quum omnes hi diuisores manifesto etiam ipsos $d$, d metiantur, atque semissis cuiusuis producti duorum factorum, quorum alter per $f$ alter per $f^{\prime}$ est repraesentabilis, per formam $\left(\frac{\pi}{2} A, \frac{x}{2} B, \frac{x}{2} C\right)$ reprae- 
sentari possit: facile perspicietur, characterem huius formae respectu cuiusuis numeri primi imparis $p$ ipsum $\frac{T}{4} D$ metientis fore $R p$, tzum si fuerit $2 R p$ atque formae $f, f^{\prime}$ respectu ipsins $p$ eundem characterem habeaxit, tum si fuerit $2 N p$ atque characteres formarum $f, f^{\prime}$ respectu ipsius $p$ oppositi; contra characterem illius formae fore $N p$, tum si $f, f^{\prime}$ habeant characteres aequales respectu ipsius $p$ atque sit $2 N p$, tum si $f$, $f^{\prime}$ habeant oppositos atque sit $2 R p$.

247. Ex solutione problematis praec. manifestum est, si $g$ sit forma primitiua ex eodem ordine et genere vt $f$, nec non $g^{\prime}$ lorma primitiua ex eodem ordine et genere vt $f i$ : formam ex $g$ et $g^{\prime}$ compositam ad idem genus pertinere ad quod pertineat forma ex $f$ et $f$ composita. Hinc sponte sequitur significatio generis ex duobus alìs generibus (siue etiam pluribus) compositi. Porro ibinde patet, si $f, f$ eundem determinantem habeant atque $f$ sit forma e genere principali, $F$ vero ex $f$ et $f$ composita: $F$ fore ex eodem genere vt $f^{\prime}$; quocirca genus principale in compositione cum aliis generibus eiusdem determinantis semper omitti poterit. Si vero reliquis manentibus $f$ non est e genere principali, $f^{\prime}$ autem forma primitiua: $F$ certo erit ex alio genere quam $f i$. Denique si $f, f^{\prime}$ sunt formae proprie primitiuae eiusdem generis, $F$ erite genere principali; si vero $f, f_{1}^{\prime}$ sunt ambae proprie primitiuae eiusdem determinantis, sed e diuersis generibus, $F$ ad genus principale pertinere non poterit. Quodsi itaque forma quaecunque proprie primitiua cum se ipsa componitur, forma 


\section{- $380-$}

inde resultans, quae etiam proprie primitiua eiusdemque determinantis erit, necessario ad genus principale pertinebit.

248. Problema. Propositis duabus formis quibuscunque $f, f^{\prime}$, e quibus composita est $F:$ e generibus furmarum $f, f^{\prime}$ definire genus formae $F$.

Sol. Sit $f=(a, b, c), f^{\prime}=\left(a^{\prime}, b^{\prime}, c^{\prime}\right)$, $" F=(A, B, C)$, porro $\mathfrak{m}$ diu. comm. max. nut merorum $a, b, c$, atque $\mathrm{m}^{\prime}$ diu. comm. max. numerorum $a^{\prime}, b^{\prime}, c^{\prime}$, ita vt $f, f^{\prime}$ sint deriuatae e primitiuis $\left(\frac{a}{\mathfrak{m}}, \frac{b}{\mathfrak{m}}, \frac{c}{\mathfrak{m}}\right),\left(\frac{a^{\prime}}{\mathfrak{m}^{\prime}}, \frac{b^{\prime}}{\mathfrak{m}^{\prime}}, \frac{c^{\prime}}{\mathfrak{m}^{\prime}}\right)$, quas denotabimus per $f, f$ resp. Iam si saltem tha forma$\operatorname{rum} f, \mathrm{fl}^{\prime}$ est proprie primitiua, diuisor comm. max.

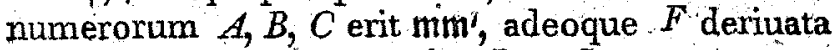
- forma primitiua $\left(\frac{A}{\mathfrak{m} \mathfrak{m}^{\prime}}, \frac{B}{\mathfrak{m}^{\mathfrak{m}}}, \frac{C}{\mathfrak{m} \mathfrak{m}^{\prime}}\right) \ldots \mathscr{F}$, nnde patet, genus formae $F$ pendere a genere formae $F$. Sed facile perspicietur, $\mathfrak{f}$ per eandem substitutionem transire in $f f$, per quam $F$ transeat in $f f$ adeoque $f$ ex $f, f^{\prime}$ esse compositam, ipsiusque genus per problema art. 246 determinari posse. - Si vero vtraque $f, f^{\prime}$ est improprie primitiua, diuisor c. m. numerorum $A, B, C$ erit $2 \mathrm{~mm}^{\prime}$, formaque $\approx$ etiamnum ex $f, f t$ composita et manifesto e proprie primitiua $\left(\frac{A}{2 m m^{\prime}}, \frac{B}{2 m m^{\prime}}, \frac{C}{2 m m^{\prime}}\right)$ deriuata. Huius itaque formae genus determinari pot. erit per art. 246 ; et quum $F$ ex eadem forma deriuata sit, ipsius genus hinc sponte innotescit.

Ex hac solutione manifestium est, theorema in art. praec. pro formis primitiuis explicatum, scilicet si. $f^{\prime}, g^{t}$ sint ex iisdem generibus resp. 


\section{$-381$}

vt $f, g$, formam ex eodem genere fore ex quo sit forma ex $f, g$ composita, generaliter pro formis quibuscunque valere.

249. Theorema. Si formae $f$, fi sunt ex iisdem ordinibus generibus et classibus vt $g$, $g$ resp.: furma ex $f$ et $f^{\prime}$ composita ex eadem classe erit vt forma ex $g$ et $g^{\prime}$ composita.

Ex hoc theoremate (cuilus veritas ex art. 239 protinus sequitur) sponte patebit significatio classis e duabus classibus datis siue etiam e pluribus compositae.

Si classis quaecunque $K$ cum classe princit pali componitur, classis $K$ ipsa prodibit, siue classis principalis in compositione cum aliis classibus eiusdem determinantis negligi potest. Ex compositione duarum classium oppositarum proprie primitiuarum semper oritur classis principalis eiusdem determinantis ( $v$. art. 243). Quum itaque quaeuis classis anceps sibi ipsa opposita sit: ex compositione cuiusuis classis ancipitis proprie primitiua cum se ipsa classis principalis eiusdem determinantis prouenit.

Propositio vltima etiam conuersa valet: scilicet si ex compositione classis proprie primitiuae $K \mathrm{cum}$ se ipsa prouenit classis principalis $H$ eiusdem determinantis, $K$ necessario erit classis anceps. $\quad \mathrm{Si}$ enim $K^{\prime}$ est classis opposita ipsi $K$, e tribus classibus $K, K, K^{\prime}$ composita erit eadem classis qua oritur ex $H$ et $K^{\prime}$; ex 'illis pronenit $K$ (quoniam $K$ et $K^{\prime}$ producunt $H$, haec can $K$ ipsam $K$ ), ex his $K^{\prime}$; quare $K$ cum $K^{\prime}$ coincidet eritque adeo classis anceps. 
Porro notetur propositio haec: Si classes $K$, $L$ oppositae sunt classibus $K^{\prime}$, et $L^{\prime}$ resp.: clctssis ex $K$, et $L$ composita classi ex $K^{\prime}$ et $L^{\prime}$ comm positae erit opposita Sint formae $f, g, f, g t$ resp. e classibus $K, L, K^{\prime}, L^{\star}$; forma $F^{\prime}$ comilo* sita ex $f, g$, atque $F^{\prime}$ composita ex $f^{\prime}, g^{\prime}$. Qmum $f^{\prime}$ ipsi $f$, atque $g^{\prime}$ ipsi $g$ improprie aequiualsizut, $F$ autem composita sit ex vraque $f, g$ directe: $F$ etiarn ex $f, g^{\prime}$ composita erit, sed ex vtrayus inuerse. Quare forma quaecunque, quar ipsi $f$ improprie aequiualet, compusita erit ex $f t^{\prime} g^{\prime}$ directe adeoque ipsi $F^{\prime}$ proprie aequinalebit (att. $238,239)$, vnde $F, G$ improprie a quindelum, classesque ad quas pertinent oppositae ermint.

Hinc sequitur, si classis anceps $K$ cum classe ancipite $L$ componatur, semper prodire classem ancipitem. Nam opposita erit classi, quae composita est e clascibus insis $K, I$ oppositis, adeoque sibi ipsi, quoniam hae classes sibi ipsae thit oppositae.

Denique obseruamus, si propositae sint disses duae quaechuque $K, L$ eiusden determis:ti.

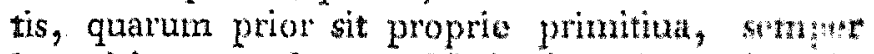
inueniri posse classem $M$ ciusten determinumis, ex qua atque $K$ composita sit $L_{\text {s }}$. Manifeste Lut $_{\text {a }}$ obtinetur, accipiendo pro $M$ clussem quate ca:aposita est ex $L$ atque classe ipsi $K$ opposita; sim mul perspicietur facillime, hanc classem vricain quae hac proprietate sit praeaita, sitho classes diuersas eiusdem det cum tatlem rasse pr. prim. compositus producere classes diuersut

Clussimm composilio commode per signum additionis, + , denotari potest, sicuti clishimm 
identitas per signum aequalitatis. In his signis propositio modo tratila exhiberi potest ita: $S \mathbf{i}$ $K^{\prime}$ est classis opposita ipsi $K$, erit $K+K^{\prime}$ classis principalis eiusdern determinantis, nndo $K+K^{\prime}$ $+L=L$; posita itaque $K^{\prime}+L=M$, erit $K+M=L$, vti desiderabatur; si vero praeter $M$ alia $M^{M}$ daretur eadem proprietate praedita, siue $K+M^{\prime}=L$, foret $K+K^{\prime}+M=L+$ $K^{\prime}=M$, nnde $M^{\prime}=M^{\prime}-$ Si plures classes identicae componuntur, hoc (ad instar multiplicationis) denotari potest praefigendo ipsarum numerum, ita rt $2 K$ idem designet vt $K+K, 5 K$ idem vt $K+K+K$ etc. Eadem signa etiam ad formas transferri possent, ita vt $(a+b+c)+$ $\left(a^{t}+b^{t}+c^{\prime}\right)$ designaret formam ex $(a+b+c)$, $\left(a^{\prime}+b^{\prime}+c^{\prime}\right)$ compositam: sed ne vel species ambigutatis oriri possit, hac abbreuiatione alkstinere malumus, praesertim quod tali signo $\sqrt{ } M\left(a_{3}\right.$ $b, c)$ significationem peculiarem iam tribuimus. - Classem $2 K$ ex duplicatione classis $K$ oriri dicemus, classem $3 X$ ex triplicatione etc.

950. Si $D$ est numerus per $m m$ diuisibilis (vbi ipsum $m$ positiuum supponimus) dabitur ordo formarum determinantis $D$ ex ordine proprie primitiuo determinantis $\frac{D}{n m m}$ dexiratus (siue duo, quando $D$ est negatiuus, nempe positiuus et negatiuus $)$ manifesto forma $\left(m, o,-\frac{\mathcal{D}}{m}\right)$ ad illum ordinem pertinebit (scilicet ad positiuum) meritoque tamquam forma simplicissima in eo considerari potest (sicuti $\left(-m, 0, \frac{D}{m}\right)$ erit simplicissima in ordine negatiuo quando $D$ neg. $)$. 


\section{$-384-$}

Si insuper est $\frac{D}{m m} \equiv \mathbf{I}$ (mod. 4 ), dabitur etiam ordo formarum det $D$ ex improprie primitiuo det, $\frac{D}{m m}$ deriuatus, ad quem manifesto forma ( $2 m ; m, \frac{m m--D}{2 m}$ ) pertinebit et pro simplicism sima in eodem habebitur. (Quândo $D$ est neg" rursus duo ordines dabuntur et in negatiuo forma $\left(-2 m,-m, \frac{D \cdot m m}{2 m}\right)$ pro simplicissirna habebitur. Ita e. g., si etiam eum casum vbi $m=1$ huc referre lubet, in quatuor ordinibus formarum det. 45 sequentes erunt simplicissimae $(1,0,-45),(2,1,-22),(3,0,-15)$ $(6,3,-6)$. Quibus ita intellectis, offert se

Problerar. Propasita forma quacungat $F e x$

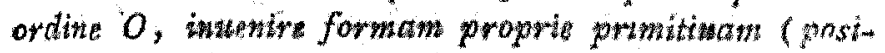
tiuom) eusdetn determinanis, ex cuits compositane cum forma in $O$ simplictssima onitur $F$.

Sol. Sit forma $F=(m a, m b, m c)$ deringtta $e$ primitiua $f=(a, b, c)$ cuits determinans $=d$, supponamusque primo, $f$ esse proprito prim mitiuam. Primo obseruamus, si forte $a$ ad $2 \mathrm{~mm}$ non sit primus, certo dari alias formas ipsi ( $a$, $b, \dot{c})$ proprie aequiualentes, quarum temini prim mi hac proprietate sint praediti. Nam per art 298 dantu mumeri ad odm primi por corman illan repraesentabiles; sit talis numerus $a^{*}=a$ as + $a b \cdot y+c y y$, supponamusque, (quod licet) $; z$ esse inter se primos; tum, acceptis 6 , it it it fiat $*-6=1$, transeat $f$ per substitutionem c, $y, \&$ in formam $\left(a^{*}, b^{\prime}, b^{*}\right)$, quae illi prow 


\section{$-385-$}

prie aequiualebit et proprietate praescripta erit praedita. Iam quum etiam $F$ et $\left(a^{\prime} m, b^{\prime} m, c^{\prime} m\right)$ proprie aequiualeant, facile perspicietur, suflicere eum casum cousiderare vbi $a$ ad $2 d m$ sit primus. Tunc $(a, b m, c m m)$ erit forma proprie primitiua (si eniur $a, \mathrm{t} b \mathrm{~m}$, crmm diuisoxem communem habereat, "hunc etiam $2 d m=2 b b m-$ $2 a \mathrm{~cm}$ implicaret) eiusdem determinantis vt $F$, confirmaturque facile, $F^{*}$ transmutari in productum e forma $(m, o,-d m)$, quae, nisi $F$ est forma negatiua, erit simplicissima ordinis $O$, in $(a, b m, c m m)$ per substitutionem $1,0,-b,-$ $c m ; o_{2} m, a, b m$, wade per criterium in obs. 4 art. 235 concluditur, $F$ ex $\left(m, o_{1}-d m\right)$ et $(a, b m$, $\mathrm{cmm}$ ) esse compositam. Quando autem $F^{\prime}$ est forma negatiua, transibit in productum e forma simplicissinna eiusdem ordinis $(-m, 0, d m)$ in positiuam ( $-a, b m,-c m m)$ per substitutionem $1,0, b,-c m, c_{1}-m,-a, b m$, adeoque ex ipsis exit composita.

Secundo, si $f$ est forma improprie primitiua, supponere licebit $a$ ad $2 d m$ esse primum; si enim haec proprietas in forma $f$ locum nondum habet, inueniri potest forma ipsi $f$ proprie aequiualens et hac proprietate praedita. Hinc antem sequitur facile, ( $a, b m, 2 \mathrm{cmm})$ esse formam proptie prinitiuam eiusdem determinantis ot $\mathcal{F}_{7}$ aeque facile confirmatur, $F$ transire in prodnctum e formis $( \pm 2 m, \pm m \pm(m-d m)),\left( \pm \frac{1}{2} a\right.$ $b m, \pm 2 c m m)$ per substitutionem $x, 0, \frac{1}{2}(1 \mp$ b), $=\mathrm{cm} ; 0, \pm 2 m_{3} \pm a_{2}(b \pm x) m_{,}$vbi signa inferiora accipienda sunt quando $F$ est forma negatiua, superiora in casibus reliquis, adeo$\mathrm{Bb}$ 
que ex his duabus formis esse compositam, quarum prior exit simplicissima ordinis $O$, pusterior forma proprie primitiua (positiua).

251. Problema. Propositis dinabns formis $F$, $f$ einsdem determinantis $D$ at ad ende'm ardinm $O$ per-

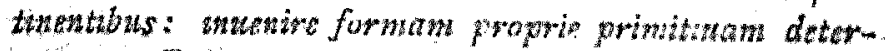
minantis $D$, quae cuen f composita producat $F$.

Sol. Sit forma simplixissima ordinis $O_{\text {; }}$ F, $f$ formae proprie primititae det. $D$, quae cum - compositae producant ipsas $F^{*}, f$ resp. $;$ dentque fi fom proprie primitian quate cum f com posita producat of. Tune forma $F$ composita erit e tribus fomis $\phi, f, f$, siue duabus $f, f$, Q. E. I .

Quaeuis itaque classis ordinis dati considerari potest tamquam composita ex quacunque classe data eiusclem ordinis et aliqua classe proprie primitiua eiusdern determinaxtis.

252. Trizonema. Pro determinante dato in

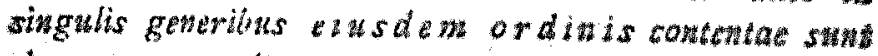
classes aeque muttae.

Dem. Pertineant genera $G$ et $H$ ad eumdem ordinem, constet $\sigma$ ex $n$ classibus $K, K_{*}$ $K^{\prime \prime} \ldots K^{n-1}$, sitque $D$ classis aliqua e genere $H^{\prime}$ Inuestigetur per art praec. classis proprie primi titu $M$ eirsdem deterninantis, ex cuats corapositione cum $K$ prodeat $L$, designenturque classes quae oriuntur ex compositione classis $M$ curn ${ }^{*}$ " $K^{\prime \prime}+\ldots K^{4-1}$ resp. per $L^{\prime}, L^{\prime \prime}{ }_{*} \cdot L^{n-1}$, Tunc ex 
obs. vltima art. 249 sequitur, omnes classes $L_{\text {, }}$ $L^{i}, Z^{\prime \prime} \ldots L^{4-1}$ esse dituersas, et per art. 248 ornnes pertinebunt ad gemu iden, $\dot{i}$. e. ad genus H. Denique perspicietur facile, $I t$ alias classes pronter lus coatinere non posse, quam quaeuis classis gineris $H$ tamquam composita considerari possit ex $M$ et alia classe eiusdem determinantis quae necessurio semper erit e genere $G$. Quocirca $H$ perinde vi $G$ continet $n$ classes diuersas, Q. E. D.

255. Theorema praecedens supponit ardinis fintitatem necrne ad ordincs diuersos est extentendum. Ita e. G fro deteminante - 17x dentur 20 clases posituae, quas reducuntur ad quature mines: his orline propre primitiuo duo conturum genewa, vituraque sex classes complectur, in ordine imyr. primituo duo genera quatuor elasses possident, sirgula biras; in or dive derituto ex 0 proprie prim det - 19 vinicum est genus quatuor classes complectens; denique 0 . deriuatus ex impr. prim. det -10 vnicum genus habet ex vna dasse constans; perinde se habent classes negatiuae. Operae itaçue pretium est, in principium generale inquirere, a quo nexus inter multitudines classium in diuersis ordinibus pendeat. Sapponamus, $K, L$, esse duas classes ex eodem ordine (positiuo) $O$ determi nantis $D$, atque $M$ classem proprie primitiuam eirusden det, ex cuits compositione cum $K$ oriatur $I$, qualis per art. 251 semper potest assignar. Iam In gitibustam casibus feri potest, vt $M$ sit vnica classis pr. primitita quae cum $K$ composita producat $L$; in aliis plures classes chuersae pr. primitituae exstare pos$\mathrm{Bb}=$ 
sunt hac proprietate praeditae. Supponamus genexaliter, dari $r$ huiusmodi classes pr. primitiuas, $M, M^{\prime}, M^{\prime \prime} \ldots M^{*-}$, quae singulae cum $K$ compositae producant eandern rlassem $L$, desiznemusque illarum complexum per $W$. Porro sit $L^{t}$ alia classis ordinis $O$ (a classe $L$ diuersa), atque $N^{N}$ classis pr. prim. det. $D$ quae cum $L$ composita efficiat $L$, designeturque complexus classium $N^{\prime}+M, N^{\prime}+M^{\prime}, N^{\prime}+M^{\prime \prime} \ldots N^{\prime}+M^{\prime}+$ (quae omnes erunt proprie primitiuae et.inter se diuersae) per $W^{\prime}$. Tunc perspicietur facile, $K$ cum classe quacunque ex $W$ compositam producere $L^{\prime}$, vnde concluditur, $W$ et $W$ nullam classem communem haberes praeterea nullo negrotio conprobatur, nullam elassem pre prisnitiuam in complexu Th non contentam dari, quae eum $K$ commposita producat ipsam $L 4$. Eadem modo patet, si $L^{\prime \prime}$ sit alia classis ordinis $O$ a classibus $L, L^{\ell}$ diuersa, dari $r$ formas pr. primitiuas tum inter se tum a formis, $W, W$ dinersas, quar singulae cum $K$ compositae ipsam $L^{\prime \prime}$ protucent, "t perinde res se habebit pro omnibus reliquis classibus ordinis 0 . Quoniam vero quatens classis pr. yrim. (positina) determinantis $D$ cum $K$ composita classem ordinis $O$ producit, facile hinc collyitur, si multitudo omnium classium oodinis 0 sit $n$, multitudinem omanium classium proprie primitiuarum (positiuarum) eitsdem determinantis fore $r n$. Habernus itaque regulaw generalem: Denotantibus $K, L$ classe quascunque ordinis 0 , at que $r$ multitudinem classium proptie primitumrum . diuersarum eiusdem determinantis, quae singulae cum $K$ compositae ipsam $L$ proctuctur, multitudo omnium classium in ordine proprie 


\section{- $389-$}

primitiuo (positiuo) $r$ vicibus maior exit quam multitudo classium ordinis 0 .

Quum classes $K, L$ in ordine $\mathrm{O}$ omnino ad libitum assumi possint, etiam classes identicas accipere licebit, et quidem e re erit ea classe vti, in qua continetur forma huius ordinis simplicissima. Quam itaque pro $K$ et $L$ assumendo, res eo reducta et, vt omnes classes proprie primitiuae assignentur, quae cum $K$ compositae ijsam $\not K$ reproducant. Huc via stemitur per sequens

254. Trrorema. Si $F=(A, B, C)$ est forma simplicissima ordanis $O$ determinantis $D$, atque $f=(a, b, c)$ forma propre primtina ezusdem determituantis: per hatuc forman f rapressentari potarit whmerns $A A$, si $F$ oritur per compositionew formatum $f, F ;$ at wice warsa $F$ ex se ipsea atque $f$ composita wit, sil $A A$ per $f$ reprotatentari potest.

Den. I. Si $F$ in productum $f F$ mansit per substitutionem $p, p^{\prime}, p^{\prime \prime}, p^{\prime \prime \prime} ; q, q^{\prime}, q^{\prime \prime}, q^{\prime \prime \prime}$; ex art 235 habenus $A$ ( $a q^{\prime \prime} q^{\prime \prime}-a b q q^{\prime \prime}+c q q$ ) $=A$, wnde $A A=a q^{\prime \prime} q^{\prime \prime}-2 b q q^{\prime \prime}+c q q$. Q.E.P.

II. Si supponitur, $A$ per $f$ repraesentaxi posse, designentur valores indeterminatarum per quos boc efficitur per $t_{i}^{\prime \prime},-q$, siue sit $A A=a q^{\prime \prime} q^{\prime \prime}-$ sbgg $q^{\prime \prime}+c q q$, ponaturque $q^{\prime \prime} a-q(b+B)=A p$, $q C=A p, q^{\prime \prime}(b-B)-q c=4 p^{*},-q^{t} C=$ Ap in, $q^{\prime \prime}{ }^{\prime}-q(b-B)=q q^{\prime \prime}(b+B)-$ $q C=A q^{n}$ Q Quo facto, facile confirmatar, $F$ $\mathrm{Bb} 5$ 
transire in productum $f F$ per substitutionem $p, p^{t}$, $p^{\prime \prime}, p^{\prime \prime \prime} ; q, q^{\prime}, q^{\prime \prime}, q^{\prime \prime \prime}$, atque adeo ex $f$ et $x^{\prime}$ compositam esse, si modo omnes numeri $p, p^{r}$ etc. sint integri. Iam per descriptionem formate simplicissimae, $A$ est vel $\alpha$ vel $\frac{x}{2} B$, adeoque $\frac{a}{A}$ integer; ibinde patet, $\frac{C}{A}$ semper esse integrum. Hinc $q^{\prime}-p, p^{\prime}, q^{\prime \prime \prime}-p^{\prime \prime}, p^{\prime \prime \prime}$ erunt integri, superestque ateo tantummodo, vt probetur $p$ et $p^{\prime \prime}$ esse integros. Fit autem $p p+\frac{2 p q B}{A}=a_{2}$ $p^{\prime \prime} p^{\prime \prime}+\frac{2 p^{\prime \prime} q^{\prime \prime} B}{A}=c$; quamobrem si $B=0$, fit $p p=a, p^{\prime \prime} p^{\prime r}=c$, et proin $p, p^{\prime \prime}$ integri, si vo ro $B=x^{\prime} A$, fit $p p+p q=a_{2} p^{\prime \prime} p^{\prime \prime}+p^{\prime \prime} q^{\prime \prime}=$ $c$, vnde aeque facile concluditur, $p$ et $p^{\prime \prime}$ to hoc quoque casu esse integros. Ex his colligitur, $F$ ex $f$ et $F$ esse compositam. Q. E. $S$.

255. Prohlema itaque eo reductum est, vt omnes classes proprie primitituas deterninamis $D$ assignare oportent, per quarum formas repratow sentari potest A.\%. Manifesto A.t repraesentari potest per quarnuis formam cuius terminus primus est vel $A A$ vel quadratum partis aliquotae ipsius $A$; vice versa autem, si $A A$ repraesentari potest per forman $f$, tribuendo ipsius indeterminatis valores cte, $\gamma^{2}$ quorum diuisor communis maximus $e$, forma $f$ per substitutionem $n, 6, y$, transibit in formam cuins terminus primus $\frac{A}{20}$, formacteo haec proprie aequiualebit forma $f$, si

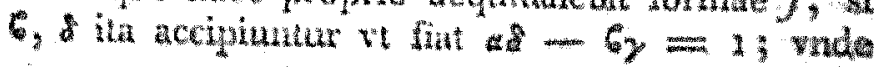


patet, in quauis classe, jer cuius formas repraesentari possit $2 A$, inuenint fomas, quarum terminus prinus sit $A A$ vel quadratum partis aliguotat ipsius $A$. Res itaque in eo versatur, vt onuas classes proprie primitiuat det. $D$ eruantur, in quibus humsmodi format occurrant, quod obtinetur stquenti modo: Sint $a, a^{\prime}, a^{\prime \prime}$ etc omnes diuirores (positiui) ipsius $A$; inuestigentur omnes valores expr. $\sqrt{ } D(\bmod a a)$ inter o et $a a-$ I incl. siti, qui sint $b, b, b u$ etc statuaturque $b b$ $-D=a a c, b b^{\prime}-D=a c^{\prime}, b n b+D=$ aact etc: complexus formarum $(a a, b, c),\left(a a_{3}\right.$ $b, c^{d}$ ) etc designetur per $V$. "Tunc facile perspicitur, in quatuis classe dat. $D$, in qua occur wat forma cuius terminus prinus an, etian aliguan fomam ex contentam esse debere. Simili modo eruantur ommes formae del. $D$, quarum terninus primus $a^{\prime} a^{*}$, medius inter o et $a^{\prime} a^{\prime}$ - incl. situs, designeturque ipsarum comple-

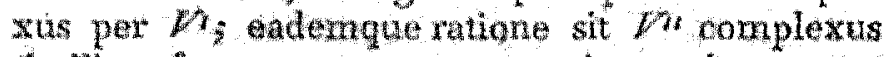
shailtum formarum quarim terminus primus allat

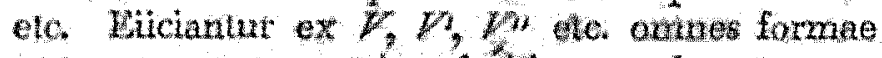
quae non sunt proprie primituae, reducantur reTiguae in classes, et, si forte plures adsint ad e mutem chassem pertinentes, in singulis classibus vna tantum retineatur. Hoc modo onnes chises quaesitate habebuntur, eritque haman multitudo ad Ynitatem, it multitulo omnitum classium proprie primituarum (posititarum) al pultududinen clas chum in ordine $U$.

Ex. Sit $D=-E 3^{1}$, atcue $O$ ordo posituus deriuatus ox ordine improprie primitiuo det - 69 , in quo forma simplicissina $(6,3,90)$ $\mathrm{BB} 4$ 
siue $A=6$. Hic $a, a^{\prime}, a^{\prime \prime}, a^{\prime \prime \prime}$ erunt $1,2,3,6$; $V$ continebit formam $(1,0,531) ; D$ has $(4,1$, $133),(4,3,135)$; Vu has $(9,0,59),(9,3,60)$, $(9,6,63) ;$ denique $V^{\prime \prime \prime}$ has $(36,3,15),(36,9,17)$, $(36,16,21),(36,21,27),(56,27,35),(36,33,45)$ : sed ex his duodecim formis sex sunt reiciendae, puta ex $V^{\prime \prime}$ secunda et tertia, ex $V^{\prime \prime}$ prima, tertia; quartá et sexta, quae omnes sunt formae deriuatae; sex reliquae omnes ad classes diuersas pertinere inueniuntur. Reuera multitudo classium. proprie primitiuarum (positiuarum) det -531 est 18 , multitudoque classium impr. primitiuarum (pos) det - 59 (siue multitudo elassium det. - $53^{1}$ ex his deriuatarum) 3 , adeoque illa ad hanc vt 6 ad 1 .

256. Solutio haec per obseruationes sequentes generales adhuc rnagis illustrabitur.

I. Si ordo 0 est deriuatus ex ordine proprie primitiuo, metietur $A A$ ipsum $D ;$ si vero $O$ est irnpr. primitiuus vel ex impr. prim. deriuatus, erit $A$ par, $D$ per $\frac{x}{4} A A$ diuisibilis et quotiens $\equiv 1$ (mod. 4$)$. Hinc quadratum cuiusuis diuisoris ipsius $A$ metietur vel ipsum $D$, vel sältem ipsum $4 D$, et in casu posteriori quotiens semper erit 1 (mod. 4).

II. $\mathrm{Si}$ a $a$ ipsum $D$ metitur, omnes valores expr. $\sqrt{ } D$ (mod $a a$ ), qui quidem inter o et $a a$ -1 iacent, erunt $0, a, 2 a \ldots a \alpha-a$, adeoque a multitudo formarum in $D$; sed inter has tot tantummodo erunt proprie primitiuae, quot numerorum $\frac{D}{a a}, \frac{D}{a a}-1, \frac{D}{a a}-4 \ldots \frac{D}{a a}-(a-1)^{2}$ 
cum a diuisorem communem non habent. Quando $a=1, V$ ex rnica forma constabit, $(1,0$, - D), quae semper erit proprie primitiua. Quando $a$ est 2 vel potestas quaecunque ipsius 2 , semissis illorum $a$ numerorum par erunt, semissis impar; quare in $V$ aderunt $\frac{\pi}{2} a$ formae proprie primitiuae. Quanda a est alius numerus primus $p$ vel potestas, numeri primi $p$, tres casus sunt distinguendi scilicet, omnes illi a numeri ad a primi erunt, adeoque omnes formae in $V$ pr. primitiuae, si $\frac{D}{a a}$ per $p$ non est diuisibilis simulque non residuum quadraticum ipsius $p$; si vero $p$ ipsum $\frac{D}{a a}$ metitur, in $V$ erunt $\frac{(p-\mathrm{I}) a}{p}$ formae pr. primitiuae; denique si $\frac{D}{a t}$ est res. quadr. ipsius $p$ per $p$ non diuisibile, in $V$ erunt $\frac{(p-2) a}{p}$ formae pr. primitiuae. Haec omnia nullo negotio dernonstrantur. Generaliter autem posito $\boldsymbol{a}=$ $2^{v} p^{z} q^{x} r c \ldots$, designantibus $p, q, r$ etc numeros primos impares diuersos, multitudo formarum pr. primitiuarum in $V$ erit $N P Q R$..., vbi statui debet $N \leq 1$ (si $v=0)$ vel $N=2^{r-1}($ si $\nu>0) ; P=p$ (si $\frac{D}{a a}$ est non residuum quadr. ipsius $p$ ) vel $P=$ $(p-1) p^{\pi-1}$ (si $\frac{D}{a a}$ per $p$ est diuisibilis) vel $\boldsymbol{P}=(p-2) p^{-1}$ (si $\frac{D}{a a}$ est res. qu. ipsius $p$ per $p$ non diuisibile); $Q, R$ etc autem eodem modo ex $q, r$ etc. suat defmiendi vt $P$ ex $p$. $\mathrm{Bb} 5$ 


\section{$-$

III. Si a ipsum $D$ non metitur, erit $\frac{4 D}{4 a}$ integer et $\equiv 1(\bmod .4)$, valoresque expr. $\sqrt{ } D$ (mod. aa) hi $\frac{\pi}{2} a, \frac{3}{2} a, \frac{5}{2} a \ldots a a-\frac{r}{2} a$, wnde multitudo formarum in $V$ erit $a$, tot autem inter ipsas erunt proprie primitiuae fuot ex numeris $\frac{D}{a a}-\frac{x}{4}, \frac{D}{a b}-\frac{2}{4}, \frac{D}{a t}-\frac{25}{4} \ldots \frac{b}{a b}-\left(a-\frac{x}{2}\right)^{2}$ ad $a$ sunt prim: Quoties $\frac{4 D}{a t} \equiv 1$ (mod 8$)$, ornnes hi, numeri erunt pares, adeoque in $V$ nulla forma pr. primitina; quando autem $\frac{4 D}{a b}=5$ (mod. 8), omnes illi numeri enunt impares; adeoque omnes formae in $V$ pr. primitiuze, si $a$ est 2 vel potestas ipsius 2, generaliter autem in hoc casu tot formae pr. primitiuae in $/$ erunt, quet illorum numerorum per nullumi diuisorem primuma imparem ipsizs $a$ sunt dinisibiles. Multitudo haec erit $N P Q R \ldots$, si $a=2 p^{*} q^{x} r^{*} \ldots$, rbi stature oportat $N=2^{p}$, ipsos $P, Q, R$ etc. antem atodem modo ex $p, q, r$ etc, deriure $x$ in casta praecedente.

IV. Hoc itaque modo multitudines formarum pr. primitiuarum in $V_{,} l, V^{\prime \prime}$ etc. dofiniri possunt; pro aggregato omnium harum multhdinurn haud difficulter ernitur sequens regula ge.

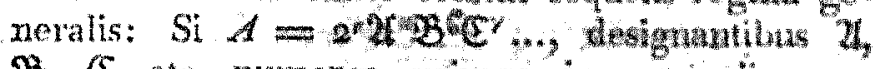
$\mathfrak{B}, \mathfrak{C}$ ete. numeros primos impares diuersos, multitudo totalis omnium formarum pr. primitiwarum in $\nu^{\prime}, \nu^{\prime}, b^{\prime \prime}$ etc, erit $=A$ nabc..., wi statua debet $\mathfrak{n}=1$ (tum si $v=0$, tum si $\frac{4 D}{2 A}$ 
$\equiv \mathrm{E}$, mod. 8), vel $\mathrm{n}=2(\mathrm{si} v>0$ simulque $\frac{1}{A A}$ integer), vel $\mathrm{n}=3\left(\mathrm{~s},>0\right.$ simulque $\frac{4 D}{A A}$ $\equiv 5$, mod. 8); porro $a=\mathfrak{N}$ (si $\mathfrak{X}$ ipsum $-\frac{4 D}{A A}$ metitur), vel $a=2 \pm 1$ (si $\mathfrak{U}$ ipsum $\frac{4 D}{\mathscr{A} A}$ non metitur, accipiendo signum superius vel inforius prout $4 A$ est non- residuum vel res. qu. ipsius $\mathfrak{F})$, denique $\mathfrak{b}, \mathfrak{c}$ etc eodern modo ex $\mathfrak{B}$, $\mathcal{E}$ derituri to $a$ ex $\mathfrak{a}$. Demonstrationem fusius hic explicare, breutias non permittit.

v. Iam quod attinet ad multitudinem elassium, quas suppeditant formae pr. primitiuae in $V, I, 7$ it etc, tres casus sequentes sunt distingtundi.

Primo, quando $D$ est numerus negatiuus, singulae format pro primititae in $V, V$ etc. constiuent classem pectharem, sine multitudo ipsa classium quaesitarum exprimetur per formulam in obseru praec traditan, duobus casibus exceptis, scilice vbi $\frac{4 D}{A A}$ vel $=-4$ vel $=-3$, sine vbi $D$ vel $=-A A$ vel $=-3 A A$ Ad demonstrationem huins theorematis manifesto ostendi tantummodo debet, fieri non posse, w pluae formate diuersae ex $r, F, V u$ ete sint proprie aequiualmtes. Supponamus itaque, $\left(h h_{\text {: }}\right.$ $v, k),(h h, i, k)$ esse duas formas diuersas $\mathrm{pr}$. primitiuas ex $\mathscr{V}, W^{\prime}, W^{\prime \prime}$ etc. ad eandem classem pertinentes, transeatcuse proor in posteriorems pes substitutionem propriam $6,6, \gamma, \delta$; nde habc- 


\section{- $396-$}

buntur aequationes os $-6 y=1$, hhot $+2 i \times y$

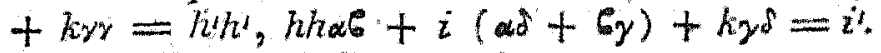
Hinc facile concluditur, primo $y$ certo non esse $=o$ (inde sequeretur, esse $a= \pm 1, h h=$ $h h^{\prime}, i^{\prime} \equiv i(\bmod h h)$ adeoque formas propositas identicas, contra hyp.); secundo, $\gamma$ dinisibi. lem esse- per diuisorem maximum communtm numerorum $h, h^{\prime}$; (ponendo enim liuno diuisorem $=r$, hic manifesto etian metietur ipsos $2 i$, $2 i^{\prime}$, ad $k$ vero erit primus; praeterea $r *$ metietur ipsum $h h^{2} k-h^{2} h^{\prime} k^{\prime}=i i-i^{2} i^{\prime} ;$ vade facile deducitur, $r$ etiam metiri insum $i-i b$ ham betiur autem $\alpha i^{\prime}-k h^{\prime} h^{\prime}=\alpha i+\gamma k$, vnde $\gamma k$ et proin etiam $\gamma$ druisibilis erit per $r$ ); tertio, esse $(\alpha h h+, i)^{2}-D_{2 \gamma}=h h h^{\prime} h^{\prime}$ Ponendo itaque o $h h+\gamma i=r p, y=r q, p$ et $q$ erunt in tegri quorum posterior non $=0$; atque $p p-$ $D_{q q}=\frac{h h h^{\prime} h^{\prime}}{r} \cdot$. Sed $\frac{h h h^{\prime} h^{\prime}}{r r}$ erit numerus minimus per $h h^{2}$ et $h^{\prime} h$ simul diuisibilis adeoque ipsum $A A$ et proin etiam ipsum ${ }_{4} D$ nutietur, quare $\frac{4 D r}{h h h^{\prime}}$ erit integer (negatimus), quem statuendo $=-\varepsilon$, erit $p p-D q q=-\frac{4 D}{}$ siue $4=\left(\frac{2 r p}{h h^{r}}\right)^{2}+e q q$, in qua aequatione pars $\left(\frac{2 r p}{h h^{2}}\right)^{2}$ tamquam quadratum ipso 4 minus necessario erit vel o vel a. In casu priori erit egq $=4$, et $D=-\left(\frac{h h^{\prime}}{r q}\right)^{2}$, vnde sequitur, $\frac{4 D}{A D}$ esse quadratum signo negatiuo affectum adeoque certo non $\equiv 1$ (mod. 4 , neque adeo 0 ordinem improprie primitium neque ex improprie prit- 


\section{$-397-$}

mitiuo deriuatum. Tinc $\frac{B}{A A}$ erit integer, vnde facile deducitur, $e$ per 4 esse diuisibilem, $q q=$ 1, $D=-\left(\begin{array}{c}h h^{\prime} \\ n^{\prime}\end{array}\right)^{2}$ atque etiam $-\frac{A A}{D^{-}}$integrum. Hinc necessario erit $D=-A A$ siue $\frac{D}{A A}=$ - 1 , quae est exceptio prima. In casu posteriori erit $e q q=5$, wnde $=5$ et $4 D=$ $-3\left(\frac{h h^{2}}{r}\right)^{2}$, hinc $\left.3 \frac{h h^{k}}{A}\right)^{2}$ erit integer, qui, quoniam per quadratum integrum $\left(h h^{A}\right)^{2}$ multiplicatus producit 3 , non poterit esse alius quam 3 ; hine $4 D=-3 A A$ siue $D=-3 A A$, quae est exceptio secunda. In omnibus igitur reliquis casibus omnes formae pr. primitiuae in $\mathscr{V}^{2} \mathscr{V}^{\prime}$, $\mathrm{V}^{\text {th }}$ etc: ad classes diuersas pertinebunt. - Pro casibus exceptis ea, quae ex disquisitione hand diffcili sed hic breuitatis caussa supprimenda resultauerunt, apposuisse sufficiat. Scillcet in priori, ex formis pr. primitiuis in $V$, $W^{\prime}$, $P^{\prime \prime}$ etc. binae semper ad eandem classem pertinebunt, in posteriori ternae, ita "t multitudo omnium classium quaesitarum in illo casu fat semissis, in hoc triens valoris expressionis in obs, praec, traditae.

Secundo quando $D$ est numerus positiuus quadratus: singulae formae pr. primitiuae in $V_{\text {, }}$ $F^{\prime}, V^{\prime \prime}$ etc. sine exceptione classem peculiarem constituunt. Supponamus enirn, $\left(h h_{h}, k\right),\left(h^{2} h^{\prime}\right.$, $i, k^{\prime}$ ) esse duas tales formas diuersas proprie aequixalentes, transeatque prior in posteriorem per substitutionem propriam $*, 6, \gamma, \&$ Tum 
patet, omnia ratiocinia pro casu praec adhibits, in quibus non supponatur $D$ esse negatium, etian hic valere. Designantibus itaque $p, q, r$ iden ve illic, etiam hic erit $\frac{4 D h r}{h h h}$ integer, at non imxplius negatiuus sed positinus insuperque quadra. tus, quo posito $=s g$, ent $\left(\frac{2 r p}{h h^{2}}\right)^{2}-g g q q=4$ Q. E. A., quia differentia duonm quadratorum nequit esse 4 , nisi quadratum minus fuexit $0_{5}$ quamobrein suppositio consistere nequit.

Pro casu tertio antem, vbi $D$ est numerus positiuus non quadratus, regulam generalem pro comparanda mrttitudine formarum pr, primitiua rum in $V, V^{\prime}, D^{\prime}$ etc. cum multitudine classitin diuersarum inde resultantium hucusque non habemus. Id quidem asserere possumus, hanc vel illi aequalem rel ipsins partern aliquotam esse; quin etiam nexum singularem inter quotiontem horum muneroum et falores minianos ipsorm $t, u$ aequationi $t-D D_{u}=A d$ satidicientes deteximus, quem hic explicars nimis probixma foret; an vero possibits sit, dilum quotisunems in ommibus casibus ex sola inspectone numerorum $D, A$ comoscere ( $w$ in casihus pratcos), de hac re nihil certi promunciare posumus. Eces quatarn exernpla, quornm mumerun quisque facile augore poterit. Pro $D=13, A=$ multitulo formarum pr. prim. in $V^{*}$ ew. eat 3 ,

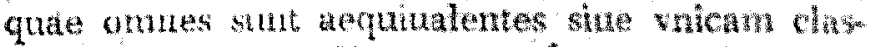

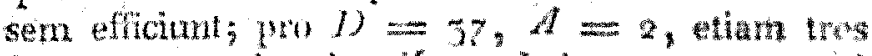

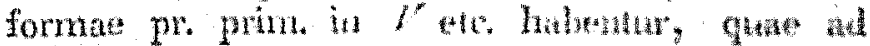
tres classes dimersas paxtineme yro $D=588_{1}$ 
$A=\tau$, habentur octo formae pr. prim. in $V$ etc. quae efficiunt quatuor clases; pro $D=869$, $A=1 z$ in $V$ etc. sunt 18 formae pr. primitiuae, totidem pro $D=1445, A=17$, sed quie pro illo determinante in duas classes discedunt, pro hoc in sex.

VI. Ex applicatione huius theoriae generaIis ad eum casum, vbi $O$ est ordo improprie printituus, colligitur, multitudinem classitm in hoc ordine contentarum fore ad mulutudinem ormium classium in ordine proprie primitiuo, v 1 ad multitudinem classinm diuersarum quas hae tres formae $(1,0,-D),\left(4,1, \frac{r-D}{4}\right)$, (4. $5, \frac{9-D}{4}$ ) efficiunt. Lt quidem hinc resultabit vnica classis, quando $D \equiv \pm(\bmod .8)$, quia in hoc casu forma secunda et tertia sunt improprie primitiuae; quando vero $D \equiv 5$ (mod. 8), illae tres forman omnes erunt propria primithat toticencue clases diuersas producent si $D$ est negatiuns, wico cast excepto, wb $D=-5$, in quo wnicam classem constituunt; denique casus vbi $D$ ) est positius (formae $8 n+5)$ ad eos pertinet, pro quibus regula genatralis hactenus desideratur. If tamen asserene possumus, illas tres formas in hro casu vel ad tres classes diuersas pertinere vel ad vuicam, numg̨uan ad duas; racile cnim perspicitur, si forme $(1,0,-D),\left(4,1, \frac{x-D}{4}\right),(4,3$, $\frac{9-D}{4}$ ) resp. pertineant ad elasses $K, K^{\prime}, K^{\prime \prime}$ foro 


\section{$-400-$}

$K+K^{\prime}=K^{\prime}, K^{\prime}+K^{\prime}=K^{\prime \prime}$, adeoque, si $K$ et $K^{\prime}$, identicae esse supponantur, etiam $K^{\prime}$ et $K^{\prime \prime}$ identicas fore, simili ratione si $K$ et $K^{\prime \prime}$ supponuntur esse identicae, etiam $K^{\prime}$ et $K^{\prime \prime}$ erunt; denique quum sit $K^{\prime}+K^{\prime \prime}=K$, ex suppositione, $K^{\prime}$ et $K^{\prime \prime}$ identicas esse, sequitur, etiam $K$ et $K^{\prime \prime}$ coincidere; vnde colligitur, vel omnes tres classes $K^{\prime}, K^{\prime}, K^{\prime \prime}$ esse diuersas, vel omnes tres identicas. E. g. Infra 600 dantur 75 numeri formae $8 n+5$, inter quos sunt 17 determinantes pro quibus casus prior locum habet siue multitudo classium in ordine pr. primitiuo ter maior est quam in impr primitiuo, puta $37,101,141,189$, $197,269,325,333,349,573,381,389,397$, $405,485,557,573$, pro 58 reliquis casus posterior valet, siue multitudo classium in vtroque ordine est aequalis.

VIr. Vix opus exit, obseruare, per disquisitionem praecedentem non solum multitudines classium in ordinibus diuersis eiusdem determinantis comparari posse, sed illam etiam ad quosuis determinantes diuersos qui rationem quadratorum inter sé teneant esse applicabilem. Scili= cet designante $O$ ordinem quemeñnque det. $d m m$, of ordinem det $d m^{\prime} m^{\prime}$, O comparari poterit cum ordine proprie primitiuo det. dimm, atque hic cum ordine deriuato ex ordine pr. prim. det. $d$, siue, quod respectu multitudinis classium eodem redit, cum hoc ordine ipso, et cum eodem prorsus simili ratione comparari poterit ordo 0 .

257. Inter omnes classes in ordine dato determinantis dati imprimis classes ancipites disqui- 
sitionem vberiorem postulant, determinatioque multitudinis harum classium ad multa alia viam nobis aperiet. Sufficit autem, hanc multitudinem in solo ordine pr. primitiuo assignare, quum casus reliqui ad hunc facile reduci possint Hoc negotium ita absoluemús, vt primo omnes formas ancipites pr primitiuas $(A, B, C)$ determirantis propositi $D$, in quibus vel $B=0$ vel $B$ $=r A$, eruere, tunc ex hanum multitudine multitudinem omnium classium ancipitum pr. primitiuarum det. $D$ inuenire doceamus.

T. Onnes formae pr. primitiuae $(A, O, C)$ determinantis $D$ manifesto inueniuntur, accipiendo pro $A$ singulos divisores ipsius $D$ (tum positiue tum negatiue) pro quibus $C=-\frac{D}{A}$ fit primus ad $A$ Quando itaque $D=1$, duaehuiusmodi formae dantur $(1,0,-1),(-1$, $0,1)$; totiden quando $D=-1$, puta $(1, o$, 1), $(-1,0,-1)$; quando $D$ est numerus primus aut numeri primi potestas (siue signo positiuo siue negatiuo), quatuor dabuntur $\left(\mathrm{t}, \mathrm{o}_{2}\right.$ $-D),(-1,0, D),(D, 0,-1),(-D)$ $o$, 1). Generaliter autem, quando $D$ per $n$ numeros primos diuersos est diuisibilis (inter quos hoc loco etiam 2 in computum ingredi debet) dabuntur omnino $2^{n+}$ hiiusmodi formae; scilicet posito $D= \pm P Q R \ldots$, designantibus $P$, $Q, R$ etc. numeros primos diuersos atit numerorum primorum diuersorum potestates quorum multitudo $=n$, valores ipsius $A$ enut $1, P, Q$, $R$ etc atque producta ex quotcunque horum numerorum; harun valorum multitudo fit treo-

Co

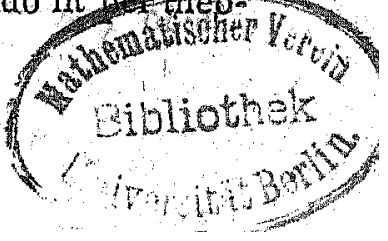




\section{$-402-$}

niam combinationum $2^{\text {a }}$, sed duplicanda est, quo. niam singulis valoribus tum signum positium tum negatiuam tribuere oportet.

II. Simili modo patet, omnes formas pr. primitiuas $(2 B, B, C)$ determinantis $D$ obtineri, si pro $B$ acciptantur ornes diuisores ipsius $D^{\prime}$ (positiue et negatiue), pro quibus $C=\frac{\pi}{2}(B-$ $\frac{D}{B}$ ) fit integer et ad $2 B$ primus. Quum itaque $C$ necessario debeat esse impar, adeoque $C C \equiv$ 1 mod. 8 , ex $D=B B-2 B C=(B-C)^{2}-C C$ sequitur, $D$ esse vel $\equiv 3$ (mod. 4 ), quando $B$ impar, vel $\equiv o$ (mod. 8 ), quando $B$ par; quoties itaque $D$ alicui numerorum $1,2,4,5,6$ sec.mod. 8 est congruus, nullae huiusmodi formae dabuntur. Quando $D$ E $3(\bmod 4), C$ fit integer et impar, quicunqué diuisor ipsius $D$ pro $B$ accipiatur; ne vero $C$ divisorem communem cum $2 B$ habeat, $B$ ita accipi debebit, vt $\frac{D}{B}$ ad $B$ fiat primus; hinc pro $D=-1$ duae formae habentur $(2,1,1)$, $(-2,-1,-1)$, generaliterque facile perspicitur, si multitudo omnium numerorum primorum ipsun $D$ metientium sit $n$, omnino emergere $2^{n}+$ formas, - Quando $D$ per 8 est divisibilis, $C$ fit integer, accipiendo pro $B$ diuisorem quemcunque parem ipsius $\frac{1}{2} D$; conditioni alteri autem, vt $C=\frac{\pi}{2} B-\frac{D}{2 B}$ ad $2 B$ sit primus, satisfit primo, accipiendo pro $B$ omnes diuisores impariter pares ipsius $D$, pro quibus $\frac{D}{B} \operatorname{cum} B$ diuisorem communem non habet, quorum mul titudo (habita ratione diuersitatis signorum) erit 
$2^{\text {n }}+$ si $D$ per $n$ numeros primos impares diversos diuisibilis esse supponitur; secundo, accipiendo pro $B$ omnes diuisores pariter pares ipsius $D$, pro quibus $\frac{D}{2 B}$ fit primus ad $B$, quorum multitudo queque erit $2^{n+1}$, ita vt in hoc casu omnino habeantur $2^{\text {nt } 2}$ huusmodi formae. Scilicet ponendo $D= \pm 2^{\mu} P Q R . .$, designante $\mu$ exponentem mąiorem quam $2, P, Q, R$ numeros primos impares diuersos aut talium numerorum primorum potestates quorum multitudo $n$ : tum pro $\frac{\pi}{2} B$, tum pro $\frac{D}{2 B}$ accipi possunt valores $1, P, Q, R$ etc productaque ex quotcunque horum numerorum, signo et positiuo et negatiuo.

Ex his omnibus colligitur, si $D$ per $n$ numeros primos impares diuersos diuisibilis supponatur (statuendo $n=0$, quando $D= \pm 1$ aut \pm 2 aut potestas binarii), multitudinem omnium formarum pre primitiuarum $(A, B, C)$, in quibus $B$ vel ovel $1, A$ fore $2^{\mathrm{n}-\mathrm{I}}$ quando $D$ aut $\mathrm{I}$ aut $=5(\bmod 8), 2^{n+2}$ quando $D \equiv 2,3,4$, 6 aut 7 (mod. 8 ); denique $\mathrm{e}^{\mathrm{n}+3}$ quando $D$ = $($ mod. 8). Quam comparando cum iis quae in art. 231 pro multitudine ormium characterum possibilium formarum primitiuarum det $D$ tradidimus, ohseruamus, fllam in omnibus casibus pracise esse duplo hac maiorem. Ceterum rato nifestum est, quando $D$ sit negatiuus, inter illas formas totidem positiuas affore quot negatiuas.

258. Omnes formae in ant praec erutae manifesto pertinent ad classes ancipites, et vice $\mathrm{C}$ C 2 
versa in quauis classe ancipite pro primitiua det. $D$ saltem vna illarum formarum vontenta esse debet, in tali enim classe certo adsunt formae ancipites et guiuis formae ancipiti pr primitiuae $(a, b, c)$ det $D$ aliqua formarum art praec. aequiualet, scilicet vel $\left(a, 0,-\frac{D}{a}\right)$ vel $(a$, $\left.\frac{\pi}{2} a, \frac{1}{4} a-\frac{D}{a}\right)$, prout $b$ vel $\equiv$ o vel $\equiv \frac{1}{2} a$ (mod. a). Problema itaque eo reductum est, vt quot classes diversas illae formae constituant inuestigemus.

Si forma $(a, 0, c)$ est inter formas art. praec, forma $(c, 0, a)$ inter easdem occurret et ab illa semper erit diuersa, vnico casu excepto $\forall b i=c= \pm 1$ adeoque $D=-1$, quem aliquantisper seponemus. Quoniam vero hae formae manifesto ad eandem classem pertinent, sufficit vnam retinere, et quidem reiiciemus eam, cuius terminus primus est maior quam tertius; eum casum vbi $a=-c=+1$ siue $D=1$ quo que seponemus. Hoc modo omnes formas $(A$, $0, C$ ) ad semissem reducere possumus, retinendo. e binis semper vaam; et in ommibus remanentibus erit $A<\sqrt{ } \pm D$

Simili nodo si inter formas art praec. occurrit forma $(2 b, b, c)$, inter easdem reperie$\operatorname{tur}(4 c-2 b, 2 c-b, c)=\left(-\frac{2 D}{b},-\frac{D}{b}\right.$, c), quae illi proprie aequiualens et ab ipsa ditersa erit, vnico quem sepónimus casu excepto. vbi $c=b= \pm 1$ siue $D=-1$. Ex his 


\section{$-405-$}

duabuis formis eam retinere sufficit, cuius terminus primus est minor quam terminus primus alterius (magnitudine aequales, signis diuersi in hoe casu esse nequeunt), vnde patet, etiam ornnes formas ( $2 B$, $B, C)$ ad semissem reduci posse, e binis tnam semper eiiciendo; et in remanentibus esse $B<\frac{D}{B}$ siue $B<$ $\sqrt{ } \pm D$. Hoe modo ex omnibus formis art. praec. semissis tantum remanet, quarum complexum per $W$ designabimus, nihilqua superest, nisi vt ostendamus, quot classes diuersae ex his formis, oriantul. Ceterum manifestum est, in eo casu vbi $D$ sit negatiuus totidem formas positiuas in $W$ affore quot negatiuas.

I. Quando $D$ est negatiuus, sirgulae formae in $W$ pertinebunt ad classes diuersas. Nam omnes formae $(A, 0, C)$ erunt reductae; similiter omnes formae $(2 B, B, C)$ reductae erunt, praeter eas in quibus $C<2 B$; in tali vero forma erit $2 O<2 B+C$; vnde (quoniam $B<\frac{D}{B}$ i. e. $B<2 C-B$, adeoque $2 B<2 C$, siue $B$ $<C), 2 C-2 B<C$ et $C-B<\frac{x}{2} C$ et proin $(C, C-B, C)$, quae manifesto illi aequiualet, forma reducta. Hoc modo totidem formae reductae habentur, quot formae habentur in $\mathrm{W}$, et quum facile perspiciatur, inter illas neque identicas neque oppositas occurrere posse, vnico casu excepto vbi $C-B=0$, in quo erit $B=C= \pm 1$, adeo que $D=-1$, quem jam seposuinus). ommes ad classes diuersas pertinebunt Hinc colligitur, multitudinem omnirum classium ancipitám pr. primitiuarum det $D$ multitudini formarum in $W$ $\mathrm{Cc} 3$ 


\section{$-406$}

seu semissi multitudinis formarum art. praec. azqualem esse; in casu excepto autem $D=-1$ per compensationem idern euenit, scilicit duae classes habentur, ad quarum alteram pertinent formae $(1,0,1),(2,1,1)$, ad alterm he $(-1,0,-1),(-2,-1,-1)$ Generaliter itaque pro determinante negatiuo multituto omnium classium ancipitum pr. prim. aequalis est multitudini omnium characterum assignabilium formanum primitiuarum huius determinantis; multitudo classium ancipitum pr. prim. posttiuarum autem semissis erit.

II. Quando $D$ est positiuus quadratus $=m \hbar$, haud difficile demonstratur, singulas formas in $W$ ad classes diuersas pertinere, sed pro hoc casu ad problematis solutiontem adhuc breuius sequenti modo peruenire possumus. Quum per art. 210 in quauis classe ancipite pr. prim. det. $h h$, neque in vlla alia, contineatur forma redum cta rna $(a, h, o)$, in qua a est ralor expr. $\sqrt{ }$ 1 (mod. oh) inter o ot $2 h-1$ incl situs: perw spicuum est, totidem classes ancipites pr. prim. det hh dari, guot valores expressos illa habeat. Ex art. 105 autern nullo negotio deducitur, mul.

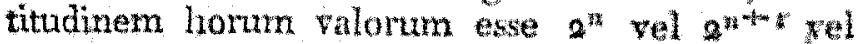
$2^{n+x}$, prout $h$ sit impar wel impariter par pel pariter par, siue prout $D=1$ vel $=4$ vel $=0(\bmod .8)$, designaute $n$ multitudinem diuisorum primomm imparium ipsius $h$ siue ipsius $D$. Tinc colligitur, rmul titudinem classium ancipitum pr. prim. semper esse semissern multitudinis ominum formarum in art. praec erutarum, situe multitudint formarum in Wr vel omnium characterum possibilium aequalem. 
III. Quando $D$ est prositiuus non quadratus, ex singulis formis $(A, B, C)$ in $I V$ contentis alias deducamus $\left(A, B^{\prime}, C^{\prime}\right)$, accipiendo $B^{\prime} \equiv$ $B$ mod. $A)$ et inter limites $\sqrt{ } D$ et $\sqrt{ } \mp \mp A$ (vbi signum superius vel inferius adhibendum, frout $A$ est pos. vel neg.) atque $C^{\prime}=\frac{B^{\prime} B^{\prime}-D}{A}$ designemusque harum complexum per $H \%$. Manif - to hae forma erunt proprie primitiuae ancipites det. $D$, atque omnes inter se dincrsae: practerea vero onnes erunt formae reductae. Quando enim $A<\sqrt{D}, B^{\prime}$ manifesto erit $<$ $\sqrt{ } D$ atque positiuus; praeterea $B^{\prime}>\sqrt{ } D \mp A$ adeoque $A>\sqrt{ } D-B^{2}$ et proin $A$, positiue acceplus, certo inter $\sqrt{D}+B^{\prime}$ et $\sqrt{ } D-B^{\prime}$ situs. Quando vero $A>\sqrt{ } D$, non poterit esse $B=$ a (quippe quas formas fiecinus), sed erit necessario $B=\frac{1}{2} A$. lime $B$ magnitudine ipsi $\frac{x}{2} A$ aem qualis, signo positiuus (quoniam enim $A<$

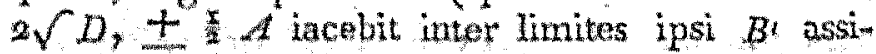
gnatos, ipsique $B$ sec mod $A$ erit congruus; quare $B^{\prime}= \pm A$ ), proin $B^{\prime}<\sqrt{ } D$, vide $a b \sqrt{ } D+D$ sine $A<\sqrt{D}+B$, quamob rem $\pm A$ necessario inter limites $\sqrt{ } D+B^{\prime}$ et $\sqrt{ } D=B$ iacebit. Denique $W$ ommes formas reductas pr. prim. ancipites det. $D$ continebit; si enim $(a, b, c)$ est huiusmodi forma, erit vel $b=0$, rel $b=$ (mod. $a$ ). In casu priori manifesto non poterit esse $b<a$ neque adeo $a>\sqrt{ } D$, quapropter forma $\left(a, 0,-\frac{D}{a}\right)$ certo contenta erit in $W$, et respondens $(a, b, c)$ in $W_{\text {; }}$ in posteriori certo erit $a<2 \sqrt{ } \mathrm{D}$, adeoque $\left(a, \frac{x}{2} a, \frac{x}{4} a-\frac{D}{a}\right)$ in $W$ contenta, atque C.c. 


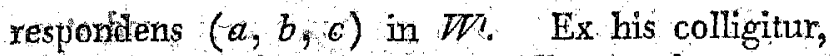
multitudinem formarum in $W$ : aequalem esse multitudini omnium formarum reductarum ancipitum pr. prim. det. $D$; quonian vero in singulis classibus ancipitibus binae formae reductae ancipites continentur (artt. 187,194 ), multitudo omnium classium ancipitum pr. prim. det. $D$ erit semissis multitudinis formarum in $W$, siue semissis omnium characterem assignabilium.

259. Multitudo classium ancipitum improprie primitiuarum determinantis dati $D$ multitudini proprie primitiuarum eiusdem det. semper est aequalis. "Sit $K$ classis princlpalis, atque $K$, $K^{\prime \prime}$ etc reliquae classes ancipites pr. primitiuae huius determinantis, $L$ aliqua classis anceps improprie primitiua eiusdem det., e. g. ea,in qua est forma $\left(2,1, \frac{x}{2}-\frac{\pi}{3} D\right)$. Prodibit itaque ex compositione classis $L$ cum $K$ classis $L$ ijsa; ex compositione classis $L$ cum $K^{\prime}, K^{\prime \prime}$ etc. prouenire supponamus classes $L, L^{\prime \prime}$ etc resp., quae mañfesto omnes ad eundem determinantem $D$ pertinebunt, atque improprie primitiuae et ancipites erunt. Patet itaque, theorema demonstratum fore, simulac probatum fuerit, omnes classes $L$, I $L^{\prime \prime}$ etc. esse diuersas, aliasque ancipites impr. prim. det $D$ praeter illas nor dari. Ad hune finem sequentes casùs distingüimus:

I. Quando multitudo classium impr primihiuarum multitudini pr primitiuarum aequalis est, quaeuis illarum oritur ex compositione classis $L$ cum classe determinata proprie primitiua, vnde necessario omnes $I_{2} L^{\prime}, L^{\prime \prime}$ etc exunt diuersae. 
Designante autem $\mathfrak{s}$ classem quamcunque ancipitem impr. prim. det. $D$, dabitur classis proprie primitiua $\Omega$ talis vt sit $\Omega+L=\Omega$; si classi $\Re$ opposita est classis $\Omega^{\prime}$, erit etiam (quoniam dasses $L$, $\mathfrak{l}$ sibi ipsae oppositae sunt) $\mathfrak{l}$ $+L=\Omega$; vnde necessario $\Omega$ cum $\Omega^{\prime}$ identica, erit, adeoque classis anceps: hinc $\Omega$ reperietur inter classes $K, K^{\prime}, K^{\prime \prime}$ etc atque $\mathfrak{R}$ inter has $L, L, L^{n}$ etc.

II. Quando multitudo - classium improprie primitiuarum ter maior est quam multitudo classium pr. primitiuarum, sit $H$ classis in qua est forma $\left(4,1, \frac{1-D}{4}\right), H^{\prime}$ ea in qua est forma (4, $\left.3, \frac{9-D}{4}\right)$, eruntque $H, H^{\prime}$ proprie primitiuae et tum inter se tum a classe principali $K$ diuersae, atque $H+H^{\prime}=K, 2 H=H^{\prime}, 2 H^{\prime}=H_{\text {; }}$ et si \& est classis quaecunque improprie primitiua det $D$, quae oritur ex compositione classis $L$ cum proprie primitiua $\Omega$, erit etiam $\&=L$ $+\hat{\pi}+H$ et $\mathcal{Q}=L+\Omega+I^{\prime}$; praeter tres classes (pr. prim. atque diuersas) $\Omega, \Omega+H, \Omega$ $+H^{I}$ aliae non dabuntur, quae cum $L$ compositae ipsam \& producant Quoniam igitur, si $\mathbb{R}$ est anceps atque $\Omega^{\prime}$ ipsi $\Re$ opposita, etiam $L+$ $\Omega^{\prime}=\Omega$, necessario $\Omega^{\prime}$ cum aliqua illarum trium classium identica erit. $\mathrm{Si} \Omega=\Omega$, erit $\Omega$ anceps; si $\Omega=\Omega+H$, erit $K=\Omega+\Omega=2 \Omega+I$ $=2(\Omega+H)$ adeoque $\Omega+H$ anceps; similiterque si $\Omega^{\prime}=\Omega+H$ erit $\Omega+H$ anceps, vade concluditur, $\&$ inter classes $L, L, L$, etc necessario reperiri Facile auten perspicitur, inter

$\mathrm{Cc} 5$ 
tres classes $\mathfrak{R}, \Omega+H, \Omega+H$ plures ancipites esse non posse; si enim tum $\Omega$ tum $\Omega+H$ ancipites essent siue cum oppositis suis $\Omega^{\prime} \mathrm{N}^{\prime}+H^{\prime}$ resp identicae, foret $\Omega+H=\Omega H^{\prime}$; eadem conclusio resultat ex suppositione, $\Omega$ et $\Omega+H^{\prime}$ esse-ancipites; deniquesi $\Omega+H, \Omega+H^{\prime}$ ancipites siue cum oppositis suis $\Omega^{\prime}+H^{\prime}, \Omega^{\prime}+H$ identicae essent, fieret $\Omega+H+\Omega^{\prime}+H=\Omega^{\prime}$ $+H^{\prime}+\Omega+H^{\prime}$, vade $2 H=2 H^{\prime}$, siue $H^{\prime}=H$. Quamobrem vnica tantum classis anceps pr. prim. dabitur, quae cum $L$ composita ipsam \& producit, adeoque omnes $L, L, L^{\prime \prime}$ etc. erunt diuersae.

Multitudo classium ancipitum in ordine deriuato manifesto aequalis est multifudini classium ancipitum in ordine primitiuo ex quo est deriuatus, adeoque per praecedentia semper poterit assignari.

260. Problema. Classis proprie primitiza $K$ determinantis $D$ oritur ex duplitatione classis proprie primitiuat $k$ eiusdem determinantis: quaeruntur o om as similes classes, ex quarum dupticatione classis $K$ oritur.

Sol. Sit $H$ classis principalis det $D$ atque II, $H^{\prime \prime}, H^{i \prime}$ etc. reliquae classes ancipites pr, primitiuae eiusdem deterninantis; classes quae ex harum compositione cum $k$ oriuntur, $k+H^{\prime}, k$ $+H^{\prime \prime}, k+H^{\prime \prime}$ etc designentur per $k^{\prime}, k^{\prime \prime}, k^{\prime \prime \prime}$ etc. Tunc omres dasses $k, k, k^{\prime \prime}$ etc erunt pr. primitiuae det. $D$ et inter se diuersae; aeque facile perspicitur, ex singularum duplicatione oriri classem $K$. Denotante autem $R$ classem quamcunque pr. prim. det. $D$, quae duplicata 


\section{$-411$}

producit classem $K$, necessario inter classes $k, k$, $k^{\prime \prime}$ etc. contenta erit. Ponatur enim $\mathfrak{A}=k+\mathfrak{j}$, ita vt $\mathfrak{S}$ sit classis pr. prim. det $D($ art. 249$)$, eritque $2 k+2 \mathfrak{S}=2 \mathfrak{k}=K=2 k$, vnde facile concluditur, $25 \mathfrak{S}$ coincidere curn classe principali, $\mathfrak{S}$ esse ancipitem siue inter $H, H^{H}, H^{\prime \prime}$ etc. contentam, atque $\Omega$ inter $k, k, k^{\prime \prime}$ etc; quamobrem hae classes completam problematis solutionem exhibent:

Ceterum manifestum est, in eo casu, ybi $D$ sit negatiuus, e classibus $k, k^{\prime}, k^{\prime \prime}$ etc. semissem fore classes positiuas, semissem regatiuas.

Quum igitur quaeuis classis pr. prim. det $D$, quae ex vllius classis similis duplicatione oriri potest, omnino ex totidem classium similium duplicatione proueniat, quot classes ancipites pr. prim, det. $D$ dantur perspicuum est, si multitudo cunctarum classium pr. prim det $D$ sit $r$, multitudo omnium classilum ancipitum pr. prim. huius det. $n$, multitudinem omnium clas sium pr. prim. eiusdem det quae ex duplicatione similis classis produci possint fore $\frac{r}{n}$. Eadem formula resultat, si, pro det negatiuo, characteres $r, n$ multitudinem classium positiuarum designant, ille omnium pr. prim., hic solarum ancipitum. Ita e. g. pro $D=-161$ multitude omnium classium pr. pr. positiuarum est 16 , multitudo ancipitum 4 , vnde multitudo omnium classium quae per duplicationem alicuius classis orini possunt debebit esse 4 . Et reuera inuenitur, 


\section{$-412-$}

omnes classes in genere principali contentas hat proprietate esse praeditas; scilicet classis principalis $(1,0,161)$ oritur ex duplicatione quatuox classium ancipitum; $(2 ; 1,18)$ ex dufulicationes classium $(9,1,18),(9,-1,18),(11,2,15)$, $(11,-2,15) ;(9,1,18)$ ex dupl. classium $(3,1,54),(6,1,27),(5,-2,33),(10,3,17)$ denique $(9,-1,18)$ ex duplicatione clasium $(3,-1,54),(6,-1,27),(5,2,33,(10,-$ $3,17)$.

26r. THeorema. Semissi omnium charatct-

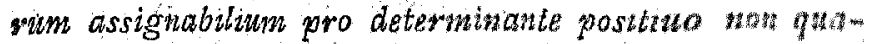
drato milla genera proprie primitian respowdro passunt; pro determinante negatiwo autem nulla grotra groprie primitiua positiua.

Dem. Sit $m$ multitudo ornnium generum proprie primitiuorum (positiuorum) detmrminan: tis $D ; k$ multitudo classium in singulis ganeribus contentarum, ita vi $\mathrm{km}$ sit multitudo omunatu clatssium proprie primitiuarum (positiunrum) $*$ "malm titudo omnium characterum diuersorum pro lowe det. assignabilium. Tunc per art 258 zumlitulo omnium classium ancipitum (positiuaman) $p$ " pringitiuarum erit $\frac{x}{2} n$; hinc per art prafse nuth titudo omnium classium pr. prim. quat ex du*

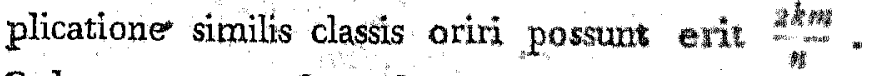
Sed per art, 247 hae classes amnes pertinent genus principale, in quo continentur $k$ classes; si itaque omnes classes generis principalis ex dayliw catione alicuius classis prouenire possume couot reuera semper locum habere in sequentibus dow 


\section{$-413-$}

monstrabitur), erit $\frac{2 k m}{n}=k$, siue $m=\frac{m}{2} n$, certo autem nequit esse $\frac{2 k m}{n}>k$ neque adeo $m$ $>\frac{T}{2} n$. Quoniam itaque multitudo omium generum pr. prim. (positiuorum) certo non est maior quam semissis omnium characterum assigrabilium: ad minimum horum semissi talia genera respondere nequeunt. Q. E. D. - Ceterum probe notandun est, hime nondum sequi, semissi omnium characterum assignabilium re uera respondere genera pr. prim. ('positiua), sed Thuius propositionis grauissimae veritas infra demum e, reconditissimis numerorum mysteriis enodari poterit.

Quum pro determinante negatiuo totidem genera negatiua semper exstent quot positiua, marifesto ex omnibus characteribus assignabilibis non plures quam semissis genexibus pr. prim ne? gatiuis competere possunt, de qua re vt et de generibus impr. prim. infra loquemur. Denique obseruanus, theoxema ad determinantes positimos quadratos non extendi, pro quikus nullo negotio perspicitur singulis characteribus assignabilibus genera reùera respondere.

262. In eo itaque casu, vbi pro deterininante mon-quadrato dato $D$ duo tantiminodo characteres diuersi assignari possunt, vnico tantum genus $\mathbf{p r}$. Primitiuum (positiuum) respondebit, (equod non poterit esse aliud quam genus principale), alter nulli formae pr. prim. (pos.) illius determinantis competet. Hoc euenit pre determinantibus - 1 ; $2,-2,-4$, numeris primis formae $4 n+1$ 
positiue, iisque formae $4 n+5$ negatiue acceptis, denique pro omnibus numerorum primorum forma $4 x+1$ potestatibus exponentis imparis positue sumtis, et pro potestatibus numerorum primorum formae $4 n+3$ positiue vel negatiue sumtis prout exponentes sunt pares vel impares. Ex hoc priticipio nethodum douam haurire possumus, non modo theorema fundamentale, sed etiam reliqua theorernata sect. praec ad residua $-1,+2,-2$ pertinentia demonstrandi, quae a metholis in sect. praec. adhibitis omnino est diursu, eleyrantiaque his nentiquam inferior aestimanda videtur. Determinantem - 4 autem, et qui sumt numerorum primorum potestates, quam nillil noux doceant, praeteribimus.

Pro determinante - 1 itaque nulla forma positiua datur cuius character sit 3,$4 ;$ pro determinarte +2 , nulla omnino forma cuits character sit 5 et 5,8 ; pro determinante -2 mulli formae positiuae competet character 5 et 7,8 ; pro determinante $+p$, si $p$ est numerus primus formae $4 n+1$, vel pro determinante - $p$, st $p$ est mimerus primus formae $4 n+3$, mulli formae pr. pr. (positiuae in casu post, competet character Np. Hinc theoremata seck. praec. sequenti modo demonstramus:

I. Est - I non residuum cuitusuis numeri (positiui) formae $4 n+5 . \quad \mathrm{Si}$ enim -1 residuum talis numeri $A$ esset, faciendo $-1=B B$ - $A C$, foret $(A, B, C)$ forma positiua det cuius character 3,4 * 


\section{$-415-$}

II: Est - I residuum cuiusuis numeri primi $p$ formae $4 n+1$. Nam character formae $(-1,0, p)$, sicuti ommim proprie primitiuanum det. $p$, eril $R p$, adeoque $-i R p$.

III. Tum +2 tum -2 est residuum chỉssuis numeri primi $p$ formae $8 n+1$. Nam vel formae $\left(8,1, \frac{x-p}{8}\right),\left(-8,1, \frac{p-1}{8}\right)$, vel hae $(8$, $\left.5, \frac{9-p}{8}\right),\left(-8,3, \frac{p-9}{8}\right)$ erunt proprie primitiuae (prout $n$ impar vel par), adeoque ipsarum character $R_{p}$; hinc $+8 R p$ et $-8 R p$, wnde etiam $2 R p,-2 R p$.

IV. Est +2 non residuum cuiusuis numer formae $8 n+3$ aut $8 n+5$. Si enim esset residuum talis numeri $A$, daretur forma $(A, B, C)$ determinantis +2 , cuits character $3 e t 5,8$.

V. Simill modo - 2 est non residutum caiusuis numen formae $8 n+5$ aut $8 n+7$, allom quin enim daretur forma $(A, B, C)$ determinantis - 2 cuius character 5 et 7,8 .

VI. Est - residuum cuiustuis numeri primi $p$ formae $8 n+3$. Hanc propositionem per methodum duplicem demonstrare licet. Primo, quum per IV sit $+q N p$, atque per $1,-1 N p$, necessario erit $+2 R p$. Demonstratio secunda petitur ex consideratione determikantis $+\mathrm{sp}$, pro quo quatuor charateres sunt assignabiles, puta $R p, x$ et 3,$8 ; R p, 5,7,8, N p, 1$, 3,8 ; Np, 5 et 7,8 , ex quibus igitur saltem duobus 


\section{$-46-$}

mulla genera respondebunt Iam formae $(1,0$, - $2 p$ ) competit character primus; formae $(-1$, $0,2 p$ ) quartus, quare qui reiici debent sunt secundus atque tertius, Quum itaque character for. mae $(p, o,-2)$ relatiue ad numerum 8 sit 1 et 3,8 , ipsius character relatiue ad $p$ non poterit esse alius quam $R p$, vnde $-2 R p$.

viI. Est +2 residuum cuiusuis numeri primi $p$ formae $8 n+7$, quod per methodum duplicem demonstrare licet Primo, quim ex I et V sit $-1 N p,-2 N p$, erit $+2 R p$. Secundo quum vel $\left(8,1, \frac{x+p}{8}\right)$ vel $\left(8,3, \frac{9+p}{8}\right)$ sit forma proprie primitiua determinantis $p$ (prout $n$ par vel inpar), ipsius character erit $R_{p}$, adeoque $8 R p$ et $a R p$.

VIII. Quilibet numerus primus $p$ formae $4 n+1$ est non residuum cuiusuis numeri imparis $q$, qui ipsius $p$ non residuum est. Patet enim, si $p$ esset residuum ipsius $q$, dari formam proprie primitiuam "determinantis $p$ cuius character $N p$.

IX. Simili modo si numerus quicunque impar $q$ est non residuum numeri primi $p$ formae $4 n+3$, erit $-p$ non residum ipsius $q$; alioquin enim daretur forma positiua pr. primitiua determinantis $-p$, cuius character $N p$.

X. Quiuis numeris primus $p$ formae $4 n+1$ est residuum cuiusuis alius numeri primi $q$, qui upsius $p$ residuum est Si etiam $q$ est formae 
$4 n+3$, erit etiam - $-q$ restutum ipsits $p$ (propter If) adeoque $p R q$ (ex IX).

XI. $\mathrm{Si}$ numerus quicunque primus $q$ est residuum alius numeri primi $p$ formae $4 n+3$, erit $-p$ residuum ipsius $q$. Si enim $q$ est formae $4^{n}+1$, ex VIII sequitur $p R q$, adeoque ( $p e r$ II), - pRq; casus autem vbi etiam $q$ est formae $4 n+3$ huic methodo se subducit, attamen facile ex consideratione determinantis $+p q$ ahsolui potest. Scilicet quum ex quatuor characteribus pro hoc determinante assignabilibus $R p$, $R q ; R p, N q ; N p, R q, N p, N q$ duobus nulla genera respondere possint, atque formarum ( 1 , $0,-p q),(-1,0, p q)$ characteres respectiue sint primus et quartus, character secundus et tertius nulli formae pr. prim. det $p q$ competere possunt. Quum itaque character formae $\left(q, o_{\text {; }}\right.$ $-p)$ resp. numeri $p$ per hyp. sit $R p$, esusdem formae character respectu numeri $q$ debet esse $R q$, adeoque - pRq. Q. E. D.

$\mathrm{Si}$ in proposs. VIII et IX, $q$ supponitur designare numerum primum, hae cum $X$ et $X I$ iunctae theorema. fundamentale sect. praec. exhibent:

263. Postquam theorema fundamentale demonstratione noua comprobauimus, earn characterum semissem, quibus nullae formae pr. primitiuae (positiuae) respondere possunt, pro determinante quocunque non quadrato dato discernere ostendemus, quod negotiun eo breuius absoluere licebit, quum ipsius fundamentum jam $D d$ 


\section{- 428}

in disquisitione arth, $1477=150$ sit contentum. Sit ce quadraturn maximum, detẹrminantem propositum $D$ metiens, atque $D=D e e$, ita $x$ tor nullum factorem quadratum implicet; porro sint $a, b, c$ etc. omnes dipisores primi impares ipsius $D^{\prime}$, adeoque $D^{\prime}$ sine respectu signi' sui rel productum ex his numeris vel duplum huius prodracti. Designetur pir s complexus charactwom particularium $\mathrm{Na}, \mathrm{Nb}, \mathrm{Nc}$ etc., solus, quando $D^{\prime} \equiv 1$ (mod. 4 ); adiuncto charactere 3,4, quando $D^{\prime} \equiv 3$ atque 2 impar aut impariter par; adiunctis his 3,8 atque 7,8 , quando $D^{\prime} \equiv 3$ atque $e$ pariter par; adiuncto vel charactere $\xi \mathrm{et}$ 5,8 , vel duobus 3,8 atque 5,8 , quando $D$ ' $\equiv 2$ (mod.8) atque e vel impar vel par; denique adiuncto vel charactere 5 et $T, 8$, vel duobus 5,8 atque 7,8, quando $D^{\prime} \equiv 6$ (mod, 8 ) atque $e$ vel par vel impar, His ita factis, omnibus characteribus integ.is, in quibus multitudo impar characteruxn particularium $s$ continetur, nulla genera proprie primitiua (positiua, determinantis $D$ respondere poterunt. In omnibus casibus characteres particulares, qui exprimunt relationem ad tales diuisores primos ipsius $D$ qui ipsum $D^{\prime}$ non metiuntur, ad generum possibilitatem vel impossibilitatem nihil conferunt. - Ex theoria combinationurn autem facilline perspicitur, hoc modo reuera semissem omnitum characterum integrorum assignabilium excludi.

Demonstratio horum praeceptornm adornatur sequenti modo. E principiis sect, praec, siue theorematibus in art. praec. denuo demonstratis sullo negotio deducitur, si $p$ sit numerus 
primus (impar positiuus) ipsum $D$ non metiens, cui aliquis e characteribus reiectis competat, $D$. implicare multitudinem imparem factorum qui sint non residua ipsius $p$, atque adeo $D$, et hinc etiam $D$, esse non residuum ipsius $p$; porro facile perspicitur, productum e numeris quotcunque imparibus ad $D$ primis, quorum nulli aliquis characterum reiectorum competat, etiam cum tali charactere consentire non posse; hinc vice versa perspicuum est, quemuis numerum imparem positium ad $D$ primum, cui aliquis characterum reiectorum conuentat, certe aliquem factorem primum eiusdem qualitatis implicare, adeoque $D$ ipsius non residuum esse. Si itaque forma proprie primitiua (positiua) determinantis $D$ daretur, alicui characterum reiectorum respondens, $D$ foret non residuum cuilusuis numeri positiui imparis ad ipsum primi per talem formam repraesentabilis, quod manifesto cum theoremate art. 154 consistere nequit.

Tamquam exempla conferantur classificationes in artt. $230 ; 231$ traditae, quarum numerum quisque pro lubitu augere poterit.

264. Hoc itaque modo pro quouis determinante non quadrato dato omnes determinantes assignabiles in duas species $P, Q$ aequaliter distribuuntur, ita vt nulli characterum $Q$ forma proprie primitiua positiua respondere possit, reHquis autem $P$, quantum quidem hucusque nouimus, nihil obstet, quominus ad tales formas pertineant. Circa has characterum species notetur imprimis propositio sequens, quae ex ipsarum 
criterio facile deducitur: Si character ex $\boldsymbol{P}$ cum charactere ex $Q$ componitur (ad normam art. 246 perinde ac si etiam huic genus responderet) prodibit character ex $Q$; si vero duo characteres ex $P$, vel duo ex $Q$ componuntur, character re. sultans ad $P$ pertinebit. Adiumento huius theo. rematis etiam pro generibus negatiuis atque improprie primitiuis semissis omnium characterum assignabilium excludi potest sequenti modo.

I. Pro determinante negatiuo $\mathcal{D}$ generà negatiua positiuis hoc respectu prorsus contraria erunt, scilicet nullus characterum $P$ pertinebit ad genus proprie primitiuum negatiuum, sed haec genera omna habebunt characteres ex $Q$. Qualdo enim $D^{\prime} \equiv 1(\bmod 4)$, erit $-D^{\prime}$ numerus positiuus formae $4 n+3$, adeoque inter $a_{3}$ $b, c$ etc. multitudo impar numerorum formae $4^{n}+3$, quorum singulorum non residuum erit - 1, vnde patet, in characterem integrum formae $(-1,0, D)$ in hoc casu ingredi multitudinem imparem characterum particularium ex $\Omega$, siue illum pertinere ad $Q ;$ quando $D^{\prime} \equiv 3$ (mod. 4 ), ex simili ratione inter $a, b, c$ etc. vel nullus numerus formae $4 n+3$ reperietur, vel duo, vel quatuor etc, sed quim vel 3 , 4 vel 7 , 8 in hoc casu occurrat inter characteres particulares formae $(-1,0, D)$, patet, characterem integrum huius formae etiam hic pertinere ad Q. Eadem conclusio aeque facile in casibus reliquis obtinetur, ita vt forma negatiua $(-3,0, D)$ semper habeat characterem ex Q. Sed quoniam haec forma cum quacunque alia pr. primitiua negatiua eiusdem det. composita similem formam 
positiuam producit, facile perspicitur, nullam formam pr. prim. negatiuam characterem ex $P$ habere posse.

II. Pro generibus improprie primitiuis (positiuis) simili modo probatur, rem vel eodem modo se habere vt in proprie primitiuis, vel contrario, prout $D \equiv 1$ vel $\equiv 5$ (mod." 8 ). Nam in casu priori erit etiam $D \equiv 1$ (mod. 8 ), nnde facile concluditur, inter numeros $a, b, c$ etc. vel nullum numerum formae $8 n+3$ et $8 n+5$ re. periri vel duos vel quatuor etc. (scilicet productum ex quotcunque numeris imparibus inter quos numeri formae $8 n+3$ et $8 n+5$ coniunctim multitudinem imparem efficiunit semper euadit vel $\equiv 3$ vel $\equiv 5$ (mod. 8$)$, productum autem ex omnibus $a, b, c$ etc., aequale esse de bet vel ipsi $D^{\prime}$ vel ipsi - $\left.D^{\prime}\right)$; hinc patet, characterem integrum formae $\left(2,1, \frac{1-D}{2}\right)$ inuol uere vel nullum characterem particularem $\mathrm{ex} \Omega$, vel duos vel quatuor etc, adeoque pertinere ad P. Iam quum quaeuis forma improprie primitiua (positiua) determinantis $D$ spectari possit tamquam composita ex $\left(2,1, \frac{1-D}{2}\right)$ atque proprie primitiua (positiua) eiusdem determinantis, perspicuum est, nullam formam improprie primitiuam (positiuam) characterem ex $Q$ in hoc casu habere posse. In casu altero, $D \equiv 5$ (mod. 8 ), omnia contraria sunt, scilicet $D$, qui etiam erit $\equiv 5$, certo multitudinem imparem facto rum-formae $8 n+3$ atque $8 n+5$ implicabit, vnde concluditur, characterem formae $(2,1$, Dd 3 
$\frac{x-D}{2}$ ), atque hinc etiam characterem cuilusuis formae improprie primitiuae (pos.) det, $D$, pertinere ad $Q$, adeoque nulli characterum $P$ genus impr. prim. pos. respondere posse.

III. Denique pro determinante negatiuo genera improprie primitiua negatiua rursus contramria sunt generibus improprie primitiuis positivis, scilicet illa non poterunt habere characterem ex $P$ vel ex $Q$, prout $D \equiv 1$ vel $\equiv 5(\bmod .8)$, siue prout $-D$ est formae $8 n+7$ vel $8 n+3$. Hoc nullo negotio deducitur inde, quod ex compositione formae $(-1,0, D)$, cuius character est ex $Q$, cum formis improprie primitiuts ne. gatiuis eiusdem determinantis formae improprie primitiuae positiuae proueniunt, adeoque, quando ab his exclusi sunt characteres $Q$, necessario ab illis exclusi esse debent characteres $P$, et contra.

265. Ex disquisitionibus artt. 257, 258 supra multitudine classium ancipitum, quibus omnia praecedertia sunt superstructa, multae aliae conclusiones attentione perdignae deduci possunt, quas breuitatis caussa supprimere oportet; sequentern tamen, elegantia sua insignem; praeterire non possumus. "Pro determinante positiuo $p$, qui est numerus primus formae $4 n+1$, vnicam tantummodo classem ancipitem proprie primitiuam dari ostendirnus; quapropter omnes format ancipites proprie primitiuae talis determinantis proprie aequiualentes erunt. $\mathrm{Si}$ itaque $b$ est mumerus integer positiuus proxime minor quam 
$\sqrt{p}$, atque $p-b b=a^{i}$, formae $\left(i, b,-a^{\prime}\right)$, $\left(-1, b, \alpha^{\prime}\right)$ proprie aequiualebunt, adeoque, quum vtraque manifesto sit forma reducta, altera in alterius periodo erit contenta. Tribuendo formae priori in periodo sua indicem $o$, index posterioris necessario erit impar (quoniam termini primi harum duarum formarum signa opposita habent); ponatur itaque $=2 n+1$. Porro facile perspicitur, si formae indicum $1,2,5$ etc. resp. $\sin t\left(-a^{\prime}, b^{\prime}, a^{\prime \prime}\right),\left(a^{n}, b^{\prime \prime},-a^{n}\right),(-$ $\left.a^{m \prime \prime}, b^{\prime \prime \prime}, a^{2 v}\right)$ etc: indicibus $2 m, 2 m-1,2 m-$ $a, 2 m-3$ etc resp. responsuras esse formas $\left(a^{l}, b,-x\right),\left(-a^{\prime \prime}, b^{\prime}, a^{\prime}\right),\left(a^{\prime \prime \prime}, b^{\prime \prime},-a^{\prime \prime}\right)$, ( $\left(-a^{\mathrm{iv}}, b^{\prime \prime \prime}, a^{\prime \prime \prime}\right.$ ) etc. Hinc colligitur, si forma indicis $m$ sit $(A, B, C)$, eandem fore $(-C, B$, - $A$ ), adeoque $C=-A$ et $p=B B+A A$. Quare quiuis numerus primus format $4 n+1$ in duo quadrata decomponi potest (quarm propositionem supra, art 182 , principis protsus diuersis deduximus), at talem decompositionem peruenire possumus per methodum simplicissi:mam et omnino viformen, scilicet per enolutionem periodi formae reductae, cuius determinans est ille numerus primus et cuins terminus primus 1, vsque ad formam, cuius termini $\mathrm{x}$ terni magnitudine sunt aequales, signis oppositi. Ita e. g. pro $p=233$ habetur $(1,15,-8)$, $(-8,9,19 ;,(19,10,-7),(-7,11,16)$ $(16,5,-13),(-15,8,13)$, atque $235=$ $64+169$. Ceterum patet, $A$ necessario fieri imparem (quoniam $(A, B,-A)$ debet esse lorma proprie primitiua), et proin $B$ parem. Quum pro determinante positiuo $p$, qui est numerus primus formae $4 n+1$, etiam in ordine $\mathrm{Dd} 4$ 


\section{- 424}

improprie primitiuo vnica tantum classis anceps contineatur, perspicuum est si $g$ sit numerus impar proxime minor quam $\sqrt{ } p$, atque $p-$ $g g=4 h$, formas reductas improprie primitiuas $(2, g,-2 h),(-2, g, 2 h)$ proprie aequiualere, adeoque alteram in alterius periodo contentam esse. Hinc per ratiocinia praecedentibus omnino similia concluditur, in periodo formae $(2, \mathrm{~g},-$ 2h) reperiri formam, cuius termini externi magnitudine aequales sint, signa habeant opposita, ita vt discerptio numeri $p$ in duo quadrata etiam hinc peti possit. Patet autem, terminos externos huius formae fore pares, adeoque medium innparem; et quum constet, numerum primurn vnico tantum modo in duo quadrata decomponi posse, forma per hanc posteriorem methodum inuenta erit vel $\left(B, \pm A_{5}-B\right)$, vel $\left(-B_{3}\right.$ $\pm A, B$, Tta in exemplo nostro pro $p=233$ habetur $(2,15,-4),(-4,13,16),(16,3$, - 14), (-14, 11, 8), (8, 13, - 8), et 293 $={ }^{169}+64$.t supra.

266. Hactenus disquisitionem nostram ad tales functiones secundi gradus restrinximnus, quae drias indeterminatas implicant, neque opus fuit, denominationem specialem ipsis tribuere. Sed manifesto hoc argumentum tamquam sectionem maxime particularem disquisitionis generalissinnae de functionibus algebraicis rationalibus integris homogenels plirium indeterminatarum et pturium dimensionum considerare, talesque functiones secundum multitudinem dimensionum in formas secundi, tertii, quarti gradus etc., se. cundum multitudinem indeterminatarum autem 


\section{$-425-$}

in formas binarias, ternarias, quaternarias etc. commode distinguere possumus. Formae itaque, hactenus simpliciter sic dictae, vocabuntur formae binariae secundi gradus; tales autem functiones vt $A x x+2 B x y+C y y+2 D x z+2 E y z+F z z$ (denotantibus $A, B, C, D, E, F$ integres datos) dicentur formae ternariae secundi gradus et sic porro. Proxime quidem Sectio praesens solis formis binariis secundi gradus est dicata; sed quoniam complures veritates ad has spectantes, eaeque pulcherrimae, adhuc supersunt, quarum fons proprius in theoria formarum ternariarum secundi gradus est querendus, breuem ad hanc theoriam digressionem hic intercalamus, in qua ex primis eius elementis ea trademus, quae ad perfectionem theoriae formarum binariarum sunt necessaria, quod geometrís acceptius fore speramus, quam si illas vel supprimeremus, vel per methodos minus genuinas erueremus. Exactiorem autem de hoc argumento grauissimo disquisitionem ad aliam occasionem nobis reseruare debemus, tum quod ipsius vbertas limites huius operis iam nunc longe egrederetur, tum quod spes est, luculentis adhuc incrementis eam in posterum locupletatum iri. Formae vero tum quaternariae, quinariae etc. secundi gradus, tum omnes superiorum graduum hoc quidem loco $\mathrm{ab}$ instituto nostro penitus exchuduntur *), sufficiatque hunc campum vastissimum geometrarum attentioni commendauisse, in quo materiem ingen-

- Pxopter hanc rationem formae binariae vel ternaxiae secuthdi gradus in sequentibus sempex surt intelligendae, guoties de talibus formis simplicitex loquenur. 
tem vires suas exercendi, Arithmeticamqque sublimiorem egregiis incrementis augendi inuenient.

267. Ad perspicuitatem multum proderit, inter tres indeterminatas, in formam ternariam ingredientes, simili modo vt in formis binariis, ordinem fixum stabilire, ita vt indeterminata pri$m a$, secunda et tertia ab inuicem distinguantur; in disponendis autem singulis formae partibus hunc ordinem semper obseruabimus, vt primum locum obtirseat ea pars quae quadratum indeterminatae primae irmplicat, in sequentibus eae quae implicant quadratum indeterminatae secundae, quadratum tertiae, productum duplum secundae in tertiam, producturn duplum primae in tertiam, productum duplum primae in secundam deinceps sequantur; denique numeros integros determinatos per quos haec quadrata et prodncta dupla multiplicata sunt eodem ordine coëfficientem primum, secundum, tertium, quartum, quintmm, sextum vocabimus. Ita $a x x+$ $a^{\prime} x^{\prime} x^{\prime}+a^{\prime \prime} x^{\prime \prime} x^{\prime \prime}+2 b x^{\prime} x^{\prime \prime}+2 b^{\prime} x x^{\prime \prime}+2 b^{\prime \prime} x x^{\prime}$ erit forma ternaria rite ordinata, cuius indeterminata prima $x$, secunda $x$, tertia $x^{\prime \prime}$, coifficiens primus $a$ etc, quartus $b$ etc. Sed quoniarn ad breuitatem multum conferet, si non semper necesse est, incleterminatas formae ternariae per literas peculiares denotare, eandern formam, quatenus ad indeterminatas non respicimus, etiam hoc modo $\left(\begin{array}{ll}a, & a^{\prime}, a^{\prime \prime} \\ b & b^{\prime}, b^{\prime \prime}\end{array}\right)$ desigmabimus.

Ponendo $b b-a^{\prime} a^{\prime \prime}=A, b^{\prime} b^{\prime}-a a^{\prime \prime}=$ $A^{\prime}, b^{\prime \prime} b^{\prime \prime}-a a^{\prime}=A^{\prime \prime}, a b-b b^{\prime \prime}=B, a b^{\prime}=$ $b b^{\prime \prime}=B^{\prime}, a^{\prime \prime} b^{\prime \prime}-b b^{\prime}=B^{\prime \prime}$, oritux alia forma 


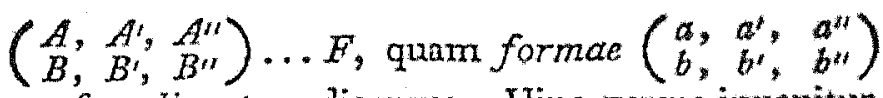
$\ldots f$, adiunctam dicemus. Hinc rursus inuenitur, denotando breuitatis caussa numerum $a b^{b} b+a^{2} b^{2} b^{t}$ $+a^{\prime \prime} b^{\prime \prime} b^{\prime \prime}-a a^{\prime} a^{\prime \prime}-2 b b^{\prime \prime} b^{\prime \prime}$ per $D, B B-A^{\prime \prime} A^{\prime \prime}$ $=a D, B^{\prime} B^{\prime}-A A^{\prime \prime}=a^{\prime} D, B^{\prime \prime} B^{\prime \prime}-A A^{\prime}=$ $a^{\prime \prime} D, A B-B^{\prime} B^{\prime \prime}=b D, A^{\prime} B^{\prime}-B B^{\prime \prime}=D^{\prime} D$, $A^{\prime \prime} B^{\prime \prime}-B B^{\prime}=b^{\prime \prime} D$, vnde patet, formae $F$ adiunctam esse formam $\left(a D, a D, a^{*} D\right)$. Num merum $D$, a cuius indole proprietates formae ternariae $f$ imprimis pendent, deterninantem huius formae vocabirnus; hoc modo determinans formae $F$ fit $=D D$, siue aequalis quadrato de terminantis formae $f$, cui adiuncta est.

Ita e. $g$. formae ternariae $\left(\begin{array}{c}29, \\ 7,-1,\end{array}, 9\right)$ adiuncta est $\left(\begin{array}{r}-68,-260,-181 \\ 217,-111, \\ 233\end{array}\right)$, viriusque determinans $=1$.

Formae ternariae determinantis o ab inuestigatione sequente omnino excludentur, quippe quae, vt in formarum ternariarum theoria, alia occasione vberius tradenda, ostendetur, specie tantum sunt temariae, reueraque binariis aequipollentes.

268. Si forma aliqua temaria $f$ determinantis $D$, cuius indeterminatae sunt $x, x^{\prime}, x^{\prime \prime}$ (puta prima $=2$ etc. in formam ternariam g deter* minantis $k$, cuits indeterminatae sunt $y, y^{\prime}, y^{\prime \prime}$, transmutatur per substitutionem talexn.

$$
\begin{aligned}
& x=y+6 y^{\prime}+y y^{\prime \prime} \\
& x^{\prime}=y^{\prime \prime}+6 y^{\prime}+y^{\prime \prime} \\
& x^{\prime \prime}=y^{\prime \prime} y+y^{\prime \prime}+y^{\prime \prime}
\end{aligned}
$$




\section{$-428-$}

vbi nouem coëfficientes $a, b$ etc omnes supponuntur esse numeri integri, breuitatis caussa neglectis indeferminatis simpliciter dicemus, $f$ trans ire in $g$ per substitutionem $(S)$

$$
\begin{aligned}
& 6, \gamma \\
& a^{\prime}, y^{\prime} \\
& \alpha^{\prime \prime}, 6 ", y^{\prime \prime}
\end{aligned}
$$

atque $f$ implicure ipsam $g$, siue $g$ sub $f$ contentam esse. Ex tali itaque suppositione sponte sequuntur sex aequatione's pro sex coefficientibus in $g$, quas apponere non erit necessarium; hin: autem rer calculum facilem sequentes conclusiones euoluuntux:

I. Designato breuitatis caussa numero ob/ $\gamma^{\prime \prime}$ $+b_{y} \alpha^{\prime \prime}+\gamma^{\prime}{ }^{\prime \prime}-2 b^{\prime \prime} \alpha^{\prime \prime}-a y^{\prime \prime}{ }^{\prime \prime}-b_{\alpha} \gamma^{\prime \prime}$ per $k_{\text {g }}$ inuenitur post debitas reductiones $E=$ $k k D$, vnde patet, $D$ metiri ipsum $E$ et quotientem esse quadratum. Patet itaque, numerum $k$ pro transformationibus formarum ternariarum simile quid esse, ac numerum $\alpha \delta^{\prime}-6 y$ in art. i 57 pro transformationibus formarum binariarum, puta radicem quadratam ex quotiente determinantium, vnde coniectare possemus, diuersitatem signi ipsius $k$ etiam hic stabilire differentiam essentialem inter transformationes atque implicationes proprias et improprias. Sed rem propius contemplando perspicuum est, $f$ transire in $g$ tiam per hanc substitutionem

$$
\begin{aligned}
& -4,-6,-y \\
& -\alpha^{\prime},-6,-y \\
& -x^{\prime \prime}-6,1
\end{aligned}
$$




\section{$-429-$}

ponendo autem in valore ipsius $k$ pro $\alpha_{,}-\star_{9}$ pro $6,-6$ etc. prodebit $-k$, quare haec substitutio substitioni $S$ dissimilis foret, et quaeuis forma ternaria, aliam vno modo implicans, eandem etiam altero modo implicaret. Talis itaque* distinctio, quoniam in formis ternariis nullum vsum habet, hic omnino proscribetur.

II. Denotando per $F, G$ formas ipsis $f, g$ resp. adiunctas, determinantur coefficientes in $F$ per coëfficientes in $f$, coëfficientesque in $G$ per valores coëfficientium formae $g$ ex aequationibus quas suppeditat substitutio $S$ notos. Exprimendo coëfficientes formae $f$ per literas, ex comparatione valorum coëfficientium formarum $F, G$ nullo negotio confirmatur, $F$ implicare formam $G$ atque in eam transmutari per substitutionem $\left(S^{\prime}\right)$

$$
\begin{aligned}
& 6^{\prime \prime} y^{\prime \prime}-\sigma^{\prime \prime} y^{\prime} g y^{\prime} \alpha^{\prime \prime}-\gamma^{\prime \prime} \alpha^{\prime}, \alpha^{\prime} b^{\prime \prime}-\alpha^{\prime \prime} b^{\prime \prime} \\
& b^{\prime \prime} \gamma-E_{\gamma}^{\prime \prime}, \gamma^{\prime \prime \alpha}-\gamma \alpha^{\prime \prime}, \alpha^{\prime \prime} \sigma-\alpha 6^{\prime \prime} \\
& 6 \gamma^{\prime}-\varepsilon^{\prime} \gamma, \gamma \alpha^{\prime}-\gamma^{\prime} \alpha, \alpha \delta^{\prime}-\alpha^{\prime} \sigma
\end{aligned}
$$

Calculum ipsum nullis difficultatibus abnoxium non adscribimus.

III. Forma $g$ per substitutionem ( $\left.S^{\prime \prime}\right)$

$$
\begin{aligned}
& 6^{\prime \prime} y^{\prime \prime}-6^{\prime \prime} y^{\prime}, 6^{\prime \prime} y-6 y^{\prime \prime}, 6 y^{\prime}-6^{\prime} y^{\prime} \\
& \gamma^{\prime} \alpha^{\prime \prime}-\gamma^{\prime \prime} \alpha^{\prime}, \gamma^{\prime \prime} \alpha-\gamma \alpha^{\prime \prime}, \gamma \alpha^{\prime}-\gamma^{\prime} \alpha \\
& \alpha^{\prime} b^{\prime \prime}-\alpha^{\prime \prime} \varepsilon^{\prime}, \alpha^{\prime \prime} b-\alpha^{\prime \prime \prime}, \alpha b^{\prime \prime}-\alpha^{\prime b}
\end{aligned}
$$

manifesto in eandem formam transmutatur, in quam $f$ transit per hanc 


$$
\begin{gathered}
430 \\
k, 0,0 \\
0, k, 0 \\
0,0, k
\end{gathered}
$$

siue in eam, quae oritur multiplicando singulos coefficientes formae $f$ per $k k$. Hanc formam designabimus per $f$.

IV. Prorsus simili modo probatur, formam $G$ per substitutionem ( $\left.S^{\prime \prime \prime}\right)$

$$
\begin{aligned}
& x, \alpha^{\prime}, z^{\prime \prime} \\
& b, c^{\prime \prime}, b^{\prime \prime} \\
& y, \gamma^{\prime}, \gamma^{\prime \prime}
\end{aligned}
$$

transire in formam, quae oritur ex $F$, multiplicando singulos coefficientes per $\mathrm{kk}$. Hanc formam exprimemus per $F$.

Substitutionem $S^{\prime \prime}$ oriri dicemus per trans. positionem substitutionis; $S i$ tunc manifesto $s$ rursus prodit ex transpositione substitutionis $S^{3 *}$; atque $S^{\prime \prime}, S^{\prime \prime}$ altera ex alterius transpositione. Substitutio $S$ commode appellari potest substitutioni $S$ adiuncta, vnde substitutioni $S$ sul adiuxta erit $S^{\prime \prime}$.

26g. Si non modo forma $f$ implicat ipsam $g$, sed etiam haec illam, formae $f, g$ aeguitulentes vocabuntur. In hoc itaque casu non modo $D$ ipsum $E$ metietur, sed etiam $E$ ipsum $D$, nde facile concluditur esse, debere $D$ $=E$. Vice versa autem, si forma $f$ implicat formam $g$ elusdem determinantis, hae duae fox- 


\section{- 451}

mae erunt aequiualentes. Erit enim (adhibenda eadem signa vt in art. praec. excipiendoque casum vbi $D=0) k= \pm 1$, adeoque forma $f^{\prime}$, in quam transit $g$ per substitutionem $S^{\prime \prime}$, cum $f$ identica, siue $f$ sub $g$ contenta. Porro patet, in hoc casu etiam formas $F, G$, ipsius $f, g$ adiunctas, inter se aequiualentes fore, posterioremque in priorem transire per substitutionem $S^{*}$. Denique vice versa, si formae $F, G$ aequiualentes esse supponuntur, atque prior transit in posteriorem per substitutionem $T$, etiam formae $f, g$ aequiualentes erunt, transibitque $f$ in $g$ per substitutionern ipsi $T$ adiunctam, atque $g$ in $f$ per eam quae oritur ex transpositions substitutionis T. Nam per has duas substitutiones resp. trarsit forma ipsi $F$ adiuncta in formam ipsi $G$ adiunctam atque haec in illam; hae duae formae autem oriuntur ex $f, g$ multiplicando singulos coëfficientes per $D$; vade nullo negotio concluditur, per easdem sulstitutiones transire $f$ in $g$, atque $g$ in $f$ resp.

270. Si forma ternaria $f$ formam ternariam $f^{\prime}$ implicat, atque haec formam $f^{\prime \prime}$ : implicabit etiam $f$ ipsam $f^{\prime \prime}$. Facillime enim perspicietur, si transeat

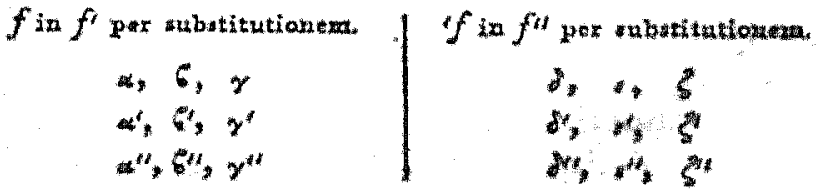

$f$ transmutatuma iri per substitutionem 


\section{$-43^{2}$}

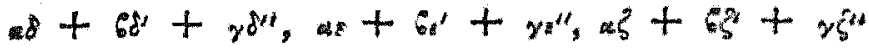

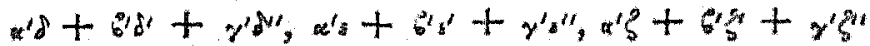

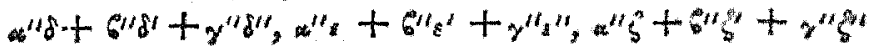

In eo itaque casu, vbi $f$ aequiualet ipsi $f^{\prime}$, atque $f^{\prime}$ ipsi $f^{\prime \prime}$, forma $f$ etiam forma $f^{\prime \prime}$ atequiualebit. - Ceterum sponte manifestum est, quomodo haec theoremata ad plures formas sint applicanda.

271. Hinc iam patet, omnes formas ternarias, perinde ac binarias, in classes distribux posşe, referendo ad classem eandem formas aequiualentes, non aequivalentes ad diuersas. Formae itaque determinantium diuersonum certo ad classes diuersas pertinebunt, et proin classes ixfinite multae formarum ternariarum dabuntur; formae autem ternariae eiusdem determinantis modo minorem modo maiorem classium mumertum efficiunt; quod vero tamquam proprietas palmaris harum formanum est considerandum, omnes formae eitusclem detarminantis dati semper constituunt clcssium multitudinem finitam. Euolutioni vberiori luuius grauissini theorematis praemittenda est explicatio sequentis differentiae essentialis, quae inter formas temarias obtinet.

Quaedam formae ternariae ita sunt comparatae, vt per ipsas sine discrimine repraesentari possint numeri positiut et negatiui, e.g forma $x x+y y-z z$, quamobrem formate indufinitat vocabuntur. Contra per alias numeri negatiui repraesentari nequeunt, sed (praeter cifram quae 
prodit, ponendo singulas indeterminatas $=0$ ) positiui tantum, vt $x x+y y+z x$, quare formae positiuae dicentur; denique per alias numeri positiui repraesentari nequeunt, it $-x x-y y$ - $z z$, vnde appellabuntur formae negatiuae; formae positiuae et negatiuae nomine communi formae definitae dicentur. Ecce iam criteria generalia, per quae haec formarum incloles discerni poterit.

Multiplicando formam ternariam $f=a x x$ $+a^{\prime} x^{\prime} x^{\prime}+a^{\prime \prime} x^{\prime \prime} x^{\prime \prime}+2 b x^{\prime} x^{\prime \prime}+2 b^{\prime} x x^{\prime \prime}+2 b^{\prime \prime} x x^{\prime}$, determinantis $D$ per $a$, denotandoque coefficiertes formae ipsi $f$ adiunctae, perinde $v$ in art. 268 per $A, A^{\prime}, A^{\prime \prime}, B, B^{\prime}, B^{\prime \prime}$, prodit $(a x+$ $\left.b^{\prime \prime} x^{\prime}+b^{\prime} x^{\prime \prime}\right)^{2}-A^{\prime \prime} x^{\prime} x^{\prime}+2 B x^{\prime \prime} x^{\prime \prime}-A^{\prime} x^{\prime \prime} x^{\prime \prime}$ $=g ;$ multiplicando denuo per $A^{\prime}$, prouent $A^{\prime}\left(a x+b^{\prime \prime} x^{\prime}+b^{\prime \prime} x^{\prime \prime}\right)^{2}-\left(A^{\prime} x^{\prime \prime}-B x^{\prime}\right)^{2}+$ $a D x t=h$. Hinc statim concluditur, si tum $A$, turn aD sint mumexi negatiui, omnes valores ipsius $h$ esse negatiuos, vide manifesto per forman $f$ tales tantummodo rumeri repraesentari poterunt, quorum signum oppositum est signo ipsius $a A^{t}$, i. e. identicum cura signo ipsius $a_{\text {, }}$ siue oppositum signo ipsius $D$. In hoc itaque casu $f$ erit forma definita, et quidem positiua vel negatiua, prout $a$ est positius rel negatiuas, sine prout $\mathcal{D}$ est negatiuus vel positiuns.

Si vero vel vterque $a D, A$ est positiuus, vel alter positiuns alter negatiuus (neuter $=0$ ), facile perspicietur, $h$ per debitam quantitatum $x, x^{\prime}, x^{\prime \prime}$ determinationem valores tum positivos turn negatiuos nancisci posse. Quare in hoo Ee 


\section{- 434. -}

casu $f$ valores tum eodem signo affectos wt $a A^{\prime}$ tum opposito, obtinere poterit, exitque adeo forma indefinita.

Pro eo casu, vbi $\not A=0$, neque vero $a=$ 0 , fit $g=\left(a x+b^{\prime \prime} x^{\prime}+b^{\prime} x^{\prime \prime}\right)^{2}-x^{\prime}\left(A^{\prime \prime} x^{\prime}\right.$ - $\left.2 B x^{\prime \prime}\right)$. Tribuendo ipsi $x^{\prime}$ valorem arbitarium (qui tamen non $=0$ ), accipiendoque $x^{\prime \prime}$ ita vt $\frac{A^{\prime \prime} x^{\prime}}{2 B}-x^{\prime \prime}$ signum idem obtineat vt $B x^{t}$ (quod fieri posse facile perspicitur, qunm $B$ nequeat esse $=0$, hinc exim foret $B B-A_{A} \mathcal{A}^{\prime \prime}$. $=a D=0$, adeoque etiam $D=0$, quem casum excludimus), erit $x^{\prime}\left(A^{\prime \prime} x^{t}-2 B x^{\prime a}\right)$ quantitas positiua, vide facile patet, $x$ ita determinari posse, vt $g$ obtineat valorem negatixum. Manifesto hi valores etiam ita accipi poterunt, vt, si desideretur, omnes sint irtegri. Denique patet, si ipsis $x^{\prime}, x^{\prime \prime}$ valores quicunque tribuanttur, Ipsum $x$ tam magnum accipi posse, it $g$ fiat positiuus. Hinc concluditur, in hoc casu formam $f$ esse indefinitam.

Denique si $a=0$, erit $f=a^{d} x^{t} x^{t}+a b x^{t} x^{d}$ $+a^{\prime \prime} x^{H} x^{\mu}+2 x\left(b^{\prime \prime} x^{\prime}+b x^{\prime \prime}\right)$. Accipiendo itaque $x^{\prime}, x^{4}$ ad lubitum, ita tamen ve $b^{\prime \prime} x^{t}+b^{\prime} x^{a t}$ non sit $=0$ (quod manifesto fieri poterit, nisi simul $b^{\prime}$ et $b^{\prime \prime}$ sint $=0$; tunc autem foret $D=0$ ), nullo negotio perspicitur, $x$ f ta determinari posse, vt $f$ obtineat valores tum positiuos, tum negatitos. Quare etiam in hocce casu $f$ exit forma indefinita

Eodem modo, vt hic ex numeris $a D, A$ indolem formae $f$ diudicauinus, etiam $a D$ et $\mathbb{A}^{34}$ 
adhiberi possunt, ita vt $f$ sit forma definita, si tum $a D$ tum $A^{\prime \prime}$ sit negatiuus; indefinita in omnibus reliquis casibus. Nec non prorsus simili modo eidem fini inseruire potest consideratio numerorum $a^{\prime} D$ et $A$, vel horum $a^{\prime} D$ et $A^{\prime \prime}$, vel horum $a^{\prime \prime} D$ et $A$, vel denique ipsorum $a^{\prime \prime} D$ et $A$.

Ex his omnibus colligitur, in forma definira sex numeros $A, A^{\prime \prime}, A^{\prime \prime}, a D, a^{\prime} D, a^{\prime \prime} D$ esse negatiuos, et quidem in forma positiua $a, a^{t}, a^{\prime \prime}$ erunt positiui, $D$ negatiuus; in negatiua autem $a, a^{\prime}, a^{\prime \prime}$ erunt negatiui, $D$ positiuus. Hinc patet, ommes formas temarias determinantis dati positini distribui in negatiuas et indefinitas; omnes autem determinantis negatiui in positiuas et indefinitas; denique formes positiuas determinantis positiui, seu negatinas determinantis negatiui omnino non dari. - Ibinde facile perspicitur, formae definitae semper adiunctam esse definitam et quidam negatiuam, indefinitae indefinitam.

Quum omnes numeri per formam ternam riam datam repraesentabiles manifesto etiam per omnes formas huic aequiualentes repraesentari possint: formae ternariae in eadem classe contentae vel omnes erunt indefinitae, vel ormnes pasitixae, vel omnes negatiuae. Quamobrem has formarum denominationes etiam ad classes. integras transferre licebit.

972. Theorema in art praec propositurn, quod omnes formae ternariae determinantis dati Ee a 


\section{- $43^{6}-$}

in multitudinem finitam classium distribuuntur, per methodum ei qua in formis binariis vi sumus analogam tractabimus, scilicet ostendendo, primo, quo pacto quaeuis forma ternaria ad formam simpliciorem reduci possit, dein, formaxum simplicissimarum (ad quas per tales reductiones perueniatur), multitudinem pro quouts doterminante dato esse finitam. Supponamus generaliter, propositam esse forman temariam $f$ $=\left(\begin{array}{l}a, a^{\prime}, a^{\prime \prime} \\ b, b^{\prime}, b^{\prime \prime}\end{array}\right)$ determinantis $D$ (a cifra diuersi), quae per substitutionem $(S)$

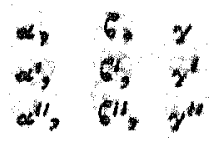

transeat in aequiualentem $g=\left(\begin{array}{c}n, m^{*}, n^{7 \prime} \\ n, \\ n_{+}^{\prime}, n^{\prime \prime}\end{array}\right)$; versabiturque negotium nostrum in eo, wt ${ }_{3}$, $\gamma$ etc. ita definiantur, vt forma $g$ simplicior euadat quarm $f$. Sint formae ipsis $f, g$ adiunctae resp. $\left(\begin{array}{l}A, A^{\prime}, A^{\prime \prime} \\ B, B, B^{\prime \prime}\end{array}\right),\left(\begin{array}{l}M, M^{\prime \prime} \\ N^{\prime}, M^{\prime \prime}\end{array}\right)$, quae designentur per $F, G$. Tunc per art. $269 . F$ transibit in $G$ per substitutionem ipsi $S$ adiunctarn, $G$ autem in $F$ per substitutionem ex transpositione ipsius

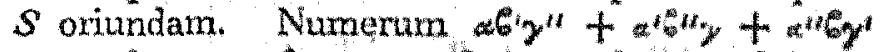
- $\alpha^{\prime \prime} \xi^{\prime} \gamma-\alpha b^{\prime \prime} y^{\prime}-\alpha^{\prime} b^{\prime \prime}$, qui esse debebit vel $=+x$ vel $=-1$, denotabimus per $k$. Quibus ita factis, obserratnus

I. Si fiat $y=0, \gamma^{\prime}=0, s^{\prime \prime}=0, w^{\prime \prime}=$ $0, \gamma^{\prime \prime}=1$, fore 


$$
\begin{aligned}
& -\quad 432 \\
& m=a \alpha+2 b^{\prime \prime} a x^{\prime}+\alpha^{\prime} \alpha^{\prime} \alpha^{\prime} \\
& m^{\prime}=a b 6+2 b^{46 b^{\prime}}+a^{\prime} b^{\prime \prime} \\
& m^{\prime \prime}=a^{\prime \prime} ; n={ }^{\prime} b^{\prime}+b^{\prime} ; n^{\prime}=b^{\prime}+b^{\prime} \\
& n^{\prime \prime}=a^{*}+b^{\prime \prime}\left(u^{b}+b^{\prime \prime}\right)+a^{\prime \prime} k^{\prime \prime}
\end{aligned}
$$

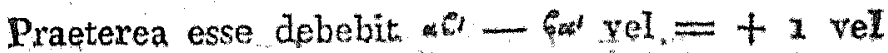
- - 1. Finc manifestum est, formam binariam $\left(a, b^{\prime \prime}, a^{\prime}\right)$, cuius determinans est $A^{\prime \prime}$, transmutari per substitutionem $\alpha, k, \alpha^{\prime}$, in formam binariam $\left(m, n^{\prime \prime}, m^{\prime}\right)$ determinantis $M{ }^{\prime \prime}$, et proin ipsi aequiualere propter -6 \pm 1 , nnde erit $M M^{\prime \prime}=A^{\prime \prime}$, quod etiam directe facile confirmatur. Nisi itaque $\left(a, b^{n}, a^{\prime}\right)$ tam est forma simplicissima in classe sua, ipsos $\ldots, \epsilon_{*}$ $\omega^{\prime}, \sigma^{\prime}$ ita determinare licebit, vt $\left(m, n^{\prime \prime}, m^{\prime}\right)$ sit Forma simplicion; et quidem e theorta aequinaIentiae formarum binariarum facile concluditur, hoc ita fieri posse, vt $m$ non sit maior quam $\sqrt{ }-A^{\prime \prime}$, si $A^{\prime \prime}$ fuerit negatius, vel non mator quam $\sqrt{A^{\prime \prime}}, \mathrm{si}^{\prime \prime}$ fuerit positiuus, vel $m=0$ si $A^{\prime \prime}=0$, tha vt in omribus casibus valor (absolutus) ipsits $m$ certe vel saltom vsque ad $\sqrt{ } \pm A^{\prime \prime}$ deprimi possit. Hoc itaque modo forma $f$ ad aliam reductur coefficientem primum, si fieri potest, minorem habentem, et cuius forma adiuncta coefficientem tertium eundem habet vt forma $F$ ipsi $f$ adiuncta. In hoc consistit reductio prima.

II. Si vero fit $\alpha=x, 6=0, \gamma=0, w^{\prime}=0$ $\alpha^{\prime \prime}=0$, erit $k=*^{\prime \prime} \gamma^{\prime \prime}-\|^{\prime}= \pm 1$ suis.itutio itaque ipsi $S$ adiuncta erit 


$$
\begin{array}{r}
-458- \\
\pm x, \quad 0, \quad 0 \\
0, y^{\prime \prime}-6 \\
0,-y^{2} \quad 6
\end{array}
$$

per quam $F$ transibit in $G$. 'Habebitur itaque

$$
\begin{aligned}
& m^{\prime \prime}=a_{2} n^{\prime}=b_{2}^{\prime \prime}+b^{\prime \prime} y_{2}^{\prime \prime} n^{\prime \prime}=b^{\prime \prime \prime}+b^{n+4}
\end{aligned}
$$

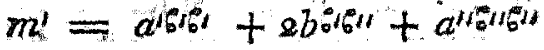

$$
\begin{aligned}
& m^{\prime \prime}=a^{\prime} y^{\prime} y^{\prime}+2 b y^{\prime} x^{\prime \prime}+a^{\prime \prime} y^{\prime \prime} y^{\prime \prime}
\end{aligned}
$$

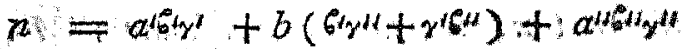

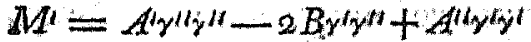

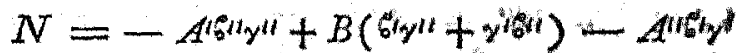

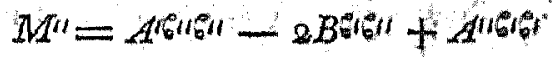

Hinc patet, formam binariam $(A, B, \not A)$, cu ius determinans est $D a$, transire per substitutio. nem ${ }^{\prime \prime},-y^{\prime}, \ldots$ - $\gamma^{\prime \prime}$ in formam $\left(M i, N, M_{i}\right)$ determinantis $D m$, adeoque (propter ${ }^{\prime \prime} y^{\prime \prime}-\gamma^{\prime \prime}$ $= \pm 1$, vel propter $D a=D m$ ipsi aequiualere. Nisi itaque $\left(A^{\prime \prime}, B, A^{\prime}\right)$ iam est forma simplicissima classis suae, coëfficientes $6^{\prime \prime}, z^{\prime}, 6^{\prime \prime \prime}, z^{\prime \prime}$ ita determinari poterurt, vt $\left(M^{\prime \prime}, N, M^{\prime}\right)$ sit simplicior, et quidem hoc semper poterit fieri ita, vt $M^{\prime \prime}$ sine respectu signi non sit maior quam $\sqrt{ } \pm \frac{a}{y} D_{\text {q. }}$. Hoc itaque modo forma $f$ reducitur ad aliam coüfficientem primum eundem habentem, sed cuins forma adiuncta coufficientem tertium si fieri, potest minorem habeat quam forma $F$ ipsi $f$ adiuncta. In hoc consistit reductio secunda.

III. Si itaque $f$ est forma temarra, ad quam heque reductio prima neque secunda est applica- 
bilis, ‥ e. quae per neutram in formam simpliciorem transmutari potest: mecessario erit tum $a a<\mathrm{vel}=4.4, \operatorname{tum} A A<\mathrm{vel}=4 a D$ sine respetu. signi. Hinc $a^{4}$ erit $<$ vel $=\frac{\times 6}{9} A A$, adeo que $a^{4}<$ vel $={ }_{27}^{64} a D, a^{7}<$ vel $=\frac{54}{\lambda \tau} D$, et $a$ $<\mathrm{vel}=\frac{43}{3} \sqrt{ } ;$ hinc rursus $A A<\mathrm{vel}=\frac{27}{2} \sqrt{3} D^{4}$ atque $A<$ vel $=\frac{4}{3} \sqrt[3]{ } D^{2}$. Quamobrem quamdiu $a$ vel $A$ hos limites adhuc superant, necessario vna aut altera reductionum praecedentium ad formam $f$ applicari poterit. - Ceterum haec conclusio non est conuertenda, quum vtique saepius accidat, vt forma ternaria, cuius coefficiens primus, atque coëfficiens tertius formae adiunctae jam sunt infra illos limites, nibilominus per vame alteramue reductionem adhuc simplicior reddi possit.

IV. Quodsi vero ad formam ternariam quamcunque datam determinantis $D$ alternis vicibus reductio prima et secunda applicantur, i. e. ad ipsam prima vel secusda, ad eam quae hinc resultat secunda vel prima, ad eam quae hinc prouénit iterum prima rel secunda etc, manifestum est, tandem necessario ad formam peruentum iri, ad quam neutra amplius applicari possit. Quum enim magnitudo absoluta tum coëfficientium primorum formantm hoc modo prodeuntium, tum coëfficientium tertiorum formarum illis adiunctarum continuto alternis vicibus eadent maneat atque decrescat, hic progressus necessario tandem alicubi finietur, quia alioquin duae series infinitae numerorum continuo decrescentium haberentur, Hine iann nacti sumus egregium theorema: Quacuis forma ternaria deterEe 4 
minantis $D$ reduct potest ad aliam aequiualentem, cuits coefficiens primus non sit maior quam $4 \sqrt[3]{ } D$, atque coëfficiens tertius formae ipsi adiunctae non maior quam $\sqrt[4]{ } \sqrt{D^{2}}$ sine respectu signi, siquidem forma proposita his propritetatibus ipsa nondum est praedita. - Ceterum loco coëfficientis primi formae $f$ atque tertii forma ipsi $f$ adiunctae prorsus simili modo tractare potuissemus vel coefficientem primum formae ipsius et secundum adiunctae; vel secundum fortnae ipsius et primum vel tertium adiunctae vel tertium formae ipsius at primum vel secundum adiunctae, quibus viis perinde ad fnem nohis propositum perueniremus: sed e re est, methom do wni constanter adhaetere, quo facilius operationes huc pertinentes ad algorithmut finom reduci possint. Denique obseruamus, duobus coefflicientibus, quos infra limites fixos deprimere doctimus, limites adhuc minores constitui posse, si formae definitae ab indefinitis separentur; hoc vero ad institutum praesens non est necessarium.

275. Ecce iam quaedam exempla, per quae praecepta praecedentia magis illustrabuntur.

$$
\begin{aligned}
& \text { Ex. 1. Sit } f=\left(\begin{array}{ll}
19,2 x_{1} & 50 \\
15,28,
\end{array}\right) \text {, eritque } F= \\
& \left(\begin{array}{r}
-825,-166,-398 \\
257,573,-370
\end{array}\right), D=-1 \text {. Quum }(19, x,
\end{aligned}
$$




\section{- $441-$}

in simpliciorem aequiualentem $(-2,1,-10)$ transmutabilis inuenitur, in quam transit per substitutionem $2,7,3,1 x$. Faciendo itaque $6^{4}$ $=2, \gamma^{\prime}=-7,{ }^{\prime \prime}=-3, \gamma^{\prime \prime}=11$, applicanda erit ad formam $f$ substitutio

$$
\begin{aligned}
& x, \quad 0, \quad 0 \\
& 0,2,-7 \\
& 0,-3, \quad 1 x
\end{aligned}
$$

per quam inuenitur transire in hanc $\left(\begin{array}{r}19,354,4769 \\ -1299,301,-82\end{array}\right) \ldots f$ Cotficiens tertius formae, huic adimctae, est - 2, "quo re spectu $f^{\prime}$ simplicior est censenda quam ${ }^{\prime 2} f$.

Ad formam $f^{\prime}$ applicari patest reductio prima. Scilicet quum forma binaria $(19,-$ $82,354)$ transmutetur in $(1,0,2)$ per substitutionem $15,4,3,1$ : applicanda erit ad formam $f$ substitutio

$$
\begin{aligned}
& 13,4,0 \\
& 3,1,0 \\
& 0,0,1
\end{aligned}
$$

per quam transit in hanc $(x, 2,4769) \ldots f^{\prime \prime}$

Ad fomam $f^{\prime \prime}$, cui adiuncta est $\left(\begin{array}{c}-513,-4513,-2 \\ -95,\end{array} 3^{2}, \quad 520\right.$, denuo applicari potest reductio secunda. Scilicet $(-2,-95,-$ $4513)$ transit per substitutionem $47,1, \ldots 1,0$ in $(-1,1,-2)$, quamobrem ad $f^{\prime \prime}$ applicanda exit substitutio 


$$
\begin{array}{rrr}
- & 449 & - \\
1, & 0, & 0 \\
0, & 47, & -1 \\
0, & 1, & 0
\end{array}
$$

per quam transit in $\left(\begin{array}{l}1,257,2 \\ 1,0,16\end{array}\right) \ldots f(1)$. Huius coefficiens primus per reductionem primam amplius dininui non potest, neque form mae, ipsi adiunctae, tertius per secundan.

Ex. 2. Proposita sit forma $\left(\begin{array}{rr}10,26,2 \\ 7,0,4\end{array}\right)$ * $f$ cui adiuncta est $\left(\begin{array}{r}-3,-20,-244 \\ 70,-28,-8\end{array}\right)$ et cuius determinans $=2$. Hic successtue reperiuntur, applicando alteruatim reductionem secun* dam et primam,

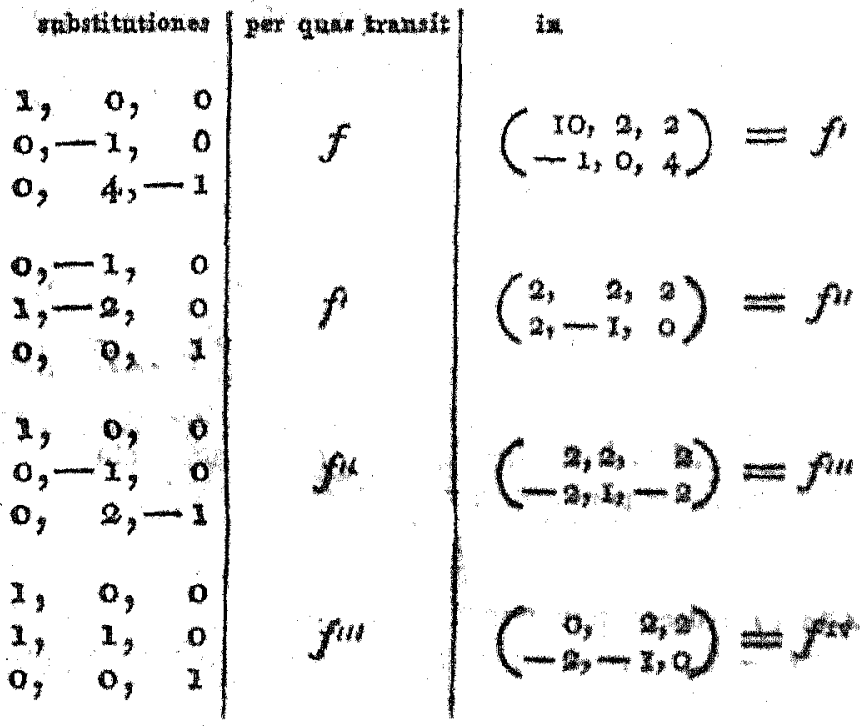


Forma $f^{\text {ro }}$ per reductioniem primam vel secundam vlterius deprimi nequit.

274. Quando forma termaria habetur, cuilus coëfficiens primus, atque formae adiunctae tertius, quantum fieri potest per methodos praecedentes sunt depressi: methodus sequens reductionnem vlteriorem suppeditat.

Adhibendo signa eadem vt in art. 272 , et ponendo ${ }^{\prime}=1, \alpha^{\prime}=0,6^{\prime}=1, u^{\prime \prime}=0$, $6^{\prime \prime}$ $=0, x^{\prime \prime}=x$, i. e. adhibenido substitutionem

$$
\begin{array}{ll}
I, & r \\
0, I, & x^{\prime} \\
0,0, & x
\end{array}
$$

erit $m=a, m^{\prime}=a^{\prime}+2 b^{\prime \prime}+a^{m}, m^{\prime \prime}=a^{\prime \prime}$ $+2 b^{\prime}+2 b^{\prime} \gamma+a \gamma \gamma+2 b^{\prime \prime} y_{\gamma}^{\prime}+a^{\prime} \gamma^{\prime} \gamma^{\prime} ; n=b 4$ $a^{\prime} \gamma^{\prime}+b^{\prime} 6+b^{\prime \prime}\left(\gamma+b^{\prime}\right)+a^{2}, n^{\prime}=b^{\prime}+$ $a v+b^{n} x^{n} n^{\text {th }}=b^{n}+a^{6}$; praeterea $N^{n}=A^{\prime \prime}$, $N=B-A^{n} \gamma_{\gamma} N^{\prime}=B^{\prime}-N_{6}-A^{n_{1}}$. Per talen Itaque sabstitutionem coefficientes $a, A^{\prime \prime}$, qui per reductiones praecedentes diminuti sunt, non mutantur; quamobrem negotium in eo versatur, vt per idoneam determinationem ipsorum $6, y, y^{*}$ depressiones in coëfficientibus reliquis obtineantur. Ad hunc finem obseruamus primo, si fuerit $A^{\prime \prime}=0$, supponi posse, essé etiam a $=0 ;$ si enim $a$ non $=0$, reductio prima adhuc semel applicabilis foret, quam cuituis formate binariae determinaxtis o aequiuateat forma talis $(0,0, h)$, sive ctùus terminus primus $=0$ (V. art. 215), Prorsus sinili retione supponere licet, 
esse etiam $A^{\prime \prime}=0$, si fuerit $a=0$, ita vt rel neuter numerorum $a, A^{\prime \prime}$ sit o vel vterque.

In casix prioxi manifestum est, ipsos $\%, \gamma$ ita determinari posse, vt sine respectu sisni $n^{t}$,

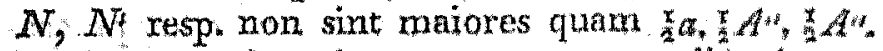
Ita in exemplo primo art praec transibit loma postrema $\left(\begin{array}{l}I, 257,2 \cdot \\ x, 0,16\end{array}\right)$, cui adiuncta esta $\left(\begin{array}{r}-5 I_{3},-2, \\ x,-16,-1\end{array}\right)$, per substitutionem"

$$
\begin{array}{rrr}
1, & -16, & 6 \\
0, & 1,-1 \\
0, & 0, & 1
\end{array}
$$

in hanc $\left(\begin{array}{c}x, x, x \\ 0,0,0\end{array}\right) \cdot f^{\mathrm{tw}}$, cui adiuncta est $\left(\begin{array}{rr}-x,-x,-x \\ 0,0,0\end{array}\right)$

In casu posteriori, vbi $a=A^{\prime \prime}=0_{2}$ adeoque etiam $b^{\prime \prime}=0$, exit $m=0, m^{\prime}=a^{\prime}, m^{\prime \prime}$ $=a^{\prime \prime}+2 b \gamma^{\prime}+2 b^{\prime} y+a^{\prime} \gamma^{\prime} y^{\prime}, n=b+a x^{\prime}+$ $b^{\prime}, n^{\prime}=b^{\prime}, n^{\prime \prime}=0$. Erit itaque $D=-$ $a^{\prime} b^{\prime} b^{\prime}=-m^{\prime} n^{\prime} n^{\prime}$; perspicieturque facile, et $v^{\prime}$ ita determinari posse, vt $n$ fiat aequalis residuo absolute minimo ipsins b secundum modulum qui est diuisor communis maximus ipsoruma $a^{\prime}, b^{\prime}, i$, e. $n$ fiat non maior quam sernissis huius diuisoris sine respectu signi, adeoque $n=$ 0 , quoties $a^{\prime}, b^{\prime}$ inter se sunt prini. Ipsis $\sigma^{*}, x^{*}$ in hunc modum determinatis, valor ipsius $*$ ita accipi poterit, vt $\mathrm{m}^{\prime \prime}$ non sit mator quat bl sine respectu signi; hoc quidem impossibile esset 
quando $b^{\prime}=0$; tunc vero foret ${ }^{\prime} D=0$, quem casum exclusimus. Ita fit pro forma postrema in ex. 2 art. praec $n=-2-6+2 \gamma^{\prime}$, rnde statuendo $6=-\hat{2}, \gamma^{\prime}=0$, fit $n=0$, porro $m^{\prime \prime}=2-2 \gamma$, et ponendo $\gamma=1, m^{\prime \prime}=$ o. Habemus itaque substitutionem

$$
\begin{aligned}
& 1,-2, \quad x \\
& 0,1,0 \\
& 0, \quad 0,1
\end{aligned}
$$

per quam forma illa transit in $\left(\begin{array}{l}0,2,0 \\ 0,-x, 0\end{array}\right) \ldots f^{*}$ *

975. Si habetur series formarum temariarum aequiualentium $f, f^{\prime}, f^{\prime \prime}, f^{\prime \prime \prime}$. etc, atque transformationes cuinsuis harum formanm in sequentem: ex transformationibus formae $f$ in $f_{z}$ formaeque $f^{\prime}$ in $f^{\prime \prime}$ per art. 270 deducitur transformatio formae of ix $f^{\prime \prime}$ ex hac atque transf formae $f^{\prime \prime}$ in $f^{\prime \prime \prime}$ sequitur transt formae $f$ in $f^{\prime \prime}$ etc, manifestoque hoc pacto transformatio formae $f$ in quamcunque aliam seriei inueniri poterit. Et quum ex transformatione formae $f$ in quamcunque aliam aequiualentem $g$ deduci possit transformatio formae $g$ in $f$ ( $S^{\prime \prime}$ ex $S$ artt. 268 , 269), hoc modo crui poterit transformatio cum iuslibet formae seriei $f^{\prime}, f^{\prime \prime}$ etc in primam $f_{*}-$ Ita pro formis exempli primi art. praec innenituntur substitutiones

$$
\begin{aligned}
& \text { 13, 4, of } 13,188,-4,15,-20, \quad 16 \\
& 6, \quad 2,-7 \quad 6, \quad 87,-2 \quad 6,-9, \quad 7 \\
& -9,-3, \quad 21-9,-x 30, \quad 5-9, \quad 4,-11
\end{aligned}
$$




\section{- $446-$}

per quas $f$ transit in $f^{\prime \prime}, f^{\prime \prime} f^{1 \mathrm{~V}}$ resp., et ex subst. vltima haec

$$
\begin{aligned}
& 1, \quad 4,4 \\
& 5, \quad 1,5 \\
& 3,-2,3
\end{aligned}
$$

per quam $f^{\mathrm{lv}}$ transit in $f$. Simili modo pro ex. 2 art. praec. prodeunt substitutiones

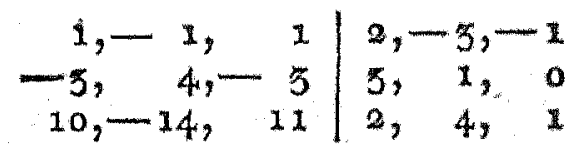

per quas resp. transit forma $\left(\begin{array}{ccc}10,26, & 2 \\ 7 & 0 & 4\end{array}\right)$ in $\left(\begin{array}{lll}0, & 2, & 0 \\ 0,-1, & 0\end{array}\right)$, atque haec in illam.

276. Throrgma. Chassium, in quas omnes formae ternariae determinantis dati distribswnitur, multitudo semper est finito.

Dem. I. Multitudo omnium formarum $\left(a, a^{\prime}, a^{\prime \prime}\right)$ determinantis dati $D$, in quibus $a$ $=0, b^{\prime \prime}=0, b$ non maior quam semissis diuisoris comm. max. numerorum $a^{\prime}, b^{\prime} ; a^{\prime \prime}$ non maior quam $b b^{\prime}$, manifesto est finita. Quoniam enim esse debet $a^{\prime} b^{\prime} b^{\prime}=D$, pro $b^{\prime}$ alli valores accipi nequeunt, quam $+1,-1$ atque radices quadratorum ipsum $D$ metientinm (si quae alia praeter I dantur) signo positiuo et negatiuo affectae, quorum valorum multitudo finita "est. Pro singulis autern valoribus ipsius' $b^{\prime}$ valor ipsins 


\section{- $447-$}

$a^{\prime}$ est determinatus, ipsorumque $b, a^{\prime \prime}$ valores manifesto limitantur ad multitudinem finitam.

II. Simili modo finita est multitudo omnium formarum $\left(\begin{array}{l}a, a^{\prime}, a^{\prime \prime} \\ b, b \\ b, b^{\prime \prime}\end{array}\right)$ determinantis $D$, in qư⿱bus $a$ non $=0$, neque maior quam $\sqrt[4]{5} \pm D$; $b^{\prime \prime} b^{\prime \prime}-a a^{4}=A^{\prime \prime}$ non $=0$ neque maior quam $4 \sqrt[3]{3}^{3} D^{2} ; b^{\prime \prime}$ non maior quam $a ; a b-b b^{4}$ $=B$ et $a^{\prime} b^{\prime}-b b^{\prime \prime}=B^{\prime}$ non maiores quam $x_{2} A^{\prime \prime}$ * Nam multitudo omnium combinationum valorum ipsorum $a, b^{\prime \prime}, A^{\prime \prime}, B, B^{\prime}$ finita exit; his vero singulis determinatis, etiam formae coefficienentes reliqui $a^{\prime}, b, b^{\prime}, a^{\prime \prime}$, coèfficientesque formae adiunctae $b b-a^{\prime} a^{\prime \prime}=A, b^{\prime} b^{\prime}-a a^{\prime \prime}=A^{\prime \prime}$ $a^{\prime \prime} b^{\prime \prime}-b b^{\prime}=B^{\prime \prime}$ determinati entant per aequationes hasce: $a^{\prime}=\frac{b^{\prime \prime} b^{\prime \prime}-A^{\prime \prime}}{a}, A^{\prime}=\frac{B B-a D}{A^{\prime \prime}}$, $A=\frac{B^{\prime} B^{\prime}-a^{\prime} D}{A^{\prime \prime}}, \quad B^{\prime \prime}=\frac{B B^{\prime}+b^{\prime \prime} D}{A^{\prime \prime}}, \quad b=$ $\frac{A B-B^{\prime} B^{\prime \prime}}{D}=-\frac{B a^{\prime}+B^{\prime \prime} b^{\prime \prime}}{A^{\prime \prime}}, b^{\prime}=\frac{A^{\prime} B^{\prime}-B B^{\prime \prime}}{D}$ $=-\frac{B b^{\prime \prime}+B^{\prime} a}{A^{\prime \prime}}, a^{\prime \prime}=\frac{b^{\prime} b^{\prime}-A^{\prime}}{a}=\frac{b b-A}{a^{\prime}}=$ $\frac{b b^{\prime}+B^{\prime \prime}}{b^{\prime \prime}}$. Iam quum omnes illae formae obtineantur, eligendo e cunctis combinationibus valorum ipsorum $a, b^{\prime \prime}, A^{\prime \prime}, B, B^{\prime}$ eas, e quibus etiam $a^{\prime}, a^{\prime \prime}, b, b^{\prime}$ valores integros nanciscuntur, illarum multitudo manifesto erit finita.

III. Cunctae itaque formae in I et II multitudinem finitam classium constituunt, quae etiam formarum ipsarum multitudine minor esse poterit, si quae ex ipsis inter se sunt aequiualen- 


\section{- $448-$}

tes. Iam quum per disquisitiones praecedentes quaeuis forma ternaria determinantis $D$ alicui ex illis formis necessario aequiualeat, i. e. ad aliquam e classibus quas hae formae constituunt pertineat: hae classes oinnes formas det. $D$ complectentur, i. e. omnes formae ternariae det. $D$ in multitudinem finitam classium distribuentior. Q. E. D.

277. Regulae, per quas omnes formae in I et II art. praec. erui possunt, ex ipsarum explicatione sponte defluunt; quare sufficiet quaedam exempla apposuisse. Pro $D=1$, formae $I$ hae sex (per ambig'nitatem signorum) pro-

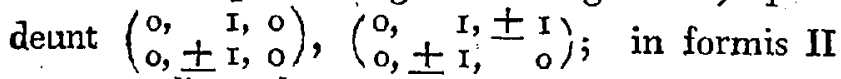
$a$ et $A^{\prime \prime}$ alios valores quam +1 et -1 habere nequeunt, pro singulis quatuor combinationum hinc oriundarum $b^{\prime \prime}, B$ et $B^{\prime}$ poni debent $=0$,

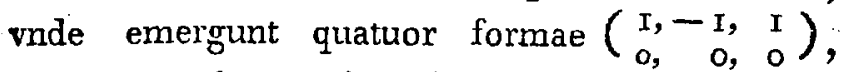
$\left(\begin{array}{r}-\mathrm{I}, \mathrm{r}, \mathrm{I} \\ 0,0,0\end{array},\left(\begin{array}{c}\mathrm{r}, \mathrm{r},-\mathrm{r} \\ 0,0,\end{array}\right),\left(\begin{array}{rr}-1,-\mathrm{r},-\mathrm{r} \\ 0, & 0,0\end{array}\right) . \quad\right.$ Simili modo pro $D=-1$ sex forma I quatuorque II habentur, $\left(\begin{array}{l}0,-\mathrm{r}, 0 \\ 0, \pm \mathrm{I}, 0\end{array}\right),\left(\begin{array}{l}0,-\mathrm{r}, \mathrm{I} \\ 0, \pm \mathrm{r}, 0\end{array}\right),\left(\begin{array}{l}\mathrm{r},-\mathrm{r},-\mathrm{I}), \\ 0,0,0\end{array}\right)$, $\left(\begin{array}{r}-\mathrm{I}, \mathrm{I},-\mathrm{I} \\ 0,0,0\end{array}\right),\left(\begin{array}{l}\mathrm{I}, \mathrm{I}, \mathrm{I} \\ 0,0,0\end{array}\right),\left(\begin{array}{rr}-\mathrm{I},-\mathrm{I}, \mathrm{I} \\ 0,0,0\end{array}\right) . \quad$ Pro $D=2$ sex formae I proueniunt $\left(\begin{array}{l}0,2,0 \\ 0, \pm 0\end{array}\right)$, $\left(\begin{array}{l}0,2, \pm I \\ 0, \pm \mathrm{r}, \mathrm{0}\end{array}\right)$, octoque formae II, $\left(\begin{array}{l}1,-\mathrm{I}, 2 \\ 0,0,0\end{array}\right)$, $\left(\begin{array}{r}-I, I, 2 \\ 0,0,0\end{array}\right), \quad\left(\begin{array}{rr}I, I,-2 \\ 0,0, & 2\end{array}\right), \quad\left(\begin{array}{r}-I,-I,-2 \\ 0,0,\end{array}\right)$,

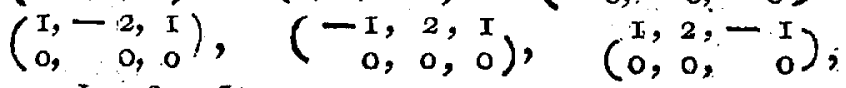
$\left(\begin{array}{rr}-1,-2,-1 \\ 0, & 0,0\end{array}\right)$. 


\section{- $449-$}

Ceterum multitudo classium ex his formis in his tribus casibus prodeuntium formarum multitudine multo minor est. Scilicet facile confirmatur

I. Formam $\left(\begin{array}{ll}0, & 1,0 \\ 0, & 1,0\end{array}\right)$ transire in $\left(\begin{array}{ll}0, & 1,0 \\ 0,-1,0\end{array}\right)$, $\left(\begin{array}{l}0, I, I \\ 0, \pm I, 0\end{array}\right),\left(\begin{array}{l}0, I,-I \\ 0, \pm I,\end{array}\right),\left(\begin{array}{l}I, I,-I \\ 0,0,0\end{array}\right)$ resp. per substitutiones

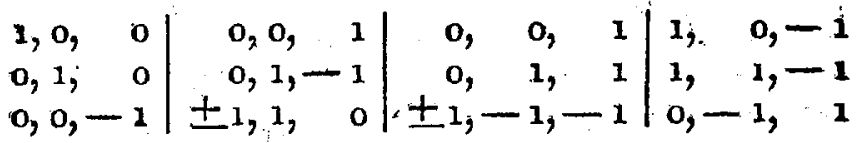

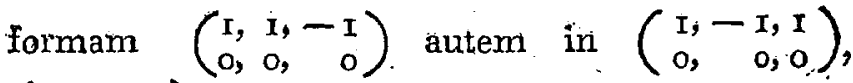
$\left(\begin{array}{r}\mathrm{I}, \mathrm{I}, \mathrm{I} \\ 0,0,0\end{array}\right)$ per solam indeterminatarum permutationem. Quare illae decem formae terna-

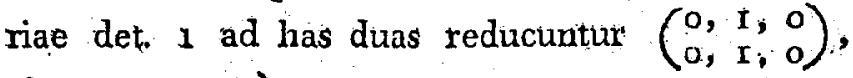
$\left(\begin{array}{r}-I,-I,-I \\ 0,0,0\end{array}\right)$; pro prióri, si magis arridet,

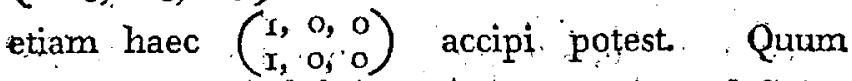
forma prior indefinita sit, posterior definita, manifestum et quamuis formam ternariani indefinitam det. 1 aequiualere formae $x x+$ zyz, quamuis definitan huic $-x x-y y$ $-z z$

II. Prorsus simili mòdo inuenitwr, quamlibet formam ternariam indefinitam determinantis $I$ aequiualere forma $-x x$ Ff 


\section{$-450$}

+ 2yz, quamlibet definitam huic $x x+y y$ $+z z$.

III. Pro determinante 2 ex octo formis (II) statim reiici possunt secunda, spxta et septima; quippe quae ex prima per solam indeterminatarum permutationem oriuntur, similique ratione etiam quinta quae e tertia, et octaua quae é quarta perinde proueniunt; tres reliquae, cum sex formis 1 , tres classes constituunt; scilicet $\left(\begin{array}{l}0,2,0 \\ 0,1,0\end{array}\right)$ transit in $\left(\begin{array}{c}0,2,0 \\ 0,-1,0\end{array}\right)$ per substitut tionem

$$
\begin{aligned}
& 1,0, \quad 0 \\
& 0,1, \quad 0 \\
& 0,0,-1
\end{aligned}
$$

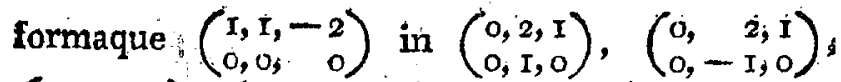
$\left(\begin{array}{rr}0,2,-1 \\ 0,1, & 0\end{array}\right),\left(\begin{array}{cc}0, & 2,-1 \\ 0,-1, & 0\end{array}\right),\left(\begin{array}{ll}1, & -1,2 \\ 0, & 0,0\end{array}\right) \cdot$ resp. per substitutiones

$$
\begin{array}{l|l|l|l|l|l}
1,0,1 & 1,0,-1 & 1,0, & 0 & 1,0,0 & 1,0,0 \\
1,2,0 & 1,2, & 0 & 1,2,-1 & 1,2,1 & 0,1,2 \\
1,1,0 & 1,1, & 0 & 1,1,-1 & 1,1,1 & 0,1,1
\end{array}
$$

Quaeuis itaque forñua ternaria determinantis 2 ad aliquam ex his tribus est reducibilis $\left(\begin{array}{lll}0, & 2,0 \\ 0, & 1, & 0\end{array}\right)$,

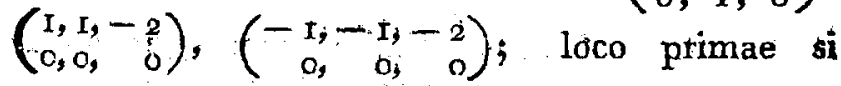
magis placet etiam $\left(\begin{array}{l}2,0,0 \\ 1,0,0\end{array}\right)$ accipi potest. Ma- 


\section{$-451-$}

nifesto autem quaeuis forma ternaria definita necessario aequiualebit tertiae $-x x-y y-2 z z$; quurn duae priores sint indefinitae; quaeuis indefinita primae vel secundae, et quidem primae $2 x x+2 y z_{2}$ si ipsius coëfficiens primus, secundus et tertius. simul sunt pares (quoniam facile perspicitur, talem formam per substitutionem quamcunque in similem formam transire, adeoque formae secundae aequitualere non posse), secundae $x x: y y-2 z z$ autem, si ipsius coëfficiens primus, secundus et tertius non simul : pares sunt, sed vinus, duo omnesue impares (in talem enim formam ex simili ratione forma prima $2 x x+$ $2 y z$ per nullam substitutionem transformabilis esse poterit).

Quod igitur in exemplis art. 273,274 euenit, vt forma definita $\left(\begin{array}{l}19,21,50 \\ 15,28,\end{array}\right)$ determiran. tis -1 ad hanc $x \dot{x}+y y+z z$, atque. forma indefinita $\left(\begin{array}{rrr}10 & 26,2 \\ 7, & 0,4\end{array}\right)$ determinantis a ad $2 x x$ - $2 y z$ siue (quod eoder redit) ad $3 x x$ * $2 y z$ reduceretur, per disquisitiones praecedentes a priori praeuideri potuisset.

278. Per formam temarịam, cuius indeterminatae sunt $x, x^{\prime}, x^{\prime \prime}$, repracsentantur tum numeri, tribuendo ipsis $x, x^{\prime}, x^{\prime \prime}$ valores determinatos, tum formae binariae per huiusmodi subs stitutiones $x=m t+n u, x^{i}=m^{i} t+n^{4} u$, " $x^{\prime \prime}$ $=m^{\prime \prime} t+n^{\prime \prime} u$, designantibus $m, n, m^{\prime}$ etc. numeros determinatos; $t, z$ indeterminatas formae repraeşentatae. Ad theoriam itıque complẹtanis formarum ternariarum requireretur solutip : . seEf 2 


\section{$-452-$}

quentium problematum: I. Inuenire omnes repraesentationes numeri dati per formam ternariam datam. II. Inuenire omnes repráesentationes formae binariae datae per ternariam datam. III. Diiudicare, vtrum duae formae ternaliae datae eiusdein determinantis aequiualentes: sint, necne, et in casu priori omnes transformationes alterius in alteram inuenire. IV. Diiudicare, vtrum forma ternaria data aliam datam determinantis maioris implicet, necne, et in casu priori omnes transformationes illius i: hanc assignare. De quibus problematibus longe difficilioribus quam anăloga in formis binariis alio loco pluribits agemus: hic disquisitionem nostram restringimus ad ostendendum, quomodo problema primum ad secundum secundimque ad tertium reduci possit; tertiuni vero pro casibus quibusdam simplicissimis formarumque binariarum theoriam imprimis illustrantibus soluere docebimus; quartum hic omnino exclùdemus.

279. Lrmma. Propositis tribus numeris integris quibuscunque $a, a^{\prime}, a^{\prime \prime}$ (qui tanten non omnes si$m u l=0$ ): inuenire sex alios $B, B^{\prime}, B^{\prime \prime}, C, C^{\prime}, C^{\prime \prime}$ ita comparatos vt fiat $B \cdot C^{\prime \prime}-B^{\prime \prime} C^{\prime}=a_{1} B^{\prime \prime} C-$ $B C^{\prime \prime}=a^{\prime} ; B C^{i}-B^{\prime} C=a^{\prime \prime}$;

Sol. Sit $\alpha$ dir. comm. max. ipsorum $a, a^{\prime}$, $a^{\prime \prime}$, accipianturque integri $A, A^{\prime}$, $A^{\prime \prime}$ ita vt fiat $A a+A^{\prime} a^{\prime}+A^{\prime \prime} a^{\prime \prime}=\alpha$. Porro accipiantur tres integri $\mathfrak{C}, \mathfrak{S}^{\prime}, \mathfrak{C}$ “ ad lubitum ea sola conditione; vt tres numeri $\mathfrak{C}: A^{\prime \prime}-\mathfrak{S}^{\prime \prime} A^{\prime}, \mathfrak{C}^{\prime \prime} A-$ C $A^{\prime \prime}{ }_{2} C^{\prime} A^{\prime}-\mathfrak{E}^{\prime} A$, quos resp. per $b, b^{\prime}, b^{\prime \prime}$ ipsorumque diuisorem communem maximim per 
designabimus, non fiant simul $=0$. Tune ponatur $a^{\prime} b^{\prime \prime}-a^{\prime \prime} b^{\prime}={ }_{\alpha b} C, a^{\prime \prime} b-a b^{\prime \prime}={ }_{a b} C^{\prime}$, $a b^{\prime}-a^{\prime} b=\alpha_{0}^{\prime} C^{\prime \prime}$, patetque ipsos $C, C^{\prime}, C^{\prime \prime}$ fore integros, Denique accipiendo integros $\mathfrak{B}$, $\mathfrak{B}^{\prime}, \mathfrak{B}^{\prime \prime}$ ita vt fiat $\mathfrak{B} b+\mathfrak{B}^{\prime} b^{\prime}+\mathfrak{B}^{\prime \prime} b^{\prime \prime}=6$, pónendo $\mathfrak{B} a+\mathfrak{R}^{\prime} a^{\prime}+\mathfrak{B}^{\prime \prime} a^{\prime \prime}=\alpha h$, et statuendo $B=\omega^{\prime}-h A, B^{\prime}=\alpha B^{\prime}-h A^{\prime}, B^{\prime \prime}=\alpha^{\prime \prime}$ - $h A^{\prime \prime}$, hi valores ipsorum $B, B^{\prime}, B^{\prime \prime}, C, C^{\prime}$, C" aequationibus praescriptis satistacient.

Inuenitur enim $a B+a^{\prime} B^{\prime}+a^{\prime \prime} B^{\prime \prime}=0, b A+$ $b ! A^{\prime}+b^{\prime \prime} A^{\prime \prime}=0$, vnde $b B+b^{\prime} B^{\prime}+b^{\prime \prime} B^{\prime \prime}=$ ab. Iam ex valoribus ipsorum $C^{\prime}, C^{\prime \prime}$ fit $\alpha b\left(B^{\prime} C^{\prime \prime}\right.$ $\left.-B^{\prime \prime} C^{\prime}\right)=a b^{\prime} B^{\prime}-a^{\prime} b B^{\prime}-a^{\prime \prime} b B^{\prime \prime}+a b^{\prime \prime} B^{\prime \prime}$ $=a\left(b B+b^{\prime} B^{\prime}+b^{\prime \prime} B^{\prime \prime}\right)-b\left(a B+a^{\prime} B^{\prime}+\right.$ $\left.a^{\prime \prime} B \cdot\right)=\alpha \zeta a$, adeoque $B^{\prime} C^{\prime \prime}-B^{\prime} C^{\prime}=a$; similique modo inuenitur $B^{\prime \prime} C-B C^{\prime \prime}=a^{\prime}$, $B C^{\prime}-B^{\prime} C=a^{\prime \prime}$. Q.E.'F. Ceterum analysis per quam haec solutio inuenta est, nec non methodus ex vna solutione omnes inueniend, hic sunt supprimendae.

2.5o. Supponamus, formam binariam att + $2 b t u+c u u \ldots \Phi$, cuius determinans $=D$, repraesentari per formam ternariam $f$ cuius indeterminatae $x, x^{\prime}, x^{\prime \prime}$, ponendo $x=n t+n u$, $x^{\prime}=m^{\prime} t+n^{\prime} u, x^{\prime \prime}=m^{\prime \prime} t+n^{\prime \prime} u$, ipsique $f$ adiunctam esse formam $F$, cuius indeterminatae $X, X^{\prime}, X^{\prime \prime}$. Tunc per calculum facile confirmatur (designando coëfficientes formarum $f, F$ per literas peculiares) siue etiam ex art, 268 . II. protinus deducitur, numerum $D$. repraesentari per $F$ ponendo $X \doteq m^{\prime} n^{\prime \prime}-m^{\prime \prime} n^{\prime}, X^{\prime}=m^{\prime \prime} n-m n^{\prime \prime}$, $X^{\prime \prime}=m n^{\prime}-m^{\prime} n$, quáe repraesentatio numeri Ff $\xi$ 


\section{$-454 \div$}

$D$ repraesentationi formae $\Phi$ per $f$ adiuncta com mode dici potest. Si valores ipsarum $X, X^{\prime}, X^{4}$ dituisorem communem non habent, 'breuitatis caussa hanc repraesentationem ipsius 1 p propriam vocabimus, sin secus impropriam, easdem denominationes etiam repraesentationi formae : per $f$, cui illa repraes. ipsius $D$ adiuncta est, tribuemus. Iam inpentio omnium repraesentationum propriarum numeri $\mathcal{D}$ per formam $F$ sequentibus momentis innititur:

I. Nulla repraesentatio ipsius $D$ per $F$ datur, quae non ex aliqua repraesentatione alicuius formae determinantis $D$ per formam $f$ deduci possit, i. e. tali repraesentationi adiuncta sit

Sit enim repraesentatio quecunque ìpsius $D$ per $F$ haec: $X=L, X^{\prime}=L^{\prime}, X^{\prime \prime}=L^{\prime \prime}$; accipiantur per lemma art. praec. $m, m^{\prime}, m^{\prime \prime}, n, n^{\prime}, n^{\prime m}$ ita vt fiat $m^{\prime} n^{\prime \prime}-m^{\prime \prime} n^{\prime}=L, m^{\prime \prime} n-m n^{\prime \prime}=L^{\prime}$, $m n^{\prime}-m^{\prime} n=L^{\prime \prime}$, transeatque $f$ per substitutionem $x=m t+n u, x^{\prime}=m^{\prime} t+n^{\prime} u, x^{\prime \prime}=m^{\prime \prime} t+$ $n^{\prime \prime} u$ in formam binariam $0=a t t+2 b t u+c u u$. Tunc facile perspicietur, $D$ fore determinantem formae $\phi$ ipsiusque repraesentationi per $f$ repraesentationem nropositam ipsius $D$ per $F$ adiunctam.

Ex. Sit $f=x x+x^{\star} x^{i}+x^{\mu} x^{\prime \prime}$, adeoque $E$ $=-X X-X^{\prime} X^{\prime}-X^{\prime \prime} X^{\prime \prime} ; D=-809$, ipsiusque repraesentatio per $F$ haec $X=1, X^{\prime}=8$, $X^{\mu}=12$; hinc inueniuntir valores ipsorum $m_{3}$ $m^{\prime}, m^{\prime \prime}, n, n^{l}, n^{\prime \prime}$ hi $=20,1,1,-12,0,1$ xesp.; atque $\phi=402 t t+482 t u+14544$. 
II. Si $\varphi, \chi$ sunt formae binariae proprie aequiualentes, quaeuis repraesentatio ipsius $D$ per $F$ alicui repraesentationi formae $\checkmark$ per $f$ adiuncta, etiam alicui repraesentationi formae $\chi$ per $f$ adiuncta erit.

Sint $p, q$ indeterminatae formae $\chi$; transeat ( in $\chi$ per substitutionem propriam $t=u p+\epsilon q$, $u=? p+\delta q$, sitque aliqua repraesentatio forma $\varphi$ per $f$ haec $x=m t+n u, x^{\prime}=m^{\prime} t+n^{\prime} u, x^{\prime \prime}$ $=m^{\prime \prime} t+n^{\prime \prime} u \ldots(R)$. Tune nullo negotio perspicitur, si ponatur $\alpha m+\gamma n=g, a m^{i}+g n^{\imath}=$ $g^{\prime \prime}, \alpha m^{\prime \prime}+y n^{\prime \prime}=g^{\prime \prime}, 5_{m}+g_{n}=h, h_{m^{\prime}}+\partial_{n^{\prime}}=$ $h^{\prime}, \mathrm{e}^{\prime} m^{\prime}+\delta n^{\prime}=h^{\prime}, \quad \bar{m} m^{\prime \prime}+\delta \dot{n}^{\prime \prime}=h^{\prime \prime}$, formam $\chi$ repraesentatum iri per $f$ statuendo $x=g p+h q$, $x^{\prime}=g^{\prime} p+h^{\prime} q, x^{\prime \prime}=g^{\prime \prime} p+h^{\prime \prime} q \ldots\left(R^{\prime}\right)$, calculoque facto inuenitur (propter $\alpha \delta-b_{\gamma}=1$ ) esse $g^{\prime} h^{\prime \prime}$ - $g^{\prime \prime} h^{\prime}=m^{\prime \prime} n^{\prime \prime}-m^{\prime \prime} n^{\prime}, g^{\prime \prime} h-g h^{\prime \prime}=m^{\prime \prime} n-m n^{\prime \prime}$, $g h^{\prime}-g^{\prime} h=m n^{\prime}-m^{\prime} n$, i. e. repraesentationibus $R, R^{i}$ eadem repraesentatio ipsius $D$ per $F$ adiuncta est.

Ita in ex. praec. formae a a quiualere inuenitur inuenitur $x=13 p p-10 p q+18 q q$, in quam illa transit per substitutionem propriam $t=-3 p$ $+q, u=5 p-2 q$; hinc inuenitur repraesentatio formae $x$ per $f$ haec $x=4 q, x^{4}=-3 p+q, x^{\prime \prime}$ $=2 p-q$, ex qua eadem numeri - 209 reprae centatio deducitur, a qua profecti eramus.

III. Denique si duae formae binariae $\rho, x$ determinantis $D$, quarum indeterminatae sunt $t, u ; p$, $q$, per $f$ repraesentari possunt, alicuique repraesentationi viús eadem repraesentatio propria ipsius $D$ 
per $F$ adiuncta est, atque alicui repraesentationi alterius, illae formae necessario erunt proprie aequiualentes. Supponamus $\Phi$ repraesentari per $f$ ponendo $x=m t+n u, x^{\prime}=m^{\prime} t+n^{\prime} u, x^{\prime \prime}=$ $m^{\prime \prime} t+n^{\prime \prime} u ; \chi$ vero statuendo $x=g p+h q$, $x^{\prime}=g^{\prime} p+h^{\prime} \bar{q}, x^{\prime \prime}=g^{\prime \prime} p+h^{\prime \prime} p$, atque esse $m^{\prime} n^{\prime \prime}-m^{\prime \prime} n^{\prime}=g^{\prime \prime} h^{\prime \prime}-g^{\prime \prime} h^{\prime}=L, m^{\prime \prime} n-m n^{\prime \prime}$ $=g^{\prime \prime} h-g h^{\prime \prime} \equiv L^{\prime}, m n^{\prime}-m^{\prime \prime}=g^{\prime} h^{\prime}-g^{\prime \prime} h$ $=L^{\prime \prime}$. Accipiantur integri $l, l^{\prime}, l^{\prime \prime}$ ita vt fiat $L l+L^{\prime} l^{\prime}+L^{\prime \prime l !}=\mathrm{I}$, ponaturque $n^{\prime \prime} l^{\prime \prime}-n^{\prime \prime l}$ $=M, n^{\prime \prime} l-n l^{\prime \prime}=M^{\prime}, n l^{\prime}-n^{\prime} l=M^{\prime \prime}$, $l^{\prime} m^{\prime \prime}-l^{\prime \prime} m^{\prime}=N, l^{\prime \prime} m-l m^{\prime \prime}=N^{\prime}, l m^{\prime}-$ $l^{\prime} m=N^{\prime \prime} ;$ denique statualur $g M+g^{\prime} M^{i}+g^{\prime \prime} M$ $=\alpha, h M+h^{\prime} M^{\prime}+h^{\prime \prime} M^{\prime \prime}=6, g N+g^{\prime \prime} N^{\prime}+$ $g^{\prime \prime} N^{\prime \prime}=\gamma, h N+h^{\prime} N^{\prime}+h^{\prime \prime} N^{\prime \prime}=\delta_{\text {a }}$. Hinc facile 'deducitur

$e m+y^{n}=g-l\left(g L+g^{\prime} L^{\prime}+g^{\prime \prime} L^{\prime \prime}\right)=g$ $6 m+\delta n=h-l\left(h L+h^{\prime} L^{\prime}+h^{\prime \prime} L^{\prime \prime}\right)=h$

similique modo $\alpha m^{\prime}+2 n^{\prime}=g^{\prime}, 6 m^{\prime}+\delta n^{\prime}=$ $h^{\prime}, u m^{\prime \prime}+y n^{\prime \prime}=g^{\prime \prime}, 6_{m}^{\prime \prime}+\delta n^{\prime \prime}=h^{\prime \prime}$. Hinc patet $m t+n u, m^{\prime} t+n^{\prime} u, m^{\prime \prime} t+n^{\prime \prime} u$ transire per substitutionem $t=\alpha p+6 q, u=\gamma p+\delta q$ ... (S) in $g p+h q, g^{\prime} p+h^{\prime} q, g^{\prime \prime} p+h^{\prime \prime} q$ resp., vnde manifestum est, \& transire per substitutionem $S$ in eandem formam, in quam $f$ transeat ponendo $x=g p+h q, x=g^{\prime} p+h^{\prime} q, x^{\prime \prime}=$ $\boldsymbol{g}^{\prime \prime} p,+h^{\prime \prime} q$, adeoque in formam $\chi$, cui itaque aequiualet. Denique per substitutiones debitas facile inuenitur $\alpha \delta^{\prime}-\sigma_{\gamma}=L l+L^{\prime \prime} l^{\prime}+L^{\prime \prime \prime}{ }^{\prime \prime}=i_{\text {, }}$ quocirca substitutio $S$ est propria, formaeque $\phi, x$ proprie aequiualentes. 
Ex his obseruationibus deriuantur regulae sequentes ad inueniendum omnes repraesentatio. nes proprias ipsius $D$ per $F$ : Euoluantur omnes classes formarum binariarum determinantis $D$, et ex singulis vna forma ad libitum eligatur; quaerantur omnes repraesentationes propriae singularum harum formarum per $f$ (reiectis is quae forte per $f$ repraesentari nequeunt), et ex singulis hisce repraesentationibus deducantur repraesentationes numeri $D$ per $F . \quad \mathrm{Ex} I$ et II manifestum est, hoc modo omnes repraesentationes proprias possibiles obtineri, adeoque solutionem esse completam; ex III, transformationes formarum e classibus diuersis certo producere repraesentationes diuersas,

281. Inuestigatio repraesentationum impropriarum numeri dati $D$ per formam $F$ ad casum praecedentem facile reducitur. Scilicet manifestum est, si $D$ per nullum quadigtum (praeter 1) diuisibilis sit, tales repraesertationes omnino non dari; sin secus, metientibus ipsum $D$ quadratis $\lambda \lambda, \mu \mu ; *$ etc., omnes repraesentationes improprias ipsius $D$ per $F$ inueniri, si omnes repraesentationes propriae numerorum $\frac{D}{\lambda \lambda}, \frac{D}{\mu \mu}, \frac{D}{\nu}$ etc. per eandem formam euoluantur, indeterminatarumque valores per $\lambda_{2}$, , etc. resp. multiplicentur.

Hoc itaque modo inuentio omnium repraesentationum numeri dati per formam ternariam datam, quae alicui formae ternariae adiuncta est, a problemate secundo pendet; ad hunc vero casum, qui primo aspectu minus late patere videri Ff 5 


\section{$-458-$}

posset, reliqui ita redueuntur. Sit $D$ numerus repraesentandus per formam $\left(g^{\prime}, g^{f}, h^{\prime}, h^{\prime \prime}\right)$, cuius determinans $\Delta_{3}$ et cui adiuncta est forma $\left(\begin{array}{l}G, G^{\prime}, G^{\prime \prime} \\ H, H^{\prime}, H^{\prime \prime}\end{array}\right)=f$. Turic huic rursus adiuncta erit $\left(\begin{array}{c}\Delta g, \Delta g^{\prime}, \Delta g^{\prime r} \\ \Delta h, \Delta h^{\prime}, \Delta h^{\prime \prime}\end{array}\right)=F$, patetque, repraesentationes numeri $\Delta D$ per $F$ (quarum inuestigatio a praecc. pendet) ommino identicas esse cum reprasesenta* tonibus numeri $D$ per formam propositam. Ceterum quando onmes coufficientes lomae $f$ diuisorem communem $p$ habent, perspicuum est, omnes coëficientes formae $F$ diuisibiles esse per $\mu \mu$, quacirca etiam $\Delta D$ per $\mu \mu$ diuisibilis esse de bebit (alioquin nullae repraesentationes daren tur); repraesentationesque numeri $D$ per for tham propositam coincident cum repraesentationibus numeri $\frac{\Delta D}{\mu \mu}$ per formarm quae oritur ex $F_{3}$ diuidendo singulos coëfficientes per an, cui formae adiuncta erit ea, quae oritur ex $f$, diüden d singulos coëfficiontes per $\mu$,

Denique obseruamus, hanc problematis prix mi solutionem in vnico casu, vbi $D=0$, non esse applicabilem; hic enim omnes formae bina* riae eleterminantis $D$ in multitudinem finitum classium non distribuuntur; infra autem hane cam S:am ex alis principiis soluernus,

282. Inuestigatio ropraesentationum formae binariae datae cuius determinans non $=0^{*}$ ) per

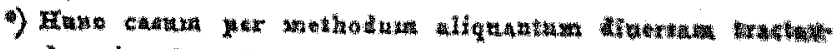

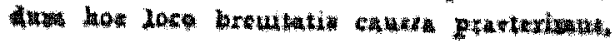




\section{$-459$}

ternariam datam pendet ab obseruationibus sequentibus:

I. Ex quanis repraesentatione propria for mae binariae $(p, q, r)=p$ determinantis $D$ per ternariam $f$ determinantis $\Delta$ deduci possunt Integri $B, B^{\prime}$ tales vt sit $B B \equiv A p, B B^{\prime} \equiv-$ $\Delta q, B^{\prime} B^{\prime}=c q(\bmod . I D)$, i. e. valor expressionis $\sqrt{ } \Delta(p,-q, r)$ (mod. $D)$ ). Ilabeatur represesentatio propria formae per $f$ harc, $x=$ $\alpha^{\prime}+b_{u}, x^{\prime}=\alpha^{\prime} t+b_{u l}, x^{\prime \prime}=\cdots t+b^{\prime \prime l u}$, (designantibus $x, x^{\prime}, x^{\prime \prime} ; t, u$ indeterminat as formarum $f, \phi)$; accipiantur integri : $\gamma^{\prime}, \boldsymbol{\gamma}^{\prime \prime}$ ita vt $\left.\left(\alpha^{\prime \prime \prime}-\alpha^{\prime \prime \prime} b^{\prime}\right) y+\left(u b^{\prime \prime}-\alpha^{\prime \prime}\right)^{\prime}\right) \gamma^{\prime}+$ $\left(\alpha_{0}^{\prime}-\alpha_{0}^{\prime}\right) \gamma^{\prime \prime}=k$ fiat vel $=+1$ vel $=$ -1 , transeatque $f$ per substitutionem

$$
\begin{aligned}
& \Leftrightarrow, \quad, \gamma \\
& \alpha^{\prime}, b^{\prime}, y^{\prime} \\
& \alpha^{\prime \prime}+b^{\prime \prime}, \gamma^{\prime \prime}
\end{aligned}
$$

in formam $\left(\begin{array}{ccc}a, & a^{\prime}, & a^{\prime \prime} \\ b, & b^{\prime}, & b^{\prime \prime}\end{array}\right)=g$, cui adiuncta sit $\left(\begin{array}{l}A, A^{\prime}, A^{\prime \prime} \\ B, B^{\prime}, B^{\prime \prime}\end{array}\right)=G$. Tunc manifestum est, fore $\left.a=p, b^{\prime \prime}=q, a^{\prime}=r, A^{\prime \prime}=I\right)$, atque $\Delta$ determinantem formae $g$; vide $B B=-p+$ $A^{\prime} D, B B^{\prime}=-\Delta q+B B(B), B \cdot B=2 r+A D$. - Ita c. g. forma $1 g t t+6 t u+4 x u u$ repraesentatur per $x x+x^{\prime \prime} x^{\prime}+x^{\prime \prime} x^{\prime \prime}$ ponendo $x=$ $3^{t}+5 u, x^{t}=3^{t}-4 u, x^{\prime \prime}=t ;$ vnde statuendo $\gamma=-1, \gamma^{i}=1, \gamma^{\prime \prime}=0$, cruitur $B$ $=-171, B^{\prime}=27$, siue valor $(\cdots 171,27)$ expr. $r-1(19,-3,41)$ (mod. $77 a$ ): 


\section{$-460-$}

Hinc iam sequitur; si $\Delta(p,-q, r)$ non sit residuum quadratum ipsius $D$, $\phi$ per nullam formam ternariam determinantis $\Delta$ proprie repraesentabilem esse posse; in eo itaque casu vhi $\Delta, D$ inter se primi sunt, $\Delta$ numerus characteristicus formae $\phi$ eșse debebit.

II. Quum $\gamma, \gamma^{\prime}, \gamma^{\prime \prime}$ infinite multis modis diuersis determinari possint, etiam alii atque alii valores ipsorum $B, B^{\prime}$ inde prodibunt, qui quem nexum inter se habeant videanuus. Ponamus, etiam $\delta, \delta^{\prime}, \delta^{\prime \prime}$ ita comparatos esse, vt $\left(\alpha^{\prime} b^{\prime \prime}-{\dot{u^{\prime}}}^{\prime \prime \sigma^{\prime}}\right) \mathcal{L}$

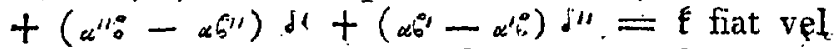
vel $=+1$ vel $=-1$, formamque $f$ transire per substitutionem.

$$
\begin{array}{lll}
\text { a, } 6, & 8 \\
\alpha^{\prime}, & 6, & \alpha \\
\alpha^{\prime \prime}, & 6 \prime \prime & \delta^{\prime \prime}
\end{array}
$$

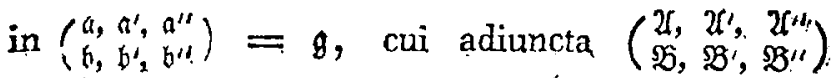
$=\mho \mathfrak{S}$. Tunc $g, g$ erunt aequiuálentes, adeoque etiam $G$ et $\mathfrak{B}$, et per applicationem praeceprorum in artt, 269,270 traditorum *) inuenitur, si statuatur $\left(6^{\prime} y^{\prime \prime}-6^{\prime \prime \prime} y^{\prime}\right) \delta+\left(6^{\prime \prime \prime} \gamma-6 \gamma^{\prime \prime}\right) \delta^{\prime \prime}+\left(6 y^{\prime}-6 \gamma^{\prime} \gamma\right) \delta^{\prime \prime}$ $=\zeta,\left(\gamma^{\prime \prime} \alpha^{\prime \prime}-\gamma^{\prime \prime} \alpha^{\prime}\right) \delta+\left(\gamma^{\prime \prime} \alpha-\gamma \alpha^{\prime \prime}\right) \delta^{\prime \prime}+\left(\gamma \alpha^{\prime}-\gamma^{\prime} \alpha\right) \delta^{\prime \prime \prime}$ = , formam $\mathcal{G}$ transire in $G$ per substitutionem.

$$
\begin{array}{lll}
k, & 0, & 0 \\
0, & k, & 0 \\
\xi & , &
\end{array}
$$

*) Eruenda ex transf. formaf $f$ in $g$, transformationem forman $g$ in $f$; ex hac atque transf. formee $f$ in $g$, transf. formate $g$ in gi denique ex hac, per treuspositionem, tranof. forr mat $\mathfrak{B S}$ in $G$. 


\section{$-461-$}

Hinc erit $B={ }_{n}{ }^{B} D+k k \mathfrak{B}, B^{\prime}=\zeta^{2} D+k k \mathfrak{B}$, adeoque, propter $k= \pm 1$, vel $B \equiv \mathscr{B}, B^{\prime}$ $\equiv \mathfrak{B}^{\prime}, \operatorname{vel} B \equiv-\mathscr{B}, B^{\prime} \equiv-B^{\prime}(\bmod . D)$. In casu priori valores $\left(B, B^{\prime}\right),\left(\mathfrak{B}, \mathfrak{B}^{\prime}\right)$ aequiualentes vocamus, in posteriori oppositos; repraesentationem formae $\phi$ autem ad quemlibet valorem expr. $\sqrt{ } \Delta(p,-q, r)(\bmod . D)$, qui ex ipsa per methodum in I deduci potest, pertinere dicemus. Hinc omnes valores, ad quos eadem repraesentatio pertinet, vel aequiualentes erunt vel oppositi.

III. Vice versa autem, si vt ante in I repraesentatio formae $\phi$ per $f$ haec $x=a t+b i c$ etc. ad valorem $\left(B, B^{\prime}\right)$. pertinet, qui inde deducitur adiumento transformationis

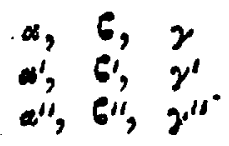

eadem quioque ad quemuis alium valorem ( $(B$, $\left.B^{\prime}\right)$ pertinebit, qui illi vel aequiualens est vel oppositus; i. e. loco ipsorum $\gamma, \gamma^{\prime}, \gamma^{\prime \prime}$ alios integros $\delta, \delta, \delta$, accipere licebit, pro quibus aequatio

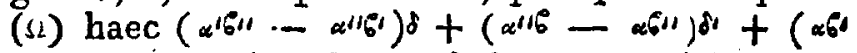
$\left.-\alpha^{\circ}\right)^{d i}= \pm 1$ locum habeat, et qui ita comparati sint, vt coëfficiens 4 et 5 in forma ei adiuncta, in quam $f$ per substitutionem $(S)$

$$
\begin{aligned}
& \alpha, 6, \delta \\
& \alpha^{\prime}, 6, \delta^{\prime \prime} \\
& \alpha^{\prime \prime}, \epsilon^{\prime \prime}, \delta^{\prime \prime} .
\end{aligned}
$$

transit, resp. fiant $=\mathfrak{B}, B^{4}$. Statuatur enim $\pm B=8+{ }^{\prime} D, \pm B^{\prime}=B^{\prime}+\zeta D$ (accipiendo 


\section{$-462$}

hic et postea signa superiora vel inferiora, prout valores $\left(B, B^{\prime}\right),\left(B, \mathfrak{B}^{\prime}\right)$ aequiualentes sunt vel oppositi), vnde $\zeta$, "erunt integri, transeatque $\sigma$ per substitutionem

$$
\begin{aligned}
& 1,0,5 \\
& 0,1, \pm \\
& 0,0, \pm 1
\end{aligned}
$$

in formam $g$, cuius determinantem esse $\Delta$, in forma adiuncta vero coufficientes of at 5 resp. $=\mathfrak{B}, \mathfrak{B}^{\prime}$ fieri facile perspicietur. l'aciondo au-

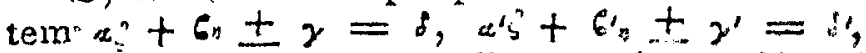
$a^{\prime \prime} \xi+b^{\prime \prime} \pm \pm \gamma^{\prime \prime}=\delta "$, nullo neegotio patobit, $f$ per substitulionem $(S)$ transire in 8 , atche atequationi (12) satisfacturn esse. (L. Ei. $D$.

283. Ex his principiis deducitur methodus sequens, omanes repraesentationes proptias formae binariat $D=p t t+2 q t u+r u u$ determinantis $D$ per temarian $f$ deterninantis a imueniendi.

I. Erunntur ornes valores diueri (i. a. non aequiualentes; 'xyprescionis $\sqrt{ }(p,-q, r)$ (mod. $D)$. Hoc problemia pro to casu, vbi . est forma primitiua atcue $\Delta$ ad $D$ primus, supra (art. 235) solutum est, casusque reliqui ad hunt facillime reducuntur, quan tamen rem fusius hic explicare breuitas non permittit. Obsemanuus tantummodo, quoties $\Delta$ ad $D$ primus sit, expressionem $\Delta(p,-q, r)$ residuum quadratioum ipsius $D$ esse non posse, nisi $\odot$ fuerit forma primitima. Supponendo enim $\triangle p=B B-D A,-\triangle q=$ $B B^{\prime}-D B^{\prime \prime}, \quad A r=B^{\prime} B^{\prime}-D A$, fit $\left(D B^{\prime \prime}-\right.$ $\left.\Delta Q)^{2}=(D A+\therefore p)(J) A+\Delta r\right) ;$ hinc, per euolutionem et substituendo $x / q$ - pr pro $D$, fit $(9 q-$ 


\section{$-463-$}

$p r)\left(B^{\prime \prime} B^{\prime \prime}-A A^{\prime}\right)-\Delta\left(A p+2 B^{\prime \prime} q+A r\right)$ $+\Delta A=0$, wnde facile concluditur, si $p, q, r$ diuisorem communem haberent, hunc etian ipsum $\Delta \Delta$ metiri; tunc vero $\Delta$ ad $D$ primus esse non posset. Quare $p, q, r$ diuisorem communem habere nequeunt, siue $\phi$ erit forma primitiua.

II. Designemus multitudinem horum valo num per $m$, supponamusque, inter cos reperiri $n$ valor's, qui sibi ipsis oppositi sint (statuendo $n$ = 0 quando tales non adsunt). Tunc manifestum est, ex $m-n$ reliquis valoribus binos semper oppositos fure (quoniam cuncti valores complete haberi supponmutur); reilciatu e binis quibusque valoribus oppositis vnus ad libitum, remanebuntque ommino valores $\frac{\pi}{2}(m+n)$. Ita e. $g$. ex octo valorihus expr. $\sqrt{ }-x \times(19,3,4.1)$ (mod. 770$)$ his $(+4,237),(171,-27),(269,-$ 83), $291,-127),(-44,-237),(-171,27)$, $(-269,83),(-291,127)$, quatuor posteriores sunt reilciendi, tamquam quatuor prioribus oppositi. Ceterum persplicuum est, si $(B, B !)$ sit valor sibi ipsi oppositus, $2 B, 2 B^{\prime}$, et proin etiam $2 \Delta p, 2: q, 2 \Delta r$ per $D$ diuisibiles fore; quodsi itaque $\triangle, D$ inter se primi sunt, etiam $2 p, 2 q, 2 r$ per $\tilde{D}$ diuisibiles erunt, et quum, per $I$, in hoc casu etiam $p, q, r$ diuisorem communem habere nequennt, etiam 2 per $D$ diuisibilis esse debebit, quod fieri nequit nisi $D$ vel $= \pm 1$, vel $=$ \pm 2. Quamobiem pro ommibus valoribus ipsius $D$ maiorit. quam 2 semper erit $n=0$, si $\Delta$ ad $D$ est primus.

III. His ita factis manifestum est, quamui repraesentationem propriam formae $p$ per, $f$ no 


\section{- 464 -}

cessario ad aliquem e valoribus remanentibus pertinere debere, et quidem ad vnicum tantum. Quare hi valores successiue surt percurrendi, repraesentationesque ad singulos pertinentes inuestigandae. $\mathrm{Vt}$ inueniantur repraesentationes ad valorem datum $\left(B, B^{\prime}\right)$ pertinentes, primo determinanda est forma ternaria $g=\left(\begin{array}{l}a, a^{\prime}, a^{\prime \prime} \\ b, b^{\prime}, b^{\prime \prime}\end{array}\right)$. cuius determinans $=\Delta$ et in qua $a=p, b^{\prime \prime}=$ $\dot{q}, a^{\prime}=r, a b-b^{\prime} b^{\prime \prime}=B, a^{\prime} b^{\prime}-b b^{\prime \prime}=B^{\prime}$; valores ipsorum $a^{\prime \prime}, b, b^{\prime}$ hinc inueniuntur adiumento aequationurn in II art. $27^{6}$, ex quibus facile perspicitur, in eo casu vbi $\Delta, D$ inter se primi sint, $b, b^{\prime}, a^{\prime \prime}$ necessario fieri integros' (nempe quoniam hi tres numeri, multiplicati tum per $D$ tum per $\Delta$ integros producunt). Iam si vel aliquis coëfficientium $b, b^{\prime}, b^{\prime \prime}$ fractus est, vel formae $f, g$ non sunt aequiualentes: nullae repraesentationes formae $\phi$ per $f$ ad $\left(B, B^{\prime}\right)$ pertinentes dari possunt; si vero $b, b^{\prime}, a^{\prime \prime}$ sunt integri, formaeque $f, g$ aequiualentes, quaeuis transformatio illius in hanc, vt

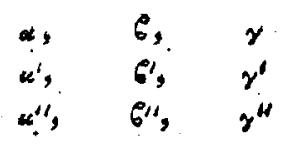

talem repraesentationem suppeditat, puta $x$ $=\alpha t+b_{u}^{\prime \prime}, x^{\prime}=\alpha^{\prime} t+{ }^{\prime} u, x^{\prime \prime}=\alpha^{\prime \prime} t+\delta^{\prime \prime} u ;$ manifestoque nulla huiusmodi repraesentatio exstare poterit, quae non ex aliqua transformatione deduci posset. Hoc itaque modo ea problematis sécindi pars, quae inuestigat repraesentationes proprias, ad problema tertiurn iam est reducta. 


\section{$-\quad 465-$}

IV. Ceterum transformationes diuersae formae $f$ in $g$ semper producuut repraesentationes diuersas, eo solo casu excepto, vbi valor $\left(B, B^{\prime}\right)$ sibi ipsi oppositus est, in quo binae transtormationes vnicam semper repraesentationem suppe: ditant. Supponendo enim, $f$ transire in $g$ etiam per substitutionem

$$
\begin{aligned}
& \alpha, 6, \delta \\
& a^{\prime}, b^{\prime}, j^{\prime} \\
& a^{\prime \prime}, 6^{\prime \prime}, \delta^{\prime \prime}
\end{aligned}
$$

(quae eandem repr. praebet vt transf. praec.), denotandoque per $k, \mathfrak{f}, ?$, n numeros eosdem vt in II art. praec., erit $B=k \xi B+n E D, B^{\prime}=$ $k^{k} B^{l}+\zeta \mathfrak{f} D$; si itaque vel vterque $k, \mathfrak{f}$ supponitur $=+1$, vel vterque $=-1$, erit (quia casum $D=0$ exclusimus) $\xi=0,{ }_{n}=0$, vade facile sequitur $\delta^{\prime}=\gamma, \delta^{\prime}=\gamma^{\prime}, \delta^{\prime \prime}=\gamma^{\prime \prime}$; quare illae duae transformationes in eo solo casu diuersae esse possünt, vbi alter numerorum $k$, est +1 , alter -1 ; tunc erit $B \equiv-B$, $B^{\prime}$ $\equiv-B^{\prime}(\bmod . D)$, siue valor $\left(B, B^{\prime}\right)$ sibi ipsi oppositus.

V. Ex is, quae supra (art. 271) de criteriis formarum definitarum et indefinitarum tradidimus, facile sequitur, si $\Delta$ sit positiuus, $D$ negatiuus, atque: $\uparrow$ forma negatiua, $g$ fieri formam definitam nega\&iuam; si vero. $\Delta$ sit positiuus, atque vel $D$ positiuus, vel $D$ negatiuus et $\phi$ forma positiva, euadere formam indefinitam. Im gutum $f, g$. certo aequiualentes esse nequeant, misi respectu huiư' qualitatis similes sint', manifestum est, for:mas binarias determinantis positiui nec non po- 
sitiuas, per ternariam negatiuam proprie repraesentari non posse, neque : formas binarias negatiuas per ternariam indefiritam determinantis positiui; sed per formam ternariam prioris posteriorisue speciei vnice binarias posterioris priorisue resp. Simili modo concluditur, per formam ternariam determinantis negatiui definitam (i. e. positiuam)' vnice repraesentari binarias positiuas, per indefinitam vnice negatiuas et formas det. positiui.

284. Quum repraesentationes impropriae formae binariae $\phi$ determinantis $D$ per ternariam $f$, cui adiuncta est $F$, eae sint, ex quibus repraesentationes impropriae numeri $D$ per formam $F$ sequuntur, $\phi$ per $f$ manifesto nequit improprie repraesentari, nisi $D$ factores quadratos implicet. Ponamus, omnia quadrata ipsum $D$ metientia (praeter i) esse $e e, e^{\prime} e^{\prime}, e^{\prime \prime} e^{\prime \prime}$ etc. (quorum multitudo finita erit, quia supponimus, non esse $D$ $=0$ ), praebebitque quaelibet repr. impr. formae $\phi$ per $f$ repraesentationem numeri $D$ per $F$, in qua valores indeterminatarum aliquem e numeris $e, e^{\prime}, e^{\prime \prime}$ etc. pro diuisore conmmuni maximo habebunt; hoc respectu breuitatis .caussa quamuis repr. impr. formae $\phi$ ad dinisorem quadratum $e e$ vel $e^{\prime} e^{\prime}$. vel $e^{\prime \prime} e^{\prime \prime}{ }^{\prime}$ etc. pertinese dicemus. Iam omnes repr. formae $\phi$ ad eundem diuisorem quadratum datum ee (cuius radicem $e$ positiue acceptam supponimus) pertinentes per regulas sequentes inueniuntir; ex quarum de. monstratione synthetica, propter breuitatem. hic. praeferenda, analysis per quam euolutae suat $\mathrm{fa}$ cile restitui poterit. 


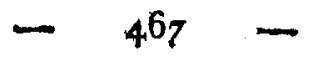

Primo eruantur omnes formae binariàe determinantis $\frac{D}{e \theta}$, quae in formam $\phi$ transeunt per substitutionem propriam talem $T=x t+\lambda u, U$ $=\mu u$, designantibus $T, U$ indeterminatas talis formae; $t, u$ indet. formae $\varphi ; \kappa, \mu$ integros positiuos (quorum productum itaque $=e$ ); $\lambda$ integrum positiuum minorem quam $\mu$ (siue etiam cifram). Hae formae, cum transformationibus respondentibus, ita inueniuntur:

Aequetur * successiue singulis diuisoribus ipsius $e$ positiue acceptis (inclusis etiam 1 et $e$ ), fiatque $\mu=\frac{\ell}{x}$; pro singulis valoribus determinatis ipsorum $x, \mu$ tribuantur ipsi $\lambda$ omnes valores integri a o vsque ad $\mu-1$, quo pacto omnes transformationes certo habebuntur. Iam forma, quae per quamuis substitutionem $T \doteq x t+\lambda u$, $U=\mu u$ in transit, inuenitur inuestigando formam in quam $\varphi$ transit per hanc $t=\frac{1}{*} T-$ $\frac{\lambda}{\ell} U, u=\frac{1}{\mu} U$; sic formae singulis transformationibus respondentes obtinebuntur; sed ex omnibus his formis eae tantum retinendae sunt, in quibus omnes tres coëfficientes euadunt integri *).

-) Si de hoc problemate fusius agere hio liceret, solutionem admodum contrahere possemus. Id statim obuium est, pro * alios diuisores ipsius $\ell$ accipere nos esse necossarium, risi quorum quadratum metiatur coüficientem primum formae $\phi$. Ceterum hoc problerin, ex qua etiam solutiones simpliciares probl, artt. 213,214 deduci possunt, alis qscarione idones reaumere nobif reterusmus. 
Secundo ponamus $\Phi$ esse aliquam ex hisce formis, quae in $\emptyset$ transeat per subst. $T=x t+$ $\lambda u, U=\mu u$; inuestigentur omnes repraesentationes propriae formae $\$$ per $f$ (si quae dantur), exhibeanturque indefinite per $x=\mathfrak{U} T+\mathfrak{B} U$, $x^{\prime}=\mathfrak{U} T+\mathfrak{B}^{\prime} U, x^{\prime \prime}=\mathfrak{u}^{\prime \prime} T+\mathfrak{B}^{\prime \prime} U^{\prime} \ldots(\mathfrak{R}) ;$ denique ex singulis $(\Re)$ deducatur repraesentatio ( $) . . x=\alpha t+b_{u}, x^{\prime}=\alpha^{\prime} t+b u, x^{\prime \prime}=$ $\alpha^{\prime \prime} t+b^{\prime \prime} u$ per aequationes $(R) \ldots \alpha={ }_{x} \mathfrak{U}, \alpha^{\prime}$ $=\times \mathfrak{U}^{\prime}, \alpha^{\prime \prime}=x^{\prime \prime}, \mathfrak{b}=\lambda \mathfrak{U}+\mu \mathfrak{B}, b^{\prime}=\lambda \mathfrak{U}^{\prime}+$ $\mu B^{\prime \prime}, \mathscr{Q}^{\prime \prime}=\lambda 22^{\prime \prime}+\mu B^{\prime \prime}$. Eodem prorsus modo, vt forma $\Phi$, tractentur formae reliquae per regulam primam inuentae (si plures adsunt), ita vt ex singulis cuiusque repraesentationibus propris aliae repraesentationes derinentur, dicoque, hoc modo prodire cunctas repraesentationes formae $\varphi$ ad diuisorem ee pertinentes; et quidem quamlibet semel talltum.

Dem. I. Formam temariam $f$ per quamuis substitutionem $(\rho)$ reuera transire in $\varphi$, tam obuium est, vt explicatione ampliori non opus sit; quamlibet autem repr. ( $\rho$ ). esse impropriam et ad diuisorem ee pertinere, inde patet, guod nu-

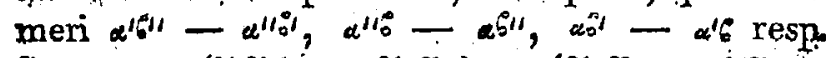
fiunt $=e\left(\mathfrak{W}^{\prime} \mathfrak{B}^{\prime \prime}-\mathfrak{X}^{\prime \prime} \mathfrak{B B}^{\prime}\right), e\left(\mathfrak{X}^{\prime \prime B}-\mathfrak{X}^{\prime \prime \prime}\right)$, $\boldsymbol{e}\left(\mathfrak{X B}^{\prime}-\mathfrak{X}^{\prime} \mathfrak{B}\right)$, vnde illorum diuisor comm. max. manifesto erit $e$ (quoniam $\Re$ est repraesentatio propria).

II. Ostendemus, ex quauis repraesentatione data $(\zeta)$ formae $\phi$, inueniri posse reprạesentacionem propriam formae determinantis $\frac{P}{b \ell}$, inter formas per regulam primam inuentas conten- 


\section{- $\quad 469$}

tae, siue ex valoribus datis ipsorum $\alpha, \alpha^{\prime}, \alpha^{\prime \prime \prime}, \varnothing, \sigma^{\prime \prime}, \sigma^{\prime \prime}$ deduci posse valores integros ipsoruim $x, \lambda, \mu$, conditionibus praescriptis, atque valores ipsorum $\mathfrak{X}, \mathfrak{Z}$, $\mathfrak{Z}^{\prime \prime}, \mathfrak{B}, \mathfrak{B}^{\prime}, \mathfrak{B}^{\prime \prime}$, aequationibus $(R)$ satisfacientes, et quidem vnico tantum modo. Primo statim patet ex tribus aequ. primis in $(R)$, pro x accipi debere diuisorem communem maximum ipsorum $\alpha, \alpha^{\prime}, \alpha^{\prime \prime}$ signo positiuo (quum enim $\mathfrak{x} \cdot \mathfrak{B}^{\prime \prime}$

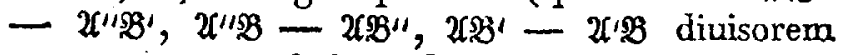
communem non habere debeart, etiam. $\mathfrak{X}, \mathfrak{W}, \mathfrak{W}^{\prime} \iota$ diu. comm. habere nequeunt); hinc etiam $\mathfrak{x}, 2 \mathfrak{L}^{\prime}$; xi' determinati erunt, nec non $\mu=\frac{1}{x}$ (quem necessario integrum fieri facile perspicitur). Ponamus, tres integros $\mathfrak{a}, \mathfrak{a}^{\prime}, \mathfrak{a}^{\prime \prime}$ ita acceptos eșse, vt. fiat $a \mathfrak{d}+\mathfrak{a}^{\prime} \mathfrak{d} \mathfrak{c}^{\prime}+\mathfrak{a}^{\prime \prime} \mathfrak{d} \mathfrak{d}^{\prime \prime}=1$, scribamusque breuitatis caussa $k$ pro $a \mathfrak{B}+\mathfrak{a}^{\prime} \mathfrak{B}^{\prime}+\mathfrak{a}^{\prime \prime} \mathfrak{B}^{\prime \prime}$. Tunc ex tribus vltimis aeq. $(R)$ sequitur, esse debere $a^{b}+a^{\prime} b^{\prime}+a^{\prime \prime b^{\prime \prime \prime}}=\lambda+{ }_{k} k$, vnde statim patet pro $\lambda$ vnicum tantummodo valorem inter limites o. et $M-$ I situm dari. Quo facto quuma etiam

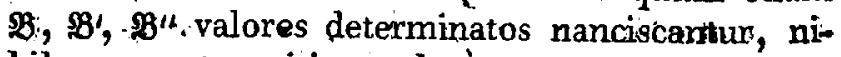
hil superest, nisi vt demonstremus hos semper bine integros euadere. Fiet autem $\mathfrak{B}=$ $\frac{x}{\mu}(6-\lambda \mathfrak{Z})=\frac{\dot{x}}{\mu}\left(6(1-a \mathfrak{C})-\mathfrak{X}\left(\mathfrak{a}^{\prime} \mathfrak{G}^{\prime}+\dot{a}^{\prime \prime} e^{\prime \prime}\right)\right)$

$-\mathfrak{X} k=\frac{\mathrm{I}}{\mu}\left(\mathfrak{a}^{\prime \prime}\left(\mathfrak{X}^{\prime \prime} \boldsymbol{b}-\mathfrak{X}^{\prime i}\right)-\mathfrak{a}^{\prime}\left(\mathfrak{X}^{b}-\mathfrak{X}^{\prime a}\right)\right)$

$-\mathfrak{a k}=\frac{\mathrm{r}}{e}\left(\mathfrak{a}^{\prime \prime}\left(\boldsymbol{a}^{\prime \prime} b-\alpha b^{\prime \prime}\right)-\mathfrak{a}^{\prime}\left(a^{\prime}-a^{\prime} b\right)\right)-$

$\mathfrak{t k}$, eritque adeo manifesto integer, similiterque facile confirmatur, etiam ipsos $\mathbb{B}^{\prime}$, $\mathbf{B}^{\prime \prime}$ valores in. tegrós nancisci. - Ex his ratrociniils colligitur, nullam repraesentationem impropriam formae $\phi$ per $f$, ad diuisorem ee pertinentem, exstare posse, $\mathrm{G}_{g} 3$ 
quae per methodum traditam rel non vel pluries obtineatur.

Quodsi iam eodem modo reliqui diuisores quadrati ipsius $D$ tractantur, repraesentationesque ad singulos pertinentes erzuntur, cunctae repratesentationes impropriae formae $\varphi$ per $f$ habebuntnur.

Ceterum ex hac solutione facile deducitur, theorema, ad finem art praec. pro repraess. propriis traditum etiam ad improprias patere, scilicet generaliter nullam formam binariam positiuam det. negatiui per ternariam negatiuam repraesentari posse etc.; patet enim, si $\$$ sit forma talis binaria, quae propter illud theorema per $f$ proprie repraesentai nequeat, etiam omnes formas determinantium $\frac{D}{\varepsilon e^{\prime}}, \frac{D}{e^{\prime} e^{\prime}}$ etc., ipsam $\oplus$ implicantes per $f$ proprie repraesentari non posse, quum hae formate onumes determinantem eoden signo affectum habeant vt $\varphi$, et, quoties hi doterminantes negatiui sunt, vel ommes cuadunt formae positiuae vel negatiuat, prout $\phi$ ad illas vel ad has pertinet.

285. De quaestionibus problnma tertium nobis propositum constituentibus (ad quod dto priora in praecc. sunt redurta), stilicet propositis duabus formis temariis eiusdem determinantis, diudicare, vtrum aequiualentes sint necne, et. in casiz priori omnes transformationes alterius in alteram inuenire, pauca tantun hoc loro inserere possumus, quuna solutio completa, quatem 
pro problematibus analogis in formis binariis tradidimus, hic adhuc maioribus difficultatibus sit obnoxia. Quamobrem ad quosdam casus particulares, propter quos praecipue haecce digressio instituta est, disquisitionem nostram limitabimus.

I. Pro determinante +1 supra ostensum est, omnes formas binarias in duas classes distribui, quarum altera omnes formas indefinitas, altera omnes definitas (negatiuas) contineat. Hinc statim concluditur, duas formas ternarias quascunque det. I aequiualentes esse, si vel vtraque sit definita rel vtraque indefinita; si vero altern sit definita, altera indefinita, aequiualentiam locum non habere (propositionis pars posterior manifesto valet generaliter pro formis determinantis cuiuscunguiv). - Simili modo duae formae quaccunque determinantis - 1 certo aequiualebunt, si vel vtraque definita est, vel vtraque indefinita. - Duae formae definitae determinantis a semper aequiualebrunt; duae indefinitae non aequiualebunt; si in altera tres coëfficientes primi omnes pares sunt, in altera vero non omnes sunt pares; in casibus reliquis (si vel vtraque tres coüfficientes primos simul pares habet, vel neutra) aequiualebunt. - Hoc modo adhuc multo plures propositiones speciales exhibere possemus, si supra (art.277) plura exempla euoluta fuissent.

II. Pro omnibus hisce casibus poterit etiam, designantibus $f, f^{\prime}$ formas ternarias aequiualentes, transformatio ma alterius in alteram inueniri. Nam pro omnibus casibus in quauis classe for. marum ternariarum multitudo satis parua formaGg 4 
rüm supra assignata est, ad quarum aliquam per methodos vniformes quacuis forma eiudern classis reduci possit; has omnes ad micam revhlure ibidem docuimus. Sit $F$ haec forma in ea classe in qua sunt $f, f^{\prime}$, poteruntque per praecepta supra tradita inueniri transformatioues fomarum $f, f^{\prime}$ in $F$, nec non formae $F^{\prime}$ in $f, f^{\prime}$. Hinc per art. 270 deduci poterunt transformationes formae $f$ in $f^{\prime}$ formaeque $f^{\prime}$ in $f$.

III. Superesset itaque tantummodo, ostendere, quo pacto ex vna transformatione: formae temariae $f$ in aliam $f^{\prime}$ omnes trankformationes possibiles deriuari possint. Hoc: problema pendet ab alio simpliciori, scilicet inuenire omnes transformationes formae ternariae $f$ in se ipsam. Nimirum si $f$ per plures substitutiones (l), ("), (7i) etc. in se ipsam et per substitutionem ( $t)$ in $f^{\prime}$ transit, patet si ad nommam art. 270 . combinetur transformatio ( $t)$ cum ( $(t), " 1),(\because ")$ rtc, prodire transformationes per quas cmmes $f$ in $f^{\prime}$ transeat; praeterea per calculum lacile probatur, qunmuis transtormationem formate $f$ in $f^{\circ}$ hoc modo deduci posse e combinatione transformationis datae $t$ formue $f$ in $f^{\prime} \mathrm{cum}$ aliqua ( +1 quilhn unica) transformatione format $f$ in se iquatn, adeoque ex combinatione transfommationis date formae $f$ in $f^{\prime}$ cum ommibus tronstormationbus formae $f$ in se ipsam oriri omnes transformationes formae $f$ in $f^{\prime}$, et quidem singulas semel tantum.

Inuestigationem omnium transformationum formae $f$ in se ipsam ad eum casum hic restrin- 
gimus, vbi $f$ est forma definita cuius coëfficientes $4,5,6$ omnes $=0 *$. Sit itaque $f=$ $\left(\begin{array}{ccc}a, & a^{\prime}, & a^{\prime \prime} \\ 0, & 0, & 0\end{array}\right)$, exhibeanturque omnes substitutiones, per quas $f$ in se ipsam transit, indefinite per

$$
\begin{aligned}
& \alpha, 6, \gamma \\
& \alpha^{\prime}, G^{\prime}, \gamma^{\prime} \\
& x^{\prime \prime}, b^{\prime \prime}, y^{\prime \prime}
\end{aligned}
$$

ita vt satisfieri debeat aequationibus $(\Omega) \ldots a \alpha a$

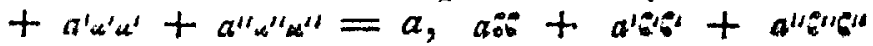
$=a^{\prime}, a_{2 \gamma}+a^{\prime} y^{\prime \prime}+a^{\prime \prime} \gamma^{\prime \prime} \gamma^{\prime \prime}=a^{\prime \prime}, a^{\prime \prime}+$

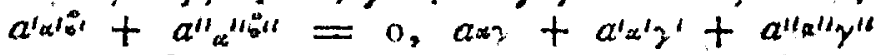
$=0, a b_{y}+a^{\prime} b_{y^{\prime}}+a^{\prime \prime} b^{\prime \prime} y^{\prime \prime}=0$. Iam tres casus sunt distinguendi:

1. Quando $a, a^{\prime}, a^{\prime \prime}$ (qui idem signum habebunt) omnes sunt inaequales, supponamus $a<a^{\prime}, a^{t}<a^{\prime \prime}$ (si alius magnitudinis ordo adest, eaedem conclusiones prorsus simili modo eruentur). Tunc aequ. prima in ( $\Omega$ ) manifesta requirit vt sit $\alpha^{\prime}=\alpha^{\prime \prime}=0$, adeoque $\alpha= \pm x_{\text {; }}$ hïrc per aequ. 4,5 erit $6=0, \gamma=0$; similiter ex aequ. 2 erit $6^{\prime \prime}=0$, et proin $\sigma^{\prime}= \pm 1$; hinc fit, per aequ. $6, \gamma^{\prime}=0$, et per $3, \gamma^{\prime \prime}=$ \pm 2 , ita vt (ob signorum ambiguitatem independentem) omnino habeantur 8 transformationes diuersae.

II. Quando e numeris $a, a^{\prime}$, $a^{\prime \prime}$ duo sunt aequales, e. $g . a^{t}=a^{t}$, tertius inaequalis, sup-

-) Cunds reliqui obi $f$ as torma dafinita ad hane reduci posvant; si varo $f$ ext forma indafinita, mothodus onvino digerse abibenda, trenaformationumque multitudo infinita eric. 
ponamus prinzo $a<a^{\prime}$. Tunc eodem modo vt in casu praec. erit $\alpha^{\prime}=0, \alpha^{\prime \prime}=0, \alpha= \pm 1$, $6=10, \gamma=0 ;$ ex aequ. $2,3,6$ autem facile deducitur, esse debere vel $k^{\prime}= \pm 1, \gamma^{\prime}=0$, $\mathrm{c}^{\prime \prime}=0, \gamma^{\prime \prime}= \pm 1$, vel $\sigma^{\prime}=0, \gamma^{\prime}= \pm 1, b^{\prime \prime}$ $= \pm i, \gamma^{\prime \prime}=0$. Si vero, secundo, $a>a^{\prime}$, eaedem conclusiones sic obtinentur: ex aequ. 2 , 3 necessario erit $\boldsymbol{b}=0, \gamma=0$, et vel $\boldsymbol{\sigma}^{l}=$ $\pm 1, \gamma^{\prime}=0,6^{\prime \prime}=0, \gamma^{\prime \prime}= \pm 1$, vel $a^{\prime}=$ $0, \gamma^{\prime}= \pm 1,6^{\prime \prime}= \pm 1, \gamma^{\prime \prime}=0$; pro suppositione vtraque ex aequ. 4,5 erit $\alpha^{\prime}=0, \alpha^{\prime \prime}=$ 0 , atque ex 1, $a= \pm 1$. Habentur itaque, pro vtroque casu, 16 transformationes diuersae. Duo casus reliqui, vbi vel $a=a^{\prime \prime}$, vel $a=a^{4}$, prorsus simili modo absoluuntur, si modo characteres $\alpha, \alpha^{\prime}, \alpha^{\prime \prime}$ in priori cum $6,6^{\prime}, 6^{\prime \prime}$, in posteriori cum $y, \gamma^{\prime}, \gamma^{\prime \prime}$ resp. commutantur.

III. Quando omnes $a, a^{\prime}, a^{\prime \prime}$ aequales sunt, aequationes $1,2,3$ requirunt, vt e tribus numeris $\alpha, \alpha^{\prime}, \alpha^{\prime \prime}$, nec non ex $6,6 \prime, 66^{\prime \prime}$, vt et ex $\nu, \gamma^{\prime}, \gamma^{\prime \prime}$ bini sint $=0$, tertius $= \pm$ I. Per aequ. 4,5 , 6 autem facile intelligitur, e tribus numeris $\alpha, e, \gamma$ vnum tantummodo $= \pm 1$ esse posse, similiterque ex $\alpha^{\prime}, 6, \gamma^{\prime}$, nec non ex $\alpha^{\prime \prime}, 6 ", \gamma^{\prime \prime}$. Quamobrem sex tantummodo combinationes dantur

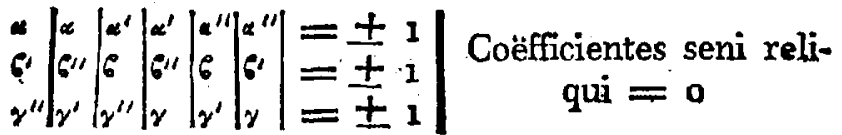

ita vt ob signorum ambiguitatem omnino 48 transformationes habeantur. - Idem typus etiam 
casus praecedentes complectitur: sed e sex columnis primis prima sola accipi debet; quando $a, a^{\prime}, a^{\prime \prime}$ omnes sunt inaequales; columna prima et secunda, quando $a^{\prime}=a^{\prime \prime}$; prima et tertia, quando $a=a^{\prime}$; prima et sexta, quando $a=a^{\prime \prime}$.

Hinc colligitur, si forma $f=a x x+a^{\prime} x^{\prime} x^{\prime}$ $+a^{\prime \prime} x^{\prime \prime} x^{\prime \prime}$ in aliam aequiualentem $f^{i}$ transeat per substitutionem $x=\delta y+y^{\prime \prime}+\delta^{\prime \prime \prime}, x^{\prime}=\delta^{\prime} y$ $+" y^{\prime}+s^{\prime \prime} y^{\prime \prime}, x^{\prime \prime}=8^{\prime \prime} y+{ }^{\prime \prime} y^{\prime}+s^{\prime \prime} y^{\prime \prime}$, ommes transf. formae $f$ in $f^{\prime}$ contineri sub schemate sequente:

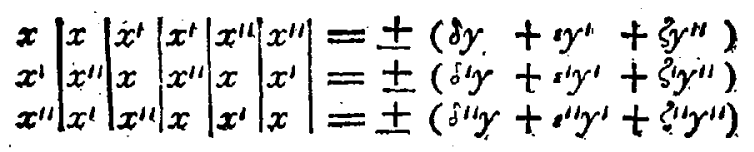

eo discrimine; vt sex columnae primae omnes adhibendae sint, quando $a=a^{\prime}=a^{\prime \prime}$; columna 1 et 2 , quando $a^{\prime \prime} a^{\prime \prime}$ aequales, $a$ inaequalis; 1 et 3 , quando $a=a^{a} ; 1$ et 6 , quando $\alpha=a^{\prime \prime} ;$ denique columna prima sola, quando $a, a^{\prime}, a^{\mu}$ ommes inaequales. In casu primo transformationum multitudo erit 48 , in secundo, ${ }_{2}$ te tio et quarto 16 , in quinto 8 .

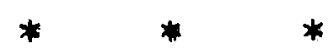

$\mathrm{Ab}$ hac succincta primorum elementorum theoriae formarum ternariarum expositione ad quasdem applicationes speciales progredimur, inter quas primum locum meretur sequens. 
286: Рвовleita. Proposita forma binaria $F$ $=\left(\begin{array}{lll}A, B, C & B\end{array}\right)$ determinantis $D$ ad genus principate pertinente: instenire formam binarian $f$, e euius dupli. catione illa oriatur.

Sol. I. Quaeratur repraesentatio propria fortrae ipsi $F$ oppositae $F^{\prime}=A T T \perp 2 B T U+$ $C U U$ per formam ternariam $x x-s y z ;$ quae sit $x=" T+6 U, y={ }^{\prime} T+{ }^{\prime} U, z=\alpha^{\prime \prime} T$ +6 6U, quod fieri posse e theoria praec. formarum ternariarum facile colligitur. Quum enim $F$ per hyp. sit a genere principali, dabitur valor expr. $\sqrt{ }(A, B, C)$ (mod. $D)$, vnde inueniri poterit forma ternaria $\phi$ determinantis 1 , in quam $(A,-\dot{B}, \dot{C})$ tamquam pars ingrediatur, cuius formae coëfficientes omnes fore integros nullo negotio perspicietur. Aeque facile intelligitur, $\varnothing$ fore formam indefinitam (quoniam per hyp. $F$ certo: non est forma negatiua); unde necéssario formae $x x-y y z$ aequiualens erit. Assignari poterit itaque transformatio huius in illam, quae repraesentationem propriam formae $F^{\prime}$ per $x x-$ sy $z$ suppeditabit. - Tunc igitur erit $A=\alpha a-$

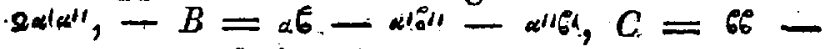
26."; porro designatis numeris $\alpha^{\prime \prime} \sigma^{\prime \prime}-\alpha^{\prime} 6, \alpha^{\prime} b^{\prime \prime}-$ $\alpha^{\prime \prime 6}, \alpha^{\prime \prime} 6-\alpha^{6 \prime}$. per $a, b, c$ resp., hi diuisorem communem non habebunt, eritque $D=$ $b b-2 a c$.

II. Hinc adiumento obseruationis vltimae art. 235 facile concluditur, $F$. transire per substituxionem $26 l, 6,6,6^{\prime \prime} ; 2 \alpha^{\prime}, \alpha, \alpha, \alpha^{\prime \prime}$ in productum formae $(2 a,-b, c)$ in se ipsam, nec non per substitutionem $A^{\prime}, 8,6,26^{\prime \prime} ; \alpha^{\prime}, \alpha, \alpha, 2 \alpha^{\prime \prime}$ in productum formae 
$(a,-b, 2 c)$ in se ipsam. Iam diuisor commue nis maximus numerorum $2 a, 2 b, 2 c$ est 2 ; sị itaque $c$ est impar, $2 a, 2 b, c$ diuisorem communem non habebunt, siue $(2 a,-b, c)$ erit forma proprie primitiua; similiter, si $a$ est impar, $(a,-b, 2 c)$ forma proprie primitiua erit; in casu priori $F$ oritur ex duplicatione formae ( $2 a$, $-b, c)$, in posteriori ex duplicatione formae $(a,-b, 2 c),($ V. concl. 4 , art. 235$)$; vius vero horum casuum certo semper locum habebit. $\mathrm{Si}$ enim vterque $a, c$ esset par, $b$ necessario foret impar; iam facile confirmatur, esse $6^{\prime \prime} a+\cdots 6$ $+b^{\prime} c=0, \alpha^{\prime \prime} a+\alpha b+\alpha^{\prime} c=0$, vnde sequeretur, $b b, \alpha b$, adeoque etiam $\alpha$ et 6 esse pares. Hinc autem $A$ et $C$ forent pares, quod esset contra hypothesin, secundum quam $F$ est forma e genere principali adeoque ex ordine proprie primitiuo. - Ceterum fieri etiam potest, vt tum $a$ tum $c$ pares sint, in quo itaque casu duae statim formae habebuntur, e quarum duplicatio-. ne $F$ oritur.

Ex. Proposita sit. forma $F=(5,2,31)$, det. - 151. Valor expressionis $\sqrt{ }(5,2,31)$ hic inuenitur $(55,22)$; hinc forma ternaria $\phi=$

$\left(\begin{array}{c}5,3 \mathrm{~T},-4 \\ \mathrm{II}, 0,-2\end{array}\right)$; huic per praecepta art. 272 aequi:valens inuenitur forma $\left(\begin{array}{l}1,1,-1 \\ 0,0,0\end{array}\right)$, quae in transit per substitutionem

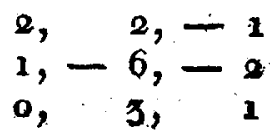




\section{- $478-$}

Hinc adiumento transformationum in art. 277 traditarum inuenitur, $\left(\begin{array}{r}1,0,0 \\ -1,0,0.0\end{array}\right)$ transire in ๑ per substitutionem

$$
\begin{aligned}
& 5,-7,-2 \\
& 2,=1,-0 \\
& 1,-9,-5
\end{aligned}
$$

Fit itaque $a=11, b=-17, c=20$; quare quum $a$ sit impar, $F$ oritur ex duplicatione formae $(11,-17,40)$ transitque in productum huius formae in se ipsam per substitutionem $-1,-7,-7,-9 ; 2,3,3, x$.

287. Circa problema in art. praec. solutum equentes adhuc annotationes adiicimus.

I. Si forma $F$ per substitutionem $p, p^{\prime}, p^{\prime \prime}$, $p^{\prime \prime \prime} ; q, q^{\prime}, q^{\prime \prime}, q^{\prime \prime \prime}$ in productum e duabus formis $(h, i, k),\left(h^{\prime}, i^{\prime}, k^{\prime}\right)$ transformatur, (vtraque vti semper supponimus proprie accepta), habebuntur aequationes, ex concl. 5 art. 235 facile deducendae: $p^{\prime \prime} / h n^{\prime}-p^{\prime} h^{\prime} n-p\left(i n^{\prime}-i^{\prime \prime} n\right)=0,\left(p^{\prime \prime}\right.$ - $\left.p^{\prime}\right)\left(i n^{\prime} i^{\prime} n\right)-p\left(k n^{\prime}-k^{\prime} n\right)+p^{\prime \prime \prime}\left(h n^{\prime}-h^{\prime} n\right)$ $=0, p^{\prime} k n^{\prime}-p^{\prime \prime} k^{\prime} n-p^{\prime \prime \prime}\left(i n^{\prime}-i^{\prime} n\right)=0$, tresque aliae ex his, per commutationem numerorum $p$, $p^{\prime}, p^{\prime \prime}, p^{\prime \prime \prime}$ cum $q, q^{\prime}, q^{\prime \prime}, q^{\prime \prime \prime}$ oriundae; $n, n^{\prime}$ sunt radices quadratàe positiuae e quotientibus prodeuntibus, si dèterminantes formarum $(h, i, k)$, $\left(h^{\prime}, i^{\prime}, k^{\prime}\right)$ per det formae $F$ diuiduntur. Si itaque hae formae sunt identicae, siue $n=n^{\prime}, h$ $=h^{\prime}, i=i^{\prime}, k=k^{\prime}$, illae aequationes transeunt in has: $\left(p^{\prime \prime}-p^{\prime}\right) h n=0,\left(p^{\prime \prime}-p\right) i n$ 
$=\mathrm{o},\left(p^{\prime \prime}-p^{\prime}\right) k n=0$, vnde erit necessario $\boldsymbol{p}^{\prime}=p^{\prime \prime}$, prorsusque simili modo $q^{\prime}=\dot{q}^{\prime \prime} \cdot-$ Tribuendo itaque formis $(h, i, k),\left(h^{\prime}, i^{\prime}, k^{\prime}\right)$ eas dem indeterminatas $t, u$, designandoque indeterminatas formae $F$ per $T, U$, transibit $F$ per substitutionem $T=p t t+2 p^{\prime} t u+p^{\prime \prime \prime} u u, U=$ $q t t+2 q^{\prime} t u+q^{\prime \prime \prime} u u$ in $(h t t+2 i t u+k u z u)^{2}$.

II. Si forma $F$ oritur e duplicatione formae $f$, orietur etiam e duplicatione cuiusuis aliae formae cum $f$ in eadem classe contentae siue classis formae $F$ e duplicatione classis formae $f(V$. art. 238). Ita in ex. art. praec. $(5,2,31)$ orietur etiam e duplicatione formae $(11,5,16)$, ipși $(11,-17,40)$ proprie aequiualentis. Ex vna classe, per cuius dupl. classis formae $F$ oritur, omnes (si plures dantur). inueniuntur adiumento probl. 260 ; in exempla nostro alia huiusmodi classis positiua non dabitur, quia vna tantummodo classis anceps proprie primitiua positiua det. - 151 exstat (puta principalis); quum e compositione classis. vnicae ancipitis negatiuae $(-1$, $0,-151)$, cum classe $(11,5,16)$ oriatur classis $(-11,5,-16)$, haec erit vnica negatiua, e cuius duplicatione classis $(5,2,31)$ oritur.

III. Quum per solutionem ipsam probl. art. praec. euictum sit, quamuis classem formarum binariarum proprie primitiuam (positiuam) ad genus principale pertinentem ex alicuius classis pr. prim. eiusdem det. duplicatione orixi posse: theorema art: 261 , per quod certi eramus, ad minimum semissi omnium characterum pro determinante non quadrato dato $D$ assignabilium 


\section{$-480$}

genera proprie primitiua (positiua) respondere non posse, eo iam ampliatur, vt praecise semissi omnium horum characterum talia genera revera respondeant, alterique ideo semissi nulla respondere possint (V. demonstr. illius theor.). Quare quum in art. 263 omnes illi characteres assignabiles in duas species $P, Q$ aequaliter distributi sint, e quibus posteriores $Q$ formis pr. prim. (positiuis) respondere non posse probatum erat, de reliquis autem $P$ incertum maneret, an singulis genera semper reuera responderent: nunc hoc dubium penitus est sublatum, certique sumus, in toto characterum complexu $P$ nullum adesse cui genus non respondeat. - Hinc facile quoque deducitur, pro. determinante negatiuo in ordine pr. prim. negatiuo, in quo omnes $P$ impossibiles solosque $Q$ possibiles esse in art, $264, I$ ostensum est, omnes $Q$. reuera possibiles esse. . Designante enim $K$ characterem quemcunque ex $Q, f$ formam arbitraxiam ex ordine pr. prim. neg. formarum det. $D$, atque $K^{\prime}$ ipsius characterem, hic erit ex $Q ;$ vnde facile perspicitur, characterem ex $K, K^{\prime}$ compositum (ad normam art. 246) ad $P$ pertinere, adeoque formas pr. primitiuas positiuas det. $D$. exstare quae ei respondeant; ex compositione talis formae cum $f$ manifesto orietir forma pr. prim. neg. det. $D$ cuius character erit $K$. - Prorsus simili ratione probatur, in ordine improprie primitiuo eos characteres qui per praecepta art. 274 II, III sali possibiles inueniuntur omnes possibiles esse, siue sint $P$. siue $Q$. - Haecce theoremata, ni vehementer fallimur, ad pulcherrima in theoria formarum binariarum sunt referenda, eo magis quod licet summa sime 
plicitate gaudeant, tamen tam recondita sint vt ipsarum demonstrationem rigorosam absque tot aliarum disquisitionum subsidio condere non liceat.

Transimus iam ad aliam applicationem digressionis praecedentis, ad discerptionem tum, numerorum tum formarum binariarum in terna quadrata, cui praemittimus sequens

288. Problema. Designante $\boldsymbol{M}$ numerum positium, inusnire conditiones swb quibus formae binariae primitiuae negatiuae determikantis $-M$ dari possint, quae sint residuc quadsatica ipsins $M$ siue pro quibus I st numerus characteristicus.

Sol. Designemus per $\Omega$ complexum omnium charactemum particularium quos praebent relationes numeri 1 tum ad singulos diuisores primos (impares) ipsius $M$ tum ad numerum 8 vel 4 quaido ipsum $M$ metitur; manifesto hi characteres erunt $R p$, $R p^{\prime}, R_{p \prime \prime}$ etc., denotantibus $p, p^{\prime}, p^{\prime \prime}$ etc., illos diuisores primos; atque 1,4 quando $4 ; 1,8$ quando 8 ipsum $M$ metitur. Praeterea vtamur literis $P, Q$ in eadem significatione. vt in att. praec. siue vt in $26 \%$. Iam distinguamus casus sequentes.

I. Quando $M$ per 4 diuisibilis est, $\Omega$ erit. character integer, patetque ex art. $233 \mathrm{~V}, 1$ talium tantummodo formarum numerum characteristicum esse posse, quarum character sit $\Omega$. Sed manifestum est, $\Omega$ fore characterem formae principalis $(1,0, M)$, adeoque ad $p$ pertinere et proin formae proprie primitiuae negatiuae competere non posse; quare quum formae impro$\mathrm{Alh}$ 
prie primitiuae pro tali det. non dentur, nullae omnino formae prim. neg. in hoc cass dantur, quae sint residua ipsius $M$.

II. Quando $M \equiv 3(\bmod 4)$, prorsus eqdern ratiocinia-valent ea sola exceptione vt in hoc casu ordo improprie primitiuus negatiuus exstet, in quo characteres $P$ vel possibiles erunt, yel impossibiles, prout $M \equiv 3$ vel $\equiv z$ (mod. 8), V. art. 264 . III. In casu igitur priori in hoc ordine genus dabitur, cuilus character sit $n$, vnde I erit numerus characteristicus omnium formarum in ipso contentarum; in casu posteriori nullae omnino formae negatiuae hac proprietate praeditae daxi poterunt.

III. Quando $M \equiv x(\bmod 4), 2$ nondum est character completus, sed iusuper accedere debet relatio ad numerum $4 ;$ patet autem, $\Omega$ necessario in characterem formae cuins zaum. char. sit 1 ingredi debere, et vice versa, formam quamuis, cuins character sit vel $\Omega ; 1,4$, vel $\Omega$; $g, 4$, habere numenm char. $x_{*} \operatorname{Iam} n ; x, 4$ manifesto est character generis principalis, qui ad P pertinet adeoque in ordine pr. prim, negatutuo impossibilis est; ex eadem ratione $s ; 3,4$ ad $Q$ pertinebit (art. 263 ), vnde ipsi in ordine pr. prim. negativo genus respondebit, cuius formae omnes habebunt dum ohax 1. Ordo improprie primitiuus in hoc casu, it in sequente, non datur.

IV. Quando $M \equiv 2(\bmod , 4)$, ad a accem dere debet relatio ad 8 , quo fiat character completus, puta vel $x$ et 3,8 , vel 5 et 7,8 , quando $M \equiv 2(\bmod , 8) ;$ et vol $x$ et 7,8 , vel 3 
5,8 , quando $M \equiv 6(\bmod .8)$. Pro casu priori character $\Omega ; 1$ et 5,8 manifesto pertinet ad $P_{3}$ adeoque $\Omega ; 5$ et 7,8 , ad $Q$, vnde ipsi recpordebit genus pr. prim. neg.; similique ratione pro posteriori mum genus in ordine pr. prist. negatiuo dabitur, cuius formae proprietate praecripta praeditae sint, puta cuivs character $42 ; 3$ et 5,8 .

Ex his colligitur, formas primitiuas negatiuas det. $-M$ quarum numerns characteristicus sit 1 dari, quando $M$ alicui numerorum $x, 2,3$, 5,6 secundum modulum 8 congruxs sit et quidem in vnico semper genere, quod improprium erit quando $M \equiv 3$; tales formas omnino non dari, quando $M \equiv 0,4$ vel 7 (mod. 8). Ceterum manifestum est, si ( $-a,-b$, -c) sit sit forma primitiua negatiua cuius num. char. $+1,(a, b, c)$ esse formam primitiuam positiuam cuius num. char. - I; hinc perspicaum est, in quinque casibus prioribus (quando $M \equiv 1,2,5,5,6)$ dari genus vnum primitiuum positiuum cuius formae habeant num. char. - 1 , et quidem, pro $M=3$, improprium, in tribus reliquis vero (quando $M \equiv 0,4,7$ ) tales formas positiuas omnino dari non posse.

289. Circa repraesentationes proprias formarum binariarum per ternariam $x x+y y+$ $z z=f$, e theoria generali in art 289 tradita colliguntur haec:

I. Forma binaria per $f$ proprie repraesentari nequit, nisi fuerit forma positina primitiua, atque - I (i. e det. format $f$ ) ipsius numerus characteristicus. Quare pro determinante positiuo, nec non pro negatiuo $-M$ quando $M$ est 
vel per 4 diuisibilis vel formae $8 n+7$, nullae formae binariae per $f$ proprie repraesentabiles dantur.

II. Si vero $\phi=(p, q, r)$ est forma positiua primitiua determinantis $-M$, atque -1 numerus characteristicus formae $\phi$, adeoque etiam oppositae $(p,-q, r)$ : dabuntur repraesentationes propriae formae $\phi$ per $f$ ad quemlibet valorern datum expr. $\sqrt{1}-(p,-q, r)$ pertinentes. Scilicet omnes coëficientes formae ternariae $g$ det- I (art. 283) necessario fient integri, $g$ vero forma definita, adeoque ipsi $f$ certo aequiualens (art. 285. I).

III Multitudo omnium repraesentationuma ad eundem valorem expr. $\sqrt{ }-(p,-q, r)$ pertinentium in omribus casibus, praeter $M=1$ et $M=a$, per art. 285 , III aeque magna est ac multitudo transformationum formae $f$ in $g$, adeoque, per art. $285,=48$; ibinde patet, si vna repraesentatio ad walorem datum gertinens habeatur, 47 reliquas inde deriuari, valores ipsorum $x, y, z$, omnitus quibus fieri potest modis tum inter se permutando turn signis oppositis afficiendo; quare omnes 48 repraesentationes unicam decompositionem formue o in tria quadrata producunt, si ad quadrata ipsa tantum, neque ad psorum ordinem radicumue signa respicitur.

IV. Posita multitudine omnium numerorum primorum imparium diuersorum ipsum $M$ metientium $=\mu$, haud difficile ex art. 255 concluditur, multitudinem omnixm valorum dituersorum expressionis $\sqrt{ }-(p,-q, r)(\bmod M)$ fore $=2^{\mu}$, e quibus per art. 285 sernissem taktum considerare oportet (quando $M>2$ ). Qua 
re multitudo omnium repraesentationum propria. rum formae $\phi$ per $f$ erit $=4.8 .2^{\mu-x}=3.2^{\mu+t-5} ;$ multitudo autern discerptionum diuersarum in terna quadiata $=2^{\text {ton }}$.

Exx. Sit $\phi=19 t t+6 t u+41 t u$, adeoque $M=770$; hic quatuor valores sequentes expr. $\sqrt{ }-(19,-3,41)(\bmod .770)$ considerare oportet (art. 285): $(39,257),(171,-27),(269,-83)$, $(291,-197)$. Vt inueniantux repraesentationes ad valorem $(50,237)$ pertinentes, primo eruitur forma temaria $\left(\begin{array}{c}39,41,2 \\ 3,6,3\end{array}\right)=g$, in quam per praecepta art $272,275 f$ transire inuenitur per substitutionem

$$
\begin{array}{r}
x,-6,-0 \\
-3,-2,-1 \\
-3,-1,-1
\end{array}
$$

vade habetux repraesentatio formae $\phi$ per $f$ haec: $x=t-6 u, y=-3 t-2 u, z=-3 t$ $-u$; repraesentationes 47 reliquas ad eundem valorem pertinentes, quae ex horum valorum permutatione signorumque conuersione oriuntur, breuitatis catussa non adscribimus. Ommes vero 48 repraesentationes eandem discerptionem for-

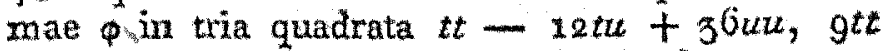
$+12 t u+4 u u, g t t+6 t u+u k u$ producunt.

Prorsus simili modo valor $(x 71,-27)$ suppeditat discerptionem in quadrata $(3 t+5 u)$; $(5 t-4 u)^{2}, t t ;$ valor $(269,-85)$ banc $(t+$ $6 u)^{2}+\left(3^{2}+u\right)^{2}+(3 t-2 u)^{2}$ denique valor $\left(29 x_{1}-127\right)$ hane $\left.t+5 u\right)+(3 t+4 u)^{2}$ $+\left(3^{t}-4 u\right)^{3} ;$ singulae hae decompositiones int 5 


\section{- $486-$}

48 repraesentationibus aequipollent. - Praeter has Ige repraesentationes autem, siue quatuor discerptiones, aliae non dabuntur, quum $77^{\circ}$ per nullum quadratum diuisibilis sit, adeoque repraesentationes impropriae exstare non possint.

290. De formis determinantis $-10 t-2$, quae quibusdam exceptionibus obroxiae exant, pacis seorsim agernus. Praemithimus obsemationem generalem, si $\phi$, $\phi^{\prime}$ sint formae binariae atquiualentes quaecunque, $(\theta)$ transformatio data illius in hanc, ex combinatione repraesentationis cuiusuis formae o per aliquam ternariam $f$ cum substitutione $(\theta)$ prodire repraesentationem formae $\varphi^{\prime}$ per $f_{i}$ porro ex repraesentationibus propris ipsius hoc modo orixi repratesentatione: proprias formae $\phi$, e diuersis duersas, denique cunctis cunctas. Haec omnia per calculum facillime comprobantur. Quare via formartum $\varphi$, totidem modis per $f$ repraesentari poterit ac altera.

1. Sit primo $\varphi=t t+u t$, atque $\varphi^{\prime}$ forma quaecunque alia binaria positiua det. -1 , cuî itaque $\phi$ aequiualebit; transeat $\phi$ in $\phi^{\prime}$ per substitutionem $t=x t^{t}+6 u^{*}, u=2 t^{*}+u_{*}^{*}$ form ma $\phi$ repraesentatur per terankam $f=x+y y$ $+z z$ ponendo $x=t, \gamma=u, z=0$; permutando $x, y$, binc emergtant sex repratesentationes, et e singulis rarsus quatuor, mutando signas ipsorum $t, u$, ita vt omnino 44 repraesentationes diuersae habeantur, quibus wnica discerptio in tria quadrata aequipollet et practer quas alias dar non posse facile perspicitur. Hine concluditur, 


\section{$-$ \\ $487-$}

etians formam $\phi^{\prime}$ vnico tantum modo in tria quadrata decomponi posse, puta in $\left(w t^{\prime}+6 u^{\prime}\right)^{2}$, $\left(y t^{\prime}+\delta u^{\prime}\right)^{2}$ et 0 , quae discerptio 24t repraésentationibus aequiualet.

II. Sit $\phi=t t+2 u t, \phi^{\prime}$ quaecunque alia forma binaria positiaa dét -2 , in quam $\phi$ transeat per substitutionem $t=\alpha t^{\prime}+6 u^{\prime}, u=$ $2 t^{t}+\delta u^{i}$. Tunc simili modo vt in casu praec. concluditur, $\varphi$, et proin etiam $\Phi$, vnico tantum modo in tria quadrata discerpi posse, puta $Q$ in $t t+w a+u u w^{\prime}$ atque $\varphi^{\prime}$ in $\left(a t^{\prime}+6 u^{\prime}\right)^{2}+\left(2 t^{\prime}\right.$ $\left.+\delta u^{u}\right)^{t}+\left(s u^{i}+\delta u^{i}\right)^{2} ;$ talem decompositionem 24. repraesentationibus aequipollere facile perspici potest.

Hinc colligitur, formas binarias determinarntium -1 et -2 respectu multitudinis repraesentationum per ternariam $x x+y y+z z$ cum aliis formis binariis omnino conuenire; quum enim in wroque casu fiat $\mu=0$, formula in art. praec.IV. tradita vtique producit 24 repraesentationes Ratio humus rei est, quod duae exceptiones, quibus tales forma obnoxiae erant, se mutuo compensant,

Theoriam generalem repraesentationum inpropriarum in art. 284 explicatam ad formam $x x+y y+z x$ applicare, breuitatis gratia supexsedermus.

291. Quaestio de inueniendis omnibus repraesentationibus propritis mumeri positiui dati $M$ per formam $x x+y y+z z$ primo per art. 281 . $\mathrm{Hh}_{4}$ 
reducitur ad inuestigationem repraesentationum propriarum numexi - $M$ per formam - $x x$ $-y x-z z=f$; hae vero per praecepta art. 280 ita eruunatur:

I. Euoluantur omnes classes formartum binariarum determinantis - $M$, quarum formae per $X X+Y Y+Z Z=F$ (cui formae ternariae adiuncta est $\mathcal{D}$ proprie repraesentari possumt Quando $M \equiv 0,4$ vel 7 (mod. 8), tales classes per art. 288 non dantur, adeoque $M$ in tria quadrata quas divisorem comraunem non trabeant discerpi nequit *). Quando vero $M \equiv x, 2$, 5 vel 6 , dahitur gexus positinum proprie primitiuum, et quando $M \equiv g_{3}$ improprie primitiuum, quad ommes illas classes comm plectetur: designemus multitudinem haxum clasm sium per $k$

II. Eligantur iam ex hisce classibus $k$ for mae ad Iubitum, e singulis val, quae sint $\phi, \psi^{\prime}$ etc.; inuestigentur ornines amnium repraesentaciones propriae per $F$, grarum itaque wrultitudo erit $32^{k-t} K=K$, designante multitudinem. factorum primorum (imparium) ipsius $M$. denique quaxis huitsmodi repraesentatione v $\boldsymbol{X}$ $=m t+n u, Y=m^{\prime}+n^{\prime} u, Z=m^{\prime \prime}+n^{\prime \prime} u$

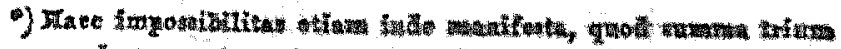

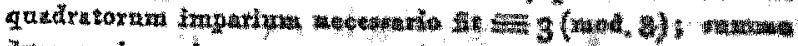

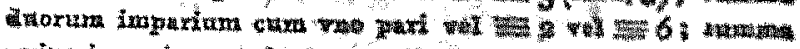

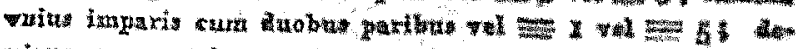

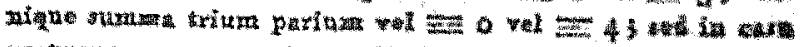

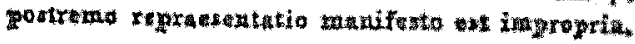


derinetur repraesentatio ipsius $M$ per $x x+y y$ $+z z$ haec $x=m^{\prime} n^{\prime \prime}-m^{\prime \prime} n^{\prime}, y=m^{\prime \prime} n-$ $m n^{\prime \prime}, z=m n^{\prime}-m i n$. In complexu harum $K$ repraesentationum, quem per $\Omega$ designemus, omnes repraesentationes ipsius $M$ necessario contentae erunt.

III. Superest itaque tantummodo, vt inquiramus, num in $\Omega$ repraesentationes identicae occurrere possint; et quum ex art. $\$ 80$, IrI iam constet, eas repraesentationes in $\Omega$, quae e formis diuersis ex et deriuatae sint, necessario dinersas esse, sola disquisitio restat, an repraesentationes diuersae einsden formae, $\ell$. $g_{*}$ ipsius $\$$, per $F$, repratsentationes identicas, numeri $M$ per $x x+y y+z z$ producere possint. Iam statim manifestum est, si inter repraesentationes ipsius $\phi$ reperiatur haec $(r), \ldots, X=m t+n u_{3} Y=$ $m^{\prime} t+n^{\prime}{ }^{\prime}, Z=m^{i t}+n^{\prime} u$, inter easdem fore hanc $(r) \ldots X=-m t-m, Y=-m^{\prime} t$ $-n^{4} u, Z=-m^{i t}-z^{\prime \prime} u$, atque ex vtraque dexiuar eandera repraesentationern ipsius $M$, quae designetur per $(R)$; examinemus itaque, num eadem $(R)$ ex alis adhuc repraesentationibus forma sequi possit. Ex art 280 , Mr facile deducitur, statiendo ibi $\chi=\varphi$, si omnes transformaliones propriae format $\varphi$ in se ipsam

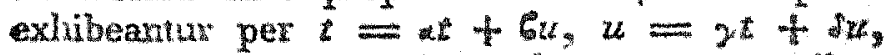
ommes eas repraesentationes formae $\phi$, e quibus $\boldsymbol{R}$ sequatur, expressum iri per $x=(\mathrm{am}+\boldsymbol{y} \boldsymbol{x}$ $+(\operatorname{m}+m) u, y=\left(4 n^{\prime}+y^{2}\right) x+\left(6 m^{4}+\right.$

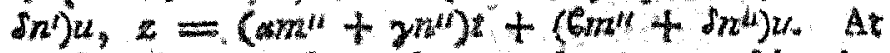
- theoria transformationum formarum binariarum det negatiui in art 779 explicata sequatur, Hh 5 
In omnibus casibus praeter $M=1$ et $\bar{N}=3$, duas tantummodo transformationes proprias formae $\varphi$ in se ipsam dari, puta $\alpha, 8, \gamma, \delta=1,0$, 0,1 et $=-1,0,0,-1$ resp. (quium enim $\varphi$ sit forma primitiua, id quod in art. 179 designabatur per $m$ erit vel 1 vel 2 , et proin, praeter casus exceptos, certo (1) locum ibi habebit). Quare $(R)$ e solis $r, r^{\prime}$ prouenire poterit, adeoque quaeuis repraesentatio propria numeri $M$ bis et non pluries in $\Omega$ reperietur, et multitudo omnium repraess propriarum diuersarum ipsius $M$ erit $\frac{x}{2} K=3 \cdot 2^{\mu+2} k_{\text {a }}$

Quod attinet ad casus exceptos, múltitudo transformationum propriarum formae $\phi$ in se ipsam. per art 179 erit 4 pro $M=1$, et 6 pro $M=3 ;$ reueraque facile confirmatur, multitudinem repraesentationum propriarum numerorum 1,3 esse ${ }_{4}^{\pi} K, \frac{x}{6} K$ resp; scilicet vterque numerus vnico tantum modo in tria quadrata discerpi potest, 1 in $1+0+0,3$ in $1+1+1$, discerptio ipsius I suppeditat sex, discerptio ipsius 3 ooto repraesentationes diuersas: $\bar{K}$ vero fit $=24$ pre $M=1($ vbi $\mu=0, k=1$ ) et $=$ 48 pro $M=3$ (via $\mu=1, k=1$ ).

Céterum obseruamus, si $h$ designet multitudinem classium in genere principali, cui multitudo classium in quouis alia genere proprie primitiuo per art: 252 aequalis est, fore $k=h$ pro $M \equiv 1,2,5$ vel 6 (modi 8 ), sed $k=\frac{x}{3} h$ pro $M \equiv 3$ (mod. 8), vinico cásu $M=3$ excepto vbi $k=h=1$. Pro numeris itaque formae $8 n+5$ multitudo repraesentationum generaliter. 
est $=2^{\mu+2} h$, quum in numero 3 dune exceptiones sese compensent.

292. Discerptiones numerorum (ve formarum binariarum supra) in tria quadrata a reprae sentationibus per formam $x x+y y+z z$ ita distinguimus, vt in illis ad solam quadratorum magnitudinem, in his vero insuper ad ipsorum ordinem radicumque signa respiciamus, adeoque repraesentationes $x=a, y=b, z=c$, et $x=$ $a^{\prime}, y=b^{\prime}, z=c^{\prime}$ pro diuersis habeamus nisi simul $d=a^{\prime}, b=b^{\prime}, c=c^{\prime}$; discerptiones autem in $a a+b b+c c$ et in $a^{\prime} a^{\prime}+b^{\prime} b^{\prime}+c^{\prime} c^{\prime}$ pro vna, si nullo ordinis respectu habito haec quadrata illis aequalia sunt. Hinc jatet,

I. Discerptionem numeri $M$ in quadrata aa $+b b+c c$ aequipollexe 48 repraesentationibus, si nullum sit $=$ 'o omniaque inaequalia; 24 autem, si vel vnum $=0$ reliqua inaequalia, nullum $=0$ atque duo inter se aequalia. Si vero in discerptione numeri dati in tria quadrata duo ex his sunt $=0$, aut vnum $=$ o reliqua aequalia, aut omnia aequalia, repraesentationibus 6 , aut 8 , aut 12 aequiualens erit; sed haec euenire nequeunt nisi in casibus singularibus vbi $M$ $=1$ aut 2 aut 3 resp, siquidem repraesentationes esse debent propriae. His exclusis supponamus, multitudinem omnium discerptionum $n \mu-$ meri $M$ in terna quadrata (divisoris communis expertia) esse $E$, atque inter has reperiri $e$ in quibus vnum quadratum, ; et $e^{\prime}$ in quibus duo. quadrata aequalid; illae etiam tamquam discer. ptiones in bina quadrata, hae tamquam discerptio- 


\section{$-492 \longrightarrow$}

nes in quadratum et quadratum duplum spectari possunt. Tunc multitudo omnium repraesentationum propriarum numeri $M$ per $x x+y y+z z$ erit $=24\left(e+e^{\prime}\right)+48\left(E-e-e^{\prime}\right)=48 E-24$ $(e+e)$. At e theoria formarum binariarum facile deducitur, $e$ fore vel $=0$ vel $=2^{\mu-8}$, prout -1 sit non-residuum vel residuum quadraticum ipsius $M$, nec non $e^{\prime}=0$ vel $=2^{\mu}-$, prout -2 non-residuum vel residuum ipsius $M$, denotante $\mu$ multitudinem factorum primorum (imparium) ipsius $M$ (v. art. 182 ; expositionem rberirem hic supprimimus). Hinc facile colligitur, fore $E=2^{\text {ma }}-2 k$, si tum -1 tum -2 sit $N \cdot R$. ipsius $M, E=2^{k-2}(k+2)$, si vterque numerus sit residuum; denique $\dot{E}=2^{\mu-2}(k+1)$, si alter residuum sit alter non-residuum. In casibus exclusis $M=1$ et $M=2$, haec formula praeberet $E=3$, quum esse debeat $E=1$; pro $M=3$ autem recte prouenit $E=1$, exceptionibus se mutua compersantibus.

Si itaque $M$ est numerus primus, fit $\mu=1$, adeoque $E=\frac{\pi}{2}(k+2)$ quando $M \equiv 1$ (mod. $8) ; E=\frac{\pi}{2}(k+1)$ quando $M \equiv 3$ aut $\equiv 5$. Haecce theoremata specialia ab ill. Le Gendre per inductionem dedecta et in commentatione egregia iam saepius laudata Hist. de $L_{A} A c$. de $P a-$ ris 1785 p. 630 sqq. proláta fuerunt, etsi sub forma aliquantum diuersa, cuius rei ratio imprimis in eo est sita, quod aequiualentiam propriam ab impropria non distinxit, et proin classes oppositas commiscuit.

II. Ad inuentionem ompium discerptionum numeri $M$ in terna quadrata (sine diu comm) 


\section{$-495 \div$}

non opus est, omnes repraesentationes proprias omnium formarum $\varphi, \phi^{\prime}, \phi^{d l}$ eruere. Primo enim facile confirmatur, omines (48) repraesentationes formae $\phi$ ad eundem valorem expr $\sqrt{ }-(p$, $-q, r)$ pextinentes (statuendo $\varphi=(p, q, r)$ ) discerptionem eandem numeri $M$ praebere, adeoque sufficere, si ma ex illis habeatur, siue quod eodem redit, si tantummodo omnes diuersae discerptiones ${ }^{*}$ ) formae $\phi$ in terna quadrata conscriptae sint, et perinde de reliquis $\varphi$ ', $\phi^{\prime \prime}$ etc. Dein si $\phi$ est e classe non ancipite, eam formans quaè e classe opposita electa est omnino praeterire licebit, siue e binis classibus oppositis vnicam considerare sufficito Quum enim prorsus arbitranium sit, quaenam forma e singulis classibus eligatur, supponamus classe opposita ei in qua est $\phi$ eligi formam ipsi oppositam, quae sit $=\phi^{\prime}$. Tunc nullo negotio perspicitur, si discerptiones propriae formae indefinite exhibeantar per $(g t+h u)^{2}+\left(g^{\prime} t+h h^{\prime} u\right)^{2}+\left(g^{\prime \prime} t+\right.$ $\left.h^{\prime \prime} u\right)^{2}$, omnes discerptiones formae $\phi^{\prime}$ expressum iri per $(g t-h u)^{2}+\left(g^{\prime} t-h^{\prime} u\right)^{2}+\left(g^{\prime \prime} t-\right.$ $\left.h^{\prime \prime u}\right)^{2}$, nec non ex his easdem discerptiones numeri $M$ deriuari ve ex illis. Denique pro eo casu vbi $\phi$ est forna e classe ancipite, attamen neque e classe principali neque torma $\left(2,0, \frac{r}{2} M\right)$ aut $\left(2,1, \frac{\pi}{2}(M+1)\right.$ aequiualens (prout $\pi$ par aut impar), e valoribus expr. $\sqrt{ }-(p,-q, r)$ semissem omittere licet; sed breutitatis caussa hocce compendium fusius hic non explicamus:Ceterum iisdem compendis etian vit possumus,

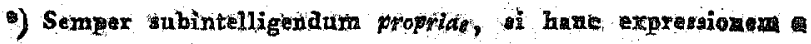

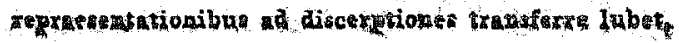




\section{- 494. -}

quando omnes repraesentationes propriae ipsius $M$ per $x x+y y+z z$ desiderantur, quum hae discerptionibus facillime enoluantur.

Exempli caussa inuestigabimus omnes discerptiones numeri $77^{\circ}$ in terna quadrata, vbi $\mu=$ $z, e=e^{\prime}=0$, adeoque $E=2 k$. Per classificationem formarum binariarum positiuarum determinantis $-77^{\circ}$, quam quoniam a quouis ad normam art 251 facile condi potest breuitatis gratia non adscribimus, inuenitur classium positiuarum multitudo $=32$, quae omnes sunt proprie primitiuae et inter 8 genera distribututur, ita vt sit $k=4$, et proin $E=8$. Genus, cuius numerus characteristicus $-x$, respectu ximmerorum $5,7,71$ manifesto characteres particulares $R_{5} ; N_{7} ; N_{1} ;$ habere debet, vnde per art 263 facile concluditur, ipsius characterem respectu numeri 8 esse debere 1 et 3,8 . Iam in eo genere cuius character $x$ et 3,$8 ; R_{5} ; N_{7}$; $N{ }_{11}$, quatuor classes reperiuntur, pro quarum repraesentantibus eligimus formas $(6,2,129),(6$, $-2,129),(19,3,41),(19,-3,41)$; classem secundam vero et quartam reiicimus, vtpote primae et tertiae oppositas. Quatuor discerptiones formae $(19,3,41)$ iam in art $\$ 89$ tradidimus, $\theta$ quibus sequuntur discerptiones numeri 770 in 9 $+3^{61}+400,16+25+729,81+400+$ $\$ 89,576+169+25$. Simill ratione inueniuntur quatuor discerptiones forme $6 t t+4 h u+$ 129uz in $(t-8 u)^{2}+(a t+u)^{2}+(t+8 u)^{2}$, $(t-10 u)^{2}+(2 t+5 u)^{2}+(t+2 u)^{2},(a t$ $-5 u)^{2}+(t+10 u)^{2}+(t+3 u)^{2},(2 t+$ $7 u)^{2}+(t-8 u)^{2}+(t-4 u)^{2}$, resp. valon- 
bus expressionis $\sqrt{ }-(6,-2,129)$ hisce oriundae $\left(48,3^{69}\right),(69,-149),(92,-159)$, $(202,-61)$; vnde prodeunt discerptiones xusmeri 770 in $225+256+289,1+144+$ $625,64+81+625, x 6+225+529$. Prae ter has octo discerptiones aliae non dantur.

Quae ad discerptiones numerorum in terna quadrata diuisores communes habentia attinent, tam facile e theoria generali art. 281 sequuntur, vt non opus sit huic rei immorari.

293. Disquisitiones praecedentes etiam sup. peditant demonstrationem theorematis famosi, omnem numerum integrum posititum in tres num meros trigonales discerpi posse, cuod a Fermatio olim inuentum est, sed cuius demonstratio rigorosa hactenus desiderabatur. Manifestum est, quamuis discerptionem numer in trigonales $x(x+1)+3 y(y+x)+z(x+x)$ producere discerptionum mumer $8 M+5$ in tema quadrata imparia $(2 x+1)^{2}+(2 y+1)^{2}+(2 z+$ $1)^{2}$, et vice versa. Quivis autem numerus integer positiuus $8 M+3$ per theoriam praecedentem in tria quadrata resolubis est, quae necessaria erunt imparia (V. annot art 29x); resolutionumque multitudo pendet tum a multitudine factorum primorum ipsius $8 M+3$, thm multitudine classium in quas formae binaxiate determinantis - $(8 M+3)$ distribuuntur Toti dem discerptiones numeri $M$ in ternas trigonam les dabuntur. Supponimus autem, $(x+x+1)$ pro valore quocunque integro ipsius $x$ tamquam txigonalem spectari, quodsi magis placeret cifram 


\section{$-496-$}

excludere, theorema ita immutare oporteret: Quiuis integer positiuus vel ipse trigonalis est, vel in duos vel in tres trigonales resolubilis. Similis mutatio in theoremate sequente facienda esset, si cifram a quadratis excludere placeret.

Ex iisdem principiis demonstratur aliud Fermatii , theorema, quemuis numertum integrum positiuum in quatuor quadrata decomponi passes. Subtrahendo a numero forma $4 n+2$ quadratum arbitraxium (illo numero xninus), a numero formae $4 \pi+1$ quadratum par, a numero form mae $4 n+3$ quadratum impar, residum in omnibus his casibus in tria quadrata resolubilis erit, adeoque numerus propositus in quatuor. Denique numerus forma an axhiberi potest per $4^{\mu} N$ ita vt $N$ ad aliquarn tritum formxartum praecedentium pertineat: resoluto autem inso $N$ in quatuor quadrata, etians $4^{\text {Hh } N}$ resolutus erit $A$ numero formae $8 n+3$ etian subduri potest quadratum radicis pariter paris, a numero formae $8 n+7$ quadratum radicis impariler pariz, à numero formae $8 n+4$ quadratum impar, residuunque in tria quadrata resolubile erit. Ceterum hocce theorema iam ab ill. La Grauge demonstratum erat, Nouv. Menn de CAc. cie Berlin $1770 \mathrm{p}, 195$, quam demonstrationern (a nostra prorsus diuersam) fusius explicauit ill. Euler in Actis Ac. Petr. DoL It. p. 48 . - Alia Fermatii thepraecedentitum quasi continuationern constituunt, quiruis numerum integrum in quinq̨ue numeros pentagonales, sex hexagonales, weytsm heptagontles etc. resolubilem esse, demonstrationo hactenus carent, aliaqua principia requirere videntur. 
294. Theofema. Designantibus $a, b, c, n t-$ oneros inter se primos quorim mulbus weque $=0$ neque per quadratum divisibils, arquatio ax $x+b y y+c z z$ $=0 \ldots(\Omega)$ resolutionem in integris non admittet (praeter hanc $x=y=z=0$ a quam non respicinus) nisi - bc, - ac, - ab resp. sint residna quadratzea ipsorum $a, b, c$, atque hi mumeri signis ina a qualibus affect; his vero guatuor conditzonibus locum habentibus, $(\Omega)$ an integris resolubuls erit.

Dem. Si (c) per iritegros omnino est resolubilis, etiam per tales valores ipsorum $x, y, z$ resolui poterit qui diuisorem communem non habent; ram valores quicunque, aequ. $\Omega$ satisfacientes, etiamnum satisfacient, si per diuisorem conmunem maximum diuidurtur: Iam supponendo $a p p+b q q+c r r=0$, atque $p, q, r$ a diuisore communi liberos, etiam inter se primi erunt; si enim $q, r$ diuisorem communem is haberent, hic ad $p$ primus esset, un autem metiretur ipsum appadeoque etiam-ipsum $a$, contra hyp, et perinde $p, r ; p, q$ inter se prini erunt. Repraesentatur itaque - app per formam binariam $b y y+c z z$, tribuendo ipsis $y, z$ valores inter se primos $q$, $r$;nde illius determinans - bc residuum quadraticum ipsius app adeoque etiam ipsius $\alpha$ erit (art. 154); eodem modo erit - $a c R b$, - $a b R c$. Quod vero $(\Omega)$ resolutionem admittere non possit, si $a, b, c$ idem signum habeant, tam obuum est vt explicatione non egeat.

Demonstrationem propositionis inuersae, quae theorematis partem secundam constituit, ita adornabimus, tt primo formam ternaxiam ipsi 
$\left(\begin{array}{ccc}a, b & b \\ a, & 0, & b\end{array}\right) \ldots f$ aequitualentem inuenire docea mus, cuius coêfficientes $2,3,4$ per $a b c$ diuisibiles sint, vnde secundo solutionem aequationis (S) deducemus.

1. Inuestigentur tres integri $A, B, C$ a diuisore communi liberi, atque ita comparati, th $A$ primus sit ad $b$ et $c ; B$ ad $a$ et $c ; C$ ad $a$ et $b ; a A A$ $+b B B+c C C$ autem per $a b c$ diuisibilis, quod efficietur sequenti modo. Sint $2, B, E$ resp. valores expressionum $\sqrt{ }-b c(\bmod a), \sqrt{ }-a c$ (mod. $b), \sqrt{-a}(\bmod c)$, qui necessario ad $a, b, c$ resp. primi erunt. Accipiantur tres integri $a, b, c$ omnino ad lubitum, modo atta tid $a, b, c$ resp. primí sint (e. g. omnes $=1$ ), determinenturque $A, B, C$ ita vit $A \equiv 6 c(\bmod b)$ et $\equiv$ $c \in(\bmod c) ; B \equiv \mathrm{c} a(\bmod c)$ et $\equiv \mathrm{a} \mathfrak{U}(\bmod$. $a), c \equiv a b$ (mod, $a$ ) et. $\equiv 68$ (mod. $b$ ). Tunc fiet $a A A+b B B+c C C \equiv a a(b a A+c b b) \equiv$ $a \mathfrak{a}(b \mathfrak{a}-\mathfrak{a} \mathfrak{x} b) \equiv$ o (mod. a) sime per $a$ diuisibilis, et perinde per $b, c$, adeoque etiam per $a b c$ diuisibilis erit. Praeterea patet, $A$ necessario fieri primum ad $b$ et $c ; B$ ad $a$ et $c ; C$ ad $a$ et $b$. Si vero hi valores ipsorum $A, B, C$ ditusorem communem (maximurn) in implicant, hic manifesto ad $a, b, c$ adeoque ad $a b c$ primus

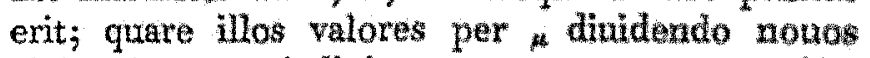
obtinebimus, qui diuisorem communem non habeburt, valorem ipsius $a A A+b B B+c C C$ etiatnnum per $a b c$ ditisiblem producent, adeoque omnibus conditionibus satisfacient.

II. Numeris $A, B, C$, hoc modo determinatis, etiam $A a, B b, C c$ dinisorem commoumems 


\section{- 499}

non habebunt. Si enim laberent dix. comm. $\mu$, hic necessario primus esset ad $a$ (quippe qui tum ad $B b$ tum ad $C c$ primus est) et similiter ad $b$ et $c$; quare $\mu$ etiam ipsos $A, B, C$ metiri deberet, contra hyp. Inueniri poternnt itaque integri $\alpha, \dot{B}, \nu$ tales vt sit $a A a+: B b+\gamma C c=x$; quaerantur insuper sex integri $*^{\prime}, 6^{4}, \gamma^{\prime}, w^{\prime \prime}, b^{\prime \prime}, 7^{\prime \prime}$ tales vi sit $b^{\prime \prime \prime} y^{\prime \prime} \gamma^{\prime \prime}=A a, \alpha^{\prime \prime}-n^{\prime \prime}=B b$, $\boldsymbol{*}^{\prime \prime} \sigma^{\prime \prime}-6^{\prime} \alpha^{\prime \prime}=C c$. Iam transeat $f$ per substitutionem

$$
\begin{aligned}
& a_{1}, a^{\prime \prime} \\
& \epsilon, b^{\prime \prime} \\
& \gamma_{2}, g^{\prime \prime}, \gamma^{\prime \prime}
\end{aligned}
$$

in $\left(\begin{array}{l}m, n^{\prime}, m^{\prime \prime} \\ n, n^{\prime}, n^{\prime \prime}\end{array}\right)=g$ (quae ipsi $f$ aequiualens erit ', dicoque $m^{\prime}, m^{\prime \prime}, n$ per abc diuisibiles fore.

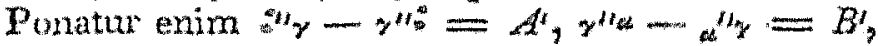

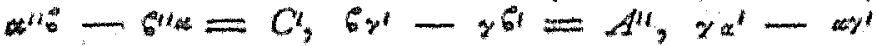
$=B^{\prime \prime},{ }^{\prime \prime}-C^{\prime \prime}=C^{\prime \prime}$, eritque of $=B^{\prime \prime C C}-$ $C^{\prime} B b, b^{\prime}=C^{\prime \prime} A a-A^{\prime \prime} C c, \gamma^{\prime}=A^{\prime \prime} B b-$ $B^{\prime \prime} A a, A^{\prime \prime}=C^{\prime} B b-B^{\prime} C c, b^{\prime \prime}=A^{\prime} C c-C_{C} A A$, $\gamma^{\prime \prime}=B \cdot A a-A B b$. Quibus valoribus in aequationibus $m^{\prime}=a^{\prime} x^{\prime}+b_{*}^{*} v^{\prime}+c y^{\prime \prime}, m^{\prime \prime}=$

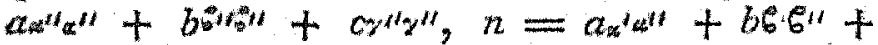
$c \gamma^{\prime} \gamma^{\prime \prime}$ substitutis, fit, secundum modulum $a_{\text {, }}$ $m^{\prime} \equiv b_{C} A^{\prime \prime} A^{\prime \prime}(B B b+C C c) \equiv o, m^{\prime \prime} \equiv$ $b c A^{\prime} A^{\prime \prime}(B B b+C C C) \equiv 0, n^{\prime} \equiv b c A^{\prime} A^{\prime \prime}(B B b$ $+C(c)=0$, i. e. $m^{\prime}, m^{\prime \prime}, n$ per $a$ diuisibiles erunt; similique modo iidem numeri per $b, c$ adeoque etiam per abc diuisibiles intreniuntur. Q. E. P.

III. Ponarnus, concimnitatis caussa, deter, minantem formarum $f, g$, is e. mumenum $-a b c$ 
$=d, m d=M, m^{\prime}=M i d, m^{\prime \prime}=M m^{n} d, n=$ $N d, n^{\prime}=N^{\prime}, n^{\prime \prime}=N^{\prime \prime}$, patetque $f$ transire per: substitutionem (S)

$$
\begin{aligned}
& \text { "d l, at, w" } \\
& \text { cd, et, } \\
& x d, x^{\prime}, x^{\prime \prime}
\end{aligned}
$$

In formam temarian $\left(\begin{array}{lll}M d, & N^{\prime} d, & M^{\prime} d \\ N d, & N^{\prime} d, & N^{*} d\end{array}\right)=g^{d}$ determinantis $d^{3}$, quae itaque sub $f$ contenta erit. Iam dico, huic formae $g^{\prime}$ necessario aequiualere hanc $\left(\begin{array}{lll}d, & 0, & 0 \\ d, & 0, & 0\end{array}\right)=g^{\prime \prime}$. Patet enim, $\left(\begin{array}{l}M, \\ N,\end{array}, N^{\prime}, N^{\prime \prime}\right)=g^{\prime \prime \prime}$ fore formam temariam determinantis 1; porro quum per hyp. $a, b, c$ eadem signa nom habeant, $f$ erit forma indefinita, vnde facile concluditur etiam $g^{i}$ et $g^{\prime \prime \prime}$ indefinitas esse debere; quare $g^{\prime \prime \prime}$ aequiualebit formate $\left(\begin{array}{lll}x, & 0, & 0 \\ x & 0, & 0\end{array}\right),($ art. 277$)$ poteritgue transformatio $\left(S^{\prime}\right)$ illixs in hanc inuexiri; manifesto autem per $\left(S^{\prime}\right)$ forma $g^{\prime}$ transibit in $g^{\prime \prime}$. Ihise etiam g" sub $f$ contenta erit, et ex combinatione subm stitutionem $(S),\left(S^{\prime}\right)$ deducetur transforametio for mae $f$ in git. Quae si fuerit

$$
\begin{aligned}
& \text { d, } 4, z^{3} \\
& \text { b) at } \\
& \text { (2) ह ह }
\end{aligned}
$$

manifestum est, duplicem solutionem aeguatiouts (.) haberi, puta $x=8, y=*^{*}, z=$, et

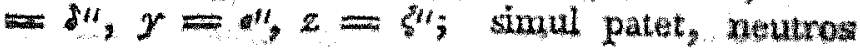




\section{$-50 x-$}

valores simul $=0$ euadere posse, quum neces

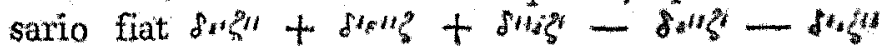
$-\delta s_{d}{ }^{\prime}=$ d. Q. E. S.

Exemplum. Sit aequatio proposita $7 x x-$ $15 y y+23 z z=0$, quate resolubilis ext quia $345 R 7,-161 R 15,105 R 23$. Habentur hic valores ipsorim $\mathfrak{A}, \mathfrak{B}$, , 5 hi $3,7,6$, faciendoque $a=b=c=x$ inuenitur $A=98, B=-$ $39, C=-8$. Hirrc eruitur substitutio

$$
\begin{array}{r}
3,5,22 \\
-1,2,-28 \\
8,25,-7
\end{array}
$$

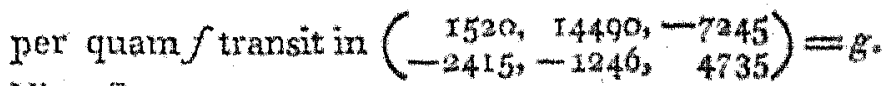
Hinc lit

$$
\begin{aligned}
& (S)=\left\{\begin{array}{rr}
7245, & 5, \quad 22 \\
-2415, & 2,-28 \\
19320, & 25,-7
\end{array}\right. \\
& g^{\prime \prime}=\left(\begin{array}{cc}
3670800, & 6,-5 \\
-1, & -1246,4735
\end{array}\right)
\end{aligned}
$$

Forma $g^{\prime \prime \prime}$ transire inuenitux in $\left(\begin{array}{lll}x, & 0, & 0 \\ x, & 0, & 0\end{array}\right)$ per substitutionem

$$
\left.\begin{array}{rr}
5, & 5, \\
-2440,-4066,-815 \\
-435,-722,-144
\end{array}\right\} *(5 y)
$$

qua cum $(S)$ combinata prodit haec:

Ii 3 


$500-$
$9,11,10$
$-1,9,-9$
$-9,4,5$

per quam $f$ transit in $g^{\prime \prime}$. Habmus itaque diplicem aequationis propositae solutionem $x=$ 11, $y=9, z=4$, et $x=12, y=-9$, $z=3$; posterior simplicior redditu diuidendo valores per diuisorem communem 5 , nnde $x=$ $4, y=-3, z=1$.

205. Pars posterior theormatis art. praec. etiam sequenti modo absolui potest. Quaeratur integer $h$ talis vt sit $a h=\mathbb{C}$ (mod. c), (characteres $2, B$, endem significatione accipinus vt in art praec.), fiatque $a t h+b$ $=c i$. Tunc lacile perspicitur, i fieri integrimo, rumerumque - $a b$ esse determinanten formae binariae $(a c, a h, i) \ldots$. . Haec forma certo non erit positiua (quam enim per hyp. $a, b, c$ eadem signa non habeant, ab ot ac simul positiui esse nequeunt; porro habebit nntrerum characteristicum - I, quod synthetice ita demonstramus: Determinentur integri $e^{*}, b^{*}$ ita vt sit $e=0(\bmod a)$ et $=23(\bmod . b) \cdot a e^{*}=$ $\mathcal{H}(\bmod a)$ et $\equiv h \mathfrak{B}(\bmod b)$, eritque $(a, b)$

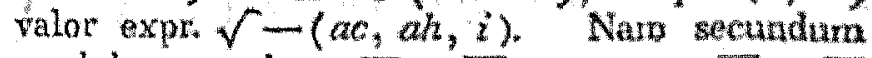
modulum $a$ erit $e=0 \equiv-a c$, $e^{\prime}=0 \equiv$ $-a h, c c e r \equiv 2 a \equiv-b c \equiv-c a^{2}$ adeoque $e^{\prime} t^{\prime} \equiv-i_{j}^{*}$ sectudum nodulum $b$ axtem

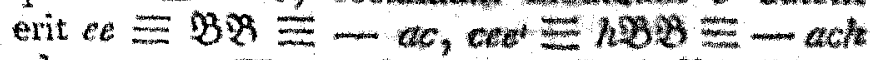
adeoque $e e^{\prime} \equiv-a h, \quad c c t=h{ }^{\prime} \equiv$ $a c h h^{2} \equiv-c c i$ adeoque $e^{t} e^{\prime} \equiv-i ;$ eaedem vero tres congruentiae quae secundum vtrumque 


\section{- $503-$}

modulum $a, b$ locum habent, etiam secundum modulum $a b$ valebunt. Hinc per theoriam formarum ternariarum facile concluditur, $\phi$ reprae-

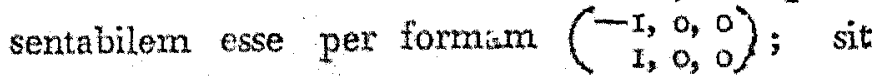
itaque $a c t u+2 a h u+i u u=-(\alpha t+b u)^{2}+$ $2(y t+\delta t)(e t+\zeta u)$, eritque, multiplicando per $c, a(c t+h u)^{2}+b u u=-c(a t+6 u)^{2}$ $+2 c(\gamma t+\delta u)(k t+3 u)$. Hinc patet, si ipsis $i$, $u$ tales valores determinati tribuantur, vt vel $\gamma t+\delta u$, vel $t+\xi u$ fiat $=0$, haberi solutionern aequationis $\Omega$, cui igitur satisfiet tum pex $x=5 c_{1}-2 h, y=x, z=\alpha x^{3}-6 x_{2}$ tum per $x=6 c-h, y={ }_{2}, z=a-6 ;$ simul manifem stum est, neque illos valores neque hos simul $=0$ fieri posse; si enim $\&-\not h=0, \gamma=0$, fieret etian $\delta=0$ atque $\phi=-(x t+u)^{2}$ vnde $a b=0$ contra lyp., et perinde de alteris. - In exeraplo nostro inuenimus formarm $\phi$ hanc $(161,-$ $63,24)$, valorem expr. $\sqrt{-}(\bmod 105)=$ (7) - 51), atque repraesentationem formae $\varphi$, per $\left(\begin{array}{r}-1,0,0) \\ 1,0,0\end{array}\right)$ hanc, $Q=-(15 t-$ $42 t)^{2}+2(11 t-4 t)(15 t-5 t)$ hinc prodeunt solutiones $x=7, y=11, z=-8$; $x=20, y=15, z=-5$, siue diuidendo per 5 et negligendo signum ipsins, $z, x=4$ a $y=3, z=1$.

Ex his duabus methodis aequationem $\Omega$ sol. uendi posterior eo prastat, quod plerumque per numeros minores absoluitur; pxior vero, quae etiam per varia artificia hic silentio praetereunda contrahi potest, elegantior videtar ea imprimis ratione, quod numeri $a, b, c$ prorsus eodem Ii 4 


\section{- $504-$}

modo tractantur, calculusque per horm permutationem quamounque nihil mutatur. Hoc swe se habet in methodo secunda, vbi calculus masime commodus plerumrue proverit, si pro $t$ accipitur minimas, pro $c$ maximus trim numerorum daterum, vti in exemplo nostro fecimus.

396. Elegans theorema in art. pmecc. explicatum primo inuenturn est ab ill. Le Gendre, Wist de l'de de Paris 1785 p. $50 \%$ atque dew monstratione pulcra (a duabus rostris ommino dinersa) munimen. Simul vero hic egregins geometra hoc loco operan dedit, demonstration nem propositionum quae cum theoremate fundamentali sect praec conueniust inde deriuare, quam ad furic seopum non itoneam nobis videri Sarn supra declaratuimus, axt 151 . Nic taque 10cus erit, hane demonstrationtem (pér se valde elegantem) breuiter exponendi iudicitque mostri rationes adiungendi. Praemittitur secquess whererm uatio: Si numeri $a, b, c$ omnes sant $=$ a (mokl. 4), acquatio $a x x+b y y+c z z=0 \ldots$ (n) solum bilis esse nequit. lacillime enim perspicitur, valorem ipsius $a x x+b y+c z z$ neceskario in hoc casu lexi vel $\equiv t$, vol $\equiv 2$, vel $\equiv 3$ (mod. 4 ) nisi omnes $x, y, z$ simul pares actipiantur; si itaque $a$ solubilis esset, bac aliter fieri non posset quam per valores pares ipsorum $x, y, z$, Q. E. A, qunniam valores quicumque aequationt S salisfacientes etiamnum satisfaciunt, si per diuisoren coramunem rnaximum ditudumtur, whele necessario ad minimum vaus impar prodire dom bet. fam casus diuersi theorenatis demonstrank di ad sequentia momenta referuntur: 


\section{$-505-$}

I. Designantibus $p, q$ ummeros primos for mao $4 n+3$ (positiuos inaequales), nequit simul esce $p R q, q R p$. Si erim possibile esset, manifestum est statuendo $1=a,-p=b,-$ $q=c$, omnes conditiones ad resolubilitatem. aequationis $a x x+b y y+c z z=0$ adimpletas esse (art 294); eadem vero per obseruationem praec, resolutionem non admittit quare suppositio consistere nequit. Hinc protinus sequitur propositio 7 , art 131 .

II. Si $\not p$ est mumerus primus formae 4 n +5 , nequit simul esse $q R p, p N q$. Alioquin enim foret $-a R g$, atque aequatio $x x+p y y-q z z$ $=$ resolubilis, quae per obs. praec resolutiom nem respuit. Hinc deriuantur casus 4 , et 5 art. $13 x$.

III. Si $p, q$ sunt mumeri primi formae 4 th +1 , nequit simul esse $p R q, q N p$. Accipiatur alits numerus primus $r$ formae $4 n+3$, qui sit residuum ipsius $q$ et cuius non residuum sit $p$. Tunc erit per casus modo (II) demonstratos $q R r, r N p$. Si itaque esset $p R q, q N q$, foret $q R R p, p R q, p q N r$ et proin - pqRr. Ifinc aequatio $p x x+q y y \rightarrow$ $r z z=0$ resolubilis esset comra obs. praec; quaxe suppositio consistere nequit. Hinc sequuntun casus 1 et 2 art 131.

Concinnius hic casusse quenti modo tractatur, Designet $r$ numerum primum format $4 n+3$ cuius non residum sit $p$. Tuno exit etiam $r N p$, adeoque: (supponendo $p R q, q N_{p}$ ) qrRp, porro - $p R q_{2}-p R r$ el proin etiam - $p f q r_{3}$ quare 


\section{- $506-$}

aequatio $x x+p y y-g r z=0$ resolubilis esset contra obs. praec. Hinc etc.

IV. Si $p$ est numerus primus formae $4 n$ $+1, q$ primus formae $4 n+3$, nequit simul esse $p R q, q N p$. Accipiatur numerus primus auxiliaris $r$ formąe $4 n+1$ qui sit anz residum vtriusque $p, q$. Thune erit (per II) qNr et (per III) $p N r$; hinc $p q R r$; si itaque essel $p R q, q N p$, haberetur etiam $p r N q,-p r R q, q r R p$ quare aequatio $p x x-q y y+r z z=0$ resolubilis es. set, Q. H.A. Hinc deriuantur casus 5 et 6 art. 751 .

$\checkmark$. Designantibus $p, q$ numeros primos for* mas $4 n+5$, nequit simul esse pNq, $q N p$. Supponendo enim fiert posse, et accipiendo mit merum primum auxiliarem $r$ formae $4 n+1$ qui sit non residuum vtriusque $p, q$ erit $q r R p$, prRq; porro (per II) $p N r$, qNr, wide pqRr ot - pqRir hinc aequatio - pxz - qry + rzz $=0$ possibilis, contra obs. praec. Hinc deduct. tur casus 8 art. 131 .

97. Demonstrationem praec proprins contemplando quisque facils intelliget, casus 1 et II ita absolutos esse vit nihil obici possit. At demonstrationes casum reliquorum innituntur existentiae numerorum auxilianiorum, qua nondum demonstrata methodus manifeto onnen vim perdit. Quas suppositionos, etsi tam speciosae sint, vt zninus attendentit derwonstratione ne opu. quidem habere videri possint, atque certe theorema demonstrandun and meximusu probatbiluta tis gratum euehant, tamen si rigar geometricus 
desideretur, neutiquam gratuito sunt admittendae. Quod quidem attinet ad suppositionern in IV et $y$, exstare numerum primum $r$ formae $4 n+3$, qui duorum aliorum primorum datorum $p, q$ non residuum sit, e Sect IV facile concluditur, omnes numeros ipso $4 p q$ minores ad ipsumque primos (quorum multitudo est $2(p-1)(q-1)$ ) in quatuor classes aequaliter distribui, quarum vna contineat non residua vtriusque $p, q$, tres reliquae residua ipsitis $p$ non residua ipsius $q$, ton residua ipsius $p$ residua ipsius $q$, residua vtriusque $p, q f$ et in singulis classibus semissem fore uumeros formae $4 n+1$, semissem formae $4 n$ +5 . Fabobrutur itaquelinter illos ${ }_{4}^{*}(p-1)(q-1)$ non residua vtriusque $p, q$ formae $4 n+1$, qui sint $g, g^{l}, g^{\prime h}$ etc.; numeri $(p-1)(q-1)$ reliqui sint $h, h^{\prime}, h^{\prime \prime}$ etc. Manifesto ommes numeri in formis $4 p q t+g, 4 p q t+g t, 4 p q t$ $+g^{\prime \prime}$ etc. $(G)$ contenti quoque erunt non residua ipsorum $p, q$ formae $4 n+1$. Inm patet, ad stuppositionem stabiliendam demonstrani tantummodo debere, sub formis $(G)$ certo continerì num meros primos, quod sane iam per se valde plausibile videtur, quum hae formae van cum his $4 p q t+h, 4 p q t+h^{t}$ etc. $(H)$ omnes xuumeros ad $4 p q$ primos adeoque etiam omnes numeros absolute primos (praeter $2, p, q$ ) comprehen dant, nullaque ratio adsit, quin numerorum pris morum series inter illas formas aequaliter distributi sint, ita yt pars octaua referantux ad $(G)$, reliqui ad $(A I)$. Attamen perspicuum est, tale ratiocinium a rigore geometrico longe abesse III. Le Gendre ipse fatetur, demonstrationem theorematis, sub tali forma $k t+t$, designantibus. 


\section{$-508-$}

$k, l$ numeros inter se primos datos, $t$ indefini tum, certo contineri numneros primos, satis difficilen vider, methodumque obiter addigitas, quae forsan illue conducere possit; multae vera disquisitiones praeliminares necessariae nobis videntur, antequam bace quidem via ad demonstrationem rigorosam peruenire liceat. - Circa aliam vero suppositionem (III, nteth. secunda) dari numerum primum $r$ formae $4^{n}+5$ cuilis non residuum sit alius numerus primus datus $p$ forma $4 n+1$, ill. Le Gendre nihil onnino adiecit. Supra demonstrauimus (art. 129), numeros primos qudrum N.R, sit $p$ certo dari, sed methodus nostra haud idonea videtur ad exi-. stentiam taliuxa namerorum primorum qui simut sint formae $4 n+5$ ostendendam (vt hic recquiritar neque vero in dem. nostra prima). Cetertura. veritatem quidem huius suppositionis ita facile probare possumus. Per ant. 287 dabitur genus posituum formarum binariarum det. $-p$, cuiuss character 3,$4 ; N_{p} ;$ sit $(a, b, c)$ talis forma atque $a$ impar (quod supponere licel). "Tum ta erit formae $4 n+5$ atque vel ipse primus vel saltem factorem primuxn $r$ formas $4 n+5$ im plieabit. Erit autem - $p R a$, adeoque stian $p R r$, vnde $p N r$. At probe ntotandum est, propp artt 263,287 theoremati fundumentali innit, adeoque circulum vitiosum fore, si qua huins pars illis superstruatur. - Dentque auppositio in methodo prima II adhue multo magis gratuita est, ita ut non opus sit plura de 1 lla hic aditicere.

Liceat obseruationem addere circa casum $V_{\text {, }}$ qui per methodum praec. quidem non satis prom 


\section{- $509-$}

batur, altamen per sequentem commode absoluitur. Si illic simul esset $p N q, q N p$, foret - p pqq, $-q R p$, vnde facile deriuatur, -1 esse xumerum characteristicum formae $(p, 0, q)$, qtae proin, (secundum theorian formarum ternariarum) per formam $x x+y y+z z$ repraesentary poterit. Sit ptt $+q u u=(\alpha t+6 u)^{2}+\left(\alpha^{\prime} t+\right.$

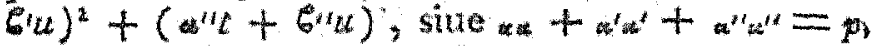

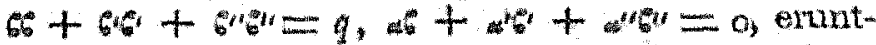
que ex aequatt. I et 2 , omnes $\|^{\prime \prime}, \alpha^{\prime \prime}, b_{*} b^{\prime}, b^{\prime \prime \prime}$ impares; tum vero manifesto aequatio tertia consistere nequit - Haud absimidi modo etiam casus II absolui potest.

298. Problema. Desighantibus $a, b, t$ mbmeros quascunque, quoram tamen wulhts $=0$ : inuenire conditoous resolubilitatis aequationis ax $x+$ byy + $c z z=0 \ldots(\omega)$.

Sol. Sint ${ }^{2}, 6, y \gamma$ quadrata maxima ipsos $b c, a c, a b$ resp. metientia, fiatque $a=6 \mathcal{L}$ $b=\alpha \beta, \gamma c=\alpha C$. Tum $A, B, C$ erunt integri, inter se primi; aequatio $(\omega)$ autem resolubilis erit vel non erit, prout haec $A X X+$ $B T Y+C Z Z=0 \ldots(S Y)$ resolutionern admittit vel non admittit, quod per art. 294 diindicari poterit.

Dem. Ponatur $b c=X * a, a c=B a, a b$ $=\mathbb{E} r \%$, eruntque $\%, 28$, (5) integri a factoribus quadratis liberi atque $\mathbb{Z}=B C, B=A C$, $=$ $A B$; hinc $x B(E)=(A B C)^{2}$, adeoque $A B C=$ $A A C=B B=(C C$ mecessatio integer. Sit rumerorum 26, AC dinisos comma. thax. $m$, atque If 


\section{$-510$}

$=g m, A \mathcal{A}=h m$, eritque $g$ primus ad $h$, nec non (quia $\mathfrak{a}$ liber a fact. qu.) ad $m$. lam fit $h \mathrm{hm}=g A A \mathbb{A}=g \mathbb{A}$, vade $g$ metietur ipsum hhm, quod manifesto impossibile est, nisi $g=$ \pm 1 . Hinc $\mathfrak{x}= \pm m, A= \pm h$, et proin in teger, et perinde $B, C$ integri erunt. $Q . E$. $P$. - Quam $\mathscr{H}=B C$ factores quadratos non imm plicet, necessario $B, C$ inter se primi esse debebunt; et similiter $A$ ad $C$ et ad $B$ primus erit Q. E. S. - Denique patet, si aequationi (n) sa* tisfaciat $X=P, \quad Y=Q, Z=R$, aequatio nem (a) resolui per $x=4 P, y=6 Q, z=$ $q R$; et vice versa si huic satistiat per $x=p_{\text {, }}$ $y=q, z=r$, illi satisfier per $X=z p, Y$ $=a, Z=\omega r$, wade vel varaque reselubilis vel neutra. Q.E. T.

299. Problema. Proposita forma tomariat $f=a x x+a^{\prime} x^{\prime} x^{\prime}+a^{\prime \prime} x^{\prime \prime} x^{\prime \prime}+2 b x^{\prime \prime}+x^{\prime \prime} b^{\prime \prime} x^{\prime \prime}+$

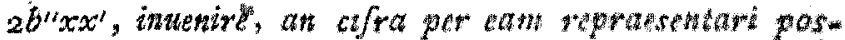
sit (per valores indetermisuatarkm qua non simul $=0$ ).

Sol. I. Quando $a=0$, valores ipsorum $x^{*}$, $x^{\prime \prime}$ ad lubitum assumi posunt, patetque ex aequatione $a^{\prime} x^{\prime} x^{\prime}+2 b x^{\prime} x^{\prime \prime}+a^{t} x^{t} x^{\prime \prime}=-2 x b^{\prime} x^{t}$ $+b^{\prime}\left(x^{\prime}\right), x$ inde valorem determinatum rationalem nancisci; quoties pro $x$ hoc modo fractio prouenit, oportet tantummodo, valores ipsorum $x, x^{\prime}, x^{\prime \prime}$ per fractionis denominatorem muliplicare, habebunturque integrt. Vnice excludenat sunt tales valores ipsoruar $x^{2}$, quil reddunt $b^{\prime} x^{\prime \prime}+b^{\prime \prime} x^{\prime}=0$, nisi simul faciant $a^{\prime \prime} x^{\prime}+$ $2 \bar{b} x^{\prime} x^{\prime \prime}+a^{\prime \prime} x^{\prime \prime} x^{\prime \prime}=0$, in quo casu $x$ ad libtuma accipi poterit, Simul patet, hoc modo amates 
solutiones possibiles obtineri posse. Ceterum is casus vbi $b^{\prime}$ et $b^{\prime \prime}=0$ huc now pertinet tunc enim $x$ in $f$ non ingreditur, siue $f$ est folma binaria, cifreque repraesentabilitas per $f$ e theoria talium formarum diindicari debet.

II. Quando vero non est $a=0$, aequationi $f=0$ aequiualebit haec $\left(a x+b^{\prime \prime} x^{\prime}+b^{\prime} x^{\prime \prime}\right)^{2}$ $-A^{\prime \prime} x^{\prime} x^{\prime}+2 B x^{\prime} x^{\prime \prime}-A^{\prime} x^{4} x^{\prime \prime}=0$, ponendo $b^{\prime \prime} b^{\prime \prime}-a a^{\prime}=A^{\prime \prime}, a b-b^{\prime} b^{\prime \prime}=B^{\prime}, b^{\prime} b^{\prime}-$ $a a^{\prime \prime}=A^{\prime}$. Iam quando hic $A^{\prime \prime}=0$, reque vero $B=0$, manifestum est, si $a x+b^{21} x^{i}+$ $b^{\prime} x^{\prime \prime}$ atque $x^{\prime \prime}$ ad lubitum assumantur, $x$ et $x^{\prime}$ inde rationaliter determivari, et quando integri non fiant, saltern multiplicatoren idoneum integros. producturum. Pro vnico valore ipsius $x^{n}$ valor ipsius $a x+b^{n} x^{4}+b x^{\prime \prime}$ non est arbitrarius sed quoque $=0$ poni debet; tunc vero $x^{\prime}$ ad lubitum assumi poterit valoremque rationalem ipsius x producet. - Quando vero simul $A^{\prime \prime}$ et $B=\mathrm{o}_{3}$ patet, si $A^{\prime \prime}$ sit quadratum $=k k$, aequationem $f=0$ reduci ad has duas theares (e quibus vel yna vel altera locum habere debet) $a x+b^{\prime \prime} x^{*}$ $+b^{\prime}+k j x^{\prime \prime}=0, a x+b^{\prime \prime} x^{\prime}+\left(b^{\prime}-k\right) x^{\prime \prime}$ - o; si vero (in eadem byp.) $A^{\prime \prime}$ est nonquadratus, manifesto solutio aequt propositae pendet ab his (quae simul locum habere debent) $x^{\prime \prime}=$ o et $a x+b^{n} x^{t}=0$.

Ceterum vix necessarim erit obseruare, mehodim in I etiam applicari posse, quando a vel $a^{i t}=0$, methodumque in II quando $A^{\prime}=0$.

III. Quando vero tec $a$ nec $A^{\prime \prime}=0$, aequatoni $f=0$ aequinalet haec $A^{\prime}\left(a x+b^{\prime \prime} x^{i}+b^{i} x^{i}\right)$ 


\section{$-512$}

$-\left(A^{\prime \prime} x^{\prime}-B x^{\prime \prime}\right)^{2}+D a x^{\prime \prime} x^{\prime \prime}=0$, designondo per $D$ determinantem format $f$ sine per Det numet um $B B-A \cdot A^{\prime \prime}$. Quando $D=0$, solutio sintili modo se habebit vt in fine casus praec,; scilicet si $A^{\prime}$ est quadratum $=k \hbar$, anqu. prop. reducitur ad has $k a x+\left(k b^{\prime \prime}-A^{\prime \prime}\right) x^{\prime}+k b^{\prime}+B x^{\prime \prime}=$ $0, k a x+\left(k b^{\prime \prime}+A^{\prime \prime}\right) x^{t}+\left(k b^{\prime \prime}-B^{\prime}\right) x^{* \prime}=0$ si vero $A$ est non quadratus, fieri debet $a x+$ $b^{\prime \prime} x^{\prime}+b^{\prime} x^{\prime \prime}=0, A^{\prime \prime} x^{\prime}-B_{x \prime \prime}=0$. Quando autem $D$ non $=0$, reducti sumus ad arejuationem $A^{\prime} t t-u t u+D a v v=0$, cutus possibilitas per art praec diudicari potest. Qhodsi haec aliter resolui nequit, quam port $t=0, t=0$, $v=0$, manfesto etiam proposita aliam solutionem non admittet, quam kanc $x=0, x^{y}=0$, $x^{\prime \prime}=0$; si vero illa aliter solubilis est, e valoribus integris quibustus ipsorma $t, u, v$ deriuabuntux, per aequationes $a x+b^{t} x^{t}+b x^{*}=t$, $A^{\prime \prime} x^{\prime}-B_{x^{\prime \prime}}=u, x^{\prime \prime}=v$, saltem valores rationales ipsorum $x, x^{\prime}, x^{*}$, e quilus si frationes inuoluunt per idoneum znultiplicatorem utem gri elici poterunt.

Quarmprimum autem vna solutio aequationis $f=0$ in integris inuenta est, problemat at cam sum I reduci, et perinde ac illic solutiones omnes exhiberi poterunt sequenti modo Satisfaciant aequationi $f=0$ valores ipsorum $x, x, x^{t}$ hi $\alpha^{\prime}, \alpha^{\prime \prime}$, quos factoribus cotamunibus liboros supponimus, accipiantur (pex arti, 40, 279) inte-

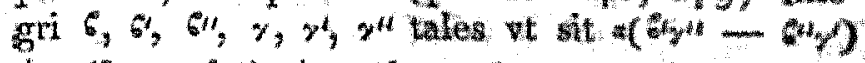

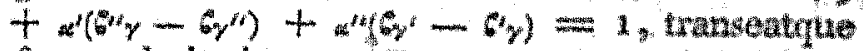

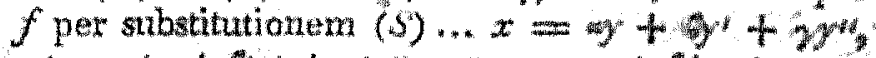

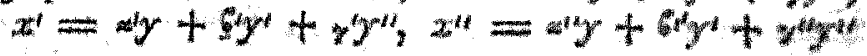


in $g=c y y+c^{\prime \prime} y^{\prime \prime} y^{\prime}+c^{\prime \prime} y^{\prime \prime} y^{\prime \prime}+2 d y^{\prime \prime} y^{\prime \prime}+2 d^{\prime \prime} y y^{\prime \prime}$ $+2 d$ lyg. Tunc manifesto erit $c=0$, atque ipsi $f$ aequinalens, vnde facile concluditur, ex omnibus solutionibus aequationis $g=0$ deriuari (pex $s$ ) omnes solutiones aequationis $f=0$ in integris, Iam ex I sequitur, omnes solutiones aequ. $g=0$ contineri sub formulis $y=$ $z\left(c p p+q d p q+c^{4} q q\right), y^{\prime}=z\left(d^{2} p p+d^{2} p q\right)$, $y^{\prime \prime}=2 x\left(d^{\prime \prime} d q+d q q\right)$, designantibus $p, q$ integros indefinitos, $z$ numerum indefinitum pro quo etiam fractiones accipi possunt, modo ita ve $\gamma, \gamma^{\prime}, y^{\prime \prime}$ integri maneant. His valaribus ipsontm $y, y^{\prime}, y^{\prime \prime}$ in $(S)$ substitutis, omnes solutiones aequ. $f=0$ in integris habebuntur. - Ita $e_{n} g$ si $f=x x$ $+x^{4} x^{4}+x^{+1} x^{\prime \prime}-4 x^{\prime \prime} x^{4}+2 x x^{\prime \prime}+8 x x^{7}$, aqque nna sollatio aequationis $f=0$ habetur $x=1, x^{*}=$ $-2, x^{\prime \prime}=1$ : faciendo $6, k, c^{\prime \prime}, x, x^{\prime \prime}=$ $0,2,0,0,0,1$ prodit $g=y^{\prime \prime}+y^{\prime \prime} y^{\prime \prime}-$ $4 y^{\prime} y^{\prime \prime}+12 y y^{\prime \prime}$. Hinc omnes solutiones aequ $g$ $=0$ in integris contenti entrit sut formala $g$ $-z(p p-4 p q+q q) \cdot y^{\prime}=18 x p q, y^{2 k}=$ 12zqq, et proin omnes solutiones aequ $f=0$ sub hac $x=-z(p p-4 p q+q q), q^{\prime}=$ $p z(p p+s p q+q q) x x^{\prime \prime}=-2(p p-4 p q$ $-1149)$.

300. E problemate art praec. sponte defluit solutio aequationis indeterminatae $a x+$ $2 b x y+c y y+2 d x+2 b y+f=0$, si valores tantummodo rationales desiderantux, quarn, si integri postulantur, supra (art 16 aq) lam ab soluimus. Nam omnes valores rationtales ipsorum $x, y$ exhiberi possunt per $\frac{t}{2}, \frac{1}{4}$, ita vt $t, w_{t}$

K K 


\section{$-514=$}

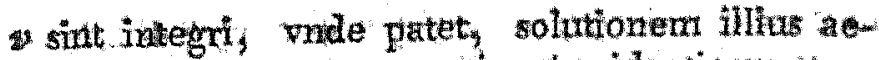
quationis per numeros ratiorales identicam enser cum solutione aequationis att $+2 b u+c u+$ $s d t u+20 u+f w=0$ per numeros integros; haec vero conuenit curn aequ in ant. praec. tractata. Excludi deberit eace sola tolutiones vi $v=0$; tales autem protienire nequsunt, quando $b b$ - $a c$ est numerus non quadratus; Ita e. $\mathrm{g}$ orones solutiones aequationis (in arr. an per integros generaliter solutas) $x x+8 x y+y y+$ $0 x-4 y+1=0$ per numeros rationales contentare erunt sub formula

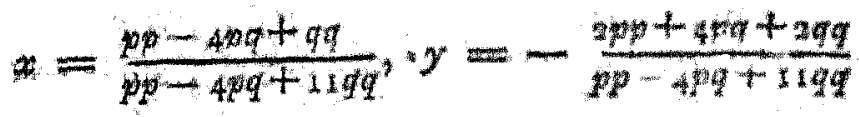

dasighantibus $p, q$ integros quosturque. - Ceterum de his duobus problematibus atetisstmo next coniunctis breuter tamtummodo hic $\mathrm{ctg}$ trmus, anu-

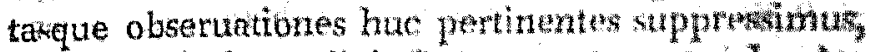
tom 'ne nimis prolixi firtemus, tum guod solutionem aliam probl art praec. halsemus, principtistgeneralioribus innixam, cuins expositionem, quata

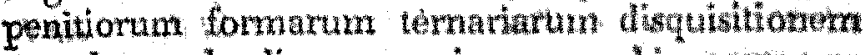
postulat, ad aliam occasionem nobis restaruar. debemus.

301. Reuertimus a formas binarias, de quibus adhuc plures proprietales singulares recensere oportet Et primo quasdam obseruatio. nes circa multitudinem generum of classium in ordine proprie primituo (posituo pro det nets) adicemus, ad quem breutiatio coussa disquistionem restringinus. 


\section{$-515=$}

Multitudo generum, in quae omnes formae (pr. prim. pos.) determinantis dati positiui vel negatiui $\pm D$ distribuzuntur, semper est $1,2,4$ vel altior potestas mumeri 2 , cuius exponens pendet a facturibus ipsitus $D$, et per disquisitiones praec. omnito a priori inueniri potest. Iam quum in serie numerorum naturali numert primal cum magis minusque compositis permixti sint, everitit, vt pro pluribus determinantibus stecessuruis $\pm D, \pm D+1, \pm(D+2)$ etc muttitudo generün nunce crescat nume decrescat, nullusque in hac serie perturbata ordo adesse videatur. Nihllominus si mulfindines generum multis dett. surcessiüs $\pm D, \pm(D+1) \ldots \pm(D+m)$ respondentes adduntur, summaçue per determinamtium multitudinem diuiditur, multitudo generum mediocris prouenit, quae circa medium determinantiurn $\pm(D+m)$ locum habere censeri potm erit, progressionemque valde regularem constithit Stapponimus attem, non modo $m$ esse satis xwagnum, sed etiam $D$ multo matorem, tt ratio determinantiom extremortun $D, D+$ me non nimis a ratione aequalitatis discreper : Regutaritas illius progressiontis ita intelligerde est: si $D^{\prime}$ est numerus multo thaior quam $D$, multiudo generum mediocris circa determinantem $\pm D^{\prime}$ seqnibiliter maior erit quarn circa $D$, si vero $D_{2}$ D' non nimis üfferunt, "etiam gerierunts multitudintes mediocres circa $D$ et $D$ ' fere aequales erunt. Ceterum multitudo mediocris genertim croa determinantem positium $+D$ semper fere aegual is inuentifur multitudini mediocr circa negatiun, eoque exactius quo mailor est $D$, quitm pro valoxe parto prior panllatum enatior equadat 


\section{$-516-$}

quam pasterior. Hae obseruationes magis illustrabuntur per exempla sequentia, twhula claspi ficationis formarum binariarum pluxes quam 4,ono determinantes compledtnte exceryta. luter contum determinantes a 801 vsque al gan reperiz

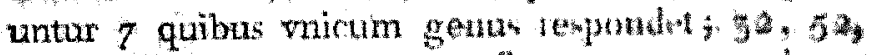
8,1 quibus resp. $2,4,8,16$. getsen re pondent; hinc omaino emergunt generis 354 , vniv mul titudo mediocris $=350$. Centua determinantes negatiti a -801 vocque ad 900 produrume genera 560 . Exempla squentia omuas desu* muntur a determinantibus neggatiuis. In centade 16 (a -1591 veque ad -1600 ) mult. med. geo nerura inuentur 389 , in centade 25 st 401 , wa

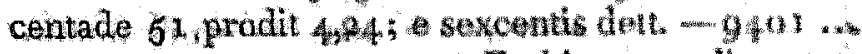
- To000 computatur 4559. Ex his exemplis pates multitudinem generum mediocrex multo levthis crescere, quam determinantes ipsos; sed quaseritur, quaeram sit lex haius progressionis? - Po disquisitionem theoreticam satis diffinem, quame hic explicare nimis prolixum foret, inuentum est, multitudinem generum medtocra circa de terminantem $+D$ vel $-D$ quam proxime ex hiberi per formular olog $D+6$, vbi $\propto, 6$ want quantitates constantes, quidem $=4=$ 0.4052847346 (designante semiperipheriam circuli cuius radius i). $6=5+3$ anh

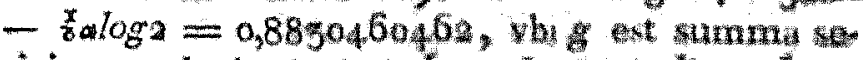
riei $1-\log (2+1)+10 \log 1+1)+\frac{1}{2}-$ $\left.\log 1+\frac{x}{3}\right)+$ etc. $=0.5772156649$ (v. Lulex Irist. Calc. Diff. p. 444); $h$ vero summa, seriet $\frac{x}{4} \log \theta+y^{2} \log 3+\frac{x}{x t} \log 4+4$ etc, quae per approximationem inuenta est $=0,9375482545 . \quad$ Ex 
hae formula patet, multitudinem mediocrem generum crescere in progressione arithmetica, si determinantes augeantur" in geometrica. Valores huius formulte fro $D=850 \frac{x}{2}, 1550 \frac{x}{2}, 245^{\circ}$, $5050 \frac{7}{2}, 9700 \frac{8}{2}$ indeniuntur 5,$627 ; 3,86 ; 4 ; 046 ;$ 4,$739 ; 4,001$, qui a multitudiaibus mediocribus supra datis parum discepant. Quo maior fuerit determinans medius, at e quo pluribus multitudo mediocris computetur, eo minus a valore formulae differet, Adiumento huius formulae etiam aggregatum multitudinum generum determinantibus successiuis $\pm D, \pm(D+1) \ldots+(D+m)$ respondentium quam proxime erui potest, si multitudines mediocres singulis respondentes computantur et in summam colliguntur, quantumuis dinersi sint extremi $D, D+m$. Haec summa erit $=u(\log D)+\log (D+1+$ etc $+\log (D+$ $m)+6(m+1)$ sine satis exacte $=((D)+m)$. $\log D+m)-(D-x) \log (D-1))+(6-\alpha)$ $(m+1)$. Hoo modo summa mult. gen pro dett.a vsque ad -100 inuenilur $=2344$ quum revera sit 235; similiter, a -1 vicque ad -2000 , $=7116,6$, quum sit $7108 \%$ a $-900 x$ xsque ad - 10000 vbi est 4595 . formula praebet $4.594,9$ qualis consensus vix exspectari potuisset.

302. Respectu multitudinis classtum (pr. primit. posit., quod semper subintelligendurn) determinantes positui prorstis aliter se habent quam negatiui ; quamobren virowque seorsim considerabinus, In to hit cum illis conumiunt, quod pro determinante dato in singulis generibus classes aeque muhas continentur, at eoque multitudo onnitura classitum aegualis eyt producto o multiKk 3 
tudine generum in multitudinem classium in singulis generibus contentarum.

Quod primo atimet ad determinantes negatiuos, multitado classium pluribus dett. successiuis $-D,-(D+1),-(D+D$ atce spondentium progressionem aeque perturbatam constituit, ao multitudo generum. Multiudo classium mediocris autern (quat defirhtione opus non habebit) valde regularter crescit, vt ex. exemplis sequentibus apparebit. Centum detersminantes a -500 vsque ad - 600 suppeditunt classes 1739 , mde multitudn madiortis $=17,29$. Similiter in centade 15 multitudo ciassium mew diocris inuepitur 28,$26 ;$ e centadibus duatuts 24 et 25 computatur 56,28 e tribus 61 , b2 et 65 prodit 58,50 e quinque $9 x: 95$, fht 71,56, den:que e quatue 96. 100 fit $735 \%$. Hate exempla osțendhnt, classium muitiudinem mediocrem Tentius quidern crescere, qum deteminatumes, multo tamen citius, quan muhitutinem mediocren generum; leui aulsm attentione cogtroscetur, illam satis exacte crosteres in ruthus radicum quadratarum e determinautibus medin, Renera per cisquisitionem thateticatn inuewamus classium multitudinem meliocren circa tetterminantem $-D$ proxime exprim per $\sqrt{ } D-4$ vbi $\nu=0,746783115=\frac{3 *}{7}$ denownte stmmarn seriei $1+\frac{x}{2}+\frac{x}{2}+\frac{x}{64}+\frac{x}{x^{3}}$ etc $s=0,2026,23675=\frac{2}{m}$ : valores modiocres cundum hanc formulam computat ab is quos supra e tabula clasificationma exsuripsinus pa- 


\section{- $519-$}

rum differunt. Adiumento huius forminlae etiam aggregatum multitudinum omnitum classium ( $\mathrm{pr}$. pr. pos.) determinantibus successizis $-D,-(D$ $+1),-(D+2) \ldots-(D+m-1)$ respondentium quarn proxime assignari potest, quantumüs extremi sint diuersi, summando multitudines mediocres illis determinantibus secundum formulam respondentes, vnde erit $=\gamma(\sqrt{ } D+\sqrt{ }(D+1)+$ etc. $+\sqrt{ }(D+m-1))+\delta m$ sine quam proxime $=\frac{2}{3}\left(\left(D+m-\frac{r}{2}\right)^{\frac{3}{2}}\left(D-\frac{x}{2}\right)^{\frac{1}{2}}\right)+2 m$. Ita e. g. illud aggregatum pro centum dett. $-1 \ldots$ - 100 ex formula computatur $=481,1$, quim revera sit $4.77 ;$ mille determinantes $-1 \ldots$ 1000 secundum tebulam suppeditart 15535 classes, formula dat 15551,4 ; millias secunda sistit classes 28603 secundam tabulam, formula praebet 28585,7 ; similiter millias tertia reuera suggerit $37 \times 12$ classes, formula dat 57074,$3 ;$ miltias decima dat 72549 per tabulam, formula $7257^{2}$.

305. Tabula deterninantium vegatiuorum secundum diuersitatem classificationum ipsis respondentium digesta multas alias obsentationes singulares offert Pro determinantibus formae $-(8 n+3)$ multitudo classium (tam earum quae in omnibus, tum earm quae in singulis generibus pr. primitiuis contentae sunt) semper ditisibilis est per 3 , vico deferminante -3 excepto, cuius rei ratio ex art. 256 , VI sponte sequitur. Pro is determinantibus, quorum formae wicum genus conficiunt, multitudo classtum semper impar est; quam enim pro tali de termainante nica trintum etassis anceps detur, puta pxincipalis, muttitudo classiun reliquarum, K k 4 
- quibus binae sernpev oppositae ertut, necessario enit pax, adeoque multitudo ommium impar; ceterum haee posterior proprietas etiam pro determinantibus posititus valet. - Porro sernes disterminantium, quibus eadem classificatio data (i. e. multitudo data tum generum tum classitm) respondet, semper abrimpi videtur, quam towerwationem satis mitran per aliquot extrom it lustramus. (Numents primus, Yomams, indicat multitudinem generum pr. prim. poss sequens mulftudinem classiun in singulis genewibus contentarum; tunc sequitur series determinatuiun, quibus illa classificatio respondet, et quoruzn signum negatium breuitatis causs omitume

$I_{1}=x, 2,3,4,7$

$1,5 \ldots 11,19,83,27,51,45,67,165$

I. $5 \cdots 47,79,105,127$

I. $7 \ldots 71,151,223,343,463,487$

II. $1 \ldots 5,6,8,9,10,12,13,15,16,18,22,95$, $28,37,58$

U. $2 \ldots 14,17,20,52,7,36,39,46,40,59,56$, $63,64,75,80,97,100,142,146,195$

IV. I. $21,24,30,33,40,42,45,48,57,60,70$, $72,78,85,88,93,102,112,150,135$ $177,190,252,435$

VIII.x.105, $100,165,168,210,240,273,280$, $319,350,345,567,385,408,460,520,760$ XVT. $.840,1520,1565,1848$

Similiter an determinantes eperiuntur (maxinns $=-1423)$, quibus chassficatio 1.9 respondet 4. maximus $=-1305$ ), quibus responde: classificatio L. 1 etc.; clasificationes 1 . $5,11.4$ 
II. 5 ; IV. 2 respondent determinantibus non pluribus quam $48,32,42,68$ resp, e quibus thaximi $-65^{2},-862,-1318,-10 \mathrm{rg}$. Quum tabula, ex qua haec exempla sumsimus, longe vltra maximos determinantes hic occurrentes producta sit*), nec vlli amplius prodierint ad illas classificationes pertinentes: nullum dubium esse videtur, quir series adscriptae reuera abruptae sint, et per analogiam conclusionem eandem ad quasuis alias classificationes extendere licebit, E. g. quum in tota milliade decima determinantium nullus se obtulerit, cui multitudo classimm infra 24 responderet; maxime est verisimile, classificationes I. 23; I. 21 etc.; If. II; If. 10 etc.; IV. 5; IV. $4 ;$ IV. 3 ; VIII. 2 iaxn ante -9000 desiisse, aut saltem perpancis determinantibus vltra I 0000 competere. Demonstrationes autem rigorosae harum obseruationum perdifficiles esse yidentur, - Non minus admiratione dignum est, quod omnes determinantes, quorum formae in $3^{2}$ aut plura genera distribuantur, ad minimum binas classes in singulis generibus habeant, adeoque classificationes XXXII. $x$, LXIV. $x$ etc. omnino excidant (minimo ex huiusmodi dett., 9240, respondet XXXII. 2); satisque probabile videtur, multitudine generum crescente continuo plures classificationes excidere. Hoc respectu 66 determinantes supra traditi, quibus classificationes I. $x$; II. $x$; IV, I; VIII. I; XVI. I Iespondent, val-

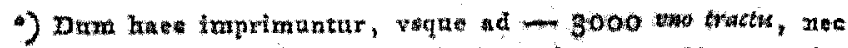

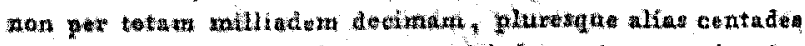

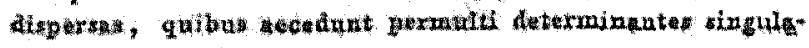
row elocto

Kk 5 
de sumt memorabiles, perspiciturque facile, illo ompes ac solos his duabus proprietatibus insiznibus gaudere, vt omnes classes formarum ad ipsos pertinentes ancipites sint, et formae quacunque in eodern generg contertae necessatio tum proprie tum improprie atquicaleant. Ceterum iidem $6_{5}$ numeri (sub aspectu paullulum diuerso cuius mentio infra fiet et cun criterio demonstratu facili) lam ab ill. Eulero traditi sunt Noww. Mem. de l'Ac. de Beriliz $177^{6}$ p. 338 .

304. Multitudo claseium pr. prünitiuarum, quas formae binariae det. positim quadrati kfo constitunat, omnino a priori assignari potest, multitudinique numerorm ad ak prinotum ipsoque minorum aequalis est; vade per rationinia non difficilia sed hic stpprimenda deducitur, multitudinem wediocrem ciskium ad tales determinantes circa $k k$ pertinentiurn proxime expimi per $\frac{8 k}{2,5}$ - Deterninantes positiui nonquatrata autem hoc respectu phatenomena prorsus singylaria offorunt. Scilicet qum clastimn moltituto

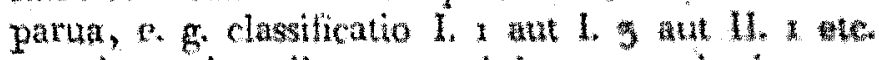
pro determinantibus negatius et quadratis patuis tantum to nox onnino cessantibus locum habeat: contua e deternimantibus pomithis non-

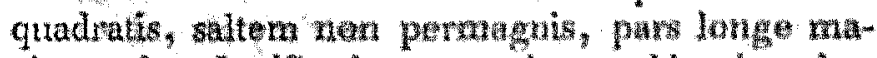
xima tales classiffcatrones praeberat, vbi snuca chassis in quouis genere continetur, ita th hat 1.3 ;

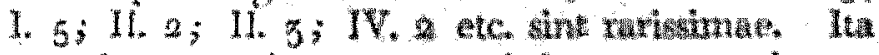

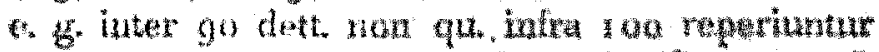
$3 x, 48, a 7$, quibus respondent clasilfoathones $\mathrm{L}$. $1, I I .1$, IV. 1 resp; rnicus tantum $(37)$ habet 
I. 3 ; duo (34, et 82 ) habent II, 2, ynus (7g) 11. 3. Attamen, determinantibus crescentibens, classum multitudines maiores sensim trequentiores fiunt; ita inter $9^{6}$ dett. non qu. a 101 vsque ad 200 duo $(101,197)$ habent 1. 3 ; quatuor $(145,146,178,194,16.2 ;$ tres $(141,148$, $189,11.5$. Ex 197 dett a 801 rsque ad y,009 tres habent $1.3 ;$ quatuor II. 2 ; quatuordecim II. 3 ; dno IL 6 ; duo Il. 6 ; quindecim IV. i2; sex IV. 3 ; dua IV. $4 ;$ quatuor VIIl. 2; reliqux 145 vnam classem in quouis genere, - Quaestio curiosa foret, nec geometrartú sagacitate indi gna, secundun quam legern determinzntes vnama classem in quouis genere babentes continuo rat riores fiant inuestigare; hactenis nee per theoriam dicidere possumus, nec per obseruationera satis certo coniecture, ftrum tandem omnino abrumpantur (quod tancer parum probabile videtur), aut "saltem infimite rort eudant, an ipsorum frequentia ad lizhitern fixum continus magis accedat Multitudo classium medtocris ut ratione parum maiori increscit, quam nuttitudo generun, longeque lentius quam radices qutadratae e determinantibus; inter 800 et 1000 illia inuenitur $=5,0 x$. Liceat his obsertationibis alian adicere, quae analogian inter determinantes positiuos et negativos quodammodo restits tuit. Scilicet inuenimus, nro determiname positiuo I) non tam multiludinem classilum ipsan , quam potius hanc multitudinem per logarithmom quantitatis $t+u \sqrt{ } D$ mautiplicatarn (dim signantibus $t, u$ numeros minimos, yraeter $\mathrm{I}, 0$, aequationi $t t-$ Duu $=1$ satisfacientes $)$ multitudini clussium pro determinante negatioo pluxia 
bus rationibus hie fhesius non exphtcandis analogan esse, atque :valorem mediocrem illius broturti ateque exacte exprimi per formulam talem $m \sqrt{ } D$ $n$; sed walores quartitatum constamium $m, n$ hat ctenuts per theoriatn determinare non liruit si quid ex aliquot centadibus determinantium inter se comparatis concludere pernissun est, $m$ parum a differe videtur. - Cetorum de prinripis disquisitiom zum praecedentilim circa valores metiocres quantitatum legen analytica non progredientum, sed ad talem legern asymptotice continuo mayis approximantium alia occasione fusius agere nobis reseruamus. Transimus iam ad aliam disquisition nem, qua classes diuersae pr. prim. eiusdem det. inter se comparabuntur, finisque huic longae sem stioni imponetur.

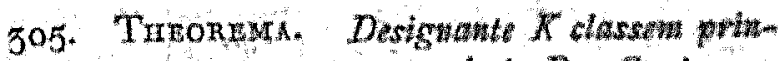
cipalem formarim determanaws dati $D, C$ classmo gutametuque aliam e genere principalt formarman ciusm

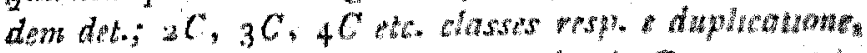
triplecatione, quadiruplicatione ete. elassis Cortus (vit at

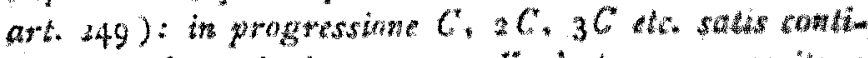

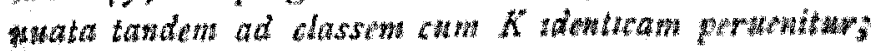

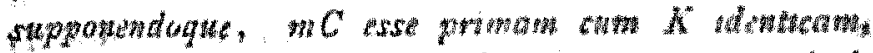

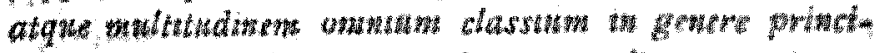

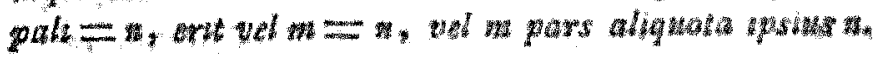

Dem. I. Quum omnes classes $K, C, 2 C_{3}$ $3 C$ etc necessario ad genus principals pertineunt (art. 247 ), classes $n+1$ priakes hains seriez $K, C, 2 C \ldots n C$ manifesto omnes dinersat esse netgueunt. Erit itaque vel $K$ cum aligna elassium $C, 2 C, 3 C \ldots n C$ ilentica, w salten dzat es Wh clussibus inter se identica. Sit $r C=\$ C$ 


\section{- $525-$}

atque $r>s$, eritque ftam $(r-1) C=$ $(s-1) C,(r-2) C=s-s) O$ atb. et $(r+1-s) C=C$, vnde $(r-s, C=K$. Q. E. P.

If. Hinc etiam protints sequitur; esse vel $m=n$ vel $m<n$, supereslque tantummodo, vit ostendaunus; in casu posteriori $m$ esse partem aliquotam ipsius $n$. Qutam classes $K_{\text {. }} C_{2} .2 C_{\ldots} \ldots$ $(m-1) C$, quarum complexum per $E$ designabimus, totum genus principale in troc casu nondutin exhauriant, sil $C$ aliqua classis huias generis in $E$ non contenta, designetunque complexus olassidm quae ex compositione ipsius $C$ cum siagulis classit bus in Corintur, puta $\mathrm{C}, \mathrm{O}+\mathrm{C}_{3} \mathrm{C}^{\prime}+2 \mathrm{C}_{\ldots} \ldots+$ $(m-1) C$, per (c. Iam facile perspicitur, ounes classes in 6 tum inter se tum ab ommibus in 6 diuersas esse ad gemus principale pertineren quodsi itaque (5) et (5) boc genus omnino extuturiunt, habebimus $n=2 m$, sin minus, erit, $2 m<n_{n}$. Sit in easu posteriori $O^{\prime \prime}$ aliqua clas sis generis principalis nea in (5 nec in contenta, designeturque complexus classium ex compositione ipsius $C^{\prime \prime}$ cum singulis classibus in $(E$ prodeuntium i. e. harum $C^{\prime \prime}, C^{r}+C, C n+$

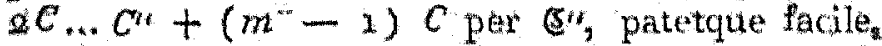
has omnes inter se et ab ommibus in $\boldsymbol{C}$ et (5) diversas esse, et ad genus principale pertinere.

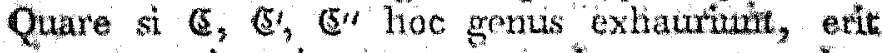
$n=5 m, \sin$ minus, $n>3 m$, in gro casu classis alia $C H$, in genere principuli contenta, neque vero in c, vel c", simili modo tractata docebit esse vel $n=4 m$ vel $n>4 m$, et sic porro. Iam qutum $n$ et $m$ sint nuweri foliti, genus principa- 


\section{$-\quad 506$}

le nacessario tandero exhaurietu, exitque $n$ maltiplum ipsius $m$, siue $m$ pars aliquota ipsius $n . Q . E_{*} S_{*}$

Ex. Sit $\left.D=-356, C=(5,2,72)^{*}\right)$. inueniturque $2 C=(20,8,21), 3 C=(4,0$, 89) $4 C=(20,-8,2 x), 5 C=5,-9,70)$, $6 C=(1,0,356)$. Hic itaque est $m=0, n$ vero pro hoc determistante est 12 . Actpuendo pro $C$ classem $(8,2,45)$, classes quingue reliquae in 6 erunt $(9,-2,40,19,2,40)$, $(8,-2,45),(7,1,21),(17,-1,21)$.

zo6. Denonstratio theos praec omnino

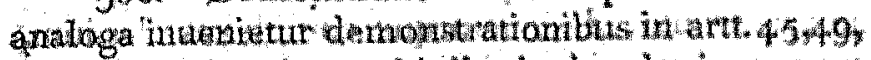
reveraque theora wultiplicationis classitum cum atgumento in Sect. III tractuto pewnagnan va

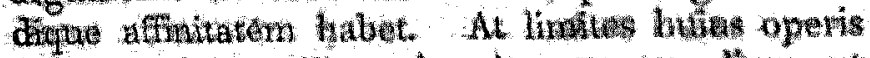

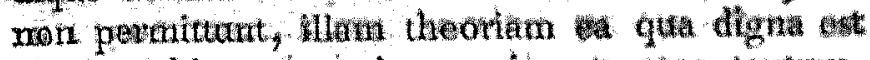
bextate hic persequi quociros patacas tantummodo obserutiones hic adicienus, eas quoque dernonatrationes quae apraratum ipwolixiorem requirerent supuritienus, disquisitionemque anpliorem ad alian vocasionem nobis reseruabianus

I. Si series $K, C, a C, 3 C$ etr. vilua (m - i) $C$ producitur, ealeden classes ikerum com partent, $\left.m C=K_{3} m+1\right) C=C_{3}(m+4)$ $C=y^{2 C}$ ate; generaliteryue (syectando concinnitatis caussa $K$ tarnquam $o(C)$ classes $g C, E \cdot C$

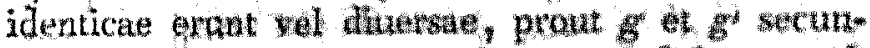
dum modulum the congrui sant vel incongriv, Classis ituque $n C$ semper idertica est cum principali $K$.

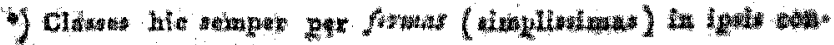

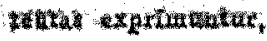


II. Complexum classium $K, C, 2 C \ldots C m$ $-1) C$, quem stupra per $(5$ designauinus, vocabithus perrodum classis $C_{3}$ quae expressio non est confundenda ctm periodis formarum reductarum det positivi non-quadrati in art. $x 86 \mathrm{sqq}$. tractatis. Patet itaque, e compositione classium quotcurnque in eadex periodo contentartm orir classem in ea periodo quoque contentam $\mathrm{g} C+$ $g^{*} C+g^{n} C$ etc $=\left(g+g^{+}+g^{n}+\right.$ etc $) C$

III. Qunm $C+(\ddot{m}-x) C=K$, classes $C$ et $(m-+) C$ oppositae erunt; i peninde $2 C$ et $6 m-2) C, \quad 3 a$ at $(m-3) C$ ete Si itaque $m$ est par, classis $3 m C$ sibi ipsa opposita ent adeoque ancops; vice versa, in \& praeter $K$ adhuc alia classis anceps occurrit puta $g C_{*}$ erit $g C=(m-g) C$ adeoque $g=(n-g)$

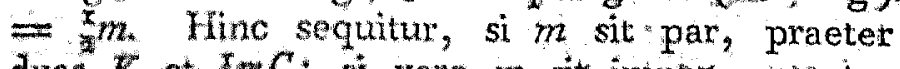
duas $K$ et $I m C_{3}$ si vero $m$ sit impar, praeter vam $K$, aliam classem ancipitem in 6 conten tam esse norl posse,

IV. Si periadus alicuius classib he in 6 contentae supponitur esse $\pi, h C_{0}, 2 h C, 5 h C$, $(\mathrm{m} t$ - $h C$, manifestum est, $n t^{\prime h}$ esse multiplum minimum ipsius $h$ per on dipisibile. Si itaque $m$ et $h$ inter se primi stant, erit $m^{\prime}=m$, duacque periodi easdom classes sed ordine diuersa dispositas continebunt gexeraliter autem designante a diuisorezx cornm max. ipsoman $m$, b erit $m^{\prime}=-$ - Hinc patet, maltudinem clas sium in periodo eaiustus classis ex c contentarun esse vel $m$ vel partem aliquotam ipsius $m$; 
et quidem tot classes in \& habebunt periodos m terminorum, quot pameri ex his o, $x_{2} 2 \ldots$. $m 2$ - I ad $m$ prini sunt, siue $4 m$, vendo signo: art $39 \%$ generaliter vero to classes in thabebutnit periodos $\frac{m}{\mu}$ terminorum, quot numeri ex his $o, I_{n} 2 \ldots \ldots m-I$ diuisorem maximum $\mu$ cum $m$ communem habent, quorum multitudinem esse $\phi \frac{m}{\mu}$ facile perspicitur Si itaque $m=n$, siue totum gemus principale sub \& contentum, dabuntur in hoc genere omnino on etosses, quaram periodi idem genus totum incladunt, et $\phi_{e}$ classes; quarum periodi ex $e$ temrinis constant, denotante fice diuxsorem quemounque ipsîs $n$. Haec conclusio generaliter valet, quando in genere primelpali vila classis datur, culus pexiodus ex $n$ terminis constat.

v. Sub eadem suppositione, systema class. sium generis primetpalis aptius disponi nequit; quam aliquam classem, periodum $n$ terminorum habentem, quasi pro basi adoptando, generisque princlpalls idsses eodem ordine collecando; quo in illius periodo progrediuntur. Qhodsi tune classi pxincipali hdex 0 adseribitur, classi quae pro basi accepta est index I et sic porro: per solam indicum additionem inueniri poterit, quaenam classis e compositione classftrm quarumcunque genenis primeipalls oriatur. Ecce exemplum pro determinante -356 , bi elassem $(9,2,40)$ pro basi accepimus: 


\section{- 529}

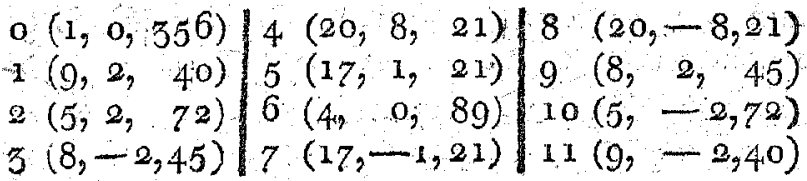

VI. Quamquam vero tum analogia cum. Sect. III; tum iriductio circa plures quam 800 determinantes negatiuos, longeque adhuc plures positiuos non quadratos instiluta maximam probabilitátem afferre videantur, illam suppositionem pro omnibus determinantibus locum habere: talis conclusio nihilominus falsa foret, et per tabulae classificationum continuationem refelleretur. Liceat, breuitatis caussa, eos determinantes, pro quibus totum genus principalé vnicae periodo includi potest, regulares vocare, reliquos vero pro quibus hoc fieri nequit irregulares. Hoc argumentum, quod ad arithmeticae sublimioris mysteria maxime recondita pertinere, disquisitionibusque difficillimis locum relinquere videtur, paucis tanturn obseruationibus hic illustrare possumus, quibus sequentem generalem praemittimus.

VII. $S i$ in genere principali classes $C, C$ occurrunt, quarum periodi ex $m, m^{\prime}$ classibus constant, atque $M$ est numerus minimus per $m$ et $m^{\prime}$ diuisibilis : in eodem genere etiam classe dabuntur, quarum periodi $M$ terminos contineant Resoluatur $M$ in duos factores $r, r$ inter se primos, quorum alter $(r)$ netiatur ipsum $m$, alter ( $r^{\prime}$ ) ipsum $m^{\prime}$ ( $v$. arti 75 ), habebitque classis $\frac{m}{r} C+\frac{m}{r^{\prime}} C=C$ proprietatem praescri- 
ptam. Supponamus enim, periodum classis $C^{\prime}$ constare ex $g$ terminis, eritque $K=g r C n=$ $g m C+\frac{g r m^{t}}{r^{\prime}} C=K+\frac{g^{r} m^{\prime}}{r^{r}} C=\frac{g^{m} m^{\prime}}{r^{\prime}} C$, vnde $\frac{g r m}{r}$ per $m$ diuisibilis esse debebit siue $g r$ per $r$, adeoque etiam $g$ per $r^{*}$. Prorsus simili modo $g$ per $r$ diuisibilis inuenitur, vnde etiam per $r r^{\prime}=M$ diuisibilis erit. Sed quum manifesto sit $M C^{\prime \prime}=K$, erit etiam $M$ per $g$ diuisibilis; quare nacessario $M=g$. Hinc aullo negotio sequitur, multitudinem maximam classium, in vlla periodo contentarum (pro det. dato), diuisibilem esse per multitudinem classium in quanis alia periodo (classis ex eodem genere prineipalt). Simul ibinde methrodus derinari potest, talem classem cuitus periodus sit quam maxima (adeoque pro det regulari totum genus principale complectatur) exuendi, methodo artt. 73,74 prorsus analoga, etsi in praxi laborem per plura artificia contrahere liceat. Quotiens $\mathrm{e}$ dinisione numeri $n$ per multitudinem classium in jeriodo maxima, qui pro determinantibus regularibus est 1, pro irregularibus semper fit integer maior quam 1 , et pro his imprimis conmodus est ad diuersas irregularitatis species exprimendas; quamobrem expontens irregularitatis dici poterit.

vIII. Hactenus regula generalis non habetur, per quam determinantes regulares $\mathrm{ab}$ irregularibus a priori distingui possent, praesertim quum inter posteriores numeri tum primi tum compositi reperiantur; sufficiat itaque quasdam 
obseruationes particulares hic adiunxisse. Quando in genere principali plures quam duae classes ancipites continentur, determinans certo est irregularis atque exponens irregularitatis par; guando vero vna tantum aut duae in illo genere adsunt, det aut regularis erit aut saltem exp. irr. impar. Omnes determinantes negatiui formae $-(216 k+27)$, vnico -27 excepto, irregulates sunt, et exp. irr per $z$ diuisibilis; idem valet de dett. negg. formae $-(1000 k+75)$ et $-(1000 k+675)$, vica -75 excepto, infinitisque alits. Si exp. irr. est numerus primus $p$, aut saltem per $p$ diuisibilis, $n$ per $p p$ diusibibilis erit, vnde sequitur, si $n$ rallum dicisorem quadratum implicet, determinantem certo esse regularem. Pro solis determinantibus quadratis positiuis $e e$ a priori semper dignosci potest, vtrum regulares sint an irregulars:s; scilicet illud euenit, quando $e$ est 1 aut 2 ant numerus primus impar aut potestas numeri primi imparis hoc in oninibus reliquis casibus. "Pro dett. negg-, irregulares continuo frequentiores endutut, quo maiores fiunt determinantes; e. $g$ in tota milliade prima tredecim irregulares reperiuntur, (signo negatiuo omisso ) $576,580,820,884,900$, quorum exp. irr. est 2, atque $243,307,339,459$, $675,755,891,974$, quorum exp, irr. 3 ; in mil liade secunda reperti sunt 13 quorum exp. irs: 2 , atque 15 quorum exp. irr. 3 ; in milliade decina 31 cum exp. irr. 2 atque 32 curn exp. ine 3. Num determinantes cum exp. irr maion quam 5 infra - 10000 occurrant, decidere nondum licet; vltra hunc limitem exponentes quicunque dati provenire possunt. Frequentiam determinantium 
negatuorum irregularium ad frequentiarn regularium continuo magis, dett. crescertibas, ad rationfern constantem appropirfquare valde probabile est, cutus deterninatio geometrarum sagacitate magnopere digna foret. - Pro determinatutibus positidis nonqquadratis irregulares multo ratiores sunt; tales; quorim exp. irr. par sit, infinite multi certo dantur (e. g. 30,26 pro quo est 2$)$; nullum quoque dubium videfur, quin tales exstent, quorum exp. irr. sit impar, etsi fateri oporteat, nullum se hacterus nobis obtulisse.

IX. De adornatione maxime commoda sy stematis classium, in geriere principali pro determinante irregulari contentarum, hic agere propter breutafem non licet; obseraamus tantammodo, qutum vica basts hic nori sufficiat, durs vel adeo plures adhuc classes hic esse accipiendas, e quarum multiplicatione et compositione ormes producantur. Hinc indices duplices aut multiplices errergent, qui eundem fere vsum praestabunt ac simplices pro regularibus. Sed banc rem alio tempore fusius tractabimus.

X. Denique obseruarnts, quum omnes proprietates in hoc art et praec considerdae imprimis a numero $n$ pendeant, qui simile quid est ac $p-1$ in Sect. III; hunc nuxnerum summa attentione dighum esse; quamobrem quam maxime optandum esset, vi inter ipstum atclue determinantem, ad quem pertinet, nexus generalis petegattir. De qua re grauissima eo minus desperandum censemus, quoniam tam successit, va- 


\section{$-533-$}

lorem mediocrèm producti ex $n$ in multitudinem generum (quae a priori assignari potest) saltem pro determinantibus negatiuis formulae analyticae subiicere (art 302 )

307. Disquisitiones artt. praecc. solas classes generis principales complectuntur, adeóque sufficiunt tuma pro dett. poss. vbi vnicum omnino genus datur, tum pro negatiuis vbi vnicum genus positiuum adest, si ad genus negatiuum respicere nolumus, Superest, vt de reliquis quoque generibus (pr. primitiuis) quaedam adiiciamus.

I. Quando in genere $G^{\prime}$ a principali $G$ (eiusdẹm det.) diuerșo vlla classis anceps datur, totidem in ipso aderunt ae in $G$. Sint in $G$ classes ancipites $L, M, N$ etc. (inter quas etiam erit classis principalis $K$ ), in $G^{\prime}$ vero hae $L^{\prime}$, $M$, $N^{\prime}$ eto, designeturque illarum complexus per $A$, complexus harum per $A$. Quum manifesto omnes classes $L+L^{\prime} M+L^{\prime}, N+L^{\prime}$ etc. ancipites diuersaeque sint, et ad $G^{\prime}$ pertineant, adeoque sub $A^{\prime}$ contentae esse debeant: multitudo classium in $A^{\prime}$ certo nequit esse minor quam in $A$; similiter quum classes $L^{\prime}+L^{\prime}, M$ $+L^{\prime}, N^{\prime}+L^{\prime}$ etc. diuersae ancipitesque sint et ad $G$ pertineant, adeoque sub $A$ contineantur, multitudo classium in $A$ nequit esse minor quam in $A^{\prime}$; quare multitudines classium in $A$ et $A^{*}$ neceśsario aequales erunt.

II. Quum multitudo omnium classium ancipitum multitudini generum aequalis sit (artt. 


\section{$-554-$}

$261,287 \mathrm{HII}$ ): manifestum est, si in $G$ vna tantum classis anceps detur, in quouis genere wnam classem ancipitem conteritam esse debere; si in $G$ duae ancipites exstent, in semissi omnium generum binas dari, in reliquis nullas; denique si in $G$ plures ancipites contineantur puta $a^{*}$ ), partem $a^{\text {tam }}$ ommium generum $a$ classes ancipites continere, reliqua nullas,

III. Sint, pro eo casu vbi $G$ duas classes anclpites continet, $G, G^{\prime}, G^{\prime \prime}$ etc. ea genera, quae binas, atque $K, H^{\prime}, F^{\prime \prime}$ etc, ea quae mullas continent, designeturque complexus illorum per complexus horum per 5 . Quum e compositione duarumi classium ancipitum semper prouentat classis anceps (art 249), nullo negotio perspicietur, e compositione duorum generum ex of semper prodire genus ex 6 . Hinc porro sequitur, e compositione generis ex cum genere ex $\mathfrak{W}$ prodire genus ex 5 ; si enim e. g. $G+H$ non ad $\hat{g}$ sed ad (S) pertineret, etiam $G^{\prime}+H+G^{\prime}$ ad $(S)$ referendum esset, Q. E. A., quoniam $G^{*}+G^{*}=Q$ adeoque $G^{\prime}$ $+I I+G^{\prime}=H$. Denique facillim intelligitur genera $Q+H, G^{\prime}+H, G^{\prime \prime}+H$ etc; vna cum his $H+H, H W+H, H H^{\prime \prime}+H$ etc omnia

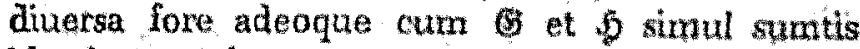
idantica sed, per ea quae modo demonstrata sunt, genera $G+H, G^{\prime}+H, G^{n}+H$ etc. omnia pertinent ad $\$$ adeoque hunc complexum exhauriunt, quare necessario reliqua $H+H$, $H^{\prime}+H, H^{\prime \prime}+H I$ etc ommia ad (s pertinebunt,

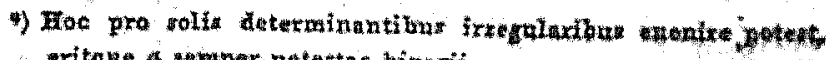

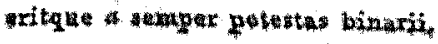


i: e. e oompositione duorum generum ex semper oritur genus ex 6 .

IV. Si $E$ est classis generis $\mathscr{V}$, a principals $G$ diuersi, patet, $2 E$, $4 E, 6 E$ ete omnes pertinere ad $G_{3}$ has vero $3 E, 5 E_{*}^{*}, Z E$ etc ad $F$. $\mathrm{Si}$ itaque periodus classis $2 E^{\circ}$ ex $m$ terminis constat: manifesto in serie $E, 2 E, 5 E$ etc classis $2 m E$, nec vlla prior, cum $K$ identica erit, siue periodus classis $E$ ex $2 m$ terminis constabit. Irine multitudo terminionum in periodo classis cutiscunque, ex alio genere quam principali, exit vel 2n vel pars aliquota ipsius $2 n$, designante $n$ malutudinem classiam in singulis generibus.

V. Sit $C$ classis data generis principalis $G_{\text {F }}$ $\vec{E}$ classis generis $\not{V}$ e cutus duplicatione $C^{*}$ ortatur (qualis semper dabitur, axt. 286), atque ornmess classes ancipites (pr. prim. eiusdem dot) $K_{*}, W_{*}$ $\mathrm{F}^{*}$ etc, enuntque onnes classes, e quartm duplicatione $C$ oritur, hae: $E(=E+K) \cdot E+K$, $E+K$ etc. quarum complexus exprimatur per $\Omega$; multitudo harum classinm acquahs ent mul* ritudini classiam ancipitum sue multitudini generum. Manifestum est, classibus in atot at genus pertiners, quot ancipites dentur in $C_{3}$ designando itaque harura multitudinem per $a_{4}$ yatet, in qunuis genere vel a classes ex a datat vel hullas. Hinc facile colligitur, quando sit $\alpha$ sas 1 , in quouis genere contineri nnam classata ex $n$ quando $a=2$, semissen oruium genemam binas classes ex $\Omega$ continere, relicha nuthas, to quidem semissem priorem vel totam com (s) coinctider (in eadera siguificatione vi supra III), posteri- 


\section{$-53^{6}-$}

arem cum 5 , vel hanc cum $\$$, illan cum $\mathfrak{f}$. - Quando $a$ adhuc maior est, semper pars $a^{\text {ta }}$ omnium generum classes $\Omega$ includent (singula $a$ classes).

VI. Supponamus iam, $C$ esse talem classem, cuius periodus ex $n$ terminis constet, perspicieturque facile, in eo casu vbi $a=2$ adeoque $n$ par, nullam ex $\Omega$ ad $G$ pertinere posse (tunc enim talis, classis in periodo classis $C$ contenta foret; si itaque esset $=r C$, siue $2 r C=C$, foret $2 r \equiv 1$ (mod $n)$ Q. E. A.); quamobren quum $G$ ad $\&$ pertineat, necessario omnes classes $\Omega$ inter genera $\mathfrak{S}$ distributa eruat. Hine colligitur, quoniam (pro det. reg.) in $G$ ommino dantur in classes periodos $n$ terminorum habentes, pro eo casu vbi $a=2$. inteniri in quouis genere $\mathfrak{5}$ omnino $2 \$ n$ classes, quarum periodi $2 n$ terminos, adeoque tum genus suum tum principale, complectantur; quando vero $a=1$, in quouis genere a principali diuerso on huiusmodi classes dabuntur.

VII. His obseruationibus methodum sequentem superstruimus, systema omnium classium pr. prim. pro quolibet determinante regulari dato (irregulares enim omnino seponimus) quam aptissime construendi. Eligatur ad lubitum classis $E$, cuius periodus an terminos, adeoque tum genus suum quod sit $V$ tum principale $G$ complectatux; classes horum duorum generum ita disponantur, vt in illa periodo progredruntur. Hoc modo res iam absoluta erit, quando plura genera quam haec duo omnino non adsunt, siue reliqua adicere non necesse videtux (e. g. pro tali det. neg, vbi duo 
tantum genera positilua dantur). Quando vero quatuor aut plura genera construenda sunt, reliqua hoc modo tractentur. Sit $V^{\prime}$ aliquod e reliquis atque $D+V=V^{\prime \prime}$, dabunturque in $V \cdot$ et $V \cdot$ duae classes ancipites (puta vel in vtroque wa, vel in altero duae in altero nulla); ex his eligatur vna $A$ ad lubitum, patetque facile, si $A$ cum singulis classibus in $G$ et $V$ componatur, prodire $2 \pi$ classes $_{*}$ diuersas ad $V^{\prime}$ et $V^{\prime \prime}$ pertinentes, adeoque haec genera omnino exhaurientes; ita haec quoque genera ordinari poterunt. - Si praeter haec qizatuor genera alia adhuc supersunt, sit Vin mum e reliquis, atque $V^{\mathrm{nv}}, \mathscr{V}^{\mathrm{V}}, \mathscr{V}^{\mathrm{vi}}$ genera ea quae prodeunt e compositione generis $V^{\prime \prime \prime}$ cum $V$, $V^{\prime}$ et $V^{\prime \prime}$. Haec quatuor genera $V^{\prime \prime \prime}=V^{\nabla D}$ quatuor classes ancipites continebunt, patetque, si ex his va $A$ eligatur atque cum singulis classibus in $G, V$, $F^{\prime}, V^{\prime \prime}$ componatur, omnes classes in $V^{\prime \prime \prime}=V^{\mathrm{vI}}$ prodire. - Si adhue plura genera supersunt, simili modo continuetur, donec omnia exhanstae sint. Patet, si maltitudo omnium generum construendorum sit $2^{\mu}$, omnino opus fore $\mu-1$ classibus ancipitibus, et quamuis classem horum generum produci posse vel e multiplicatione classis $E$, vel e compositione, classis, e tali compositione ortae, cum na pluribusue ancipitibus. Ecce duo exempla, per quae haec praecepta illustrabuntur; plura de vsu talis constructionis, vel de artificiis per quae labor subleuari potest hic adiicere non licet. 


\section{$538-$ \\ 1. Determinans $-16 \mathrm{I}$.}

Quatuor genera positiua, in sipgulis quaterna classex.
$G$
$x, 4 ; \pi ; * 23$
$(1,0,161)=\pi$
$(0,1,18)=2 E$
$(2,1,54)=4 E$
$(9,-1,18)=6 E$
VI
3. $4 ; x ; \pi 23$
$(3,1,54)=E$
$(6,-1,07)=3 E$
(6, 3,27$)=5 F$
$(3,-1,54)=\pi$
3. $4{ }^{2} 7 \times 2 \times$
(7, 0,23$)=A$
$(11,-2,15)=A+2 E$
$(14,7,15)=A+4 E$
thid
* 4; $\mathrm{N}_{7} ; \mathrm{N}_{23}$
$(10,5,27)=A+D$
(5, 2,35$)=A+3 E$
$(5,-3,53)=A+5 E$
$(11,2,15)=A+6 E$
$(10,-5,17)=A+7^{E}$

\section{Determinans -546 .}

Octo genera positiua; in singulis ternae classte,

$G$
I et s, 8; R3; RT; RI3
$(1,0,546)=K$
$(22,-2,25)=2 E$
$(22,2,25)=4 E$

$x * 3,8 ; \mathrm{N}_{3} ; \mathrm{R} 7 \mathrm{~N}$

$(2,0,275)=A$

$(1,1,-2,50)=A+2 E$

$(1,2,50)=A+4 E$
Tr

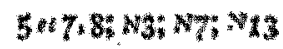

$(6,2,110)=E$

$(2, a, 26)=35$

$(5,-9,120)=5$

Fis

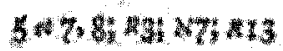

$(10,2,55)=4+2$

$(15,0,49)=4+5$

$(10,-2,55)=A+5 C$ 


\section{$-539-$}
7 III
$r * 3 \quad 8 ; N 3 ; N T ; R \times 3$
$(3,0,182)=A^{\prime}$
$P^{\mathrm{ry}}$
$(17,7,35)=A^{2}+2 E$
$(15,-3,37)=A^{\prime}+E$
$(17,-7,35)=A^{\prime}+4 E(15,3,37)=A^{\prime}+5 E$
$5 * 2 \cdot 8 ; * 3 ; R 7 ; 213$
$\gamma^{4}$

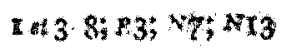
$(6,0,9 x)=A+A^{2}$
$F^{\mathrm{rr}}$
$(19,9,33)=A+A^{\prime}+2 E$
$(19,-9,53)=A+A^{\prime}+4 E$
5*7.8*3;7\%15
$(23,1,29)=A+A+E$
$(1,0,26)=A+A+3 E$
$(23,-1,29)=A+A^{4}+5 E$ 


\section{$S$ ล}

VARIAE DISQVISITIONVM PRAECEDENTYVM APPLICATIONES.

508. Quam fertilis sit arithmetica sublumio ritatibus, quae in alis quoque matheseos partibus

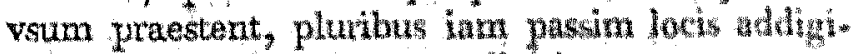
tauimus; quasdawn vero applicationes, quate expom sitionem ampliorem merentur, seorsm tracksta

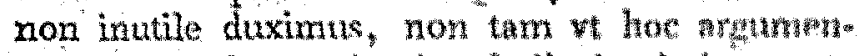

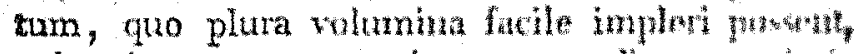

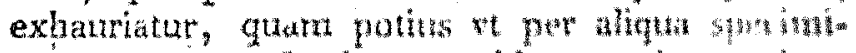
na illustretur. In hates quatem sethens: promo de resolatione fractionum in simpliciores agernus

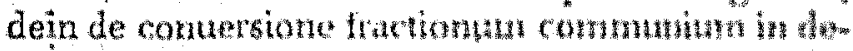

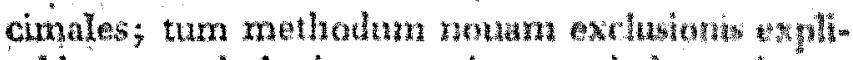
cabimus, solutioni aequhtionum indeternituatu.

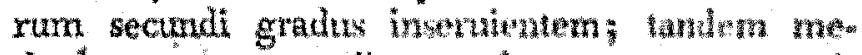

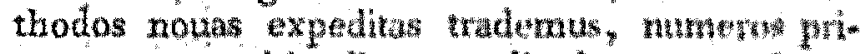
mos a compogitis dignoscendi, horumque vactom res explorandi. In sectione sequeate autem

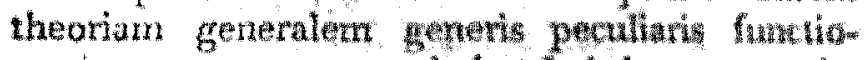
num, per totam andysin latisime saturnis,

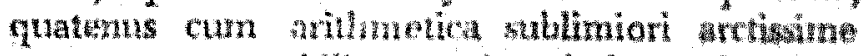

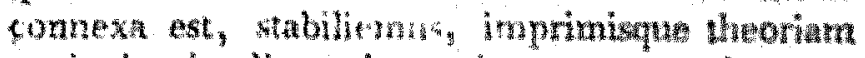
sectionis circuli, cuizs prisha tantum elenenta 
hactemus innotuerunt, nouis incrementis amplificare studebimus:

309. ProbeBma. Fractionem $\frac{m}{n}$, cuitus denon minator nest quodurtum e duobus mumeris inter se primis $a, b$, iw dus ditus discerpere, quarum denonititatoress sint $a . b$.

Sol. Sint fractioures quaesitae $\frac{x}{a}, \frac{y}{b}$, fiexque debebit $b x+a y=m$; hinc $x$ erit radix congruentiae $b x \Longrightarrow m($ mod. $a)$, quae per sect, II erui poterit, $y$ vero liet $=\frac{m-b x}{a}$.

Ceterum constat, congruentiam $b x \equiv m$ radices infinite multas, sed secundum $a$ congruas, habere, wilca vero tantum positiua minorque quam a dabitur, fieni autem potest etiam, it $y$ euadat negatious. Vix necesse erit monere, $y$ etiam per congruentiam ay $\equiv m(\bmod . b)$, atque $x$ per aequationem $x=\frac{m-a y}{b}$ inueniri posse E. g. proposita fractione $\frac{58}{7}$, erit 4 valor expr. $\frac{58}{17}(\bmod .7)$; vnde $\frac{58}{7}$ resoluitur in $\frac{4}{7}+\frac{2}{1 x^{*}}$

310. Si fractio $\frac{m}{n}$ proponitur; cuius denominator $n$ est productum e factoribus quotcunque inter se primis $a, b, c, d$ etc.: per art praec. primo in duas resolui potest, quarum denominatores sint $a$ et $b c d$ etc.; secunda iterum in duas denominatorum $b$ et $c d$ etc; posterior rursus in duas et sic porro, rnde tandem fractio proposita 


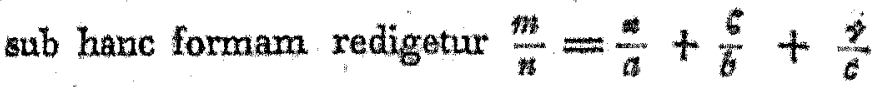
$+\frac{\delta}{d}$ etc. Numeratores $k, 2, d$ etc manifesto pasitiuos ac denominatoribus suis minores accipere licebit, praeter vitimum, qui reliquis determinatis non amplius est arbitrarits, atque etiam negatiuns aut denominatore maior fieri potest (siquidem non supponimus $m<n$ ). Tum plerumque e re erit, ipsurn sub formam $\mp k$ redigere, ita vt a sit positius ac minor quam $e, k$ vero integer. Dexique patet, $a, b, c$ êtc. ita accipi posse, vt sint vel mumeri urimi vel numerorum primonum potestates.

$E x$ Fractio $\frac{20 \pm}{9}$, cuins denominator $=$

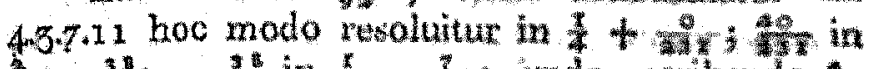

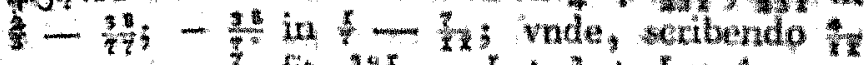

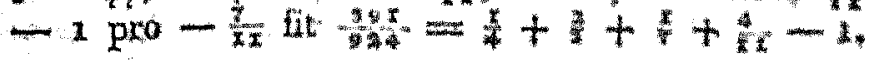

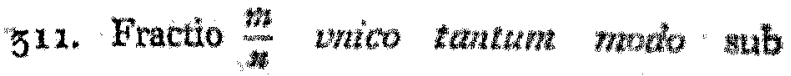
formarn $\frac{b}{a}+\frac{b}{b}+$ atc $\mp k$ reduci potest ita vt $\ldots, 6$ etc sint positiui ac minores quam a $b$ etc. scilicet supponendo $\frac{m}{a}+\frac{b}{b}+$ $\frac{y}{c}+$ etc $F=\frac{z^{\prime}}{a}+\frac{b}{b}+\frac{y^{*}}{6}+$ etc $\pm k^{\prime}$, atque etiam al, posituos ac minores quam $a, b$ ete, necessahio erit $=-6=$ $\epsilon_{y}, y=$ : etc, $k=k$. Multuplicando onim pect. $m=a b c$ etc., patet theri $m=$ whed tate $E$ abcd etc. (mod a), vade quoniam bed etcid a primus 
est, necessario $\equiv \alpha^{\prime}$ adeoque $\alpha=\alpha^{\prime}$, et perinde $C=b$ etc., rnde etiam sponte $k=k^{\prime}$ Iam quum prorsus arbritrarium sit, cuiusnam denominatoris numerator primus supputetur, manifestum est, omnies numeratores ita inuestigarf posse, vt to in art. praec. puta 6 per congruentiam bacd etc. $\equiv m$ (mod. $b$ ), $\gamma$ per hanc zabd etc. $\equiv m$ (mod. $c$ ) etc.; summa ompium fractionum sic inuentarum vel propositae $\frac{m}{n}$ aequalis erit, vel differentia numerus integer $=k$, qua via simul confirmationem calculi nanciscimur. Ita in ex. art praec. valores expr. $\frac{39 x}{23}$ (nod. 4),

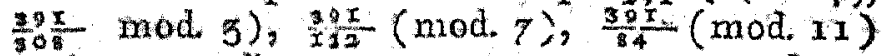
statim suppeditant numeratores $1,2,1,4$ denominatoribus $4,3,7,11$ respondentes, summaque harum fractionum propositam vnitate superare inuenitur.

312x Demunxtro. Si fractio communis in decimalem conuertitur, seriem figurarum decimalium *) (excluso si quis adest numero integro), siue finita sit, siue in infinitum excurrat, fractionis mantissam vocaxnus, expressionem, alias tantummodo apud logarithmos vsitatam, in significatione lationi accipientes. Ita e. g. fractionis mantissa est 125 , mantissa fractionis $\frac{37}{75}$ 287\%, fractionis $\frac{2}{37}$ mantissa $054.054 \ldots$ in inf.

Ex hac definitione statim patet, fractiones eiusdem denominatoris $\frac{l}{q}, \frac{m}{m}$ easdem vel diuersas

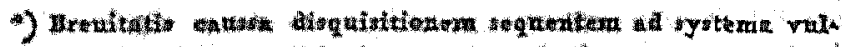

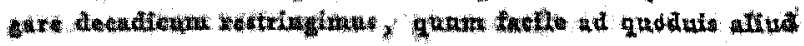

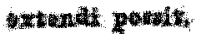


mantissas habere, prout numeratores $l, m$ secumdum $m$ congrui sint vel incongrui. Mantissa finita non mutatur, si ad dextram cifrae quotcunque apponanwir. Mantissa fractionis $\frac{x}{n}$ obtinetur, rescindendo a mantissa fractionis $\frac{m}{n}$ figuram primam et generaliter mantissa fractionis $\frac{\text { ICm }}{n}$ inuenitur rescindendo, figuras primas mantissae ipsius $\frac{m}{n}$. Mantissa fractionis $\frac{1}{n}$ statim figura significatiua (i. e. a cifra diuersa) incipit, sì $n<10$; si vero $n=10$ vel $<10$, multitudoque figurarum e quibus constat est, $k$, primae $k-i$ figurae mantissae ipsius $\frac{I}{n}$ erunt cifrae atque demum sequens $k^{\text {ta }}$ erit significatiua. Hinc fadile deducitur, si $\frac{l}{n}, \frac{m}{n}$ mantissas diuersas habeant (i. e. si $l, m$ sec. $n$ incongrui), has certo in primis $k$ figuris conuenire non posse, sed saltem in $k^{k a}$ discrepare debere.

313. Problema. Dato denominatore fractionis - atque primis $k$ figuris ex ipsius mantissa. inuenire mumeratoren m; quem ipso n minorem supponimus.

Sol. Considerentur illae $k$ figurae tamquam numerus integer, qui per $n$ multiplicetur. productumque per $10^{b}$ diuidatur (siue $k$ vltimae figurae resecentur). Si quotiens est integer (siue figurae resectae cifrae /, ipse manifesto erit numerator quaesitus atque mantissa data completa 


\section{- $545-$}

sin minus, numerator quaesitus erit integer proxi. me maior, siue ille quotiens vnitate auctus, post. quam figurae decimales sequentes reiectae sunt. Ratio huius regulae tam facile ex is quae ad finem art. praec. obseruauimus cognoscitur, vt explicatione vberiori opus non habeat.

Ex. Si constat, duas figuras primas mantissae fractionis, cuius denominator 23 , esse 59 , habemus productum $83.59=1557$, a quo duas yltimas figuras abiiciendo, vnitatemque addendo, numerator quaesitus prodit $=16$.

314. Inchóamus a consideratione talium fractionum, quarum denominatores sunt numeri primi vel numerorum primorum potestates, posteaque reliquas ad has reducere ostendemus. Et primo statim obseruarnus, mantissam fractionis $\frac{a}{p^{a}}$ (cuius numeratorem a per numerum primum $p$ non diuisibilem esse semper supponimus) finitam esse, atque ex $\mu$ figuris constare, si $p=2$ aut $=5 ;$ in casu priori haec mantissa, tam quam numerus integer considerata, erit $=5^{\mu} a$ in posteriori $=2^{\mu} a$. Haec tam obuia sunt, vt expositione non egeant.

Si vero $p$ est alius numerus primus, $10^{r} a$ per $p^{\text {re }}$ numquam diuisibilis erit, quantumuis magnus accipiatur $r$, vinde sponte sequitur, mantissam fractions $F=\frac{a}{p^{k}}$ nececessario in infinitum progredi. Supponamus, $10^{\mathrm{e}}$ esse potestatem Mra 


\section{- $546-$}

infimam numeri 10 , quae vnitati secundum mo-

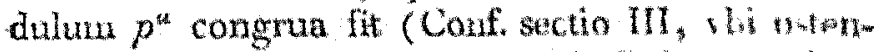
dimus, $e$ vel numero $(p-1) p^{\text {man }}$ aequalem vel ipsius partem aliquotam esse:, perspirisoturo que facile, etiam $20^{\circ} a$ fore mmerum, in strito $10 a, 100 a, 1000 a$ te primum, qui ipsi $a$ sem cundum eurdem modulum sit congutus, lima quam per art. 312 mautissae fractionum " "a rooa ... ro a oriantur, demendo mantiscae fractiphis $F$ figuram primam, dnas ... $e$ figuras primas resp., manifestum est, in hac wantissa post primas e figuras, neque prus, easdem ittrum repeti. Has primus e figuras, quabus infinities repetitis mantissa formata est, pertodum hum mantissae sive fractionis $F$ vocare possumus, patetque magnitudinem perioti, i. e multitudi nem figurarem e quibus constat, quat, est $=4$ a numeratore a omnino indeputenam ase,

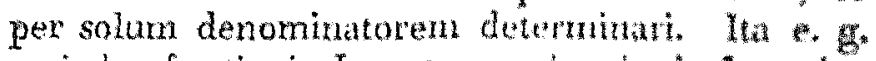
periodus fractionis ${ }_{x}^{x}$ est 09 , Hactionis perion dus $4.2857^{*}$ ).

315. Simulac igitur frationis alcututu pe riodus habetur, mantissa al figuras quotcunque produci poterit. Porro patet, si luwrit $b=100$ $\left(\bmod p^{4}\right)$, periodum fractionis orit, priw mae $>$ figurae periodi fractions $F$ (stmponerulo

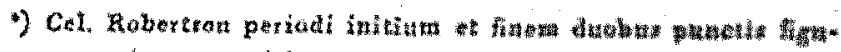

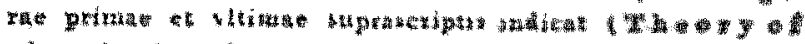

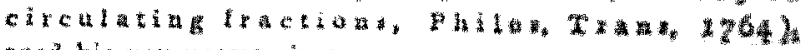

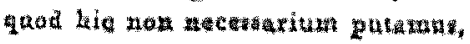




\section{- 547 -}

$x<e$ quod licet) reliquis $e-\lambda$ postscribantur, adeoque cum periodo fractionis $F$ simul periodos omnium fractionum haberi, quarum numeratores iysis $10 a, 200 a, 1000 a$ etc. secundum denominatorem $p^{\mu}$ sint congrui. Ita e. g. quum $6 \equiv$ $5.10^{2}(\bmod .7)$, periodus fractionis $\frac{6}{8}$ statim e periodo fractionis $\frac{3}{7}$ fit 857142 .

Qnoties itaque pro modulo $p^{k}$ numerus xo est radix primitiua (artt. 57,89 ), e pexiodo fractionis $\frac{x}{p^{k}}$ protinus deduci poterit periodus cuiusuis alius fractionis $\frac{m}{p^{\mu}}$ (cuirus numerator $m$ per $p$ non diuisibilis), tot figuras ab illa a laeua resecando et ad dextram restituendo, quot vnitates labet index ipsius $m$, numera to pro basi accepto. Hinc perspicuum est, quanobrem in hocce casu numerus to in tabula I semper pro basi acceptas sit ( $\mathrm{v}$. art. 72 ).

Quando vero Io non est radix primitiua, e periodo fractionis $\frac{I}{p^{4}}$ earum tantummado fractionum periodi exscindi possunt, quarum numeratores alicui potestati ipsius to secundum $p^{\mu}$ sunt congrui. Sit $10^{8}$ potestas infima ipsius 10 vritati secundum $p^{*}$ congrua, $(p-1) p^{\mu-x}=e f$, atque talis radix prirritiua $r$ pro basi accepta, vt. index numeri to liat $f$ (art. 71 ). In hoc itaque systemate numeratores fractionum, quarum periodi e periodo fractionis $\frac{\mathrm{r}}{\mathrm{P}^{\mathrm{m}}}$ exscindo possunt, habebunt indices $f$, of, $3 f \ldots \ldots e f-f$; simili modo 
e periodo fractionis $\frac{r}{p^{u}}$ deduci possunt periodi fractionum, quarum numeratores lor, 10or, looor etc. indicibus $f+1,2 f+1,3 f+1$ etc. respondentes; e periodo fractionis cum numeratore rr (cuius index 2) deducentur periodi fractionum cum numeratoribus quorum indices $f+2,3 f+2$, $3 f+2$ etc.; generaliterque e periodo tractionis cum numeratore $\gamma^{i}$ deriuari poterunt periodi fractionum cum numeratoribus, quorum indices $f+i$, of $+i, 3 f+i$ etc. Hinc facile colligitur, si tantummodo periodi fractionum cum numeratoribus $1, r, r r, r^{3} \ldots r^{f-1}$ habeaniur, omnes reliquas inde per solam transpositionem deduci posse adiumento regulae sequentis: Sit index numeratoris $m$ fractionis propositae $\frac{m}{p^{u}}$, in systemate vbi $r$ pro basi acceptus est, $=\dot{i}$ (quem supponimus minorem quam $\left(p-1 \cdot p^{u-1}\right)$; fiat (diuidendo per $f$ ) $i=a f+6$ ita vt $a, 6$ sint integri positiui (siue etiam o) atque $E<f$; quo facto orietur periodus fractionis $\frac{m}{p^{\mu}}$ e periodo fractionis cuius numerator $r^{6}$ (adeoque 1 , quando $b=0$ ), collocando huiús primas figuras post reliquas (adeoque hanc ipsam periodum retinendo, quando $\alpha=0$ ). Haec sufficienter declarabunt, cur in condenda tabula $I$ normam in art. $7^{2}$ explicatam sequuti simus.

316. Secundam haec principia pro omnibus denominatoribus formae $p^{\mu}$ infra 1000 tabulam periodorum necessariarum construximus, quam integram siue etiam viterius continuatam 
occasione data publici iuris faciemus. Hoc loco tabula III vsque ad 100 tantum producta tamquam specimen sufficiat, quae explicatione vix opus habebit. Pro iis denominatoribus, vbi 10 est radix primitiua, periodos fractionum cum numeratore 1 exhibet (puta pro $7,17,19, .25,29$; $47,59,61,97) ;$ pro reliquis, $f$ periodos numeratoribus $1, r, \operatorname{rr} \ldots r f-r$ respondentes; quae per numeros adscriptos (0), (1), (a) etc. sunt distinctae; pro basi $r$ semper eadem radix primitiua adoptata est $\mathrm{vt}$ in tabula I. Hinc igitur periodus fractionis cuiusuis cuius denominator in bac tabula continetur adiumento praeceptorum art. prace. erui poterit, postquam numeratoris index per tabulam I est computatus. Ceterum pro denominatoribus tam paruis negotium aeque facile absque tabula I absoluere 'poterimus,' si per diuisionem vulgarem tot figuras initiales mantissae quaesitae computamus, quot per art. 313. necessariae sunt, vt ab omnibus aliis eiusdem denominatoris distingui possit (pro tabula III non plures quam 2), omnesque periodos denominatori dato respondentes perlustramus, vsquedum ad illas figuras initiales perueniamus, quae periodi initium haud dubie indicabunt; monere tamen oportet, illas figuras etiam separatas esse posse, ita vt prima (vel plures) finem alicuius periodi constituant, reliqua vel reliquae eiusdem initium.

Ex. Quaeritur periodus fractionis $\frac{12}{19}$. Hic pro modulo 19 per tab. I habetur ind. $12=$ 2 ind. $2+$ ind $3=39 \equiv 3$ (mod. 18 art. 57); quare quum pro hoc casu vnica tantum periodus numeratori I respondens habeatur, 
huius tres primas figuras ad finem transincare oportet; vnde fit periodus quaesita 651578947 68421052 - Aeque facile periodi initiun duabus primus figuris 63 inuentum fuisset.

$\mathrm{Si}$ periodus fractionis $\frac{5}{33}$ desideratur, fit pro modulo 53 , ind. $45=2$ ind. $5+$ ind. $5=49$ multitudo periodorum hic est $4=f$, atume 49 $=12 f+1$, quare a periodo curn (1) simuata 12 primae figurae postponendae crum vitimate periodusque quaesita fit $81.90,566037755$ * Hztu* rae initiales 84 in hoc casu sepuratae sunt in tabula.

Obseruabimus adhuc, adiumento talulae III etiam numerum inueniri posse, qui pro modulo dato (in ipsa sub denominatoris titulo contento) indici dato respondeat, yt in art 59 polliciti su* mixs. Patet enim per praecc, inueniri posse pew riodum fractionis cuius numeratori (like in cognitus sit) index datus respondeat; sufficit at* tem, tot figuras initiales huius periodi excerpere, quot figuras babet denominator; ex illis per art 313. eruetur numerator siue numerus quatesitu indici dato respondens.

3.7. Per praecedentia mamtissa fractionü cuiuscunque, cuius denominator est numerus prits mus aut numeri primi potestas intra limites ta bulae, ad figuras quoteanque sine computo erut potest; sed adiumento disquistiomm in intio huius sectionis tahulae ambitus multo latus patert omnesque fructiones, quarum denominatores sunt producta e numeris primis aut primonim pote* 
states intra ipsius limitem, complectitur. Quum enim talis fractio in alias decomponi possit, quarum denominatores sint hi factores, atque has in fractiones decimales ad figuras quotcurque conuertere liceat, restat tantummodo, vt hae, in surnmam vniantur. Ceterum vix opus erit monere, summae sic prodeuntis figuram vltimam iusto minorem euadere posse; manifesto autem defectus ad tot vaitates adscendere nequit, quot fractionis particulares adduntur, vnde hae ad aliquot figuras viterius computare conueniet, quam fractio proposita iusta desideratur. Exempli caussa considerabimus fractionem $\frac{609938035 \mathrm{I}}{1271808720}$ $=F^{*}$ ), cuius denominator est productum e numeris $16,9,5,49,13,47,59$. Per praecepta supra data inuenitur $F=1+\frac{\pi \pi}{\pi 6}+\frac{4}{9}+$ $\frac{4}{5}+\frac{22}{49}+\frac{5}{33}+\frac{7}{47}+\frac{52}{59}$, quae fractiones particulares ita vit sequitur in decimale conuertuntur:

$$
\begin{aligned}
& 1=1 \\
& \text { ir }=0,625 \\
& \frac{4}{5}=0,8 \\
& \frac{4}{9}=0,4444444444 \quad 4444444444 \quad 44 \\
& \frac{22}{49}=0,4489795918 \quad 3673469387 \quad 75 \\
& \frac{5}{x^{3}}=0,3846153846 \quad 1538461538 \quad 46 \\
& \frac{z}{47}=0,1489361702 \quad 127659574468 \\
& \frac{52}{59}=0,88135593^{22} \quad 0338983050 \quad 84 \\
& F=4,7958315233 \quad 1271954166 \quad 17
\end{aligned}
$$

- Hraec fractio est rna $e x$ iis, quae ad radicem quadratam ex 23 quam prcxime appropinquant, et quitem excessus est minor quem septem vilitates in loco figura decimalis vigerimae. 
Defectus huius summae a iusto certo minor est quinque vnitatibus in figura vltima vigesima secunda, quare viginti primae inde mutari nequeunt. Cálculum ad plurés figuras producendo, pro duabus figuris vltimis 17 prodit $1893936 \ldots$ - Ceterum vel nobis non monentibus quisque videbit, hanc methodum, fractiones communes in decimales convertendi, ei potissimum casui accommodatam 'esse, vbi multae figurae decimales desiderentur; quando enim paucae sufficiunt, diuisio vulgaris siue logarithmi aeque expedite plerumque adhiberi poterunt.

318. Quum itaque resolutio talium fractionum, quarum denominatores e pluribus numeris primis diuersis compositi sunt, ad eum casum iam reducta sit, vbi denominator est primus aut primi potestas: de illarum mantissis pauca tantum adiiciemus. Si denominator factorem 2 et 5 non continet, mantissa etiam hic e periodis constabit, quoniam pro hoc quoque casu in serie 1o, 100, 1000 ad terminum, wnitati secundum denominatorem congruum, tandem peruenitur, simulque huius termini exponens, qui per art. $9^{2}$ facile determinari poterit, periodi magnitudinem, a numeratore independentem, indicabit, siquidem hic ad denominatorem prinus fuerit. - Si vero denominator est formae $2^{2} 5^{6} N$, designante $N$ numerum ad 10 primum, $\alpha$ et $b$ numeros quorum vnus saltem non est $o_{\text {, }}$ Iractionis mantissa post primas \& vel 6 figuras (prout \& vel - maior) e periodis constare incipiet, cum periodis fractionum cum denominatore $N$ respectu longitudinis conuenientibus; hoc 
facillime inde deriuatur, quod illa fractio in duas alias cum denominatoribus $2^{\infty} 5^{6}$ et $N$ resolubilis est, quarum prior post prrmas a vel $b$ figuras abrumpetur. - Ceterum de hoc argumento multas alias obseruationes adiicere possemus, praesertim circa artificia, talem tabulam vt III quam. citissime construendi, quas breuitatis caussa eo lubentius hoc loco supprimimus, quum plara huc pertinentia tum a cel. Robertson l. c., tum a cel. Bernoulli (Nouv. Memo de l'Ac. de Berlin 1771) iam sint tradita.

319. Congruentiae $x x \equiv A(\bmod m)$, quae conuenit cum aequatione indeterminata $x x$ $=A+m y$, possibilitatem in sect. IV (art. 14,6) ita tractauimus, vt nihil amplius desiderari posse videatur; respectu inuestigationis incognitae ipsius autem, iam supra (art. 152 ) obseruauimus, methodos indirectas directis longe esse praeferendas: Si $m$ est numerus primus (ad quem casum reliqui facile reduountur), tabulam indicum I (cum III secundum obs: art 316 combinatam) ad hünc finem adhibere possemus, vt in art. 60 generalius ostendimus: haec vero methodus intra tabulae limites restricta foret. Propter has rationes methodum sequentem generalem ac expeditan arithmeticae amatoribus haud ingratam fore speramus.

Ante omnio obseruamus sufficere, si ii tantummodo valore sipsius $x$ habeantur, quî sint positiui atque non maiores quam $\frac{2}{2} m$, quim quiuis alius horum alicui vel ipsi vel negative sumto secundum modulum $m$ congruus sit; pro tali Mm 5 
vero valore ipsius $x$ valor ipsius $y$ necessario in ter limites $-\frac{A}{m}$ et $\frac{r}{4} m-\frac{A}{m}$ contenus erit. Methodus itaque, quae statim so offert, in 10 consisteret, vt pro singulis valuribus iprins $y$ inn tra hos limites contentis, quorum comply anm

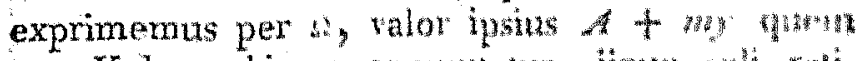
per $V$ denotabinus computemr, iigue soll wht neantur, pro quibus $P$ tit qualratum Quatolo $m$ est numerts parnus (o. g. intra +0$)$, hor an* tamen tars breue est, vt contractione vix opus habeat; quando autem $m$ est mayrmx, labur purr merfodum exclusionis sequenten, quantusn h. bet, abbreuiari poterit.

320. Sit $E$ numerts arbitratus intewer ad $m$ primus ac mator. quam a; omnia etus now residua quadratica diuersa (i. $\theta$. secundurn the

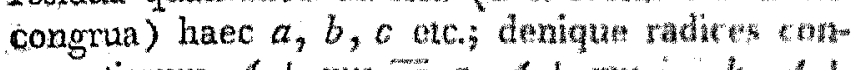
gruentiarum $A+m y=a_{3} A+m y^{*} b_{*}+$

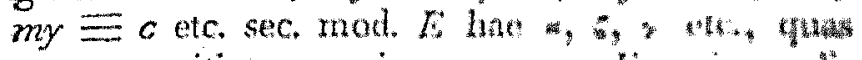

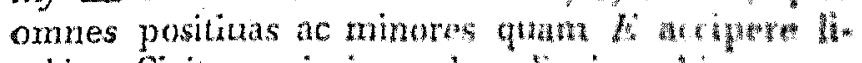
cebit. Si itaque ipsi $y^{*}$ valur allu

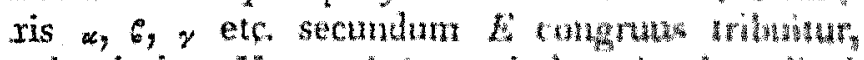

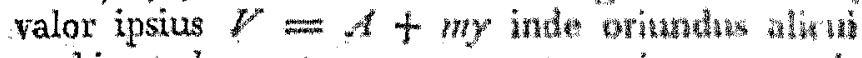
ex his $a, b, c$ etc congruas ot proin nut

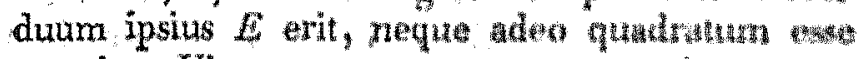

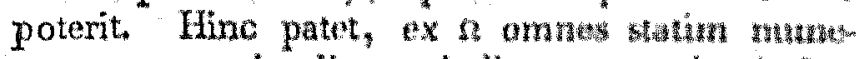

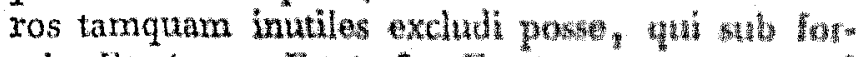

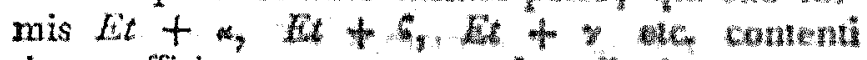

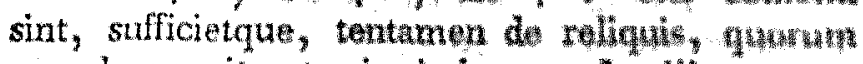

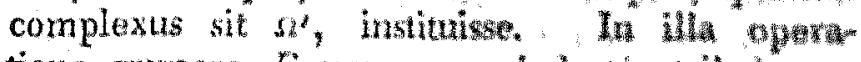

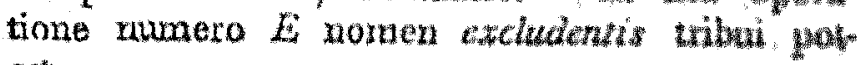
est. 
Accipiendo autem pro excladente numerum idoneum alium $E^{\prime}$, prorsus simili modo inuenientur tot numeri $\alpha i,{ }^{6}, \gamma$ ! etc., quot non residua diuersa quadratica habet; quibus $y$ secundum modulum $E^{\prime}$ congruus esse nequit. Quare denuo ex $\Omega^{4}$ eicere licebit omnes numeros sub formis $E^{\prime} t+\alpha^{\prime}, E^{\prime} t+E^{\prime}, E^{\prime} t+\gamma^{\prime}$ etc contentos. Hoc modo continuari poterit, alios aliosque semper excludentes adhibendo, donec multitudo numerorum ex $\Omega$ tantum deminuta fuerit, vt non difficilius videatu, omnes superstites tentamini reuera subiicere, quam exclusiones nouas instituere.

$E x$. Proposita aequatione $x x=22+97 y_{2}$ limites valorum ipsius $y$ erunt - $\frac{2}{3} \frac{2}{7}$ et $24 \frac{x}{4}$

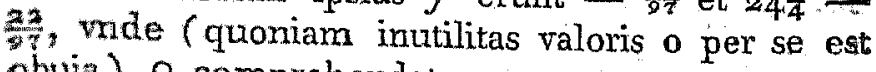
obuia) $\Omega$ comprehendet numeros $1,2,3 \ldots 24$. Pro $E=3$ habetur vicum non residurm $a=2$; vnde fit $=I$; excludendi sunt itaque ex $\Omega$ omnes numeri formae $3 t+1$; multitudo remanentum $\Omega$ exit 16 . Simili modo pro $E=4$ habetur $a=a, b=3$, vnde $x=0,6=1 ;$ quare reiui debent numeri formae $4 t$ et $4 t+1$ restantque hi octo $2,3,6,11,14,15,18,23$. Perinde pro $E=5$ reiiciendi inueniuntur numeri formarum $5 t$ et $5 t+3 i$ remanentque, hi $2,6,11,14$. Excludens 6 remoueret numeros formarum $6 t+\mathrm{I}$ et $6 t+4$, hi vero (qui cum murneris formae $3^{t}+x$ conueniunt) iam absunt. Exciudens 7 eiicit numeros farmarum $7 t+2$, $7^{t}+3,7^{t}+5$, ac relinquit hos 6,11 , 14 Hi pro $\eta$ substituti producunt resp. $D=$ $604,1089,1380$, e quibus valor secundus solus est cuadratus, winde $x \pm 37$. 


\section{$-\quad 55^{6}-$}

321. Quum operatio cum excludente $E$ instuta e valoribus ipsius $V$, valoribus ipsius $y$ in $\Omega$ respondentibus, omnes eos releget, qui sunt non residua quadratica ipsius $I$, residua voro eiusdem numeri non attingat; facile intelligitur, vsum excludentium $E$ et $2 E$ nilil differre, si $E$ sit impar, quum in hoc casu $E$ et at batem residua et non residua habeant. Hinc patet, si. successiue numeri $3,4,5$ etc. tamquam excludentes adhibeantur, numeros impariter pares 6 , 10, 14 etc. tamquam superfluos practereundos esse. 'Porro perspicuum est, operationern duplicem, cum excludentibus $E, E$ ' institutam, omnes cos valores ipsius $V$ remouere, qui vel strusquat $E, E^{\prime}$ vel vnius non residua sint, eosque qui sint, ptriusque residua remanere. Iam qumm in eo casu, vbi $E$ et $E^{\prime}$ diuisorem communem non habent, illi numer eiecti ormues sint non residua, atque hi superstites residua producti $E E^{\prime}$, manifestum est, vsum excludentis $E E^{\prime}$ in hoc casu ommino tantumdem effoctre, ac vsum duorum $E, E^{\prime}$, adeoque illum, post hunc, superfluum fieri. Quare eos quoque excludentes omnes praeterire licebit, qui in duos factores inter se primos resolui possunt, sufficietque its wi, qui sunt vel numeri primi (ipsum $m$ non metientes) vel primorum potestafes. Denique mantfestum est, post rsum excludentis $p^{4}$, qui sit. patestas numeri primi $p$, excludentem $p$ seu $p$, quando $r<\mu$ superfluurn fier; qutum trim $p^{\mu}$ inter valores ipsius $V$ sola sui residua reliquerit, a potiori non-residua ipsius $p$ atut potestatis caiusuis inferioris $p^{\prime}$ non amplius aterunt. Si vero $p$ aut $p^{\nu}$ iam ante $p^{\text {h }}$ adhibitus est, hic manufesto 


\section{$\div 55 \%-$}

tales tantumi valores ipsins $\nu$ eilcere potést, qui simul sunt residua ipsius $p$ (aut $p^{v}$ ) atque non residua ipsius $p^{\mu}$; quare huiusmodi tantum nonresidua ipsius $p^{\mu}$ pro $a, b, c$ etc accipere sufficiet.

322. Computus humerorum of, $\dot{6}, \gamma$ etc. cuivis excludenti dato $E$ respondentium multum contrahitur per obseruationes sequentès. Sint $\mathfrak{A}, \mathfrak{B}$, $\boldsymbol{S}$ etc. radices congruentiarum $m y \equiv a_{i}$ $m y \equiv b, m y \equiv c$ etc. (mod. $E$ ) atque $k$ radix huius $m y \equiv-A$, patetque fieri $a \equiv \mathfrak{I}+k$,

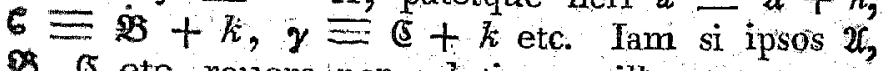
8, (5) etc. reuera per solutionem illarum congruentiarum eruere oporteret, haec via ipsos $a, b, \gamma$ etc. inueniendi nibil vtique breuior foret, quam ea quam supra ostendimus: sed illud neutiquam est necessarium. Si enim, primo, $E$ est numerus primus, atque $m$ residuium qu ipsius $E$, patet per art. 98 , ipsos $\mathfrak{U}, \mathfrak{B}$, $\mathfrak{S}$ etc, qui sunt valeres expr. $\frac{a}{m}, \frac{b}{m}, \frac{c}{m}$ etc. (mod. $E$ ), fieri non residua diuersa ipsius $E$, adeoque cum ipsis $a_{2}$ C, y etc. omnino conuenire, abstrahendo ab ipsorum ordine, cuius nihil hic refert; si vero in eadern suppositione $m$ est non-residuum ipsius $\boldsymbol{E}$, mumeri $\mathfrak{a}, \mathfrak{B}$, 5 etc cum omnibus residuis quadraticis, abiecto o, conuenient. Si $E$ est quadratum numeri primi (imparis), $=p p$, atque $p$ lam tamquam excludens applicatus, sufficit per art. praec., pro $a, b, c$ etc. ea non residua ipsius $p p$ asșumere qui sunt residuta ipsits $p$; i. e. numeros $p, 2 p, 3 p \ldots p p-p$ (scilicet omnes numeros infra $p p$ praeter o qui per $p$ sunt diudsibi- 


\section{- $558-$}

Ies); hinc vero facile perspicitur, pro $\mathfrak{x}, \mathbb{B}$, $\mathbb{C}$ etc. ómnino eosdem numeros prouenire debere, aliter tantum dispositos. Similiter si post applieationem excludentium $p$ et $p p$ ponitur $E^{*}=\mu^{*}$, sufficiet pro $a, b, c$ etc accipere producta singulorum nonresidnorum ipsius $p$ in $p p$, vnde pro ZC, $\mathfrak{B}_{2}$, 5 etc. prouenient vel iidem numeri, vel producta ipsius $p p$ in singula residua ipsizss $p$ praeter $o$, prout $m$ est residum vel non resim duum ipsius $p$. Generaliter accipiendo pro $E$ potestatem quamcunque numeri primi puta $p^{\text {tu }}$, omnibus inferioribus iam applicatis, pros $\chi, B$, o etc. prodibunt producta ipsius $p^{-1}-1$ wal in ormons numeros ipso $p$ minores, o semper excepto, quando $\mu$ par, vel in omnia nou residua ipsitus $p$ minora quam $p$, quando $m$ impar atque $m / p_{3}$ vel in omnia residua, quando $m \mathrm{~Np}_{0}-\mathrm{Si} E$ $=4$, adeoque $a=2, b=3$, pro $x, 8$ lathew mus. vel 2 et 3 vel 2 et $x$, protat ma 1 atut $\equiv 3(\bmod .4)$. Si post ssun axd. \& stutuitue

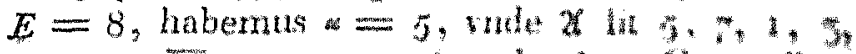
protat $n \equiv 1,3,5,7$ (nod. 8 ). Goneraliter autern, si $E$ est fotesias allor quatenaque bi marii puta $2^{\mu}$, inferioribus iasa applicanis, pout

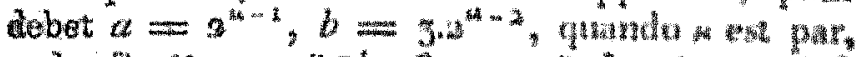

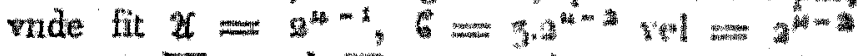
prout $m \equiv 1$ vel $\equiv 3$, quanto vero sest im

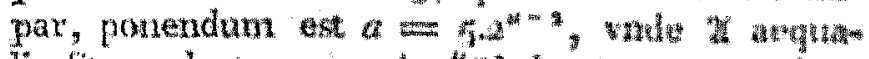

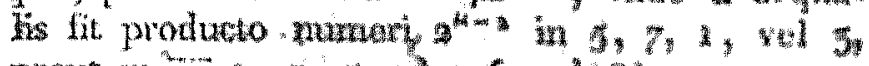
prout $m=1,5,5$ vel 7 (mod 8 ).

Ceterum perit facile comminiscentur appa-

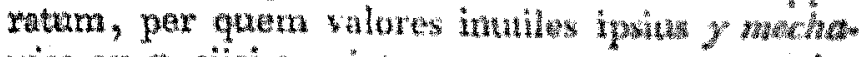
race ex a elici possint, postguan pro tot exche 
dentibus quot necessarii videntur numeri $~ ", \epsilon, z$ etc. sunt computati; sed de hac re sicut de alits artificiis laborem contrahendi hic agere non licet

323. Omnes repraesentationes numeri dati $A$ per formam binariam $m x x+n y y$, siue solutiones aequationis indeterminatae $m x x+n y^{y} y=$ $A$, in sectione $V$ methodo genterali inuenire docuimus, cuius breuitas quoque nihil desiderandum relinqueré videtir, si omnes valores expr: $\sqrt{-} m$ secundum modulum $A$ ipsum, et per suos factores quadratos diuisurn, iam habentur; hic auteras prö eo casu, vbi $m n$ est positiuus, solutionera explicabimus, directa multo expeditiorem, si ad hanc illos valores antea computare oportet. Strpponemus autem, numeros $m, n$ et $A$ esse posiciuos atque inter se primos, qutum casus reliqua ad humc facile possint redrci. Manifesto quoquo sufficit, valores positiuos ipsorum $x, y$ eruere, qurun reliqui inde per solam signorum mutationeern dedutanttr.

Perspicttrm est, $x$ ita comparatum esse debere, vt $\frac{A-m x x}{q}$, pro quo scribemus $F$, positiurts, integer, et quadratxs guadat. Conditio prima requirit, ut $x$ non sit maior quam $\sqrt{m}_{m}^{A}$; secunda ian per se locum habet quando $n=1$, elioquin requirit, vt valor expr. $\frac{A}{n}(\bmod n)$ sit residurum quadraticum ipsirs $n$, designandoqre omnes valores dittersos expr. $\sqrt{\frac{A}{n}}$ (mod. $\left.n\right)$ per $\pm r, \pm r$ etc., $x$ sub aliqua formarum $n t$ 


\section{$-560-$}

$+r, n t-r, n t+r^{t}$ etc. contentus esse debebit. Simplicissimum itaque foret, omnes numetos harum formarrm infra limitem $\sqrt{ } \frac{A}{m}$, qdos rum complexum per $\Omega$ exprimemus, pro $x$ substittuere, eosque solos retinere, pro quibus $V$ fit quadratum. Hoc tentamen, quantum lubeat, contrahere, in art. sq. docebimus.

324. Methodus exclusionum, per quam hoc efficiemts, perinde ac in disqu. praec. in eo consistit, vt plures numeros, etiam hic excludentes vocandos, ad lubitum accipiamus, pro quibusnam valoribus ipsius $x$ valor ipsitus $V$ fiat non residutum qu horum excludentium inuestigemus, talesque $x$ ex $\Omega$ eiliciamus. Per ratiocinia is quae in art 321 exposuimus omnino analoga apparet, tales tantrum excludentes adhibendos esse, qui sint numeri primi aut numerormm primorum potestates, et pro excludente posterioris generis ea $\operatorname{tanttm}$ ipsitus non residua a valoribus ipsitus $\nu$ arcenda, quae sint residua omnium potestatum inferiorum eiusdem numeri primi, siquidem exclusio cum his iam est instituta.

Sit itaque excludens $E=p^{\mu}$ (includendo etiàm errm castum vbi $\mu=1$ ), vbi $p$ est numerus primus ipsum $m$ non metiens, supponamusque $\left.{ }^{*}\right) p^{i}$ esse summam potestatem eiusdem nus meri primi per quam $n$ sit dikisibilis. Sint $a, b_{3}$

-) Breuitatis crussa duos castas, in quibus $n$ per $p$ est diuisibilis ae non dinisibilis, simul complectimax, in pastexioks $\longrightarrow$ o pozere oportet. 


\section{$-56 \mathrm{i}-$}

ce etc. nơn residua quadratica ipsius $E$ (ombià, quando $u=1$; necessaria siue ea quae sunt iresidua potestatum inferiorum, quando $\mu>1$ ) Computentur radices congruentiarum $m z \equiv \hat{A}$ $-n a, m z \equiv A-n b, m z \equiv A-n c$ etc. $\left(\bmod . E p^{\nu} \equiv p^{\mu+\nu}\right)$, quae sint $\alpha, b, 2$ etc., patetque facile, si pro quo valore ipsius $x$ fiat $x x \equiv{ }^{\prime}{ }^{2}\left(\bmod . E p^{\circ}\right)$, valorem respondenten ipsins $V$ fieri $\equiv a$ (möd. $E$ ) siue non residuum ipsius $E$, similiterque de numeris reliquis 6,2 etc.; aeque facile vice versa perspicitur, si quis valor ipsius $x$ producat $V \equiv a$ (mod. $E$ ), pro eodem fieri $x x \equiv \alpha \cdot\left(\bmod E p^{\prime}\right)$, adeoque ommes valores ipsius $x$, pro quibus $x x$ nulli numerorum $\propto, \xi, \gamma$ etc. sec. mod. Ep congruus sit, talès valores ipsius $\mathscr{V}$ próducere, qui nulli numberorum $a, b, c$ etc. sec. mod. $E$ sint côngrüi. Eligantur iam e vumeris $\alpha, b, \gamma$ etc. omnia residua quadratica ipsius $E p^{*}$, quae sint $g, g^{\prime}, g^{\prime t}$. etc., computentur valores expressionum $\sqrt{g}$, $\sqrt{ } g^{\prime \prime}, \sqrt{ } g^{\prime \prime}$ etc. (mod. $\left.E p^{\prime}\right)$, ponamusque hinc predire $t h, \pm h^{\prime}, \pm h^{h}$ etc. His ita factis mainfestum est, omines numeros formarum Ept $t$ $\pm h, E p^{\prime} t \pm h^{\prime}, E p^{\prime} t \pm h^{\prime \prime}$ etc. ex $\Omega$ tuto eiici posse, nullique valori ipsius $x$ in $\Omega$ post hanc exclusionem remanenti valorem ipsius $\nu$ sub formis $E u+a, E u+b, E u+c$ etc. contentum respondere pósse. Ceterum manifestum est, tales valöres ipsius $\dot{V}$ iam per se e nuffo valore ipsius $x$ prodire posse, quaindo iater numeros a, $b, y$ etc. nuilla résidua qu. ipsius $E p$ inueniantur, adeogue in hoc casu numerum $E$ tamquam excludentem applicari, non posse. Huirismodi excludentes, quot placet, adhiberi, 


\section{$-562-$}

atque sic numeri in $\Omega$ ad lubitum diminui pos sunt.

Videamus iam, aninon etiam numeros primos ipsum $m$ metientes, táliumue numerorum potestates tamquam excludentes adhibere liceat Sit $B$ valor expr. $\frac{A}{n}$ (mod $m$, patetque, $V$ sempex ipsi $B$ secundum mod. $m$ congruum fieri, quicunque valor pro $x$ accipiatur, adeoque ad possibilitatem aequ prop. necessario requiri, vt $B$ sit residtrum quadraticum ipsius $m$. Designante itaque $p$ diuisorem quencunque primum imparem ipsius $m$, qui per hyp. ipsos $n$ et $A$, adeoque etiam ipsum $B$ non metietur, pro valore. quocunque ipsius $x$ erit $V$ residuum ipsius $p$ adeoque etiam cutuscunque potestatis ipsius $p$; quamobrem $p$ ipsiusque potestates nequeunt excludentium loco haberi - Prorsus simili ratione quando $m$ per 8 est divisibilis ad, aequ prop. possibilitatem necessario requiritur vt sit $B \equiv \mathrm{x}$ (mod. 8 ), wride etiam $V$ pro valore quocunque ipsius $x$ fiet $\equiv 1$ (mod. 8 ), et proin binarii potestates ad exclusionern non idoneae. - Quando autem $m$ per 4 neque vero per 8 est diuisibilis, ex simili ratione esse debebit $B \equiv 1$ (mod. 4), adeoque valor expr. $\frac{A}{n}(\bmod 8)$ vel 1 vel 5 designetur per $C_{\text {. }}$ Nullo negotio perspicietur, pro valore pari ipsius $x$ hic fieri $\not \partial \equiv C_{\text {; }}$ pro impari, $P \equiv C+4$ (mod 8 , vade patet, valores pares reiiciendos esse, quando $C=5$; impares, quando $C=1$. Denique quando $m$ per 2 , neque vero per 4 est diuisibilis, sit wt an- 


\section{$-563-$}

te $C$ valor expr. $\frac{A}{n}(\bmod .8)$, qui erit $1,3,5$, vel 7 ; atque $D$ valor huirus $\frac{\frac{T}{2} m}{n}(\bmod , 4)$, qui erit 1 vel 3 . Iam quum valor ipsius $V$ manifesto semper fiat $\equiv C-2 D x x$ (mod. 8), adeoque pro $x$ pari $\equiv C$, pro impari $\equiv C-$ $2 D$, facile hinc colligitur, reiiciendos esse omnes valores impares ipsius $x$, quando $C=1$; omnes pares, quaindo $C=3$ et $D=1$, aut $C$ $=7$ et $D=3$, atque valores remanentes omnes producere $\nu \equiv 1(\bmod .8)$ siue residuum cuiusuis potestatis binarii; in casibus reliquis autem, puta quando $C=5$, aut $C=3$ et $D=5$, aut $C=7$ et $D=1$, fiet $V \equiv 3,5$ vel 7 (mod. 8), siue $x$ accipiatur par siue impar, vnde liquet, in his casibus aequationem prop. solutionem omnino non admittere.

Ceterum quim prorsus simili modo, vi hic valorem ipsius $x$ per exclusiones inuenire docuimus, etiam, mutatis mutandis, valorem ipsius $y$ elicere possimus, methodum exclusionis ad problematis propositi solutionem duobus semper modis applicare licebit (nisi $m=n=1$, vbi coincidunt), e quibus si plerimque est praeferendus, pro quo $\Omega$ terminorum multitudinem minorem continet, quod facile a priori aestimari poterit. - Denique vix necesse erit obseruare, si post aliquot exclusiones omnes numeri ex $\Omega$ abierint, hoc vt certum indicium impossibilitatis aequationis propositae esse considerandum.

325. Ex. Proposita sit aequatio $3 x x+$ $455 y y=10857368$, quam duplici modo solue$\mathrm{Na}$ 


\section{$-504-$}

mus, primo inuestigando valores iqkitus $a$, deixt valores ipsius $y$. Limes illorum in hor cast wst $\sqrt{3619120} \frac{2}{3}$, qui cadit inter 1902 et 1903 : valor expr. $\frac{A}{3}(\bmod .455)$ est 354 atcue valores expr. $\sqrt{354}(\bmod 455)$ hi $\pm 82,+152, \pm 175$ \pm 212 . Hinc $\Omega$ conklit e 53 numuris sequentibus: $82,152,173,212,247,282,30 \%, 3734$ $557,607,628,667,698,737,758,824,924$, $1062,1083,2122,1155,1192,1213,1283$, $1447,1517,1538,1577,1608,1677,1668$, 1738 , 1902. Numerus 3 in hor" cossa ad exclusionem adhiberi nequit, quia ipssm $n$ unetitur. Pro excludente 4 , habemus $a=a, b=3$, onde

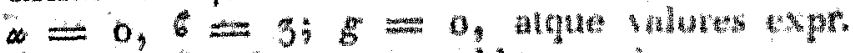
$\sqrt{8}\left(\bmod _{4} 4\right)$ hos o at 2 ; binc sequitur, ombers

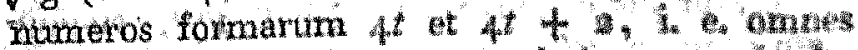
pares ex $\Omega$ eiiciendos esse; designentur (sedito

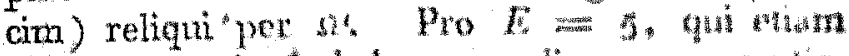
ipsum $n$ metiut, habemus raflices congusuenta

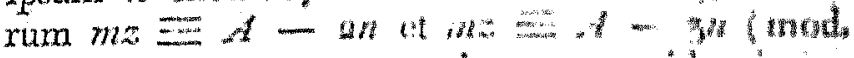

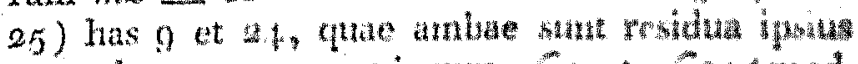
25 , valoresque expressionum $\sqrt{9}$ et $\sqrt{ }$ at 4 (mod. 25) fiunt $\pm 3, \pm 73$ eiectis ex 5 ondibu mu meris formarum $25 t \pm 3,05 t+7$ restats hi decem (2.24): $173,573,537,667,75 \%, 1063$

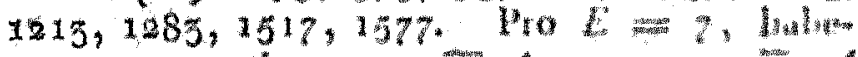

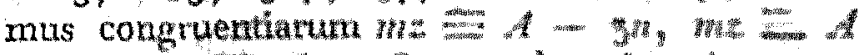
$-5 n, m z \equiv A-6 n$ (mod. 4n) rat cor $3 a_{3}$ 39,18 , quae omne sunt residua ipsiass 14 , do que valores expr. $\sqrt{3}, \sqrt{39}, \sqrt{18}(\mathrm{~mm}$, 49)

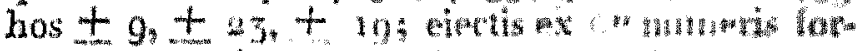

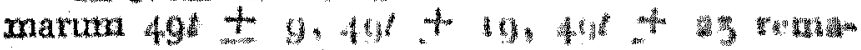
nent hi quinque ( 


\section{- $565-$}

1517. Pro $E=8$ habemus $a=5$, vnde $\alpha=$ 5 , qui est non residuum ipsius 8 ; quare excludens 8 non potest adhiberi. Numerus 9 ex eadem ratione praetereundus est vt 3 . Pro $E=$ 11 numeri $a, b$ etc. fiunt $2,6,7,8,10 ; 2=$ 0 ; rnde numeri $a_{2} b$ etc. $=8,10,5,0,1$, e quibus tres sunt residua ipsius il puta $0, x, 5$; hinc deducitur, ex $\Omega$ 'l! reiiciendos esse numeros formarum $11 t, 11 t \pm 1,11 t \pm 4$, quo facto remanent $537,1083,1213$. Quos teritando prodeunt pro $V$ resp. valores $21961,16+29,14.16 x$, - quibus secundus ac tertius soli sunt quadrata. Quare aequ. prop. duas solutiones per valores positixos ipsorum $x, y$ admittit, $x=1083, y$ $=127$, et $x=1213, y=x 9$.

Ir. Si alteram eiusdem aequationis incognitam per exclusiones indagare placet, ponatur haec sub formam $455 x x+7 y y=0857362$, commutando $x$ cum $y$, vt omnia signa artt. 323 , 324 retinere liceat. Times valorum ipsius $x$ hic cadit inter 154 et 155 ; valor expr. $\frac{A}{n}$ (mod. $n$ ) est 1 ; valores buius $\sqrt{1}(\bmod , 3)$ sunt +1 et - 1. Quare $\Omega$ continet omnes numeros farmanum $3 t+1$ et $3 t-1$, i. e. omnes per 3 non diuavibiles vsque ad $15 \%$ incl, quorum multitudo est 103 ; applicando autem praecepta supra data inuenitur 
pro excl. reiiciendos esse numeros formarum

\begin{tabular}{l|l}
3 & $9 t+4$ \\
4 & $4 t, 4 t+2$ siue omnes pares \\
9 & $27 t+1,27 t+10$ \\
11 & $11 t, 11 t+1,11 t+5$ \\
17 & $17 t+3,17 t+4,17 t+5,17 t+7$ \\
19 & $19 t+2,19 t \pm 3,19 t+8,19 t+9$ \\
23 & $23 t, 23 t+5,23 t+7,23 t+9,23 t+10$
\end{tabular}

Wis deletis superstites inueniuntur II $9, I_{27}, 157$, e quibus duo priores soli ipsi $V$ valorem quadratum conciliant, easdemque solutiones suggerunt, ad quas supra peruenimus.

326. Methodus praecedens iam per se tam expedita est, vt vix quidquam optandum relinquat, attamen per multifaria artificia magnopere adhuc contrahi potest, e quibus hic pauca tantum attingere licet. Restringemus itaque disquisitionem ad, eum casim, vbi excludens est numerus primus impar insum $A$ non metiens, siue talis primi potestas, praesertim quoniam casus reliqui vel ad hunc reduci vel methodo analoga tractari possunt. Supponendo primo, excludentem $E=p$ esse numerum primum ipsos $m, n$ non metientem, atque valores expr. $\frac{A}{m},-\frac{a}{m}$, $-\frac{n b}{m}-\frac{n c}{m}$ etc (mad. p) resp. $k, \mathcal{X}, \mathfrak{B}$, (E) etc.: numeri $\propto, \varphi, \gamma$ ete imueniuntur per congruentias $=k+2,6=k+2,2=$ $\mathcal{k}+(5$ etc $(\bmod p)$ Numeri $\mathfrak{A}, \mathfrak{B}, 6$ ete autern per artificium ei prorsus simile quo in art. 322 vsi sumus sine congruentiamu computatio- 


\section{$-5^{6} 7-$}

ne erui possunt, et vel cum omnibus non-residuis, vel cum omnibus residuis ipsius $p$ (praeter o) conuenient, prout valor expr. - $\frac{m}{n}$ (mod. $\left.p\right)$, siue (quod hic eodem redit) numerus $-m n$ est residuum vel non residuum ipsius $p$. Ita in ex. II art. praec. pro $E=17$ fit $k=7$; $m n=-{ }^{3} 6_{6} 6_{5} \equiv \mathrm{I}_{2}$ est non residuum ipsius I7; hino numeri $\mathfrak{x}, \mathfrak{B}$ etc. erunt $\mathrm{x}, 2,4,8,9$, $I 3$, I 5, I 6 adeoque numeri $\alpha, 6$ etc. 8,9 , IT, I5, I6, $3,5,6$; ex his rẹsidua sunt 8,9, I5, $\mathrm{x} 6$, vnde $\pm h, h^{\prime}$ etc. fiunt $\pm 5,3,7,4-$ Quibus saepius occasio est huinsmodi problemata soluendi, commoditati suae eximio consulent, si pro pluribus numeris primis $p$, valores ipsorum $h, h^{\prime}$ etc. singulis valoribus ipsorum $k,(\mathrm{I}, 2$, $3 \ldots p-$ I ) responderites, in duplici suppositione (puta vbi $-m n$ est residuum et vbi non-residuuin ipsius $p$ ) computent. Ceterum obseruamus adhuc, multitudinem numerorum $h,-h, h^{\prime}$ etc. semper esse $\frac{x}{2}(p-r)$, quando vterque numerus $k$ et $m n$ sit residuum vel vterque non resilluum tpsitus $p ; \frac{x}{2}(p-3)$, quando prior $R$, posterior $N . R$; $\frac{x}{2}(p+\mathrm{x})$, quando prior $N R$, posterior $R$; sed demonstrationem huius theorematis ne nimis prolixi fiamus supprimere debemus.

Quod autem, secundo, eos casus attinet, whi $E$ est numerus primus ipsum $n$ metiens, aut potestas numeri primi (imparis) ipsum $n$ metientis seu non metientis, hi adhuo expeditius tractari possunt. Ommes hos casus simul complectemur, omnibusqus art. 524 signis retentis ponemus $n=n^{\prime} p^{\prime}$, ita wt $n^{\prime}$ per $p$ non sit di$\operatorname{Nn} 4$ 


\section{- $568-$}

uisisibilis. Numeri $a, b, c$ etc erunt producta numeri $p^{\mu-x}$ vel in omnes numeros ipso $p$ minores (prater 0 ), vel in amnia non residua ipsius $p$ infra $p$, prout $\mu$ est par vel fmpar; exprimantur indefinite per up $p^{\mu-x}$. Sit $k$ valor expro $\frac{A}{m}\left(\bmod p^{a+1}\right)$ ). eritque per $p$ non diuisibilis, quia eadem proprietás in $A$ supponitur; porro patet, omnes $\alpha, \vec{b}, \gamma$ etc. ipsi $k$ sec. mod, $p$ congxuos fieri, adeoque $p^{\dot{a}}$ nihil ex $\Omega$ excludere, si $k N p ;$ si vero $k R p$ adeoque etiam $k R p^{\mu}+$, sit $r$ valor expr. $\sqrt{ } k\left(\bmod . p^{\mu+1}\right)$, qui per $p$ non erit diuisibilis, atque e valor huius $-\frac{n !}{2 m r}$ (mod. $p$ ), eritque $\alpha \equiv r r+$ serap (mod. $\left.p^{4+}\right)$, vnde facile colligitur, „ esse residoum ipsius $p^{\mu+\gamma}$, atque valores expr. $\sqrt{ }$. $\left(\bmod p^{\mu+}+r\right)$ fieri $\pm\left(r+e a p^{\mu}\right)_{j}$ hinc omnes $h, h ! h^{\prime \prime}$ etc. exprimentur per $r+$ $e p^{a+r-r}$. Denique nullo negotio hinc concluditur, numeros $h ; h^{\prime}, h^{\prime \prime}$ etc. oriri ex additione numeri $r$ cum productis numeri $\dot{p}^{u+r-x}$ vel in orimes numeros infia $p$ (praeter $o$ ), puta quanda $\mu$ par; vel in omnia non residua ipsius $p$ infra hunc limitem, quando $\mu$ impar atque $e R p_{s}$ siue quod hic eodem redit quando $-2 m r n ' R p$; vel in omnia residua (praeter o), quando $\mu$ impar atgre $-2 m r n^{\prime}+\mathrm{Np}$.

Cetemm simulac pro singulis excludentibus, quos applicare placet, numeri $h, h^{\prime}$ etc. sunt eruti, exclusionem ipsam etiam per operationes mechanicas perficere licebit, quales quisque barum rerum peritus facile proprio marte excogitare poterit, si operae pretium esse videbitur. 
Tandem obseruare debermus, quamuis aequationem $a x x+2 b x y+c y y \doteq M$, in qua $b b-a c$ negatiuus $=-D$, facile ad eam formam quam in praecc consideraumus reduci posse. Designando enim diuisorem communem maximum numerorum $a, b$ per $m$, et ponendo $a=$ $m a^{\prime}, b=m b^{\prime}, \frac{D}{m}=a^{\prime} c-m b^{\prime} b=n, a^{\prime} x+b^{\prime} y=$ $x^{\prime}$, aequ. illa manifesto aequiualet huic $m x^{\prime} x^{\prime}+n y y$ $=a^{\prime} M$, quae per praecepta supra tradita solui poterit. Ex huius autem solutionibus ea tantum erunt retinendae, in quibus $x^{\prime}-b y$ per $a^{\prime}$ fit diuisibilis, siue vnde $x$ valores integros nanciscitur.

327. Quemadmodum solutio directa aequationis $a x x+2 b x y+c y y=M$ in sect. $V$ con tenta valores expr. $\sqrt{(b b-a c)}(\bmod M)$ notos supponit, ita vice versa pro ea casu, vbi $b b$ - ac est negatiuus, solutio indirecta in praecc. exposita methơdum expeditissimam subministrat, illos valores eruendi, quae, praesertim pro valare permagno ipsius $M$, methodo art. 322 sqq. longe est praeferenda. Supponemus autem, $M$ esse numerum primum, aut saltem ipsius factores, si compositus esset, adhuc incognitos; si enim constaret, numerum primum $p$ ipsum $M$ metiri, atque esse $M=p^{\prime \prime} M$, ita vt $M$ factorem $p$ non amplius implicet, longe commodius foret, valores expr, $\sqrt{ }(b b-a c)$ pro modulis $p^{u}$ et $M$ sigillatim explorare (priores ex valoribus secundum modulum $p$, art. I o I), valoresque see mod. $M$ ex homm combinatione deducere (art 105 ). 
Quaerendi sint itaque omnes valores expr. $\sqrt{ }-D(\bmod \cdot M)$, whi $D$ et $M$ positiui stypansututur, atque $M$ sub forma dinisorum ipsins $+D$ contentus (art. I f 7 sqy.), aliognin enim a priori constaret, nullos numzeros expressisuni propositae satisfacere posse. Sint valores quarsiti, quibus bini semper oppositi erunt, $\pm r, \pm$, $+r^{\prime \prime}$ etc., atque $D+r=M h, D+r^{*}=$ $M h^{\prime}, D+r^{\prime \prime} r^{\prime \prime}=M h^{\prime \prime}$ etc; porro designewntur classes ad quas formae $(M, r, h),(M,-r$, $h),\left(M, r^{\prime}, h\right),(M,-r, h),\left(M, m_{n}, h\right)$ $\left(M,-r^{\prime \prime}, h\right)$ etc, pertinent, resp. per $\mathfrak{C},-$, $\mathbb{E}^{\prime},-\mathfrak{E}^{\prime}, \mathbb{E}^{\prime \prime},-\mathbb{E}^{\prime \prime}$ etr., ipsaramque romplyxus per B. Hae classes quidem, generaliter low quendo, tamquam incognitae sunt spectansuas ;

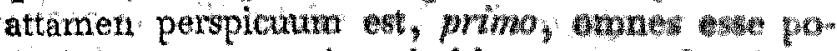
sitivas atque proprie primitivas, securndo omnes ad idem genus pertinere, tuius characters ox ittdole numeri $M$, i. e ex ipsius rolationtusas ad

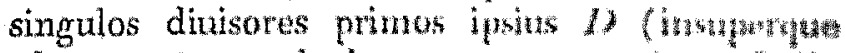
ad 4 aut 8 quando hae sunt newessarian's latile cognosti possit (art. 230: Qmam stuppositura

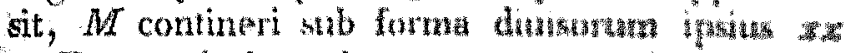
$+D$, a priori certi esse possumats, haic eharacteri necessario genus pos. pr. pr. formuarum determ $-D$ respondere, etiamsi forsan expresas oni $\sqrt{ }-D$ (mod. $M)$ saticferi nequeat; quuus itaque hoc genus sit noturn, ommes clastevel fir

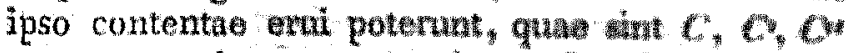
etc., atque ipsarum complexus $G$. Patat igitsur, singulas classes $5,-6$ to carm aliqua clasese in $G$ tdenticas esse debere; fieri potest quaguse, vt pluxes classes in 6 inter se, adeoeque cum detn in $G$ ideaticae sint, et catuado $G$ vaicato 


\section{$-\quad 571$}

classem continet, certo omnes in $\&$ cum hac conuenient. Quare si e classibus $C, C^{\prime}, C^{\prime \prime}$ etcformae (simplisşimae) $f, f^{\prime}, f^{\prime \prime}$ etc. eliguntur, (vna e singulis): e singulis classibus in $\mathfrak{G}$ vaa forma inter has reperietur. Iam si $a x x+2 b x y$ $+c y y$ est forma in classe 5 contenta, dabuntur duae repraesentationes numeri $M$ per ipsam ad valorem $r$ pertinentes, et si vna est $x=m_{m}$ $\gamma=n$, altera enit $x=-m, y=-n$; vnicus casus excipi debet, vbi $D=x$, in quo quatuor repraesentationes dabuntur (v. art 180).

Ex his colligitur, si omnes repraesentationes numeri $M$ per singulas formas $f, f^{\prime}$, fu etc. in-. uestigentur (per methodum indirectam in praece traditam), atque hinc valores expr. $\sqrt{ }-D$ (mod. $M$ ) ad quos singulae pertinent deducantux (art. 15* sqq), omnes valores huius expressionis inde obtineri, et quidem singulos bis, aut; si $\mathcal{D}=1$, quater. $Q$. $E . F$. Si quae formae inter $f, f$ etc reperiuntur, per quas $M$ reprae. sentari nequit, hoc est indicium, ipsas, ad nullam classem in (s) pertinere, adeoque negligendas esse: si vero $M$ per nullam illarum formarum repraesentari potest, necessario $-D$ debebit esse non residuum quadraticum ipsius $M$. Circa has operationes teneantur adlux obseruationes sequentes.

I. Repraesentationes numeri $\boldsymbol{M}$ pex formas $f$, $f$ etc, quas hic adhibemus, subintelliguntur esse tales, in quibus indeterminataxum valores inter se primi sunt; si quae aliae se offerunt, in quibus hi valores diuisorem commuaem $\mu$ has 


\section{- $572-$}

bent /quod tunc tantummodo accidere potest, ybi $\mu$ metitur ipsum $M$, certoque accidet, quanda $-D \mathrm{R}_{\mu \mu}^{M}$ ) hae: ad institutum praesens omnino négligi debent, etsi alio respectu vtiles esse pos* sint.

II. Ceteris paribus labor manifesto eo faciLior erit, quo minor est multitudo classium $f, f^{\prime}$, $f^{\prime \prime}$ etc.; adeoque breuissimus, quando $D$ est vnus e 65 numeris in art. 303. traditis, pro quibus in singuís generibus trica tantum classis dátur.

III. Quum binae semper huiusmodi reprae. sentationes $x=m, y=n ; x=-m, y=$ - $n$ ad eunden valorem pertineant, perspicuum est, sufficere, si eae tantumimodo repraesentationes considerentur, in quibus $y$ positiuus. Tales taque repraesentationes diuersae semper valoribus diuersis expr. $\sqrt{ }-D(\bmod M)$ respondent, vade multitudo omnium valorum diuersum mulțiudini omnium talium repraesentationum prodeuntium aequalis erit (semper excipiendo casum $D=1$, vbi illa huis-semissis prit),

IV. Quoniam, simulac alter duorum valoirum oppositorum $+r_{2}-r$, cognitus est, alter sponte innotescit, operationes adhuc aliquantum abbreuiari possunt; si valer $r$ obtinetur e repraesentatione numeri $M$ per fornam in classe $C$ contentam, i, e. si $c=C_{i}$ valor oppositus $-r$ manifesto emerget e repraesentatione per formam, in classe ipsi $C$ opposita contentam, 


\section{- $573-$}

qua differens erit a classe $C$, nisi haec est anceps. Hinc sequitur, quando non orrines classes in $G$ ancipites sint, e reliquis semissem tantum considerare oportere, puta e binis oppositis quibusque vnam; alteram negligendo, e qua valores iis, quos prior suppeditauit, oppositos resultare iam absque calculo praeuidere licet. Quando autem $C$ est anceps, ambo valores $r$ et $-r$ si- $^{\text {in }}$ mul inde emergent; puta, si ex $C$ classis anceps $a x x+2 b x y+c y y$ electa est, atque valor $r$ prodiit e repr $x=m, y=n$, valor $-r$ prodibit ex hac $x=-m-\frac{2 b n}{n}, y=m_{0}$

V. Pro eo casu vbi $D=1$, vnà tántun classis omnino datur, e qua formam $x x+y y$ electam esse supponere licebit. Quodsi valor $r$ ex repraesentatione $x=m, y=n$ prouenit idem ex his prodibit $x=-m, y=-n$; $x=n, y=-m, x=-n, y=m, o p$ positusque $-r$ ex his $x=m, y=-n ; x$ $-m_{3} y=n ; x=n_{5} y=m ; x=-n, y$ $=-m ;$ quare ex his acto reprr., quae viicam discerpptionem coristituturit, vna sufficit, si modo valori inde resultanti oppositum associex mits:

VI. Valor expr. $\sqrt{ }-D(\bmod M)$, ad quem repr: haec $M=a m m+2 b m n+c h n$ pertinet, per art 155 est $(m b+n c)-\nu(m a+$ $n b$ ) siue numerus quicunqute have secundum $M$ congruus; ipsis $\mu$, v ita acceptis vit fiat $\mu m+v r$ - 1. Designando itague talem valorem per rit $m w \equiv m(m b+n c)-(M-m n b-$ 


\section{- 574}

$z m c) \equiv(\mu n++m)(m b+n c) \equiv m b+n c$ (mod. $M$ ). Hinc patet, $v$ esse valorem expr. $\frac{m b+n c}{m}(\bmod M)$, similique modo inuenitur, $v$ esse valorem expr. $-\frac{m a+n b}{n}(\bmod . M)$. Hae formulae saepenumero ei ex qua deductae fuerunt praeferendae sunt.

328. Exempla. I. Quaeruntur omnes valores expr. $\sqrt{ }-1365(\bmod 5428681=M)$; numerus $M$ hic est $\equiv 1,1,1,6,1$ (mod. $4,3,5,7,15)$ adeoque sub forma diuisorum ipsorum $x x+1, x x+3, x x-5$, et sub forma non diuisorum 1 psorum $x x+7, x x-13$, et proin sab forma diüsorum ipsius $x x+13^{65}$ contentus characterque generis in quo classes \&S reperientur erit 1,$4 ; R_{3} ; R_{5} ; N_{7} ; N_{13}$. In hoc genere vnica classis continetur, e "qua eligimus formam $6 x x+6 x y+229 y y$; vt omnes repraesentationes numeri $M$ per hanc inueniantur, ponemus $2 x+y=x !$, vnde fieri debebit $3 x^{2} x^{\prime}+455 y y=2 M$. Haec aequatio quatuor solutiones admittit in quibus $y$ est positiuus, puta $y=127, x^{\prime}= \pm 1085, y=119, x^{y}=$ \pm 1213. Hinc prodeunt quatuor solutiones aequ. $6 x x+6 x y+229 y y=\pi$, in quibus $y$ positituutús,

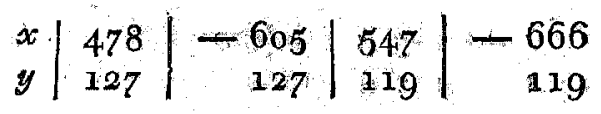

Solutio prima dat pro $v$ valorem expr. $\frac{30517}{478}$ sit 
tae $-\frac{3249}{127}(\bmod . M)$, vnde inuenitur 2350978 , secunda producit valorem oppositum - 2350978 ; tertia hunc 2600262 , quarta oppositum 2600262

II. Si quaerendi sunt valores expr. $\sqrt{-}$ $286(\bmod .4272943=M)$, character generis in quo classes $\mathcal{B}$ contentae sunt, inuenitur 1 et 7,$8 ; R_{11} ; R_{13} ;$ quare erit genus principale $;$ in quo tres classes continentur, per formas ( 1,0 , $286),(14,6,23),(14,-6,23)$ exhibitae; ex his tertiam, vtpote secundae oppositam negligere licet. Per formam $x x+286 y y$ duae re praesentationes numeri $M$ inueniuntur, in quibus $y$. positiuus, puta $y=103, x= \pm 111$; vnde prodeunt valores expr. propositae hi 1495445, - 1493445. Per formam (14, 6, 23) autem $M$ non repraesentabilis inuenitur, vnde concluditur, praeter duos valores inuentos alios non dari.

III. Proposita expr. $\sqrt{ }-70$ (mod 9.97331), classes \& contentae esse debebunt in genere cuius character 3 et $5.28 ; R_{5} ; N_{7}$; in hoc vnica classis reperitur cuius forma repraesentans haec $(5,0,14)$. At calculo instituto inuenitur, numerum 997331 per formam $(5,0$, 14) non esse repraesentabilem, quamobrem 70 necessario erit non residuum qu, illius numeri.

329. Problema, numeros primos a compositis dignascendi, hosque in factores suos primos resoluendi, ad grauissima ac vilissima toti- 
us arithmeticae pertinere, et geometrarum tum veterum tum recentiorum industriam ac sagacitatem occupauisse, tam notum est, vt de hac re copiose loqui superflum foret. Nihilominus fateri oportet, omnes methodos hucusque prolatas vel ad casus valde speciales restrictas esse, vel tam operosas et prolixas, vt iam pro numeris talibus, qui tabularum a viris meritis constructarum limites non excedunt, i. e. pro quibus. methodi artificiales superuacuae sunt, calculatoris etiam exercitati patientiam fatigent, ad maiores autem plerumque vix applicari possint. Etsi vero illae tabulae, quae in omnium manibus versantur, et quas subinde adhue vlterius contimuatum ir sperare licet, in plerisque casibus vulgo occtrrentibus vtique sufficiant: tamen calculatori perito occasio haud raro se offert, e numerorum magnorum resolutione in factores magna emolumenta capiendi, quae temporis dispendium inediocre largiter compensent; praetereaque scientiae dignitas requirere videtur, vt omnia subsidia ad solutionem problematis tam elegantis ac celebris sedulo excolantur. Propter has rationes non dubitamus, quin duae methodi sequentes, quarum efficaciam ac breuitatem longa experientia confirmare possumus, arithmeticae amatoribus haud ingratae sint futurae. Ceterum in problematis natura fundatum est, vt methodi quaecanque continuo prolixiores euadant, quo maiores sunt numert ad quos applicantur; ațtamen pro methodis sequentibus difficultates perlente increscunt, numerique e septem, octo vel adeo adhuc plaribus figuris constantes praesertim per secundam felici semper successu tra 
ctati fuerunt, omnique celeritate, quam pro tantis numeris exspectare aequum est, qui secundum omnes methodos hactenus notas laborem, etiam calculatori indefatigabili intolerabilem, requirerent:

Antequam methodi sequentes in rsum vocentur, semper vtilissimum est, diuisionem numeri cuiusque propositi per aliquot numeros primos minimos tentare, puta per $2,3,5,7$ etc. vsque ad ig aut adhuc vlterius, non solum, ne poeniteat, talem numerum quando diuisor est per methodos subtiles ac artificiosas eruisse, quî multo facilius per solam divisionem inueniri po* tuisset*), sed etiam, quod tunc, vbi nulla diuisio successit, applicatio methodi secundae residuis ex illis diuisionibus ortis gagno cum fructu vtitur. Ita e. g. si numeras $314159^{2} 6_{5}$ in factores suos resoluendus est, diuisio per $z$ bis succedit, posteaque etiam diuisiones per 5 et 7 , vude habetur $314159265=9 \cdot 5 \cdot 7.997351$, sufficitque bumerum $99 \% 351$, qui per 11, 13, 17, 19 non diuisiblis inuenitur, examini subtilio ri subiicere. Similiter proposito numero 43429448 , factorem 8. aaferemus, methodosque magis artificiales ad quotientem 5428681 applicabimus.

330. Fundamentum Merhod parmat est theorema, quemuis numerum pasitium seu negatium, qui alius numeri $M$ residum quadraticum sit, etian residum cuitusuts dituisoris

-) Eo magie, quod inter sex moneros, generaliter loquendo, Tix vmus per omnes $2,3,5 \ldots$ Ig zon diuisibilis rogeritur, 
ipsizs $M$ esse. Vulgo notum est, si $M$ per nullum numerum primum infra $\sqrt{ } M$ diuisibilis sit, certo $M$ esse primum; si vero omnes numeri primi infra hunc limitem, ipsum $M$ metientes sint $p, q$ etc. numerum $M$ vel ex his sulis (ipsorumue potestatibus) compositum esse, vel vnum tantum alium factorem primum maiorem quam $\sqrt{ } M$ implicare posse, quá inuenitur, diuidendo ipsum $M$ per $p, q$ etc. quaties licet. Designando itaque complexum omnium numerorum primorum infra $\sqrt{ } M$ (exclusis is, per quos diuisio frustra iam tentata est) per 4 , manifesto sufficit, si omnes diuisores primi iusius $M$, in $a$ contenti, habeantur. Iam si alicunde constat, numerum aliquem $r$ (nom-quadratum) esse residuuno quadraticum ipsius $M$, nullas certo numerus primus cuius $N R$. $r$ dinisor ipsius $M$ esse poterit; quare ex $\Omega$ omnes huiusmodi numbers primos (qui plerumque omnium semissem fero efficient) eiicere licebit. Si insuper de alio num mero non quadrato, $r$, constat, i ipsum esse rem siduum ipsus $M$, e numeris primis in $\Omega$ post priman exclusionem relictis iterum eos excludere poterimus, quorum $N . R$ est $r^{*}$, qui rursus illoram semissem fere conficient, siquidem residua $r$ et $r_{\text {, }}$ sunt independentia, (i. e. nis alterum necessario per se est residuum omnium numerorum, quorum residuum est alterum, quod eueniret quando $r^{\prime}$ esset quadratam). Si adhue alia residua ipsius $M$ noti sunt, ${ }^{\mu}$, put etc, quae ornnia reliquis sunt independentia *, cum singulis ex-

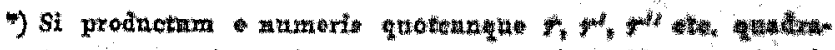

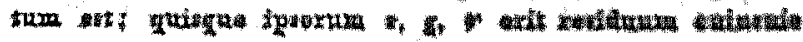


chusiones similes institui possunt, per quas multitudo numerorum in $\Omega$ rapidissime diminuetur, ita vt mox vel omnes deleti sint, in quo casu $M$ certo erit numerus primms, vel tam pauci restent (inter quos, tmines diuisores primi ipsius $M$, si quos habet, manifesto reperientur), vt diuisio per ipsos nullo negotio tentari possit. Pro numero millionem non multum superante plerumque sex aut septem; pro numero ex octo aut nouem figuris constante, nouem aut decem exclusiones abunde sufficient. Duo iam sunt de quibus agere oportebit, primo quomodo residua ipsius $M$ idonea et satis multa inumiri possint, deinde quo pacto exclusionem ipsam commodissime perficere liceat. Sed ordinem harum quaestionum inuertemus, praesertin quoniam secunda docebit, qualia potissimum residua ad hunc finem sint commoda.

531. Numeros primos quorum residuum est numerus datus $r$ (quem per nullum quadratum diuisibilem supponere licet $)$; $a b$ fis quorum non residuum est, sine diuisores expr. $x x-r$ a non diuisoribus distinguere, in sect. IV copiose docuimus, omnes priores sub certis huiusmodi formulis $r z+a, r z+b$ etc, aut talibus $4 r z+a$, $4 r z+b$ etc. contentos esse, posterioresque sub aliis similibus, Quoties $r$ est numerus valde paruus, exclusiones adiumento harum formularum percommode perfici possunt; e. g. excludendi erunt

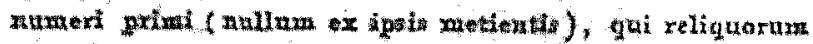

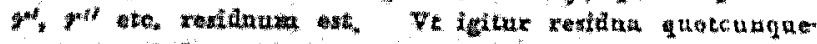

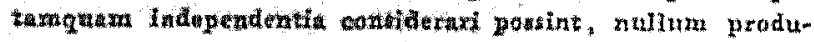

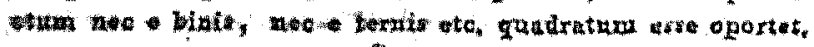




\section{- $580-$}

omnes numeri formae $4 z+3$, quando $r=$ -1 ; omnes numeri formarum $8 z+3$ et $8 z$ +5, quando $z=2$ etc. Sed quum non semper in potestate sit, huiusmodi residua numeri propositi $M$ inuenire, neque formularum applicatio pre valore magno, ipsius $r$ satis commoda sit, ingens lucrum est, laboremque exclusionis mirifice subleuat, si pro multitudine satis magna numerorum . $(r)$ per quadratum non diuisibilium tum positiuorum tum negatiuorum tabula jam constructa habetur, in qua numeri primi quorum residua sunt illi singuli $(r)$ ab iis quorum non residua sunt distinguuntur. Talis tabula perinde adornari poterit ac specimen ad calcem huius operis adiectum suppraque iam descriptum; sed vt ad institufum praesens vtilitatem satis amplam praestet, numeri primi in margine positi (moduli) longe vlterius puta saltem vsque ad 1000 aut ad 10000 continuati esse debeint, praetereaque commoditas multum augetur, si in facie etiam numeri compositi et negatiui recipiuntur, etsi hoc non sit absolute necessarium, vt e sect. IV perspicuum est. $\Delta d$ summun autem commoditatis fastigium vsús talis tabulae euehetur, si singulae columellae verticales e quibus constat exsecantur lamellisque ant baculis ( $\mathrm{Ne}-$ perianis similibus) agglutinantur, ita vt eae quae in quouis casu sunt necessariae i. e. quae numeris $r, r^{\prime}, r^{\prime \prime}$ etc., residuis numeri propositi in factores resoluendi, respondent, separate examinari pqssint. Quibus iuxta tabulae colnmnam primam (quae modulos exhibet) rite positis, i. e. ita, vt loca singulorum baculorum eiderm numero primo columnae primae respondentium 
cum hoc in directum iaceant, siue in eadem li nea horizontali siti sint: manifesto ei numeri primi, qui post exclusiones cum residuis $r, r^{\prime}, r^{\prime \prime}$ ex $\Omega$ remanent, per solam inspectionem immediate cognosci poterunt $;$ nimirum hi conuenient cum iis in columna prima, quibus in onnibus baculis, adiacentibus lineolae respondent, reícique debent omnes, quibus in vllo bacillo spatium vacuum adiacet. Per exemplum haec sufficienter illustrabuntur, Si alicunde constat, numeros $-6,+13,-14,+17,+37,-53$ esse residua ipsius $99733^{1}$, consociandae erunt columna prima (quae in hoo casu vsque ad 997 continuata esse debet, i. e. rsque ad numerum primum proxime minorem quam $\left(99733^{1}\right.$ ) atque lamellae, in quarum facie numeri -6 , + 13 etc. sunt suprascripti. Ecce partem schematis hoc modo prodeuntis:

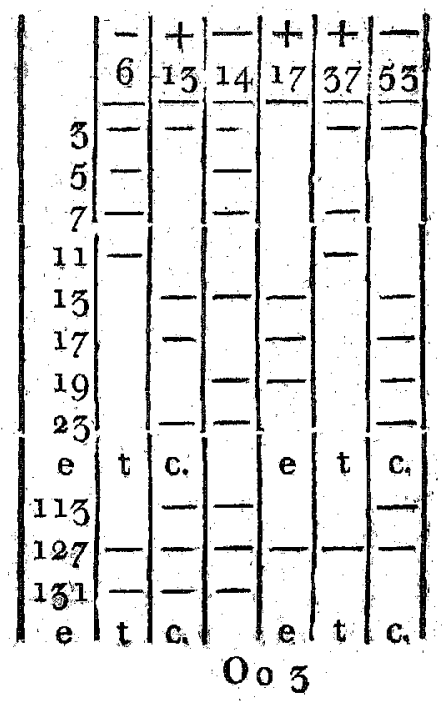


Quemadmodum hic ex sola inspectione cognoscitur, ex is numeris primis qui in hac schematis parte continentur solum 127 post exclusiones cum residuis $-6,13$ etc. in $\Omega$ relinqui, ita schema integrum vsque ad 997 extensum ostendit, omnino nullum allium ex $\Omega$ remanere; diuisione autem tentata, $99733^{1}$ per 127 reuera diuisibilis inuenitur. Hoc itaque modo ille numerus in factores primos $127 \times 7853$ resolutus habetur *).

Ceterum ex hac expositone abunde colligitur, praesertim vtilia esse residua non nimis magna, aut saltem in factores primos non nimis magnos resolubilia, quum tabulae auxiliaris vsus immediatus non vltra numeros in facie positos pateat, vsusque mediatus tales tantum complectatur, qui in factores in tabula contentos resolui possunt.

352. Ad inuenienda residua numeri dati $M$ tres methodos diuersas trademus, quarum expositioni duas obseruationes praemittimus, quarum adiumento e residuis, ninus idoneis simpliciora deriuari possint. Primo, si numerus akk per quadratum $k k$ diuisibilis (quod ad $M$ primum esse supponitur) est residuum ipsius $M$, etiam a erit residuum; propter hanc rationem residua

- Auctar paratan satis amplum tabulae hic escriptae, quem ad rsum suuv construondum curauit, publici iurio lubentex faceret, si paucitas eorum, quibins rsui esse potest, suptibus talis incepti sustentandis sufficeret. Si quis interea arithmetical atorator, principiis probe penetratis, proprio maxte talem tabulam sibi condere opta atictoz magrae roluptati sibi lucet, ornilia cum ed emolnment co artificie per literas communicare. 
per magna quadrata diuisibilia aeque vilia sumt ac parua; omniaque residua per methodos sequentes suppeditata, a factoribus suis quadratis statim liberata supponemus. Secundo si duo pluresue numeri sunt residua, etiam productum ex ipsis residutum erit. Combinando hanc obseruationem cum praec, persaepe e pluribus residuis quae non omnia sunt satis simplicia aliud admodum simplex deduci potest, si modo illa multos factores communes implicant. Hane ob caussam talia quoque residua valde sunt opportuna, quae $\mathrm{e}$ multis factoribus non nimis magnis composita sunt, conuenietque omnia statim in factores suos resoluere Vis harum obswruationum melius per exempla vşumque frequenten quam per praecepta percipietur.

I. Methodus simplicissima, iisque, qui per frequentem exercitationem iam aliquatn dextexitatem sibi conciliauerunt, commodssima, consistit in eo, vt $M$ aut generalius multiplum quodcunque ipsius $M$ quomodocunque in duas partes decomponatur $k M=a+b$ (siue vtraque sit positiua siue altera positiua altera negatima) quarum productum signo mutato erit residum ipsius $M_{;}$erit enim - $a b \equiv a a \equiv \bar{b} b$ (mod. $M$ ), adeoque $-a b R M$. Numeri $a, b$ ita accipiendi sunt, yt productum per quadratum magnum diuisibile quotiensque vel paxaus vel saltem in factores non nimis magnos resolubilis euadat, quod semper non difficile effici poterit. Imprimis commendandum est, vt pro $a$ accipiatur vel quadratum, vel quadratum duplex, vel triplex etc. a numero $M$ numero vel paruo 0.4 
vel in factores commodos resolubill discrepans. Ita. e. g. inuenitur $99733^{1}=999^{2}-2.5,67=$ $994^{2}+5.11 .13^{2}=2.706^{2}+3.17 .3^{2}=3.575^{2}$ $+11.31 .4=3.577^{2}-7.134^{2}=3.578^{2}-$ $7.19 .37=11.299^{2}+2.5 .5 .29 \cdot 4^{2}=11.301^{2}+$ 6. $11^{2}$ etc. Hinc habentur residua sequentia $2.5 .672-5.11,-2.3 .17,-3.11 .31,3.7 .13 \%$ $87.19 .37,-3.3 .5 .11 .29 ;$ discerptio vltima suppe ditat residuum - 5.11 quod iam habemus. Pro residuis - $3.11 .31,-3.5 .11 .29$ haec adoptare possumus $3.5 .31,2.3 .29$, ex illorum combinatione cum -5.1 .1 oriunda.

II. Methodus secunda et tertia inde petuntur, quod, si dua formae binariae $(A, B, C)$, $\left(A^{\prime}, B^{\prime}, C^{\prime}\right)$ einsdem deterninantis II , aut - $M$, aut generalius $\pm k M$, ad idem genus pertinent, numeri $A A^{i}, \overline{A C}, A C$ surit residua ipsius $k M$; hoc nullo negotio inde perspicitur, quod numerus quiuis characteristicus vrius formae, puta $m_{\text {w }}$ etiam est numerus char. alterius, adeoque $m A d$, $m C, m \& 4, m C$ omnes residua ipsius $h M$. Si itar que $\left(a, b, a^{\prime}\right)$ est forma reducta determinantis positiui $M$ aut generalius $k M$, atque $\left(a^{\prime}, b^{\prime}, a^{\prime \prime}\right)$, $\left(a^{\prime \prime}, b^{\prime \prime}, a^{\prime \prime \prime}\right)$ etc. formae ex ipsius pexiodo, adeoque ipsi aequiualentes et a potioni sub eodem genere contentae: numeri $a a^{\prime}, a a^{\prime \prime}$, $a a^{\prime \prime \prime}$ etc. omnes erunt residua ipsius $M$. Computus multitudinis magnae formarum talis periodi facilime adiumento algonithmi art. 187. instituitur; residua simplicissima plerumque prodeunt statueada $a=1$; ea quae factores nimis magnos implicant, erunt rejicienda. Ecce initia periodorum, farmarum $\left(1,998,-13^{2} 7\right)$ et $(1,1412$, 


\section{$-685$}

- 918), quarum determinantes sunt $99733 x_{\text {. }}$ 1994662:
$(1,998,-1327)$
$(1,1412,-918)$
$(-1327,329,670)$
$(670,341,-1315)$
$(-918,1342,211)$
$(-1315,974,37)$
$(211,1401,-151)$
$(37,987 ;-626)$
$(-626,891,325)$
$(-151,1317,1723)$
$(325,734,-141 i)$
$(-1411,677,382)$
$(382,851,-715)$
$(1723,406,-1062)$
$(-1062,656,1473)$
$(1475,817,-901)$
$(-901,985,1137)$
etc.

Sunt itaque residua numeri 997351 omnes numeri -1327 , 670 etc, negligendo autem ea, quae factores nimis magnos implicant, haecce habemus: $2.5 .67,37,13,-17.83 ;-5.11 .13$, $-2.3 .17,-2.59,-17.53 ;$ residuum 2.5 .67 s nec non hoc - 5.11, quod e combinatione tertii cum quinto euoluitur, iam supra erueramus.

III. Si $C$ est classis quaecunque formarum det neg. - $M$ siue generalius - $k M$, a principali diuersa, ipsiusque periodius haec $2 C, 3 C$ etc. (art. 307.): classes $2 C, 4 C$ etc ad genus principale pertinebunt; hae vero, $3 C,{ }_{5} C$ etc. ad idem genus vt $C$. Si itaque $(a, b, c)$ est forma (simplicissima) ex $c$ atque $\left(a^{\prime}, b^{\prime}, c^{\prime}\right)$ forma ex aliqua classe illius (periodi puta ex $n C$, erit vel $a^{\prime}$, tel acl residum ipsius, $M$, prout $n$ par vel impar (in cast priori manifesto etiam $c^{\prime}$, in posterioni $a c^{\prime}$, cot et $c c^{\prime}$ ). Elualutio periodi, i. e. formarum simplicissimarum in ipsius classibus, mira facilitate perficitur, quando $a$ est valde parius,

O 5 


\section{$-586-$}

praesertim quando est $=3$, quod semper efficere licet, quando $k M \equiv 2(\bmod .3)$. Ecce initium periodi classis, in qua est forma $(3, x$, 332444)

$$
\begin{array}{c|c}
C(3,1,332444) & 6 C(729,-209,1428) \\
2 C(9,-2,110815) & 7 C(476,209,2187) \\
5 C(27,7,36940) & 8 C(1027,342,1085) \\
4 C(81,34,12327) & 9 C(932,-437,1275) \\
5 C(243,34,4109) & 10 C(425,12,2347)
\end{array}
$$

Hinc promanant residua (inutilibus reiectis) $3.476,1027,1085,425$ siue (tollendo factores quadratos) $3.7 .17,13.79,5.7 .31,17$, e quorum combinatione apta cum octo residuis in II inuentis facile eruuntur duodecio sequentia -2.3 , $13,-2.7,17,37,-53,-5.11,79,-83$, $-2.59,-2.5 .51,2.5 .67$, sex priores sunt Lidem quibus in art. 331 vsi sumus. Adiici potuissent residua 19 et -29 , si 'ea quoque in vsum vocare voluissemus, quae in I reperta sunt; reliqua illic eruta $a b$ is quae hic euoluimus iam. sunt dependentia.

353. METHODUS SECUNDA, numerum datum $M$ in factores resoluendi, petitur e consideratione valorum talis expr. $\sqrt{-D}(\bmod , M)$, obseruationibusque sequentibus innititur.

I. Quando $M$ est numerus primus aut potestas numeri primi (imparis ipsumque $D$ non metientis), erit $-D$ residuum vel non residuum ipsius $M$, prout $M$ vel in forma diuisorum vel in forma non diuisorum ipsius $x x+D$ con-

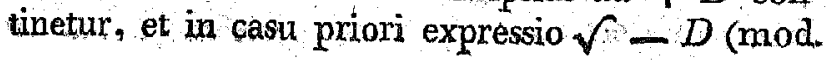


$M$ ) duos tantummodo valores diuersos habebit, qui oppositi erunt.

II. Quando vero $M I$ est compositus, puta $=p p^{\prime} p^{\prime \prime}$ etc., designantibus $p, p^{\prime}, p^{\prime \prime}$ etc. numeros primos (diuersos impares ipsumque $D$ non metientes) aut talium numerorum potestates: $-D$ tunc tartummodo residuum ipsius $M$ erit, quando est residuum singulorum $p, p^{\prime}, p^{\prime \prime}$ etc., i. e. quando hi numeñ omnes in formis dituisorum ipsius $x x+D$ continentur. Designando autem valores expr. $\sqrt{ }-D$ sec modulos $p, p^{\prime}$, $p^{\prime \prime}$ etc. resp. per $\pm r, \pm r^{\prime}, \pm r^{\prime \prime}$ etc., onnes \&alores eiusdem expressionis sec. mod. $M$ orientur, eruendo numeros qui $i$ secundum $p$ sint $\equiv r$ aut $\equiv-r$, secundum $p^{\prime}$ aut $\equiv r^{\prime}$ aut $\equiv-r^{\prime}$ etc, quocirca ipsorum multitudo fiet $=q^{\mu}$, designante $\mu$ multitudinem numerorum $p, p^{\prime}, p^{\prime \prime}$ etc. Quodsi itaque hi valores sunt $R,-R, R^{\prime}$, $-R^{\prime}, R^{\prime \prime}$ etc, sponte erit $R \equiv R$ secundum omnes $p, p^{\prime}, p^{\prime \prime}$ etc., sed secundum nullos $R_{\xi} \equiv$ - $R^{\prime}$, rnde diuisor communis maximus numeri $M$ cum $R-\dot{R}$ erit $M$, et $\mathbf{1}$ diu. comm. max. ipsius' $M$ cum $R+R$ '; 'sed valores duo nec identici nec oppositi vt $R$ et $R^{\prime}$ necessario secundum vnum pluresue numerorum $p, p^{\prime}, p^{\prime \prime}$ etc., neque vero secundum omines, congrui erunt, et secundum reliquos $R \equiv-R^{\prime}$; hinc illoram productum erit diuisor communis maximus numerorum $M$ èt $R-R^{\prime}$, productumque horum d. c. $\mathrm{m}$. ipsorum $M$ et $R+R^{\prime}$. Hinc facile sequitur, si omnes diuisores communes maximi ipsius $M$ cum differentiis inter singulos valores expr. $\checkmark$ $D(\bmod M)$ atque aliquem valorem datum 
computentur, horum complexum continere numeros $1, p, p^{\prime}, p^{\prime \prime}$ 'etc. atque omnia producta $\mathrm{e}$ binis, ternis etc. hortum numperorum. Hoc itaque modo e valoribus illius expressionis numeros $p, p^{\prime \prime}, p^{\prime \prime}$ etc. eruere licebit.

Ceterum quum methodus art. 327 singulos hosce valores ad valores expressionum huius formae $\frac{m}{n}(\bmod M)$ reducat, ita vt denominator $n$ ad $M$ primus sit: ad institutum praesens ne necessarium quidem est, has ipsas computare. Nam: diu. conm. max. numeri $M$ cum differentia inter $R$ et $R^{\prime}$ qui cum $\frac{m}{n}, \frac{m}{k^{\prime}}$ conueniunt manifesto etiana erit diu. comm. max. ipsorum $M$ et $n n^{i}(R-$ $\left.R^{\prime}\right)$, sive ipsorum $M$ et $m n^{\prime}-m^{\prime} n$, quippe cui $m n^{\prime}\left(R-R^{\prime}\right)$ secundutm modulum $M$ est congruus.

354. Applicatio obseruationum praecc. ad problema de quo agimus duplici modo institui potest; prior non solum decidet, ${ }_{2}$ trum numerus propositus $M$ primus sit an compositus, sed in hoc casu etiam factores ipsos, suppeditat; poste rior autem eatenus praestat, quod plerumque calculum expeditiorem permittit, sed factores ipsos numerorum compositorum, quos quoque a primis protinus distinguit, interdum non profert nisi jluries repetatur,

I. Inuestigetur numerus negatiuus $-D$, qui sit residuum quadraticum ipsius $M$, ad quem finem methodi in art. $33^{2}$ sub I et II traditae adhiberi potenuth Per se quidem arbitrarium 
est, quidnam residuum eligatur, neque híc vt in methodo praec. opus est, vt $D$ sit numerus paruus; sed calculus eo breuior erit, quo minor est multitudo classium formarum binariarum in singulis generibus pr. pr. det. $-D$ contentaruirt; quanobrem imprimis talia residua qui inter 65 numeros art. 3.03 continentur, si qui se offerunt, opportúna erunt. Ita pro $M=997331$ ex omnibus residuis negatiuis supra erutis hoc -102 maxime idoneum esset. Eruantur omnes valores diuersi expr. $\sqrt{ }-D$ (mod. $M$; quodsi duo tantum proveniunt (oppositi), $I$ C certo erit vel hat merus primus vel numeri primi potestas; si piltres, puta $2^{u}, \pi T$ compositus erit ex $\mu$ numeris primis, aut primorum potestatibus, diueršis, qui factores per methodim art praec. erui poterunt. Vtrum vero hi factores numeri primi sint an primorum potestates, tumo per se fácillimum erit dignoscere; tum etiam via ipsa per quam valores expr. $\sqrt{ }-D$ iniéniuntur, omnes numeros primos, quorum potestas aliqua ipsum $M$ metin tur, sponte indicat; scilicet si diuisibilis est per quadratum numeri primi i $r$; ille calculus certo etiam vnam pluresue repraesentationes tales nuneri $M, M=a m m+2 b m+c m$, pro duxerit, in quibus diuisor comm. max numerarum $m, n$ est $n$ (et quidem ideo, quod in hoe casu $-D$ etiam est residurm ipsius $\frac{M}{\pi \pi}$, Quando vero nulla repraesentatio prodiit, in qua $m$ et $n$ diuisorem communem habent, boc certum indicium est, $I$ per mullum quadratum diuisibilem esse adeoque omines $p, p^{\prime}, p^{\prime \prime}$ etc. numeros primos, 
Ex. Per methodum supra traditam inueniuntur quatuor valores expr. $\sqrt{ }-102$ (mod, $997351)$ cum valoribus harum $\pm \frac{5664}{1 \times 3}, \pm \frac{2874}{3}$ conuenientes; diuisores communes maximi 997331 cum his $3.1664-113.2824$ et $3.1664+113.2824$ siue cum $3 \times 4$ 120 et 324 I 04 , exuuntur hi 7853 et $\mathrm{x} 27$, vnde $99733^{\mathrm{I}}=\mathrm{I} 27.7853$, vt supra.

II. Accipiatur aliquis numerus negatiuus - $D$ talis, vt $M$ contentus, sit in forma diuisorum ipsius $x x+D$; per se arbitrarium est, quis huiusmodi numerus eligatur, sed commoditatis caussa imprimis videndum est, vt multitudo classium in generibus det. $-D$. sit quam maxime parua. Ceterum inuentio talis numeri nulli difficultati obnoxia est, si tentando adeatur; nam plerumque inter multitudinem considerabilem numerorum tentatorum pro totidenr fere $M$ in forma diuisorum continetur, ac in forma non diuisorum. Quare maxime e re exit, tentamen. a 65 numeris art. 303 inchoare (et quidem a maximis), et si eueniret, vi nullus idoneus esset (quod tamen generaliter loquexdo inter 16384 casus semel tantum accidit), ad alios progredi, vbi classes binae in singulis genexibus continentuf. - Tunc inuestigentur valores expr. $\sqrt{ }-D$ (mod. $M$ ) si qui incenimutur, factores ipsius $M$ prorsus eodem modo inde deducantur vt supra; si vero nulli valores prodeunt, adeoque $-D$ estnon residuum ipsius $M$, certo $M$ neque numerus primus neque numeri primi potestas esse poterit. Quodsi in hoc casu faetores ipsi desiderantur, vel eandem operationem repetere oportet, alios valores pro $D$ accipiendo, vel ad methodum aliam confugere 


\section{- $59 \mathrm{X}-$}

Ita e. g. tentamine facto 99733 I contentus innenitur in forma non diuisorum ipsorum $x x$ $+\mathrm{r} 848, x x+1365, x x+\mathrm{x} 320$, sed in forma diuisorum ipsius $x x+840$; pro valoribus expr: $\sqrt{-840}$ (mod. $99733^{\prime}$ ) prodeunt expr. $\pm \frac{x_{27}}{I_{0}}$, $\pm \frac{3288}{125}$, vnde iidem factores deducuntur vt ante. - Si quis plura exempla desiderat, art. 328 consulat, vbi primum docet esse $542868 \mathrm{I}=$ 307.17683 ; secundum, 4272945 esse numerum primum; tertium, $99733^{1}$ certe e pluribus primis compositum esse.

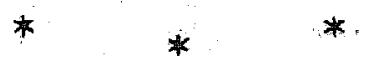

Ceterum limites huius operis praecipua tantum momenta vtriusque methodi factores inuestigandi hic exsequi permiserunt; disquisitionem vberiorem vna cum pluribus tabolis auxiliaribus aliisque subsidits aliae occasioni reseruamus. 
SECTIO SETTIMA

\section{DE AEQVATIONIBVS CRRCVLT SE- CTIONES DEFINIENTIBVS.}

335. Tnter incrementa splendidissima, mathesi per recentiorum labores adiecta, theoria functionum a circulo pendentitum procul dubio locum imprimis insignem tenet. Cui mirabili quantitatum generi, ad qtrod in disquisitionibus maxime heterogeneis saepissime deferimur, curiusque subsidio nulla vniuersae matheseos pars carere potest, summi geometrae recentiores industriam sagacitatemque suam tam assidue impenderunt, disciplinamque tam vastam inde efformauerunt; vt parum exspectari potuisset, vllam huius theoriae partem, nedum elementarem atque in limine quasi positam, grauium adhuc. incrementorum capacem esse. Loquor de theoria functionum trigonometricarum, arcubus cum peripheria commensurabilibus respondentium, siue de theoria polygonorum regularium, critus quam parua pars hucusque enucleata sit, sectio praesens patefaciet. Mirari possent lectores, talem disquisitionem in hocce potissimum opere, disciplinae primo aspectu maxime heterogeneae imprimis dicato, institui; sed tractatio ipsa abun=- 
de declarabit, quam intimo nexu hoc argumentum cum arithmetica sublimiori coniunctum sit.

Ceterum principia theoriae, quam exponere aggredimur, multo latius patent, quam hic extenduntur. Namque non solum ad functiones circulares, sed pari successu ad multas alias functiones transscendentes applicari possunt, e. g. ad eas quae ab integrali $\int \frac{d x}{\sqrt{(}\left(1-x^{4}\right)}$ pendent, praetereaque etiam ad varia congruentiarum genera: sed quoniam de illis functionibus transscendentibus amplum opus peculiare paramus, de congruentis, autem in continuatione disquisitionum arithmeticarum copiose tractabitur, hoc loco solas functiones circulares considerare visum est. Imo has quoque, quas summa generalitate amplecti liceret, per subsidia in art. sq. exponenda ad casum simplicissimum reducemus, tum breuitati consulentes, tum vt principia plane noua huius theoriae eo facilius intelligantur.

336. Designando circuli peripheriam siue quatuor angulos rectos per $\boldsymbol{P}$, supponendoque $m, n$ esse integros, atque $n$ productum e factoribus inter se primis $a, b, c$ etc.. angulus $A=$ $\frac{m P}{n}$ per art. 310 sub hanc formam reduci potest $A=\left(\frac{\alpha}{a}+\frac{6}{b}+\frac{\gamma}{c}+\right.$ etc. $) P$, functionesque trigonometricae ipsi respondentes e functionibus ad partes $\frac{\alpha P}{a}, \frac{\varepsilon P}{b}$ etc. pertinentibus per methodos notas deducentur. Quoniam itaque pro $a, b_{3}$ - etc. numeros primos aut numerorum primo$P_{p}$ 
rum potestates accipere licet: manifesto sulficit, sectionem circuli in partes, quarum muhthulo est numerus primus aut primi potestas, consitut:rare, polygonumque $n$ laterum e polygonis $a$. $b_{\text {, }}$ $c$ etc. Iaterum protinus habebitur. Allamera loo locó disquisitionem ad eum castun restringenus, vbi circulus in partes diuidendus ast, tuarma multitudo est numerus primus (impary), squtu uti praesertim ratione inducti. Constat, functioness circulares angulo $\frac{m P}{p p}$ respondentes e funtimibus ad $\frac{m P}{p}$ pertinentibus per solutionern ataqua-

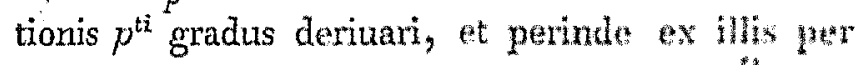

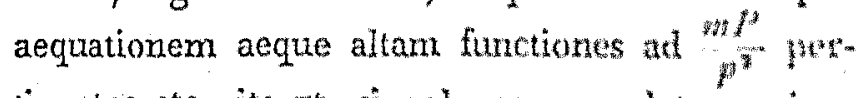
tinentes etc., ita vt, si polygonum $p$ latehum ium habeatur, ad determinationexn polygoni $p^{\lambda}$ hetrow rum necessario solutio $\lambda$ - 1 aequativunan $p^{\text {sh }}$

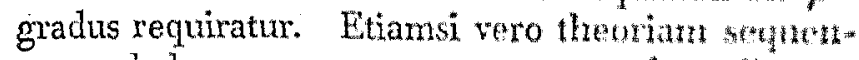
tem ad hunc quoque casum extrntere liferets tamen hac via non minus ad totidem atevtutiv. nes $p^{\text {ti }}$ gradus delaberemur, quae, statutew numerus primus, ad inferioxes deprimut molo modo possunt. Ita a. infra ostendetur, pon lygomim ${ }^{2} 7$ laterum seonfetrice constum poss: sed ad determinationern polygoni 284 laterum aequationem $17^{\text {mi }}$ gradus nullo modo etaiture licet.

337. Satis constat, functiones trigonomets cas ommium angulorum $\frac{k p}{n}$, denotando per $k$ indefinite omnes numeros $0, x, 2 \ldots n-1$, per 


\section{- $595-$}

radices aequationum $n^{\text {ti }}$ gradus exprimi, puta si* nus per radices huius (I) *

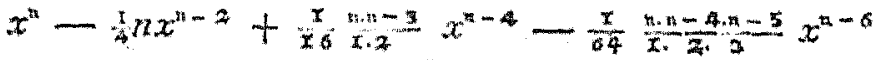

$$
\begin{aligned}
& \text { t etc. } \pm \frac{x}{2^{n}-x} n x-0
\end{aligned}
$$

cosinus per radices huius (II)

$$
\begin{gathered}
x^{n}-\frac{x}{4} n x^{n-2}+\frac{x}{x \cdot n-3} x^{n+4}-\frac{x}{64} \frac{n-4 \cdot n}{x \cdot 2 \cdot 5} x^{n-6} \\
+ \text { etc. } \pm \frac{1}{2^{n}-x} n x-1=0 .
\end{gathered}
$$

denique tangentes per radices tiuius (III)

$$
\begin{gathered}
x^{n}-\frac{n \cdot n-x}{x \cdot 2} x^{n-2}+\frac{n+x-x-2 \cdot n-3}{x \cdot 2} x^{n-4}-\text { etc } \pm \\
n x=0
\end{gathered}
$$

Hae aequationes (quae generaliter pro quouis valore impari ipsius $n$ valent, If vero pro pari quoque), pnnendo $n=2 m+1$, facile ad gradum $m^{\text {turs }}$ deprimuntur; scilicet I et III, dividerdo partem a laxua per $x$ et substituendo $y$ pro x.x. Aequatio II autem mazifesto radicen $x=$ $x(=\cos 0)$ implicat, et e reliquis binae semner aequales sunt $\left(\cos \frac{p}{n}=\cos \frac{(n-x) P}{n}\right.$, $\cos \frac{2 p^{P}}{n}=\cos \frac{(n-2) P}{n}$ etc.); quare ipsius pars a laeva per $x-1$ dinisibilis, quotiensque quadratum exit, cuius radicem quadratam extrahendo, arquatio If reducitur ad hanc

$$
\begin{aligned}
& x^{m}+\frac{x}{2} x^{m-x}-\frac{x}{4}(m-1) x^{m-z}-\frac{x}{1}(m-q) x^{m-x}
\end{aligned}
$$

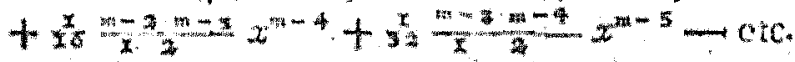

$$
\begin{aligned}
& =0
\end{aligned}
$$

cenius radices exunt cosinus angulorum $\frac{P}{\eta}, a^{p}$,

$$
\text { Ppo }
$$




\section{- $596-$}

$\frac{3 P}{n} \ldots \frac{m P}{n}$. Vlteriores reductiones harum dequationum, pro eo quidem casu vli $n$ est numserus primus, hacterius non habebantur.

Attamen nulla harum aequationum tam tractabilis et ad institutun aostrum tam idonea quam haec $x^{\mathrm{n}}-1=0$, cuili, sadices ctum radicibus illarum arctissime connexas esere contht. Scilicet, scribendo breutatis caussa it pro quathitate imaginaria $\sqrt{ }-1$, radices atruatioun $x^{*}$ $1=0$ exhibentur yer $\cos \frac{k p}{n}+i \sin { }^{p}$ $=r$, vbi pro $k$ accipiendi sumt ommes numeti $0,1,2 \ldots n-1$. Quocirca quum sit $=$ $\cos \frac{k p}{n}-i \sin \frac{k p}{n}$, radices aeguntionis 1 exhibe-

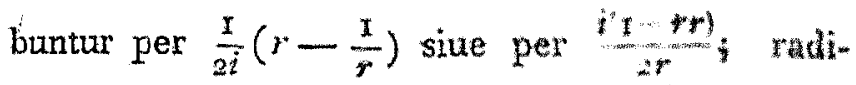
ces aequationis II per $\frac{x}{2}\left(r+\frac{r}{r}\right)=\frac{I+m}{2 r}$; dun que radices aequationis III $p e \frac{1}{1+m}$. Hanc ob caussam disquisitionem corsiderution atouationis $x^{\prime \prime}-1=0$ superstruemus, iftenn $n$ ese numerum primum imparem suppontalo. Ne ves ro inuestigationum ordinem intmrtmpere oporteat, sequens lemma hic praenittimus.

358. Prosrama. Dala aeguatiowe $(W)$... $2^{\mathrm{m}}+d z^{\mathrm{m}-\mathrm{x}}$ etc $=0$, inuentre atequationem (W), cuius ralice's sint potestates $x^{\text {tar }}$ radhum aequationis ( $F$ ), destignante $A$ exponentera intem grum posütum datum. 
Sol. Designatis radicibus aequationis $W F$ per $a, b, c$ etc, radices arqu. Ih esse debebunt $a^{\lambda}, b^{\lambda}, c^{\lambda}$ atc. Fer theorema notum Newtoniaman e coefficientibus aequ. If inuenire licet aggregata quarumalibet potestatum radicum $a, b, c$ etc. Quaternun itaque summae $a^{\lambda}+b^{\lambda}+c^{\lambda}+$ etc., $a^{2 \lambda}+b^{2 \lambda}+c^{2 \lambda}$ etc. etc. vsque ad $a^{\mathrm{m} \lambda}+$ $b^{2}{ }^{\lambda}+c^{m \lambda}+e l c$, nde via inuersa per idem theorema coefficiontes aequ. WW deduci poterunt. Q. E. $I F$ - Simul hinc liquet, si ormes coëfficitentes in $W$ sint rationales, omnes quoque in II rationales enadere. Alia quidem via probari potest, si illi omres integri sint, etiam hos omnes integros fieri; huic autem theoremati, ad institutum rostrum non adeo necessario, hic non immoramur.

359. Aequatio $x^{n}-x=0$ (in suppositione semper ablinc subintelligenda, $n$ esse numerum primum imparem) vnicam radicem realem implicat, $x=1 ; n-1$ reliquae, quas aequatio $x^{n-x}+x^{n-x}+$ etc. $+x+1=0 \mathrm{com}-$ plectitur, omnes sunt imaginariae; harum comple$\because n$ per $\Omega$, functionemque $x^{n-x}+x^{n-x}+$ etc. $+x$. +1 per $X$ denotabimus. Si itaque $r$ est radix quaccunque $\mathrm{ex} \Omega$, erit $1=r^{\mathrm{n}}=r^{2 \text { etc., et }}$ generaliter $r^{\text {to }}=1$ pro quouis valore integro ipstis $c$, positino sel negatio; hinc perspicuum est, si $\lambda, \mu$ sint integri secundum in congrut, fore $r^{A}=r^{*}$. Si vero $\lambda$, $\mu$ sec. mod. $n$ incongrui stut, $r^{\lambda}$ et $r^{-4}$ invequales erunt; in hoa enim casu integer, ita accipi potest vt fiat ( $\lambda-$ H) ${ } \equiv 1(\bmod n)$, vnde $r^{(\lambda-\mu) *}=r$, adeo que $r^{\lambda-\mu}$ certo non $=1$. Porro patet, quamuis Pp 3 


\section{$-598$}

potestatem ípsius $r$ etiam radicem aeq̧u. $x^{n}-x$ $=0$ esse; quocirca quum quantitates $1\left(=r^{\circ}\right)$, $r, r r \ldots r^{r-1}$ omnes sint diuersue, hae axhibetunt omnes radices aequ. $x^{\mathrm{a}}-1=0$, et proin hat $r, r r, r^{3} \ldots r^{n-x}$ cum $\Omega$ coincident. Facile line generalius colligitur, $\Omega$ conuenire cum $r^{*}, r^{* *}$, $r^{30} \ldots r^{(n-x) e}$, si $c$ sit integer quicunque per $n$ non dinisibilis, positiuns sen regatiuns. Frit itaque $X=\left(x-r^{2}\right)\left(x-r^{2 n}\right)\left(x-r^{3}\right) \cdot \ldots(x-$

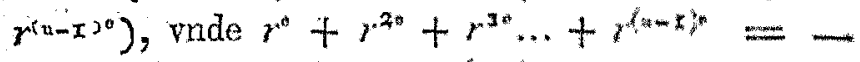
1 , et $1+r^{0}+r^{20} \ldots+r^{\text {mo-1: }}=0$. Duas ram dices tales vt $r$ et $\frac{Y}{r}\left(=r^{m-x}\right)$, ant generaliter $r^{o}$ et $r^{-8}$ reciprocas vocabimus; mimifestum est, productum ex duobus factoribus simplicibus $x-r$ et $x-\frac{x}{r}$ fieri reale $=x x-2 x \cos$ $+x$, ita vt angulus $\omega$ vel angulo $\frac{P}{f}$ vel alloux multiplo eius sit aequalis.

"340. Quoniam itarue, wa ratice ox $\Omega$ per $r$ expressa, omnes radices aequ. $2^{n}-1=0$ per potestates ipsius $r$ exprinumur, froductum, e pluribus radicibus huius atgu. quomodormentes conflatum, per $r^{\lambda}$ exhiberi poterit, ita ot $A$ s.t. vel $o$, vel positiurs et $<h$. Designando itaque per $\phi(t, u, v, \ldots)$ functionem algebraicam ra tionalem integram indeterminatarum $t, u, v$ etc.,

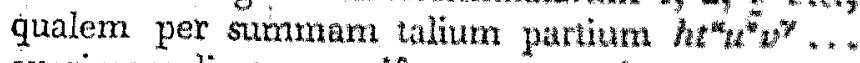
exprimere licet: manifestum est, sit pro $t, u, v$ etc. quaedam e radicibus aequ. $x^{n}-x=0$ substituantur, puta $t=a, u=b, v=c$ etc., $\Phi(a, b, c \ldots)$ sub forman $A+A r+A^{n} r+$ $A^{\prime \prime \prime} r^{3} \cdots+\mathscr{A}^{4-x} r^{4-x}$ reduci posse, itat wt cuefficientes $A, A^{\prime}$ etc. (e quibus etiam aliqui deesse adeo- 


\section{- $599-$}

que $=0$ fieri possunt) sint quantitates determinatae, insuperque omnes hos coüfficientes integros fieri, si ommes coefficientes determinati in $\Phi(t, u, u \ldots), i$. e. omnes $f_{t}$ sint integri. Quodsi vero postea pro $t, u, v \ldots$ substituuntur $a a, b b$, co... resp., quaeuis pars vt $h t^{*} t^{6} t^{*}{ }^{*} \ldots$, quae intea reducebatur ad $r^{\sigma}$, nunc fiet $r^{2 \sigma}$, vnde facile concluditur, fieri $\varphi(a a, b b, c c \ldots)=A$ $+A^{\prime} r r+A^{\prime \prime} r^{4}+A^{\prime \prime \prime} r^{6} \ldots+A^{n} r^{2 n-7}$. Perinde (rit generaliter, pro valore quocunque integro ipsius $\lambda, \phi\left(a^{\lambda}, b^{\lambda}, c^{\lambda} \ldots\right)=A+A^{\prime} \gamma^{\lambda}+$ $A^{\prime \prime} r^{2 \lambda} \ldots+A^{\prime} r^{(n-x) \lambda}$, quae propositio maximi est momenti, fundamentumque disquisitionum sequentium constituit. - Hinc sequitur etiam $\phi(\mathrm{x}, \mathrm{x}$, $x \ldots)=\psi\left(a^{n}, b^{n}, c^{n} \ldots\right)=A+A^{i}+A^{\prime \prime} \ldots+A^{n} ;$ nec non $\phi(a, b, c \ldots)+\varphi(a a, b b, c c \ldots)+\phi\left(a^{3}\right.$, $\left.b^{n}, c^{3} \ldots\right) \ldots+\$\left(a^{n}, b^{n}, c^{n} \ldots\right)=n \mathcal{A}$, quae itaque summa semper fit integer per $n$ diuisibilis, quando omnes coefficientes determinati in $\varphi(t$, $u, v, \ldots$ ) sunt integri.

34.1. Theorma. Si functio $X$ per functionem inferioris gradus $P=x^{\lambda}+A x^{\lambda}-x+$ $B x^{\lambda}-2 \ldots+K x+L$ est diutisibilis, coifficientes $A, B \ldots L$ omnes integri esse nequeunt.

Dem. Sit $X=P Q$, atque $\$$ complexus radicum aequationis $\supseteqq=0, \mathfrak{Q}$ complexus radicum aequationis $\mathfrak{Q}=0$, ita vt sa constet ex $\mathfrak{P}$ et 2 simul sumtis. Porro-sit $\mathbb{R}$ complexus radicum ipsis reciprocarum, of complexus radicum ipsis o reciprocarum, sintque radices quae continentur in $\mathfrak{R}$ radires aequationis $R=0$ $\operatorname{Pp} 4$ 


\section{$-600-$}

(quam fieri $x^{2}+\frac{K}{L} x^{\lambda-x}+$ etc. $+\frac{A}{L} x+1$ $=0$ facile perspicitur), eaeque quae continentur in $\subseteq$ radices aequationis $\mathcal{S}=0$. Manifesto etiam radices $\Re$ et $\widetilde{S}$ iunctae complexum $\Omega$ efficient, ac erit $R S=X$. Iam quatuor casus distinguimus.

I. Quando $M$ conuenit cum $\Re$ adeoque $P$ $=R$. In hoc casu manifesto binae semper radices in $\$$ reciprocae erunt, adeoque $P$ productum ex $\frac{x}{2} \lambda$ factoribus talibus duplicibus $x x-$ $2 x \cos \omega+1$; quum talis factor sit $=(x-$ $\cos \omega^{2}+\sin \omega^{2}$, facile perspicietur, $P$ pro vaIore quocunque reali ipsius $x$ necessario valorem realem obtinere. Sint aequationes, quarum radices sunt quadrata, cubi, biquadrata ... potestates $n-1^{\text {tae }}$ radicum in $\mathfrak{g}$ resp. hae $P=0$, $P^{\prime \prime}=0, P^{\prime \prime \prime}=0, \ldots P^{\prime}=0$, sintque valores functionum $P, p_{\prime}^{\prime}, P^{\prime \prime} \ldots P^{\prime}$, quos obtinent statuendo $x=1$, resp. $p, p^{\prime}, p^{\prime \prime} \ldots p^{y}$, tunc per ante dicta $p$ erit quantitas positiua et prorsus simili ratione etiam $p^{\prime}, p^{\prime \prime}$ etc. positiuae erunt: Quum itaque $p$ sit valor functions $(1-t)(1$ - u) $(1-v)$ etc, quem obtinet ponendo pro $\boldsymbol{t}, u, v$ etc radices in $\$ p^{\prime}$ valor eiusdem, statuendo pro $t, u, v$ etc. quadrata illarum radicum etc, insuperque valor pro $t=1, u=1$, $v=1$ etc. manifesto fiat $=0$ summa $p+p^{2}$ $+p^{\prime \prime} \ldots+p^{\prime}$ erit integer per $n$ diuisibilis. Praeterea facile perspicietur, productum $P P^{\prime} P^{\prime \prime}$ ... fieri $=X^{\lambda}$, adeoque pp'p"... $=n^{\lambda}$.

Iam si omnes coëfficientes in $P$ rationales essent, onmes quoque in $P^{\prime}, P_{i}$ etc. per art. 338 
rationales euaderent; per ant. 42 autem cunctì hi coëfficientes necessario forent integri. Hinc etiam $p, p^{\prime}, p^{\prime \prime}$ etc. omnes integri forent, quosum productum quum sit $n^{\lambda}$, multitudo vero $n$ - $1>\lambda$, necessario quidam ex ipsis (saltem $n-1-\lambda$ ) esse debebunt $=1$, reliqui vero ipsi $n$ vel potestati ipsius $n$ aequales. Quodsi itaque $g$ ex ipsis sunt $=1$, summa $p+p+$ etc. manifesto erit $\equiv g(\bmod . n)$ adeoque certo. per $n$ non diuisibilis. Quare suppositio consistere neqúit.

II. Quando $\mathfrak{B}$ et $\Re$ non quidem coincidunt, attamen quasdem radices communes continent, sit $\mathfrak{I}$ harum complexus atque $T=0$ aequatio cuius radices sunt. Tunc $T$ erit diuisor communis maximus functionum $P, R$ (vt e theoria aequationum constat). Manifesto autem binae semper radices in $\mathfrak{Z}$ reciprocae emunt, vnde per ante demonstrata omnes coefficientes in $T$ rationales esse nequeunt. Hoc vero certo eueniret, si omnes in $P$ adeoque etiam omnes in $\boldsymbol{R}$ rationales essent, vt e natura operationis', diuisorem comm. max. inuestigandi sponte sequitur. Quaro suppositio est absurda.

III. Quando $\&$ et $\&$ vel coincidunt, vel saltem radices communes implicant, prorsus eodem modo omnes coëfficientes in $Q$ rationales esse nequeunt; fierent vero rationales, si omnes in $P$ rationales essent; hoc itaque est impossibile

IV. Si vero neque $\$$ cum $\Re$, neque $\Omega$ cum is vllam radicem communem habet, omnes Pps 


\section{- $602-$}

radices 9 necessario reperientur in $\mathbb{G}$, ommestue $\Omega$ in $\Re$; mde erit $P=S$ et $Q=R$. (uan* obrem $X=P Q$ erit productum $* x$ in 11 , i. e. ex $x^{\lambda}+A x^{\lambda-x} \ldots+h x+1$. in $x^{x}+\frac{h^{*}}{L} x^{x-x}$ $\therefore+\frac{A}{L} x+\frac{\mathrm{r}}{I}$, vnde statuendo $x=1$, fit $n L=(1+A \ldots+\pi+L)^{2}$. Inm si ommas coëfficientes in $P$ rationales, adeoque per art. 12 etiam integri essent, $l_{\text {s }}$ qui cobifinentern vitimum in $X i_{+}$e vuitatem metiri deberest nepessatrio foret $= \pm 1$, vade $\pm n$ esset numerus quadratus. Quod quum hypothesi reptamet, sup. positio consistere nequit.

Ex hoc itaque theoremate liquet, quomolocunque $X$ in factores resoluatur, horum coenteren. tes partim saltem irrationales fieri, adeoque atim ter, quam per asquationen elethatan, detwrixnari non posse.

342. Propositum disquistionmm satents um, quod paucis decluratisse haud intile etit, eo tendit, vt $X$ in factores continuo plutes tianDATIM resoluatur, et quidem ita, ve luoram coêfficientes per aequations's ordinis quanan infim mi determinentu, vsque dum hoc modo ad fa" ctores simplices sine ad radices a ipsas pertuentatur. Scilicet ostendemus, si numerus $n-1$ quomodocunque in factores integros $\ldots, 6,2,4$ resoluatur (pro quibus singulis murmeros primos accipere licet), $X$ in a factores $\frac{-1}{2}$ dimearsiom num resolui posse, quorum couficientus per 


\section{$-603-$}

quationem $a^{5}$ gradus determinentur; singulos hos factores iterum in 6 alios $\frac{n-1}{x^{6}}$ dimensionum adiumento aequationis $6^{i}$ gradus etc., ita vt designante multitudinem faclorum o, $b, y$ etc. inuentio radicum $\Omega$ ad resolutionum , aequationum $a^{i \mathrm{i}}, \mathbf{6}^{\mathrm{ti}}, \gamma^{\mathrm{ti}}$ etc. gradus reducatur. E g. pro $n=$ $I 7$, vbi $n-x=2.2 .2 .2$, quatuor atquationes quadraticas soluere oportebit; pro $n=73$ tres quadraticas duasque cubicas.

Quum in sequentibus persaepe tales potestates radicis $r$ considerandae sint, quarum exponentes rursus sunt dignitates, huiusmodi exnressiones autem non sine molestia typis describantur: ad facilitandam impressionem sequenti in posterum abbreuiatione vtemur. Pro $r, r r, r$ etc. scribemus [1], [2], [5] etc, generaliterque pro $r^{\lambda}$, denotante $\lambda$ integrum quentunque, [A]. 'Tales itaque expressiones penitus determinatae nondum sunt, sed fiunt, simnlac pro $r$ sine [1] radix determinata ex $\Omega$ accipitur. Erunt itaque generaliter $[\lambda],[\mu]$ aequales vel inaequales, prout $\lambda, \mu$ secundum modultum $n$ congrui sunt vel incongrui; porro $[0]=1 ; \cdot \lambda] \cdot[\mu]=[\lambda+$ $\mu] ;[\lambda]^{r}=[\lambda] ;$ summa $[0]+[\lambda]+[2 \lambda] \ldots+$ $[(n-1) \lambda]$ vel o vel $n$, prout $\lambda$ per $n$ hon diuisibilis est vel diuisibilis.

34.5. Si, pro modulo $n, g$ est numerus talis, qualem in sect. III radictm primititum diximus, $n-1$ mumeri $1, g, g 6 \cdot \ldots g^{\text {ma }}$ his $1, a$, $5 \ldots n-1$ secundum mod. $n$ congrui erum, etsi alio ordine, puta quinis numerus vaus seriet 


\section{$-604-$}

congruum habebit in altera. Hinc sponte kequitur, radices $[\mathrm{I}],[g],[g g] \cdots\left[g^{\mathrm{sin}}\right]$ cum $\Omega$ coincidere; et prorsus simili modo guneralius

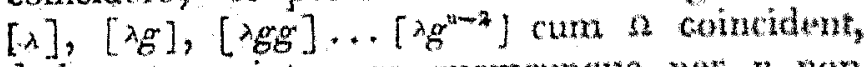
designante $\lambda$ integrum quemcunque por $n$ non diuisibilem. Porro quun sit $g^{\ln x}=1$ (mod, $n$, nullo. negotio perspicietur, duas radices [s $\left.g^{* t}\right]$ $\left[\lambda g^{\prime}\right]$ identicas vel diuessas esse, prout pas "st. cundum $n-1$ congrui sint vel incongrub.

Si itaque $G$ est alia radix primitiua, ratices $[1],[g] \cdots\left[g^{\mathrm{n}-2}\right]$ etiam cum his $[1],[G] \ldots$ $\left[G^{n-2}\right]$ contenient, si ad ordinem non rapiritur. Sed praeterea facile probatur, st $c$ sil thuisor ipsius $n-1$, atque ponatur $n-1=0$ s $\mathrm{g}^{*}=\bar{h}, G^{*}=H$, etiam f numeros $1, h_{,} h$ $\ldots h f^{-1}$ his $1, H, H^{*} \ldots h J^{-1}$ secundum $n$ com gruos esse (sine respectu ordinis: Supponamans

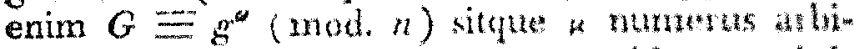
trarius positiuus et $<f$ atque - residuma minti mum ipsius $\mu$ (mod. $f$ ). 'luns arit of

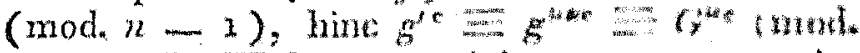

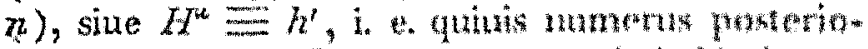
ris seriei $1, H, H^{2}$ etc congrum habelit is serie $1, h, h h \ldots$, " perinde vice vers. - Hhuc manifestum est, $f$ radices $[1],[h],|m|, \ldots|h m|$ identicas esse cum his [1], $[H],\left[H^{*}, \ldots\left[H^{*}\right]\right.$,

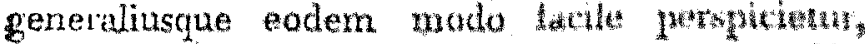
$[\lambda],[\lambda / h],[\lambda h h] \ldots(\lambda h-x]$ cum $1,1, h$, $\left[\lambda H^{2}\right] \cdots[\lambda / f(-x]$ conuenire. Aggregatton whin $f$ radicum $[\lambda]+[x h]+$ etc $+\left[x_{t}-x\right)$ quast,

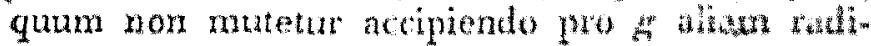
cem primitiuam, tamequan indepentens a g con siderandum est, per $(j, \lambda)$ designtatimus; earum- 


\section{$-$

dem radicum complexum vocabimus periodum $(f, x)$, vbi ad radicum ordinem non respicitur *). - In exhibenda tali periodo e re erit, singulas radices e quibus constat ad expressionem simplicissimam reducere, puta pro numeris $\lambda, \lambda h, \lambda h h$ etc. residua minima sec. mod, $n$ substituere, securdum quorum rxagnitudinem, si placet, etiam periodi partes ordinari poterunt.

E. g. Pro $n=19$, vbi 2 est radix primitiua, periodus $(6,1)$ constat e radicibus $[1],[8]$, $[6.4],[512],[4096],[32768]$, siue $[1],[7],[8]$, $[11],[12],[18]$. Similiter periodus $(6,2)$ constat ex $[2],[5],[5],[14],[16],\left[{ }^{7}\right]$. Perind $x$. $(6,3)$ cum praec. identica inuenitur. Periodus $(6,4)$ continet $[4],[6],[9],[10],[15],[15]$.

344. Circa huiusmodi periodos statim se offerunt obseruationes sequentes:

I. Quum sit $\lambda h f \equiv \lambda, \lambda h r+\mathrm{x} \equiv \lambda h$ etc. (mod. $n$ ), manifestum est, ex issdem radicibus, e quibus constet $(f, \lambda)$, etiam constare $(f, \lambda h)$, $(f, \lambda h h)$ etc.; generaliter itaque designante $\left[\lambda^{i}\right]$ radicem quamcunque ex $(f, \lambda)$, haec periodus cum $\left(f, x^{\prime}\right)$ omuino identica erit. Si itaque duae periodi ex aeque multis radicibus constantes (quales similes dicemus) vllam radicem commumem habent, manifesto identicae erunt. Quare fien nequit, vt duae radices in aliqua periodo simul contineantur, in alia simili vero ma earum tantum reperiatur; porro patet, si duat radices

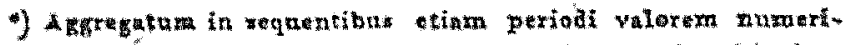

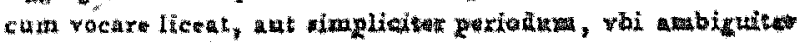
4. 


\section{- $606-$}

[x], [x] ad eandem periodun $f$ terminorum pertineant, valorem expr. $\frac{\lambda^{\prime}}{\lambda}(\bmod n)$ alicui potestati ipsius $h$ congruum esse, siue supponi posse $\lambda^{\prime} \equiv \lambda g^{\prime \bullet}(\bmod n)$.

II Si $f=n-1, a=1$, periodus ( $f$, 1.) manifesto cum $\Omega$ coincidit; in reliquis vero casibus $\Omega$ ex $e$ periodis $(f, 1),(f, g),(f, g g:)$ ... $\left(f, g^{c-1}\right)$ compositus erit. Hae periodi itaque omnino inter se diuersae erunt, patetque quamuis alian similem periodum $\left(f_{\lambda}, \lambda\right)$ cum harum aliqua coincidere, siquidem $[\lambda]$ ad $\Omega$ pertineat, j.e. si $x$ per $n$ non diuisibilis sit. Periodus $(f, 0)$ autem aut $(f, k n)$ manifesto ex $f$ vnitatibus est composita Aeque facile perspicitur, si $\lambda$ sit numerus quicunque per $n$ non diuisibilis, etiam complexum $e$ periodorum $(f, \lambda),(f, \lambda g),(f, \lambda g g)$ ... $\left(f, g^{g} g^{a-1}\right)$ cum $\Omega$ conuenire - Ita e. g. pro. $n=19, f=6, \Omega$ constat e tribus periodis $(6,1),(6,2),(6,4)$, ad quarum aliquam quaeuis alia similis, praeter $(6,0)$, reducitur.

III. Si $n-1$ est productum e tribus nummeris positiris $a, b, c$, manifestum est, quamuis periodum $b c$ terminorum ex $b$ periodis $c$ terminorum compositam esse, puta $(b c, \lambda)$ ex $(c$,

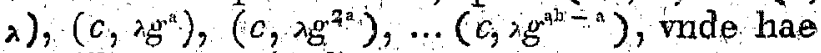
sub illa contentae dicentur. Ita pro $n=19$ periodis $(6,1)$ constat 'e tribus $(2,1),(2$, $8),(2,7)$, quarum prima continet radices $r$, $r^{\text {I8 }}$; secunda $r^{8}, r^{\text {II }}$, tertia $r^{\tau}, r^{\text {Iq }}$.

545. Theorema. Sint $(f, \lambda),(f, \mu)$ duae periodi similes, identicae aut ditiersae, constetque $(f, \lambda)$ e radicibus $[\lambda],[\lambda i],[\lambda ']$ etc. 
Tunc productum ex $(f, \lambda)$ in $(f, \mu)$ erit aggre gatum $f$ periodorum similium puta $=(f, x+$ $\left.\mu^{\mu}\right)+\left(f, \lambda^{\prime}+\mu\right)+\left(f ; \lambda^{\prime \prime}+\mu\right)+e t c .=W$.

Dem. Sit vt supra $n-1=e f ; g$ radix prinaitiua pro modulo $n$, atque $h=g^{\mathrm{e}}$, vnde per praecedentia erit $(f, \lambda)=(f, \lambda g)=(f, \lambda h h)$ etc. Hinc productum quaesitum erit $=[\mu] \cdot(f, \lambda)$ $+[\mu h] \cdot(f, \lambda h)+[\mu h h] \cdot(f, \lambda h h)+$ etc. adeoque $=$

$[\lambda+\mu]+[\lambda \hbar+\mu] \ldots+\left[\lambda h f^{-1}+\mu\right]$

$+[\lambda h+\mu h]+[\lambda h h+\mu h] \ldots+[\lambda h f+\mu h]$ $+[\lambda h h+\mu h h]+\left[\lambda h^{3}+\mu h h\right] \ldots+[\lambda h \dot{f}+1+\mu h h]$ etc.

quae expressio omnino ff radices continet. Quodsi hic singulae columnae verticales seorsim in summam colliguntur, manifesto prodit $(f, \lambda+\mu)$ $+(f, \lambda h+\mu) \ldots+\left(f, \lambda h^{f-1}+\mu\right)$, quam expressionem cum $W$ conuenire nullo negotio perspicitur, quum numeri $\lambda, \lambda^{\prime}, \lambda^{\prime \prime}$ etc per hyp. ipsis $\lambda, \lambda h, \lambda h h \ldots \lambda h f^{-1}$ secundum modulum $n$ congrui esse debeant (quonam orline hic nihil interest) adeoque etiam $\lambda+\mu, \lambda^{\prime}+\mu, \lambda^{\prime \prime}+\mu$ etc. ipsis $\lambda$. $+\mu, \lambda h+\mu, \lambda h h+\mu \ldots \lambda h^{f i}+\mu_{0} \quad$ Q. E. $D$.

Huic theoremati adiungimus corollaria ser quentia:

I. Designante $k$ integrum quemcunque, pro, ductum ex $\left(f, k_{\lambda}\right)$ in $\left(f, k_{k}\right)$ erit $=(f, k(\lambda$ $+\mu))+(f, k(\lambda+\mu))+\left(f, k(\lambda \mu+\mu)+\right.$ etc $^{\prime}$

II. Quum singulae partes, e quibus $W$ constat, vel cum aggregato $(f, 0)$, quod est $=$ 
$f$, vel cum aliquo ex his $(f, 1),(f, g),(f, g g)$ $\ldots\left(f, g^{0-1}\right)$ conueniant, $W$ ad formam sequentem reduci poterit $W=a f+b(f, x)+b^{4}(f$, $g)+b^{\prime \prime}(f, g g) \ldots+b^{g}\left(f, g^{a-1}\right)$, vbi coëfficientes $a, b, b^{\prime}$ etc. erunt integri positiui ( siue etiam quidam $=0$ ): porro patet, productnm ex $(f, k \lambda)$ in $(f, k, k)$ tunc fieri $=a f+\bar{b}(f, k)+$ $b(f, k)+b^{i}(f, k g) \ldots+b^{f}\left(f, \mathrm{~kg}^{e-1}\right)$. - Ita e. g. pro $n=19$ productum ex aggregato $(6,1)$ in se ipsum, siue quadratum huius aggregati fit $=$ $(6,2)+(6,8)+(6,9)+(6,12)+(6,13)$ $+(6,19)=6+2(6,1)+(6,2)+2(6,4)$.

III. Quum productum ex singulis partibus ipsius $W$ in periodum similem $(f, y)$ ad forman analogan reduci possit, manifestum est, etiam productum e tribus periodis $(f, \lambda) \cdot(f, \mu) \cdot(f, \mu)$ per $c f+d(f, g) \ldots+d^{7}\left(f, g^{a-1}\right)$ exhiberi posse, et coëfficientes $c, d$ etc. integros ac positiuos (siue $=0$ ) euadere, insuperque pro valore quocunque integro ipsius $k$ fieri $\left(f, k_{\lambda}\right) \cdot\left(f, k_{\mu}\right) \cdot\left(f, k_{\nu}\right)=$ $c f+d(f, k)+d^{\prime}(f, k g)+$ etc. Perinde hoc theorema ad producta e periodis similibus quotcunque extenditur, nihilque interest, siue hae periodi-omnes diuersae sint, siue partim aut cunctae identicae.

IV. Hinc colligitur, si in functione quacunque algebraica rationali integra $F=\phi\left(t, x_{g}\right.$ $v$...) pro indeterminatjs $t, u, v$ etc resp. substituantur periodi similes $(f, \lambda),(f, \mu) ;(f, r)$ etc. eius valorem ad formam $A+B(f, 1)+$ $B^{\prime}(f, g)+B^{\prime \prime}(f, g g) \ldots+B^{x}\left(f, g^{e-1}\right)$ reducibisem esse, coëfficientesque $A, B, B^{\prime}$ etc. omnes 


\section{$-609-$}

integros fieri, si omnes coëfficientes determinati in $F$ sint integri; si vero postea pro $t, u, v$ etc. resp. substituantur $(f, k \lambda),\left(f, k_{\mu}\right),\left(f, k_{v}\right)$ etc., valorem ipsius $F$ reduci ad $A+B(f, k)+$ $B^{\prime}(f, k g)+$ etc.

346. Throrema. Supponendo, $\lambda$ esse $\mathrm{nu}$ merum per $n$ non diuisibilem, et scribendo breuitatis ergo p pro $(f, \lambda)$, quaeuis alia similis periodus $\left(f,{ }_{\mu}\right)$, vbi etiam $\mu$ per $n$ non diuisibilis supponitur, reduci poterit sub formam talem a + $6 p+\gamma p p \ldots+\phi_{p} p^{-x}$, ita vt coëfficientes $a, b$ etc. sint quantitates determinatae rationales.

Dem. Designentur ad abbreuiandum periodi $(f, \lambda g),(f, \lambda g g),\left(f, \lambda g^{\text {i3 }}\right)$ etc. vsque ad $(f$, $\left.\lambda g^{o-x}\right)$, quarum multitudo est $e-1$, et cum quarum aliqua $(f, \mu)$ necessario conueniet, per $p^{\prime}, p^{\prime \prime}, p^{\prime \prime \prime}$ etc. Habetur itaque statim aequatio $0=1+p+p^{\prime}+p^{\prime \prime}+p^{\prime \prime \prime}$ etc. ... (I); euol. uendo autem secundum praecepta art. praec. valores potëstatum ipsius $p$ vsque ad $e-1^{\text {tam }}, e-$ 2 aliae tales promanabunt:

$0=p p+A+a p+a^{\prime} p^{\prime}+a^{\prime \prime} p^{\prime \prime}+a^{\prime \prime \prime} p^{\prime \prime \prime}+$ etc. (II) $0=p^{3}+B+b p+b^{\prime} p^{\prime}+b^{\prime \prime} p^{\prime \prime}+b^{\prime \prime \prime} p^{\prime \prime \prime}+$ etc. (III) $0=p^{4}+C+c p+c^{\prime} p^{\prime}+c^{\prime \prime} p^{\prime \prime}+c^{\prime \prime \prime} p^{\prime \prime \prime}+$ etc. (IV)

etc.

vbi omnes coëfficiènte's $A, a, a^{\prime}$ etc. $B, b, b^{\prime}$ etc. etc. erunt integri, atque, quod probe notandum est et ex art. praec. sponte sequitur, a $\lambda$ orminio inidependentes; i. e. eaedem aequationes etiamnum valebunt, quicunque alius valor ipsi $\lambda$ tribuatur; haec annotatio manifesto etiam ad aequi? I extenditur, si modo $\lambda$ per $n$ non diuisibilis accipiatur - Supponamus $(f, \mu)=p^{\prime} ;$ facillim Qq 
enim perspicietur, si $(f, \mu)$ cam alia perio do ex $p^{\prime \prime}, p^{\prime \prime \prime}$ etc. conueniat", ratiocinia sequentibus prorsus analoga adhiberi posse. Quum multitudo aequationum $I, I I$, III etc. sit $e-I$, quantitates $p^{\prime \prime}, p^{\prime \prime \prime}$ etc., quarum multitudo $\perp e$ - 2, per methodos notas inde eliminari possunt, ita vt prodeat aequatio talis ( $Z$ ') ab ipsis libera $0=\mathfrak{U}+\mathfrak{B} p+\mathfrak{S} p p$ etc. $+\mathfrak{M} p^{e-1}+\mathfrak{R} p^{\prime}$ quod ita fieri poterit, vt omnes coëfficientes $\mathfrak{R}$, $\mathfrak{B} \ldots \Re$ sint integri atque certe non omnes $\equiv 0$. Iam si hic non est $\mathfrak{g} t=0$, protinus liquet, $p^{\prime}$ inde ita vt in theoremate enunciatum est determinari. Superest itaque, vt demonstremus, $\mathfrak{R}$ $=0$ fieri non posse.

Supponendo esse $\mathfrak{R}=0$, aequatio $Z$. fit $\mathfrak{m} p^{-x}+$ etc. $+\mathfrak{B} p+\mathfrak{X}=0$, cui, quum vltra gradum $e-1^{\text {thm }}$ certo ron ascendat, plures quam $e-1$ valores diuersi ipsius $p$ satisfacere nequeunt. At quum aequationes, e quibus $Z$ deducta fuit, a $\lambda$ sint independentes, liquet, etiam $Z$ a $\lambda$ non pendere, siue locum habere, quicunque integer per $n$ non diuisibilis pro $\lambda$ accipiatur. Quare aequ. $Z$ satisfiet, cuicunque ex $e$ aggregatis $(f, 1),(f, g),(f, g g) \ldots\left(f, g^{c-x}\right)$ aequaLis statuatur $p$, vnde sponte sequitur, haec aggregata omnia inaequalia esse non posse, sed ad minimum duo inter se aequalia esse debere. Contineat vnum e duobus talibus aggregatis aequalibus radices $\left[\delta^{n}\right],\left[q^{\prime}\right],\left[\xi^{\prime \prime}\right]$ etc., alterum has $[\eta],\left[\eta^{\prime}\right],\left[\eta^{\prime \prime}\right]$ etc., supponamusque (quod licet), omnes numeros $\zeta, \zeta^{\prime}$, $\xi^{\prime \prime}$ etc, $n ; n^{\prime}$, $\eta^{\prime \prime}$ etc. esse positiuos et $<n$; manifesto omnes etiam diuersi erunt, nullusque $=0$. Designetux functio $x^{2}+$ 
$x^{5^{\prime}}+x^{5^{\prime \prime}}+$ etc. $-x^{n}-x^{\eta^{\prime}}-x^{x^{\prime \prime}}-$ etc. cuius terminus summus non vltra $x^{\mathrm{n}}-\mathrm{r}$ ascendet, per $Y$, patetque fieri $Y=0$ si statuatur $x=$ [1]; hinc $Y$ implicabit factorem $x-$ [I], quem cum functione in praec. per $X$ denotata communem habebit; hoc vero absurdum esse facile monstrari poterit. $\mathrm{Si}$ enim $Y$ cum $X$ vllum factorem communem haberet, diuisor communis $m a$ ximus functionum $\dot{X}, \quad$, quem certo vsque ad $n-1$ dimensiones ascendere non posse iam inde patet, quod $Y$ per $x$ est diuisibilis), omnes coëfficientes suos rationales haberet, vt e natura operationum, diuisorem communem maximum duarum talium functionum inuestigandi quarum coëfficientes omnes sunt rationales; sponte sequitur." Sed in art. 341 ostendimus, $X$ implicare non posse factorem pauciorum quam $n$ - 1 dimensionum, cuius coefficientes omnes sint rationales: quamobrem suppositio, esse $\mathfrak{N}=0$, consistere nequit.

Ex. Pro $n=19, f=6$, fit $p p=6+$ $2 p+p^{\prime}+2 p^{\prime \prime}$, vnde et ex $0=1+p+p^{\prime}+$ $p^{\prime \prime}$ deducitur $p^{\prime}=4-p p, p^{\prime \prime}=-5-p$ + pp. Quare $(6,2)=4-(6,1)^{2},(6,4)$ $=-5-(6,1)+(6,1)^{2} ;(6,4)=4-$ $(6,2)^{2},(6,1)=-5-(6,2)+(6,2)^{2}$ $(6,1)=4-(6,4)^{2},(6,2)=-5-$ $(6,4)+(6,4)^{2}$.

347. Theorema Si $F=\phi(t, u, v \ldots)$ est functio in uariabilis*) algebraica vationalis integra

-) Functiones inuariabiles eas voeari constat, gof

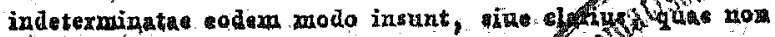




\section{$-61 \%$}

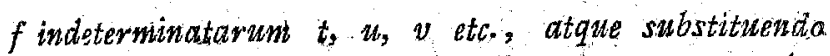
pro his $f$ radices in periodo $(f, \lambda)$ contentas valor ipsius $F$ per praecepta art. 340 ad formam $A+$ $A[1]+A^{i}[2]+{ }_{\text {etc. }}=W$ reducitur: radices quae in hac expressione ad eandem periodum quamingue $f$ terminorum pertinent coofficientes aequales habebunt.

Dem. Sint $[p],[q]$ duae radices ad vnain eandemque periodum pertinentes, supponanturque $p, q$ positiui et minores quam $n$, ita vt demonstrare oporteat, $[p]$ et $[q]$ in $W$ eundem coëflicientem habere. Sit $q \equiv p g^{r e}(\bmod . n)$; sint porro radices in $(f, \lambda)$ contentae $[\lambda],\left[\lambda^{\prime}\right]$, $\left[\lambda^{\prime \prime}\right]$ etc., vbi numeros $\lambda . \lambda^{\prime}, \lambda^{\prime \prime}$ etc positiuos et minores quam $n$ supponimus; denique sint residua minima positiva numerorum $\lambda g^{\prime p^{e}}, \lambda g^{\prime y_{6}}, \lambda^{\prime \prime \prime}$ etc., secundum modulum $n$, haec $\mu, \mu^{t}, \mu^{\prime \prime}$ etc., quae manifesto cum numeris $\lambda, \lambda^{\prime}, \lambda^{\prime \prime}$ etc. identici erurit, etsi ordine transposito. Iam ex art. 340 patet, $\Phi\left(\lambda g^{\prime e}, \lambda^{\prime} g^{\prime 2}, \lambda^{\prime \prime} g^{\prime \circ} \ldots\right)=$ (I) reduci ad $A+A^{\prime}\left[g^{\text {re }}\right]+A^{\prime \prime}\left[2 g^{\prime \theta}\right]+$ etc. aut ad $A+$ $A^{\prime}[\theta]+A^{\prime \prime}\left[\theta^{\prime}\right]+$ elc $=(W t)$, designando per $\theta, \theta$ etc. residua minima numerorum $g^{t \theta}, 2 g^{y_{\theta}}$ etc. secundum modulum $n$, vnde manifesțum est, $[q]$ habere eundem coëfficientem in (IVI), quem $[p]$ habeat in $(W)$. Sed nulla negotio perspicitur, ex euolutione expressionis (I) idem prouenire atque ex enolutione huius $\phi\left(\mu, \mu, \mu^{\prime \prime}\right.$ etc. $)$ quoniam $\mu=\lambda g^{\prime \mathrm{c}}, \mu^{\prime} \equiv \lambda^{\prime} g^{\mathrm{je}}$ etc $(\bmod n)$, haec vero expressio idem praducit ac baec $\phi\left(\lambda, \lambda^{\prime}, \lambda^{\prime \prime}\right.$

mutantur, quomodocunque indeterminatae inter se permutentur; chiusmodi sunt e. g, summa ommium, productim á omuibúa sunimą gioductoxum binis eto 


\section{- $615-$}

etc.) , quoniam numeri $k, \mu^{\prime}, \mu^{\prime \prime}$ etc ordine tantum ab his $\lambda, \lambda^{\prime}, \lambda^{\text {th }}$ etc. discrepant, cuius in functione inuariabili nibil interest. Hinc colligitur, $W$ i omnino identicam fore cum $W T$; quamobrem radix $[q]$ eundem coëfficientem in $W$ habebit vt $[p]$. Q. E. $D$.

Hinc manifestum est, W reduci. posse sub formam: $A+a(f ; 1)+a^{\prime}(f, g)+a^{\prime \prime}(f, g g)$ $\ldots+a^{a}\left(f, g^{a-x}\right)$, ita vt coêfficientes $A, a \ldots a^{b}$ sint quantitates determinatae, quae insuper integri erunt, si omnes coëfficientes rationales in $F$ sunt integri, - Ita e. g. si $n=19, f=6, \lambda$ $=1$, atque functio $\otimes$ designat aggregatum productorum e binis indeterminatis, eius valor reducitur ad $5+(6,1)+(6,4)$.

Porro facile perspicietur, si postea pro $t, u_{*}$ v etc. radices ex alia periodo $(f, k \lambda)$ substituantur, valorem ipsius $F$ fieri $A+a(f, k)+a^{\prime}\left(f_{i}\right.$ $k g)+a^{i \prime}(f, k g g)+$ etc.

348. Quum in aequatione quacunque $x$ $x^{f^{-I}}+6 x f^{-2}-y^{f^{-3}} \ldots=0$ coèfficientes $a_{2}$ $\dot{b}, \boldsymbol{\gamma}$ etc sint functiones inuariabiles radicum, puta a summa omnium, 6 summa productorum e binis, $\gamma$ summa productorum e ternis etc.: in aequatione cuius radices sunt radices in periodo $\left(f_{*}\right.$ a) contentae coëfficiens primus erit $=(f, \lambda)$, singuli reliqui vero sub formam talem $A+a(f, 1)$ $+a^{t}(f, g) \ldots+a^{s}\left(f, g^{e-1}\right)$ reduci poterunt, vbi omnes $A, a, a^{\prime}$ etc erunt integri praetereaque patet, aequationem cuins radices sint radices in quacunque alia periodo $(f, k \lambda)$ contentae ex illa Qq 5 


\section{$-614-$}

deriuari, si in singulis coefficientibus pro $(f, \mathbf{1})$ substituatur $(f, k) ;$ pro $(f, g),(f, k g)$ et generaliter pro $(f, p),(f, k p)$. Hoc itaque modo assignari poterunt $e$ aequationes $z=0, z^{\prime}=0$, $z^{\prime \prime}=0$ etc. quarum radices sint radices contentae in $(f, 1)$, in $(f, g),(f, g g)$ etc, quamprimum $e$ aggregata $(f, 1),(f, g),(f, g g)$ etc. innotuerunt, aut potius quamprimum vnum quodcunque eorum inuentum est, quoniam per árt. praec. ex vno omnia reliqua rationaliter deducere licet. Quo pacto simul functio $X$ in $e$ factores $f$ dimensionum resoluta habetur: productum enim e functionibus $z, z^{\prime}, z^{\prime \prime}$ etc. manifesto erit $=X$.

Ex. Pro $n=19$ summa omnium radicum in periodo $(6,1)$ est $=(6,1)=\alpha$; summa productorum e binis fit $=3+(6,1)+(6,4)$ $=6$; similiter summa productorum e ternis inuenitur $=2+2(6,1)+(6,2)=7$; summa productorum e quaternis $=3+(6,1)+$ $(6,4)=\delta$; summa productorum e quinis = $(6,1)=i$ productum ex omnibus $=1$; quare aequatio $z=x^{6}-\alpha x^{5}+6 x^{4}-\gamma x^{3}+$ $\delta x x-x x+1=0$ omnes radices in $(6,1)$ contentas ccmplectitur. Quodsi in coëfficientibus $\star 6,2$ etc pro $(6,1),(6,2),(6,4)$ resp. substituantu: $(6,2),(6,4),(6,1)$, prodibit aequatio $z=0$, quae radices in $(6,2)$ conplectetur; et si eadem commutatio hic denuo applicatur, habebitur aequatio $z^{\prime \prime}=0$, radices in $(6,4)$ complectens, productumque $z z^{\prime} z^{\prime \prime}$ erit $=X$.

349. Plerumque commodius est, praeserim quoties $f$ est numerus magnus, coëfficientes 
- $\gamma$ etc. secundum theorema Newtonianum e summis potestatum radicum deducere. Scilicet sponte patet, summam quadratorum radicum in $(f, \lambda)$ contentarum esse $=\left(f_{i} 2 \lambda\right)$, summam cuborum $=(f, 3 \lambda)$ etc Scribendo itaque breuitatis caussa pro $(f, \lambda),(f, 2 x),\left(f, 3^{\lambda}\right)$, etc. $q$, $q^{\prime}, q^{\prime \prime}$ etc. erit. $a=q, 2^{\prime \prime}=\alpha-q^{\prime}, 3^{\gamma}=\varepsilon q$ - $a q^{\prime}+q^{\prime \prime}$ etc., vbi producta e duabus periodis per art 345 statim in summas periodorum sint conuertenda. Ita in exemplo nostro, scribendo pro $(6,1),(6,2),(6,4)$ resp. $p, p^{\prime}, p^{\text {wa }}$ fiunt $q^{\prime}, q^{\prime}, q^{\prime \prime}, q^{\prime \prime \prime}, q^{\mathrm{N}}, q^{\mathrm{V}}$ resp. $=p, p^{\prime}, p^{\prime}, p^{\prime \prime}$, $p^{\prime}, p^{\prime \prime} ;$ hinc $\alpha=p, 26=p p-z^{\prime}=6+2 p$ $+2 p^{\prime \prime} ; 3 y=\left(3+p+p^{\prime \prime}\right) p-p p^{\prime}+p !=$ $6+6 p+3 p^{\prime} ; 4 \delta=\left(2+2 p+p^{\prime}\right) p-(3$ $\left.+p+p^{\prime \prime}\right) p^{\prime}+p p^{\prime}-p^{\prime \prime}=12+4 p+4 p^{\prime \prime}$ etc. Ceterum sufficit semissem coèfficientium santum hoc modo computare; etenim non difficile probatur, vltimos ordine inuerso primis vel àequales esse puta vitimum $=1$, penultimum $=\alpha$, antepenultimum $=6$ etc, vel ex iisdem resp, deduci, si pro $(f, 1),(f, g)$ eto substituantur $(f,-1),(f,-g)$ etc. siue $\left(f_{i} n-g\right)$, $(f, n-1)$ etc Casus prior locum habet quando $f$ est par; posterior quando $f$ impar; coèfficiens. vltimus autem semper fit $=1 . \quad$ Fundamentum huius rei innitituf thesoremati art $79 ;$ sed breui tatis caussa huic argumențo non inmoramur.

350. TuEOREMA. Sit $n-r$ productrome tri-s Gis integris positiuts $\alpha, b, \gamma$; constet periodus (by,

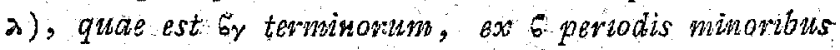
$\gamma$ tapminorunze izs $(\gamma, \lambda),\left(y, \lambda^{\prime}\right),\left(\% \lambda^{\prime \prime}\right)$ etc., supponamusque, si in functione. 6 indeteminatarim, si- 
militer affecta ot in art. praec., puta in $F=\varphi(t, u$, v...) pro indeterminatis $t, u, v$ etc substituantur aggregata $(\gamma, \lambda),\left(\gamma, \lambda^{\prime}\right),\left(\gamma, \lambda^{\prime \prime}\right)$ etc. resp., eius volorem per praecepta art. praec. 7 cduci ad $A+a(\gamma$, I) $+a^{\prime}(\gamma, g) \ldots+a \zeta\left(\gamma, g^{x \sigma \ldots \alpha}\right) \ldots+a a^{\alpha}(\lambda$, $\left.g^{x^{6}-\mathrm{x}}\right)=W$. Tum dico, si $F$ sit functio inuariabitis, ens periodos in $W$, quat sub eatem pexiodo or terminorum contentae sint, i. e. generaliter tales ( $\gamma$, $\left.g^{\mu}\right)$ et $\left(y, g^{x-1-k}\right)$ designante integrum quemcunque, coêfficientes eosdem habituras esse.

Dem. Quum periodus $\left(\xi \gamma, \lambda g^{a}\right)$ identica sit cum hac $(6 y, \lambda)$, minores hale $\left(\gamma, \lambda g^{\alpha}\right),(\gamma$, $\left.\lambda^{\prime} g^{\alpha}\right),\left(\gamma, \lambda^{\prime \prime} g^{\alpha}\right)$ etc., e quibus manifesto prior constat, necessario cum iis conuenient e quibus posterior constat, etsi alio ordine. Quodsi itaque, illis pro $t, u, v$ etc resp. substitutis, $F$ in Wh transire supponitur, $W$ coincidet cum $W$. At per art. 347 erit $W^{\prime}=A+a\left(\gamma, g^{\prime \prime}\right)+a^{\prime}(\gamma /$ $\left.g^{\alpha+1}\right) \ldots+a^{5}\left(\gamma, g^{\alpha}\right) \ldots+a^{\alpha}\left(\gamma, g^{\alpha \beta}+\alpha-x\right)=$ $A+a\left(\gamma, g^{\alpha}\right)+a^{\prime}\left(\gamma, g^{\alpha+1}\right) \ldots+a^{\xi}(\gamma, 1) \ldots$ $+a^{\theta}\left(\gamma, g^{\alpha-r}\right) ;$ quare quum haec expressio cum $W$ conuenire debeat, coëficiens primus, secundus, tertius etc. in $W$ (incipiendo ab $a$ ) necessario conueniet cum $\alpha+1^{\text {to }}+2^{\text {to }}, a^{4}+3^{\text {to }}$ etc. vnde nullo negotio concluditur, generaliter coëfficientes periodorum $\left(\gamma, g^{\mu}\right),\left(\gamma, g^{\alpha+\mu}\right),(\gamma$, $\left.g^{2 \alpha+\mu}\right) \ldots\left(\gamma, g^{\alpha+\mu}\right)$, qui sunt $\mu+x^{\text {tua }}, \alpha+$ $\mu+I^{\text {thus }}, 2 a+\mu+1^{\text {this }} \ldots v \alpha+\mu+1^{\text {tus }}$, inter se conuenire debere. Q. E. D.

Hinc manifestum est, $W$ reduci posse ad formam $A+a(6, \gamma)+a^{\prime}(6 \gamma, g) \ldots+a\left(\sigma_{\gamma}\right.$ $\left.g^{x-x}\right)$, vbi omnes coefficientes $A, a$ etc. integri 
erunt, si omnes coêfficientes determinati in $F$ sunt integri. Porro facile perspicietur, si posted pro indeterminatis in $F$ substituantur 6 periodi $\%$ terminorum in alia periodo $b_{\gamma}$ terminorum puta in $\left(\zeta_{\gamma}, \lambda k\right)$ contentaé, quae manifesto erunt $(\gamma$, $\lambda k),\left(\nu, \lambda^{\prime} k\right),\left(\nu, \lambda^{\prime \prime} k\right)$ etc., valorem inde prodeuntem fore $A+a\left(b_{\gamma}, k\right)+a^{t}\left(b_{\gamma}, g h\right) \ldots+$ $a^{2}\left(b_{y}, g_{i}^{\alpha-x} k\right)$.

Ceterrum patet, theorema ad eum quoque casum extendi posse, vbi $\alpha=1$, siue $6_{\gamma}=n$ - 1 ; scilicet hic omnes coêfficientes in $t V$ aequales erunt, vide $W$ reducetur sub formam $A$ $+a(6 \gamma, 1)$

351. Retentis itaqué omnibus signis art. praec., manifestum est, singulos coëfficientes aequationis, cuius radices sunt 6 aggregata $(\gamma, \lambda)$, $\left(v, \lambda^{\prime}\right),\left(\gamma, \lambda^{\prime \prime}\right)$ etc., sub formam talem $A+$ $a(6 y,-1)+a^{\prime}(6 y, g) \ldots+a^{y}\left(6 y, g^{\alpha-x}\right)$ reduci posse, atque numeros $A$, a etc. omnes fieri integros, aequationem autem, cuius radices sint 6 periodi $\gamma$ terminorum in alia periodo $\left(6_{y}, k_{x}\right)$ contentae, ex illa deriuari, si vbique in coëfficientibus pro qualibet periodo $\left(\zeta_{y}, \mu\right)$ substituatur $\left(\sigma_{y}, k_{\mu}\right)$. Si igitur $\alpha=1$, omnes 6 periodi $\gamma$ terminorum determinabuntur per aequationem $6^{\text {th }}$ gradus, cuius singuli coêfficientes sub formam $A+a\left(\sigma_{\gamma}, 1\right)$ rediguntur, adeoque sunt quantitates cognitae; quoniam $\left(6^{6}, 1\right)=$ $(n-1,1)=-1$. Si vero $\alpha>1$, coëfficientes aequationis, cuius radices sunt omnes periodi $\gamma$ terminorum in aliqua periodo data $b_{\gamma}$ ter minorum contentae, quantitates cọnitáe erunt,

Qq5 
simulac valores numerici omnium a periodorum 6r terminorum innotuerunt. - Ceterum calculus coëfficientium harum aequationum saepe commodius instituitur, praesertim quando G/ non est valde paruus, si primo summae potestatum radicum eruuntur, ac dein ex his per theorema Newtonianum coèfficientes deducuntur, simili modo vt supra art. 34.9 .

Ex. I. Quaeritur pro $n=19$ aequatio cuius radices sint aggregata $(6,1),(6,2),(6$; 4). Designando has radices per $p, p^{\prime}, p^{\prime \prime}$ resp., et aequationem quaesitam per $x^{3} A x x+B x$ $-C=0$, fit $A=p+p^{\prime}+p^{\prime \prime}, B=p p^{\prime}+$ $p p^{\prime \prime}+p^{\prime} p^{\prime \prime}, C=p p^{\prime} p^{\prime \prime}$. Hinc $A=(18,1)$ $=-19$ porro habetur $p p^{\prime}=p+2 p^{\prime}+3 p^{\prime \prime}$ $p p^{\prime \prime}=2 p+3 p^{\prime}+p^{\prime \prime}, p^{\prime \prime}=3 p+p^{\prime \prime}+2 p^{\prime \prime}$, vnde $B=6\left(p+p^{\prime}+p^{\prime \prime}\right)=6(18,1)=-6$; denique fit $c=\left(p+2 p^{\prime}+3 p^{\prime \prime}\right) p^{\prime \prime}=3(6,0)$ $+11\left(p+p^{\prime}+p^{\prime \prime}\right)=18-11=7$; quare aequatio quaesita $x^{3}+x x-6 x-7=0$ Vtendo methodo altera habemus $p+p^{\prime}+p^{\prime \prime}=$ $-1 ; p p=6+2 p+p^{\prime}+2 p^{\prime \prime}, p p^{\prime}=6+$ $2 p^{\prime}+p^{\prime \prime}+2 p, p^{\prime \prime} p^{\prime \prime}=6+2 p^{\prime \prime}+p+2 p^{\prime}$, vnde $p p+p^{\prime} p^{\prime}+p^{\prime \prime} p^{\prime \prime}=18+5\left(p+p^{\prime}+p^{\prime \prime}\right)_{1}=13 ;$ similiterque $p^{3}+p^{\prime 3}+p^{\prime 3}=36+34\left(p+p^{\prime \prime}\right.$ $\left.t p^{\prime \prime}\right)=2$, hinc per theorema Newtonianum eadem aequatio deriuatur vt ante

II. Quaeritur pra $n=19$ aequatio cuivs radices sint aggregata $(2,1),(2, z),(2,8)$. Quibus resp. per $q, q^{\prime}, q^{\prime \prime}$ designatis, inuenitur $q^{\prime}+q^{\prime}+q^{\prime \prime}=(6,1), q q^{\prime}+q q^{\prime \prime}+q^{\prime} q^{\prime \prime}=$ $(6,1)+(6,4), q q^{\prime} q^{\prime \prime}=\mathbf{2}+(6,2)$, wnde, 
retentis signis ex. praec, aequatio quaesita erit $x^{3}-p x x+\left(p+p^{\prime \prime}\right) x-2-p^{\prime}=0$. Aequatio cuius radices sunt aggregata $(2,2),(2$, $3),(2,5)$, sub $(6,2)$ contenta, e praecedente deducitur, substituendo pro $p, p^{\prime}, p^{\prime \prime}$ resp. $p^{\prime}, p^{\prime \prime}$, $p$, eaảemque substitutione iterum facta, prodit aequatio, cuius radices sunt aggregata $(2,4),(2$, $6),(2,9)$ sub $(6,4)$ contenta

352. Theoremata praecedentia cum consectariis annexis praecipua totius theoriae momenta continent, modusque valores radicum $\Omega$ inueniendi paucis iam tradi poterit.

Ante omnia áceipiendus est numerus $g$, qui pro modulo $n$ sit radix primitiua, residuaque minima potestatum ipsius $g$ vsque ad $g^{x-2}$ secundum modulum $n$ eruenda. Resoluatur $n+1$ in factores, et quidem, si problema ad aequationes gradus: quarm infimi reducere lubet, in factores primos; sint hi (ordine prorsus arbitrario) $4, b, y \ldots \xi_{\text {; }}$ ponaturque $\frac{n-I}{a}=b \gamma \ldots s=a, \frac{n-I}{a b}=$ $x \ldots s=b$ etc Distribuantur omnes radices $\Omega$ in a periodos a terminorum; hae singulae russus in 6 periodos $b$ terminorum; hae singulae denuo in $\gamma$ periodos etc. Quaeratur per art. $35^{\circ}$ aequatio $a^{\text {th }}$ gradus $(A)$, cuius radices sint illa aggregata $a$ terminorum, quorum itaque valores: per resolutionem huius aequationis innotescent.

At hic difficultas oritur, quum incertum videatur, cuinam radici aequationis (A) quoduis aggregatum aequale statuendum sit, puta quae 
nam radix per $(a, 1)$, quaenam per $(a, g)$ etc. denotari debeat: huic rei sequenti modo remedium afferri poterit. $\operatorname{Per}(a, 1)$ designari potest radix quaecunque aequationis $(A)$; quum enim quaeuis radix huius aequ. sit aggregatum $a$ radicum ex $\Omega$, omninoque arbitrarium sit, quaenam radix ex $\Omega$ per $[1]$ denotetur, manifesto supponere licebit, aliquam ex is radicibus, e quibus radix quaecunque data aequ. $(A)$ constat, per [1] exprimi, vnde illa radix aequ. $(A)$ fiet $(a, 1)$; radix $[1]$ vero hinc nondum penitus determinatur, sed etiamnum prorsus arbitrarium seu indefinitum manet, quamnam radicem $e x$ is quae $(a, 1)$ constituunt pro $[1]$ adoptare velitrus. Simulac vero $(a, 1)$ determinatum est, etiam omnia reliqua aggregata $a$ terminorum *ationaliter inde deduci poterunt (art 346 ). Hinc simul patet, vnicam tantummodo radicern per huius resolutionem eruere oportere. - Potest etiam methodus sequens, minus directa, ad hunc finem adhiberi. Accipiatur pro [1] radix determinata, i. e. ponatur $[1]=\cos \frac{k P}{n}+i \sin \frac{k P}{n}$, integro $k$ ad lubitum electo, ita tamen vt per $n$ hon sit diuisibilis; quo facto etiam [2], [3] etc. radices determinatais indicabunt, vnde etiam aggregata $(a, 1),(a, g)$ etc. quemtitates determinatas designabunt. Quibus e tabulis sinuum leui tantum calamo computatis, putá ea praecisionene, $\mathrm{vt}$ quae maiore qraeue minora sint decidi passit, nullum dubium superesse poterit, quibusnam signis singulae radices aequ. $(A)$ sint distinguendae.

Quando hoc modo omnia a aggregata $a$ terminorum innenta sunt, inuestigetur per art. 3 to 
aequatio $(B) 6^{\text {ti }}$ gradus, cuius radices sint $6 \mathrm{ag}-$ gregata $b$ terminorum sub $(a, 1)$ contenta; coêfficientes huius aequationis omnes erunt quantitates cognitae Quum adhuc arbitrarium sit, quaenam ex $a=6 b$ radicibus sub $(a, 1)$ contentis per [1] denotetur, quaelibet radix data aequ. (B) per $(b, 1)$ exprimi poterit, quia nanifesto supponere licet, aliquam $b$ radicum e quibus composita est per [1] denotari. Inuestigetur itaque vna radix quaecunque aequationis $(B)$ per eius resolutionem, statuatur $=(b, 1)$, deriuen, turque inde per art. 346 ornnia reliqua aggregata $b$ terminorum. Hoc modo simul calcul confirmationem nanciscimur, quium semper ea aggregata $b$ terminorum, quae ad easdem periodos $a$ terminorum pertinent, summas notas conficere debeant. - In quibusdam casibus aeque expeditum esse potest, $a-1$ alias aequationes $\zeta^{\mathrm{ti}}$ gradus, eruere, quarum radices sint resp. singula 6 aggregata $b$ terminorum in reliquis periodis a terminorum, $(a, g),(a, g g)$ etc contenta, atque omnes radices tum harum aequationum tum aequationis $B$ per resolutionem inuestigare: tunc vero simili modo vt supra adiumento tabulae sinuum decidere oportebit, quibusnam periodis $\tilde{b}$ terminorum singulae radices hoc modo prodeuntes aequales statui debeant. Ceterum ad hocce iudicium varia alia artificia adhiberi possunt, quae hoc loco complete explicare non licet; vnum tamen, pro eo casu vbi $\varepsilon=2$, quod imprimis vtile est, ac per exempla breuius quam per praecepta declarari poterit, in exempli sequentibus cognoscere licebit. 
Postquam hoc modo valores omnium $\alpha 6$ aggregatorum $b$ terminorum inuenti sunt, prorsus simili modo hinc per aequationum $\mathcal{2}^{\text {ti }}$ gradus ommia $\alpha$ aggregata $c$ terminorum determipari poterunt. Scilicet vel vnam aequationem $r^{\text {ti }}$ gradus cúins radices sint $\gamma$ aggregata $c$ terminorum sub $(b, 1)$ contenta, per art. 350 eruere; per eius resolutionem vnam radicem quancunque elicere et $=(c, 1)$ statuere, tandernque hinc per art. 346 omnia reliqua similia aggregata deducere oportebit; vel simili modo omnino 46 aequationes $r^{\text {ti }}$ gradus euoluere, quarum radices sint resp. $x$ aggregata $c$ terminorum in singulis periodis $b$ termiriorum contenta, valores omnium radicum omnium harum aequationum per resolutionem extrahere, candemque ordinem harum radicum perinde vt supra adiumento tabitlae sinum, vel, pro $y=2$, per artificium infra in exemplis ostendendum determinare.

Hoc modo pergendo, mañifesto tandem ominia $\frac{n 1}{\zeta}$ aggregata $\zeta$ terminorum habebuntur; euoluendo itaque per art. 348 aequationem $\zeta^{\text {ti }}$ gradus, cuius radices sint ? radices ex $a$ in $(\zeta, 1)$ contentae, huius coefficientes omnes erunt quantitates cognitae; quodsi per resolutionem via eius radix quaecunque elicitur, hanc $=[1]$ statuere Jicebit, omnesque reliquae radices $\Omega$ per huius potestates habebuntur, $\mathrm{Si}$ 'magnis placet, etiam omnes radices jllius aequationis, per resolutionem erui, praetereaquae per solutionem $\frac{n-x}{\zeta}-1$ aliarum aequationum gradus, quae resp, omnes $\zeta$ radices in singulis reliquis péxio- 
dis 's terminorum contentas exhibent, omnes reliquae radices $\Omega$ inueniri poterunt.

Ceterum patet, simulac prima aequatio $(A)$ soluta sit, siue simulac valores onnium * aggregatorum $a$ terminorum habeantur, etiam resolutionem functionis $X$ in a factores $a$ dimensionum per art. 34.8 sponte haberi; porroque post solutionem aequ. $(B)$, siue postquam valores omnium $\alpha^{b}$ aggregatorum $b$ terminorum inuenti sint, singulos illos factores iterúm in $\epsilon$, siue $X$ in ${ }^{b}$ factores $b$ dimensionum resolui etc.

353. Exemplum primum pro $n=19$. Quum hic fiat $n-1=3: 5.2$, inuentio radicum $\Omega$ ad solutionem duarum aequationum cubicarum vniusque quadraticae est reducenda. Hoc exemplum eo facilius intelligetur, quod operationes necèssariae ad maximam partem in praecedentibus iam sunt contentae. Accipiendo pro radice primitiua $g$ numerum 2 , residua minima eius potestatum haec prodeunt (exponentes, potestatum in serie prima residuis surt suprascripti).

0.1.2.3. 4. 5.6. 7.8. 9.10.11.12.13.14.15.16.17 1.2.4.8.16.13.7.14.9.18.17.15.11. 3. 6.12. 5.10

Hinc per artt. 344,345 facile deducitur distributio sequens omnium radicum $s$ in tres periodos senorum, harumque singularum in ternas binorum terminorum: 


$$
\Omega=(18,1)\left\{\begin{array}{r}
62,1)\left\{\begin{array}{l}
(2,1) \ldots[1],[18] \\
(2,8) \ldots[8],[11] \\
(2,7) \ldots[7],[12]
\end{array}\right. \\
(6,2)\left\{\begin{array}{l}
(2,2) \ldots[2],[17] \\
(2,16) \ldots[3],[16] \\
(2,14) \ldots[5],[14]
\end{array}\right. \\
(6,4) \begin{cases}(2,4) \ldots[4],[15] \\
(2,13) \ldots[6],[13] \\
(2,9) \ldots[9],[10]\end{cases}
\end{array}\right.
$$

Aequatio $(A)$, cuius radices sunt aggregata $(6,1),(6,2),(6,4)$, muenitur $x^{3}+x x-$ $6 x-7=0$, cuius nna radix eruitur $=$ $1,2,218761623$. Hanc per $(6,1)$ exprimendo fit $(6,2)=4-(6,1)^{2}=2,5070186441$, $(6,4)=-5-(6,1)+(6,1)^{2}=-$ 2,2851424818. Hinc $X$ in tres factores $6 \mathrm{di}$ mensionum resoluta erit, si hi valores in art. 348 substituurntur.

Aequatio $(B)$, cuius radices sunt aggregata $(2, x),(2,7),(2,8)$, prodit haec $x^{3}-(6$, 1) $x x+((6,1)+(6,4)) x-2-(6,2)$ - o siue

$$
\begin{gathered}
x^{3}+1,2218761623 x x-3,5070186441 x- \\
4,5070186441=0
\end{gathered}
$$

cuius vna radix elicitur $-1,3545631453$, quam per $(2, I)$ exprimemus. Per methodum art. 346 autern inueriuntur aequationes sequentes, vbi breuitatis caussa $q$ pro $(2, x)$ scribitur: $(2,2)=$ $q q-2,(2,3)=q^{3}-3 q,(2,4)=q^{4}-$ $4 q q+2,(2,5)=q^{5}-5 q^{2}+5 q,(2,6)=$ 
$q^{6}-6 q^{4}+9 q q-2,(2,7)=q^{7}-7 q^{9}$ $14 q^{3}-7 q,(2,8)=q^{3}-8 q^{3}+20 q^{4}-$ $16 q q+2,(2,9)=q^{9}-9 q^{7}+27 q^{5}-$ $309^{3}+99$. Commodius quam per praecepta art. 346 hae aequationes in casu praesenti per reflexiones sequentes euolui possunt. Supponendo $[1]=\cos \frac{k P}{19}+i \sin \frac{k P}{19}$, fit $[18]=$ $\cos \frac{18 k P}{19}+i \sin \frac{18 k P}{19}=\cos \frac{k P}{19}-i \sin \frac{k P}{19}$, adeoque $(2,1)=2 \cos \frac{k P}{19} ;$ nec non generaliter $[\lambda]=\cos \frac{\lambda k P}{19}+i \sin \frac{\lambda k P}{19}$, adeoque $(2, \lambda)=[\lambda]+\left[18^{\lambda}\right]=[\lambda]+[-\lambda]=$ $2 \cos \frac{\lambda k P}{19} \quad$ Quare si $\frac{\pi}{2} q=\cos \omega$, erit $(2,2)$ $=2 \cos 2 \cdots,(2,3)=2 \cos 3$ etc., vnde per aequationes notas pro cosinubus angulorum multiplicium eaedem formulae vt supra deriuantur. Iam ex his formulis valores sumerici sequentes eliciuntur:

$(2,2)=-0,1651586909(2,6)=0,4909709743$ $(2,3)=1,5782810188(2,7)=-1,7589475024$ $(2,4)=-1,9727226068(2,8)=1,8916344834$ $(2,5)=1,0938963162](2,9)=-0,8033908493$

Valores ipsorum $(2,7),(2,8)$ etiam ex àequatione $(B)$, cuius duae reliquae radices sunt, elici possunt; dubiumque, vtra harum radicum fiat $(2,7)$ et vtra $(2,8)$, vel per calculum approximatum secundum formulas praecc., vel per tabulas sinuum tolletur, quae obiter tantum consultae ostendunt, fieri $(2,1)=2 \cos \omega$ po. 
rièndo $\omega=\frac{7}{19} P$, wide fieri oportet $(2,7)=$ $2 \cos \frac{49}{19} P=2 \cos \frac{8}{19} P$, et $(2,8)=2 \cos \frac{56}{x 9} P$ $=2 \cos \frac{I}{19} P_{0}-$ Similiter aggregata $(2,2),(2$, $3),(2,5)$ etiam per aequationem $x^{3}-(6$, 2) $x x+((6,1)+(6,2)) x-2-(6,4)$ 0 , cuius radices sunt, inuenire licet, incertinudoque, quaenam radices illis aggregatis resp. aequales statuendae sint, prorsus eodem modo remouebitur, vt ante? et perinde etiam ag gregata $(2,4),(2,6),(2,9)$ per aequationem $x^{3}-(6,4) x x+((6,2)+(6,4)) x-2$ $-(6,1)=0$ elici poterunt.

Denique $[1]$ et $[18]$ sunt radices aequationis $x x-(2,1) x+1=0$, quarum altera fit $=\frac{r}{2}(2,1)+i \sqrt{ }\left(1-\frac{x}{4}(2,1)^{2}\right)=\frac{x}{2}(2$, 1) $+i \sqrt{ }\left(\frac{1}{2}-\frac{I}{4}(2,2)\right)$, altera $=\frac{x}{2}(2,1)-$ $i \sqrt{ }\left(\frac{7}{2}-\frac{\pi^{2}}{4}(2,2)\right)$, hinc valores numerici $=$ $-0,6772815716+0,7357239107 i$. Sedecim radices reliquae vel ex euolutione potestatum vtriusuis harum radicum, vel e solutione octo aliarum similium aequationum deduci possunt, vbi in methodo posteriori vel per tabulas sinuum vel per artificium in ex. sq. explicandum decidi debebit, pro vtra radice parti imaginariae signum positiuum et pro ytra negatiuum praefigendum sit. Hoc modo inuenti sunt valores sequentes, vbi signum superius radici priori, inferius posterioxi respondere supponitur. 


\section{- 627.}

[1] et $[18]=-0,6772815716 \pm 0,73572591072$

$[2]$ et $[17]=-0,0825793455 \mp 0,9965844930 i$

[3] et $[16]=0,7891405094+0,6142127127 i$

[4] et $[15]=-0,9863613034+0,1645945903 i$

$[5]$ et $[14]=0,5469481581 \mp 0,8371664783 i$

$[6]$ et $[13]=0,2454854871 \pm 0,9694.002659 i$

[7] et $[12]=-0,87947375^{12} \mp 0,4759473930 i$

[8] et $[: 1]=0,9458172417+0,3246994692 i$

[9] et $[10]=-0,4016954247 \pm 0,9557733267 \dot{ }$

354. Exemplum secundum pro $n=17$. Hic habetur $n-I=2.2 .2 .2$, quamobrem calculus radicum $\&$ ad quatuor aequationes quadraticas reducendus erit Pro radice primitiua hic accipiemus vumerum 3 , cuius potestates residua minima sequentia secundum modulum I 7 sup. peditant:

0.t.2. $5 \cdot 4.5 \cdot 6.7 \cdot 8 \cdot 9.10 .1$ I.I 2.13 .14 .95 I.3.9.1 0.15.5.I5.II.I6.14.8.7. 4.12. 2. 6

Hiric emergunt distributiones sequentes complexus $\Omega$ in periodos duas octonorum, quatuor quaternorum, octo binorum terminorum:

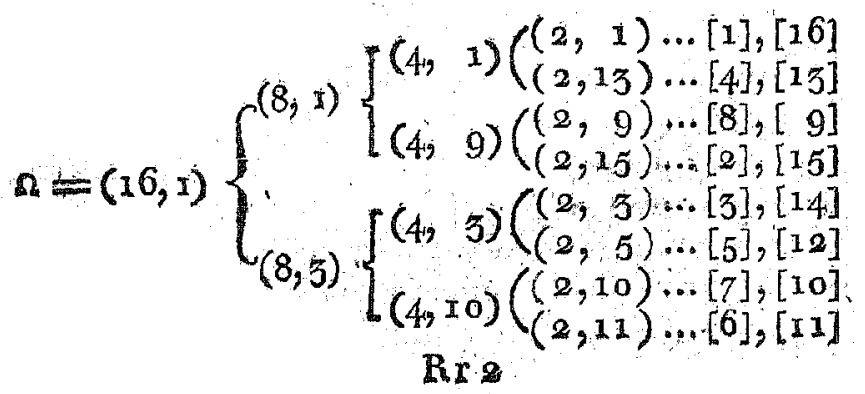


Aequatio $(A)$, cuius radices sunt aggregata $(8,1),(8,3)$, per praecepta art $35 \mathrm{I}$ inuenitur haec $x x+x-4=0$; huius radices computantur $-\frac{x}{2}+\frac{1}{2} \sqrt{I 7}=1,5615528128$, et $-\frac{x}{2}-x \sqrt{17}=-2,5615528128$; priorem statruemus $=(8,1)$, vnde necessario posterior ponenda erit $=(8,3)$.

Porro aequatio, cuius radices sunt aggregata $(4, I)$ et $(4,9)$, eruitur haec $(B): x x-(8$, I) $x-1=0$; huius radices sunt $\frac{T}{2}(8,1)$ $\pm \frac{\pi}{2} \sqrt{ }\left(4+(8,1)^{2}\right)=\frac{x}{2}(8,1) \pm \frac{1}{2} \sqrt{ }(12$ $+3(8, I)+4(8,3))$; eam in qua quantitati radicali signum positiuum tribuitur, et cuins valor numericus est $2,04948 \times 1777$, statuemt's $=$ $(4,1)$, vnde sponte altera, vbi quantitas radicalis negatiue sumitur et cuius valor est $0,4879^{2} 83649$, per $(4,9)$ exprimi debebit. Aggregata autem reliqua quatuor terminorum, puta $(4,3)$ et ( 4, ro) duplici modo indagari possunt. Scilicet primo per methodum art. 346 , quae formulas sequentes suppeditat, vbi ad abbreuiandum pro $(4, I)$ scribitur $p$ :

$$
\begin{aligned}
& (4,3)=-\frac{3}{2}+3 p-\frac{\pi}{2} p^{3}=0,3441507314 \\
& (4,10)=\frac{3}{2}+2 p-p p-\frac{\pi}{2} p^{3}=-2,9057035442
\end{aligned}
$$

Eadem methodus etiam hanc formulam largitur $(4,9)=-\mathrm{I}-6 p+p p+p^{7}$, vnde valor idem elicitur quem ante tradidinus. Secundo vero aggregata $(4,3),(4$, I0) etiam per ressolutionem aequationis cuius radices sunt determinare licet, quae aequatio fit $x x-(8$, $5-1=0$, vnde eius radices sunt $\frac{1}{3}(8,3)$ 
$\pm \frac{\pi}{2} \sqrt{ }\left(4+(8,5)^{2}\right)$, siue $\frac{\pi}{2}(8,3)+\frac{\pi}{2} \sqrt{(12}$ $+4(8, I)+3(8,3))$ et $\frac{T}{2} \sqrt{ }(8,3)-\frac{\pi}{2} \sqrt{ }(I 2$ $+4(8, \mathrm{r})+3(8,3))$; dubium vero, vtram radicem per $(4,3)$ et vtram per $(4$, io $)$ exprimere oporteat, per artificium sequens cuius mentionem in art. $35^{2}$ iniecimus tolletur. Euoluatur productum ex $(4, I)-(4,9)$ in $(4$, $3)-4$, to $)$, vnde emergere inuenietur 2 ( 8 , I) $\left.-2(8,3)^{*}\right)$; iam huius expressionis valor manifesto est positiuus puta $=+2 \sqrt{ } 17$, praetereaque etiam producti factor primus $(4, I)-$ $(4,9)$ positiuus est puta $=+\sqrt{ }\left(T_{2}+3(8\right.$, I) $+4(8,3))$, quare necessario etiam alter factor $(4,3)-(4$, Io $)$ positiuus esse debebit, et proin $(4,3)$ radici priori in qua signum positiuum radicali praefigitur, et $(4$, ro) posteriori aequale statui. Ceterum hinc iidem valores numerici deriuantur vt supra.

Cunctis aggragatis quatuor terminorum in. uentis progredimur ad aggregata duorum terminorum. A equatio $(C)$, cuius radices sunt haec $(\varepsilon, I),(2,13), \operatorname{sub}(4, I)$ contenta, eruitur haec $x x-(4, \mathbf{I}) x+(4,3)=0$; huius radices sunt $\frac{x}{2}(4, x) \pm \frac{1}{2} \sqrt{(}\left(-4(4,3)+(4, x)^{2}\right)$ siue $\left.\frac{r}{2}(4, I) \pm \frac{r}{2} \sqrt{(4+}+(4,9)-2(4,3)\right)$; eam vbi quantitas radicalis positiue sumitur et cuius valor reperitur $=\mathrm{I}, 8649444588$, statuimus $=(2, \mathrm{l})$,

7) Vera indoles huius artificii in eo consistit, quad a priori praeuideri poterat, hocee productum" euolutum.aggręgat quatuor terminorum non continere sed per sola aggregata octo texminorum exhiberi posse, cuius rei rationew hic breuitatis eauss pretereundam periti facillime deprehem. dest.

$\operatorname{Rr} 3$ 
nude $(2,13)$ aequale fiet alteri, cuius valor $=$ 0,1845367 189. - Si aggregata reliqua duorum terminorum per methodum art. 346 inuestigare: placet, pro $(2,2),(2,3),(2,4),(2,5),(2$, $6),(2,7),(2,8)$ eaedem formulae adhiberi poterunt, quae in ex. praec. pro quantitatibus similiter disignatis tradidimus, puta $(2,2)$, (siue $(2,15)),=(2, I)^{2}-2$ etc." Si vero magis arridet, binas per resolutionem aequationis quadraticae computare, pro his $(2,9),(2$, I5) inuenitur aequatio $x x-(4,9) x+(4$, Io) $=0$, cuius radices euoluuntur $\frac{\pi}{2}(4,9) \pm$

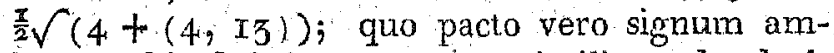
biguum hic definire oporteat, simili modo decidetur vt supra. Scilicet per euolutionem producti: $(2, I)-(2,13)$ in $(2,9)-(2,15)$ producitur $-(4, x)+(4,9)-(4,3)+(4$, Io); quod quum manifesto sit negatiuum, factor $(2, I)-(2, I 3)$ vero positiuus, necessario $(2$, 9) - $(2$, I5) negatiuus esse debebit, quocirca in expressione ante data signum superius positiuum pro $(2,15), \operatorname{pro}(2,9)$ inferius negatiuum adoptandum erit. Hinc computatur $(2,9)=$

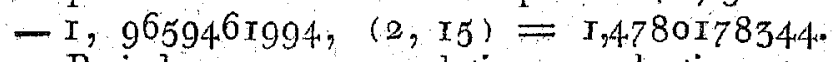
- Perinde quum ex enolutione producti ex (2, I) $-(2,73)$ in $(2,3)-(2,5)$ prodeat $(4,9)$ $-(4,10)$, adeoque quantitas positiua, factorem $(2,3)-(2,5)$ positiuum esse concludimus; hinc simili calculo vt ante instituto inuenitur

$$
\begin{aligned}
(2,3)= & \frac{r}{2}(4,3)+\frac{x}{2} \sqrt{ }(4+(4,10)-2(4,9))= \\
& 0,891476715 \\
(2,5)= & \left.=\frac{\pi}{2} 4,3\right)-\frac{x}{2} \sqrt{ }(4+(4,10)-2(4,9))= \\
& -0,5473259801
\end{aligned}
$$




\section{- 631}

Denique per operationes omnino analogas erus itur

$$
\begin{aligned}
(2,10) & =\frac{T}{2}(4,10)-\frac{\pi}{2} \sqrt{ }(4+(4,3)-2(4,1)) \\
& =-1,7004342715 \\
(2,11) & =(4,10)+\frac{\pi}{2} \sqrt{2}(4+(4,3)-2(4,1)) \\
& =-1,20526927^{28}
\end{aligned}
$$

Superest yt ad radices $\Omega$ ipsas descendamus. Aequatio $(D)$ cuius radices sunt [1] et [16] prodit $x x-(2, k) x+1=0$, vnde radices $\left.\frac{x}{2}(2,1) \pm \frac{x}{2} \sqrt{(}(2,1)^{2}-4\right)$ aut potius $\frac{1}{2} \sqrt{(2,1)}$ $\pm i \sqrt{ }\left(4-(2,1)^{2}\right)$ siue $\frac{x}{2}(2,1) \pm \frac{x}{2} i \sqrt{ }(2-$ $(2,15))$; signum |superius pro [1], inferius pro. [16] adoptamus. Quatuordecim reliquae radices vel per potestates ipsius [1] habebuntur; vel per resolutionem septem aequationum quadraticarum, quae singulae binas exhibent, vbi incertitudo de signis quantitatum rùdicalium per idem. artificium tolli poterit vt in praecedentibus. Ita [4] et [13] sunt radices aequationis $x x-(2$, 13) $x+1=0$, adeoque $\frac{x}{2}(2,13) \pm \frac{\pi}{2} i \sqrt{ }(2$ $-(2,9))$; per enolutionem producti ex $[1]-$ [16] in $[4]-[13]$ autem prodit $(2,5)-(2,3)$, adeoque quantitas realis negatiua, quare quum [1] $-[16]$ sit $+i \sqrt{ }(2-(2,15))$, is e productum ex imaginaria $i$ in realem positiuam, etiam [4] $-[13]$ esse debet productum ex $i$ in realem positiuan propter $i i=-1$; hinc colligitur, pro [4] signum superius, pro [13] inferius accipiendum esse. Simili modo pro radicibus [8] et [9] inuenitur $\frac{\tau}{2}(2,9) \pm \frac{x}{2} i f(2-(2,1))$, vbi, quoniarn productum ex $[1]-[16]$ in $[8]-$ [9] fit $(2,9)-(2,10)$ adeoque negatium, $\mathrm{Pr}_{4}$ 
pro [8] signum superius, pro [9] inferius arcipere oportet. Corraputando nesmde radires reliquas, sequentes valores numericus obtinemos, whi radicibus prioribus signa superiora, posteriuribus inferiora respondere subintelligerdum est:

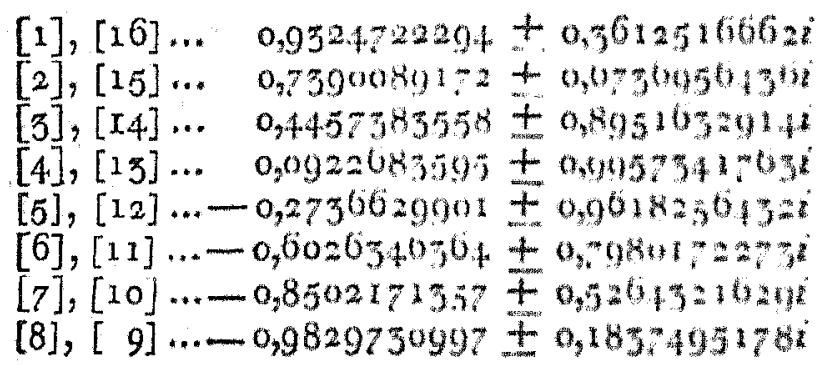

*

* $\quad *$

Possent quidem ea quae in praecc. stum traw dita ad solutionem aequationis $x^{*}-1=0$

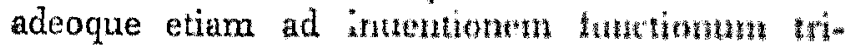

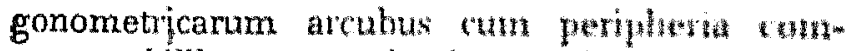

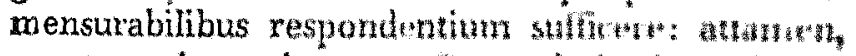

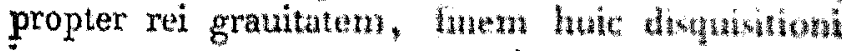

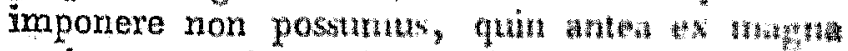
copia quum olseruationum hoc argamentum it. lustrantium tum positionum ex afrimun vel inde pendentium quaedan hic annectamus, luns quae talia potissimum eligemu, qua sine

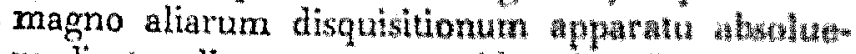

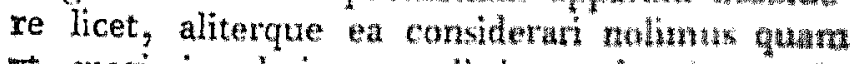
vpecimina huius amplisima doctratate, in posterum copiose pertractandae. 


\section{- $633-$}

355. Quum $n$ semper supponatur impar, erit 2 inter factores ipsius $n=1$, complexusque $\Omega$ ex $\frac{T}{2}(n-1)$ periodis duorum terminorum formatus. Talis periodus, vt $(2, \lambda)$, e yadicibus $[\lambda]$ et $\left[\lambda g^{\frac{T}{2}(n-1)}\right]$ constabit, denotante $g$ vt supra radicem primitinam quarncurique pro modulo n. Sed fit $\sigma^{\frac{r}{2}(n-1)} \equiv-1(\bmod \cdot n)$ adeoque $\lambda g^{\frac{T}{2}(n-I)} \equiv-\lambda$ (V. art. 62), vnde $\left[\lambda g^{\frac{x}{2}(n-1)}\right] \equiv[-\lambda]$. Quare supponendo $[\lambda]=$
$\cos \frac{k P}{n}+i \sin \frac{k P}{n}$, et proir $[-\lambda]=\cos \frac{k P}{n}$ $-i \sin \frac{k P}{n}$, fit aggregatum $(2, \lambda)=2 \cos \frac{k P}{n}$. Vnde hoc loco hanc tantummodo conclusionem deducimus, valorem cuiusuis aggregati duorum terminorum esse quantitatem realem. Quum quaeuis periodus, cuius terminorum multitudo par $=2 a$, in $a$ periodos binorum terminorum discerpi possit, patet generalius, valorem cuiusuis aggregati cuius terminorum multitudo par semper esse quantitatem realem. Quodsi itaque in art. 352 inter factores $a, \hbar, \gamma$ etc. binarius ad vltimum locum reseruatur, omnes operationes vsquedum ad aggregata duorum terminorum perueniatur per quantitates reales absoluentur, imaginariaeque tunc demum introducentur, quando $a b$ his aggregatis ad radices ipsas progredieris.

356. Summam attentionen merentur aequationes auxiliares, per quas pro quolibet valore ipsius $n$ aggregata complexum $\Omega$ corstituentia determinantur, quae mirum in mocium cum proprietatibus maxime reconditis numeri $n$ $\operatorname{Rr} 5$ 


\section{$-634-$}

connexae sunt. Hoc vero loco disquisitionem ad duos casus sequentes restringemus: primo de aequatione quadratica cuius radices sunt aggregata $: \frac{T}{2}(n-1)$ terminorum, secundo, pro eo casu vbi $n-1$ factorem 3 implicat, de cubica cuius radices sunt aggregata $\frac{1}{3}(n-1)$ terminorum agemus,

Scribendo breuitatis caussa m pro $\frac{x}{2}(n-1)$ et designando per $g$ radicem primitiuam quamcunque pro modulo $n$, complexus $\Omega$ e duabus periodis $(m, 1)$ et $(m, g)$ constabit, continebitque prior radices $\left.[1],[\mathrm{gg}],\left[g^{4}\right]\right]^{\prime} \ldots\left[g^{u-3}\right]$, posterior has $[g],\left[g^{3}\right],\left[g^{5}\right] \ldots\left[g^{n-2}\right]$. Supporiendo residua ninima positiua numerorum $g g_{*}$ $g^{1 .} \ldots g^{n-3}$ secunduin modulum $n$ esse, ordine arbitrario, $R, R^{\prime}, R^{\prime \prime}$ etc.; nec non residua horum $g, g^{3}, g^{5} \ldots g^{n-2}$ haec $N, N^{\prime}, N^{\prime \prime}$ etc., radices e quibus ( $\mathrm{m}, \mathrm{I}$ ) constat conuenient cuma his $[1],[R],\left[R^{\prime}\right],\left[R^{\prime \prime}\right]$ etc., radicesque periodi $(m, g)$ cum his $\left[N^{\top}\right],\left[N^{\prime}\right],\left[N^{\prime}\right]$ etc. Iam patet, omnes numeros $1, R, R^{\prime}, R^{\prime \prime}$ etc. esse residua quadratica numeri $n$, et quum omnes duersi ipsoque $n$ minores sint ipsorumque multitudo $=\frac{x}{2}(n-1)$ adeoque multitudini cunctorum residuorum positiuorum ipsius $n$ infra $n$ aequalis, haec residua cum illis nunieris omnino. contuerilent. Hinc sponte sequitur, omnes numeros $N, N^{\prime}, N^{\prime \prime}$ etc, qui tum inter se tum ab ipsis $I, R, R$ etc diuersi sunt, et cum his simul sumti omnes numeros $1,2,3 \ldots n-I$ exhaumint, cum ommibus non residuis quadraticis positiuis ipsius $n$ infra $n$ conuenire debere. Quodsi iam supponitur, aequationem cuius ra- 
dices sunt aggregata $(m, 1),(m, g)$ esse $x x$ $-A x+B=0$, fit $A=(m+x)+(m, g)$ $=-\mathrm{I}, B=(\mathrm{m}, \mathrm{I}) \times(\mathrm{m}, \mathrm{g})$. . Productum ex $(m, I)$, in $(m, g)$ per art. 345 fit $=(m$, $N+\mathrm{r})+(m, N+\mathrm{I})+\left(m, N^{\prime \prime}+\mathrm{I}\right)+$ etc. $=W$, atque hinc reducetur' sub formam talem $a(m, 0)+b(m, r)+g(m, g) . \quad A d$ determinationem coefficientium $\alpha, \varepsilon, \gamma$ obseruamus, primo, fieri $\alpha+6+\gamma=m$ (scilicet quoniam multitudo aggregatorum in $W$. est $\Longrightarrow$ $m) ;$ secundo, esse $:=\gamma$ (hoc sequitur ex art. $35^{\circ}$ quum productum $(m, \mathrm{I}) \times(m, g)$ sit functio inuariabilis aggregatorum $(m, I),(m, g)$, e quibus aggregatum maius $(n-1,1)$ compositum est)s. tertio, quum onnes numeri $N+1$, $N^{\prime}+\mathrm{I}, N^{\prime \prime}+\mathrm{r}$ etc. infra limites 2 et $n+\mathbf{r}$ excl. contineantur, manilestum est, vel nullum aggregatum in $W$ ad $(n, 0)$ reduci adeoque esse $\alpha=0$, quando inter pumeros $N, N^{\prime}, N^{\prime \prime}$ etc. non occurrat $n-I$, vel vnum puta ( $m$, $n)$, et proin haberi $\alpha=1$, quando $n-1$ in ter numeros $N, N^{\prime}, N^{\prime \prime}$ etc. repersatur. Hinc colligitur, in casu priori fieri $e=0, b=\gamma$ $=\frac{1}{2} m$, in posteriori $a=1,6=2=\frac{1}{2}(m$ - I), simul hinc sequitur, quim numeri 6 et $\gamma$ necessario fiant integri, casum priorem locum habere, siue $n-\mathrm{r}$ (aut quod idem est $-\mathrm{x}$ ) inter non residua ipsius $n$ non reperiri, quanda $m$ sit par siue $n$ formae $4 k+1 ;$ casum posteriorem vero adesse, siue $n-I$ aut -1 inter non residua ipsius $n$ reperini, quoties $m$ sit impar. siue $n$ formae $4 k+3 *$; Hinc productum

*) Hóc modo napti sumus demonstrationexa nounn theorematis, - I esse residuum omminur namerorum primotan 
quaesitum fit, propter $(m, o)=m,(m, 1)+$ $(m, g)=-\mathrm{r}$, in . casu priori $=-\frac{3}{2} m$, in posteriori $=$ a $(m+n)$, adeoque aequatio quaesita in illo casu $x x+x-{ }_{4}^{x}(n-1)$ $=0$, cuius radices sunt $-\frac{x}{2} \pm \sqrt{n}$, in hac vero $x x+x+\frac{x}{4}(n+1)=0$, cuins radices $-\frac{r}{2} \pm \frac{\pi}{2} i \sqrt{n}$.

- Quaecunque itaque radix ex $\Omega$ pro $[1]$ ato. ptata est, differentia inter summas s[s] on

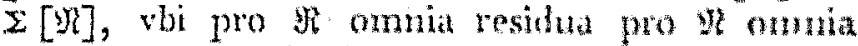
non residua quadratica positiua ipsius $"$ infra $n$ substituenda sunt, erit $= \pm \sqrt{n}$, wo $n=x$, et $= \pm i \sqrt{ } n$, pro $n=3($ mod, 4$)$ Nec non hinc facile sequitur, denotante $k$ integrum qumm-

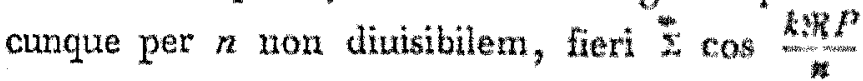
$-\Sigma \cos \frac{k 9 P}{s}= \pm \sqrt{n}$ et $\Sigma \sin \frac{k g P}{k n}$ $\Sigma \sin \frac{k \Re P}{n}=0$ pro $n \equiv I$ (mod. \& ; contra pro $n \equiv 3$ (mod. 4 ) differentiam illun $=0$, hanc $= \pm \sqrt{n}$, quat theoremata propter solegantiam suam valde sunt memuabilia. Ceterum obsertamus, signa stiperiora sempers vatero quando pro $k$ acciphistur vnitas ant generwilas residum quadraticum ipsits $n$, inferitona qquando pro $k$ non residum assumatur, nes: non hateces theoremata salua vel potius aucta eleguma sua

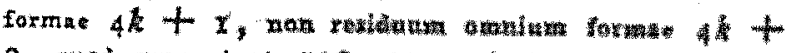

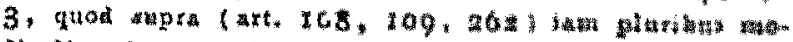

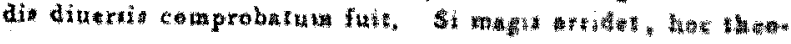

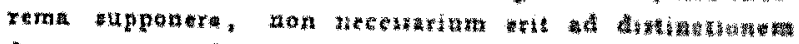

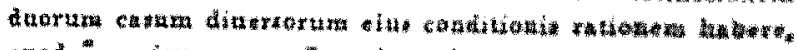

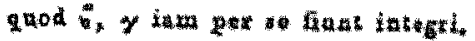




\section{- $637-$}

etiam ad valores quosuis compositos ipsius 72 extendi posse: sed de his rebus quae altioris sunt indaginis hoc loco tacere earumque cunsiderationem ad aliam occasionem novis reseruare oportet.

357. Sit àequatio $m^{\text {ti }}$ gradus, cuius radices sunt $m$ radices in periodo $(m, 1)$ contentae haec $x^{m}-$ $a x^{m-1}+b x^{m-2}-$ etc. $=0$ siue $z=0$, eritque $a$ $=(m, x)$, singulique reliqui coëfficientes $b$ etc.

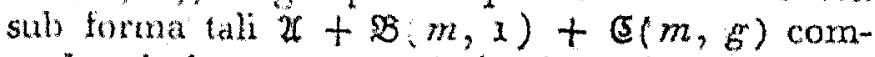
prellensi, ita ve $\mathbb{X}, 98$, E sint integri (art. 34.8 ); denotanduque per $z$ functionem, in quan $z$ transit, si pro $(m, x)$ vbique substituitur $(m, g)$, pro $(m, g)$ vero $(m, g g)$ sine quod idem est $(m, 1)$, radices aequationis $z^{\prime}=0$ erint radices in $(m, g)$ contentae, productumque $z z^{\prime}=$ $\frac{x^{n}-1}{x-1}=X . \quad$ Potest itaque $z$ ad formam talem $R+S(m, x)+T(m, g)$ reduci, vibi $R, s, T$ entnt functiones integrae ipsius $x$, quarum omnes coüficientes etiam integri erunt; quo facto habebitur $z^{\prime}=R+S(m, g)+T^{\prime}(m, x)$. Hinc fit stribendo breuitatis caussa $p$ et $q$ pro $(m$, I) et $m, g)$ resp., $2 z=2 R+(s+T)(p+q)$ $-(T-S)(p-q)=2 R-S-T-(T$ - S) $(p-q)$ similiterque $2 z^{\prime}=2 R-S-T$ $+(T-S)(p-q)$, wnde ponendo $2 R-S-$ $T^{\prime}=Y, T-S=Z$, fit $4 X=Y Y-(p-$ $q)^{2} z Z$, adesque quum $(p-q)^{2}= \pm n, 4, X$ $=Y Y+n Z Z$, signo superiori valente quando $n$ est formae $4 k+1$, inferiori quando $n$ formae $4 k+3$. Hoc est theorema, cuins demonstrationem supra (art. 124) polliciti surnus. Terminos duos summos functionis $Y$ semper fiexi 
$2 x^{m}+x^{m-1} ;$ summumque functionis $Z, x^{m-x}$ facile perspicietur; coéfficientes reliqui autem, qui manifesto omnes erunt integri, variant pro diuersa indole numeri $n$, nec formulae analyticae generali subiaci possunt.

Ex. Pro $n=17$ aequatio cuius radices sunt octo adices in $(8,1)$ contentae per praecepta art. 348 eruitur $x-p x+(4+p+2 q) x^{5}-(4 p$ $+3 q) x^{5}+(6+3 p+5 q) x^{4}-(4 p+3 q) x^{3}+$ $(4+p+2 q) x x-p x+1=0$, vnde $R=$ $x^{3}+4 x+6 x+4 x x+1, S=-x^{7}+$ $x^{5}-4 x+3 x^{4}-4 x^{3}+x x-x, T=2 x^{6}$ $-3 x+5 x-3 x^{3}+2 x x$, atque hinc $Y=$ $2 x^{5}+x^{7}+5 x^{5}+7 x^{3}+4 x+7 x^{3}+5 x x$ $+x+2, Z=x^{7}+x^{6}+x^{5}+2 x^{4}+x^{3}+$ $x x+x$. Ecce adhuc alia quaedarn exempla:

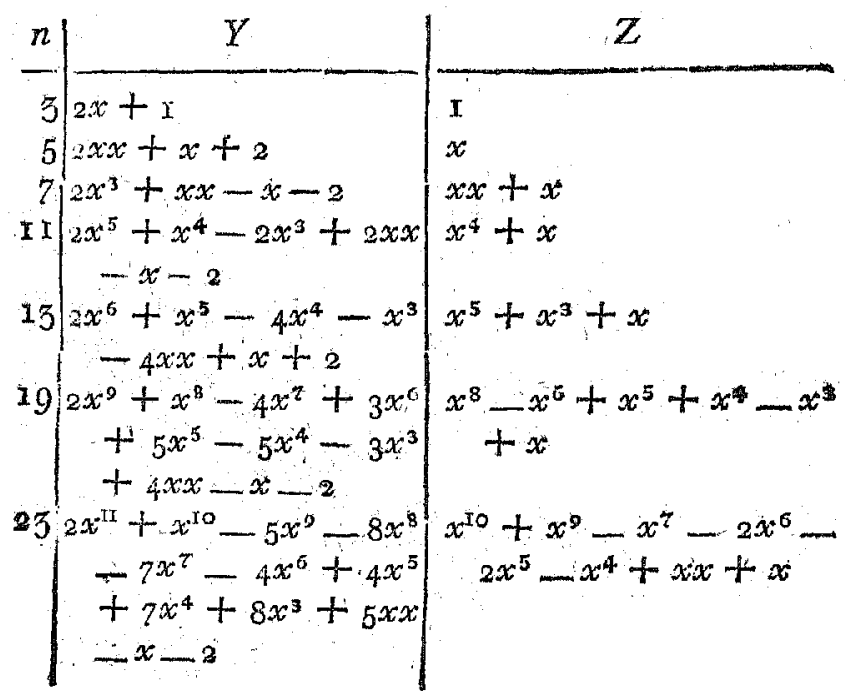


358. Progredimus ad considerationem aequationum cubicarum; per quas in eo casu bi $n$ est formae $3 h+1$ tria aggregata $\frac{x}{3}(n-1)$ terminorum conplexum $\Omega$ componentia determinantur. Sit $g$ radix primitiua quaecunque pro modulo $n$, atque $\frac{5}{3}(n-1)=m$, qui erit integer par. Tunc tria aggregata e quibus $\Omega$ constat erunt $(m, 1),(m, g),(m, g g)$ pro quibus resp. scribemus $p, p^{\prime}, p^{\prime \prime}$, patet que primum continere radices $[\mathrm{I}],\left[\mathrm{g}^{3}\right],\left[\mathrm{g}^{\mathrm{j}}\right]$ $\ldots\left[g^{n-4}\right]$, secundum has $[g],[g-4] \ldots\left[g^{n-3}\right]$, tertium has $\left[g g^{\prime}\right],\left[g^{S}\right] \ldots\left[g^{2-2}\right]$. Supponendo, aequationem quaesitam esse $x^{3}-A x x+B x-$ $C=0$, fit $A=p+p^{\prime}+p^{\prime \prime}, B=p p^{\prime}+$ $p^{\prime} p^{\prime \prime}+p p^{\prime \prime}, C=p p^{\prime} p^{\prime \prime}$, vnde protinus habetur $A=-1$. Sint residua minima positiua numerorum $g^{3}, g^{5} \ldots g^{n-+}$ secundum modulumi $n$

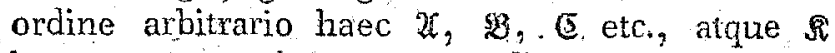
ipsorum complexus superadiecto numelo $\mathrm{I}$; similiter sint $\mathfrak{k}^{\prime}, \mathfrak{B}^{\prime}$, $\mathfrak{E}^{\prime}$ etc, residua minima numerorum $g, g \div, g^{7} \ldots g^{n-3}$, atque $n^{\prime}$ illorum complexus; denique $\mathfrak{X}^{\prime \prime}, 9 \mathbb{3}^{\prime \prime}$, $\mathrm{S}^{\prime \prime}$ etc. residua minima ipsorum $g g, g^{r}, g^{8} \ldots g^{n-2}$ et $\mathfrak{N}^{\prime \prime}$ eorum complexus, vnde omnes nutheri in $\Omega, a, \Re^{\prime \prime}$ diuersi erunt et cum his $1,2,3 \ldots n-1$ conuenient. Ante omna hic obseruandum est, numerum $n-$ I necessario in $\Omega$ reperiri, quippe quem esse residuum ipsius $g_{2}^{\frac{3}{2} \text { h }}$ facile perspicitit. Hinc facile quoque consequitur, dnos numeros tales $h, n-h$ semper in eodem trium complexuum $\Omega, \Re^{\prime}, \Omega^{\prime \prime}$ reperiri $i$, si enim alter est residuum potestatis $g^{\lambda}$, alter erit residuum potestatis $g^{\lambda}+\frac{3}{2} m$, aut huius $g^{\lambda}-\frac{3}{2} m$ si $\lambda>\frac{3}{2} m$. Denotemus hocce signo (RI) multitudinem nume- 
rorum in seriei $1,2,3 \ldots n-1$ qui tum ipsi tum simul numeri proximi vnitate maiores in $\mathscr{\Uparrow}$ continentur; similiter sit ( $\left(\mathrm{SI}^{\prime}\right)$ mulattudo nuinerorum in eadem serie qui ipsi in $\Omega$ proxime sequentes vero in $\Omega^{\prime}$ continentur, vude simul significatio

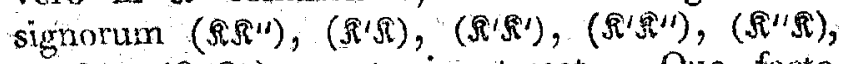

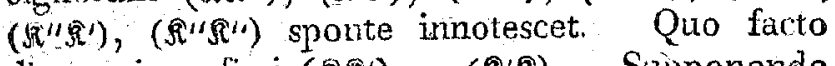
dico primo, fieri $\left(\Omega^{\prime} \mathbb{R}^{\prime}\right)=\left(\mathbb{R}^{\prime} \mathfrak{R}\right)$. Supponendo erim, $h^{\prime}, h^{\prime}, h^{\prime \prime}$ etc. esse omnes numeros seriei I, $2,3 \ldots n-\mathbf{I}$ qui ipsi in $\Re$ proxime maiores $h+1, h^{\prime}+1, h^{\prime \prime}+I$ etc. autem in $\Omega^{\prime}$ continentur, et quorum ideo multitudo $=\left(\Omega\left(\Omega^{\prime}\right)\right.$, manifestum est omnes numeros $n-h-\mathrm{r}$, $n-h^{\prime}-\mathrm{I}, n-h^{\prime \prime}-I$ etc. in $\Re^{\prime}$ contineri, proxime maiores vero $n-h, n-I^{\prime}$ etc. in $\Omega$; quare quum tales numeri omnino dentur $\left(\Omega^{\prime}(\Omega)\right.$, certo nequit esse $\left(\Omega^{\prime} \Omega\right)<\left(\Omega \Omega^{\prime}\right)$, et perinde demonstratur, esse nón posse $\left(\Omega^{\prime}\right)<\left(\Omega^{\prime}(\Omega)\right.$, quocirca hi numeri necessario aequales erunt. Prorsus eodem modo probatur ( $\left(\Omega^{\prime \prime}\right)=\left(\Omega^{\prime \prime}(\mathfrak{\Omega}),\left(\Omega^{\prime} \Omega^{\prime \prime}\right)\right.$ $=\left(\Omega_{1}^{\prime \prime} \Omega^{\prime}\right)$. Secundo, quum necessario quemuis numerum ex $\mathfrak{A}$, maximo $n-1$ excepto, sequi debeat proxime maior vel in $\Omega$, vel in $\Re^{\prime}$ vel in $\Omega^{\prime \prime}$ contentus, summa ( $(\Omega \Re)+\left(\Omega^{\prime} \Omega^{\prime}\right)+\left(\Omega \Omega^{\prime \prime}\right)$ fiet aequalis multitudini omnium numerorum in vaitate deminutae puta $=m-\mathrm{r}$, et simili ratione erit $\left(\mathbb{R}^{\prime} \mathbb{R}+\left(\mathfrak{R}^{\prime} \mathbb{R}^{\prime}\right)+\left(\mathfrak{R}^{\prime} \mathfrak{R}^{\prime \prime}\right)=\left(\mathbb{R}^{\prime \prime} \mathfrak{R}\right)+\right.$

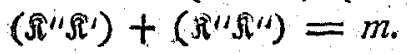

His ita praeparatis euoluimus per praecepta art. 345 productum $p p^{\prime}$ in $(m, \mathfrak{a}+1)+\left(m, \mathfrak{B}^{\prime}\right.$ $+I)+m,(s+I)+$ elc, quam expressionem facile perspicietur reduci ad $(\mathfrak{N} / \mathcal{R}) p+\left(\mathfrak{N}^{\prime} \mathfrak{N}^{\prime}\right) p^{\prime}$ $+\left(\Omega^{4}\right) p^{\prime \prime}$, et quum per art. 345 I productum 


\section{- 64.}

ptp" ex illo oriatur, substituendo pro $(m, 1)$, $(m, g),(m, g g)$ resp. $(m, g),(m, g g)$, $\left(m g^{3}\right)$ i. e. pro $p, p^{\prime}, p^{\prime \prime}$ resp. $p^{\prime}, p^{\prime \prime}, p$, fiet $p^{\prime} p^{\prime \prime}=\left(\Re(\Re) p^{\prime}+\left(\mathfrak{\Omega}^{\prime} \mathfrak{\Omega}^{\prime}\right) p^{\prime \prime}+\left(\Re^{\prime \prime} \mathfrak{\Omega}^{\prime \prime}\right) p\right.$, et prorsus simili modo $p^{\prime \prime} p=(\mathfrak{N} \mathbb{N}) p^{\prime \prime}+\left(\Omega^{\prime} \Omega\right)_{p} p$ $+\left(\Omega^{\prime} \Omega^{\prime \prime}\right) p^{\prime}$. Hinc protinus sequitur primo $B=$ $m\left(p+p^{\prime}+p^{\prime \prime}\right)=-m$; secundo quum simili ratione, vt antea $p p^{\prime}$ exolutum est, etiam

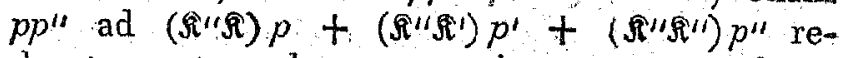
ducatur, atque haec expressio cum praecedente identica esse debeat, necessario erit $(\Omega \| \Re)=$ $\left(\boldsymbol{\Omega}^{\prime} \Omega^{\prime}\right)$ et $\left(\boldsymbol{\Omega}^{\prime \prime} \boldsymbol{\Omega}^{\prime \prime}\right)=\left(\Omega^{\prime} \mathfrak{\Omega}\right)$. Hinc colligitur, statuendo $\left(\Omega^{\prime} \Omega^{\prime \prime}\right)=\left(\Omega^{\prime \prime} \Omega^{\prime}\right)=a,\left(\Omega^{\prime \prime} \Omega^{\prime \prime}\right)=\left(\Omega^{\prime} \Omega\right)$ $=\left(\Omega^{\prime}\right)=b,\left(\Omega^{\prime} \Omega^{\prime}\right)=\left(\Omega^{\prime \prime}\right)=\left(\Omega^{\prime \prime}\right)=c_{2}$ fieri $m-1=(\hat{R})+\left(\hat{M} \hat{N}^{\prime}\right)+\left(\hat{\Omega} \hat{\Omega}^{\prime \prime}\right)=(\hat{R} \Omega)$ $+b+c$, atque $a+b+c=m$, vnde ( $(\Omega)$ $=a-1$, ita vt illae nouem quantitates incognitae ad tres, $a, b, c$ siue potius propter aequationem $a+b+c=m$ ad duas reductae sint Denique patet, quadratum $p p$ euolui in $(m, 1+I)+(m, \mathfrak{X}+1)+(m, \mathfrak{B}+1)+$ $(m, \sigma+1)+$ etc; inter partes huius expressionis reperietur $(m, n)$ quae reducitur ad $(m, 0)$ siue ad $m$, reliquas vero facile perspicietur reduci ad $(\Re \Re) p+\left(\Omega \Omega^{\prime}\right) p^{\prime}+\left(\Omega \Omega^{\prime \prime}\right) p^{\prime \prime}$, vnde habetur $p p=m+(a-1) p+b p^{\prime}+c p^{\prime \prime}$.

Hoc itaque modo per disquisitiones praecedentes quatuor hasce reductiones nacti sumus:

$$
\begin{array}{ll}
p p=m+(a-I) p+b p^{\prime}+c p^{\prime \prime} \\
p p^{\prime}= & b p+c p^{\prime}+a p^{\prime \prime} \\
p p^{\prime \prime}= & c p+a p^{\prime}+b p^{\prime \prime} \\
p^{\prime} p^{\prime \prime}= & a p+b p^{\prime}+c p^{\prime \prime}
\end{array}
$$




\section{$-642-$}

tbi inter tres incognitas $a, b, c$ aequatio conditionalis $a+b+c=m \ldots$ (I) intercedit, insuperque certum est ipsas esse numeros integros. Hinc colligitur $C=p \times p^{\prime} p^{\prime \prime}=a p p+b p p^{\prime}+$ $c p p^{\prime \prime}=a m+(a a+b b+c c-a) p+(a b$ $+b c+a c) p^{\prime}+(a b+b c+a c) p^{\prime \prime}$. At quum $p p^{\prime} p^{\prime \prime}$ sit functio inuariabilis aggregatorum $p, p^{\prime}$, $p^{\prime \prime}$, coëfficientes per quos haec in expr. praec. multiplicata sunt necessario aequales erunt (art. $350)$, vnde habetur aequatio noua $a a+b b+$ $c c-a=a b+b c+a c \ldots$ (II), atque hinc $c=a m+(a b+b c+a c)\left(p+p^{\prime}+p^{\prime \prime}\right)$, siue (propter I, et $\left.p+p^{\prime}+p^{\prime \prime}=-1\right), C=a a$ - $b c . .$. (III). Iam etsi $C$ hic a tribus incognitis pendeat, inter quas duae tantum aequationes habentur, tamen hae, adiumento conditionis ex qua $a, b, c$ sunt integri, ad plenam determinationem, ipsius $C$ sufficiunt Quod vt ostendamus, aequationem II ita exhibemus $12 a+12 b$ $+12 c+4=3^{6 a a}+3^{6 b b}+3^{6 c c}-3^{6 a b}$ $-36 a c-36 b c-24 a+12 b+12 c+4$ pars prior, per I, fit $=12 m+4=4 n$; posterior vero reduritur ad $\left(6 a-3^{b}-3^{c}-2\right)^{2}$ $+27(b-c)^{2}$, aut scribendo $k$ pro $2 a-b-$ $c$, ad $(3 k-2)^{2}+27(b-c)^{2}$. Hinc patet, numerum $4^{n}$ (i. e. generaliter quadruplum cuiuslibet primi formae $3^{n}+1$ ) per formam $x x+$ $27 y y$ repraesentari posse, quod quidem sine difficultate e theoria generali formarum binariarum deduci potest, attamen satis mirum est, talem discerptionem cun valoribus ipsarum $a, b, c$ cohaerere. At numeras $4 n$ semper vnico tantum modo in quadratum et quadratum $27^{\text {plex }}$ discerpi potest, 
qund ita demonstramus *). Si supponatur $4 n$ $=t t+27 u t=t^{\prime} t^{\prime}+27 u^{\prime} w^{\prime}$, fieret primo $\left(t t^{\prime}-27 u u^{\prime}\right)^{2}+27\left(t u^{\prime}+t^{\prime} u\right)^{2}=16 n n$, secundo $\left(t t^{\prime}+27 u u^{\prime}\right)^{2}+27\left(t w^{\prime}-t^{\prime} u\right)^{2}=16 \mathrm{mn}$, tertio $\left(t u^{\prime}+t^{\prime} u\right)\left(t u^{\prime}-t^{\prime} u\right)=4 n\left(u^{\prime} u^{\prime}-u u ; ;\right.$ ex aequatione tertia sequitur, ipsum $n$, quoniam est numerus primus, alterutrum numerorum $t u$ ' + $t^{\prime} u, t u^{\prime}$ - t'u metiri; e prima et secunda vero patet, vtrumque hunc numerum esse minorem quam $n$; quare is quem $n$ metitur necessario esse debet $=0$, adieque etiam $u^{\prime} u^{\prime}-u$ - u $=0$, vade $u^{\prime} u^{\prime}=u u$, et $t^{\prime} t^{\prime}=t t, \mathbf{i}_{\mathrm{i}}$ e. duae illae discerptiones non different. $S_{i}$ itaque dis cerptionem ipsius $4 n$ in quadratum et quadratum $27^{\text {plex }}$ notam supponimus (quain vel per methodum directam sect. $V$ vel per indirectam in artt. 325,324 traditam eruere licet) puta si habetur $4^{n}=M M+27 N N$, quadrata ( $3^{k}-$ $2)^{2},(b-c)^{2}$ determinata exunt, et loco aequationis II duas iam nacti erimus. Sed facile patet, non solum quadratum $(3 k-2)^{2}$ sed étiam radicem ipsam $3 k-2$ penitus determinatam esse; quum enim necessario sit vel $=$ $+M$ vel $=-M$, ambiguitas inde tolletur quod $k$ fieri debet integer, quamobrem statuetur $z^{k}$ $-2=+M$ vel $=-M$, prout $M$ est formae $3 z+1$ vel $3 z+2 * *$ j. Iam quum fiat $k$

*) Magis directe hacce propositio principio sect V probar posset.

*) Manifesto $M$ nequit esse formae $3 x$, alioquin enim $4 *$ per 3 divisibilis endadet. - Ad ambiguitatern, vtrum $b-t$ statui debeat $=N, \quad n=-N$, hic non opus est xespicere, megue etiam per rei, naturap yllo medo Ss 
$=2 a-b-c=5 a-m$, erit $a=k(m+$ k), $b+c=m-a=f(2 m-k)$, wade $C$ $\left.=a a-b c=c a-\frac{1}{4}(b+c)^{2}+\frac{1}{4} b-c\right)^{2}$ $=\frac{x}{y}(m+k)^{2}-{ }_{3}^{x}(2 m-k)^{2}+\frac{7}{4} \mathrm{~V} V={ }_{x}^{k} k k$ $+\frac{r}{3} k m+3 N N$, atque sic omnes cowfluientes. aequ. quaesitae inuenti. Q. L. F - Inec furmula adhuc simplicior euadit, si pro $N . V$ witus valor ex aequ. $(3 k-2)^{2}+2 y \cdot v y=4 t=$ $12 m+4$ substituitur, vnde elicitur calculo bacto $c=\frac{x}{5}(m+k+3 k m)=\frac{3}{3}(m+k n)$. Idem valor etiam ad $(3 k-2) N N+k^{4}-23 k$ $+k-k m+m$ reduci potest, gquke expressio, ad vsum quidem minus illonea, protims mon" strat, $C$ vr par est certo euadere inteyrum.

Ex. Pro $n=10$, fit $4 n=49+97$, sndo $3 k-2=+7, k=3, C=3(6+57)=7$ et aequatio quaesita $x^{5}+x x-6 x-7=0$ vt supra (art. 352). - Simili moda par nt $7,13,31,37,43,61,67$ valor ipsites $A$ enat tur resp. $1,-1,2,-3,-2,1,-1, k$ kn de $C=1,-1,8,-11,-8,9,-3$.

Ceterum etsi problema in boc art. soltutum satis intricatum sit, tamnen id supprimere "noiuk miss, tum propter solutionis elegantiam, tum quod variis artificis in vem vocundis octutonem de dit, quae in aliis quoque quanestionibus ingigni curn fructu adhibexi poterunt.

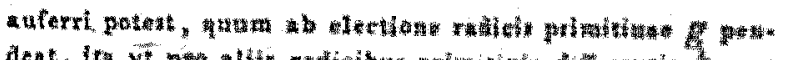

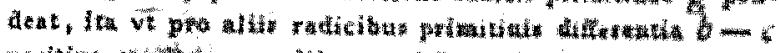

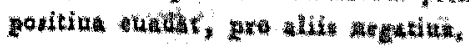




\section{$-645$}

359. Disquisitiones praecc. circa inuentionem aequationum auxiliarium versabantur: iam de earum solutione proprietatem magnopere insignem explicabimus. Constat, omnes summorum geometrarum labores, aequationum ordinem quartum superantium resolutionem generalem, siue (vt accuratius quid desideretur definiam) AFFECTARVM REDVCTIONEM AD pVRAS, inueniendi 'semper hactenus irritos fuisse, et vix dubium manet, quin hocce problema non tam analyseos hodiernae vires superet, quam potius aliquid impossibile proponat (Cf. quae de hoc argumento anotauimus in Demonstr: noua etc. p. 22). Nihilominus certum est, innumeras aequationes affectas cuiusque gradus dari, quae talem reductionem ad puras admittant, geometrisque gratum fore speramus, si nostras aequationes auxiliares semper huc referendas esse ostenderimus. Sed propter amplum ambitum huius disquisitionis, praecipua tantum momenta, quae ad possibilitatem ostendendam necessaria sunt, hoc loco tradimus, vberioremque tractationem qua hoc argumentum perdignum est ad aliud tempus differimus. Praemittendae sunt quaedam obseruationes generales circa radices aequ. $x^{\mathrm{e}}-1$ $=0$, quae eum quoque casum complectantur, vibi $e$ est numerus compositus.

1. Exhibentur hae radices (vt ex libris elementaribus notum est) per $\cos \frac{k P}{e}+i \sin \frac{k P}{e}$, vi pro $k$ accipiendi sunt $e$ numeri $0,1,2,3 \ldots$ $e-1$, aut quicunque alii his secundum modix$\operatorname{lum} e$ congrui. Vna radix, pro $k=0$ aut ge- 
neraliter pro $k$ per $\epsilon$ diutsibili fit $=1 ;$ cuiuis alii valori ipsius $k$ radix $a b$ i diuersa respondet.

II Quum sit $\left(\cos \frac{k P}{b}+i \sin \frac{k p^{2}}{e}\right)^{\lambda}=$ $\cos \frac{\lambda k P}{\varepsilon}+i \sin \frac{\lambda k P}{B}$, patet, si $R$ sit radix ta lis quae respondeat valori ipsius $k$ ad $e$ primo, in progressione $R, R R, R^{3}$ etc. terminum $e^{\text {tum }}$ quidem esse $=1$, omnes antecedentes vero ab 1 diuersos Hinc statim sequitur, omnes $e$ quantitates $1, R, R R, R^{3} \ldots R^{-1}$ inaequales esse, et quum manifesto omnes aequationi $x^{0}-1$ = o satisfaciant, exhibebunt omnes radicos huius aequationis,

III. Denique in eadem suppositione aggregatum $1+R^{\lambda}+R^{2 \lambda} \ldots+R^{\lambda(0)}$ fit $=0$, pro quouis valore integro ipsius $\lambda$ per $e$ non diuisibili, etenim est $=\frac{1-R^{\lambda \theta}}{I-R^{\lambda}}$, cuius fractionis numerator fit $=0$, denominator vero non $=0$. Quando vero $\lambda$ per $e$ dinisibilis est, illud aggregatum manifesto fit $=e_{\text {. }}$

360. Sit, vt semper in praece, n numerus primus, $g$ radix primitiua pro modulo $n$, atque $n-1$ productum e tribus integris positiuis; breuitatis caussa disquisitionem ita statim instituemus, vt etiam ad casus vbi a aut $\gamma=1$ pateat; quando $\gamma=1$, pro aggregatis $(\gamma, i)$, $(2, g)$ etc. radices, $[1]$, $[g]$ etc accipere oportebit. Supponamus itaque, ex omnibus a aggregais $6 y$ terminorum cognitis $(6,1),(6, g)$, 
$(6 y, g g) \ldots\left(b y, g^{\alpha-1}\right)$ deducenda esse aggregata $y$ terminorum, quod negotium supra ad aequationem affectam $6^{\text {ti }}$ gradus reduximus, nunc vero per puram aeque altam absoluere docebimus. Ad abbreuiandum pro aggregatis $(r, 1)$, $\left(\gamma, g^{\alpha}\right),\left(\nu, g^{2 \alpha}\right) \ldots\left(\gamma, g^{\alpha \omega}-\alpha\right)$, quae sub $(\xi \gamma$, 1) contenta sunt, scribemus $a, b, c . \therefore m$ resp.; pro his $(\gamma, g),\left(\gamma, g^{\alpha+1}\right) \ldots\left(\gamma, g^{a_{b}-\alpha+1}\right)$ sub $\left(\epsilon_{\gamma}, g\right)$ contentis resp. $a^{\prime}, b^{\prime} \ldots m^{\prime}$; pro his $(\gamma, g g),\left(\gamma, g^{x+2}\right) \ldots\left(\nu, g^{\alpha b-a+2}\right)$ resp. $a^{u}$, $b^{\prime \prime} . . . m^{\prime \prime}$ etc. vsque ad ea quae sub $\left(6 y, g^{\alpha-1}\right)$ continentur.

I. Iam designet $R$ indefinite radicem aequationis $x^{6}-1=0$, supponamusque ex euolutione potestatis $6^{\text {the }}$ functionis $t=a+R b+$ $R R C, \ldots+R^{6-1} m$ oriri per praecepta art. 345

$$
\begin{aligned}
& N+A a+B b+C c \ldots+M m \\
& +A^{\prime} a+B^{\prime} b^{\prime}+C^{\prime} C^{\prime} \cdots+M^{\prime} m^{\prime} \\
& +A^{\prime \prime} a^{\prime \prime}+B^{\prime \prime} b^{\prime \prime}+C^{\prime \prime} C^{\prime \prime} \cdots+M^{\prime \prime} m^{\prime \prime} \\
& + \text { etc. }=X^{\prime}
\end{aligned}
$$

vbi omnes coëfficientes. $N, A, B$, $A^{\prime}$ etc. erunt functiones rationales integrae ipsius $R$. Supponantur etiam potestates $6^{\text {ta }}$ duarum aliarum functionum $u=R^{b} a+R b+R R c \ldots+R^{6-x} m$, $u^{r}=b+R c+R^{u} d \ldots+R^{b-2} m+R^{b-x} a$. resp. euolui in $U$ et $U$, perspicieturque facile ex art 350 , quum $u$ oriatur ex $t$ commutando aygregata $a, b, c \ldots m$ resp. cum $b, c, d \ldots a_{n}$ fore $U^{\prime \prime}=$ 


$$
\begin{aligned}
& -648 \quad- \\
& N+A b+B c+C d \ldots+M a^{\prime} \\
& +A^{\prime} b^{\prime}+B^{\prime} c^{\prime}+C^{\prime} d^{\prime} \ldots+M^{\prime} a^{\prime} \\
& +A^{\prime \prime} b^{\prime \prime}+B^{\prime \prime} C^{\prime \prime}+C^{\prime \prime} d^{\prime \prime} \ldots+M^{\prime \prime} a^{\prime \prime} \\
& + \text { etc. }
\end{aligned}
$$

Porro patet quum sit $u=R u^{\prime}$, fore $U=$ $R^{\bullet} U^{\prime}$, quare propter $R^{\bullet}=1$ coëfficientes correspondentes in $U$ et $U^{\prime}$ aequales erunt; denique quum $t$ et $u$ in eo tantum differant, quod $a$ in $t$ per vnitatem, in $u$ per $R^{5}$ multiplicatur, facile intelligetur, omnes coëfficientes correspondentes (i. e. qui eadem aggregata multiplicant) in $T$ et $U$ aequales esse, et proin etiam omnes coëfficientes correspondentes in $T$ et $U$. Hinc tandem colligitur $A=B=C$ etc $=M ; A$ $=B^{\prime}=C^{\prime}$ etc,,$A^{\prime \prime}=B^{\prime \prime}=C^{\prime \prime}$ etc, etc., $\mathrm{nn}$ de $T$ reducitur ad formam talem $N+A(6 \gamma, 1)+$ $A^{\prime}(\zeta \gamma, g)+A^{\prime \prime}(6 \gamma, g g)$ etc, vbi singuli coëfficientes $N, A_{2} A^{\prime}$ etc. sub formam talem reducere licet $p R^{0-1}+p^{\prime} R^{b^{-2}}+p^{\prime \prime} R^{0-3}+$ etc. ita ot $p, p^{\prime}, p^{\prime \prime}$ etc. sint numeri integri dati.

II. Si pro $R$ accipitur radix determinata aequationis $x^{6}-1=0$ (cuius solutionem iam haberi supponimus), et quidern talis cuius nulla inferior potestas quam $\boldsymbol{t}^{\text {th }}$ vnitati aequalis est, etiam $T$ quantitas determinata erit, ex qua $t$ per aequationem puram $t^{6}-T=0$ deriuare licet. At quum haec aequatio 6 radices habeat, quae erunt $t, R t, R R t \ldots R^{:-1} t$, dubium videri potest, quamnam radicem adoptare oporteat. Hoc vero prorsus rbitrarium esse, ita facile apparebit. Meminisse oportet, postquam omnia aggregata 
4. terminorum determinata sint, radicem [1] eatenus tantum definitam esse, vt aliqua ex $\zeta_{y}$ radicibus in $(6: x, 1)$ contentis hoc signo deriotari debeat; et perin omnino arbitrarium esse, quidnam ex $6 \mathrm{ag}-$ gregatis ipsum $(\xi, 1)$ constituentibus per $a$ designare velimus. Quodsi iam, aliquoaggregato determinato per $a$ expresso supponatur fieri $t=\mathfrak{z}$, facile perspicietur, si postêa aggregatum id, quod modo, designabatur per $b$, per $a$ denotare lubeat, ea quae antea exant $c, d \ldots \ldots a, b$ nunc fier $b, c \ldots m, a$, adeoque valorem ipsius $t$ nunc $=\frac{\mathfrak{I}}{R}=\mathfrak{I}^{6-\mathbf{r}}$. Simili modo si per $a \mathrm{id}$ aggregatum exprimere placet quod ab initio erat $c$, valor ipsius $t$ fiet $=\mathfrak{I} R^{\circ}-2$, et ita porro $t$ cuicunque quantitatum $\mathfrak{I}, \mathfrak{I} R^{\mathfrak{b}-\mathfrak{x}}, \mathfrak{I} R^{\mathfrak{b}-2}$ etc.aequalis censeri potest, i. e. cuilibet radici aequ. $x^{0}=$ $T=$ o, prout aliud alihdue aggregatum sub $(6 y, 1)$ contentum per $(r, 1)$ expressum supponatur. $Q . E . D$.

III. Postquam quantitas $t$ hoe modo determinata est, $6 \div 1$ alias inuestigare oportet, quae ex $t$ prodeunt, si in eics expressione pro $R$ successiue $R R, R^{3}, R^{4} \ldots R^{6}$ substituuntur, puta $i^{\prime \prime}=a+R R b+R^{4} c \ldots+R^{2 b-2} m, t^{\prime \prime}=a$ $+R^{3} b+R^{6} c \ldots+R^{3}{ }^{6-3} m$ etc. Vltima quidem iam habetur, quum manifesto fiat $=a+b+$ $c \ldots+m=(6 \gamma, 1) ;$ reliquae vero sequenti modo erui possunt. Si per praecepta art. 345 , simili modo vt $t^{6}$ antea in $\mathrm{I}$, productum $t^{6-t^{\prime}} t^{\prime}$ euoluitur, probabitur per methodum praecedenti prorsus analogam, quod inde prodeat ad formam talem $\mathfrak{n}+\mathfrak{x}(6 y, 1)+\mathfrak{A}(6 \%, g)+$ 
$\mathfrak{X}^{\prime \prime}\left(\zeta v, g g^{\prime}\right)$ etc. $=T^{t}$ reduci posse, ita vt $\mathfrak{R}$, $\mathfrak{W}$, $\mathfrak{X}^{\prime}$ 'ete. sint functiones rationales integrae ipsius $\mathbb{R}$, adeoque $T$ quantitas nota, vnde habebitur ' $t$ ' $=\frac{T: t}{T}$ Prorsus eodem modo, si ex euolutione producti $t^{t}-3 t^{\text {th }}$ prodire supponitur $T^{\prime \prime}$, haec expressio similem formam habebit et proin ex eius valore noto deriuabitur $t$ " per aequation nem $t^{\prime \prime}=\frac{T^{\prime \prime} t^{3}}{T^{3}}$, perinde $t^{\prime \prime \prime}$ per aequationem talem innuenietur $t^{\prime \prime \prime}=\frac{T^{u / t^{4}}}{T}$ ita vt $T^{\prime \prime \prime}$ sit quantitas nota etc.

Haec methodus non foret applicabilis, si fieri posset $t=0$, vnde etiam esse deberet $T$ $=T^{\prime}=T^{4}$ etc. $=0$; sed probari potest hoc esse impossibile, etsi demonstrationem propter pralixitatem hoc loco supprimere oporteat. Dantur etiam artificia peculiaria per quae fractiones $\frac{T^{\prime}}{T}, \frac{T^{\prime}}{T}$ etc in functiones rationales integras ipsins $R$ conuertere licet, nec non methodi breuiores pro eo casu vbi $\alpha=1$ valores ipsarum $t^{\prime}, t^{\prime \prime}$ etc. exuendi, quae omnia hic silentio praeterire debermus.

IV. Denique simulac $t, t^{\prime}$, th etc. inuentae sunt, hababitar statim per obs. III art praec. $t+$ $t^{\prime}+t^{\prime \prime}+$ etc. $=8 a_{a}$ vade valor ipsius a notus. erit, ex quo per art. 346 valores omaium reliquorum aggregatorum y terminorum derinari poterunt - Valores ipsorum $b, c, d$ etc. etiam per aequationes sequentes elici possunt, quarum ratio cuinis attendenti facile patebit: $6 b=R^{6-x} t+$ 
$R^{6-2} t^{\prime}+R^{2-3} t^{\prime \prime}+$ etc,, $6 C=R^{25-2} t+R^{2 b^{2}} t+$ $R^{26-6} t^{\prime \prime}$ etc, $6 d=R^{30-3} t+R^{36-6} t^{t}+$ etc. ete.

Ex magno numero obseruationum ad disquisitionem praec. pertinentium hic vram tanfum attingimus. Quod attinet ad sohtionem aequationis purae $x^{5}-T=0$, facile patet, $T^{*}$ in plerisque casibus valorem imaginarium $P+$ tQ habere, vnde illa solutio partim a sectione anguli (cuins tangens $=\frac{Q}{p}$ ) partion a sectione rationis (rnitatis ad $\sqrt{ }(P P+Q Q))$ in 6 partes, vt constat, pendebit. Vbi valde mirabile. est (quod tamen fusius hic non exsequimur), valorem ipsius $\sqrt[f]{ }(P P+Q Q)$ semper rationaliter per quantitates iam notas exprimi posse, ita yt, praeter extractionem radieis quadraticae, ad solutionem sola sectio anguli requiratur, e. g. pro ${ }^{\circ}=3$ sola trisectio. anguli, quwarn pro plerisque aliis aequationibus cubicis, quarum radices omnes reales sunt, simul: anguli et rationis trisectio euitari nequeat.

Tandem quum nihil obstet, quo minus statuamus $\alpha=1, \gamma=1$ adeoque $6=n-1$; manifestum est, şolutionem aequationirs $x^{\mathrm{n}}-1$ - o statim reduci posse ad solutionem aequationis purae $n-1^{\mathrm{t}}$ gradus $x^{n-1}-T=0$, vbi $T$ peradices aequationis $x^{\mathrm{n}}-\mathrm{I}-1=0$ deterninabitur, Vnde adiumento obseruationis moda factae colligitur, sectionem circuli integri in $n$ partes: requirere $i^{\circ}$ sectionem circuli integri in $n-1$ partes, $2^{\circ}$ sectionem alius arcus, qui llla sectione facta construi potest, in $n-1$ partes, $3^{\circ}$ extraetionem vnius radicis quadraticae, et quidem ostendi potest, hanc semper esse $\sqrt{ } n_{\text {. }}$. 
361. Superest vt nexum inter radices $\Omega$ atque functiones trigonometricas angulorum $\frac{P}{n}$, $\frac{2 P}{n}, \frac{3 P}{n} \ldots \frac{(n-x) P}{n}$ adhuc propius contemplemur. Methodus quam pro inueniendis radicibus. $\Omega$ exposuimus ita comparata est, vt adhuc incertum relinquat (nisi tabulae sinuum inter là-, borem ita vt supra diximus consultae fuerint, quod tamen minus directum foret), quaenam radices singulis illis angulis respondeant i. e. quaenam radix sit $=\cos \frac{P}{n}+i \sin \frac{P}{n}$, quaenarm $=\cos \frac{2 P}{i 2}+i \sin \frac{2 P}{n}$ etc. Haec vero incertitudo facile discutitur, reflectende, cosinus angulorum $\frac{P}{n}, \frac{2 P}{n}, \frac{3}{n}, \ldots \frac{(n-1) P}{2 n}$ continuo decrescere (siquidem etiam signorum ratio habeatur), sinưs omnes positiuos esse; angulos $\frac{(n-x) P}{n}, \frac{(n-z) P}{n}$, $\frac{(n-3) P}{n} . . \frac{(n+\mathrm{r}) P}{2 n}$ vero eosdem resp. cosinus habere vt illos, sinus autem negativos ceterum magnitudine absoluta sinubus illorum aequales. Quare e radicibus $\Omega$ duce istae, quae partes reales maximas (inter se aequales) habent, respondebunt angulis $\frac{P}{n}, \frac{(n-1) P}{n}$, et quidem priori ea vbi quantitas imaginaria $z$ per quantitatem positiuam, posteriori ea vbi $i$ per quantitatem negatiuam multiplicata est. Ex $n-3$ reliquis radicibus istae rursus quae maximas partes reales habent angulis $\frac{2 P}{n}, \frac{(n-2) P}{n}$ respondebunt et sic porro. 


\section{- $653-$}

- Simulac ea radix cui angulus $\frac{P}{n}$ respondet agnota est, eae quae angulis reliquis respondent etiam inde distingui poterunt; quod, si illa supponatur esse $=,[1]$ angulis $\frac{2 P}{n}, \frac{3 P}{n}, \frac{4 P}{n}$ etc. manifesto respondebunt radices $\left[\mathfrak{2 \lambda}^{\lambda}\right],\left[\mathrm{K}^{\lambda}\right],\left[4^{\lambda}\right]$ etc. Ita in exemplo art. 353 illico videtur, angulo $\frac{x}{x_{9}} P$ aliam radicem respondere non posse quam hanc [11] anguloque ${ }_{10} \mathcal{P} P$ radicem [8]; similiter angulis $\frac{\frac{2}{19}}{19} P, \frac{\mathrm{x} z}{\mathrm{x} 9} P, \frac{3}{\mathrm{x} 9} P, \frac{16}{19} P$ etc. respondent radices $[3],[16],[14]$, [5] etc. In exemplo art. 354 angulo ${ }_{T}^{T} P$ manifesto respondet radix $[1]$, angulo $\frac{2}{x z} P$ haec [2] etc. Hoc itaque modo. cosinus et sinus angulorum $\frac{P}{n}, \frac{2 P}{n}$ etc plene determinati erunt,

362. Quod vero attinet ad reliquas functiones trigonometricas horum angulorum, possent eae quidem e cosinubus et sinubus respondentibus per methodos vulgo notas facile deriuari, puta secantes et tangentes, diuidendo vnitatem et sinus per rosinus; nec non cosecantes et cotangentes, diuidendo vnitatem et cosinus per sinus. Sed commodius plerumque idem obtinetur adiumento formularum sequentium absque diuisionibus per meras additiones. Sit angulus: quicunque ex his $\frac{P}{n}, \frac{2 P}{n} \ldots \frac{(n-\mathrm{r}) P}{n}$ atque $\cos \omega+\ddot{i} \sin \omega=R$, vnde $R$ erit aliqua e radicibus $\Omega, \cos \omega=\frac{\mathrm{x}}{2}\left(R+\frac{\mathrm{T}}{\mathrm{a}}\right)=\frac{\mathrm{x}+R R}{2 R}, \sin \omega=$ $\frac{\mathrm{x}}{2 i}\left(R-\frac{\mathrm{I}}{\mathrm{s}}\right)=\frac{i(\mathrm{I}-R R)}{2 R}$. Hinc fit $\sec \omega=$ 


\section{$-654 \rightarrow$}

$\frac{2 R}{I+R R}$, tang $(\omega)=\frac{\left.i^{\prime} I-R R\right)}{I+R R}, \operatorname{cosec} \omega=\frac{2 R i}{R R-I}$, cotang $\omega^{\prime}=\frac{\langle(R R-\mathrm{I})}{R R-\mathrm{I}}$. Iam numeratores harum quatuor fractionum ita transformare ostendemus, vt per denominatores diuisibiles euadant.

I. Propter $R=R^{\mathrm{n}+\mathrm{x}}=R^{2 \mathrm{n}+\mathrm{x}}$, fit $2 R$ $=R+R^{2 n+x}$, quam expressionem per $1+$ $R R$ diuisibilem esse patet, quum $n$ sit numerus impar. Hinc fit sech $=R-R^{3}+R^{5}-R^{2} \ldots$ $+R^{2 n-x}$, adeoque (quum propter $\sin \omega=\sin 2 n-$ 1), $\sin 3 n=-\sin (2 n-3) \omega$ etc. manifesto fiat $\sin \omega-\sin 5 \omega+\sin 5 \omega+\sin (2 n-1) \omega=0)$, sec $\omega$ $=\cos \omega-\cos 3 \omega+\cos 5 \omega \ldots+\cos (2 n-1) \omega$, siue tandem, (quoniam $\cos \omega=\cos \left(2 n^{\prime}-1\right) \omega$, $\cos 3 \omega=\cos (2 n-3) \omega \mathrm{etc}),=2(\cos \omega-\cos 3 \omega$ $+\cos 5 \omega \ldots \mp \cos (n-2) \omega) \pm \cos n \omega$, signo signo stiperiori vel inferiori valente prout $n$ est formae $4 k+1$ vel $4 k+3$. Manifesto haec formula etiam ita exhiberi potest

$$
\begin{aligned}
& \sec \omega= \pm(1-2 \cos 2 \omega+2 \cos 4 \omega \cdots \pm \\
& 8 \cos (n-1) \omega) .
\end{aligned}
$$

II. Simili modo substituendo $1-R^{20+12}$ pro $1-R R$, prodit tangw $=i(\mathrm{I}-R R+$ $R^{4}-R^{1} \ldots-R^{3 \mathrm{n}}$ ), siue (quoniam $1-R^{2 \mathrm{n}}$ $=0, R R-R^{2 n-2}=2 i \sin 2 \omega, R^{4}-R^{2 m-4}$ $=2 i \sin 4$ (etc.)

$$
\underset{+}{\operatorname{tang} \omega}=2(\sin 2 \omega-1) \omega) . \sin 4 \omega+\sin 6 \omega \ldots
$$

III. Quum habeatur $i+R R+R^{*} \ldots+$ $R^{2 n-2}=0$ fit $n=n-1-R R-R+\ldots$ 
$R^{21-3}=(1-1)+(1-R R)+(1-R 4)$ $\cdots+\left(1-R^{2 n-2}\right)$ cuius aggregati partes singulae per $1-R R$ sunt diuisibiles. Hinc $\frac{n}{I-R R}=1+(1+R R)+(1+R R+R)$ $\cdots+\left(1+R R+R^{4} \ldots+R^{2 n-4}\right)=(n-1)$ $+(n-2) R R+(n-3) R 4 \ldots+R^{2 n-4}$; quocirca multiplicando per 2 , subtrahendo $o=$ $(n-1)\left(1+R R+R 4 \ldots+R^{2 n-2}\right)$ rursus que per $R$ multiplicando fit $\frac{2 m R}{I-R R}=(n-$ 1) $R+(n-3) R^{3}+(n-5) R^{5} \cdots-(n$ - 3) $R^{2 n-3}-(n-1) R^{2 n-\mathrm{r}}$, vnde protinus deducitar $\operatorname{cosec} \omega=\frac{x}{n}((n-\mathrm{I}) \sin s+(n-$ 3) $\sin 3 * \ldots-(n-1) \sin (2 n-1) \omega)=$ $\frac{2}{n}((n-1) \sin w+(n-3) \sin 3 n-$ etc + $2 \sin (n-2) \omega)$, quae formula etiam ita exhi beri potest

$$
\begin{aligned}
& \text { cosec }=-\frac{2}{n}(2 \sin 2 \omega+4 \sin 4 \omega+ \\
& 6 \sin 6 \omega+(n-1) \sin (n-1) 0 \%
\end{aligned}
$$

IV. Multiplicando valorem ipsius $\frac{n}{I-R R}$ supra traditum per $1+R R$ et substrahendo o $=(n-1)\left(1+R R+R^{4} \ldots+R^{2 n-2}\right)$, prodit $\frac{n(1+R R)}{x-R R}=(n-2) R R+(n-4) R^{4}$ $+(n-6) R^{6} \ldots-(n-2) R^{2 n-2}$, unde sta tim sequitur cotang $\%=\frac{T}{3 n}((n-2) \sin 2 \omega+(n$ $-4) \sin 40+(n-6) \sin 6 \ldots-(n-2)$ $\sin (n-2) \omega)=f((n-2) \sin 2 \omega+(n$ -4) $\sin 40 \ldots+3 \sin (n-3) \omega+\sin (n-$ 


\section{$-.656$}

1) w), quam forraulam etiam hocce modo exhibere licet

$$
\begin{aligned}
& \text { cotang } \omega=-\frac{2}{n}(\sin w+3 \sin 3 w \ldots+(n \\
& -2) \sin (n-2) w
\end{aligned}
$$

363. Quemadmodum, supponendo $n-1$. $=e f$, functio $X$ in $e$ factores $f$ dimensionem resolui potest; simulac valores omnium $e$ aggregatorum $f$ terminorum innotuerunt (art. 34.8 ): ita tunc etiam, supponendo $Z=0$ esse aequationem $n-1^{\text {if }}$ ordinis cuius radices sint sinus aut quaelibet aliae functiones trigonometricae aegularum $\frac{P}{n}, \frac{2 P}{n}, \frac{(n-1) P}{n}$, functio $Z$ in $e$ factores $f$ dimensionum resolui poterit, cuius rei praecipua momenta haec sunt.

Constet $\Omega$ ex $e$ periodis $f$ terminorum his $(f, \mathrm{I})=P, P^{\prime}, P^{\prime \prime}$ etc., periodusque $P$ e radicibus $[r],[a],[b],[c]$ etc.; $P^{\prime}$ ex his' $\left[a^{\prime}\right],\left[b^{\prime}\right]$, $\left[c^{\prime}\right]$ etc, ; $P^{\prime \prime}$ ex his $\left[a^{\prime \prime}\right],\left[b^{\prime \prime}\right],\left[c^{\prime \prime}\right]$ etc. etc. Respondeat radici [1] angulus $\omega$, adeoque radicibus $[a],[b]$ etco anguli $a w, b_{w}$ etc, radicibus $\left[a^{\prime}\right],\left[b^{\prime}\right]$ etc. anguli $a^{\prime} \omega, b^{\prime} \omega$ etc., radicibus $\left[a^{\prime \prime}\right],\left[b^{\prime \prime}\right]$ etc. anguli $a^{\prime \prime \alpha}, b^{\prime \prime}{ }_{\omega}$ etc. etc: perspicieturque facile, omnes: hos angulos simul sumtos cum angulis $\frac{P}{n}, \frac{2 P}{n}, \frac{3 P}{n} \cdots \frac{(n-\mathrm{x}) P}{n}$ respectu functionum trigonometricarum *), con-

-) Hoc respectu dno anguli conueniunt, quorum aiffexẹntia vel pexipheriae integrae vel alicui cius multiplo aequalie ast, quales secunid un periphexia m confruos rocare possemus, si congruentiain sensi aliquantum latiori intelligere Iuberet, 


\section{$-657-$}

uenire. Quodsi itaqüe furictio de qua agitur per characterem $\phi$ angulo praefixum denotetur; productum ex $e$ factoribus $x-\Phi \omega, x-\phi a \omega$ etc. statuatur $=Y$, productum ex his $x-\phi a^{\prime} \omega, x$ - $\phi b^{\prime} \omega$ etc. $=Y^{\prime}$, productum ex his $x-\phi a^{\prime \prime} \omega_{g}$ $x-\phi b^{\prime \prime} \omega$ etc. $=Y^{\prime \prime}$ etc.: necessario erit productum $Y Y Y^{\prime \prime} \ldots=Z$. Superest iam, vt demonstremus, omnes coëfficientes in functionibus $Y, Y^{\prime}, Y^{\prime \prime}$ etc. ad formam talem $A+B(f, 1)$ $+C(f, g)+D(f, g g) \ldots+L\left(f, g^{e-1}\right)$ reduci posse, quo facto manifesto omnes pro cognitis habendi erunt, simulac valores omnium aggregatorum $f$ terminorum innotuerunt: hoc sequent modo efficienus.

Sicuti $\cos \omega=\frac{x}{x}[1]+\frac{\pi}{2}[1]^{n-1}, \sin \omega=-$ $\frac{\pi}{2} i[1]+\frac{\pi}{2} i[1]^{n-1}$, ita per art. praec. reliquae quoque functiones trigonometricae anguli $\omega$ ad formam talem reduci possunt $\mathfrak{X}+\mathfrak{B}[1]+\mathfrak{E}[1]^{2}$ $+\mathfrak{D}[1]^{3}+$ etc, nulloque negotio perspicietur, functionem anguli $k$ tunc fieri $=\mathfrak{x}+\mathfrak{B}[k]$ $+\mathfrak{C}[k]^{2}+\mathfrak{D}[k]^{3}+$ etc. denotante $k$ integrum quemcunque. Iam quum singuli coëfficientes in $Y$ sint functiones rationales integrae inuariabiles ipsarum $\phi^{\prime}, \Phi a \omega, \phi b \omega$ etc, perspicuum est, si pro his quantitatibus valores sui substituantưr, singulos coëfficientes fieri functiones rationales integras inuariabiles ipsarum $[1],[a],[b]$ etc.; quamobrem per art. $34 \%$ ad formam $A+B\left(f_{2}\right.$ 1) $+C(f, g)+$ etc. reducentur. Et prorsus simili ratione etiam omnes coëfficientes in $Y^{\prime}, Y^{\prime \prime}$ etc. ad formam similem reducere licebit. $Q . E_{.} D$.

364. Circa problema art. praec. quasdam adhuc obseruationes adjicimus. 
I. Quum singuli coëfficientes in $Y$ sint functiones tales radicum in periodo $P^{\prime}$ (quam $\doteq$ $\left(f, a^{\prime}\right)$ statuere licet) contentarum, quales functiones radicum in $P$ sunt coëfficientes respondentes in $Y$, ex art. 347 manifestuma est, $Y^{\prime}$ ex $Y$ deriuari posse, si modo vbique in $Y$ pro $(f, 1)$, $(f, g),(f, g g)$ etc. resp. substituantur $\left(f, a^{\prime}\right)$, $\left(f, a^{\prime} g\right),\left(f, a^{\prime} g g\right)$ etc. Et perinde $Y^{\prime \prime}$ ex $Y$ deriuabitur substituendo vbique in $Y$ pro $(f, 1)$, $\left(f, g,(f, g g)\right.$ etc. resp. $\left(f, a^{\prime \prime}\right),\left(f, a^{\prime \prime} g\right)_{,}$ $\left(f, a^{\prime} g g\right)$ etc. etc. Simulatque igitur functio $Y$ euoluta est, reliquae $Y^{\prime}, Y^{\prime \prime}$ etc. nullo negotio inde sequuntur.

II. Supponendo $Y=x^{f}-\alpha x^{f-1}+6 x^{f-2}$ - etc, coêfficientes $a$, $b$ etc. erunt resp. summa radicum aequ. $Y=0$ i. e. quantitatum $Q \omega, \phi a a$, $\$ b$ w etc, summa productorum e binis etc. At plerumque hi coëfficientes multo commodius eruntur per methodum ei quae art. 34.9 tradita est similem, computando summam radicum $+\omega,+\alpha \omega, \psi b \omega$ etc, summam quadratorum, cuborum etc, atque hinc per theorema Newtonianum. illos coëfficientes deducendo. - Quoties \$ designat tangentem, secantem, cotangentem aut cosecantem adhuc alia compendia dantur, quae tarners silentio hic praeterimus.

III. Considerationem peculiarem meretur is casus vbi $f$ est numerus par, adeoque quaeuis periodus $P, P, P^{\prime}$ etc. ex ${ }^{r} f$ periodis binorum terminorum composita. Constet $P$ ex his $(2,1)$ $(2, a),(2, b),(2, c)$ etc., conuenientque numeri $1, a, b, c$ etc atque $n-1, n-a$; $n-b, n-c$ etc. simul sumti, cum his $i, a, b, c$ 


\section{$-659$}

etc, aut saltem (quod hic eodem redit) his secundurm modulum $n$ congrui erunt. Sed est $\varphi(n-$ i) $\omega= \pm \phi \omega, \phi(n-\mathfrak{a}) \omega= \pm \phi a \omega$ etc., signis superioribus valentibus quoties $\bar{\phi}$ designat cosinum aut secantem, inferioribus quando $\phi$ exprimit sinum, tangentem, cotangentem aut cosecantem. Hinc colligitur, in duobus casibus prioribus inter factores e quibus compositus est $x$ binos semper aequales, adeoque $T$ quadratum esse, et quidem $T=$ $y y$, si $y$ ponatur aequalis producto ex $x-\phi \omega$, $x-\varphi a \omega, x-\varphi b \omega$ etc. Similiter in iisdem casibus functiones reliquae $2 \prime q^{\prime \prime}$ etc. quadrata erunt, et quidem supponendo $P$ constare ex ( 2 , $\left.a^{\prime}\right),\left(2, b^{\prime}\right),\left(2, c^{\prime}\right)$ etc.; $p^{\prime \prime} \operatorname{ex}\left(2, a^{\prime \prime}\right),\left(2, b^{\prime \prime}\right)$ s ( $\left.2, a^{\prime \prime}\right)$ etc. etc., productum ex $x-\phi a^{\prime} a, x-$ $\phi b^{\prime} \omega, x-\phi c^{\prime} \omega$ etc. esse $=y^{\prime}$, productum ex $x$ $-\varphi \mathfrak{a}^{\prime \prime}, x-\phi \mathfrak{b}^{\prime \prime}{ }^{\prime \prime}$ etc., $=y^{\prime \prime}$ etc. erit $\mathfrak{x}^{\prime}=$ $y^{\prime} y^{\prime}, x^{\prime \prime}=y^{\prime \prime} y^{\prime \prime}$ etc.; nec non etiam functio $Z$ quadratum erit (conf. supra art. 337 ), et radix producto ex $y, y^{\prime}, y^{\prime \prime}$ etc. aequalis. Ceterum facile perspicietur, $y^{\prime}, y^{\prime \prime}$ etc. perinde ex $y$ deriuari, vt $x, x^{\prime}$ etc. ex $\mathscr{T}$ sequi ante in I diximus; nec non singulos coëfficientes in $\gamma$ quoque ad formam $A+B(f, \mathrm{r})+C(f, g)+$ etc. posse, quum summae singularum potestatum aequ. $y=0$ manifesto sint semisses potestatum aequ. $\mathscr{T}=0$, adeoque ad talem formam reducibiles. - In quatuor casibus posterioribus autem $x$ erit productum e factoribus $x x-(\varphi \cdot)^{2}, x x-\left(\varphi a_{v}\right)^{2}$, $x x-(\Phi b \dot{b})^{2}$ etc, adeoque formae $x f-\lambda f^{-2}$ $+\mu x^{f-4}-$ etc., patetque coëfficientes $\lambda, \mu$ etc. e summis quadratorum, biquadratorum etc. radicum $\varphi_{w}, \phi \omega, \phi a w, \phi \mathfrak{b}_{w}$ etc. deduci posse; et similiter se habebunt functiones $I^{\prime}, \mathscr{X}^{\prime \prime}$ 'etc. 


\section{$-660-$}

Ex. I. Sit $n=17, f=8$ atque designet cosinum. Hine fit $Z=\left(x^{8}+{ }_{4}^{8} x^{4}-{ }^{7} x^{n}-\right.$

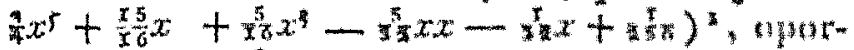
tetque adeo $\sqrt{ } Z$ in duos factures quaterno um dimensionum $y, y^{\prime}$ resoluere, Peritudus $p=$ $(8, x)$ constat ex $(2,1),(2,9),(2,13,(2$, 15) vnde $y$ erit producturn e: fattoribus $x$ $x-\varphi 9 \omega, x-015 \%, x-915 \%$. Subsitukndo $\frac{\pi}{2}[k]+\frac{x}{2}[n-k]$ pro qkw, inu? $\left.\phi 13^{2}+\phi 15^{\alpha}=1{ }^{x}(8,1) ; \quad(\phi \omega)^{2}+606 \omega\right)^{2}+$ $(\phi 13 \omega)^{2}+\left(\$ 15^{\infty}\right)^{2}=2+4(8,1) ;$ perinde summa cuborum $={ }^{3}(8,1)+{ }_{n}^{3}(8,3)$, summa biquadratorum $=1 \frac{8}{2}+\frac{3}{x}, 8,1 ;$ hine per theorema Newtonianum coifticientibus in $y$ determinatis prodit $y=x^{4}-{ }_{2}^{x}(8,2) x^{4}+{ }_{4}^{7}(28,1+$ $2(8,3): x x-3\left((8,1)+5(8,3) x+\frac{4}{5}((8,1)+\right.$ $(8,3) ; y^{\prime}$ vero ex $y$ deriuatur commutando $(8$, I) cum $(8,3)$; substituendo itatque pro $(8,1)_{2}$ $(8,3)$ valores $-\frac{x}{2}+\frac{x}{2} \sqrt{7} 7,-\frac{x}{2}-\frac{1}{4} \sqrt{17} \mathrm{fit}_{\mathrm{i}}$

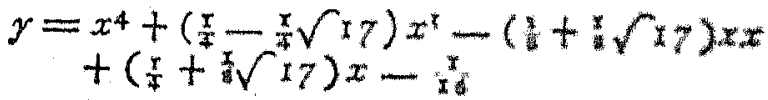

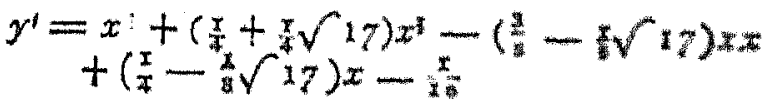

Simili modo $\sqrt{ } Z$ in quatuor factores binarurn dimensionum resolui potest, quorum primus erit $\left(x-\phi^{21}\right)(x-\phi 13$ ) $)$, secundus $(x-49$ w) $(x-\phi 15 \alpha)$, tertius $(x-\phi)\left(x-x_{0}\right)$, quar. tus $\left(x-\phi_{10 \omega}\right)(x-\phi 1)$, omnesque cotflicientes in his factoribus per quatuor aggregata $(4,1)(4,9),(4,5),(4,10)$ expratri goterunt. Manifesto autem productum * factore 


\section{$-661$}

primo in secundum erit $y$, productum e textio in quartum $y^{\prime}$.

Ex. II. Si, omnibus reliquis manentibus, - sinum indicare supponitur, ita vt $Z=x^{56}$ $\frac{x 7}{4} x^{14}+\frac{x \times 9}{x 6} x^{x 2}-\frac{27 x}{37} x^{60}+\frac{935}{250} x^{4}-\frac{36 x}{512} x^{6}$ $+{ }_{2048}^{357} x^{4}-\frac{5 x}{4096} x x+8 x^{3} 38$ in duos factores 8 dimensionum $y, y^{t}$ resoluere oporteat, exit $y$ productum e quatuor factoribus duplicibus $x x-$ $\left(\Phi_{\omega}\right)^{2}, x x-(\varphi 9)^{2}, x x-(\varphi 13 *)^{2}, x x-$ $(\$ 15)^{2}$. Iam quum sit $\phi k \omega=-\frac{1}{3} i[k]+\frac{1}{2} i[n$ $-k]$, erit $(\phi k \omega)^{2}=-\frac{x}{4}[2 k]+\frac{x}{2}[n]-\frac{x}{4}[2 \pi$ $-2 k]=\frac{x}{2}-x[2 k]-\frac{x}{4}[2 n-2 k]$; hinc deducitur summa quadratorum radicum $\varphi^{\omega}, 9^{\omega}$, $\phi 13^{\circ}, \Phi_{15}$ haec $2-{ }_{4}{ }_{4}(8,1)$, earundem biquadratorum summa $=\frac{1}{2}-\frac{3}{x 6}(8,1)$, summa potestatum sextarum $=\frac{\pi}{4}-\frac{9}{27}(8,1)-\frac{1}{64}(8,3)$, summa octauarum $\frac{3}{8}-\frac{z^{2}}{2}(8,1)-\frac{1}{3}(8,3)$. Hinc fit $y=x^{8}-\left(2-x(8,1) x^{6}+(1-\right.$ $\left.\frac{1}{78}(8,1)+\frac{7}{3}(8,3)\right) x^{4}-\left(\frac{x}{2}-\frac{2}{64} 8,1\right)+$

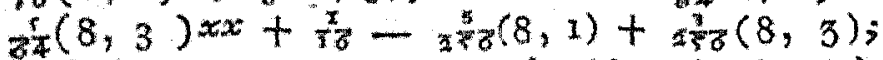
$y^{\prime}$ deriuatur ex y commutando $(8, x),(8,3)$, ita te per substitutionem valorum horum aggregatorum habeatur

$$
\begin{aligned}
& y=x^{8}-\left(\frac{17}{8}-\frac{\pi}{8} \sqrt{ } \sqrt{ }+x^{5}+\left(\frac{5 \pi}{32}-\frac{7}{32} \sqrt{17}\right) x^{4}\right. \\
& -\left(\frac{17}{32}-\frac{7}{64} \sqrt{27}\right) x x+\frac{17}{250}-\frac{x}{64} \sqrt{x} 7 \\
& y^{\prime}=x^{8}-\left(\frac{17}{8}+\frac{x}{8} \sqrt{x} 7\right)^{x^{5}}+\left(\frac{51}{32}+\frac{7}{32} \sqrt{ } 7\right) x^{4}- \\
& \left(\frac{17}{32}+\frac{7}{64} \sqrt{ } \sqrt{7}\right) x x+\frac{17}{256}+\frac{1}{64} \sqrt{ } \times 7
\end{aligned}
$$

Perinde $Z$ in quatuox factores resolui potest, quo$\mathrm{T}+3$ 
rum coëfficientes per aggregata quatuor terminorum exprimi possunt, et quidem productum $\theta$ duobus erit $y$, productum e duobus reliquis $y^{\prime}$.

365. Reduximus itaque, per disquisitiones praecedentes, sectionem circuli in $n$ partes, si $n$ est numerus primus, ad solutionem tot aequationum, in quot factores resoluere licet numerum $n-1$, quarum aequationem gradus per magnitudinem factorum determinantur. Quoties itaque $n-I$ est potestas numeri 2 , quod euenit pro valoribus ipsius $n$ his $3,5,17,257,65537$ etc., sectio circuli ad solas aequationes quadraticas reducetur, functionesque trigonometricae angulorum $\frac{P}{n}, \frac{2 P}{n}$ etc. per radices quadraticas plus minusue complicatas (pro magnitudine ipsius $n$ ) exhiberi poterunt; quocirca in his casibus sectio circuli in $n$ partes, siue descriptio polygoni regularis $n$ laterum manifesto per constructiones geometricas absolui poterit. Ita e. g. pro $n=I 7$ ex artt. 354, $36 \mathrm{I}$ facile pro cosinu anguli $\frac{\mathrm{x}}{\mathrm{I} z} \boldsymbol{P}$ expressio haec deriuatur:

$$
\begin{aligned}
& -\frac{x}{16}+\frac{T}{16} \sqrt{1} 7+\frac{1}{16} \sqrt{ }(34-2 \sqrt{I} \eta)-\frac{1}{8} \sqrt{ }(17 \\
& +3 \sqrt{17}-\sqrt{(34}-2 \sqrt{17})-2 \sqrt{(34+2 \sqrt{17}))}
\end{aligned}
$$

cosinus multiplorum illius anguli formam similem, sinus autem vno signo radicali plus habent. Magnopere sane est mirandum, quod, quum iam Euclidis temporibus circuli diuisibilitas geometrica in tres et quinque partes nota fuerit, nihil his inuentis interuallo 2000 annorum adie. ctum sit, omnesque geometrae tamquam certum 


\section{$-663-$}

pronunciauerint, praeter illas sectiones easque quae sponte inde demanant, puta sectiones in 15 , $3.2^{\mu}, 5.2^{\mu}, 15.2^{\mu \nu}$ nec non in $2^{\mu}$ partes, nullas alias per constructiones geometricas absolui posse. - Ceterum facile probatur, si numerus primus $n$ sit $=2^{m}+1$, etiam exponentem $m$ alios factores primos quam numerum 2 implicare non posse, adeoque vel $=1$ vel $=2$ vel altiori potestati numeri 2 aequalem esse debere; si enim $m$ per vllum numerum imparem $\zeta$ (vnitate maiorem) diuisibilis esset atque $m=\zeta_{n}$, foret $2^{m}+1$ diuisibilis per $2^{n}+1$, adeoque necessario compositus. Omnes itaque valores ipsius $n$, pro quibus ad meras aequationes quadraticas deferimur; sub forma $2^{2^{\prime}}+1$ continentur; ita quinque numeri $3,5,17,257,65537$ prodeunt statuerido $v=0,1,2,3,4$ siue $m=1,2,4,8$, 36. Neutiquam vero pro omnibus numeris sub illa forma contentis sectio circuli geometrice perficitar, sed pro tis tantum qui sunt numeri primi. Fermatius quidem inductione deceptus affirmauerat, omnes numeros sub illa forma contentos necessario primos esse; at ill. Fuler hanc regulam lam pro $v=5$ siue $m=32$ erroneam esse, numero $2^{3 / 2}+1=4294967297$ factorem $64 \mathrm{x}$ inuoluente, primus animaduertit.

Quoties autem $n-\mathrm{r}$ alios factores primos praeter 2 implicat, semper ad aequationes altiores deferimur; puta ad vnam pluresue cubicas quando 3 semel aut pluries inter factores primos ipsius $n-1$ reperitur; ad aequationes quinti gradus, quando $n-I$ diuásibilis est per 5 etc, omNipve RIGORE DEMONSTRARE POSSVMVS, HAS AEQVATIONES T t 4 


\section{$-66_{4}-$}

ELBVATAS NVLLO MODO NEC RVITARI NEC AD INDRTORES REDVCr pOSs, etsi limites huius operis hanc demonstrationem hic tradtre non patiantur, quod tamen monendum esse duximus, nt: quis adhuc alias sectiones praeter eas quas theoria nostra suggerit, e. g. sectiones in $7,11,13,10$ etc. partes, ad constructiones geomatricas perducere speret, tempusque inutiliter terat.

366. $\mathrm{Si}$ circulus in $a^{*}$ partes secandus est, designante $a$ numerum primum, manilesto hoc geometrice perficere licet, quando $a=2$, nem que vero pro vllo alio valore ipsius $a$, siquidem $a>$ I; tunc enim praeter eas aequationes quae ad sectionem in a partes requiruntur necessario adhuc - I alias $a^{\text {t }}$ gradus soluere oportet; etiam has nullo modo nec eutare nec deprimere licet. Gradus itaque aequationum necessariarum e factoribus primis numeri $(a-I) a^{\alpha-x}$ generaliter (scilicet pro eo quoque casu vbi $=1$ ) cognosci possunt.

Denique si circulus in $N=a^{*} b^{6} c^{\psi} \ldots$ partes secandus est, denotantibus $a, b, c$ etc. numeros primos inaequales, sufficit, sectiones in $a^{*}, b^{6}, c^{2}$ etc. partes perfecisse (art. $33^{6}$ ); quare vt gradus aequationum ad hunc finem necessariarum cognoscantur, factores primos numerorum (a I) $a^{\alpha-x},(b-1) b^{b-1},(c-I) c^{y-x}$ etc., siue quod hic eodem redit producti ex his numeris considerare oportet. Obseruetur, hoc productum exprimere multitudinem numerorum ad $N$ pri morum ipsoque minorum (ant. 58). Geometrice itaque sectio tunc tantummodo absoluitur, 
quando hic numerus est potestas binarii; quando vero factores primos alios quam 2 puta $p, p^{\prime}$ etc. implicat; aequationes gradus $p^{\prime \prime}$, $p^{\prime \text { ti }}$ etc. nullo modo euitari possunt. ITinc colligitur generaliter, vt circulus geometrice in $N$ partes diuidi possit, $N$ esse debere vel 2 aut altiorem potestatem ipsius 2, vel numerum primum formae $2^{m}+1$, vel productum e pluribus huiusmodi numeris primis, vel productum ex vno tali primo aut pluribus in 2 aut potestatem altiorem ipsius 2; siue breuius, requiritur, vt $N$ neque vllum factorem primum imparem qui nori est formae $2^{m}+x$ implicet, neque etian vllum factorem primum formae $2^{m}+\mathrm{x}$ pluries. Huiusmadi valores ipsius $N$ infra 300 reperiuntur hi 38 :

s, $3,4,5,6,8,10,12,15, \times 6, \times 7,20,24,30$, $3^{2}, 34,40,48,5 \mathrm{x}, 60,64,68,80,85,96$, x02, $120,128,136,160,170,192,204,240,255$, $256,257,272$. 


\section{ADDITAMENTA.}

$A_{d}$ art. 28. Solutio aequationis indeterminatae $a x=b y \pm 1$ non primo ab ill. Eulero (vt illic dicitur) sed iam a geometra $17^{\mathrm{mi}}$ saeculi $\mathrm{Ba}$ chet de Meziriac, celebri Diophanti editore et commentatore, perfecta est, cui ill. La Grange hunc honorem vindicauit (Add. à l'Algebre d' Euler p. 525, vbi simul methodi indoles indicata est ). Bachet inuentum suum in editione secunda libri Problêmes plaisans et delectables qui se fant par les nombres, 1624 , tradidit; in editione prima (à Lyon 1612), quam solam mihi videre licuit, nondum exstat, verumtamen iam annunciatur.

Ad artt. 151, 296, 297. Ill. Le Gendre demonstrationem suam denuo exposuit in opere praedaro Essai d'une theorie des nombres p. 214 sqq., attamen ita, vt nihil essentiale mutatum sit: quamobrem haec methodus etiamnum omnibus obiectionibus in art. 297 prolatis obnoxia manet. Theorema quidem (cui vna suppositio innititur), in quauis progressione arithmetica $l, l+k, l+2 k$ etc, numeros primos reperiri, si $k$ et $l$ diuisorem 


\section{$-667-$}

communem non habeant, fusius in hoc opere consideratum est $p, 12 \cdot s q$ : : sed rigori geometrico nondum satisfactum esse videtur. Attamen tunc quoque, quando hoe theorema plene demonstratum erit: suppositio altera supererit (dari numeros primos formae $4 n+3$, quorum non residuum quadraticum sit numerus primus datus formae $4 n+1$ positiue sumtus), quae an rigorose demonstrari possit, nisi theorema fundamentale ipsum iam supponatur, nescio, Ceterum obseruare oportet, ill. Le Gendre hanc posteriorem suppositionem non tacite assumsisse, sed. ipsum quoque eam non dissimulauisse, p. 221.

Ad artt. 288:293. De eodem argumento, quod hic tamquam applicatio specialis theoriae formarum ternariarim exhibetur, et respectu xigoris et generalitatis ita absolutum esse videtur, vi nihil amplius desiderari possit, ill. Le Gendre in parte III operis sui p. $32 \mathrm{r}=400$ disquisitionem multo ampliorem instituit*). Principiis et methodis vsus est a nostris prorsus diuersis: attamen hac via compluribus difficultatibus implicatus est, quae effecerunt, vt theoremata palmaria demonstratione rigorosa munire non licuerit. Has difficultates ipse candide indicauit: sed ni fallimur hae quidem facilius forsan auferri poterunt, quam ea, quod in hac quoque disquisitione theorema modo memoratum (In quauis progressione

*) Vel nobis non monontibus lectores cauebunt, ne nostras form mas ternarias cum eo, quod ill. Le Gendre forme trinaixe d'un nombre dixit, confundaat, Scilicet pex hane expressionexa andicalít decompositionem nume is id tria gúadrata, 


\section{$-668-$}

arithmetica etc.) suppositum est, p. 372 annot. in fine.

Ad art. 306 VIII. In chiliade tertia determinantium negatiuorum reperti sunt 37 irregulares, inter quos 18 habent indicem irregularitatis. 2, et 19 reliqui indicem 3 .

Ad eundem X. Quaestionem hic propasitam plene soluere nuper successit, quam disquisitionem plures partes tum Arithmeticae sublimioris tum Analyseos mirifice illustrantem in continuatione huius operis trademus quam primum licebit. Eadem docuit, coefficientem $m$ in art 504. p. 504 . esse $=y^{*}=0,3458847616$, designante $\gamma$ eandem quantitatem vt in art. 302 , et * vt ibidem semicircumferentiam circuli cuius radius 1 .

* * *

In regulam art. 256 IV error irrepsit, quï vt emendetur, in linea pell. p. 394, pro Anabs ... legatur $\frac{A \text { nnk...; }}{20285 .:}$ ot in linea vit verba tum st $=0$, tum, in l. prima et secunda p. 395 vero thaec $>>0$ simulque deleantur. 


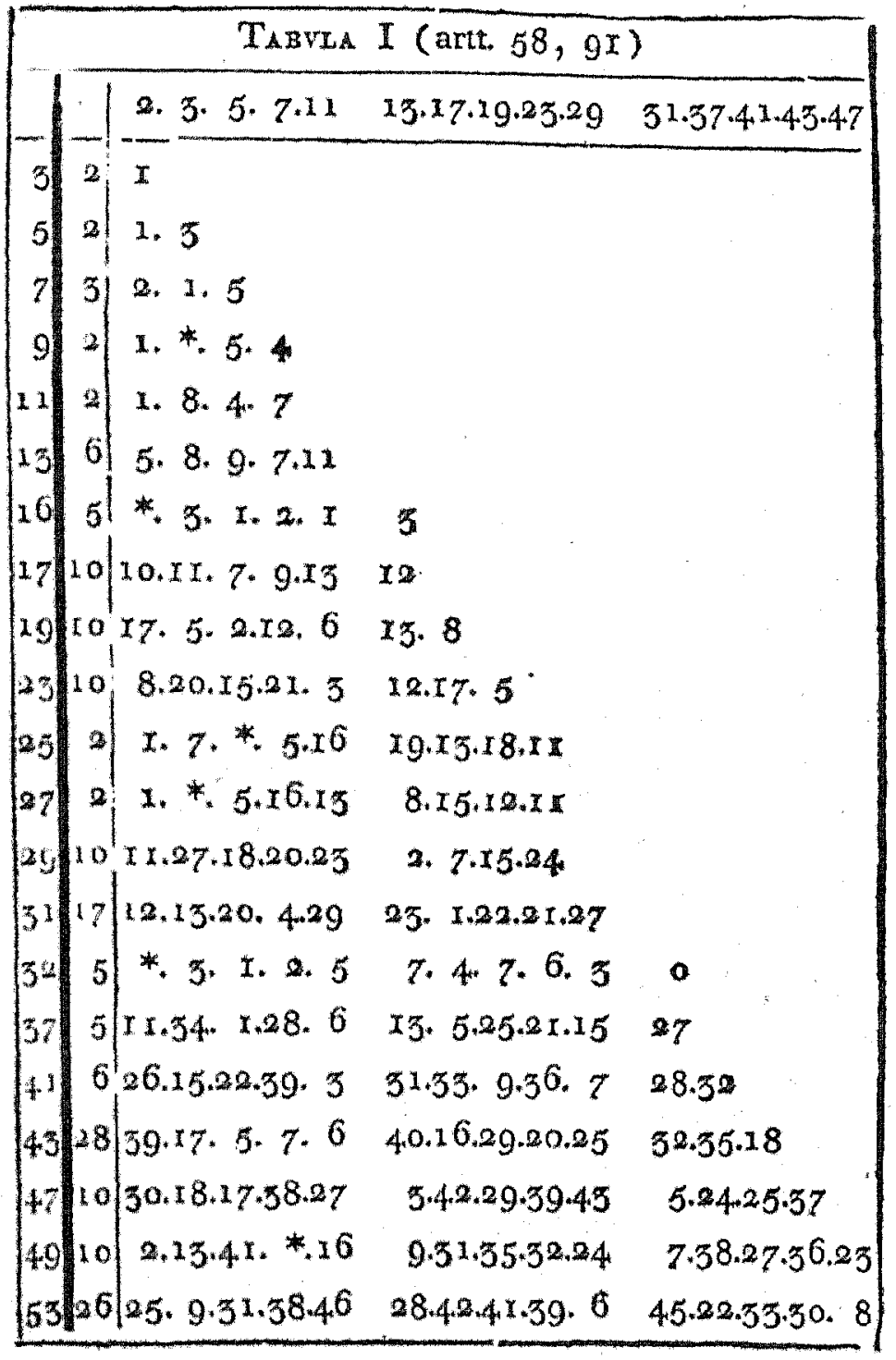




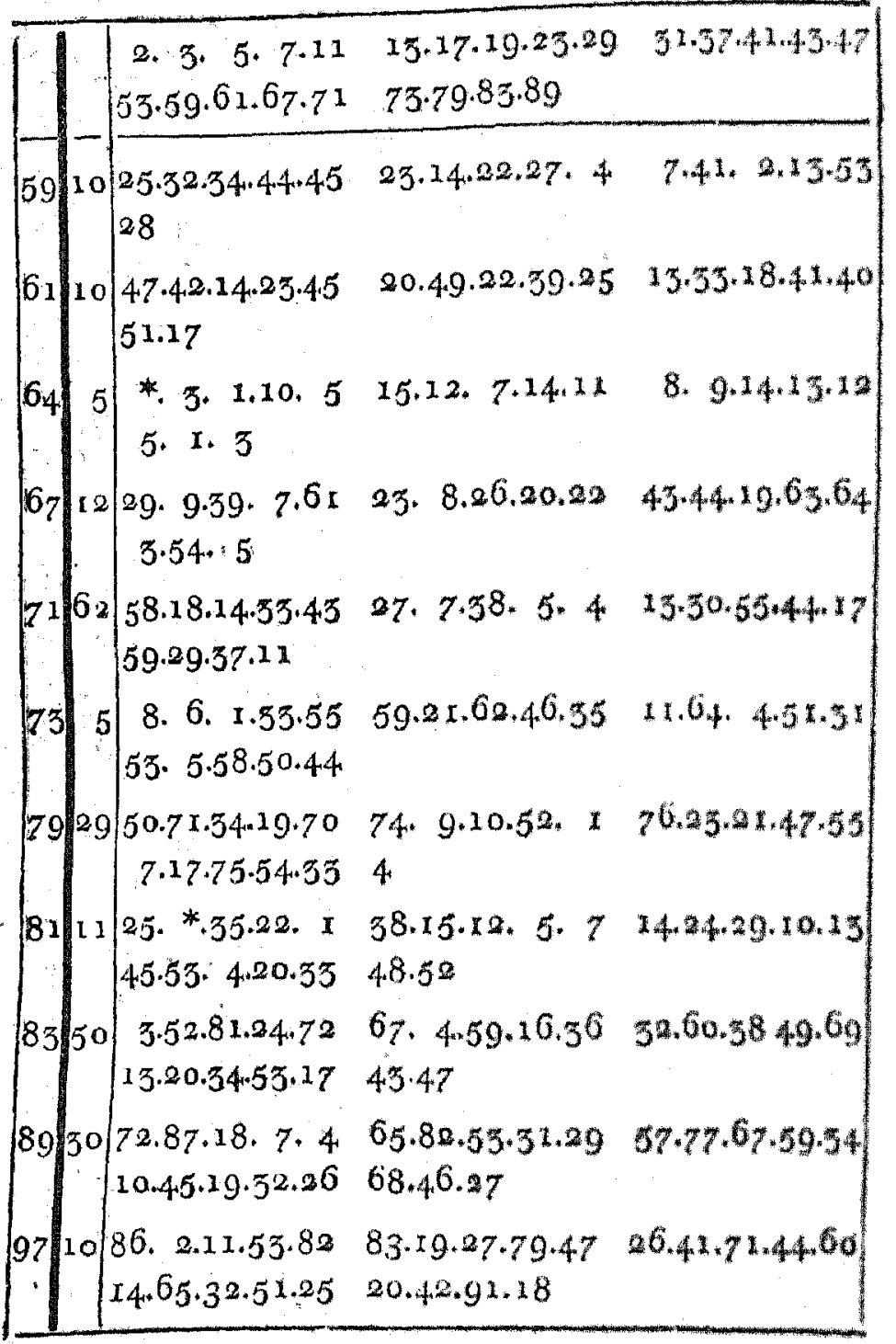




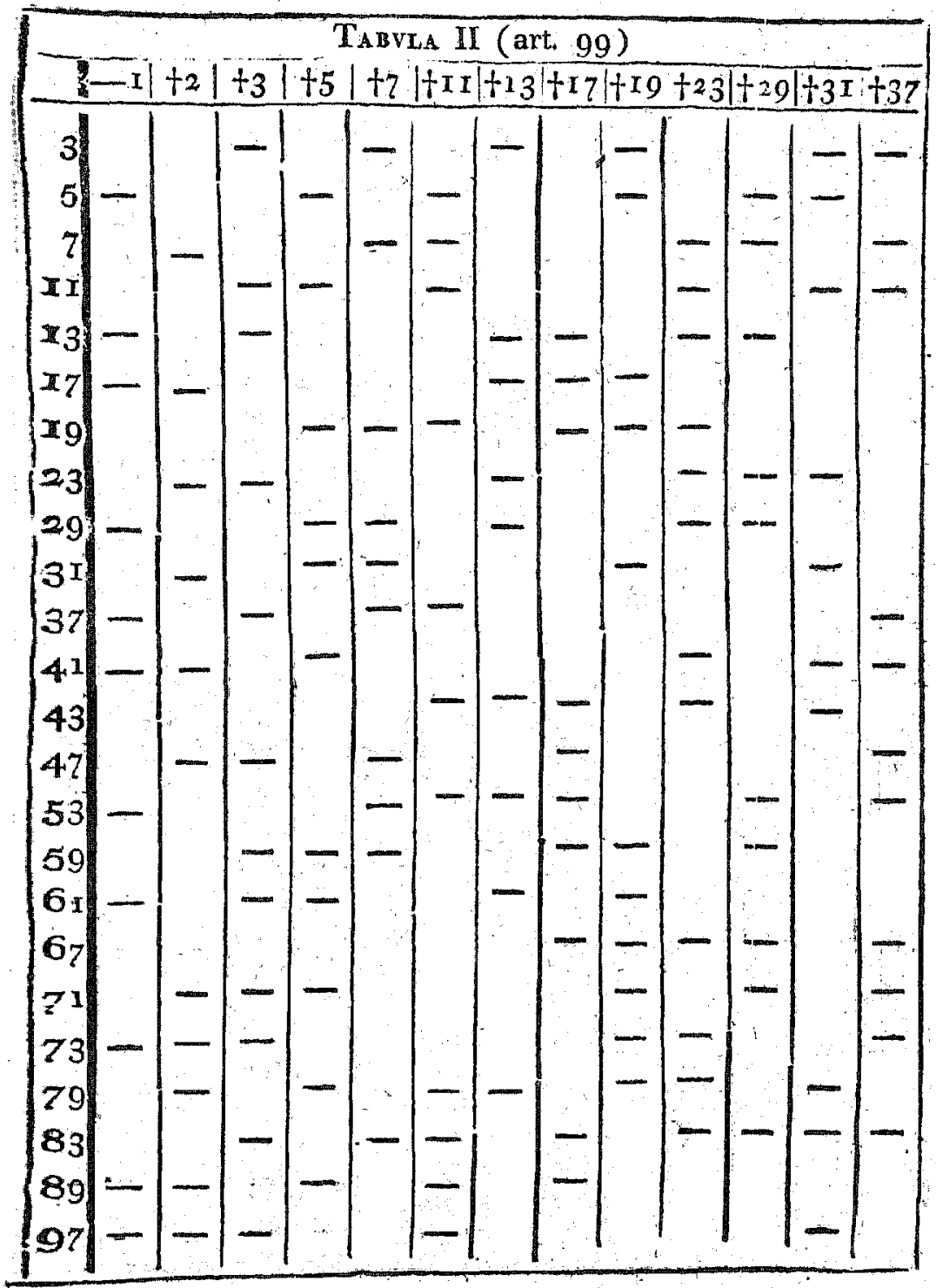




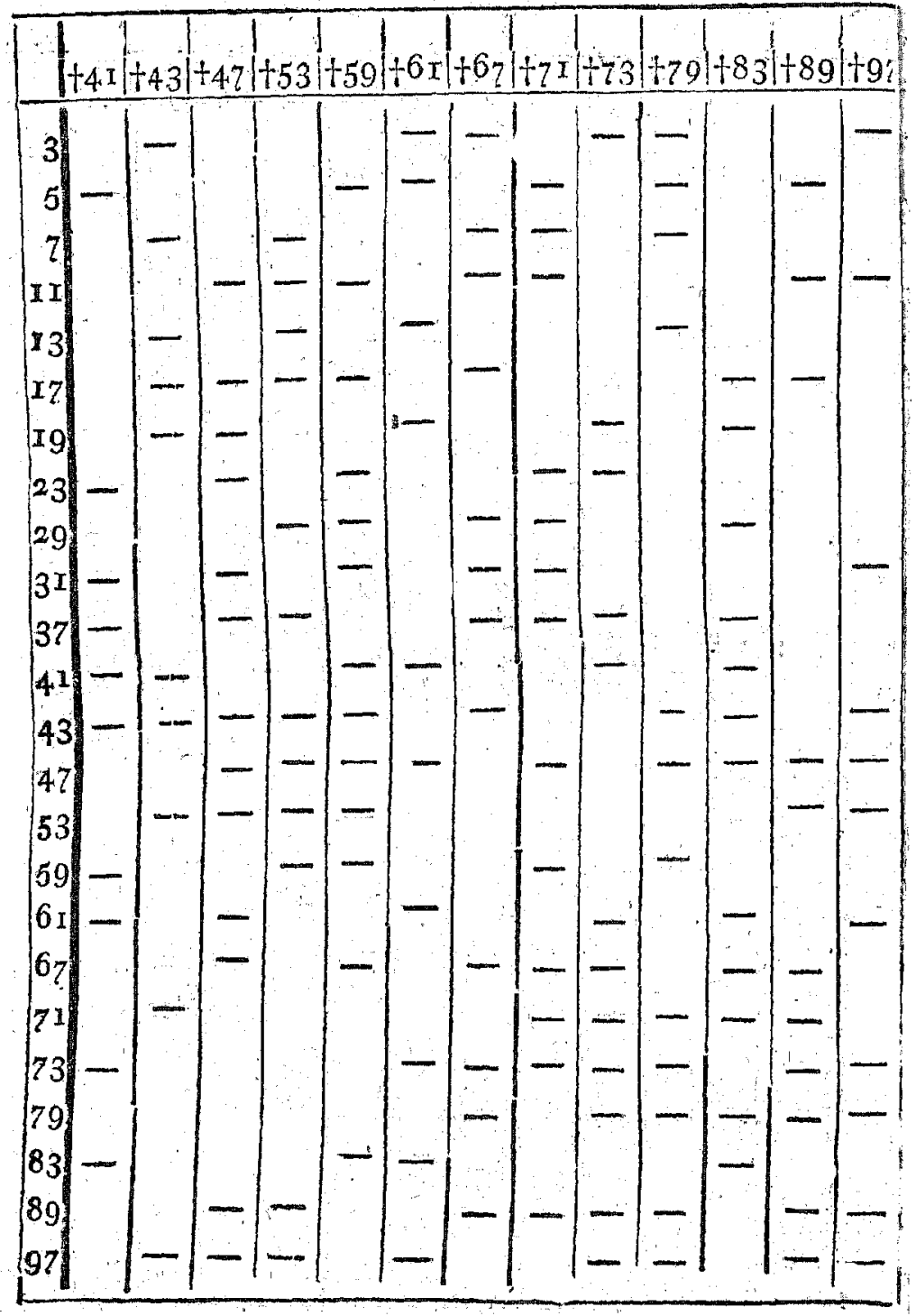




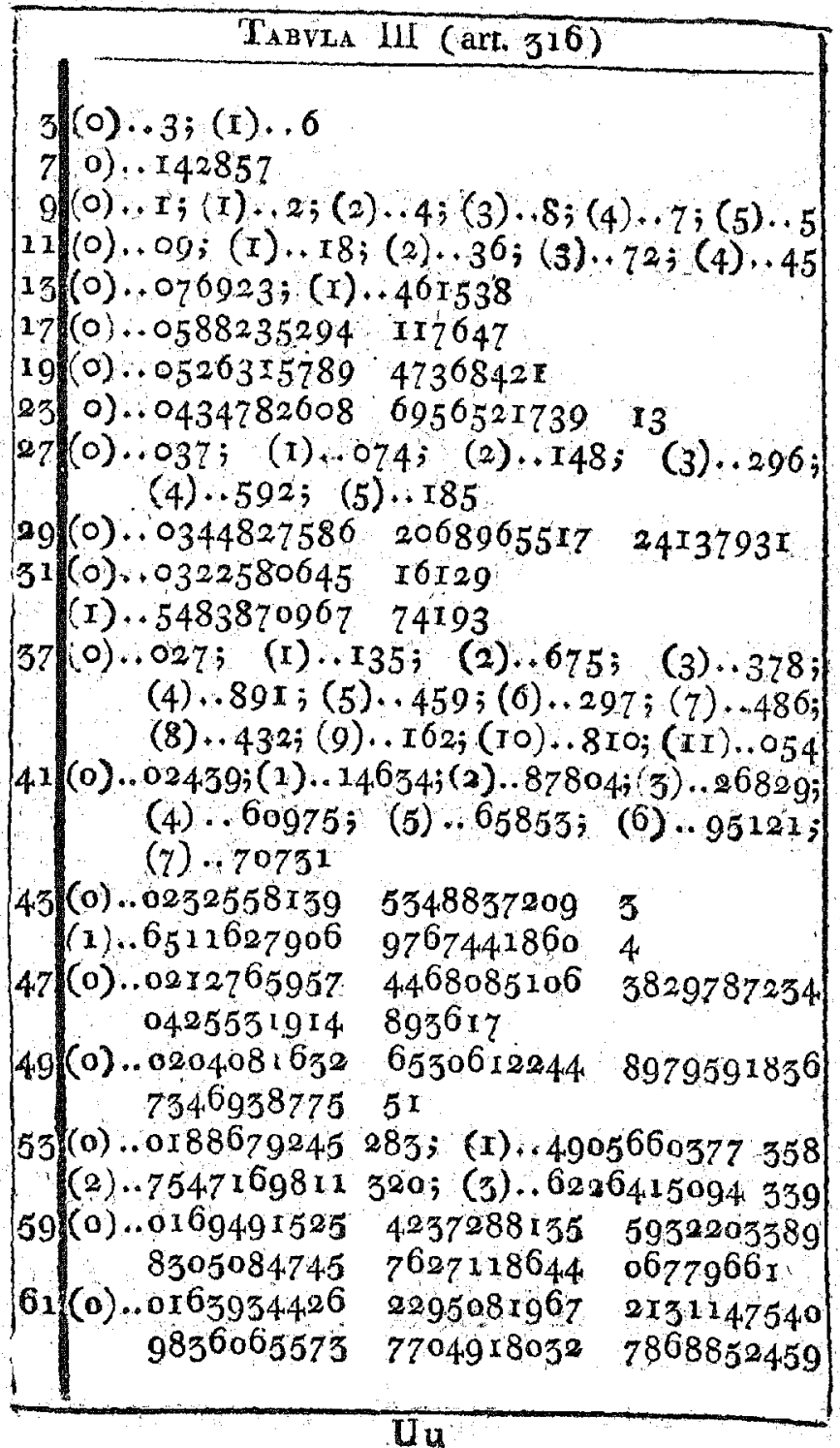

Tabvea III (art. $3^{16}$ )

$3(0) . .3 ;(x) . .6$

7 o) : . 142857

$11(0) . .09 ;(1)+18 ;(2) . .36 ;(3) .+72 ;(4), .45$

$13(0)+076923 ;(x)+.46 \mathrm{r}_{538}$

17 (o) $.0588235294 \quad$ II 2647

$19(0) .0526355789 \cdot 4736842 \mathrm{~F}$

23 0)..0434782608 6956521739

27 (0)..037; (1) ..074; (2).. $448 ;$ (3)..296;

(4) $.592 ;(5) \div 185$

$29(0) .0344827586 \quad 2068965517 \quad 2413793 \mathrm{r}$

$31(0)+0322580645$ I6r 29

(I) $+5483870967 \quad 74193$

(4). $.89 x ;(5) \ldots 459 ;(6)+297 ;(7) . .486$;

(8)..432; (9)..162; (IO)..810; (II)..054

(4) 609750 (5) $658550(6)$ (7)

(7). .70731

$43(0) .00232558 \times 39$

(1)..6511627906

53488372093

9767441860

45

4468085100

(9536r

.

897991856 $8 \pi$ 


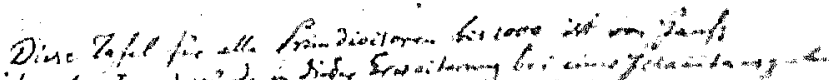

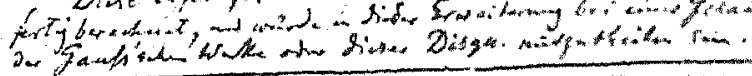

67 (0).0149253731 $343^{2853820} 8955225880$ 597

1).. $179104477^{6} \quad 119402985^{\circ} \quad 7462686567$ 164

71 o) .0140845070 4225352112 6/60563580 28,69

(c) $.8732394366 \quad 1971830985 \quad 9154929577$ 46478

$75(0) .01369863 ;(x) .06849315 ;(2) \cdot 34246575$

(3). $.71232876 ;(4) . .56 \times 64383 ;(5) . .80821917$

$(6) .04109589 ; 7) \ldots 20547945 ;(8) . .02739726$

79 (0)..or 26582278 481; (1)..3670886075 949

(2)..6455696202 53x;(3)..7215189873417

(4) $+9240506309113 ;(5) \cdot .797468354+303$

$81(0), .012345679 ;(1) .1358024 .09$

(2)..493827160; (3) . 432098765

4). $.7530864,19 ; 5) \cdot .283950617$

$83(0) .0120481927 \quad 71084332349397590561$ $4,45783 \times 395 \quad 3$

(1). $6024096385 \quad 5421686746 \quad 9870518072$ $2891566265 \quad 0$

$89(0) .0112359550 \quad 5^{617977528} \quad 0898876404$ $4943820224 \quad 7191$

1). $.3370786516 \quad 85393^{2} 5^{842} \quad 6966292134$ 8314606741.5730

$97(0)_{1} .0103492783 \quad 5051646391 \quad 7525773195$ $8762886597 \quad 9381443298 \quad 9090721649$ $4845360824 \quad 7422680412 \quad 3711340206$ $1855^{67} 7$

Goslartak ex Ofeicina E. W. G. Kinchizk. 


\section{Errata Typographica.}

P. 2. 1. 9 a calce pro $\frac{A-a}{m}$ l. $\frac{a-A}{m}$ p. 4.1 .20 pro $A b$ 1. $A B$. P. 6. 1. I9 factores noo et $c$ quos iungere oportuisset separati sunt. Similiter aliis passim locis P. 16.1 . plt pro +1. P. 161 penult. literae a et $B$ commate soparentur. $P, 19.3$. a. c. $l_{\text {o }}$ $\frac{b-a v}{m}$. P. 20 l. 3 pro m 1. n. Tbid 1. I6 pro $30 x^{\prime}$ 1. $38 x^{\circ}$. P. 22. 1. ro, I2, rg et 20 pro $A v 1 . a+A v$. Ib. 1. I9 pro $\frac{C}{\delta}, \frac{C}{\varepsilon}$ Ib. 1 2r pro $\frac{A B C}{\varepsilon}$, $\frac{A B C}{\delta \varepsilon}$ P. 3 I 1, 20 1. $\phi N, \varphi P$. P. 331.18 pro, M $1=M_{\text {。 }}$ P. 38. 1. pen. l. incongruas. $P$. 39. I. I8 deleatur debevi. P. 40 1. 24 l. Comm, nox P. 43 1. ro incongruae. Ib. 1. 22 pro praec. 1. 45 P. 48.1 .3 pro $p-I$ factores l. factores numeri $p-$ I. P. 52 l. 14 pro $\equiv 1 .=$ P. 551.9 pro - 1. I. P. 591.171 . et $\sqrt{A}$. Ib. $1.251 . x^{\delta}$. p.60l. 51 posterioris. P.621.5 lo vnusque valor.Ib. $1.7 \mathrm{lin} / \mathrm{hi}$ : $1, r, \mathrm{rr} . \mathrm{Ib}$. I. 13 pro vnitati 1. ipsi $A$. P. 651.31 etiam km - I per $\frac{t}{d}$ p. 69 1. 4 a. c. in fractionis numeratore pro $p 1 . p-I_{0}$. 701. I6 1. elegimus. P. 78 1. I7 pro $p 1 . p-\mathrm{x}$. P. 82 l. I3 I. $x^{7} \equiv$ I. Ib. 1. 20 pro $p^{y-1}$ I. $p^{y}-$ I. P. 83 1. 201 . mod $p^{\mu+2+2}$. Ib. 1. vlt. pro $t$ l. $\varepsilon$. P. 841.6 pro indeterminatum 1. integrum. P. 85 l. 4 et $Z$ a calce pro $\mu$ l. $n_{*}$ P. 88 l. I pro $m+$ I $1 . m+2$. P. 89 l. 3 pro +7 l. +5 ant $8 n+7$. P. 921.6 a c. pro $\frac{r}{2}(m-r)^{2}$ l. $\left(\frac{T}{2} m-r\right)^{2}$. P. 93 art. 95 cum praecedente vniacur, numerusque 95 sequenti praeponatur. P. 97 L. 8 pro 95 l. 96. P. 99 l. 7 pro $\mu$ l. $m$. P. Ioo l. 8 a. c. 1 ipsius $p^{n}$. P. ror 1.3 pro $2 x+I 1$. t I. P. ro5 I. 8 a. c. 1. Sect praec. P., Ioz l. I l. numerorum prinorum $\mathrm{P}$, ro8 inter l. penult et vit. exciderunt haec: $4 n+1$; impar quando $p$ est formae. P. IIL 1. 7. insere numerum 43 P. II9 1 . I3 I, nonresidaum impar. P. I20 l, I7 l, numerorum imparium. 
P. 123 1.6 1. numeri qui ipsivis 7 . Ib. $\%, 7,6$ et 5 a. c. pro $\left(a^{2}-a\right)^{2}\left(1,\left(a^{2}+a\right)^{2}\right.$. Ib. 1. vlt. pro I 1. - I. P. I24 $l_{1}$ I et 2 pro -1 . t. P. 126 l. Io l. Praemittimus. Ib. $l_{\text {. }}$ I3 pro idem 1. eadem. P. 127 l 3 a. c. $1 . n=h e+f$. P. 1281. vlt. déle (I) et. P. Izol. $z$ et 8 pro $a-\operatorname{rrl} b-b-r$. P. I3 1 II factor primus debet esse $\frac{\pi}{m}$ I. $\quad$ P. I33 1 . vit. pro a 1. b. P $\mathrm{I}_{34}$ in theor. 44 signum superius debet esse t, inf. - . P. I40 l. 8 pro † $A$ 1. $-A$ et pro $-A$ i. $+A$. 146 1. I7 pro $a^{\prime}$. p et pro $p$ l. $a$. P. $\mathrm{I}_{48}$ 1. 3 a. c. $1 .-p R h$ Ib. 1. pen. 1. sequitur. P. I55 1.22 pro primorum 1 priorum. P. I6 I l. I $1 . x x-$ $A$ contentorum impar est, $B$ quoque in forma non diuisorum contentus erit. P. I66 I. 4 pro comperti 1. experti. P. r68 1. 9 pro v l. \&. Ib. 1. vit. pro $\mu m^{\prime \prime}$ 1. $\mu^{\prime} m^{\prime}$. P. $\mathrm{r} 701.4$ pro $x=1 . y=$ P. 174 1. I4 pro $+y^{\prime} 1 .-y^{\prime}$. P. 177 in aequ. 9 ante parenth. excidit factor $D$. : P. 183 fin. in valoribus 2 et 4 ipsarum $x$ et $y$ signa + mutentur in -...'P. 1861.4 pro

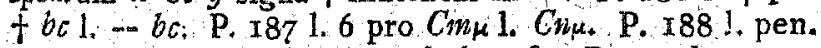
pro 8 1. 7. P. I89 1. Io pro 6 $e^{\prime}$ 1. - - 6'e. P. I92 l. x I pro $\mu m^{\prime}$ 1. $\mu^{\prime} m m^{\prime}$. Ib. 1. 15 1. $\mu^{\prime}, x^{\prime}, m^{\prime}, n^{\prime}$. P. 194 l. $\mathrm{r}_{3}$ pro $u^{\prime} b$ l. $m^{\prime} b$, linea sq. vero pro $m^{\prime} b$ l. $n^{\prime} b$. P. I95 1. 3 a.c. pro $m b$ l. $n b$. Ib. 1. pen. pro $n c$ I. $n b$. P. 204 l. ro pro $\rightarrow 1$ <. P. 205 1. r9 et 20 l: externi. P. 207 l. I pro $a_{0}$ $a^{\prime}, a^{\prime \prime}$ l. a, $a^{\prime \prime}, a^{\prime \prime \prime}$. P. 210 1. 9 pro q 1. u. P. 213 1. 6 post adevque insere haec forma. Ib. I. 7 l. ( $\pm_{2}$ $\pm 1, \pm 2$ ). Tb. 1 . I2 pro I 1. 2. P. $2211.61 .73=25$ +48 P. 2221.2 pro $r D$ l. $r D+B$. Tb. l. Ir pro a i. a. P.223l.2rpro $\angle C$ 1. $\supset$ C. P. 2251.9 pro $B$ 1. b. P. 227 l. 18 pro $a$ 1. $-a_{0}$ P. 2281 . 3 et 2 a. c. pro $\pm 5, \mp 55, \pm 51$. $\mp 5, \pm 15$, T5. P. 232 1. 5 pro $b 1$. $b^{\prime}$ Ib. $L^{\prime} 6$ pro $b+b^{\prime \prime} 1 . b^{\prime \prime}+b^{\prime \prime \prime}$. P. 233 1. 4 forma prima debet esse $\left(1,8,-\right.$ I $\left._{5}\right)$, P. 237

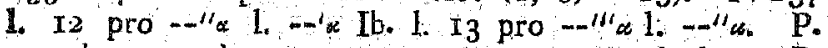
240 1. II verbum contra ponatur post semicolon. $P$. 24I 1. 3 pro $n m$ I. $\mathrm{nm}^{\prime}, \mathrm{P}, 242$ in aequ. 5 pro $a A^{\prime}$ 1. $a^{\prime} A$, in aequ. 6 pro $a^{\prime} A$ l. $a^{\prime} A^{\prime}$ p. 2531.4 pro F 1. 5 . P. 2601 . pen pro $m$ 1. n. P. 26 r 1.8 pro a uu l. aa'un. P. 268 1. I2 et I3 pro hac, illa l. his, âllis Ib. 1. 4. a, c. pro $a^{\prime}$ 1. $a$ Tb. 1, vit pro $a 1$. 
a. P. 263 l. $91 . U$ vero fit $=[2,2,7,2,2]=$ $\frac{\pi}{3}[2,7,2,2,7]$. P. 272 1. 17 pro $u^{\lambda}+1$. $u^{\prime}+$. P. 278 1. II 1 . non primus. Ib. 1 . I4 pro +121 . $-12 \mathrm{Ib}$. 1. 22 pro $-121 .+$ I2. Ib. l. vlt pro $+1,--$. P. 279 1. 5 pro -1 to P. 2801.22 pro $r$ l. 3. P. 2821 . 1. I6 l. $(a, b, c)$. Ib. 1.3 a. c. pro $2 b \alpha_{a j} 1.2 b_{\alpha \gamma \delta}$ et pro $24 \delta 1$, $27 \delta_{*}$ P. 284 1. 3 pro $=4$ 1. +4 . P. 297 1. 7 pro $=1 . r=$ Ib. 1 I2 1 . quos. P. 2991 . I3

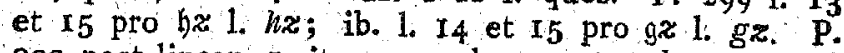
300 post lineam 5 , iterumque 1 . 7. post verbum nimero insere $f(b b-a c c)^{2}-\ldots$. P. $3^{\circ} 71.3$ et bis in 1.5 pro $f$ l. $m f$, P. 3 rg 1. II a. c. pro tum si 1 , si tum.'P. $3^{28} 1$. I7 pro 27 1. 23. P. 3351.8 pro $91 .-9$. P. 347 l. 5 pro $q^{\prime \prime}$ l. $q^{\prime \prime \prime}$. P. 348 l. 5 pro $q^{\prime} q^{\prime}$ l. $p^{\prime} q^{\prime}$ et 1.6 pro $q^{\prime \prime} q^{\prime \prime} 1 . p^{\prime \prime} q^{\prime \prime}$. Ib. $1.20 \mathrm{t} k=$ I. P. 3601 . II, I2 pro $n \mathfrak{R}, n^{\prime \mathfrak{N}}$ I. $n \mathfrak{N}, n^{\prime} \mathfrak{R}$. Ibid, in valore ipsius $(5,8)$ mita - in t. P. $36 \mathrm{r}$ l. 20 pro $p q^{\prime}-q p^{\prime} 1$. $p q^{\prime} \ldots$ qp. P. $3^{63} 1$ 1. 3 pro $\mathfrak{F}$ l. $\mathscr{F}^{2}$. P. $3^{66} \mathrm{l}$. I pro $p^{\prime \prime \prime} 1$, $\mathfrak{p}^{\prime \prime \prime}$.

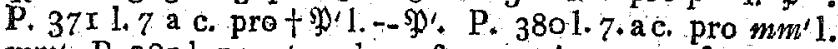
$\mathrm{mm}^{\prime}$. P. 38I l. I post verbum formam insere ex $\mathrm{f}^{\prime}, \mathrm{g}^{\prime} \mathrm{com}-$ positam. P. 3871 . 2o pro quatuor l. tres. P. 3881 1. I 2 pro $W$ 1. W. P. 3891.7 a c. pro $A$ l. $A A$. P. 3901.51 . $B$ est vel o vel $\frac{x}{2} A$, Ibid. 1.9 , Ix et 12 pro $a 1$. $a$ $\frac{q q C}{A}$, et l. Io, 11 et I3 pro $c 1 . c-\frac{q^{\prime \prime} q^{\prime \prime} C}{A}$, P. $3941 \times 6$ pro $2,1.2^{\gamma}$. P. 399 1. 2 pro 869 1. 867 . Ib 1 . I2 post classium insere proprie primitiuarum, P. $406 \mathrm{l}$. II a c. pro expressos 1: expressio. P. 408 I. 9 pro omnium characterem 1. multitudinis omnium characterum. P. 4Io 1 . 4 pro $H^{\prime} 1_{0}+H^{\prime}$. P. $4 \mathrm{r} 51.5$ a c. pro $+2 R p 1.2 R p$. P. 4 I6 1. $x_{3}$ pro $p$ 1. - p. Ibid post l. vlt. omissa sunt haec: $4 n+1$, hoc statim sequitur ex VIII; si vero $q$ est formae: P. 419 l. 8 a c. pro determinantes l. characteres. P. 4201.9 a c. 1 . vel 3 , 4 , vel 3,8 , vel 7,8 . P. 430 1. $1_{3}$ pro substitutionis; $S i$ L. substitutionis $S$; P. 4371,21 past certe insere vel infra. P. 442 in forma $f$ pro 41 . -4 . P. 444 l. II et Io a c. deleantur signa negatiua. P. 453 . 6 pro ah 1. $h$. P. 458 l. I 1 . quae forma adinncta erit ei, quae oritur etc. P. 4631 . I 7 et 18 pro 44 1. 39. P. $47 \times 1.21$ pro semicolo scribe comma P. 4771 . 3 a c. pro - I l. I. P. 4781.9 pro $-171 .+17 . \mathrm{Ib} .1$. 
II pro 9 1. -18 et pro I 1. 2. Ib, 1. vit. pro $p 1 . p p^{\circ}$. P. 479 l. 13, 22 et 23 pro 5 1. -5 . Ib. 1. I4 pro - I7 1. I7. P. 49 I l. 1o a c. 1.6 , aut I2, aut 8. P. 495 l. 3 pro -61 1. 6I. P. 496 I. I3 l. resolubile Ib. 1.5 a c. l. theoremata quae praecedentium. P. 505 l. II post verbum formae omissa sunt haec: $4^{n}+1, q$ numerus primus formae. P. 5 II l. 15 adde: puta pro $x^{\prime \prime}=0$ Ib. 1. vlt. et p. sq. $1.5,8$ et II pro $A^{\prime}$ l. $A^{\prime \prime}$. P. 5 I3 1. 9 pro $d^{\prime \prime} d q$ l. $d^{\prime \prime} p q$. P. 516 I. I4 pro 4 , on 1.4 , o3. Ib. 1. 7 a c. $1.6=2 u g$ t. P. $5 \times 7.23$ pro 7 ro8 I. 7 1ia. P. 5 ig 1. I8 1. 37092 classes, formula dat 37074,3 . P. 542 1. pen pro $m=1 . n=$ P. 5451.8 et 9 pro 591.69 , et pro $1557 \mathrm{~L} 1587$. P. 550 l. potestatibus. P. $55 \mathrm{r}$ 1. 20 I. $\frac{x}{\Gamma} \frac{5}{6}=0,6875$. P. 554 l. 3 a ca prositl. fit. P. $55^{6}$ deleatur comma post sint. $P .5^{6} 3$ l. 9 a c. pro si l. is. P. 568 1. I3 pro zerap 1. zerap". Ib. 1. I6 pro eape 1. eap. Ib. 1. 18 1. $2 \mathrm{ep} \mathrm{p}^{\mu+1+x}$. P. 579 l. 24 pro omnes 1. scilicet omnes. P. 584 1. ro $1 .-2.3 .5 .1$ r.29. P. 5861.16 l. priora snnt eadem. P. 5901.2 pro ro2 1. 408. P. 59 r 1. vit. 1. alii. P. 5991.6 a c. pro 9 1. P; ibid. 1. 5 a c. pro 5 1. Q. P. 607 1. 6 pro $\lambda g$ l. $\lambda h$. P. 608 1. 8. del. $b(f$, k). Ib. 1. 17 pro $d(f, g)$ l. $d(f, I)$. P. $616 I_{\text {. I }}$ I et 4 pro

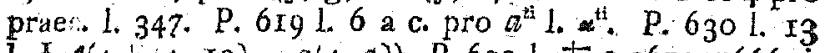
l. $\left.\frac{x}{2} r\left(4+14, x_{3}\right)-2(4,3)\right)$. P. $63^{2} 1 . \pm 0,3612416662 i_{\text {. }}$ P. 638 in valore ipsius $\mathscr{T}$ pro $n=13$ terminis $4 x^{4}$ et $4 x x$ signum t praefigi debet. P. 646 I. 7 a c. post positints insere \&, $6, y$. P. 647 l. 5 a c. pro $R^{\prime \prime} 1 . R^{2}$. P. 653 L. 4 pro $=,[x] L_{+}=[\lambda]$, P. 654 l. 2 numerator pro cot. w esse debet $i(R R+\mathrm{r})$.

Erratorum multitudinem lector beneuolus, qui quam difficile in huiusmodi scriptis a typothetis tali lam bori minus adsuetis auctore absente euitentur nouerit, benigne ignoscet, et si quae alia vel minoris momenti vel sensum non turbantia offenderit, facile corriget. 


$$
\begin{aligned}
& \text { Dnderdio: } \\
& \text { p. } 110.111 \\
& 118.120 .123 \\
& 132 \\
& .49 .16 d \\
& -529
\end{aligned}
$$

"notione neque notatione" p.75.

$1848: p \div 20$

.$A^{2}$ (neque AA) p.10.4. 



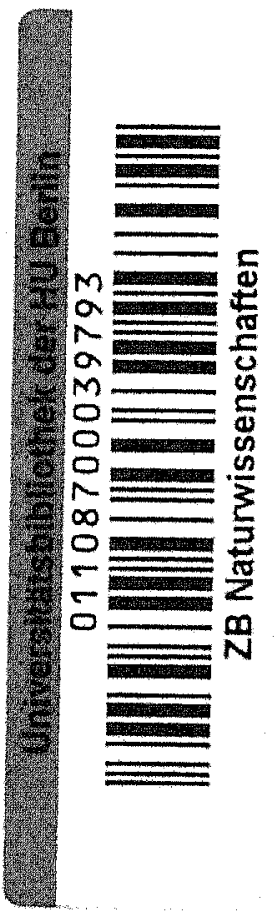





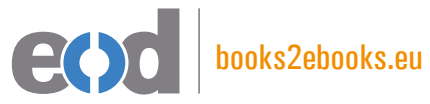

www.books2ebooks.eu 